\title{
MOLECULAR MOBILITY AND OXYGEN PERMEABILITY IN \\ AMORPHOUS $\beta$-LACTOGLOBULIN FILMS
}

by

\section{KASI VISALAKSHI SUNDARESAN}

\author{
A Dissertation submitted to the \\ Graduate School-New Brunswick \\ Rutgers, The State University of New Jersey \\ in partial fulfillment of the requirements \\ for the degree of \\ Doctor of Philosophy \\ Graduate Program in Food Science \\ written under the direction of \\ Dr. Richard D. Ludescher \\ and approved by
}

New Brunswick, New Jersey

January, 2008 


\title{
ABSTRACT OF THE DISSERTATION \\ Molecular Mobility and Oxygen Permeability in Amorphous $\beta$-Lactoglobulin Films \\ by
}

\author{
KASI VISALAKSHI SUNDARESAN \\ Dissertation Director: Dr. Richard D. Ludescher
}

Edible films and coatings are developed to extend the shelf life of food products. Our overall objective is to understand how molecular mobility modulates diffusion rates and thus chemical reactivity in films made from amorphous $\beta$-lactoglobulin. The phosphorescence emission spectra and lifetimes of the triplet probe erythrosin B embedded in the $\beta$-Lg films provide measures of the modes, rates, and distribution of molecular mobility in the film, providing the molecular detail necessary to connect food quality and stability to molecular structure and mobility. The mobility contours generated from this research provided us with information about the onset temperature and level of molecular mobility required to support permeability of atmospheric oxygen. In $\beta-\mathrm{Lg}-$ based binary matrices, sugars (sucrose, trehalose, maltose), plasticizers (glycerol, sorbitol, maltitol and PEG-400), fatty acids (palmitic acid, caprylic acid) and protein (BSA) were selected to investigate how variations in composition influence the molecular mobility and oxygen permeability in amorphous $\beta-\mathrm{Lg}$ matrix. Further more complicated $\beta$ - Lg - based ternary matrices (maltose and maltitol) and (PEG and sucrose) were generated inorder to gain a deeper understanding of edible films. 
In pure $\beta$-Lg films there was linear correlation between molecular mobility and oxygen permeability. Various additives showed different results with respect to mobility and permeability. The addition of sucrose, maltose, maltitol and trehalose greatly reduced the mobility and the permeability of the $\beta$-Lg matrix. Glycerol exhibited an antiplasticization effect and showed decreased mobility at a molar ratio of 1:1 glycerol/ $\beta$-Lg. PEG greatly enhanced the permeability of $\beta$-Lg matrix. Fatty acids palmitic acid and caprylic acid had a rigidification effect on the matrix with no change in permeability. We were able to detect dynamic synergies in $\beta$-Lg maltose and maltitol mixtures, whereby these sugar- polyol mixtures at equal ratios anti-plasticized the $\beta$-Lg matrix and at unequal ratios plasticized the matrix. The tertiary matrix comprising of $\beta$-Lg, PEG 400 and sucrose brought about a substantial reduction in the permeability. A better understanding of the mobility in these complex matrices will help improve the effectiveness of $\beta-\mathrm{Lg}$ in barrier applications in real food systems. 


\section{Acknowledgement}

First and foremost, I want to thank my advisor Dr. Richard Ludescher, for his guidance during my research and study at Rutgers University. His perpetual energy and enthusiasm in research is a motivation to all his students, including me. I am grateful to have him as my advisor; his flexibility and open door policy was tremendously helpful in shaping this thesis into a valuable piece of work.

I would like to thank the members of my committee, Dr. Qingrong Huang, Dr. Paul Takhistov and Dr. Joel Friedman, for their helpful input and guidance.

I would also like to express my appreciation to my labmates Sonali Shirke, Yumin You, Tom Nack, Rashmi Tiwari, Melinda Ligneres, Sanaz Jalalian, Andrew Draganski and Xiang Zhang. I have learned a great deal from all of them. Thanks for making Lab 309, a great and fun place to work.

My deepest gratitude goes to my family, especially my husband, parents-in-law and parents, for their unflagging love and support throughout my life; this dissertation is simply impossible without them. I would like to extend special thanks to my son Saimahesh Srinivasan for his love and for making my life meaningful and pleasurable.

To all my friends I would like thank you for keeping me in your prayers and being my sincere well wishers. 


\section{DEDICATION}

This dissertation is dedicated to my dear husband, my best friend and partner in life for your encouragement, patience and love.

Let the journey continue... 


\section{TABLE OF CONTENTS}

\section{Page}

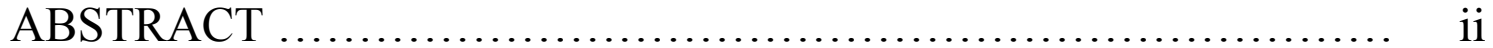

ACKNOWLEDGEMENT

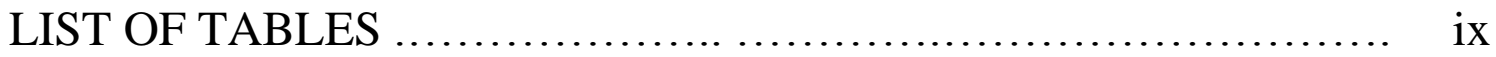

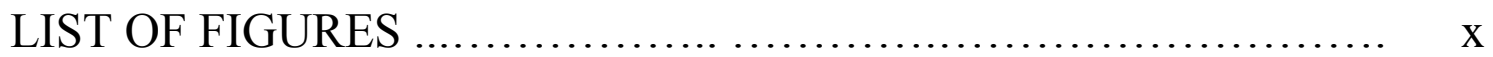

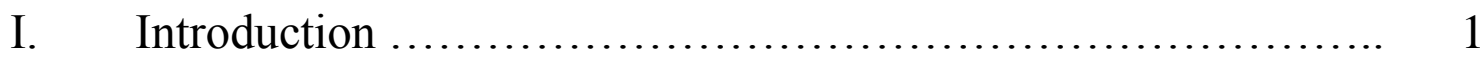

II. Molecular mobility and oxygen permeability in amorphous $\beta$ Lactoglobulin ( $\beta$-Lg) films

a. Introduction ..................................... 36

b. Materials and methods ............................ 39

c. Results ........................................ 44

d. Discussion ....................................... 50

e. Conclusion ........................................ 56

f. References ........................................ 57

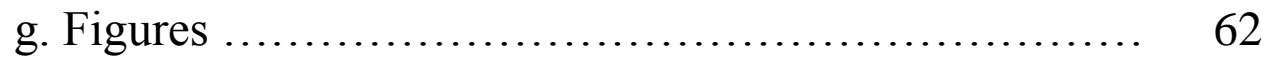

III. Molecular mobility and Oxygen permeability in amorphous $\beta$ Lactoglobulin ( $\beta$-Lg) Films with various sugars

a. Introduction ................................... 70

b. Materials and methods ............................. 73

c. Results ............................................ 81

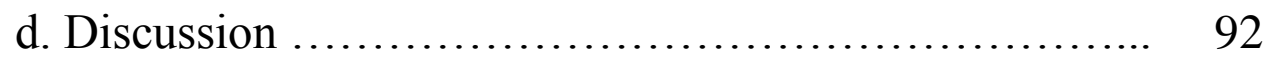

e. Conclusion ...................................... 100

f. References ........................................ 101

g. Figures ........................................ 107 
IV. Dynamic site heterogeneity in amorphous $\beta$-Lactoglobulin ( $\beta$ $\mathrm{Lg})$ and sucrose / $\beta$-Lactoglobulin $(\beta$-Lg) from spectral heterogeneity in Erythrosin B phosphorescence

a. Introduction ..................................... 157

b. Materials and methods ............................ 159

c. Results ....................................... 163

d. Discussion ......................................... 168

e. Conclusion ............................................ 176

f. References ......................................... 178

g. Figures ......................................... 181

V. Molecular Mobility and Oxygen Permeability in amorphous $\beta$ Lactoglobulin Films with various plasticizers

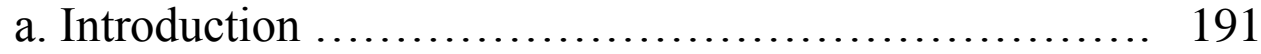

b. Materials and methods ............................. 194

c. Results ...................................... 200

d. Discussion ........................................ 214

e. Conclusion ..................................... 226

f. References ...................................... 227

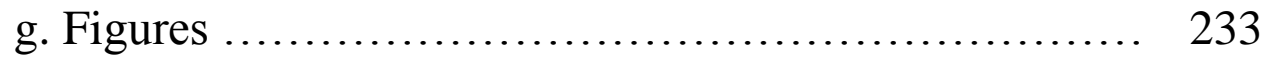

VI. Molecular Mobility and Oxygen Permeability in Composite Films with $\beta$-Lactoglobulin $(\beta$-Lg) /Plasticizer mixtures

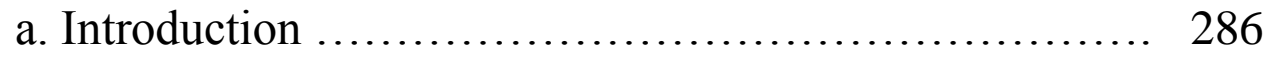

b. Materials and methods ............................ 289

c. Results .......................................... 295

d. Discussion ........................................... 304

e. Conclusion ..................................... 314

f. References ....................................... 315

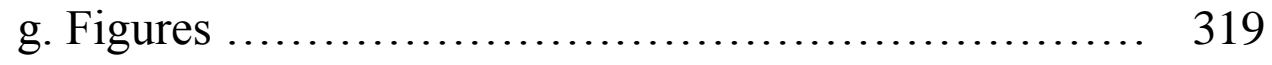


VII. The effect of bovine serum albumin (BSA) on the molecular mobility and oxygen permeability of the amorphous $\beta$-Lactoglobulin $(\beta-\mathrm{Lg})$ matrix.

a. Introduction ....................................... 334

b. Materials and methods ............................ 337

c. Results .......................................... 342

d. Discussion ....................................... 352

e. Conclusion ....................................... 361

f. References ......................................... 363

g. Figures ........................................ 366

VIII. Effect of fatty acids on the molecular mobility and permeability of amorphous $\beta$-Lactoglobulin ( $\beta$-Lg) films

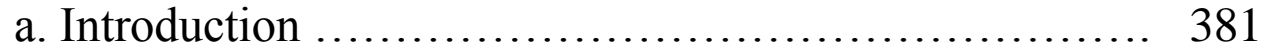

b. Materials and methods ............................... 384

c. Results ........................................ 392

d. Discussion ......................................... 398

e. Conclusion .......................................... 404

f. References .................................... 405

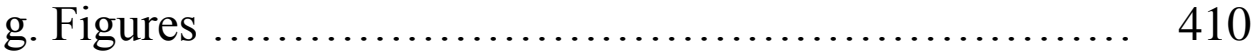

IX. Study to characterize the use of retinol and retinal as phosphorrescent probes in the amorphous $\beta$-lactoglobulin $(\beta-\mathrm{Lg})$ matrix

a. Introduction ....................................... 417

b. Materials and methods ............................... 419

c. Results .......................................... 421

d. References...................................... 428

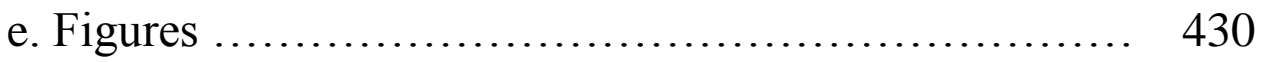

X. Summary conclusion and Future work ..................... 438

CURRICULUM VITA 448 


\section{LIST OF TABLES}

Table I-1: Modes of molecular mobility of proteins in solution..........

Table III-1: The estimated $\mathrm{k}^{\circ}$ TS1 values for erythrosin B in sucrose / $\beta$ Lg films (a) trehalose/ $\beta$-Lg films (b) maltose/ $\beta-\operatorname{Lg}$ (c) films............

Table III-2: Calculated $\Delta \mathrm{E}_{\mathrm{TS}}$, the energy gap between triplet and singlet state in sucrose/ $\beta-\mathrm{Lg}$ (a) trehalose/ $\beta$ - Lg (b) maltose/ $\beta$ - $\mathrm{Lg}$ (c) films

Table III-3: Apparent activation energy $\left(\mathrm{E}_{\mathrm{A}}\right)$ for de-excitation of the triplet state, calculated from the slope of a $\ln \left(\mathrm{k}_{\mathrm{TS} 0}\right)$ versus $1 / \mathrm{T}$ and activation energy $E_{A}$ for oxygen permeability $\mathrm{k}_{\mathrm{Q}}\left[\mathrm{O}_{2}\right]$ in sucrose / $\beta-\mathrm{Lg}$ films(a) trehalose / $\beta$-Lg films (b) maltose/ $\beta$-Lg films(c)..............

Table V-1: The estimated maximum $\mathrm{k}^{\circ}$ TS1 values in Glycerol / $\beta$-Lg (a) so sorbitol / $\beta$-Lg (b) maltitol/ $\beta$-Lg (c) PEG/ $\beta$-Lg (d) films.........

Table V-2: Calculated $\Delta \mathrm{E}_{\mathrm{TS}}$, the energy gap between triplet and singletstate in glycerol/ $\beta$ - Lg (a) sorbitol/ $\beta$-Lg (b) maltitol/ $\beta$ - Lg (c) PEG / $\beta$ $\operatorname{Lg}(d)$ films

Table VII-1: Activation energies of pure $\beta-\mathrm{Lg}, \mathrm{BSA}$ and BSA $\beta-\mathrm{Lg}$, mixtures.

Table IX-1: Instrumental parameters used to characterize the phosphorescent properties of the probes retinol and retinal. Variation of delay time with emission wavelength (a) Variation of gate time with emission wavelength (b) Variation of excitation wavelength with emission wavelength (c) .... 


\section{LIST OF FIGURES}

Figure I-1: Functions of Edible films................................

Figure I-2: Edible film functionality ..............................

Figure I-3: Structure of Bovine $\beta$-Lactoglobulin ..................... 6

Figure I-4: Attributes and molecular mobility ...................... 8

Figure I-5: Macromolecular Dynamic Transitions.................... 11

Figure I-6: Dynamic transition in Proteins............................ 13

Figure I-7: Erythrosin B ....................................... 21

Figure I-8: Jablonski Diagram...................................... 22

Figure II-1: The effect of temperature on the delayed emission spectra of Erythrosin B dispersed in amorphous $\beta-\mathrm{Lg}$ films. Spectra were collected every $10^{\circ} \mathrm{C}$ from -20 to $110^{\circ} \mathrm{C}$ (curves from high to low

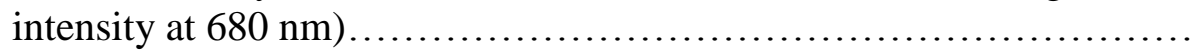

Figure II-2: The effect of temperature on the emission maximum ( $\square$, left hand scale) and bandwidth ( $\boldsymbol{\Lambda}$, right hand scale) for phosphorescence from Erythrosin $\mathrm{B}$ in amorphous $\beta$-Lg films. Parameters were determined from analysis of the delayed emission spectra (as in Figure 1) using a log normal bandwidth function

Figure II-3: The effect of temperature on the phosphorescence emission intensity of Erythrosin B in amorphous $\beta$-Lg films equilibrated against nitrogen ( $\boldsymbol{\bullet})$ and air $(\mathbf{\Lambda})$. Intensity was determined from analysis of the phosphorescence emission band using a log normal bandwidth function.

Figure II-4: Phosphorescence emission intensity decays of Erythrosin B dispersed in $\beta-\operatorname{Lg}$ films equilibrated against nitrogen ( $\square$ ) and air $(\Delta)$ at $20{ }^{\circ} \mathrm{C}$. Solid lines show the results of a fit to the data using a stretched exponential decay function with $\tau=0.557 \mathrm{~ms}$ and $\beta=0.835$ for film in nitrogen and $\tau=0.369 \mathrm{~ms}$ and $\beta=0.810$ for film in air. The bottom graph 
is a plot of the modified residuals ((Data-Fit)/Data1/2) for the fit in nitrogen (solid line) and in air (dotted line)

Figure II-5: The effect of temperature on the lifetime for Erythrosin B dispersed in $\beta$ - Lg films equilibrated against nitrogen ( $\boldsymbol{\square})$ and against air $(\boldsymbol{\Delta})$; the lifetime was determined from analysis using a stretched exponential decay function

Figure II-6: The effect of temperature on the stretching exponent $\beta$ for Erythrosin B dispersed in $\beta$-Lg films equilibrated against nitrogen ( $\boldsymbol{\square}$ ) and against air $(\boldsymbol{\Delta}) ; \beta$ was determined from analysis using a stretched exponential decay function..........................................

Figure II-7: Arrhenius plot of the effect of temperature on the triplet state deexcitation rates for intersystem crossing followed by vibrational relaxation to the ground state $\mathrm{k}_{\mathrm{TS} 0}\left({ }^{*}\right)$ and for oxygen quenching $\mathrm{k}_{\mathrm{Q}}\left[\mathrm{O}_{2}\right]$ $(\Delta)$

Figure II-8: Dependence of the oxygen quenching rate $\mathrm{k}_{\mathrm{Q}}\left[\mathrm{O}_{2}\right]$ on the

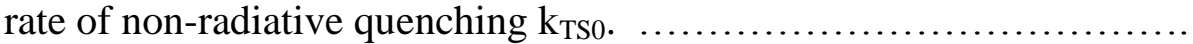

Figure III-1a: The effect of temperature on the delayed emission spectra of Erythrosin B dispersed in amorphous sucrose / $\beta$-Lg films (sucrose: $\beta-\operatorname{Lg} 10: 1)$

Figure III-1b: The effect of temperature on the delayed emission spectra of Erythrosin B dispersed in amorphous trehalose/ $\beta-\mathrm{Lg}$ films (trehalose: $\beta$-Lg 10:1).

Figure III-1c: The effect of temperature on the delayed emission spectra of Erythrosin B dispersed in amorphous Maltose/ $\beta$-Lg films. (Maltose: $\beta-\operatorname{Lg} 10: 1)$

Figure III-2a: The effect of temperature on the emission maximum for phosphorescence from Erythrosin B in amorphous sucrose / $\beta-\mathrm{Lg}$ films

Figure III-2b: The effect of temperature on the emission maximum for phosphorescence from Erythrosin B in amorphous trehalose/ $\beta$-Lg films.

Figure III-2c: The effect of temperature on the emission maximum for phosphorescence from Erythrosin B in amorphous maltose / $\beta$-Lg films.

Figure III-3a: The effect of temperature on the bandwidth for phosphorescence from Erythrosin B in amorphous sucrose $/ \beta$-Lg films... 
Figure III-3b: The effect of temperature on the bandwidth for phosphorescence from Erythrosin $\mathrm{B}$ in amorphous trehalose/ $\beta-\mathrm{Lg}$ films

Figure III-3c: The effect of temperature on the bandwidth for phosphorescence from Erythrosin B in amorphous maltose / $\beta$-Lg films.

Figure III-4a,b,c,d,e,f: The effect of temperature on the lifetime for Erythrosin $\mathrm{B}$ dispersed in $\beta$ - $\mathrm{Lg}$ in various molar ratios of sucrose equilibrated against nitrogen $(\diamond)$ and against air $(\mathbf{\square})$; the lifetime was determined from analysis using a stretched exponential decay function. The text box in the graph refers to the molar ratio of sucrose: $\beta-\operatorname{Lg} . \ldots \ldots$.

Figure III-4g: Comparison plot depicting the effect of temperature on the lifetime for Erythrosin B dispersed in $\beta-\mathrm{Lg}$ in various molar concentrations of sucrose equilibrated against nitrogen.................

Figure III-4h: Comparison plot depicting the effect of temperature on the lifetime for Erythrosin B dispersed in $\beta-\mathrm{Lg}$ in various molar concentrations of sucrose equilibrated against air

Figure III-5a, b, c, d, e, f, g: The effect of temperature on the lifetime for Erythrosin $\mathrm{B}$ dispersed in $\beta$ - Lg in various molar concentrations of trehalose equilibrated against nitrogen and against air. The lifetime was determined from analysis using a stretched exponential decay function. The text box in the graph refers to the molar ratio of trehalose: $\beta$ -

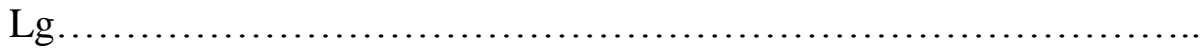

Figure III-5h: Comparison plot depicting the effect of temperature on the lifetime for Erythrosin B dispersed in $\beta-\mathrm{Lg}$ in various molar concentrations of trehalose equilibrated against nitrogen..............

Figure III-5i: Comparison plot depicting the effect of temperature on the lifetime for Erythrosin B dispersed in $\beta-\mathrm{Lg}$ in various molar concentrations of trehalose equilibrated against oxygen...............

Figure III-6a, b, c, d, e: The effect of temperature on the lifetime for Erythrosin $\mathrm{B}$ dispersed in $\beta-\mathrm{Lg}$ in various molar concentrations of maltose equilibrated against nitrogen and air. The lifetime was determined from analysis using a stretched exponential decay function. The text box in the graph refers to the molar ratio of maltose: $\beta-\operatorname{Lg} \ldots \ldots$.

Figure III-6f: Comparison plot depicting the effect of temperature on the lifetime for Erythrosin B dispersed in $\beta-\mathrm{Lg}$ in various molar 
concentrations of maltose equilibrated against nitrogen.

Figure III-6g: Comparison plot depicting the effect of temperature on the lifetime for Erythrosin B dispersed in $\beta-\mathrm{Lg}$ in various molar concentrations of maltose equilibrated against air....

Figure III-7a: The effect of temperature on the stretching exponent $\beta$ for Erythrosin B dispersed in sucrose $\beta$ - Lg films equilibrated against nitrogen. $\beta$ was determined from analysis using a stretched exponential decay function

Figure III-7b: The effect of temperature on the stretching exponent $\beta$ for Erythrosin B dispersed in sucrose $\beta$ - $\mathrm{Lg}$ films equilibrated against air...

Figure III-8a: The effect of temperature on the stretching exponent $\beta$ for Erythrosin B dispersed in trehalose $/ \beta$ - $L$ g trehalose films equilibrated against nitrogen

Figure III-8b: The effect of temperature on the stretching exponent $\beta$ for Erythrosin $\mathrm{B}$ dispersed in trehalose $/ \beta-\mathrm{Lg}$ films equilibrated against air

Figure III-9a: The effect of temperature on the stretching exponent $\beta$ for Erythrosin B dispersed in maltose $/ \beta-\mathrm{Lg}$ films equilibrated against nitrogen.........................................................

Figure III-9b: The effect of temperature on the stretching exponent $\beta$ for Erythrosin $\mathrm{B}$ dispersed in maltose $\beta$ - $\mathrm{Lg}$ films equilibrated against nitrogen

Figure III-10a: The oxygen quenching rate $\mathrm{k}_{\mathrm{Q}}\left[\mathrm{O}_{2}\right]$ as a function of temperature in the $\beta$ - $\mathrm{Lg}$ and sucrose/ $\beta-\mathrm{Lg}$ films

Figure III-10b: The oxygen quenching rate $\mathrm{k}_{\mathrm{Q}}\left[\mathrm{O}_{2}\right]$ as a function of temperature in the $\beta$ - $\mathrm{Lg}$ and trehalose/ $\beta$ - $\mathrm{Lg}$ films

Figure III-10c: The oxygen quenching rate $\mathrm{k}_{\mathrm{Q}}\left[\mathrm{O}_{2}\right]$ as a function of temperature in the $\beta$-Lg and maltose/ $\beta$-Lg mixtures

Figure III-11a: Plot of the effect of temperature on non-radiative decay from $\mathrm{T} 1$ to $\mathrm{S} 0\left(\mathrm{k}_{\mathrm{TS} 0}\right)$ in amorphous sucrose $/ \beta$-lg films

Figure III-11b: Plot of the effect of temperature on non-radiative decay 
from $\mathrm{T} 1$ to $\mathrm{S} 0\left(\mathrm{k}_{\mathrm{TS} 0}\right)$ in amorphous trehalose/ $\beta-\mathrm{Lg}$ films

Figure III-11c: Plot of the effect of temperature on non-radiative decay from $\mathrm{T} 1$ to $\mathrm{S} 0\left(\mathrm{k}_{\mathrm{TS} 0}\right)$ in amorphous maltose / $\beta$ - $\lg$ maltose films........

Figure III-12a: Emission energy as a function of concentration of sucrose at various temperatures in $\beta$-lg films. Data from Figure III-2a replotted versus molar ratio.

Figure III-12b: Zoom in effect of emission energy as a function of concentration of sucrose at 0,20 and $40{ }^{\circ} \mathrm{C}$ Data from Figure III-2a replotted versus molar ratio.

Figure III-13a: A plot of the effect of change in concentration of trehalose in emission energy at various temperatures. Data from Figure III-2b replotted versus molar ratio

Figure III-13b: Zoom in effect of the change in concentration of trehalose in emission energy at 0,20 and $40^{\circ} \mathrm{C}$. Data from Figure III$2 \mathrm{~b}$ replotted versus molar ratio.

Figure III-14a: A plot of the effect of change in concentration of maltose in emission energy at various temperatures. Data replotted from Figure III-2c versus molar ratio...

Figure III-14b: Zoom in effect of the change in concentration of maltose in emission energy at 0,20 and $40^{\circ} \mathrm{C}$. Data replotted from Figure III$2 \mathrm{c}$ versus molar ratio

Figure III-15a: Effect of change in concentration of sucrose in $\mathrm{k}_{\mathrm{Q}}\left[\mathrm{O}_{2}\right]$ at various temperatures. Data replotted from Figure III-10a versus molar ratio

Figure III-15b: Zoom in effect of the change in concentration of sucrose in $\mathrm{k}_{\mathrm{Q}}\left[\mathrm{O}_{2}\right]$ at 0,20 and $40{ }^{\circ} \mathrm{C}$.. Data replotted from Figure III-10a versus molar ratio

Figure III-16a: $\mathrm{k}_{\mathrm{Q}}\left[\mathrm{O}_{2}\right]$ as a function of concentration of trehalose at various temperatures. Data repotted from Figure III-10b.

Figure III-16b: Zoom in effect of the change in concentration of trehalose in $\mathrm{k}_{\mathrm{Q}}\left[\mathrm{O}_{2}\right]$ at 0,20 and $40^{\circ} \mathrm{C}$. Data repotted from Figure III$10 \mathrm{~b}$ 
Figure III-17a: A plot of the effect of change in concentration of maltose in $\mathrm{k}_{\mathrm{Q}} \mathrm{O}_{2}$ at various temperatures Data replotted from Figure $10 \mathrm{c}$

Figure III-17b: Zoom in effect of the change in concentration of maltose in $\mathrm{k}_{\mathrm{Q}}\left[\mathrm{O}_{2}\right]$ at 0,20 and $40{ }^{\circ} \mathrm{C}$ Data repotted from Figure III-10c

Figure III-18a: Effect of change in concentration of sucrose in $\mathrm{k}_{\mathrm{TS} 0}$ at various temperatures. Data replotted from Figure III-11a ..............

Figure III-18b: Zoom in effect of change in concentration of sucrose in $\mathrm{k}_{\mathrm{TS} 0}$ at 0,20 and $40{ }^{\circ} \mathrm{C}$. Data replotted from Figure III-11a

Figure III-19a: A plot of the effect of change in concentration of trehalose in $\mathrm{k}_{\mathrm{TS} 0}$ at various temperatures. Data replotted from Figure III$11 \mathrm{~b}$

Figure III-19b: Zoom in effect of the change in concentration of trehalose in $\mathrm{k}_{\mathrm{TS} 0}$ at 0,20 and $40^{\circ} \mathrm{C}$. Data replotted from Figure III- $11 \mathrm{~b}$.

Figure III-20a: A plot of the effect of change in concentration of maltose in $\mathrm{k}_{\mathrm{TS} 0}$ at various temperatures. Data replotted from Figure 11c.

Figure III-20b: Zoom in effect of the change in concentration of maltose in $\mathrm{k}_{\mathrm{TS} 0}$ at 0,20 and $40{ }^{\circ} \mathrm{C}$ Data replotted from Figure $11 \mathrm{c} \ldots \ldots \ldots \ldots$

Figure III-21a: Dependence of the oxygen quenching rate $\mathrm{k}_{\mathrm{Q}}\left[\mathrm{O}_{2}\right]$ on the rate of non-radiative quenching $\mathrm{k}_{\mathrm{TS} 0}$ in sucrose $\beta$ - $\mathrm{Lg}$ films. The number

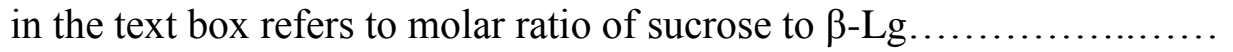

Figure III-21b: Dependence of the oxygen quenching rate $\mathrm{k}_{\mathrm{Q}}\left[\mathrm{O}_{2}\right]$ on the rate of non-radiative quenching $\mathrm{k}_{\mathrm{Ts} 0}$ in sucrose $\beta$ - $\mathrm{Lg}$ films. The number in the text box refers to molar ratio of sucrose to $\beta-\mathrm{Lg}$.

Figure III-21c: Dependence of the oxygen quenching rate $\mathrm{k}_{\mathrm{Q}}\left[\mathrm{O}_{2}\right]$ on the rate of non-radiative quenching $\mathrm{k}_{\mathrm{Ts} 0}$ in sucrose $\beta$ - $\mathrm{Lg}$ films. The number in the text box refers to molar ratio of sucrose to $\beta-\mathrm{Lg} \ldots \ldots \ldots \ldots \ldots \ldots \ldots$

Figure III-21d: Dependence of the oxygen quenching rate $\mathrm{k}_{\mathrm{Q}}\left[\mathrm{O}_{2}\right]$ on the rate of non-radiative quenching $\mathrm{k}_{\mathrm{TS} 0}$ in sucrose $\beta$ - $\mathrm{Lg}$ films. The number

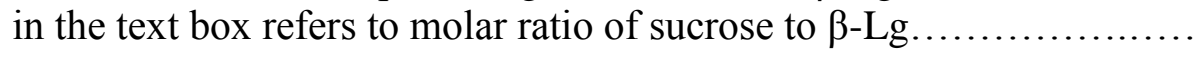

Figure III-21e: Dependence of the oxygen quenching rate $\mathrm{k}_{\mathrm{Q}}\left[\mathrm{O}_{2}\right]$ on the rate of non-radiative quenching $\mathrm{k}_{\mathrm{TS} 0}$ in sucrose $\beta-\mathrm{Lg}$ films. The number in the text box refers to molar ratio of sucrose to $\beta-\mathrm{Lg}$. 
Figure III-21f: Dependence of the oxygen quenching rate $\mathrm{k}_{\mathrm{Q}}\left[\mathrm{O}_{2}\right]$ on the rate of non-radiative quenching $\mathrm{k}_{\mathrm{TS} 0}$ in sucrose $\beta$-Lg films. The number

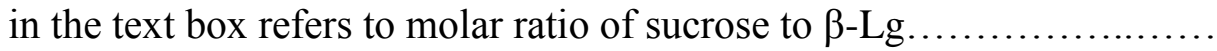

Figure III-22a: Dependence of the oxygen quenching rate $\mathrm{k}_{\mathrm{Q}}\left[\mathrm{O}_{2}\right]$ on the rate of non-radiative quenching $\mathrm{k}_{\mathrm{TS} 0}$ in $\mathrm{t}$ rehalose $\beta-\mathrm{Lg}$ films. The number in the text box refers to molar ratio of trehalose to $\beta$-Lg.........

Figure III-22b: Dependence of the oxygen quenching rate $\mathrm{k}_{\mathrm{Q}}\left[\mathrm{O}_{2}\right]$ on the rate of non-radiative quenching $\mathrm{k}_{\mathrm{TS} 0}$ in trehalose $\beta$ - $\mathrm{Lg}$ films. The number in the text box refers to molar ratio of trehalose to $\beta-\mathrm{Lg} . . . . . . .$.

Figure III-22c: Dependence of the oxygen quenching rate $\mathrm{k}_{\mathrm{Q}}\left[\mathrm{O}_{2}\right]$ on the rate of non-radiative quenching $\mathrm{k}_{\mathrm{TS} 0}$ in trehalose $\beta$ - $\mathrm{Lg}$ films. The number in the text box refers to molar ratio of trehalose to $\beta-\mathrm{Lg} . . . \ldots \ldots$.

Figure III-22d: Dependence of the oxygen quenching rate $\mathrm{k}_{\mathrm{Q}}\left[\mathrm{O}_{2}\right]$ on the rate of non-radiative quenching $\mathrm{k}_{\mathrm{TS} 0}$ in trehalose $\beta$ - Lg films. The number in the text box refers to molar ratio of trehalose to $\beta-\mathrm{Lg} . . . \ldots \ldots$.

Figure III-22e: Dependence of the oxygen quenching rate $\mathrm{k}_{\mathrm{Q}}\left[\mathrm{O}_{2}\right]$ on the rate of non-radiative quenching $\mathrm{k}_{\mathrm{TS} 0}$ in trehalose $/ \beta-\mathrm{Lg}$ films. The number in the text box refers to molar ratio of trehalose to $\beta-\mathrm{Lg} . . . \ldots \ldots$.

Figure III-22f: Dependence of the oxygen quenching rate $\mathrm{k}_{\mathrm{Q}}\left[\mathrm{O}_{2}\right]$ on the rate of non-radiative quenching $\mathrm{k}_{\mathrm{TS} 0}$ in trehalose/ $\beta-\mathrm{Lg}$ films. The number in the text box refers to molar ratio of trehalose to $\beta-\operatorname{Lg} \ldots \ldots \ldots \ldots$

Figure III-22g: Dependence of the oxygen quenching rate $\mathrm{k}_{\mathrm{Q}}\left[\mathrm{O}_{2}\right]$ on the rate of non-radiative quenching $\mathrm{k}_{\mathrm{TS} 0}$ in trehalose $/ \beta-\mathrm{Lg}$ films. The number in the text box refers to molar ratio of trehalose to $\beta-\mathrm{Lg} . . . \ldots \ldots$.

Figure III-23a: Dependence of the oxygen quenching rate $\mathrm{k}_{\mathrm{Q}}\left[\mathrm{O}_{2}\right]$ on the rate of non-radiative quenching $\mathrm{k}_{\mathrm{TS} 0}$ in maltose $\beta$-Lg films. The number in the text box refers to molar ratio of maltose to $\beta$-Lg.................

Figure III-23b: Dependence of the oxygen quenching rate $\mathrm{k}_{\mathrm{Q}}\left[\mathrm{O}_{2}\right]$ on the rate of non-radiative quenching $\mathrm{k}_{\mathrm{TS} 0}$ in maltose $/ \beta$ - Lg films. The number

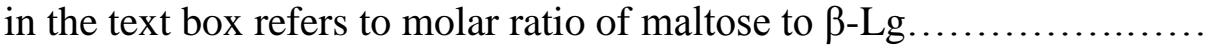

Figure III-23c: Dependence of the oxygen quenching rate $\mathrm{k}_{\mathrm{Q}}\left[\mathrm{O}_{2}\right]$ on the rate of non-radiative quenching $\mathrm{k}_{\mathrm{TS} 0}$ in maltose $/ \beta$ - $\mathrm{Lg}$ films. The number in the text box refers to molar ratio of maltose to $\beta$ - $\mathrm{Lg} . . . \ldots \ldots \ldots \ldots \ldots$ 
Figure III-23d: Dependence of the oxygen quenching rate $\mathrm{k}_{\mathrm{Q}}\left[\mathrm{O}_{2}\right]$ on the rate of non-radiative quenching $\mathrm{k}_{\mathrm{TS} 0}$ in $\beta$ - $\mathrm{Lg}$ maltose films. The number

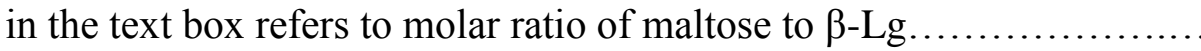

Figure III-23e: Dependence of the oxygen quenching rate $\mathrm{k}_{\mathrm{Q}}\left[\mathrm{O}_{2}\right]$ on the rate of non-radiative quenching $\mathrm{k}_{\mathrm{TS} 0}$ in maltose/ $\beta-\mathrm{Lg}$ films. The number in the text box refers to molar ratio of maltose to $\beta-\mathrm{Lg} . . . \ldots \ldots$.

Figure IV-1: Phosphorescence emission spectra for Erythrosin B in amorphous $\beta-\mathrm{Lg}$ films collected as a function of delay time in ms........

Figure IV-2: Evolution of phosphorescence emission peak frequency with time following excitation in amorphous $\beta-\mathrm{Lg}$ film .................

Figure IV-3: Evolution of phosphorescence emission peak frequency with time following excitation in amorphous sucrose / $\beta$ - $\mathrm{Lg}$ film (Ratio of $10: 1$

Figure IV-4: Evolution of phosphorescence emission bandwidth with time following excitation in amorphous $\beta$ - Lg films

Figure IV-5: Evolution of phosphorescence emission bandwidth with time in amorphous sucrose / $\beta$ - Lg films (Ratio 10:1) ....

Figure IV-6: Effect of emission wavelength on the Erythrosin B phosphorescence lifetimes in amorphous $\beta$-Lg films determined from analysis using a stretched exponential model.

Figure IV-7: Effect of emission wavelength on the Erythrosin B phosphorescence lifetimes in amorphous sucrose / $\beta$ - Lg film determined from analysis using a stretched exponential model

Figure IV-8: Effect of emission wavelength on the stretching exponent $(\beta)$ characterizing the intensity decay transients of Erythrosin B phosphorescence in amorphous $\beta$ - $\mathrm{Lg}$ films determined from analysis using a stretched exponential model...................................

Figure IV-9: Effect of emission wavelength on the stretching exponent $(\beta)$ characterizing the intensity decay transients of Erythrosin B phosphorescence in amorphous sucrose $\beta$ - Lg film determined from analysis using a stretched exponential model.

Figure IV-10: The rate constant for non-radiative decay of the triplet state to S0 $\left(\mathrm{k}_{\mathrm{TS} 0}\right)$ plotted as a function of emission wavelength from Erythrosin B in $\beta$-Lg film. 
Figure IV-11: The rate constant for non- radiative decay of the triplet state to $\mathrm{S} 0\left(\mathrm{k}_{\mathrm{TS} 0}\right)$ plotted as a function of emission wavelength from Erythrosin B in sucrose/ $\beta$-Lg films

Figure IV-12: Apparent activation energy Ea for non- radiative decay rate of Erythrosin B in amorphous $\beta-\mathrm{Lg}$ films as a function of emission wavelength (calculated from an Arrhenius analysis of the data from figure 10 using the three lowest and highest temperatures respectively)..

Figure IV-13: Apparent activation energy Ea for non- radiative decay rate of Erythrosin B in amorphous sucrose/ $\beta-\mathrm{Lg}$ films as a function of emission wavelength (calculated from an Arrhenius analysis of the data from Figure 11).

Figure IV-14: Comparative plot of lifetime as a function of emission wavelength following excitation in amorphous $\beta-\operatorname{Lg}$ and $\beta$-Lg sucrose films at $20^{\circ} \mathrm{C}$ and $65^{\circ} \mathrm{C}$

Figure IV-15: Comparative plot of $\beta$ as a function of emission wavelength following excitation in amorphous $\beta-\mathrm{Lg}$ and $\beta-\mathrm{Lg}$ sucrose films at $20^{\circ} \mathrm{C}$ and $65^{\circ} \mathrm{C}$

Figure IV-16: Comparative plot of peak frequency with delay time following excitation in amorphous $\beta-\operatorname{Lg}$ and $\beta-\mathrm{Lg}$ sucrose films at $25^{\circ} \mathrm{C}$

Figure IV-17: Comparative plot of FWHM with delay time following excitation in amorphous $\beta-\mathrm{Lg}$ and $\beta-\mathrm{Lg}$ sucrose films at $25^{\circ} \mathrm{C}$

Figure V-1a: The effect of temperature on the delayed emission spectra of Erythrosin B dispersed in amorphous $\beta-\mathrm{Lg}-$ glycerol films (Glycerol: $\beta$-Lg 10:1)

Figure V-1b: The effect of temperature on the delayed emission spectra of Erythrosin B dispersed in amorphous $\beta-\mathrm{Lg}-$ sorbitol films

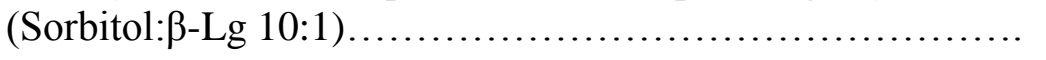

Figure V-1c: The effect of temperature on the delayed emission spectra of Erythrosin B dispersed in amorphous $\beta-\mathrm{Lg}-$ Maltitol films

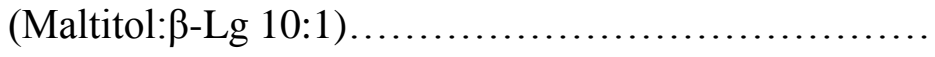

Figure V-1d: The effect of temperature on the delayed emission spectra of Erythrosin B dispersed in amorphous $\beta$ - Lg - PEG films (PEG 400: $\beta$ $\operatorname{Lg} 10: 1) \ldots \ldots \ldots \ldots \ldots \ldots \ldots \ldots \ldots \ldots \ldots \ldots$. 
Figure V-2a: The effect of temperature on the emission maximum for phosphorescence from Erythrosin B in amorphous glycerol / $\beta$-Lg films.

Figure V-2b: The effect of temperature on the emission maximum for phosphorescence from Erythrosin B in amorphous sorbitol/ $\beta$-Lg films...

Figure V-2c: The effect of temperature on the emission maximum for phosphorescence from Erythrosin B in amorphous maltitol/ $\beta-\mathrm{Lg}$ films ............................................................

FigureV-2d: The effect of temperature on the emission maximum for phosphorescence from Erythrosin B in amorphous PEG $400 / \beta-\mathrm{Lg}$

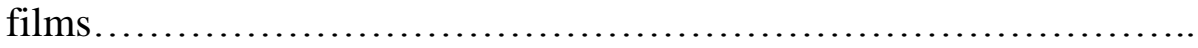

Figure V-3a: The effect of temperature on the bandwidth for phosphorescence from Erythrosin B in amorphous glycerol/ $\beta$-Lg films.

Figure V-3b: The effect of temperature on the bandwidth for phosphorescence from Erythrosin B in amorphous sorbitol $/ \beta$-Lg films...

Figure V-3c: The effect of temperature on the bandwidth for phosphorescence from Erythrosin B in amorphous maltitol/ $\beta$-Lg films...

Figure V-3d: The effect of temperature on the bandwidth for phosphorescence from Erythrosin B in amorphous PEG / $\beta$-Lg films.....

Figure V-4a,b,c,d,e: The effect of temperature on the lifetime for Erythrosin $\mathrm{B}$ dispersed in $\beta-\mathrm{Lg}$ in various molar concentrations of glycerol equilibrated against nitrogen $(\bullet)$ and against air $(\mathbf{\square})$; the lifetime was determined from analysis using a stretched exponential decay function. The text box in the graph refers to the molar ratio of

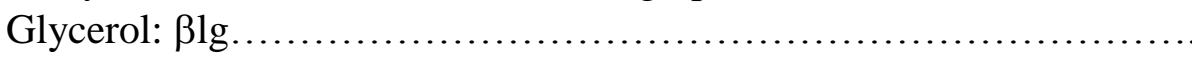

Figure V-4f: Comparison plot depicting the effect of temperature on the lifetime for Erythrosin B dispersed in $\beta-\mathrm{Lg}$ in various molar concentrations of glycerol equilibrated against nitrogen..................

Figure V-4g: Comparison plot depicting the effect of temperature on the lifetime for Erythrosin B dispersed in $\beta-\mathrm{Lg}$ in various molar concentrations of glycerol equilibrated against oxygen..................

Figure V-5a,b,c,d,e: The effect of temperature on the lifetime for Erythrosin $\mathrm{B}$ dispersed in $\beta$ - $\mathrm{Lg}$ in various molar concentrations of sorbitol equilibrated against nitrogen $(\bullet)$ and against air ( $(\mathbf{)}$; the 
lifetime was determined from analysis using a stretched exponential decay function. The text box in the graph refers to the molar ratio of Sorbitol: $\beta$-Lg.

Figure V-5f: Comparison plot depicting the effect of temperature on the lifetime for Erythrosin B dispersed in $\beta$-Lg in various molar concentrations of sorbitol equilibrated against nitrogen.................

Figure V-5g: Comparison plot depicting the effect of temperature on the lifetime for Erythrosin B dispersed in $\beta-\mathrm{Lg}$ in various molar concentrations of sorbitol equilibrated against oxygen.................

Figure V-6a,b,c,d,e: The effect of temperature on the lifetime for Erythrosin $\mathrm{B}$ dispersed in $\beta-\mathrm{Lg}$ in various molar concentrations of maltitol equilibrated against nitrogen $(\bullet)$ and against air $(\boldsymbol{\square})$; the lifetime was determined from analysis using a stretched exponential decay function. The text box in the graph refers to the molar ratio of Maltitol: $\beta$-Lg.................................................

Figure V-6f: Comparison plot depicting the effect of temperature on the lifetime for Erythrosin B dispersed in $\beta-\mathrm{Lg}$ in various molar concentrations of maltitol equilibrated against nitrogen................

Figure V-6g: Comparison plot depicting the effect of temperature on the lifetime for Erythrosin B dispersed in $\beta-\mathrm{Lg}$ in various molar concentrations of maltitol equilibrated against oxygen.................

Figure V-7a,b,c: The effect of temperature on the lifetime for Erythrosin $B$ dispersed in $\beta-\mathrm{Lg}$ in various molar concentrations of PEG 400 equilibrated against nitrogen $(\bullet)$ and against air $(\mathbf{\square})$; the lifetime was determined from analysis using a stretched exponential decay function. The text box in the graph refers to the molar ratio of PEG 400: $\beta$ $\mathrm{Lg}$

Figure V-7d: Comparison plot depicting the effect of temperature on the lifetime for Erythrosin B dispersed in $\beta$ - Lg in various molar concentrations of PEG 400 equilibrated against oxygen................

Figure V-7e: Comparison plot depicting the effect of temperature on the lifetime for Erythrosin B dispersed in $\beta-\mathrm{Lg}$ in various molar concentrations of PEG 400 equilibrated against oxygen.................

Figure V-8a: Beta as a function of temperature in glycerol $/ \beta$-Lg films equilibrated against nitrogen 
Figure V-8b: Beta as a function of temperature in $\beta-\mathrm{Lg} /$ glycerol films equilibrated against oxygen....

Figure V-9a: Beta as a function of temperature in sorbitol $/ \beta-\mathrm{Lg}$ films equilibrated against nitrogen

Figure V-9b: Beta as a function of temperature in sorbitol $/ \beta$-Lg films equilibrated against oxygen.

Figure V -10a: Beta as a function of temperature in maltitol / $\beta$-Lg films equilibrated against nitrogen

Figure V-10b: Beta as a function of temperature in maltitol $/ \beta$-Lg films equilibrated against oxygen......................................

Figure V-11a: Beta as a function of temperature in PEG / $\beta-\mathrm{Lg}$ films equilibrated against nitrogen........................................

Figure V-11b: Beta as a function of temperature in PEG / $\beta$-Lg films equilibrated against oxygen........................................

Figure V-12a: The oxygen quenching rate $\mathrm{k}_{\mathrm{Q}}\left[\mathrm{O}_{2}\right]$ as a function of temperature in glycerol/ $\beta$-Lg mixtures

Figure V-12b: The oxygen quenching rate $\mathrm{k}_{\mathrm{Q}}\left[\mathrm{O}_{2}\right]$ as a function of temperature in the $\beta$ - $\mathrm{Lg} /$ sorbitol mixtures.

FigureV-12c: The oxygen quenching rate $\mathrm{k}_{\mathrm{Q}}\left[\mathrm{O}_{2}\right]$ as a function of temperature in the $\beta$ - $\mathrm{Lg}$ and $\beta$ - $\mathrm{Lg}$ maltitol mixtures

Figure V-12d: The oxygen quenching rate $\mathrm{k}_{\mathrm{Q}}\left[\mathrm{O}_{2}\right]$ as a function of temperature in the $\beta$ - $\mathrm{Lg}$ and $\mathrm{PEG} / \beta$-Lg mixtures....................

Figure V-13a: Plot of the effect of temperature on non-radiative decay from $\mathrm{T} 1$ to $\mathrm{S} 0\left(\mathrm{k}_{\mathrm{TS} 0}\right)$ in amorphous glycerol $/ \beta$-Lg films

Figure V-13b: Plot of the effect of temperature on non-radiative decay from $\mathrm{T} 1$ to $\mathrm{S} 0\left(\mathrm{k}_{\mathrm{TS} 0}\right)$ in amorphous sorbitol/ $\beta$ - $\mathrm{Lg}$ films

Figure V-13c: Plot of the effect of temperature on non-radiative decay from $\mathrm{T} 1$ to $\mathrm{S} 0\left(\mathrm{k}_{\mathrm{TS} 0}\right)$ in amorphous maltitol/ $\beta-\mathrm{Lg}$ films

Figure V-13d: Plot of the effect of temperature on non-radiative decay from $\mathrm{T} 1$ to $\mathrm{S} 0\left(\mathrm{k}_{\mathrm{TS} 0}\right)$ in amorphous $\beta$-Lg PEG 400 films. ... 
Figure V-14a,b,c,d: Dependence of the oxygen quenching rate $\mathrm{k}_{\mathrm{Q}}\left[\mathrm{O}_{2}\right]$ on the rate of non-radiative quenching $\mathrm{k}_{\mathrm{TS} 0}$ in glycerol $/ \beta-\mathrm{Lg}$ films. The number in the text box refers to molar ratio of glycerol to $\beta$ Lg.

Figure V-15a,b,c,d,e: Dependence of the oxygen quenching rate $\mathrm{k}_{\mathrm{Q}}\left[\mathrm{O}_{2}\right]$ on the rate of non-radiative quenching $\mathrm{k}_{\mathrm{TS} 0}$ in sorbitol/ $\beta$-Lg films. The number in the text box refers to molar ratio of sorbitol to $\beta$ $\operatorname{Lg}$

Figure V-16a,b,c,d,e: Dependence of the oxygen quenching rate $\mathrm{k}_{\mathrm{Q}}\left[\mathrm{O}_{2}\right]$ on the rate of non-radiative quenching $\mathrm{k}_{\mathrm{TS} 0}$ in maltitol/ $\beta$-Lg films. The number in the text box refers to molar ratio of maltitol to $\beta$ $\operatorname{Lg}$

Figure V-17a,b,c: Dependence of the oxygen quenching rate $\mathrm{k}_{\mathrm{Q}}\left[\mathrm{O}_{2}\right]$ on the rate of non-radiative quenching $\mathrm{k}_{\mathrm{TS} 0}$ in $\mathrm{PEG} / \beta-\mathrm{Lg}$ films. The number in the text box refers to molar ratio of PEG 400 to $\beta$-Lg..........

Figure V-18a: Emission energy as a function of concentration of glycerol at various temperatures in $\beta$ - Lg films. Data replotted from

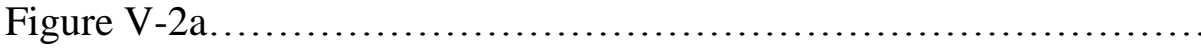

FigureV-18b: Zoom in plot of emission energy as a function of concentration of glycerol at various temperatures in $\beta-\mathrm{Lg}$ films. Data

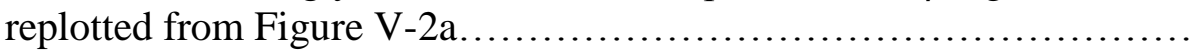

Figure V-19a: A plot of the effect of change in concentration of glycerol in $\mathrm{k}_{\mathrm{TS} 0}$ at various temperatures. Data replotted from Figure V-13a...

Figure V-19b: Zoom in plot of the effect of change in concentration of glycerol in $\mathrm{k}_{\mathrm{TS} 0}$ at various temperatures. Data replotted from Figure V-13a.

Figure V-20a: $\mathrm{k}_{\mathrm{Q}}\left[\mathrm{O}_{2}\right]$ as a function of concentration of glycerol at various temperatures in $\beta$-Lg films Data replotted from Figure V- 12a...

Figure V-20b: Zoom in plot of $\mathrm{k}_{\mathrm{Q}}\left[\mathrm{O}_{2}\right]$ as a function of concentration of glycerol at various temperatures in $\beta$ - $\mathrm{Lg}$ films .Data replotted from

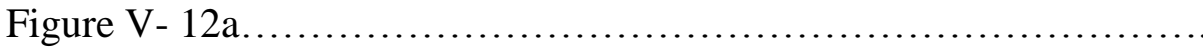

Figure V-21a: Emission energy as a function of concentration of sorbitol at various temperatures in $\beta-\mathrm{Lg}$ films Data replotted from Figure V- 2b................................................. 
Figure V-21b: Zoom in plot of emission energy as a function of concentration of sorbitol at various temperatures in $\beta$-Lg films. Data replotted from Figure V-2b.

Figure V-22a: A plot of the effect of change in concentration of sorbitol in $\mathrm{k}_{\mathrm{TS} 0}$ at various temperatures Data replotted from Figure V- 13b...

Figure V-22b: Zoom in plot of the effect of change in concentration of sorbitol in $\mathrm{k}_{\mathrm{TS} 0}$ at various temperatures Data replotted from Figure $\mathrm{V}-13 \mathrm{~b}$.

Figure V-23a: $\mathrm{k}_{\mathrm{Q}}\left[\mathrm{O}_{2}\right]$ as a function of concentration of sorbitol at various temperatures in $\beta-\mathrm{Lg}$ films Data replotted from Figure V-12b....

Figure V-23b: Zoom in plot of $\mathrm{k}_{\mathrm{Q}}\left[\mathrm{O}_{2}\right]$ as a function of concentration of sorbitol at various temperatures in $\beta$ - $\mathrm{Lg}$ films. Data replotted from

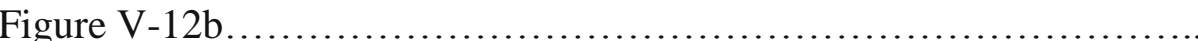

Figure V-24a: Emission energy as a function of concentration of Maltitol at various temperatures in $\beta$ - $\mathrm{Lg}$ films Data replotted from Figure V-2c.

Figure V-24b: Zoom in plot of emission energy as a function of concentration of maltitol at various temperatures in $\beta$ - $\mathrm{Lg}$ films. Data replotted from Figure V- 2c.

Figure V-25a: A plot of the effect of change in concentration of maltitol in $\mathrm{k}_{\mathrm{TS} 0}$ at various temperatures. Data replotted from Figure V-13c...

Figure V-25b: Zoom in plot of the effect of change in concentration of maltitol in $\mathrm{k}_{\mathrm{TS} 0}$ at various temperatures Data replotted from Figure $\mathrm{V}-13 \mathrm{c}$

Figure V-26a: $\mathrm{k}_{\mathrm{Q}}\left[\mathrm{O}_{2}\right]$ as a function of concentration of maltitol at various temperatures in $\beta$-Lg films. Data replotted from Figure V-12c...

Figure V-26b: Zoom in plot of $\mathrm{k}_{\mathrm{Q}}\left[\mathrm{O}_{2}\right]$ as a function of concentration of maltitol at various temperatures in $\beta-\mathrm{Lg}$ films. Data replotted from Figure V-12c.

Figure V-27a: Emission energy as a function of concentration of PEG at various temperatures in $\beta-\mathrm{Lg}$ films. Data replotted from Figure V-2d.....

Figure V-27b: Zoom in plot of emission energy as a function of concentration of PEG at various temperatures in $\beta-\mathrm{Lg}$ films Data 
replotted from Figure V-2d.

Figure V-28a: A plot of the effect of change in concentration of PEG 400 in $\mathrm{k}_{\mathrm{TS} 0}$ at various temperatures. Data replotted from Figure V-13d.

Figure V-28b: Zoom in plot of the effect of change in concentration of PEG400 in $\mathrm{k}_{\mathrm{TS} 0}$ at various temperatures Data replotted from Figure V$13 \mathrm{~d}$

Figure V-29a: $\mathrm{k}_{\mathrm{Q}}\left[\mathrm{O}_{2}\right]$ as a function of concentration of PEG 400 at various temperatures in $\beta$ - Lg films Data replotted from Figure V-12d....

Figure VI-1: The effect of temperature on the delayed emission spectra of Erythrosin B dispersed in $\beta-\mathrm{Lg} / \mathrm{PEG} / \mathrm{suc}$ films $(\beta-\mathrm{Lg} / \mathrm{PEG} / \mathrm{Suc}$ at a ratio of $1: 10: 10)$.

Figure VI-2: The effect of temperature on the emission maximum for phosphorescence from Erythrosin B in $\beta$-Lg/PEG/suc films (The ratios in the text box refer to the ratio of $\beta$-Lg: PEG: suc in the films).......

Figure VI-3: The effect of temperature on the bandwidth for phosphorescence from Erythrosin B in $\beta$-Lg/PEG/suc films (The ratios in the text box refer to the ratio of $\beta-\mathrm{Lg}: \mathrm{PEG}:$ suc in the films).....

Figure VI-4(a,b): The effect of temperature on the lifetime for Erythrosin B dispersed in amorphous $\beta$ - $\mathrm{Lg} / \mathrm{Peg}$-suc films in various molar concentrations of sucrose equilibrated against nitrogen $(\downarrow)$ and against air ( $\mathbf{\square})$; the lifetime was determined from analysis using a stretched exponential decay function. The text box in the graph refers to the molar ratio of $\beta$-Lg:PEG:suc

Figure VI-4c: Comparison plot depicting the effect of temperature on the lifetime for Erythrosin B dispersed in $\beta$-Lg /PEG /suc films equilibrated against nitrogen (The ratios in the text box refer to the ratio of $\beta$ - $\mathrm{Lg}$ : PEG : suc in the films).

Figure VI-4d: Comparison plot depicting the effect of temperature on the lifetime for Erythrosin B dispersed in $\beta$-Lg /PEG /suc films equilibrated against air (The ratios in the text box refer to the ratio of $\beta$ $\mathrm{Lg}$ : PEG : suc in the films)

Figure VI-5a: Beta as a function of temperature in $\beta$-Lg /PEG /suc films equilibrated against nitrogen (The ratios in the text box refer to the ratio of $\beta$-Lg : PEG : suc in the films).... 
Figure VI-5b: Beta as a function of temperature in $\beta$-Lg /PEG /suc films equilibrated against oxygen (The ratios in the text box refer to

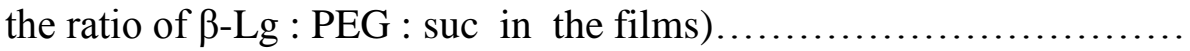

Figure VI-6: The oxygen quenching rate $\mathrm{k}_{\mathrm{Q}}\left[\mathrm{O}_{2}\right]$ as a function of temperature in $\beta$ - $\mathrm{Lg} / \mathrm{PEG} / \mathrm{suc}$ films (The ratios in the text box refer to the ratio of $\beta-\mathrm{Lg}: \mathrm{PEG}:$ suc in the films)........................

Figure VI-7: Plot of the effect of temperature on non-radiative decay from $\mathrm{T} 1$ to $\mathrm{S} 0\left(\mathrm{k}_{\mathrm{TS} 0}\right)$ in $\beta$ - $\mathrm{Lg} / \mathrm{PEG} / \mathrm{suc}$ films (The ratios in the text box refer to the ratio of $\beta$-Lg : PEG : suc in the films)................

Figure VI-8(a,b): Dependence of the oxygen quenching rate $\mathrm{k}_{\mathrm{Q}}\left[\mathrm{O}_{2}\right]$ on the rate of non-radiative quenching $\mathrm{k}_{\mathrm{TS} 0}$ in $\beta-\mathrm{Lg} / \mathrm{PEG} / \mathrm{suc}$ films (The ratios in the text box refer to the ratio of $\beta-\mathrm{Lg}$ : PEG : suc in the films)....

Figure VI-9: The effect of temperature on the delayed emission spectra of Erythrosin B dispersed in amorphous $\beta-\mathrm{Lg} / \mathrm{maltose} /$ maltitol mixture (Ratio of $\beta$-Lg: maltose: maltitol 1:5:5).

Figure VI-10: The effect of temperature on the emission maximum for phosphorescence from Erythrosin B in composite films from $\beta$ - $\mathrm{Lg} /$ maltose / maltitol (The ratios in the text box refer to the ratio of $\beta$-Lg: maltose: maltitol in the films)

Figure VI-11: The effect of temperature on the band width for phosphorescence from Erythrosin B in composite films from $\beta-\mathrm{Lg} /$ maltose / maltitol (The ratios in the text box refer to the ratio of $\beta$-Lg: maltose : maltitol in the films)....

Figures VI-12(a,b,c): The effect of temperature on the lifetime for Erythrosin B dispersed in composite films from $\beta$ - $\mathrm{Lg} /$ maltose / maltitol mixtures equilibrated against nitrogen $(\diamond)$ and against air $(\mathbf{\square})$; the lifetime was determined from analysis using a stretched exponential decay function. (The ratios in the text box refer to the ratio of $\beta-\mathrm{Lg}$ : maltose : maltitol in the films) ...................................

Figure VI-12d: Comparison plot depicting the effect of temperature on the lifetime for Erythrosin B dispersed in composite films from $\beta-\mathrm{Lg}$ /maltose /maltitol mixtures equilibrated against nitrogen. (The ratios in the text box refer to the ratio of $\beta$ - $\mathrm{Lg}$ : maltose : maltitol in the films)...

Figure VI-12e: Comparison plot depicting the effect of temperature on the lifetime for Erythrosin B dispersed in composite films from $\beta$-Lg / 
maltose / maltitol mixtures equilibrated against air. (The ratios in the text box refer to the ratio of $\beta$ - $\mathrm{Lg}:$ maltose : maltitol in the films)......

Figure VI-13a: Beta as a function of temperature in composite films from $\beta$ - Lg /maltose /maltitol mixtures equilibrated against nitrogen (The ratios in the text box refer to the ratio of $\beta-\mathrm{Lg}:$ maltose : maltitol in the films).

Figure VI-13b: Beta as a function of temperature in composite films from $\beta-\mathrm{Lg} / \mathrm{maltose} / \mathrm{maltitol}$ equilibrated against air (The ratios in the text box refer to the ratio of $\beta-\mathrm{Lg}$ : maltose : maltitol in the films).......

Figure VI-14: The oxygen quenching rate $\mathrm{k}_{\mathrm{Q}}\left[\mathrm{O}_{2}\right]$ as a function of temperature in $\beta$ - $\mathrm{Lg} / \mathrm{maltose} / \mathrm{maltitol}$ films..........................

Figure VI-15: Plot of the effect of temperature on non-radiative decay from $\mathrm{T} 1$ to $\mathrm{S} 0\left(\mathrm{k}_{\mathrm{TS} 0}\right)$ in $\beta$ - $\mathrm{Lg} /$ maltose / maltitol films.................

Figure VI-16: Peak frequency as a function of molar ratio of maltose/ maltitol in the composite films. Data re-plotted from Figure V-10

Figure VI-17: $\mathrm{k}_{\mathrm{TS} 0}$ as a function of molar ratio of maltose/maltitol in the composite films. Data re-plotted from Figure VI-14

Figure VII-1: The effect of temperature on the delayed emission spectra of Erythrosin B dispersed in amorphous BSA / $\beta$ - Lg films (BSA: $\beta$ - Lg $1: 1)$

Figure VII-2: The effect of temperature on the emission maximum for phosphorescence from Erythrosin B in amorphous BSA/ $\beta$ - Lg films.....

Figure VIII-3: The effect of temperature on the bandwidth for phosphorescence from Erythrosin B in amorphous BSA/ $\beta$ - Lg films

FigureVII-4a,b, c: Lifetime as function of temperature in amorphous BSA/ $\beta$ - Lg films equilibrated against N2 $(\lambda)$ and air $(\boldsymbol{\square})$ : The lifetime was determined from analysis using a stretched exponential decay function.

Figure VII-4d: Comparison plot depicting the effect of temperature on the lifetime of Erythrosin B dispersed in BSA/ $\beta$ - Lg films equilibrated against nitrogen .The lifetime was determined from analysis using a 
stretched exponential decay function.

Figure VII-4e: Comparison plot depicting the effect of temperature on the lifetime of Erythrosin B dispersed in BSA/ $\beta$ - Lg / films equilibrated against air. The lifetime was determined from analysis using a stretched exponential decay function.

Figure VII-5a: The effect of temperature on the stretching exponent $\beta$ for Erythrosin B dispersed in BSA/ $\beta$ - Lg films equilibrated against nitrogen. $\beta$ was determined from analysis using a stretched exponential decay function.

Figure VII-5b: The effect of temperature on the stretching exponent $\beta$ for Erythrosin B dispersed in BSA/ $\beta$ - Lg films equilibrated against nitrogen. $\beta$ was determined from analysis using a stretched exponential decay function.

Figure VII-6: The oxygen quenching rate $\mathrm{kq}\left[\mathrm{O}_{2}\right]$ as a function of temperature in $\beta$ - Lg and BSA mixtures....

Figure VII-7: Arrhenius plot of the effect of temperature on oxygen quenching rate $\mathrm{k}_{\mathrm{Q}}\left[\mathrm{O}_{2}\right]$ in amorphous BSA/ $\beta$-Lg films.................

Figure VII-8: Plot of the effect of temperature on non radiative decay from $\mathrm{T} 1$ to $\mathrm{S} 0\left(\mathrm{k}_{\mathrm{TS} 0}\right)$ in amorphous BSA/ $\beta$ - Lg films

Figure VII-9: Arrhenius plot of the effect of temperature on non radiative decay from $\mathrm{T} 1$ to $\mathrm{S} 0\left(\mathrm{k}_{\mathrm{TS} 0}\right)$ in amorphous $\mathrm{BSA} / \beta$ - $\mathrm{Lg}$ films...

Figure VII-10: Effect of emission wavelength on the Erythrosin B phosphorescence lifetimes in amorphous BSA/ $\beta-\mathrm{Lg}$ mixtures determined from analysis using a stretched exponential model ....

Figure VII-11: Comparitive plot of the effect of emission wavelength on the Erythrosin B phosphorescence lifetimes in amorphous $\beta$-Lg, BSA and BSA/ $\beta$ - $\mathrm{Lg}$ mixtures determined from analysis using a stretched exponential model at $20^{\circ} \mathrm{C}$.

Figure VII-12: Effect of emission wavelength on the stretching exponent $(\beta)$ characterizing the intensity decay transients of Erythrosin $\mathrm{B}$ phosphorescence in amorphous BSA $\beta-\mathrm{Lg}$ mixtures determined from 
analysis using a stretched exponential model

Figure VII-13: Effect of emission wavelength on the stretching exponent $(\beta)$ characterizing the intensity decay transients of Erythrosin $\mathrm{B}$ phosphorescence in amorphous BSA $\beta$ - Lg mixtures determined from analysis using a stretched exponential model.

Figure VII-14: Phosphorescence emission spectra for Erythrosin B in amorphous BSA/ $\beta-\mathrm{Lg}$ mixtures collected as a function of delay time....

Figure VII-15: Evolution of phosphorescence emission peak frequency with time following excitation in amorphous BSA/ $\beta-\mathrm{Lg}$ mixtures........

Figure VII-16: Evolution of phosphorescence emission bandwidth with time following excitation in amorphous BSA/ $\beta$ - Lg mixtures.

Figure VII-17: Variation of activation energy $\left(E_{A}\right)$ for quenching of phosphorescence lifetime of Erythrosin B in amorphous BSA/ $\beta-\mathrm{Lg}$

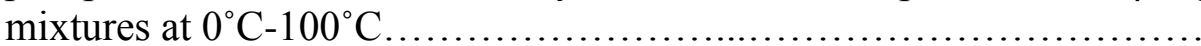

Figure VII-18a, b, c: Plot of the dependence of the radiative collisional quenching rate $\mathrm{k}_{\mathrm{TS} 0}$ to oxygen quenching rate $\mathrm{k}_{\mathrm{Q}}\left[\mathrm{O}_{2}\right]$ in $\mathrm{BSA} / \beta$ - $\mathrm{Lg}$ films

Figure VIII-1a: The effect of temperature on the delayed emission spectra of Erythrosin B dispersed in amorphous $\beta$-Lg- palmitic acid

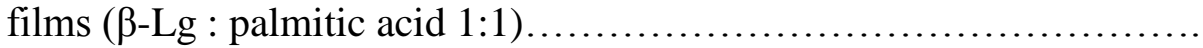

Figure VIII-1b: The effect of temperature on the delayed emission spectra of Erythrosin B dispersed in amorphous $\beta$ lg- caprylic acid films

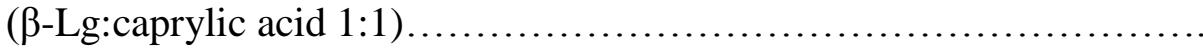

Figure VIII-2: The effect of temperature on the emission maximum for phosphorescence from Erythrosin B in amorphous fatty acid / $\beta-\mathrm{Lg}$ films

Figure VIII-3: The effect of temperature on the bandwidth for phosphorescence from Erythrosin B in amorphous fatty acid/ $\beta$ - Lg films 
Figure VIII-4a: Comparison plot depicting the effect of temperature on the lifetime of Erythrosin B dispersed in fatty acid/ $\beta$-Lg films equilibrated against nitrogen

Figure VIII-4b: Comparison plot depicting the effect of temperature on the lifetime of Erythrosin B dispersed in fatty acid/ $\beta$ - Lg films equilibrated against air

Figure VIII-5a: The effect of temperature on the stretching exponent $\beta$ for Erythrosin B dispersed in fatty acid / $\beta$ - Lg films equilibrated against nitrogen. $\beta$ was determined from analysis using a stretched exponential decay function.

Figure VIII-5b: The effect of temperature on the stretching exponent $\beta$ for Erythrosin B dispersed in fatty acid/ $\beta$-Lg films equilibrated against nitrogen. $\beta$ was determined from analysis using a stretched exponential decay function.

Figure VIII-6: The oxygen quenching rate $\mathrm{kq}\left[\mathrm{O}_{2}\right]$ as a function of temperature in $\beta$ - $\mathrm{Lg}$ and fatty acid $/ \beta$ - $\mathrm{Lg}$ mixtures

Figure VIII-7: Plot of the effect of temperature on non radiative decay from $\mathrm{T} 1$ to $\mathrm{S} 0\left(\mathrm{k}_{\mathrm{TS} 0}\right)$ in amorphous fatty acid / $\beta$ - Lg films

Figure VIII 8: Arrhenius plot of the effect of temperature on non radiative decay from $\mathrm{T} 1$ to $\mathrm{S} 0\left(\mathrm{k}_{\mathrm{TS} 0}\right)$ in amorphous fatty acid/ $\beta$ - $\mathrm{Lg}$ films.

FigureVIII-9a: Dependence of the oxygen quenching rate $\mathrm{k}_{\mathrm{Q}}\left[\mathrm{O}_{2}\right]$ on the rate of nonradiative quenching $\mathrm{k}_{\mathrm{TS} 0}$ in caprylic acid/ $\beta-\mathrm{Lg}$ films.

FigureVIII-9b: Dependence of the oxygen quenching rate $\mathrm{k}_{\mathrm{Q}}\left[\mathrm{O}_{2}\right]$ on the rate of nonradiative quenching $\mathrm{k}_{\mathrm{TS} 0}$ in palmitic acid / $\beta-\mathrm{Lg}$ films

Figure IX-1a: Emission spectrum of retinol in $\beta-\operatorname{Lg}(1: 4)$ as a function of gate time.

Figure IX-1b: Emission spectrum of retinol in $\beta-\mathrm{Lg}$ (1:4) (pure $\beta-\mathrm{Lg}$ subtracted) as a function of gate time. 
Figure IX-2a: Emission spectrum of retinol in $\beta-\operatorname{Lg}(1: 4)$ as a function of delay time.

Figure IX-2b: Emission spectrum of retinol in $\beta-\mathrm{Lg}$ (1:4) (pure $\beta-\mathrm{Lg}$ subtracted) as a function of delay time

Figure IX-3a: Emission spectrum of retinol in $\beta-\operatorname{Lg}(1: 1)$ as a function of excitation wavelength

Figure IX-3b: Emission spectrum of retinol in $\beta-\mathrm{Lg}$ (1:1) (pure $\beta-\mathrm{Lg}$ subtracted) as a function of excitation wavelength

Figure IX-4a: Emission spectrum of retinol in $\beta-\operatorname{Lg}(1: 4)$ as a function of excitation wavelength.

Figure IX-4b: Emission spectrum of retinol in $\beta-\mathrm{Lg}$ (1:4) (pure $\beta-\mathrm{Lg}$ subtracted) as a function of excitation wavelength

Figure IX-5a: Emission spectrum of retinal in $\beta \lg (1: 4)$ as a function of gate time.

Figure IX-5b: Emission spectrum of retinal in $\beta \lg (1: 4)$ (pure $\beta$ - $\mathrm{Lg}$ subtracted) as a function of gate time

Figure IX-6a: Emission spectrum of retinal in $\beta-\operatorname{Lg}(1: 4)$ as a function of delay time.

Figure IX-6b: Emission spectrum of retinal in $\beta-\mathrm{Lg}$ (1:4) (pure $\beta-\mathrm{Lg}$ subtracted) as a function of delay time

Figure IX-7a: Emission spectrum of retinal in $\beta-\operatorname{Lg}(1: 1)$ as a function of excitation wavelength

Figure IX-7b: Emission spectrum of retinal in $\beta$-Lg (1:1) (pure $\beta-\mathrm{Lg}$ subtracted) as a function of excitation wavelength....

Figure IX-8a: Emission spectrum of retinal in $\beta-\operatorname{Lg}(1: 4)$ as a function of excitation wavelength

Figure IX-8b: Emission spectrum of retinal in $\beta \lg (1: 4)$ (pure $\beta-\mathrm{Lg}$ subtracted) as a function of excitation wavelength 


\section{$\underline{\text { Introduction }}$}

Edible films

Edible coatings are very promising systems for the future improvement of food quality and preservation during processes and storage. Due to recent increases in ecological consciousness a considerable amount of research has been diverted towards the potential development and exploration of new frontiers in the field of edible food technology. Edible films are defined as a thin layer of edible material formed on a food as a coating or placed on or between food components (Krochta and DeMulder-Johnston, 1997). Edible films and coatings are typically natural polymers obtained from agricultural products such as animal and vegetable proteins and are perfectly biodegradable, and thus are environmentally friendly (Gennadios et al., 1994; Torres et al., 1994).

The use of edible films in food products seems new, but edible films and coatings first covered food products many years ago. Wax has been used to delay dehydration of citrus fruits in China since the twelfth and thirteenth centuries (Guilbert, et al., 1986). Over the last 40 years, a great number of studies on the formulation, application, and characterization of edible films and coatings have been reported in both the scientific and patent literature.

Edible films and coatings have been applied on meat, poultry, seafood, fruits, vegetables, grains, candies, heterogeneous and complex foods, or fresh, cured, frozen, and processed foods because of the potential for these films to enhance food quality, food safety, and product shelf life. Edible films provide possibilities for improving the quality of heterogeneous foods by limiting the migration of moisture, lipids, flavors/aromas, and colors between food components, carrying functional food ingredients (antioxidants, 
antimicrobials, flavor, etc), or improving the mechanical integrity or handling characteristics (Debeaufort et al., 1998). Various biodegradable protein and polysaccharide-based edible films can potentially serve as packaging materials (Khwaldia et al., 2004). Edible films can be intelligent packaging because they are both active and selective and have wide potential use.

Edible films are generally made from proteins, polysaccharides and lipids used alone or together. Proteins can form a continuous matrix and due to this property they have been widely employed to make edible films. Protein-based films have more interesting mechanical and barrier properties than polysaccharide. Many protein-based films have been tested: corn zein (Parris et al., 1997), wheat gluten (Gontard, et al., 1992), peanut (Jangchud et al., 1999), soy protein isolate (Brandenburg et al., 1993), fish proteins (Tanaka et al., 2001), whey protein isolate (Mc Hugh et al., 1994), casein (Avena et al., 1993), etc.

Viable edible films and coatings can be produced using milk proteins. The film forming ability of caseins and whey proteins has been studied extensively in the last decade. It has been shown that edible films and coatings can be formed from total milk proteins or components of milk protein. Researchers have stated that whey protein-based films are bland in flavor and transparent (Chick and Ustunol 1998; Miller and Krochta 1997; McHugh and Krochta 1994; Gennadios et al., 1994). These films and coatings may retard moisture loss, are good oxygen barriers, show good tensile strength and moderate elongation (Figure I-1, 2). In addition to their nutritive value milk proteins have several key factors for effective performance in edible films. Furthermore considerable interest exists in finding new uses for milk proteins due to their industrial surplus (Khwaldia et 
al., 2004). The functional properties of whey proteins are of substantial and growing importance to the food industry. The major fractions of whey proteins are $\beta$ Lactoglobulin (57\%), $\alpha$-lactalbumin (19\%), bovine serum albumin (7\%), immunoglobulins (13\%) and specific polypeptides (4\%). Whey protein isolate is capable of forming transparent films and coatings that provide excellent oxygen, aroma and oil barrier properties at low relative humidity (McHugh and Krotcha, 1994; Mate and Krotcha, 1996; Miller and Krotcha, 1997).

\section{Figure I-1}

\section{Functions of Edible films}

Organic vapors, aroma, solvents

Water vapor

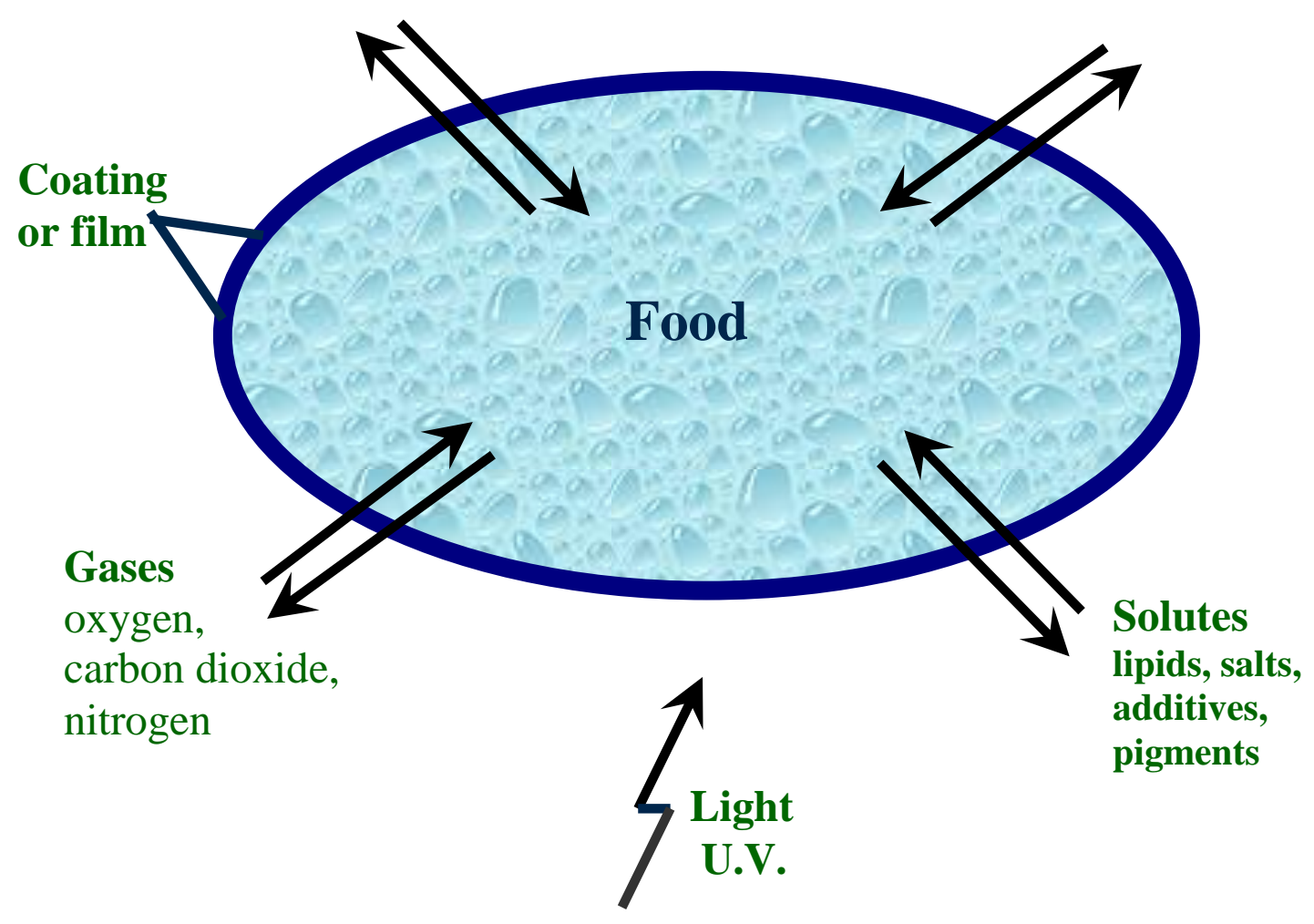




\section{Figure I-2}

\section{Edible film functionality}

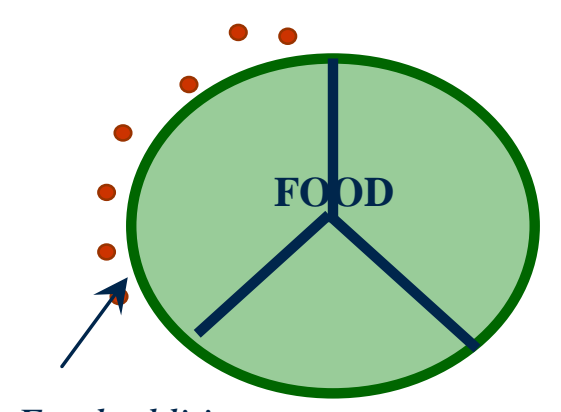

Food additives
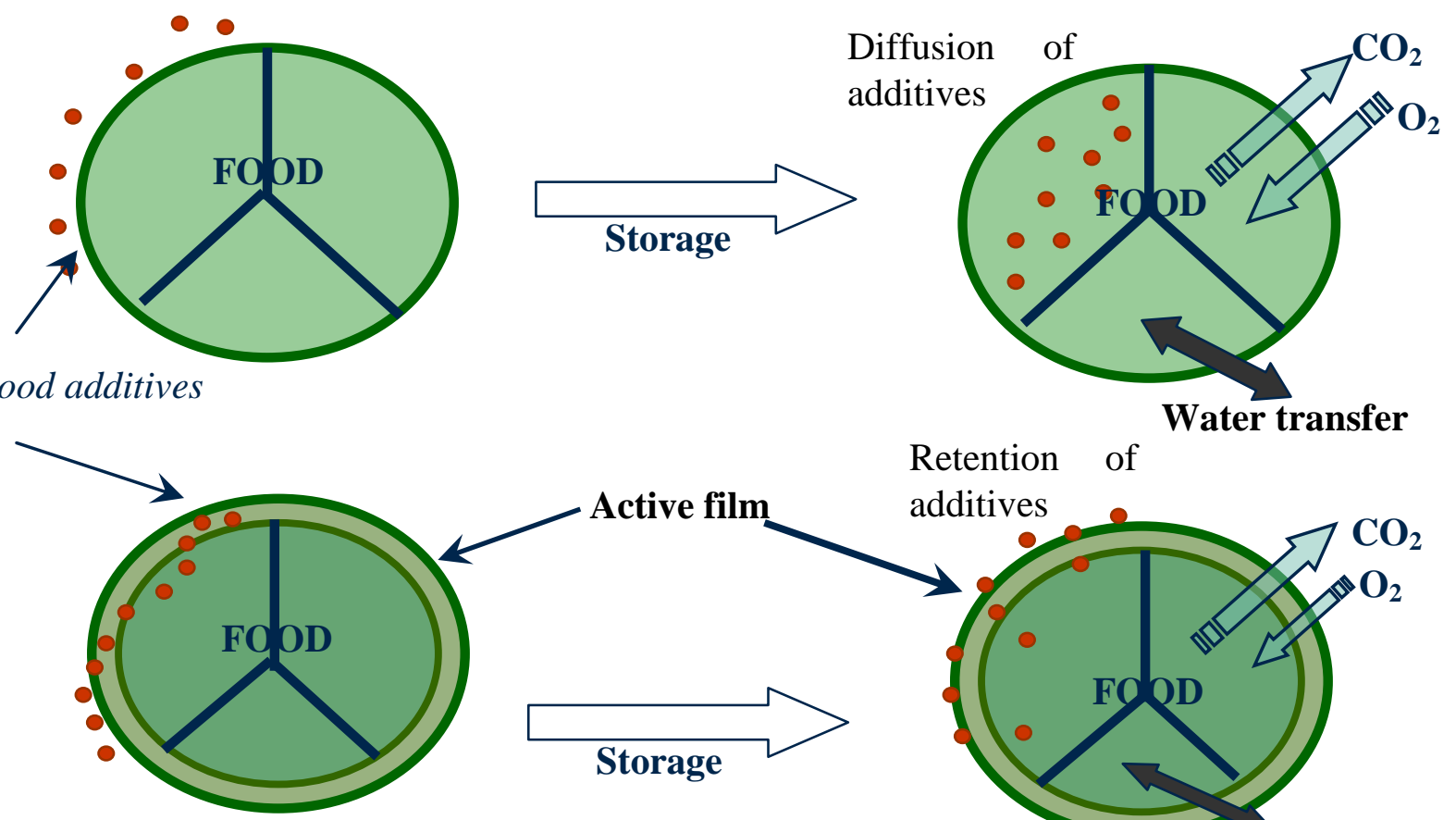

Retention of additives

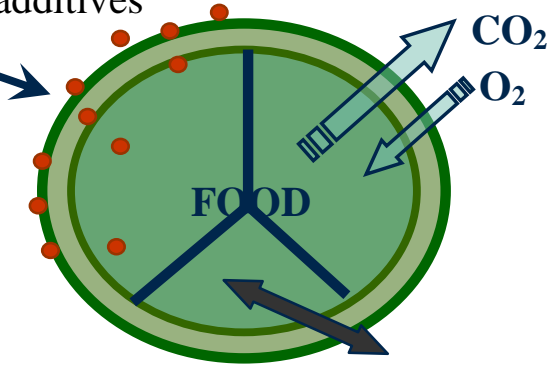

Controlled Water transfer 


\section{$\beta$-Lactoglobulin}

$\beta$-Lactoglobulin $(\beta-\mathrm{Lg})$ is the most abundant protein in bovine whey, constituting almost 50\% of the total protein (Dybing and Smith 1991); therefore, its properties are very important to the functionality of milk and isolated whey proteins. Monomeric $\beta-\mathrm{Lg}$ is a water soluble, globular protein of $18,300 \mathrm{Da}$, corresponding to a polypeptide of 162 residues for all genetic variants. At physiological $\mathrm{pH}$ it is a dimer but dissociates into monomers below pH 3.0 (Blanch et al., 1999). This globular protein contains two disulfide bonds (C106-C119 and C66-C160) and one free cysteine (C121) (Wada et al., 2006) (Figure I-3). The unusual properties of these labile cysteines influence many of its functionalities in foods (Kinsella and Whitehead, 1989). $\beta$-Lg has a protein fold composed of two antiparallel $\beta$-sheets and shows a remarkable similarity to plasma retinol-binding protein (Papiz et al., 1986); it is a member of the lipocalin super family of transporters of hydrophobic molecules. Its physiological role is still unclear and, it has been demonstrated that $\beta$-Lg binds a variety of hydrophobic molecules such as retinol, fatty acids, triglycerides, protoporphyrin IX and ANS (Dufour et al., 1990; Fugate and Song, 1980; Collini et al., 2000; D’Alfonso et al., 1999; Andrade and Costa, 2002)

$\beta$-Lg film properties such as transparency, water vapor permeability, oxygen permeability, and mechanical properties are identical to the properties of whey protein isolate films (Mate and Krochta, 1996; Anker et al., 1998). Heat denaturation opens the $\beta$-Lg globular structure, exposes sulfhydryl and hydrophobic groups, induces oxidation of free sulfhydryls, and promotes disulfide bond interchange and hydrophobic bonding thereby forming water-insoluble edible films (McHugh et al., 1994; Mate and Krochta, 1996). 
Figure I-3

\section{$\underline{\text { Structure of Bovine } \beta \text {-Lactoglobulin }}$}

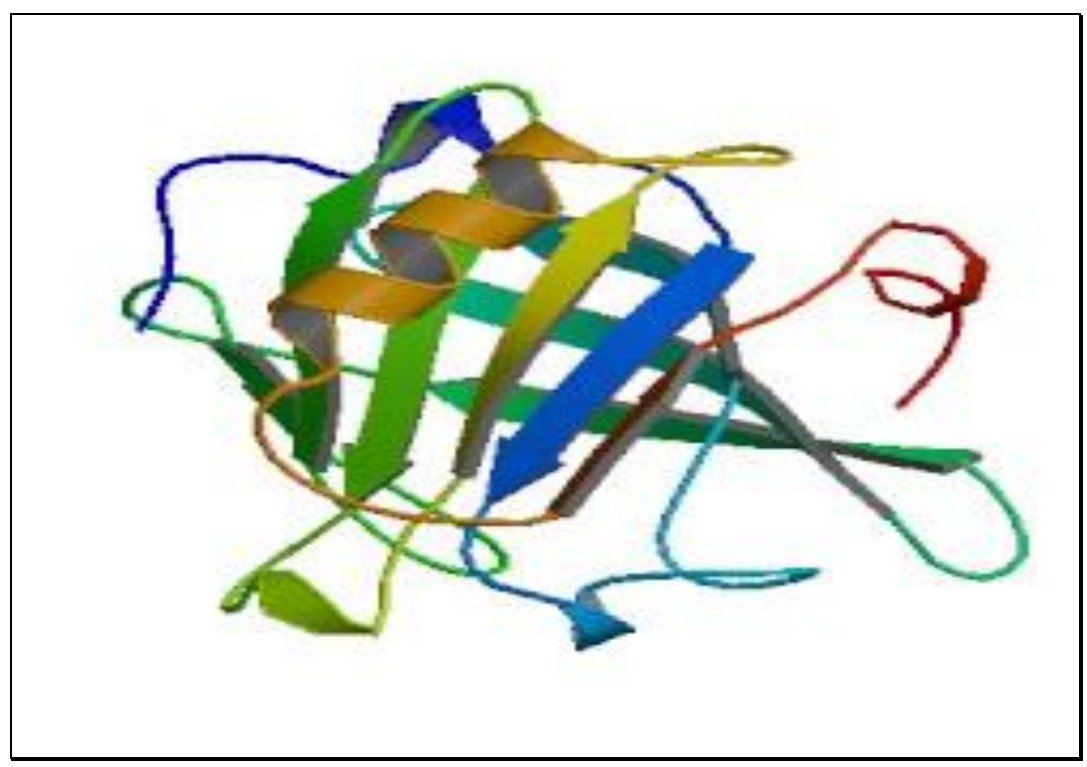

(Brownlow et al., 1997

\section{Significance of studying the amorphous glassy state}

A glass is defined as a super-cooled liquid with an extremely high viscosity typically in the order of $10^{14} \mathrm{~Pa}$ s (Franks, 1991). Their molecular motions are restricted to vibrations and short-range rotational motions (Sperling, 1986). Within the glassy state the mobility is mainly locally restricted to vibrations of atoms or reorientation of small groups of atoms and thus does not involve the surrounding matrix. Amorphous solids can be obtained through physical processes such as rapid cooling, extrusion and drying (Zallen, 1998) or can be generated unintentionally by routine manufacturing practices such as coating, granulation, milling and lyophilization (Hancock and Zografi, 1997). The glassy state is of immense importance and it has been known for a long time that 
stabilization of many macromolecules is greatly enhanced in this state (Roos, 1995; Slade and Levine, 1995).

The presence of an amorphous state has very important implications for storage of pharmaceutical dosage forms (Hancock and Zografi 1997). The formation of glasses in seeds is thought to be responsible for the prolonged survival of these tissues in the dry state (Sun, et al., 1998; Buitink et al., 1998). The shelf life of food materials has been associated with the presence of a glassy state (Roos, 1995). Edible films made from proteins are amorphous protein solids. It has been suggested that the glassy state plays a vital role to ensure the preservation of the structure and activity of proteins in the course of long-term storage (Franks, 1990). Amorphous protein solids are glassy when dry and rubbery when moist and exhibit unusually broad thermal transitions between these states.

\section{Glass transition and Molecular mobility}

The molecular mobility of the components of amorphous solid foods can be viewed as a fundamental molecular determinate of the macroscopic physical, chemical, and sensory qualities of foods. 


\section{Figure I-4}

\section{$\underline{\text { Attributes and molecular mobility }}$}

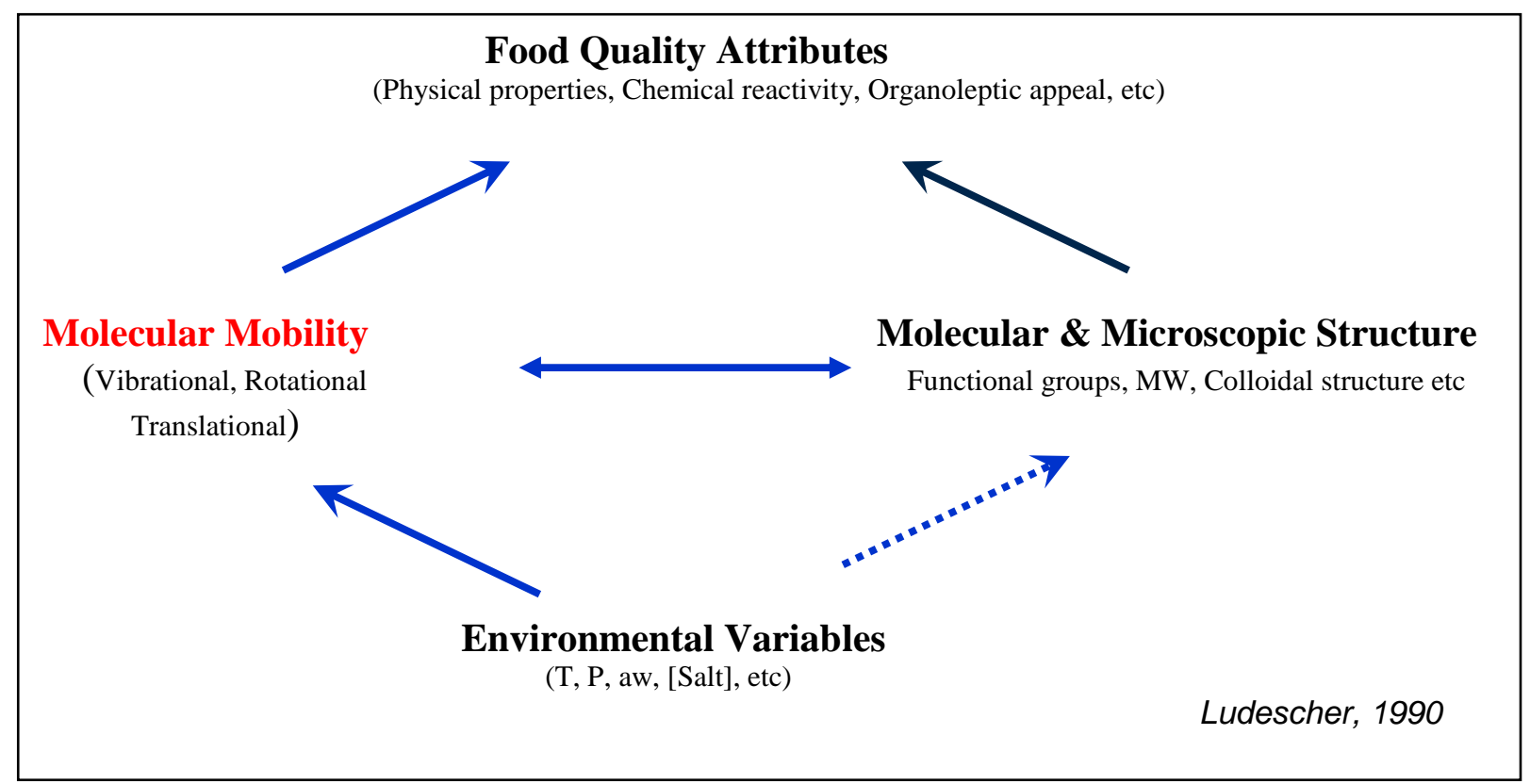

"Environmental variables such as temperature, pressure, water activity, salt concentration, as well as the presence of other solutes, affect the quality of amorphous foods primarily by modulating the molecular mobility of the components and only secondarily, if at all, by modulating their molecular and microscopic structure" (Ludescher, 1990). Molecular mobility is an important temperature dependent factor and is often considered to be one of the main factors that affect rates of deteriorative changes and shelf life of food materials. Molecular mobility is related to phenomena such as diffusion, viscosity and the glass transition. In amorphous solids, due to their high viscosity, diffusion is often the rate limiting step and changes in viscosity that influence diffusion significantly influences the reaction rate (Karel and Saguy, 1991; Craig et al., 2001). 
Slade and Levine (1991) suggested that diffusion in amorphous foods is governed by the glass transition. Their hypothesis assumed that at temperatures below the glass transition the rates of chemical reactions are extremely slow due to restricted molecular mobility and slow diffusion. The glass transition marks the onset of the $\alpha$ relaxation. But the glass transition cannot be considered the absolute threshold for molecular mobility. Molecular motions still persist in the glassy state with a lower amplitude and cooperativity than for the glass transition. Transport of water and small molecules takes place even in the glassy state at a significant rate. Chang et al. (1996) examined the stability of freeze dried recombinant interleukin-1 receptor antagonist in a variety of matrices and found that protein de-amidation and aggregation occurred in some formulations below Tg. Srickley and Anderson (1996) studied the mechanism of insulin stability in amorphous freeze-dried powders. They found that hydrolytic de-amidation and dimerization proceeded in both the glassy and rubbery states, however they showed different sensitivity to mobility.

Below the normal glass transition amorphous or partially crystalline polymers also exhibit secondary relaxations. These are normally given the notations $\beta, \gamma, \delta$, etc., in decreasing order of occurrence (Figure I-5). These sub Tg relaxations have amplitudes smaller than the $\alpha$ relaxations associated with major backbone chain movements (i.e., the primary glass transition). The existence of secondary relaxations is indicative of mobility at temperatures below the glass transition temperature $(\mathrm{T}<\mathrm{Tg})$. The physical reason or origin of a particular secondary relaxation, however, remains unclear. For example, $\beta$ relaxations in amorphous polymers have frequently been attributed to the motion of specific side-groups attached to the main polymer chain or to so-called "crankshaft" 
rotation in regions of the molecule where density and intermolecular forces are at a minimum (Roberts and White, 1973; Boyd, 1985; Simatos et al., 1995)

Edible films are affected by the glass transition as both mechanical and barrier properties are strongly affected by temperature, ambient humidity and plasticizer content. In an extensive study on polyol plasticized pullulan starch blends, the apparent activation energy for permeability was higher above the glass transition than below (Biliaderis et al., 1999). 


\section{Figure I-5}

\section{Macromolecular dynamic transition}

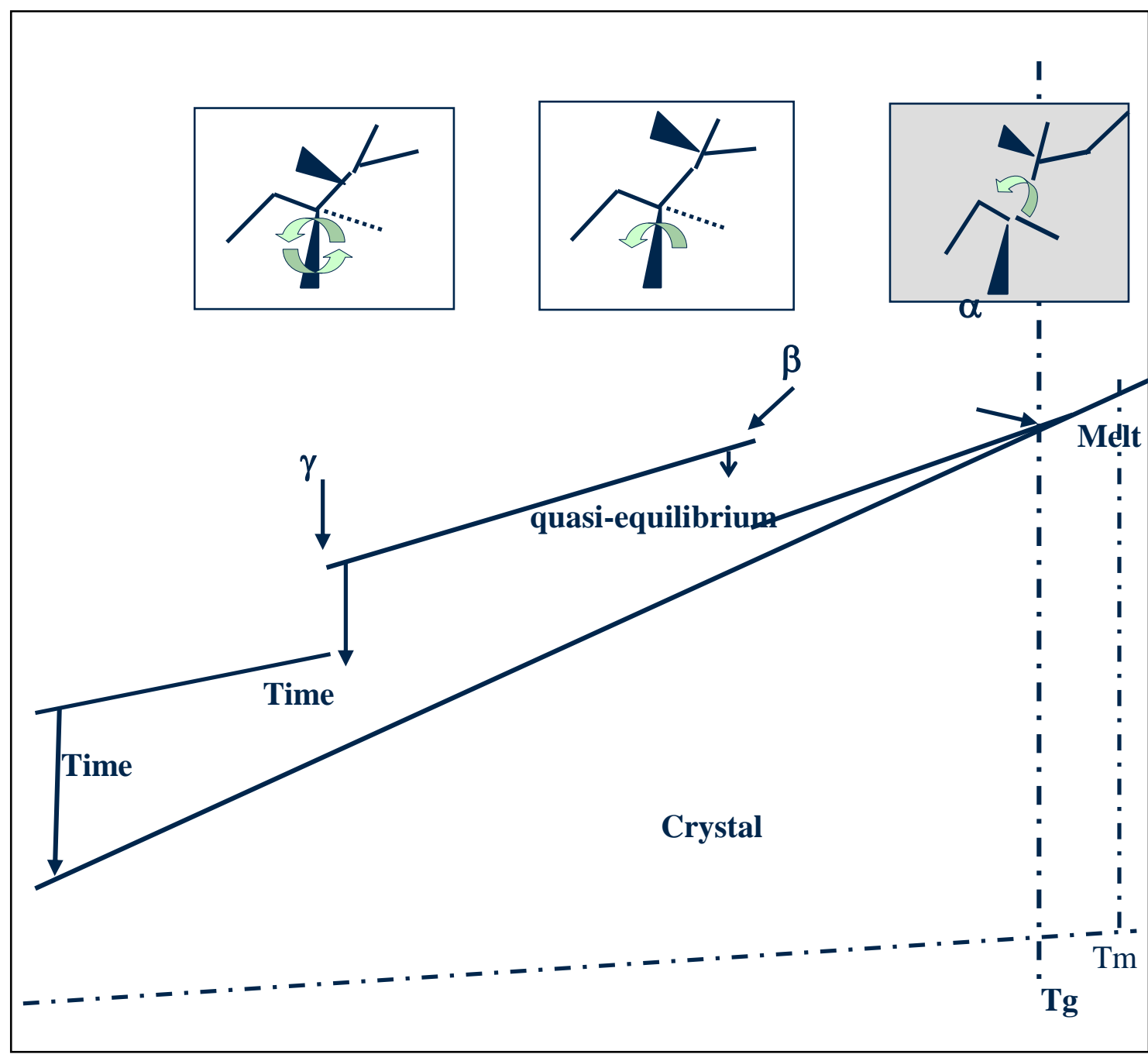




\section{Motions in proteins}

In solution proteins demonstrate many degrees of molecular motions (Table I- 1). Motions in proteins cover time scales from femtoseconds (individual bond vibrations) to picoseconds (small group fluctuations) to nano- and microseconds and longer (collective motions of groups of bonded and nonbonded atoms) and corresponding distance scales from fractions of an Angstrom to nanometers.

\section{Table I-1}

\section{Modes of molecular mobility of proteins in solution}

\begin{tabular}{|l|l|}
\hline Time scale & Dynamic event \\
\hline Picosecond $\left(10^{-12} \mathrm{~s}\right)$ & $\begin{array}{l}\text { Bond vibrations, rotational motion of } \\
\text { water, side chain rotations }\end{array}$ \\
\hline Nanosecond $\left(10^{-9} \mathrm{~s}\right)$ & $\begin{array}{l}\text { Segmental motions (small proteins) } \\
\text { Rotational motions (small proteins) }\end{array}$ \\
\hline Microsecond $\left(10^{-6} \mathrm{~s}\right)$ & $\begin{array}{l}\text { Segmental motion (large proteins) } \\
\text { Rotational motion of large/asymmetric } \\
\text { proteins) } \\
\text { Flexing of filamentous proteins }\end{array}$ \\
\hline Millisecond $\left(10^{-3} \mathrm{~s}\right)$ & $\begin{array}{l}\text { Conformational changes /Enzyme } \\
\text { turnover }\end{array}$ \\
\hline Second (s) & Protein folding \\
\hline
\end{tabular}


"Fully hydrated proteins undergo a substantial change in their dynamical fluctuations when cooled near the temperature called the dynamical transition $T_{d}$, the socalled glass transition in proteins which is reminiscent of the $\alpha, \beta$ motions in glasses. Above $T_{d}$, dynamic behavior is dominated by highly temperature dependent, large-scale, anharmonic, collective motions of bonded and non-bonded atoms superimposed on small amplitude vibrational motions, whereas below $T_{d}$, nearly small-amplitude, harmonic vibrations exist with a temperature dependence similar to the harmonic atomic vibrations in small molecule crystals" (Hill et al., 2005). Broad thermal softening transitions have been seen in calorimetric studies of lysozyme, hemoglobin and myoglobin (Sartor and Johari, 1994) (Figure I-6).

Figure I-6

$\underline{\text { Dynamic Transition in Proteins }}$

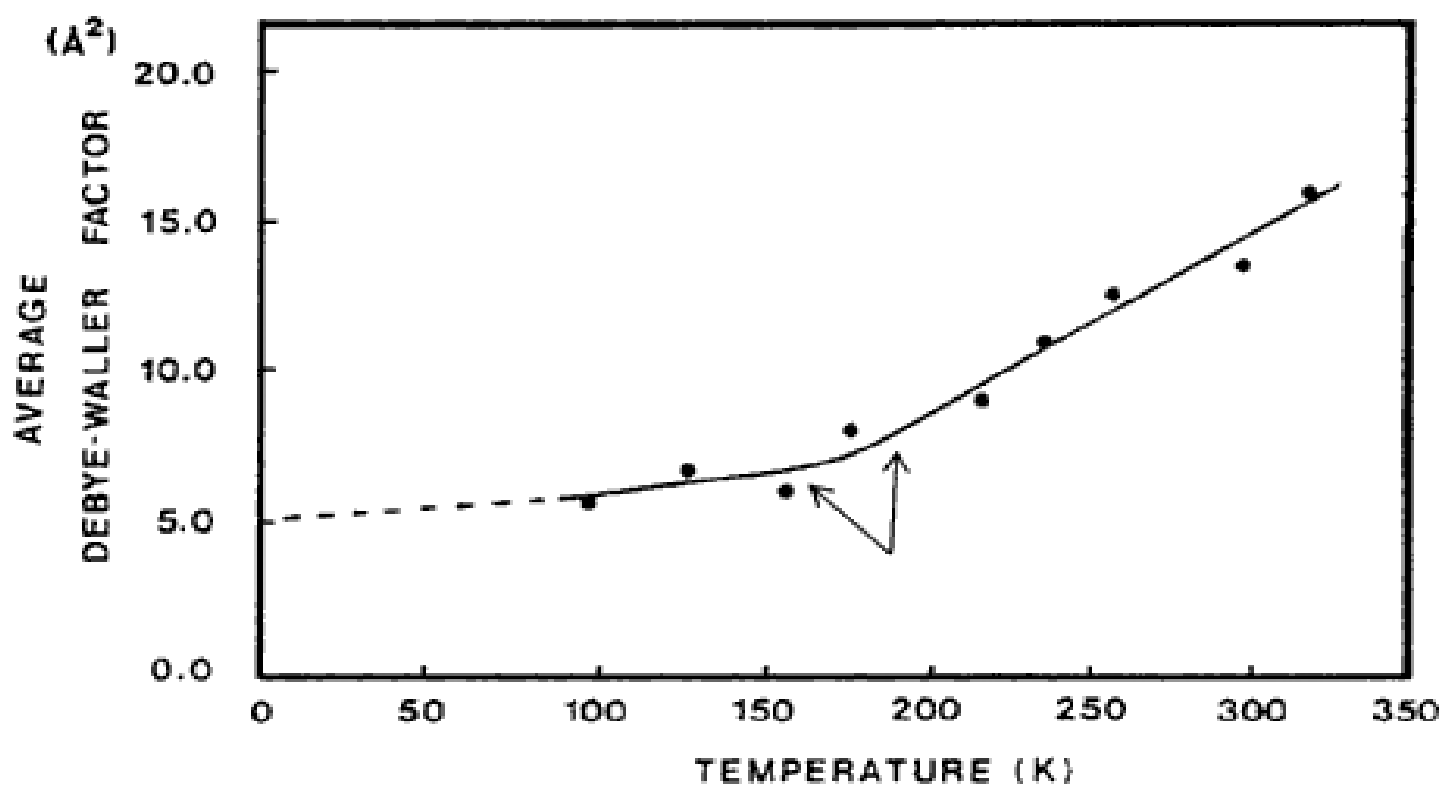

Figure I-6 Plot of the average Debye-Waller factor for crystalline ribonuclease A as a function of temperature. The arrows indicate the dynamic transition region of the curve (Hill et al., 2005) . 
Although these transitions have been observed, very little research has been devoted to studies of these vital transitions in actual food matrices. Since changes in relaxation time can be an indicator of the tendency for physical or chemical instability in the amorphous state, it is important to be able to measure such a parameter as a function of temperature, relative humidity, and other factors that might impact on molecular mobility. Temperature dependence particularly can be useful when one wants to obtain some measure of the expected molecular mobility over extended periods of time. These studies have the potential to illustrate complex chemical reactions, molecular diffusion and other important quality attributes in amorphous food materials.

Studies have used dielectric relaxation (Gangasharan and Murthy, 1993; Chan et al., 1986; Faivre et al., 1999; Richert, 2001), mechanical spectroscopy (Faivre et al., 1999), Fourier transform infrared spectroscopy (Wolkers et al., 1998), nuclear magnetic resonance (Moran and Jeffrey, 1999; Van Dusschoten et al., 1999) and electron spin resonance (Buitink et al., 2000) to study the molecular mobility in amorphous food matrices.

The water vapour permeability (WVP) is extensively studied in edible films (Erdohan et al., 2005; Wan et al., 2005; Khwaldia et al., 2004; Choi et al., 2001; Mate and Krotcha 1996; Sobral et al., 2001; Sothornvit et al., 2000; Tanada et al., 2002) because of the ease of its measurement. But very few studies have looked into various gas permeation techniques. 


\section{Oxygen permeability}

Oxygen is involved in many degradation reactions in foods, such as fat and oil rancidity, microorganism growth, and enzymatic browning and vitamin loss. The permeability of protein films to oxygen is thus a fundamental aspect of their functionality. Oxygen permeability (OP) is related to its diffusion coefficient (D) and solubility (S) with the familiar relationship related to Henry's law: $\mathrm{P}=\mathrm{DS}$. Oxygen permeability in amorphous protein films increases dramatically with increases in temperature (Gennadios et al.,1993) and relative humidity (Simon-Lukasik and Ludescher, 2004; Mc Hugh and Krotcha, 1994). Thus, many packaging strategies seek to exclude oxygen to protect the food product (Gontard et al., 1996). The development of edible films to reduce the oxidation or the respiration in food systems is important to prolong the shelf lives of foods. On the other hand, the permeability to oxygen and carbon dioxide is essential for respiration in living tissues such as fresh fruits and vegetables. So, moderate barrier coatings are more appropriate. The measurement of permeabilities of stand-alone films to water vapor and oxygen are important tools for the development of functional edible films.

\section{Measurements of oxygen permeability}

There are various techniques used to measure oxygen permeability. Today, the ASTMapproved method using a dynamic permeation cell with a sensitive oxygen-specific coulometric detector is the method of greatest commercial use. A commercial instrument, which conforms to the ASTM method, is the MOCON Ox-Tran apparatus (Modern Controls Inc. Minneapolis, MI, USA). Oxygen permeability is measured as follows:- Films were placed on a stainless steel mask within an open testing area. 
Nitrogen gas flows on one side and pure oxygen gas flows on the other at the above condition. Oxygen permeability is calculated by dividing the oxygen transmission rate by the difference in oxygen partial pressure between both sides of the film and multiplying by the average film thickness.

The ASTM method was further modified by Ayranci and Tunc (2002) for the measurement of permeability in methylcellulose films. The design was mainly based on the ASTM standard (1988). The well-known method of iodimetry was applied for the determination of the amount of permeated $\mathrm{O}_{2}$, instead of using an oxygen analyzer as in the ASTM standard, which uses a coulometric sensor. The OP of the film was then calculated by

$$
\mathrm{OP}=\mathrm{m} * \mathrm{~d} / \mathrm{A} * \mathrm{t} * \Delta \mathrm{P}
$$

where $\mathrm{m}$ is the mass of $\mathrm{O}_{2}$ permeated through the film with a thickness of $\mathrm{d}$ and an area of $A$ over the measured time interval t. $\Delta \mathrm{P}$ is the difference in $\mathrm{O}_{2}$ pressure between the two sides of the film. The above-mentioned methods, however, cannot be used to directly measure diffusion through edible films in situ, on food.

A new method for measuring the oxygen permeability of common polymer membranes based on an amperometric sensor was developed by Campenella et al. (2005). The system was successfully applied to food wrapping and storing films. This method is based on the measurement of oxygen concentration by means of an amperometric oxygen sensor (Clark electrode), covered with the polymer film and secured with a proper cap. Measurements are carried out under a constant flow of oxygen. 
Ambient oxygen ingress rate (AOIR) is one alternative method for measuring oxygen transmission rate, which is based on measuring the increase in oxygen concentration over time inside the whole package (Larsen et al., 2000; Moyls et al., 1992).

The measurement of oxygen permeability requires instruments which may not be easily available. But owing to the existing complexity in these kinds of measurements, the present work aims at utilizing a simple, non-invasive technique called luminescence spectroscopy to measure the oxygen permeability of edible films.

Despite the obvious fact that that both mechanical and barrier properties of edible films are governed by temperature, humidity and plasticizer content (Le Meste et al., 2000), rarely do studies of oxygen permeability through edible films or amorphous biomaterials make an attempt to investigate the molecular mechanisms controlling oxygen transport (Nack and Ludescher, 2006). The importance of these studies for developing rational methods to formulate edible films with appropriate functionality is clear and obvious. Luminescence spectroscopy provides a powerful set of tools for investigating the molecular mechanisms that control oxygen permeability through amorphous proteins (Simon-Lukasik and Ludescher, 2004; Lukasik and Ludescher, 2006). The proposed method was applied to measure the oxygen permeability of films of various compositions with the aim of finding the optimum composition to minimize oxidative degradation of foods.

The principle governing this research is as follows: Oxygen is a powerful quencher of both fluorescence and phosphorescence and luminescence quenching has been used as an effective method for the probing of molecular oxygen in polymeric 
media (Masoumi et al., 1996). Oxygen molecules react efficiently with free radicals and excited electronic states so even traces of oxygen greatly reduce the lifetime. Collisions between the excited triplet state and ground state oxygen molecule have a high probability of transfer of energy to the latter thus returning the excited molecules to the ground state without emission. Measured lifetimes are indicative of the various complex processes occurring in the matrix.

Since lifetime is little affected by temperature (Strambini et al., 1985) it can be inferred that increase in molecular diffusion and the presence of quencher species like oxygen is responsible for its decrease. Previous studies have examined the potential of the use of phosphorescence quenching by oxygen in glassy polymers (Charlesworth, 1996; Korolev et al., 1999).

A study by Buettner (1964) in the gelatin matrix showed that comparison of the probe emission lifetime in the presence and absence of oxygen provides a facile and rapid indicator of oxygen quenching. Simon-Lukasik and Ludescher, (2004) looked into the use of phosphorescence quenching of erythrosin B to effectively study oxygen diffusion in the amorphous gelatin matrix. We have used the same technique to characterize oxygen permeability in the amorphous $\beta$-Lactoglobulin matrix.

\section{Hypothesis}

We hypothesize that oxygen permeability in amorphous $\beta$-Lg films are modulated by specific local modes of vibrational and rotational molecular mobility in the glassy and the rubbery state. The composition of the matrix affects oxygen permeability by modulating the mobility in the matrix. 


\section{Sensitivity of Luminescence measurements to Molecular mobility}

Luminescence spectroscopy can be successfully used to study the molecular mobility of amorphous solids; measurements of emission intensity, energy (wavelength), and polarization can provide direct information about molecular structure and mobility (Ludescher, 2001). It is one of the most promising technologies in the fields of biology and medicine because of its high sensitivity and ease of use (Slavik, 1994).

Luminescent spectroscopy uses spectroscopic probes, which are organic molecules with well-characterized spectroscopic properties that can provide information not only about the molecule but also about the surrounding medium. The dye molecule can be used as a tiny reporter placed close to the object of interest. Probes can supply a large amount of information in the form of excitation spectrum, emission quantum yield, lifetime, polarization etc. On the molecular level this information involves data on the rotational and translational motions of the molecule as well as the surrounding medium (Slavik, 1994).

Luminescence probes are ideally suited to monitor the molecular mobility of specific components (protein, carbohydrate, lipid) at specific sites in foods (Strasburg and Ludescher, 1995; Lakowicz, 1999). Luminescence spectroscopy in a microscope can potentially generate detailed three-dimensional maps of the distribution of molecular mobility in complex heterogeneous foods. Such maps should provide the molecular detail necessary for detailed physical-chemical models connecting food quality and stability to molecular structure and mobility.

Phosphorescence is especially useful for probing molecular mobility on the millisecond and longer time scale, which corresponds to the modes of local and 
segmental motion seen in solid phase proteins (Shah and Ludescher, 1993), due to the long lifetime of the triplet state $\left(10^{-4}-10 \mathrm{~s}\right)$. Phosphorescence is effectively and efficiently quenched by collision with molecular oxygen. It is thus possible to probe both the presence of oxygen and specific molecular properties of a matrix using an appropriate phosphorescent probe molecule.

Luminescence spectroscopy thus can provide a powerful set of tools for investigating the molecular mechanisms that control oxygen permeability through amorphous proteins (Simon-Lukasik and Ludescher, 2004; Lukasik and Ludescher, 2006 $\mathrm{a}, \mathrm{b})$.

Erythrosin B (Ery B; tetraiodofluorescein Figure I-7) is perhaps the most widely used phosphorescent probe of the molecular mobility of water-soluble and membranebound proteins because of its large extinction coefficient and high phosphorescence quantum yield even in aqueous solution. The Ludescher lab has successfully used Ery B phosphorescence to monitor molecular mobility in amorphous solid sugars and sugar alcohols (Shah \& Ludescher, 1995; Pravinata et al., 2005; Shirke et al., 2005, 2006; Shirke \& Ludescher, 2005), in solid gelatin (Simon-Lukasik and Ludescher, 2004; Lukasik \& Ludescher, 2006a,b), and in bovine serum albumin (Nack and Ludescher, 2006). 


\section{Figure I-7}

\section{$\underline{\text { Erythrosin B }}$}

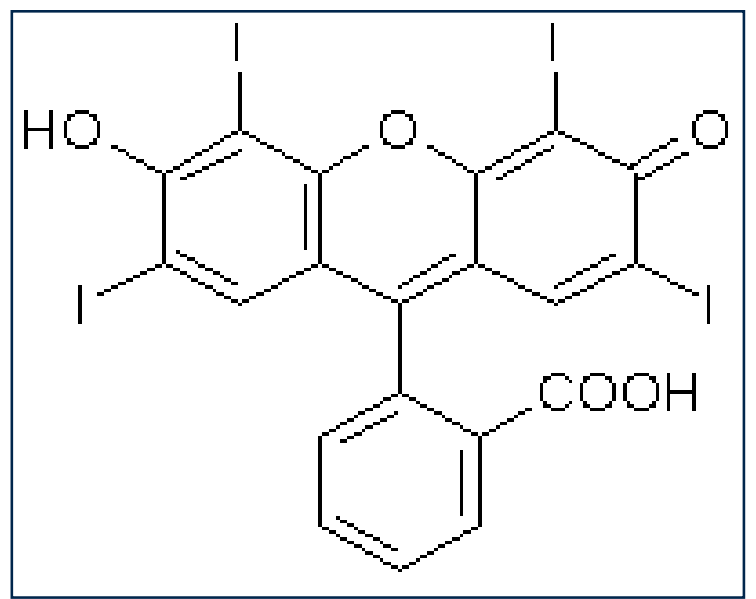

\section{Photophysics of luminescence}

Luminescence is a general term used to describe what occurs when a probe molecule absorbs photons of higher energy (excitation) and subsequently exits photons of lower energy (emission). This phenomenon is a function of a molecule's structure, which in luminescent molecules is usually characterized by an extended delocalized $\pi$-orbital system. 
Figure 1-8

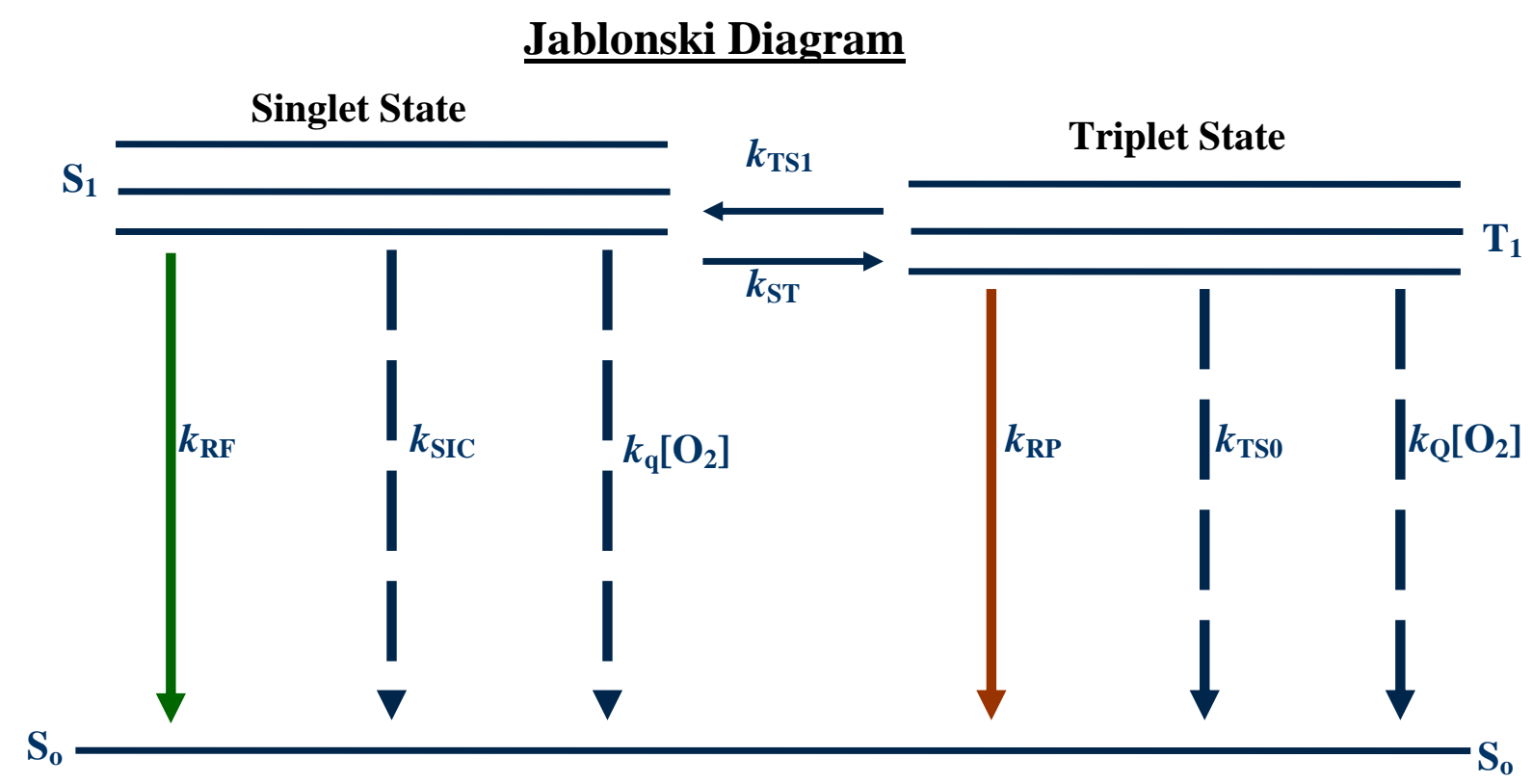

Abbreviations used :

$\mathbf{k}_{\mathbf{R F}}$ Rate of fluorescence emission

$\mathbf{k}_{\mathbf{R P}}$ Rate of phosphorescence emission

$\mathbf{k}_{\text {ST }}$ Rate of intersystem crossing from singlet to triplet state.

$\mathbf{k}_{\text {TS1 }}$ Rate of intersystem crossing from triplet to singlet state.

$\mathbf{k}_{\text {SIC }}$ Rate of interval conversion from $S_{1}$ state to $S_{0}$ state

$\mathbf{k}_{\text {TS0 }}$ Rate of intersystem crossing from triplet state $T_{1}$ to ground state $S_{0}$

$\mathbf{k}_{\mathbf{Q}}\left[\mathbf{O}_{2}\right]$ Rate of collisional quenching due to oxygen 
The Jablonski energy level diagram depicted in Figure I-8 is a schematic representation of the electronic energy levels (ground and excited) of the molecule. It summarizes the various photophysical events occurring during luminescence. During light absorption the ground state molecule makes an essentially instantaneous transition $\left(\sim 10^{-15} \mathrm{~s}\right)$ to one of the many vibrational levels of the first excited state $\left(\mathrm{S}_{1}\right)$; excess vibrational energy is rapidly $\left(\sim 10^{-12} \mathrm{~s}\right)$ dissipated to the solvent matrix to relax to the lowest vibrational level of $\mathrm{S}_{1}$. The molecule can return to the ground sate with emission of energy (a photon), this phenomenon is called fluorescence.

Along with fluorescence (rate $\mathrm{k}_{\mathrm{RF}}$ ), the excited state may engage in several deexcitation processes including non-radiative decay to $S_{0}$ through exchange of vibrational energy to the matrix (rate constant $\mathrm{k}_{\mathrm{SIC}}$ ), intersystem crossing from $\mathrm{S}_{1}$ to $\mathrm{T}_{1}$ (rate $\mathrm{k}_{\mathrm{ST}}$ ) and collisional quenching rate $\mathrm{k}_{\mathrm{q}}\left[\mathrm{O}_{2}\right]$. Along with phosphorescence (rate $\mathrm{k}_{\mathrm{RP}}$ ) occurs the deexcitation processes including non-radiative decay from $T_{1}$ to $S_{0}$ (rate $k_{T S 0}$ ), reverse intersystem crossing from $\mathrm{T}_{1}$ to $\mathrm{S}_{1}\left(\right.$ rate $\mathrm{k}_{\mathrm{TS} 1}$ ) and collisional quenching by oxygen $\mathrm{k}_{\mathrm{Q}}[\mathrm{O} 2]$.

The rate constants $\mathrm{k}_{\mathrm{RF}}, \mathrm{k}_{\mathrm{RP}}$ are fixed by the probe structure and largely unaffected by environments (Turro, 1991; Birks, 1970). The rate of quenching the triplet state by oxygen $\mathrm{k}_{\mathrm{Q}}\left[\mathrm{O}_{2}\right]$ is the product of a quenching constant $\mathrm{k}_{\mathrm{Q}}$ and the oxygen concentration $\left[\mathrm{O}_{2}\right]$ and this value is easily calculated from the difference of the lifetime's values under oxic and anoxic conditions. The non-radiative processes that depopulate the triplet state $\left(\mathrm{k}_{\mathrm{TS} 0}\right.$, and $\left.\mathrm{k}_{\mathrm{Q}}\left[\mathrm{O}_{2}\right]\right)$ are of great interest, because they give molecular information about the processes within the matrix. 


\section{Emission intensity}

Luminescence emission intensity is directly proportional to the emission quantum yield, $\Phi=$ photons emitted/photons absorbed. The emission quantum yield reflects the relative rates of the several de-excitation pathways available to the excited state of a chromophore. The quantum yield for fluorescence is given by the following ratio of rate constants (Lakowicz, 1999). The term $\mathrm{k}_{\mathrm{q}}\left[\mathrm{O}_{2}\right]$ describes the rate of collisional quenching of the excited state of the probe by oxygen.

$$
\Phi_{\mathrm{F}}=\mathrm{k}_{\mathrm{RF}} /\left(\mathrm{k}_{\mathrm{RF}}+\mathrm{k}_{\mathrm{SIC}}+\mathrm{k}_{\mathrm{ST}}+\mathrm{k}_{\mathrm{q}}\left[\mathrm{O}_{2}\right]\right)
$$

In the absence of specific quenchers in the local environment, the typical situation for fluorescence, Eq. (1) simplifies to:

$$
\Phi_{\mathrm{F}}=\mathrm{k}_{\mathrm{RF}} /\left(\mathrm{k}_{\mathrm{RF}}+\mathrm{k}_{\mathrm{SIC}}+\mathrm{k}_{\mathrm{ST}}\right)
$$

In the case of phosphorescence, the quantam yield for phosphoresence $\Phi_{\mathrm{P}}$ is the product of the quantam yield for $T_{1}$ formation $\left(\Phi_{T}\right)$ times the probability of emission from $T_{1}\left(q_{P}\right)$ (Hurtubise, 1990):

$$
\Phi_{\mathrm{P}}=\Phi_{\mathrm{T}} \mathrm{q}_{\mathrm{P}}=\left\{k_{\mathrm{ST}} /\left(k_{\mathrm{RF}}+k_{\mathrm{SIC}}+k_{\mathrm{ST}}+k_{\mathrm{Q}}\left[O_{2}\right]\right)\right\}\left\{k_{R \mathrm{P}} /\left(k_{R \mathrm{P}}+k_{T S O}+\mathrm{k}_{\mathrm{TS} 1}+k_{\mathrm{Q}}\left[O_{2}\right]\right)\right\}
$$

The quantam yield for delayed fluorescence in the absence of oxygen is the product of the quantam yield of fluorescence and the probabilities of intersystem crossing from $S_{1}$ to $\mathrm{T}_{1}$ and also from $\mathrm{T}_{1}$ to $\mathrm{S}_{1}$ (Duchowicz et al., 1998)

$$
\Phi_{\mathrm{DF}}=\Phi_{\mathrm{F}}\left\{k_{\mathrm{ST}} /\left(k_{\mathrm{RF}}+k_{\mathrm{SIC}}+k_{\mathrm{ST}}\right)\right\}\left\{k_{T S I} /\left(k_{R \mathrm{P}}+k_{T S O}+\mathrm{k}_{\mathrm{TS} 1}\right)\right\}
$$

By comparing the emission intensities of delayed fluorescence and phosphorescence it is possible to obtain information about the rate constants for intersystem crossing $\mathrm{k}_{\mathrm{TS} 1}$. 


$$
\mathrm{I}_{\mathrm{DF}} / \mathrm{I}_{\mathrm{P}}=\Phi_{\mathrm{F}}\left\{\mathrm{k}_{\mathrm{ST}} / \mathrm{k}_{\mathrm{F}}\right\}\left\{\mathrm{k}_{\mathrm{TS} 1} / \mathrm{k}_{\mathrm{P}}\right\} /\left\{\mathrm{k}_{\mathrm{ST}} / \mathrm{k}_{\mathrm{F}}\right\}\left\{\mathrm{k}_{\mathrm{RP}} / \mathrm{k}_{\mathrm{P}}\right\}=\Phi_{\mathrm{F}} \mathrm{k}_{\mathrm{TS} 1} / \mathrm{k}_{\mathrm{RP}}
$$

Where $\mathrm{k}_{\mathrm{F}}$ and $\mathrm{k}_{\mathrm{P}}$ are the total decay rates for the de-excitation of the singlet and triplet state, respectively.

This ratio is highly temperature dependent due to the additional energy required to go from the $T_{1}$ to the $S_{1}$ energy level and can be described by the following equation where $\Delta \mathrm{E}_{\mathrm{TS}}$ is the effective energy gap between the triplet and singlet states.

$$
\Phi_{\mathrm{F}} \mathrm{k}_{\mathrm{TS} 1} / \mathrm{k}_{\mathrm{RP}}=\left\{\Phi_{\mathrm{F}} / \mathrm{k}_{\mathrm{RP}}\right\} \mathrm{k}_{\mathrm{TS} 1}{ }^{\circ} \exp \left(-\Delta \mathrm{E}_{\mathrm{TS}} / \mathrm{RT}\right)
$$

The slope of the Van't Hoff plot of the natural log of the ratio of delayed fluorescence $\left(I_{D F}\right)$ to phosphorescence $\left(I_{P}\right)$ intensity versus inverse temperature provides a measure of $\Delta \mathrm{E}_{\mathrm{TS}}$ (Duchowicz, et al., 1998).

Phosphorescence intensity decays are non-exponential and a stretched exponential decay function has been shown to be appropriate to describe the wide distribution of relaxation times (Champion et al., 2000; Richert, 2000).

$$
I(t)=I_{0} \exp \left[-(t / \tau)^{\beta}\right]+c
$$

Where $\mathrm{I}_{0}$ is the initial amplitude, $\tau$ is the stretched exponential lifetime, $\beta$ is an exponent varying from $0 \sim 1$ and characterizing the distribution of lifetime and $\mathrm{c}$ is a constant. The use of a stretched exponential model provides a direct measurement of a continuous distribution of lifetimes, which is appropriate for describing a complex glass possessing a distribution of relaxation times for the dynamic molecular processes.

The phosphorescence lifetimes were used to calculate the rate constants associated with the various processes that depopulate the excited triplet state. In an 
amorphous solid in the absence of oxygen the temperature dependence of $k_{P}$ can be written as follows:

$$
\mathrm{k}_{\mathrm{P}}(\mathrm{T})=\mathrm{k}_{\mathrm{RP}}+\mathrm{k}_{\mathrm{TS} 1}(\mathrm{~T})+\mathrm{k}_{\mathrm{TS} 0}(\mathrm{~T})
$$

But in the presence of oxygen the de-excitation of the triplet state is also influenced by the quencher species. The term $\mathrm{k}_{\mathrm{Q}}\left[\mathrm{O}_{2}\right]$ describes the rate of collisional quenching of the excited state of the probe by oxygen. Thus in the presence of oxygen $\mathrm{k}_{\mathrm{P}}(\mathrm{T})$ includes an additional term $\mathrm{k}_{\mathrm{Q}}\left[\mathrm{O}_{2}\right]$.

$$
\mathrm{k}_{\mathrm{P}}(\mathrm{T})=\mathrm{k}_{\mathrm{RP}}+\mathrm{k}_{\mathrm{TS} 1}(\mathrm{~T})+\mathrm{k}_{\mathrm{TS} 0}(\mathrm{~T})+\mathrm{k}_{\mathrm{Q}}(\mathrm{T})\left[\mathrm{O}_{2}\right]
$$

This equation can be used to calculate $\mathrm{k}_{\mathrm{Ts} 0}$, the rate of collisional quenching to the ground state where $\mathrm{k}_{\mathrm{RP}}, \mathrm{k}_{\mathrm{TS} 1}, \mathrm{k}_{\mathrm{TS} 0}$ and $\mathrm{k}_{\mathrm{Q}}\left[\mathrm{O}_{2}\right]$ are known. $\mathrm{k}_{\mathrm{RP}}$, the radiative decay rate of the triplet state, is $41 \mathrm{~s}^{-1}$ for erythrosin B (Duchowicz et al.,1998; Lettinga et al., 2000). The value of $\mathrm{k}_{\mathrm{TS} 1}(\mathrm{~T})$ was estimated using $\mathrm{k}^{\circ} \mathrm{TS} 1 \approx 6.5 \times 10^{7} \mathrm{~s}^{-1}$ based on the data from (Duchowicz et al., 1998).

$\mathrm{k}_{\mathrm{TS} 1}$, the rate of reverse intersystem crossing from the excited triplet state to the excite singlet state, depends on $\Delta E_{T S}$, the energy gap between $S_{1}$ and $T_{1}$

$$
\mathrm{k}_{\mathrm{TS} 1}(\mathrm{~T})=\mathrm{k}^{\mathrm{o}} \mathrm{TS} 1 \exp \left(-\Delta \mathrm{E}_{\mathrm{TS}} / \mathrm{RT}\right)
$$

In order to study the oxygen diffusion in the $\beta$-Lactoglobulin matrix $\mathrm{k}_{\mathrm{Q}}\left[\mathrm{O}_{2}\right]$ can easily be calculated from the difference between the decay rate in the presence of oxygen and the absence of oxygen

$$
\mathrm{k}_{\mathrm{Q}}\left[\mathrm{O}_{2}\right]=1 / \tau(\text { Air })-1 / \tau_{0}(\text { Nitrogen })
$$




\section{Emission energy (wavelength)}

Both absorption and emission are distributed over a wide range of energies and thus wavelengths. The energy of an electronic transition is also quite sensitive to dipolar interactions between a polar chromophore and the molecules in its immediate environment (the solvent/matrix shell) (Ludescher et al., 2001). Because both absorption and emission occur on time scales $\left(10^{-15} \mathrm{~s}\right)$ much faster than the molecular motions of solvent molecules $\left(10^{-12} \mathrm{~s}\right)$, the non-equilibrium solvent shells surrounding the newly formed excited or ground states raise the energies of these states; the rate and extent of dipolar relaxation due to molecular motion in the non-equilibrium solvent shells thus has a dramatic effect on the emission energy (wavelength) distribution. In solutions of high viscosity, and especially in amorphous rubbery and glassy solids, solvent relaxation is slow because molecular mobility is slow; solvent relaxation may occur on the microsecond time scale or longer. The time scale for relaxation in such cases is often comparable to or longer than the phosphorescence lifetime. So phosphorescence spectroscopy can be an effective tool to study the dipolar relaxation in a protein matrix (Ludescher et al., 2001).

Edible protein films are the active subject of research in the field of food technology. Applied research on solid state proteins in edible film systems has focused primarily on practical aspects, particularly mechanical and barrier properties. However, these studies have failed to look at the finer molecular details pertaining to edible films. A complete picture of dynamic events occurring in a protein matrix, and a correlation to the critical modes of molecular motion is necessary inorder to most effectively manipulate food materials to ensure optimal functionality. Edible protein films are often 
plasticized by small molecular weight compounds. These small molecular weight compounds can have a dramatic impact on the film's functionality. So, the present study aims at looking into the effect different additives that are potentially used in edible films on the molecular mobility and oxygen permeability of the $\beta$-Lg matrix. This dissertation involves the application of a versatile non- intrusive spectroscopic technique that can be potentially used to detect and measure molecular mobility and permeability in a wide variety of food and food ingredients. As compared to other, more traditional analytical methods these luminescence studies provide a unique perspective that will ideally help optimize the use of $\beta$-Lg in emerging food applications.

\section{$\underline{\text { Research Objectives }}$}

The objectives of this study are as follows:

Objective 1: Investigate the local molecular mobility and oxygen permeability in amorphous $\beta$-Lactoglobulin films as a function of temperature using phosphorescence of erythrosin B

Objective 2: Detect the effect of sugars on the molecular mobility and oxygen transport of the amorphous $\beta$-Lactoglobulin matrix

Objective 3: To compare the site heterogeneity in amorphous $\beta$-Lactoglobulin and $\beta$ Lactoglobulin sugar mixtures

Objective 4: To study the effect of various polyols on the matrix mobility and oxygen permeability in $\beta$-Lactoglobulin films

Objective 5: To study the molecular mobility in composite films of $\beta$-Lactoglobulin with plasticizer and sugar and sugar- sugar alcohol mixtures 
Objective 6: Detect the effect of a globular protein bovine serum albumin (BSA) on the molecular mobility and oxygen permeability of the amorphous $\beta$-Lactoglobulin matrix

Objective 7: Use phosphorescent techniques to study the effect of fatty acids on the molecular mobility and oxygen permeability in the amorphous $\beta$-Lactoglobulin matrix as a function of temperature.

Objective 8: Use of Retinol / Retinal as a phosphorescent probe in the amorphous $\beta$ Lactoglobulin matrix 


\section{$\underline{\text { References }}$}

Andrade, S. M. and Costa, S. M. B. (2002). Spectroscopic studies on the interaction of a water soluble porphyrin and two drug carrier proteins. Biophysical Journal. 82, 1607-1619.

Anker, M., Stading, M., and Hermansson, A. (2001). Aging of Whey Protein Films and the Effect on Mechanical and Barrier Properties. J. Agric. Food Chem. 49, 989-995.

Avena-Bustillos, R. J. and Krochta, J. M. (1993). Water vapor permeability of caseinate-based edible films as affected by $\mathrm{pH}$, calcium crosslinking and lipid content. J. Food Sci. 58, 904-907.

Ayranci, E. and Tunc, S. (2003/3). A method for the measurement of the oxygen permeability and the development of edible films to reduce the rate of oxidative reactions in fresh foods. Food Chemistry. 80, 423-431.

Biliaderis, C. G., Lazaridou, A., and Arvanitoyannis, I. (1999). Glass transition and physical properties of polyol-plasticized pullulan-starch blends at low moisture. Carbohydr. Polym. 40, 29-47.

Blanch, E. W., Hecht, L., and Barron, L. D. (1999). New insight into the pH-dependent conformational changes in bovine $\beta$ - lactoglobulin from Raman optical activity. Protein Science. 8, 1362-1367.

Brandenburg, A. H., Weller, C. L., and Testin, R. F. (1993). Edible films and coatings from soy protein. J. Food Sci. 58, 1086-1089.

Buettner, A. V. (1964). Flash photolysis in thin films of gelatin and other polymers. Journal of Physical Chemistry. 68, 3253-3259.

Buitink, J., Claessens, M. M. A. E., Hemminga, M. A., and Hoekstra, F. A. (1998). Influence of Water Content and Temperature on Molecular Mobility and Intracellular Glasses in Seeds and Pollen. Plant Physiol. 118, 531-541.

Campanella, L., Antiochia, R., Dragone, R., and Lavagnini, I. (2005). Determination of oxygen permeability of food wrapping films by an amperometric sensor. Int. J. Environ. Anal. Chem. 85, 959-969.

Chan, R. K., Pathmanathan, K., and Johari, G. P. (1986). Dielectric relaxations in the liquid and glassy states of glucose and its water mixtures. J. Phys. Chem. 90, 6358-6362.

Chang, B. S., Beauvais, R. M., Dong, A., and Carpenter, J. F. (1996). Physical factors affecting the storage stability of freeze-dried interleukin-1 receptor antagonist: Glass transition and protein conformation. Archives of Biochemistry and Biophysics. 331, 249-258. 
Charlesworth, J. M. and Gan, T. H. (1996). Kinetics of Quenching of Ketone Phosphorescence by Oxygen in a Glassy Matrix. J. Phys. Chem. 100, 14922-14927.

Chick, J. and Ustunol, Z. (1998). Mechanical and barrier properties of lactic acid and rennet precipitated casein-based edible films. J. Food Sci. 63, 1024-1027.

Choi, W. and Han, J. H. (2001). Physical and mechanical properties of pea-protein-based edible films. J. Food Sci. 66, 319-322.

Collini, M., D'Alfonso, L., and Baldini, G. (2000). New insight on $\beta$-lactoglobulin binding sites by 1-anilinonaphthalene-8-sulfonate fluorescence decay. Protein Science. 9, 1968-1974.

Craig, I. D., Parker, R., Rigby, N. M., Cairns, P., and Ring, S. G. (2001). Maillard reaction kinetics in model preservation systems in the vicinity of the glass transition: experiment and theory. J. Agric. Food Chem. 49, 4706-4712.

Cuq, B., Gontard, N., Cuq, J., and Guilbert, S. (1997). Selected Functional Properties of Fish Myofibrillar Protein-Based Films As Affected by Hydrophilic Plasticizers. J. Agric. Food Chem. 45, 622-626.

D'Alfonso, L., Collini, M., and Baldini, G. (1999). Evidence of heterogeneous 1anilinonaphthalene-8-sulfonate binding to $\beta$-lactoglobulin from fluorescence spectroscopy. Biochimica et Biophysica Acta - Protein Structure and Molecular Enzymology. 1432, 194-202.

Debeaufort, F., Quezada-Gallo, J., and Voilley, A. (2002). Edible films and coatings as aroma barriers. Protein-Based Films Coat. 579-600.

Duchowicz, R., Ferrer, M. L., and Acuña, A. U. (1998). Kinetic Spectroscopy of Erythrosin Phosphorescence and Delayed Fluorescence in Aqueous Solution at Room Temperature. Photochemistry and Photobiology. 68, 494-501.

Dufour, E., Marden, M. C., and Haertle, T. (1990). b-Lactoglobulin binds retinol and protoporphyrin IX at two different binding sites. FEBS Lett. 277, 223-226.

Dybing, S. T. and Smith, D. E. (1991). Relation of chemistry and processing procedures to whey protein functionality: a review. Cultured Dairy Products Journal. 26, 4-9, 11-12.

Erdohan, Z. O. and Turhan, K. N. (2005). Barrier and mechanical properties of methylcellulosewhey protein films. Packag. Technol. Sci. 18, 295-302.

F. Franks. (1990). Cryo. Lett. 11, 93-100.

Faivre, A., Niquet, G., Maglione, M., Fornazero, J., Jal, J. F., and David, L. (1999). Dynamics of sorbitol and maltitol over a wide time-temperature range. European Physical Journal B: Condensed Matter Physics. 10, 277-286. 
Franks, F., Hatley, R. H. M., and Mathias, S. F. (1991). Materials science and the production of shelf-stable biologicals. BioPharm. 4, 38-42.

Fugate, R. D. and Song, P. (1980). Spectroscopic characterization of b-lactoglobulin-retinol complex. Biochimica et Biophysica Acta, Protein Structure. 625, 28-42.

Gangasharan and Murthy, S. S. N. (1993). Study of a-, b-, and g-relaxation processes in some supercooled liquids and supercooled plastic crystals. J. Chem. Phys. 99, 9865-9873.

Gennadios, A., McHugh, T.H., Weller, C.L., and Krochta, J.M. (1994.). In Edible Coatings and Films to Improve Food QualityTechnomic Publishing Co.,Inc., Lancaster, PA.

Gennadios, A., McHugh, T. H., Weller, C. L., and Krochta, J. M. (1994). Edible coatings and films based on proteins. Edible Coat.Films Improve Food Qual. 201-277.

Gontard, N., Thibault, R., Cuq, B., and Guilbert, S. (1996). Influence of relative humidity and film composition on oxygen and carbon dioxide permeabilities of edible films. Journal of Agricultural and Food Chemistry. 44, 1064-1069.

Gontard, N., Guilbert, S., and Cuq, J. L. (1992). Edible wheat gluten films: influence of the main process variables on film properties using response surface methodology. J. Food Sci. 57, 190-5, 199.

Guilbert, S. (1986). Food packaging and preservation. Elsevier Applied Science, London. 371.

Hancock, B. C. and Zografi, G. (1997). Characteristics and Significance of the Amorphous State in Pharmaceutical Systems. Journal of Pharmaceutical Sciences. 86, .

Hill, J. J., Shalaev, E. Y., and Zografi, G. (2005). Thermodynamic and dynamic factors involved in the stability of native protein structure in amorphous solids in relation to levels of hydration. J. Pharm. Sci. 94, 1636-1667.

Hurtubise, R. J. (1990). Phosphorimetry:Theory,Instrumentation and applications. .

Jangchud, A. and Chinnan, M. S. (1999). Properties of peanut protein film: sorption isotherm and plasticizer effect. Lebensmittel-Wissenschaft und -Technologie. 32, 89-94.

Karel, M. and Saguy, I. (1991). Effects of water on diffusion in food systems. Adv. Exp. Med. Biol. 302, 157-173.

Khwaldia, K., Banon, S., Desobry, S., and Hardy, J. (2004). Mechanical and barrier properties of sodium caseinate-anhydrous milk fat edible films. International Journal of Food Science and Technology. 39, 403-411.

Kinsella, J. E. and Whitehead, D. M. (1989). Proteins in whey: chemical, physical, and functional properties. Adv. Food Nutr. Res. 33, 343-438. 
Korolev, V. V. and Bazhin, N. M. (2000). Processes of luminescence quenching in study of molecular oxygen diffusion in glassy matrices. Spectrochim. Acta, Pt. A: Mol. Biomol. Spectrosc. 56A, 2501-2507.

Krochta, J.M. and De-Mulder-Johnston, C.D. (1997). Edible and biodegradable polymer films: Challenges and opportunities. Food Technol. 51, 7.

Lakowicz, J. R. (1999). Principles of fluorescence spectroscopyKluwer Academic/Plenum Press, Dordrecht/New York.

Larsen, H., Kohler, A., and Magnus, E. M. (2000). Oxygen ingress rate method - An alternative method to Ox-tran for measuring oxygen transmission rate of whole packages. Packag. Technol. Sci. 13, 233-241.

Ludescher, R. D. (1990/7). Molecular dynamics of food proteins: experimental techniques and observations. Trends in Food Science \& Technology. 1, 145-149.

Lukasik, K. V. and Ludescher, R. D. (2006/1). Effect of plasticizer on dynamic site heterogeneity in cold-cast gelatin films. Food Hydrocolloids. 20, 88-95.

Lukasik, K. V. and Ludescher, R. D. (2006/1). Molecular mobility in water and glycerol plasticized cold- and hot-cast gelatin films. Food Hydrocolloids. 20, 96-105.

Lukasik, K. V. and Ludescher, R. D. (2005). Effect of plasticizer on dynamic site heterogeneity in cold-cast gelatin films. Food Hydrocoll. 20, 88-95.

Lukasik, K. V. and Ludescher, R. D. (2005). Molecular mobility in water and glycerol plasticized cold- and hot-cast gelatin films. Food Hydrocoll. 20, 96-105.

Mate, J. I. and Krochta, J. M. (1996). Comparison of Oxygen and Water Vapor Permeabilities of Whey Protein Isolate and b-Lactoglobulin Edible Films. J. Agric. Food Chem. 44, 30013004.

McHugh, T. H., Aujard, J. F., and Krochta, J. M. (1994). Plasticized whey protein edible films: water vapor permeability properties. J. Food Sci. 59, 416-19, 423.

Miller, K. S. and Krochta, J. M. (1997). Oxygen and aroma barrier properties of edible films: a review. Trends Food Sci. Technol. 8, 228-237.

Moran, G. R. and Jeffrey, K. R. (1999). A study of the molecular motion in glucose/water mixtures using deuterium nuclear magnetic resonance. J. Chem. Phys. 110, 3472-3483.

Moyls, L., Hocking, R., Beveridge, T., and Timbers, G. (1992). Exponential decay method for determining gas transmission rate of films. Trans. ASAE. 35, 1259-1265. 
Nack, T. J. and Ludescher, R. D. (2006). Molecular Mobility and Oxygen Permeability in Amorphous Bovine Serum Albumin Films. Food Biophysics. 1557-1858.

Papiz, M. Z., Sawyer, L., and Eliopoulos, E. E. (1986). The structure of $\beta$-lactoglubulin and its similarity to plasma retinol-binding protein. Nature. 324, 383-385.

Parris, N. and Coffin, D. R. (1997). Composition Factors Affecting the Water Vapor Permeability and Tensile Properties of Hydrophilic Zein Films. J. Agric. Food Chem. 45, 1596-1599.

Pravinata, L. C., You, Y., and Ludescher, R. D. (2005). Erythrosin B Phosphorescence Monitors Molecular Mobility and Dynamic Site Heterogeneity in Amorphous Sucrose. Biophys. J. 88, 3551-3561.

Richert, R. (2001). Spectral selectivity in the slow b-relaxation of a molecular glass. Europhys. Lett. 54, 767-773.

Roos, Y. H. (1995). Phase Transitions in Foods.

Sartor, G. and Johari, G. P. (1994). Calorimetric studies of the kinetic unfreezing of molecular motions in hydrated lysozyme, hemoglobin, and myoglobin. Biophys. J. 66, 249-258.

Shah, N. K. and Ludescher, R. D. (1995). Phosphorescence Probes of the Glassy State in Amorphous Sucrose. Biotechnol. Prog. 11, 540-544.

Shirke, S., Takhistov, P., and Ludescher, R. D. (2005). Molecular Mobility in Amorphous Maltose and Maltitol from Phosphorescence of Erythrosin B. J. Phys. Chem. B. 109, 16119-16126.

Simon-Lukasik, K. V. and Ludescher, R. D. (2004/7). Erythrosin B phosphorescence as a probe of oxygen diffusion in amorphous gelatin films. Food Hydrocolloids. 18, 621-630.

Slade, L. and Levine, H. (1995). Glass transitions and water-food structure interactions. Adv. Food Nutr. Res. 38, 103-269.

Slavik, J. (1994). Fluorescent probes in cellular and molecular biologyCRC Press, Boca Raton, FL.

Sobral, P. J. A., Menegalli, F. C., Hubinger, M. D., and Roques, M. A. (2001). Mechanical, water vapor barrier and thermal properties of gelatin-based edible films. Food Hydrocoll. 15, 423-432.

Sothornvit, R. and Krochta, J. M. (2000). Water vapor permeability and solubility of films from hydrolyzed whey protein. J. Food Sci. 65, 700-703. 
Srickley, R. G. and Anderson, B. D. (1996). Solid-state stability of human insulin I. Mechanism and the effect of water on the kinetics of degradation in lyophiles from $\mathrm{pH} 2-5$ solutions. Pharm. Res. 13, 1142-1153.

Strasburg, G. M. and Ludescher, R. D. (1995). Theory and applications of fluorescence spectroscopy in food research. Trends Food Sci. Technol. 6, 69-75.

Tanada-Palmu, P. S. and Grosso, C. (2002). Edible wheat gluten films: Development, mechanical and barrier properties and application to strawberries (Fragaria ananassa). Boletim do Centro de Pesquisa e Processamento de Alimentos. 20, 291-308.

Tanaka, M., Iwata, K., Sanguandeekul, R., Handa, A., and Ishizaki, S. (2001). Influence of plasticizers on the properties of edible films prepared from fish water-soluble proteins. Fisheries Science. 67, 346-351.

Torres, J. A. (1994). Edible films and coatings from proteins. IFT Basic Symposium Series. 9, 467-507.

Van Dusschoten, D., Tracht, U., Heuer, A., and Spiess, H. W. (1999). Site Specific Rotational Mobility of Anhydrous Glucose near the Glass Transition As Studied by 2D Echo Decay 13C NMR. Journal of Physical Chemistry A. 103, 8359-8364.

Wada, R., Fujita, Y., and Kitabatake, N. (2006/6). Effects of heating at neutral and acid pH on the structure of [beta]-lactoglobulin A revealed by differential scanning calorimetry and circular dichroism spectroscopy. Biochimica et Biophysica Acta (BBA) - General Subjects. 1760, 841-847.

Wan, V. C., Kim, M. S., and Lee, S. (2005). Water vapor permeability and mechanical properties of soy protein isolate edible films composed of different plasticizer combinations. $J$. Food Sci. 70, E387-E391.

Wolkers, W. F., Alberda, M., Koornneef, M., Léon-Kloosterziel, K. M., and Hoekstra, F. A. (1998). Properties of proteins and the glassy matrix in maturation-defective mutant seeds of Arabidopsis thaliana. Plant Journal. 16, 133-143. 


\section{Chapter II: Molecular mobility and oxygen permeability in amorphous $\beta$ - Lactoglobulin films ${ }^{1}$}

\section{Introduction}

Edible films are able to control mass transfer and thus extend shelf life and otherwise improve the quality of foods and pharmaceuticals (Krochta, 1997); thus, they show much promise to not only reduce the use of synthetic, non-biodegradable packaging materials but also, perhaps, to introduce new functionalities. Upon drying, proteins can form a continuous solid matrix with appropriate mechanical and barrier properties for use as edible films. Edible films have been made from corn zein (Parris \& Coffin, 1997), wheat gluten (Gontard, Guilbert, \& Cuq, 1992), peanut globulins (Jangchud \& Chinnan, 1999), soy globulins (Brandenburg, Weller, \& Testin, 1993), casein (Avena-Bustillos \& Krochta, 1993), and whey proteins (McHugh, Aujard, \& Krochta, 1994), among others (Krochta \& De Mulder-Johnston, 1997). Whey proteins are especially appropriate raw materials for edible films due to their useful functionality and their widespread availability in relatively pure form as a byproduct of cheese manufacture worldwide.

$\beta$-Lactoglobulin $(\beta$ - $\mathrm{Lg})$ is the most abundant protein in bovine whey, comprising almost $50 \%$ of the total protein (Dybing \& Smith, 1991); therefore, its properties are very important to the functionality of milk and isolated whey proteins. $\beta$ - $\mathrm{Lg}$ is a water soluble, globular protein of $18,300 \mathrm{Da}$, corresponding to a polypeptide of 162 residues for all genetic variants. At physiological $\mathrm{pH}$ it is a dimer, but dissociates into monomers below pH 3.0 (Fugate \& Song, 1980). It has five cysteine residues, three of which form two possible disulfide bonds; the unusual properties of these labile cysteines influence many of its functionalities in foods (Kinsella \& Whitehead, 1989). $\beta$-Lg has a protein fold 
composed of two antiparallel $\beta$-sheets and shows a remarkable similarity to plasma retinol-binding protein (Papiz, Sawyer, \& Eliopoulos, 1986); it is a member of the lipocalin super family of transporters of hydrophobic molecules.

Oxygen is responsible for many degradative reactions in foods and pharmaceuticals including growth of microorganisms, enzymatic browning, and the oxidation of specific molecules including proteins, vitamins, unsaturated fatty acids, and others (Fennema, 1996). The permeability of protein films to oxygen is, therefore, a fundamental aspect of their functionality. A number of techniques have been used to measure gas permeability through solid materials (McHugh et al., 1994); most of these techniques, however, can only measure transport through autonomous (free standing) films. Despite the obvious fact that both mechanical and barrier properties of edible films are governed by temperature, humidity and plasticizer content (Le Meste, Champion, Roudaut, Blond, \& Simatos, 2002), few studies of oxygen permeability through edible films or amorphous biomaterials make any attempt to investigate the molecular mechanism(s) controlling oxygen transport (Nack \& Ludescher, 2006). The importance of such studies for developing rational methods to formulate novel edible films with appropriate functionality is evident.

Luminescence spectroscopy provides a powerful set of tools for investigating the molecular mechanisms that control oxygen permeability through amorphous proteins (Lukasik \& Ludescher (2006a) and Lukasik \& Ludescher (2006b); Simon-Lukasik \& Ludescher, 2004). Luminescence data are unique to a given probe or class of probes (Slavik, 1994). Depending on the molecular structure and the presence of specific functional groups, such as carboxylic acids and amines, luminescence probes may be 
sensitive to polarity, $\mathrm{pH}$, or matrix mobility, thus providing highly specific and detailed information about the local molecular environment surrounding the probe. In addition, phosphorescence is effectively and efficiently quenched by collision with molecular oxygen. It is thus possible to probe both the presence of oxygen and specific molecular properties of a matrix using an appropriate phosphorescent probe molecule.

Due to the long lifetime of the triplet state $(10-4-10 \mathrm{~s})$, phosphorescence is especially useful for probing molecular mobility on the millisecond and longer time scales and is thus sensitive to the modes of local and segmental motion seen in solid protein (Shah \& Ludescher, 1993). Erythrosin B (Ery B; tetraiodofluorescein) is perhaps the most widely used phosphorescent probe of the molecular mobility of water-soluble and membrane-bound proteins because of its large extinction coefficient and high phosphorescence quantum yield even in aqueous solution. We have successfully used Ery B phosphorescence to monitor molecular mobility in amorphous solid sugars and sugar alcohols (Pravinata, You, \& Ludescher, 2005; Shah \& Ludescher, 1995; Shirke \& Ludescher (2005a) and Shirke \& Ludescher (2005b); Shirke, Takhistov, \& Ludescher, 2005), in solid gelatin (Lukasik \& Ludescher (2006a) and Lukasik \& Ludescher (2006b); Simon-Lukasik \& Ludescher, 2004), and in amorphous solid bovine serum albumin (Nack \& Ludescher, 2006). Here, we report a study of the effect of temperature on the molecular mobility and oxygen permeability in amorphous films of $\beta$ - $\mathrm{Lg}$ using Ery B phosphorescence. These data provide insight into the molecular mechanism(s) controlling oxygen permeability in this protein matrix as well as providing indications of a softening transition within the protein film that modulates oxygen permeability. 


\section{Materials and methods}

Sample preparation. $\beta$-lactoglobulin $(\beta-\mathrm{Lg})$ was purchased from Sigma Chemical Co (St. Louis, MO); solutions were prepared in distilled de-ionized water at a concentration of $\sim 10 \mathrm{mg} / \mathrm{mL}$. In order to define and minimize the presence of counter ions, this solution was extensively dialyzed using 12-14 $\mathrm{kDa}$ molecular weight cutoff cellulose tubing (Spectrum, Houston, TX) at $5^{\circ} \mathrm{C}$, first against $0.1 \mathrm{M} \mathrm{KCl}$ and then against distilled de-ionized water. The dialyzed solution was then filtered through a 0.2 $\mu \mathrm{m}$ Acrodisc membrane filter (Pall Corporation, Ann Arbor, MI) to remove particulate matter. The protein concentration was determined after dialysis by absorbance at $280 \mathrm{~nm}$ using an extinction coefficient of $0.96(\mathrm{~g} / \mathrm{L})^{-1} \mathrm{~cm}^{-1}$. The solution used to make films had a protein concentration of $6-8 \mathrm{mg} / \mathrm{mL}$.

Erythrosin B (EryB; tetraiodofluorescein), purchased from Molecular Probes, Inc. (Eugene, OR), was dissolved in spectrophotometric grade $\mathrm{N}, \mathrm{N}$-dimethylformamide (DMF; Aldrich Chemical, Milwaukee, WI) to make either $10 \mathrm{mM}$ or100 mM stock solutions. The solvent DMF was selected for probe stability during long time storage and the concentrations were selected in order to simplify the addition of probe to the protein solutions. Aliquots of dye stock were added to protein solutions to give a molar ratio of EryB: $\beta-\operatorname{Lg}$ of $4: 1$.

Preparation of protein films. To make glassy films, $15 \mu \mathrm{l}$ of protein/erythrosin solution were spread on approximately one third of a quartz slide $(30 \times 13.5 \times 0.6 \mathrm{~mm}$; custom made by NSG Precision Cells, Farmingdale, NY). Before use the quartz slides were soaked in Terg-A-Zyme (Alconox, Inc., NY) soap solution overnight to remove surface impurities, washed with de-ionized water, soaked in ethanol for 1-2 hours and 
dried with acetone. Occasionally the slides were soaked in acid for thorough cleaning. The protein/erythrosin films, $\sim 0.05 \mathrm{~mm}$ thick when dried, were visually checked for good spreading, uniformity, and consistency. The slides were stored at room temperature against the desiccant DrieRite and kept in an atmosphere of $\mathrm{P}_{2} \mathrm{O}_{5}$ in order to maintain $0 \%$ $\mathrm{RH}$. The slides were stored in a desiccator for at least a week and protected from light to prevent any photobleaching of the erythrosin B. The desiccant was refreshed as necessary.

Luminescence measurements. All measurements were conducted using a Cary Eclipse spectrophotometer (Varian Instruments, Walnut Creek, CA). The temperature was controlled using a TLC 50 thermoelectric heating/cooling system (Quantum Northwest, Spokane, WA) with a sample compartment fitted with a jacketed cover. For luminescence measurements necessitating the absence of oxygen (which is a phosphorescence quencher) the samples were flushed with nitrogen gas for at least 15 minutes prior to collecting data. An oxygen free nitrogen stream was generated by passage of high purity nitrogen through a Supelco (Bellefonte, PA) carrier gas purifier.

Quartz slides containing protein film were placed on-end on the diagonal of a standard $1 \mathrm{~cm}$ x $1 \mathrm{~cm}$ quartz fluorescence cuvette which was capped with a lid having inlet and outlet ports for gas lines. The outside of the cuvette was flushed with dry air to prevent condensation on the faces of the cuvette for experimental conditions below room temperature.

Delayed luminescence emission spectra were collected from 535-800 nm (10nm bandwidth) using excitation of $525 \mathrm{~nm}$ (10nm bandwidth) over the temperature range from $-20^{\circ} \mathrm{C}$ to $120^{\circ} \mathrm{C}$. For the scan, each data point (collected at $1 \mathrm{~nm}$ interval, $0.1 \mathrm{~s}$ 
averaging time) was collected from a single lamp flash with a $0.2 \mathrm{~ms}$ delay, $0.5 \mathrm{~ms}$ gate time and $4.0 \mathrm{~ms}$ total decay time.

For lifetime measurements as a function of temperature samples were excited at $540 \mathrm{~nm}(20 \mathrm{~nm}$ bandwidth) and emission transients collected at $690 \mathrm{~nm}(20 \mathrm{~nm}$ bandwidth) at temperatures ranging from $-20^{\circ} \mathrm{C}$ to $120^{\circ} \mathrm{C}$. Before collecting data, films were equilibrated at each temperature for 15 minutes. The measurements were taken in the presence or absence of air (under a nitrogen purge). Each time-resolved decay transient was the average of 100 cycles and for each cycle data was collected from a single lamp flash with a delay of $0.1 \mathrm{~ms}$, a $0.02 \mathrm{~ms}$ gate time, and $4.0 \mathrm{~ms}$ total decay time. All experiments were done multiple times and the averages were used for data analysis and interpretation; the reported errors are standard deviations.

Data Analysis. Phosphorescence lifetimes were determined by nonlinear leastsquares analysis with the program NFIT (Island Products, Galveston, TX). Fits were judged acceptable if fit parameters had satisfactory fit errors and if data points were randomly distributed about the fit curve; most data sets had $\chi^{2} \leq 1.0$ and $\mathrm{R}^{2}$ in the range of 0.99-1.0. Intensity decays $(\mathrm{I}(\mathrm{t}))$ were clearly non-exponential and were analyzed using a stretched exponential model function.

$$
I(t)=I(0) \exp \left[-(t / t)^{\beta}\right]+c
$$

Where $\mathrm{I}(0)$ is the initial intensity at time zero, $\tau$ is the Kohlrausch-Williams-Watt lifetime, $\beta$ is the stretching exponent, a term characterizing the distribution of decay times, and $\mathrm{c}$ is a constant. The heterogeneity in the matrix is modeled by $\beta$; as $\beta$ decreases below unity, the intensity decay is less single exponential and the distribution of decay times becomes broader (Lindsey and Patterson, 1980; Richert et al., 2000) 
Delayed luminescence spectra collected from 535-800 nm were converted to intensity versus frequency $\left(\mathrm{I}(\mathrm{v})\right.$, with $v$ in $\left.\mathrm{cm}^{-1}\right)$ and fit to a sum of two log-normal functions using the program NFIT; all fit parameters were independent for each emission band. The log normal fitting function (Maroncelli and Fleming, 1987) is as follows:

$$
\mathrm{I}(v)=\mathrm{I}_{\mathrm{o}} \exp \left\{-\ln (2)\left[\ln \left(1+2 \mathrm{~b}\left(v-v_{\mathrm{m}}\right) / \Delta\right) / \mathrm{b}\right]^{2}\right\}
$$

In this equation, $\mathrm{I}_{\mathrm{o}}$ is the maximum emission intensity, $v_{\mathrm{m}}$ is the frequency (in $\mathrm{cm}^{-1}$ ) of the emission maximum, $\Delta$ is a linewidth parameter, and $\mathrm{b}$ is an asymmetry parameter. The bandwidth ( $\Gamma$, full width at half maximum) of the emission band is related to $b$ and $\Delta:$

$$
\Gamma=\Delta\{\sinh (\mathrm{b}) / \mathrm{b}\}
$$

Photophysical scheme. The phosphorescence lifetimes were used to calculate the rate constants associated with the various processes that depopulate the triplet state. Our analysis of the delayed emission is similar to the photophysical scheme for erythrosin B outlined by Duchowicz et al. (1998) using slightly different nomenclature. The measured phosphorescence lifetime $(\tau)$ is the inverse sum of all possible deexcitation rates for the triplet state $\mathrm{T}_{1}$.

$$
1 / \tau=\mathrm{k}_{\mathrm{P}}=\mathrm{k}_{\mathrm{RP}}+\mathrm{k}_{\mathrm{TS} 1}+\mathrm{k}_{\mathrm{TS} 0}+\mathrm{k}_{\mathrm{Q}}\left[\mathrm{O}_{2}\right]
$$

Here $k_{R P}$ is the rate of radiative decay to the ground state, $k_{T S 1}$ is the rate of reverse intersystem crossing to $\mathrm{S}_{1}, \mathrm{k}_{\mathrm{TS} 0}$ is the rate of intersystem crossing to the singlet manifold followed by vibrational relaxation to $\mathrm{S}_{0}$, and $\mathrm{k}_{\mathrm{Q}}\left[\mathrm{O}_{2}\right]$ is the rate of oxygen quenching (assumed negligible in the absence of oxygen). The radiative decay rate has a value of 41 $\mathrm{s}^{-1}$ for erythrosin B (Duchowicz et al., 1998; Lettinga et al., 2000). 
Reverse intersystem crossing is a thermally activated process that has an exponential dependence on the energy gap $\Delta \mathrm{E}_{\mathrm{TS}}$ between $T_{1}$ and $\mathrm{S}_{1}$.

$$
\mathrm{k}_{\mathrm{TS} 1}(\mathrm{~T})=\mathrm{k}^{\circ} \mathrm{TS} 1 \exp \left(-\Delta \mathrm{E}_{\mathrm{TS}} / \mathrm{RT}\right) .
$$

The ratio of the intensity of delay fluorescence ( $\left.I_{D F}\right)$ to phosphorescence $\left(I_{P}\right)$, where $I_{D F}$ and $\mathrm{I}_{\mathrm{P}}$ are determined from analysis of emission spectra using the log normal function (equation 2), is proportional to the rate of reverse intersystem crossing (Duchowicz et al., 1998). A plot of $\ln \left(I_{D F} / I_{P}\right)$ versus $1 / T$ thus has slope of $-\Delta E_{T S} / R$. The value of $k_{T S 1}(T)$ was calculated using equation 5 with $\mathrm{k}^{\circ}{ }_{\mathrm{TS} 1}=6.5 \times 10^{7} \mathrm{~s}^{-1}$ (Duchowicz et al., 1998).

The magnitude of $\mathrm{k}_{\mathrm{Q}}\left[\mathrm{O}_{2}\right]$ was calculated from the difference between the decay rate (equation 4) in the presence and absence of oxygen:

$$
\mathrm{k}_{\mathrm{Q}}\left[\mathrm{O}_{2}\right]=1 / \tau\left(\mathrm{O}_{2}\right)-1 / \tau\left(\mathrm{N}_{2}\right)
$$

The measured phosphorescence intensity $\left(I_{P}\right)$ is proportional to the product of the quantum yield for formation of the triplet state $\left(\mathrm{Q}_{\mathrm{T}}\right)$ and the probability of emission from the triplet state $\left(\mathrm{q}_{\mathrm{P}}\right)$. Assuming that $\mathrm{Q}_{\mathrm{T}}$ is constant:

$$
\mathrm{I}_{\mathrm{P}} \propto \mathrm{q}_{\mathrm{P}}=\mathrm{k}_{\mathrm{RP}} /\left(\mathrm{k}_{\mathrm{RP}}+\mathrm{k}_{\mathrm{TS} 1}+\mathrm{k}_{\mathrm{TS} 0}+\mathrm{k}_{\mathrm{Q}}\left[\mathrm{O}_{2}\right]\right)
$$

Since $\mathrm{k}_{\mathrm{RP}}$ is constant, this expression indicates that the decrease in intensity with temperature reflects an increase in the sum $\mathrm{k}_{\mathrm{TS} 1}+\mathrm{k}_{\mathrm{TS} 0}+\mathrm{k}_{\mathrm{Q}}\left[\mathrm{O}_{2}\right]$. 


\section{$\underline{\text { Results }}$}

\section{Analysis of emission spectra}

Delayed emission spectra of Ery B, dispersed in optically clear amorphous films of dry $\beta-\mathrm{Lg}$ at a mole ratio of 0.25 probe per protein, were collected over the temperature range from -20 to $110^{\circ} \mathrm{C}$ (Fig. 1). The long wavelength emission band reflects phosphorescence from the excited $\mathrm{T}_{1}$ state while the short wavelength band reflects delayed fluorescence from the excited $S_{1}$ state that has been repopulated by reverse intersystem crossing from $\mathrm{T}_{1}$ (Parker, 1968). These spectra showed the decrease in the phosphorescence intensity $\left(I_{P}\right)$ and increase in the delayed fluorescence intensity $\left(I_{D F}\right)$ with increasing temperature expected for a thermally stimulated process. Plots of $\ln \left(\mathrm{I}_{\mathrm{DF}} / \mathrm{I}_{\mathrm{P}}\right)$ versus $1 / \mathrm{T}$ (using the maximum intensity determined from fitting spectra to a log-normal function; Eq. (2) in Section 2), which were linear with $\mathrm{R}^{2} 0.998$, were used to calculate $\Delta \mathrm{E}_{\mathrm{TS}}$, the energy gap between $\mathrm{T}_{1}$ and $\mathrm{S}_{1}$ (data not shown). $\Delta \mathrm{E}_{\mathrm{TS}}$ was $35.2 \pm 0.5$ $\mathrm{kJ}$ mol-1 in the presence of air and slightly lower, $33.6 \pm 0.8 \mathrm{~kJ} \mathrm{~mol}^{-1}$, in the presence of nitrogen; the nitrogen value is similar to that for Ery B in amorphous bovine serum albumin films equilibrated against nitrogen $(32.9 \pm 0.5 \mathrm{~kJ}$ mol-1; Nack \& Ludescher, 2006). These values are significantly different from those for Ery B in amorphous sucrose $\left(31.6 \pm 0.4 \mathrm{~kJ} \mathrm{~mol}^{-1}\right)$, in water $\left(36.9 \pm 0.6 \mathrm{~kJ} \mathrm{~mol}^{-1}\right)$, or in $66 \mathrm{wt} \%$ aqueous sucrose $\left(36.9 \pm 1.0 \mathrm{~kJ} \mathrm{~mol}^{-1}\right.$ ) (Pravinata et al., 2005), in ethanol $\left(28.5 \pm 2.5 \mathrm{~kJ} \mathrm{~mol}^{-1}\right.$; Duchowicz et al., 1998), or in polyvinyl alcohol (41.2 $\pm 0.4 \mathrm{~kJ} \mathrm{~mol}^{-1}$; Lettinga et al., 2000), but similar to those seen in maltose $\left(32.7 \pm 1.1 \mathrm{~kJ} \mathrm{~mol}^{-1}\right)$ and maltitol $\left(34.2 \pm 0.9 \mathrm{~kJ} \mathrm{~mol}^{-1}\right)$ (Shirke et al., 2005), and in lactose $\left(34.1 \pm 0.3 \mathrm{~kJ} \mathrm{~mol}^{-1}\right)$ and lactitol $\left(34.0 \pm 0.3 \mathrm{~kJ} \mathrm{~mol}^{-1}\right)$ (Shirke, You, \& 
Ludescher, 2006), suggesting that matrix properties modulate somewhat the singlettriplet energy gap.

The delayed fluorescence and phosphorescence bands shifted to longer wavelength (lower energy) at higher temperature; the delayed fluorescence emission maximum was $553 \mathrm{~nm}$ at $-20^{\circ} \mathrm{C}$ and red-shifted to $563 \mathrm{~nm}$ at $110^{\circ} \mathrm{C}$ while the phosphorescence emission maximum was $688 \mathrm{~nm}$ at $-20^{\circ} \mathrm{C}$ and red-shifted to $700 \mathrm{~nm}$ at $110^{\circ} \mathrm{C}$. The emission maximum $(\mathrm{vm})$ and bandwidth $(\Gamma)$ were determined by fitting the delayed emission spectra to a sum of two log-normal functions. The behavior of the delayed fluorescence band reflected an excited state process involving a three state transition $\left(\mathrm{S}_{1} \rightarrow \mathrm{T}_{1} \rightarrow \mathrm{S}_{1}\right)$ and will not be discussed; the temperature dependence of the phosphorescence emission maximum and bandwidth are plotted in Fig II- 2. The phosphorescence emission maximum decreased with an increase in temperature; this decrease was small at low temperature but somewhat larger above $20^{\circ} \mathrm{C}$. A decrease in the emission energy with temperature can be attributed to an increase with temperature in the rate of dipolar relaxation around the excited triplet state (Pravinata et al., 2005; Richert, 2000). The emission bandwidth, a measure of the extent of inhomogeneous broadening due to the presence of probes in multiple sites with different emission energies, increased with increasing temperature. This increase was gradual at low temperature and increased with increasing temperature, indicating that the increase in dipolar relaxation rate was accompanied by an increase in the width of the distribution of energetically distinct environments. The increase in error at higher temperatures reflects the uncertainty involved in estimating the bandwidth of emission bands with very low intensity 
Analysis of emission lifetimes

The phosphorescence intensity decreased monotonically with increasing temperature in nitrogen and in air (Fig III- 3), indicating that the triplet state quenching rates increased with temperature (Eq. (7), Section 2). Oxygen did not quench the phosphorescence at $-20^{\circ} \mathrm{C}$ as the emission intensity of individual films was the same when equilibrated against air and against nitrogen; the data are thus plotted as intensity normalized to unit intensity at $-20^{\circ} \mathrm{C}$. Oxygen quenching was activated at higher temperature, however, and the extent of quenching, as reflected in the decrease in emission intensity, increased with an increase in temperature. The "shoulder" seen at $40^{\circ} \mathrm{C}$ in the intensity curve collected in the presence of air was not seen in the comparable thermal behavior of the lifetime data (Fig.II-5) and is, therefore, not considered to be physically meaningful.

In an effort to quantify the extent of oxygen quenching as a function of temperature, time-resolved intensity decays were measured from -20 to $120^{\circ} \mathrm{C}$ in films equilibrated against nitrogen and against air. Representative intensity decays at $20^{\circ} \mathrm{C}$ in the presence of air and nitrogen are plotted in Fig. 4 along with fits using a stretched exponential model function (Eq. (1), Section 2); the modified residuals for this fit varied randomly about zero, indicating that this model function provides a statistically reasonable description of the decay as well as a theoretically reasonable description of the dynamics of an amorphous solid (Nack \& Ludescher, 2006; Pravinata et al., 2005). The stretched exponential decay model provided a satisfactory fit for data collected at all temperatures from -20 to $120^{\circ} \mathrm{C}$ with modified residuals that varied randomly about zero and $\mathrm{R}^{2}$ values that ranged from a high of 0.9999 at $-20^{\circ} \mathrm{C}$ to a low of 0.9975 at $120^{\circ} \mathrm{C}$. 
The stretched exponential lifetime $\tau$ and stretching exponent $\beta$ are plotted versus temperature for films equilibrated against nitrogen and against air in Fig II-5 and Fig II-6.

The phosphorescence lifetime decreased monotonically with increasing temperature in nitrogen; the decrease was gradual at first and steeper at higher temperature indicating that the non-radiative decay rates were thermally activated. This lifetime behavior is similar to that seen in films of bovine serum albumin (Nack \& Ludescher, 2006) as well as in amorphous sucrose (Pravinata et al., 2005), maltose and maltitol (Shirke et al., 2005), and lactose and lactitol (Shirke et al., 2006), although the lifetime was shorter at low temperature than that seen in either BSA or the amorphous sugars. The lifetime at $-20^{\circ} \mathrm{C}$ was identical within error in films equilibrated against nitrogen, $0.567 \pm 0.018$, and against air, $0.559 \pm 0.006 \mathrm{~ms}$, confirming that oxygen quenching was negligible at this temperature. However, the lifetime was significantly lower in the presence of air at $-10^{\circ} \mathrm{C}$ and all higher temperatures, indicating that oxygen quenching was significant at this temperature and above, and decreased to $0.15 \pm 0.005$ and $0.04 \pm 0.005 \mathrm{~ms}$ in nitrogen and air, respectively, at $120^{\circ} \mathrm{C}$. Oxygen quenching was thus also activated in films at higher temperature.

The stretching exponent $\beta$ is a measure of the dynamic heterogeneity in the matrix; values of $\beta$ close to 1 indicate that the matrix is dynamically homogenous while smaller values of $\beta$ indicate increasing dynamic heterogeneity (Lindsey \& Patterson, 1980). The value of $\beta$ in nitrogen was $0.87 \pm 0.004$ at $-20^{\circ} \mathrm{C}$, decreased gradually to $0.80 \pm 0.013$ at $60^{\circ} \mathrm{C}$, and then decreased more dramatically to 0.7 at $100^{\circ} \mathrm{C}$ and above; $\beta$ was identical within error in nitrogen and air at all except the highest temperatures. These values of $\beta$ are similar to the values in amorphous films of bovine serum albumin (Nack 
\& Ludescher, 2006), but significantly smaller than those seen for Ery B dispersed in amorphous sugars over the same temperature range (Pravinata et al., 2005; Shirke, Takhistov, \& Ludescher (2005) and Shirke, You, \& Ludescher (2006)).

\section{Photophysical rate constants}

Analysis of lifetimes in terms of the underlying photophysical rate constants for de-excitation of the triplet state provides additional insight into the effect of temperature on matrix mobility and oxygen quenching in $\beta$-Lg films. In the absence of oxygen, the total rate of decay $k_{P}(=1 / \tau)$ is the sum of the rates for radiative decay $k_{R P}$, for reverse intersystem crossing $\mathrm{k}_{\mathrm{TS} 1}$, and for vibrational relaxation due to collisional quenching $\mathrm{k}_{\mathrm{TS} 0}$. The radiative decay rate is $41 \mathrm{~s}^{-1}$ and constant for Ery B (Duchowicz et al., 1998; Lettinga et al., 2000). The reverse intersystem crossing rate $\mathrm{k}_{\mathrm{TS} 1}(\mathrm{~T})$, estimated as described in Section 2 (Eq. (5)), was $7 \mathrm{~s}^{-1}$ at $-20^{\circ} \mathrm{C}$ and increased exponentially with temperature to $2220 \mathrm{~s}^{-1}$ at $120^{\circ} \mathrm{C}$; this value was used to calculate $\mathrm{k}_{\mathrm{TS} 0}$ as a function of temperature from $\mathrm{k}_{\mathrm{P}}$ in the presence of nitrogen; since we potentially overestimate $\mathrm{k}_{\mathrm{TS} 1}$ this calculated value may underestimate $\mathrm{k}_{\mathrm{TS} 0}$. The oxygen quenching rate $\mathrm{k}_{\mathrm{Q}}\left[\mathrm{O}_{2}\right]$ was calculated directly from the difference between $\mathrm{kP}$ in the presence of air and nitrogen (Eq. (6), Section 2). The $\log$ of $\mathrm{k}_{\mathrm{TS} 0}$ and $\mathrm{k}_{\mathrm{Q}}\left[\mathrm{O}_{2}\right]$ are plotted versus inverse temperature in Fig. II-7.

The magnitude of $\mathrm{k}_{\mathrm{TS} 0}$ reflects internal factors associated with the mechanism by which the excited $T_{1}$ state of Ery $B$ is coupled to highly excited vibrational states of the $\mathrm{S}_{0}$ ground state as well as external factors associated with the mechanism by which the ground state vibrational energy can dissipate from the excited probe into the surrounding matrix (Fischer, Gafni, Steel, \& Schauerte, 2002; Vanderkooi \& Berger, 1989). Because 
the efficiency of this external vibrational dissipation is related to the overall mobility of the matrix, the magnitude of $\mathrm{k}_{\mathrm{TS} 0}$ provides a measure of matrix mobility. The magnitude of $\mathrm{k}_{\mathrm{TS} 0}$ was $1710 \mathrm{~s}^{-1}$ at $-20^{\circ} \mathrm{C}$; this value was slightly larger than that for Ery $\mathrm{B}$ dispersed in amorphous BSA films ( $1600 \mathrm{~s}^{-1}$; Nack \& Ludescher, 2006) or amorphous sugar films ( $1650 \mathrm{~s}^{-1}$; Pravinata et al., 2005; Shirke, Takhistov, \& Ludescher (2005) and Shirke, You, \& Ludescher (2006)). $\mathrm{k}_{\mathrm{TS} 0}$ increased gradually at low temperature and more dramatically at high temperature; linear fits to the Arrhenius plot at low $\left(-10-50^{\circ} \mathrm{C}\right)$ and high $\left(90-120^{\circ} \mathrm{C}\right)$ temperature had $\mathrm{R}^{2}$ values of 0.977 and 0.978 and gave activation energies of 2.35 and $21.4 \mathrm{~kJ} \mathrm{~mol}^{-1}$, respectively. These values were significantly higher than the comparable values of 1.4 and $7.9 \mathrm{~kJ}$ mol-1 for the activation energy for $\mathrm{k}_{\mathrm{TS} 0}$ in BSA films at low and high temperature (Nack \& Ludescher, 2006). A break in the Arrhenius plot indicative of a dynamic transition in the $\beta$-Lg film occurred at $81^{\circ} \mathrm{C}$ (determined by extrapolation of the linear regions of the Arrhenius plots at low and high temperature).

Since the oxygen quenching rate is the product of $\mathrm{k}_{\mathrm{Q}}$, which is proportional to the oxygen diffusion rate through the matrix, and $\left[\mathrm{O}_{2}\right]$, the concentration of oxygen in the protein film, it is proportional to the permeability of oxygen through the protein film (Nack \& Ludescher, 2006). The quenching rate $\mathrm{k}_{\mathrm{Q}}\left[\mathrm{O}_{2}\right]$ was negligible at temperatures below $0^{\circ} \mathrm{C}$, and increased gradually at low temperature and dramatically at high temperature; the downward curvature in the Arrhenius plot at low temperature reflects the onset of oxygen quenching at $-10^{\circ} \mathrm{C}$. Linear fits to the Arrhenius plot at low $\left(10-60^{\circ} \mathrm{C}\right)$ and high $\left(90-120^{\circ} \mathrm{C}\right)$ temperature had $\mathrm{R}^{2}$ values of 0.9843 and 0.9997 and gave activation energies of 20.8 and $54.8 \mathrm{~kJ} \mathrm{~mol}^{-1}$, respectively. A similar analysis of oxygen 
quenching in BSA films gave a single activation energy of $29.9 \mathrm{~kJ} \mathrm{~mol}^{-1}$ (Nack \& Ludescher, 2006). The break in the plot indicative of a transition in the rate of oxygen permeability occurred at $75^{\circ} \mathrm{C}$ (determined by extrapolation of the linear regions of the Arrhenius plots at low and high temperature), only slightly lower than the temperature of the transition in $\mathrm{k}_{\mathrm{TS} 0}$.

Dramatic increases with temperature in the rates for both oxygen quenching and matrix mobility suggest that these two phenomena reflect the same underlying physical change in the matrix. A plot of $\mathrm{k}_{\mathrm{Q}}\left[\mathrm{O}_{2}\right]$ versus $\mathrm{k}_{\mathrm{TS} 0}$ illustrates this interdependence (Fig. 8); oxygen permeability was a biphasic linear function of $\mathrm{k}_{\mathrm{TS} 0}$ over nearly three orders of magnitude change in $\mathrm{k}_{\mathrm{Q}}\left[\mathrm{O}_{2}\right]$. Analysis of the linear equations for fits to regions of this plot at low $\left(\mathrm{R}^{2}=0.989\right)$ and high $\left(\mathrm{R}^{2}=0.996\right)$ values of $\mathrm{k}_{\mathrm{TS} 0}$ indicated that the onset of oxygen quenching occurred at $\mathrm{k}_{\mathrm{TS} 0}=1682 \mathrm{~s}^{-1}$ and the transition point occurred at $\mathrm{k}_{\mathrm{TS} 0}=2245 \mathrm{~s}^{-1}$, with slopes of 3.96 below and 8.82 above $2245 \mathrm{~s}^{-1}$. The temperature corresponding to $2245 \mathrm{~s}^{-1}$ was $65^{\circ} \mathrm{C}$, indicating that the transition in this plot of $\mathrm{k}_{\mathrm{Q}}\left[\mathrm{O}_{2}\right]$ versus $\mathrm{k}_{\mathrm{TS} 0}$ occurred at a slightly lower temperature than the transitions seen in the Arrhenius plots of the individual rate constants $\mathrm{k}_{\mathrm{TS} 0}$ and $\mathrm{k}_{\mathrm{Q}}\left[\mathrm{O}_{2}\right]$.

\section{$\underline{\text { Discussion }}$}

The phosphorescence emission energy, intensity, and lifetime of Ery B are sensitive to two modes of molecular mobility within the amorphous solid matrix: the relaxation of matrix dipoles around the excited $\mathrm{T}_{1}$ triplet state and molecular collisions with the excited state (Pravinata et al., 2005; Shirke, Takhistov, \& Ludescher (2005) and Shirke, You, \& Ludescher (2006)). Dipolar relaxations stabilize the triplet state and thus 
lower the energy of the phosphorescence emission while molecular collisions with the excited triplet state dissipate excess vibrational energy of the probe into the matrix and thus lower the emission intensity and lifetime. In addition, as with all triplet probes, the intensity and lifetime of Ery B phosphorescence emission are exquisitely sensitive to contact quenching by the triplet ground state of molecular oxygen (Nack \& Ludescher, 2006; Simon-Lukasik \& Ludescher, 2004). The direct measurement of these modes of molecular mobility as well as the oxygen quenching constant, a rate proportional to oxygen permeability through the matrix, using the same spectroscopic probe enables a direct and unambiguous correlation between a macroscopic transport property and the underlying molecular mobility in the matrix, providing insight into the molecular mechanisms controlling oxygen permeability through an amorphous protein matrix.

\section{Matrix molecular mobility}

The thermal response of the peak emission energy and bandwidth reflects the affect of temperature on the rate of dipolar relaxation around the excited triplet state. Despite a slight change in slope in the $v \mathrm{~m}(\mathrm{~T})$ curve near room temperature, there is no direct evidence of a dynamic transition in either the peak emission energy or bandwidth data. This contrasts with the results of a similar study of mobility and oxygen permeability in amorphous films of bovine serum albumin using Ery B phosphorescence where a distinct break was seen in the $v m(T)$ curve (Nack \& Ludescher, 2006). This difference may reflect subtle differences in the manner in which the Ery B probe interacts with the protein; BSA, for example, has a specific binding site for Ery B (Garland \& Moore, 1979) while $\beta$-Lg apparently does not. 
The distinct break in the Arrhenius plot of the collisional quenching rate kTSO, however, does provide direct evidence of a dynamic transition near $80^{\circ} \mathrm{C}$ in amorphous $\beta$-Lg. The increase in slope above $80^{\circ} \mathrm{C}$ could reflect denaturation which occurs near $78^{\circ} \mathrm{C}$ for $\beta$ - $\mathrm{Lg}$ in aqueous solution (DeWit \& Klarenbeek, 1984). Two lines of argument militate against this interpretation, however. First, the lifetime versus temperature curves are superimposable during heating and cooling (data not shown); such behavior would be unlikely following protein denaturation. Second, since protein denaturation temperatures are know to increase dramatically, as much as $40^{\circ} \mathrm{C}$, upon dehydration (Fujita \& Noda (1981a) and Fujita \& Noda (1981b)), it is unlikely that the thermal melting temperature of $\beta-\mathrm{Lg}$ would be nearly the same in the dry solid as in solution.

Another possibility is that this break reflects a glass-like transition within the amorphous dry protein (Hill, Shalaev, \& Zografi, 2005). Hydrated globular proteins undergo a dynamical transition (Td) at $200 \mathrm{~K}$, a phenomenon well documented by X-ray diffraction analysis of thermal B-factors (Rasmussen, Stock, Ringe, \& Petsko, 1992), neutron scattering (Cusack, 1989; Cusack, Smith, Finney, Tidor, \& Karplus, 1988; Doster, Cusack, \& Petry, 1990; Loncharich \& Brooks, 1990; Smith, 1991), and differential scanning calorimetry (Barkalov, Bol'shakov, Gol'danskii, \& Krupyanskii, 1992). This transition involves a change in protein motions from small amplitude, harmonic vibrations of covalently bonded atoms to large scale, anharmonic, collective motions of non-bonded atoms. Due to its similarity to the high-temperature dynamical transition seen in small molecules and polymers, this transition is often referred to as a glass transition (Ringe \& Petsko, 2003). Although it is not known whether Td is 
modulated by water in a manner comparable to $\mathrm{Tg}$, $\mathrm{Td}$ does appear to increase considerably upon dehydration (Hill et al., 2005).

The softening transition seen at $80^{\circ} \mathrm{C}$ may thus correspond to a glass transition within the $\beta$ - $\mathrm{Lg}$ molecules in the film. This interpretation is supported by similarities between the phosphorescence response of Ery B to the effect of heating in amorphous $\beta$ Lg and in amorphous sugars through their glass transition (Pravinata et al., 2005; Shirke, Takhistov, \& Ludescher (2005) and Shirke, You, \& Ludescher (2006)). Within this interpretation, the activation energy (EA) for collisional quenching, $\mathrm{k}_{\mathrm{TS} 0}$, below $80^{\circ} \mathrm{C}$, $2.35 \mathrm{~kJ} \mathrm{~mol}^{-1}$, is the activation energy for motions within the glassy protein that activate vibrational relaxation of the highly excited probe while the activation energy above $80^{\circ} \mathrm{C}$, $21.4 \mathrm{~kJ} \mathrm{~mol}-1$, is the activation energy for motions within the rubbery protein that activate probe vibrational relaxation. The small value of EA in the glass suggests that the motions that activate vibrational relaxation are small amplitude, localized modes of molecular mobility; in the rubber, on the other hand, the significantly ( 9-fold) larger value of EA suggests that the modes of protein motion involve significantly larger segments of the protein structure.

The value of $\beta$, which provides an estimate of the width of the distribution of lifetimes, decreased approximately linearly with temperature. Given the dependence of the lifetime on $\mathrm{k}_{\mathrm{TS} 0}$, this decrease in $\beta$ reflects a broadening in the distribution of dynamically distinct environments with different values of $\mathrm{k}_{\mathrm{TS} 0}$. The protein matrix at higher temperature thus had both a faster rate of collisional quenching (larger value of $\mathrm{k}_{\mathrm{TS} 0}$ ) and a broader distribution of environments with distinct values of $\mathrm{k}_{\mathrm{TS} 0}$ (smaller $\beta$ ). 
The lower value of $\beta$ in the presence of air at high temperature may reflect the additional heterogeneity associated with variations in the oxygen quenching constant, $\mathrm{k}_{\mathrm{Q}}\left[\mathrm{O}_{2}\right]$.

\section{Oxygen permeability}

Oxygen is a powerful quencher of both fluorescence and phosphorescence and luminescence quenching has been used as an effective method for probing the presence of molecular oxygen in polymeric media (Charlesworth \& Gan, 1996; Guillet \& Andrews, 1992; Jones, 1968; Masoumi et al., 1996) following the initial study showing that comparison of the triplet lifetime of a probe in the presence and absence of oxygen provided a facile and rapid indicator of oxygen quenching in the gelatin matrix (Buettner, 1964). We have recently used phosphorescence quenching of Ery B to correlate the dynamic properties of the gelatin matrix with the oxygen diffusion rate (Lukasik \& Ludescher, 2006a; Simon-Lukasik \& Ludescher, 2004) while other studies have shown the potential of Ery B as an oxygen sensor in sol-gel silica (Lam, Chan, \& Lo, 2001).

In the present study, the lifetime in the presence of nitrogen and oxygen were indistinguishable at $-20^{\circ} \mathrm{C}$, indicating that oxygen quenching was negligible at that temperature, while the lifetime was lower in the presence of oxygen at higher temperatures. There thus appeared to be an onset temperature for oxygen permeability within the $\beta$-Lg matrix; this onset temperature is the origin of the extensive downward curvature seen in the Arrhenius plot of $\mathrm{k}_{\mathrm{Q}}\left[\mathrm{O}_{2}\right]$ ) at low temperature. Ignoring this lowtemperature artifact, the Arrhenius plot of oxygen quenching was also biphasic with a break point at $75^{\circ} \mathrm{C}$, only slightly lower than the break point temperature seen in the Arrhenius plot of $\mathrm{k}_{\mathrm{TS} 0}$. Given the clearly linear, but also biphasic, relationship between 
the oxygen quenching constant, $\mathrm{k}_{\mathrm{Q}}\left[\mathrm{O}_{2}\right]$, and the collisional quenching constant, $\mathrm{k}_{\mathrm{TS} 0}$, it appears that the transition seen in the oxygen permeability reflects that same underlying softening in the protein matrix. The $\beta$ - $\operatorname{Lg}$ matrix is thus more rigid and less permeable to oxygen at low temperature when it is in an apparent glassy state and less rigid and more permeable to oxygen in an apparent rubbery state at high temperature. The activation energies for the molecular motions that control oxygen permeability are $20.8 \mathrm{~kJ} \mathrm{~mol}^{-1}$ in the glassy and $54.8 \mathrm{~kJ} \mathrm{~mol}^{-1}$ in the rubbery matrix. These values are significantly larger than the activation energies for non-radiative quenching of the triplet state $\left(\mathrm{k}_{\mathrm{TS} 0}\right)$ and surely reflect the more complex modes of molecular motion required to activate the diffusive transport of a molecule of oxygen compared to those required to dissipate the vibrational motion of an excited chromophore.

The correlation between $\mathrm{k}_{\mathrm{Q}}\left[\mathrm{O}_{2}\right]$ and $\mathrm{k}_{\mathrm{TS} 0}$ suggests that the onset condition for oxygen permeability may be a level of molecular mobility within the protein matrix rather than a temperature per se. A similar onset condition was seen in a comparable study of oxygen permeability and molecular mobility in amorphous films of bovine serum albumin using Ery B phosphorescence (Nack \& Ludescher, 2006). The magnitude of $\mathrm{k}_{\mathrm{Ts} 0}$ corresponding to onset of oxygen permeability was similar but not identical in the two protein matrixes, $1682 \mathrm{~s}^{-1}$ in $\beta-\mathrm{Lg}$ and $1550 \mathrm{~s}^{-1}$ in BSA.

The similarities in the relationship between oxygen permeability and molecular mobility between $\beta-\mathrm{Lg}$ and BSA suggest that the basic mechanisms controlling oxygen permeability are similar in the two protein matrixes; nonetheless, the unique features of this relationship are presumably related to the specific structure of the proteins and the 
manner in which they interact to form the amorphous solid matrix. Additional research, currently underway in our laboratory, will reveal much about the role that protein structure plays in modulating the complex interplay of molecular mobility and oxygen transport.

\section{$\underline{\text { Conclusion }}$}

This study demonstrates how spectroscopic data provided by the luminescent probe Ery B dispersed throughout a thin film of amorphous $\beta$-Lg can provide insight into the molecular mechanism(s) controlling oxygen transport through the protein matrix. Analysis of the phosphorescent lifetime of the probe in the absence and presence of oxygen gives the rate of non-radiative decay, $\mathrm{k}_{\mathrm{TS} 0}$, a quantitative measure of the matrix molecular mobility, and the rate of oxygen quenching, $\mathrm{k}_{\mathrm{Q}}\left[\mathrm{O}_{2}\right]$, which is proportional to the oxygen permeability. The thermal behavior of these two rates indicates that the $\beta$ - $\mathrm{Lg}$ matrix undergoes a dynamic, softening transition near $80^{\circ} \mathrm{C}$, which does not appear to be related to protein denaturation. The linear dependence of $\mathrm{k}_{\mathrm{Q}}\left[\mathrm{O}_{2}\right]$ on $\mathrm{k}_{\mathrm{TS} 0}$ above a threshold value and ranging over nearly 3 -orders of magnitude change in the amplitude of oxygen quenching provides compelling evidence that oxygen transport is controlled by the local molecular mobility of the matrix. Such mechanistic studies will be useful in developing rational methods for manipulating and controlling oxygen transport through protein and other biomolecular matrixes that are based on detailed biophysical mechanisms of the transport process. 


\section{$\underline{\text { References }}$}

Avena-Bustillos \& Krochta (1993) R.J. Avena-Bustillos and J.M. Krochta, Water vapor permeability of caseinate-based edible films as affected by $\mathrm{pH}$, calcium crosslinking and lipid content, Journal of Food Science 58 (1993), pp. 904-907.

Barkalov, Bol'shakov, Gol'danskii, \& Krupyanskii (1992) I.M. Barkalov, A.I. Bol'shakov, V.I. Gol'danskii and Y.F. Krupyanskii, Glass transition effects in water-protein systems, Doklady Akademii Nauk 326 (1992), pp. 1083-1087.

Brandenburg, Weller, \& Testin (1993) A.H. Brandenburg, C.L. Weller and R.F. Testin, Edible films and coatings from soy protein, Journal of Food Science 58 (1993), pp. 1086-1089.

Buettner (1964) A.V. Buettner, Flash photolysis in thin films of gelatin and other polymers, Journal of Physical Chemistry 68 (1964), pp. 3253-3259.

Charlesworth \& Gan (1996) J.M. Charlesworth and T.H. Gan, Kinetics of quenching of ketone phosphorescence by oxygen in a glassy matrix, Journal of Physical Chemistry 100 (1996), pp. 14922-14927.

Cusack (1989) S. Cusack, Low frequency dynamics of proteins: Comparison of inelastic neutron scattering results with theory, Chemica Scripta 29A (1989), pp. 103-107.

Cusack, Smith, Finney, Tidor, \& Karplus (1988) S. Cusack, J. Smith, J. Finney, B. Tidor and M. Karplus, Inelastic neutron scattering analysis of picosecond internal protein dynamics. Comparison of harmonic theory with experiment, Journal of Molecular Biology 202 (1988), pp. 903-908.

DeWit \& Klarenbeek (1984) J.N. DeWit and G. Klarenbeek, Effects of various heat treatments on structure and solubility of whey proteins, Journal of Dairy Science 67 (1984), pp. 2701-2710.

Doster, Cusack, \& Petry (1990) W. Doster, S. Cusack and W. Petry, Dynamic instability of liquidlike motions in a globular protein observed by inelastic neutron scattering, Physical Review Letters 65 (1990), pp. 1080-1083.

Duchowicz, Ferrer, \& Acuña (1998) R. Duchowicz, M.L. Ferrer and A.U. Acuña, Kinetic spectroscopy of erythrosin phosphorescence and delayed fluorescence in aqueous solution at room temperature, Photochemistry and Photobiology 68 (1998), pp. 494-501. Dybing \& Smith (1991) S.T. Dybing and D.E. Smith, Relation of chemistry and processing procedures to whey protein functionality: A review, Cultured Dairy Production Journal 26 (4-9) (1991), pp. 11-12.

Fennema (1996) O.R. Fennema, Food chemistry (3rd ed.), Marcel Dekker, Inc, New York (1996). 
Fischer, Gafni, Steel, \& Schauerte (2002) C.J. Fischer, A. Gafni, D.G. Steel and J.A. Schauerte, The triplet-state lifetime of indole in aqueous and viscous environments: Significance to the interpretation of room temperature phosphorescence in proteins, Journal of the American Chemical Society 124 (2002), pp. 10359-10366

Fugate \& Song (1980) R.D. Fugate and P. Song, Spectroscopic characterization of $\beta$ lactoglobulin-retinol complex, Biochimica et Biophysica Acta: Protein Structures 625 (1980), pp. 28-42. View Record in Scopus | Cited By in Scopus (81)

Fujita \& Noda (1981a) Y. Fujita and Y. Noda, The effect of hydration on the thermal stability of ovalbumin as measured by means of differential scanning calorimetry, Bulletin of the Chemical Society of Japan 54 (1981), pp. 3233-3234.

Fujita \& Noda (1981b) Y. Fujita and Y. Noda, Effect of hydration on the thermal stability of protein as measured by differential scanning calorimetry: Chrymotrypsinogen A, International Journal of Peptide and Protein Research 18 (1981), pp. 12-17.

Garland \& Moore (1979) P.B. Garland and C.H. Moore, Phosphorescence of protein-bound eosin and erythrosin: A possible probe for measurements of slow rotational mobility, Biochemical Journal 183 (1979), pp. 561-572.

Gontard, Guilbert, \& Cuq (1992) Gontard, N., Guilbert, S., \& Cuq, J.L. (1992). Edible wheat gluten films: Influence of the main process variables on film properties using response surface methodology. Journal of Food Science, 57, 190-195, 199.

Guillet \& Andrews (1992) J.E. Guillet and M. Andrews, Studies of oxygen diffusion in poly(styrene-co-1-naphthyl methacrylate) by phosphorescence quenching, Macromolecule 25 (1992), pp. 2752-2756.

Hill, Shalaev, \& Zografi (2005) J.J. Hill, E.Y. Shalaev and G. Zografi, Thermodynamic and dynamic factors involved in the stability of native protein structure in amorphous solids in relation to levels of hydration, Journal of Pharmaceutical Sciences 94 (2005), pp. $1636-1667$.

Jangchud \& Chinnan (1999) A. Jangchud and M.S. Chinnan, Properties of peanut protein film: Sorption isotherm and plasticizer effect, Lebensmittel-Wissenschaft und- Technologie 32 (1999), pp. 89-94.

Jones (1968) P.F. Jones, The use of phosphorescence quenching for determining permeabilities of polymeric films to gases, Journal of Polymer Science: Polymer Letters 6 (1968), pp. 487-491. Full Text via CrossRef

Kinsella \& Whitehead (1989) J.E. Kinsella and D.M. Whitehead, Proteins in whey: Chemical, physical, and functional properties, Advances in Food and Nutrition Research 33 (1989), pp. 343-438. 
Krochta (1997) J.M. Krochta, Edible protein films and coatings. In: S. Damodaran and A. Paraf, Editors, Food proteins and their applications Vol. 80, Marcel Dekker, Amsterdam (1997), pp. 529-549.

Krochta \& De Mulder-Johnston (1997) J.M. Krochta and C.D. De Mulder-Johnston, Edible and biodegradable polymer films: Challenges and opportunities, Food Technology 51 (1997), pp. 61-74.

Lam, Chan, \& Lo (2001) S.K. Lam, M.A. Chan and D. Lo, Characterization of phosphorescence oxygen sensor based on erythrosin B in sol-gel silica in wide pressure and temperature ranges, Sensors and Actuators B: Chemical B73 (2001), pp. 135-141.

Le Meste, Champion, Roudaut, Blond, \& Simatos (2002) M. Le Meste, D. Champion, G. Roudaut, G. Blond and D. Simatos, Glass transition and food technology: A critical appraisal, Journal of Food Science 67 (2002), pp. 2444-2458.

Lettinga, Zuilhof, \& Van Zandvoort (2000) M.P. Lettinga, H. Zuilhof and M.A.M.J. Van Zandvoort, Phosphorescence and fluorescence characterization of fluorescein derivatives immobilized in various polymer matrices, Physical Chemistry Chemical Physics 2 (2000), pp. 3697-3707.

Lindsey \& Patterson (1980) C.P. Lindsey and G.D. Patterson, Detailed comparison of the Williams-Watts and Cole-Davidson functions, Journal of Chemical Physics 73 (1980), pp. 3348-3357. Full Text via CrossRef

Loncharich \& Brooks (1990) R.J. Loncharich and B.R. Brooks, Temperature dependence of dynamics of hydrated myoglobin: Comparison of force field calculations with neutron scattering data, Journal of the Molecular Biology 215 (1990), pp. 439-455.

Lukasik \& Ludescher (2006a) K.V. Lukasik and R.D. Ludescher, Molecular mobility in water and glycerol plasticized cold- and hot-cast gelatin films, Food Hydrocolloids 20 (2006), pp. $96-105$

Lukasik \& Ludescher (2006b) K.V. Lukasik and R.D. Ludescher, Effect of plasticizer on dynamic site heterogeneity in cold-cast gelatin films, Food Hydrocolloids 20 (2006), pp. $88-95$.

Maroncelli \& Fleming (1987) M. Maroncelli and G.R. Fleming, Picosecond solvation dynamics of coumarin 153: The importance of molecular aspects of solvation, Journal of Chemical Physics 86 (1987), pp. 6221-6239.

Masoumi et al. (1996) Z. Masoumi, V. Stoeva, A. Yekta, Z. Pang, I. Manners and M.A. Winnik, Luminescence quenching method for probing the diffusivity of molecular oxygen in highly permeable media, Chemical Physics Letters 261 (1996), pp. 551-557. McHugh, Aujard, \& Krochta (1994) McHugh, T.H., Aujard, J.F., \& Krochta, J.M. (1994). 
Plasticized whey protein edible films: Water vapor permeability properties. Journal of Food Science, 59, 416-419, 423.

Nack \& Ludescher (2006) T.J. Nack and R.D. Ludescher, Molecular mobility and oxygen permeability in amorphous bovine serum albumin films, Food Biophysics 1 (2006), pp. 151-162.

Papiz, Sawyer, \& Eliopoulos (1986) M.Z. Papiz, L. Sawyer and E.E. Eliopoulos, The structure of $\beta$-lactoglubulin and its similarity to plasma retinol-binding protein, Nature 324 (1986), pp. 383-385.

Parker (1968) C.A. Parker, Photoluminescence of solutions, Elsevier Publishing, Amsterdam (1968).

Parris \& Coffin (1997) N. Parris and D.R. Coffin, Composition factors affecting the water vapor permeability and tensile properties of hydrophilic zein films, Journal of the Agricultural Food Chemistry 45 (1997), pp. 1596-1599.

Pravinata, You, \& Ludescher (2005) L.C. Pravinata, Y. You and R.D. Ludescher, Erythrosin B phosphorescence monitors molecular mobility and dynamic site heterogeneity in amorphous sucrose, Biophysics Journal 88 (2005), pp. 3551-3561. Rasmussen, Stock, Ringe, \& Petsko (1992) B.F. Rasmussen, A.M. Stock, D. Ringe and G.A. Petsko, Crystalline ribonuclease A loses function below the dynamical transition at $220 \mathrm{~K}$, Nature 357 (1992), pp. 423-424.

Richert (2000) R. Richert, Triplet state solvation dynamics: Basics and applications, Journal of Chemical Physics 113 (2000), pp. 8404-8429.

Ringe \& Petsko (2003) D. Ringe and G.A. Petsko, The "glass transition" in protein dynamics: What it is, why it occurs, and how to exploit it, Biophysical Chemistry 105 (2003), pp. $667-680$.

Shah \& Ludescher (1995) N.K. Shah and R.D. Ludescher, Phosphorescence probes of the glassy state in amorphous sucrose, Biotechnology Progress 11 (1995), pp. 540-544.

Shah \& Ludescher (1993) N.K. Shah and R.D. Ludescher, Influence of hydration on the internal dynamics of hen egg white lysozyme in the dry state, Photochemistry and Photobiology 58 (1993), pp. 169-174.

Shirke \& Ludescher (2005a) S. Shirke and R.D. Ludescher, Molecular mobility and the glass transition in amorphous glucose, maltose, and maltotriose, Carbohydrate Research 340 (2005), pp. 2654-2660. 
Shirke \& Ludescher (2005b) S. Shirke and R.D. Ludescher, Dynamic site heterogeneity in amorphous maltose and maltitol from spectral heterogeneity in erythrosin B phosphorescence, Carbohydrate Research 340 (2005), pp. 2661-2669.

Shirke, Takhistov, \& Ludescher (2005) S. Shirke, P. Takhistov and R.D. Ludescher, Molecular mobility in amorphous maltose and maltitol from phosphorescence of erythrosin B, Journal of Physical Chemistry B 109 (2005), pp. 16119-16126.

Shirke, You, \& Ludescher (2006) S. Shirke, Y. You and R.D. Ludescher, Molecular mobility and dynamic site heterogeneity in amorphous lactose and lactitol from erythrosin B phosphorescence, Biophysical Chemistry 123 (2006), pp. 122-133.

Simon-Lukasik \& Ludescher (2004) K.V. Simon-Lukasik and R.D. Ludescher, Erythrosin B phosphorescence as a probe of oxygen diffusion in amorphous gelatin films, Food Hydrocolloids 18 (2004), pp. 621-630.

Slavik (1994) J. Slavik, Fluorescent probes in cellular and molecular biology, CRC Press, Boca Raton, FL (1994).

Smith (1991) J.C. Smith, Protein dynamics: Comparison of simulations with inelastic neutron scattering experiments, Quarterly Review of Biology 24 (1991), pp. 227-291.

Vanderkooi \& Berger (1989) J.M. Vanderkooi and J.W. Berger, Excited triplet states used to study biological macromolecules at room temperature, Biochimica et Biophysica Acta: Bioenergetics 976 (1989), pp. 1-27. 
Figure II-1

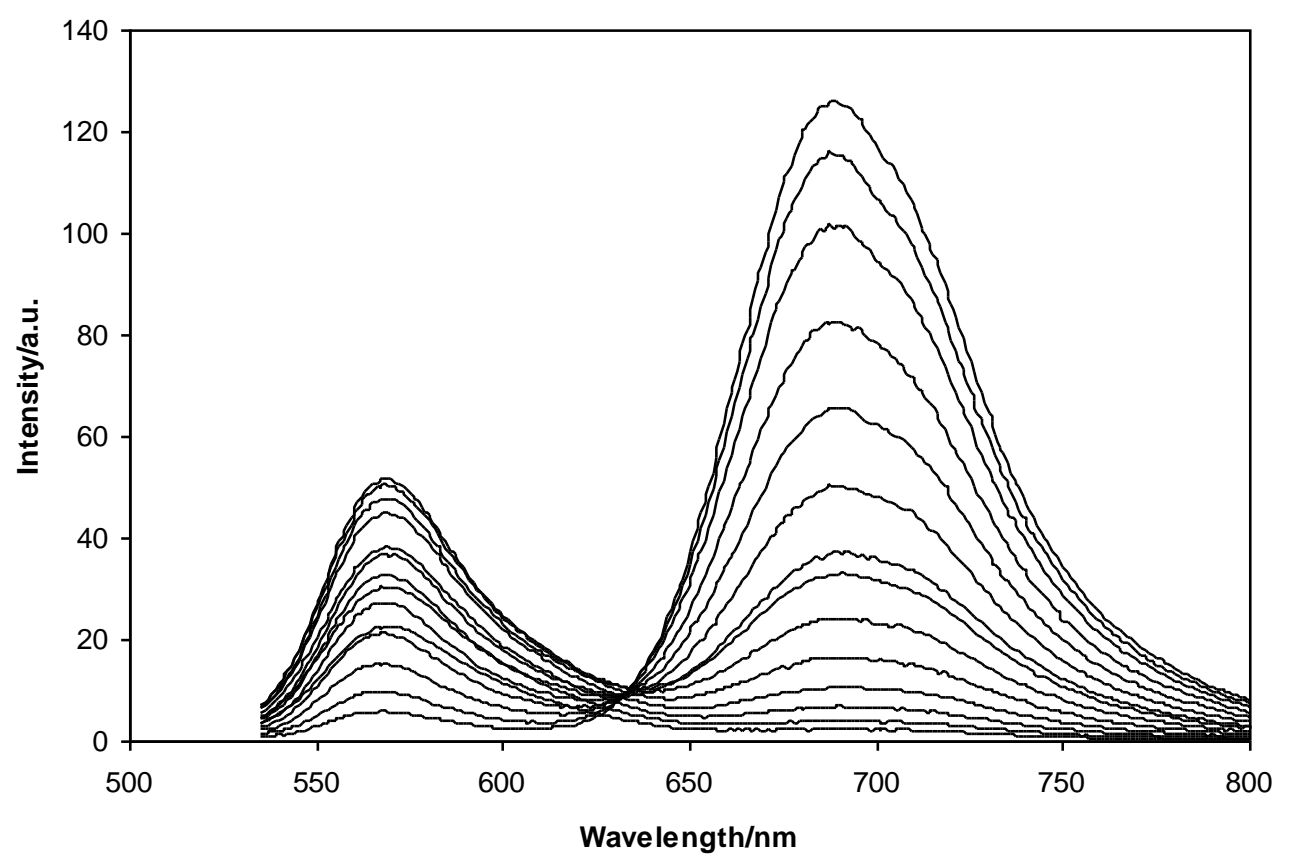

Figure II-1: The effect of temperature on the delayed emission spectra of Erythrosin B dispersed in amorphous $\beta$ - Lg films. Spectra were collected every $10^{\circ} \mathrm{C}$ from -20 to $110^{\circ} \mathrm{C}$ (curves from high to low intensity at $680 \mathrm{~nm})$. 
Figure II-2

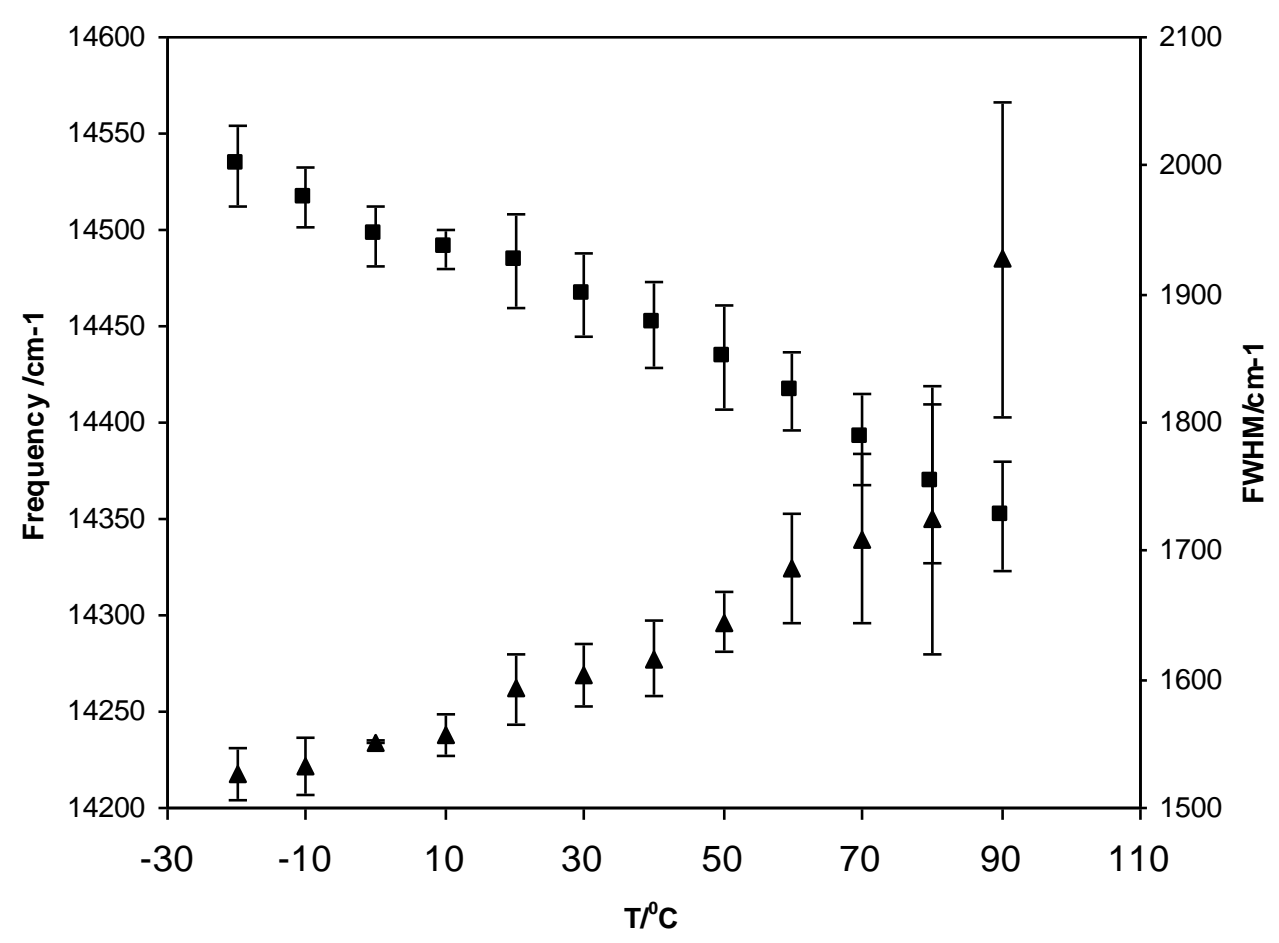

Figure II-2: The effect of temperature on the emission maximum ( $\mathbf{\square}$, left hand scale) and bandwidth ( $\mathbf{\Lambda}$, right hand scale) for phosphorescence from Erythrosin B in amorphous $\beta$-Lg films. Parameters were determined from analysis of the delayed emission spectra (as in Figure 1) using a log normal bandwidth function. 
Figure II-3

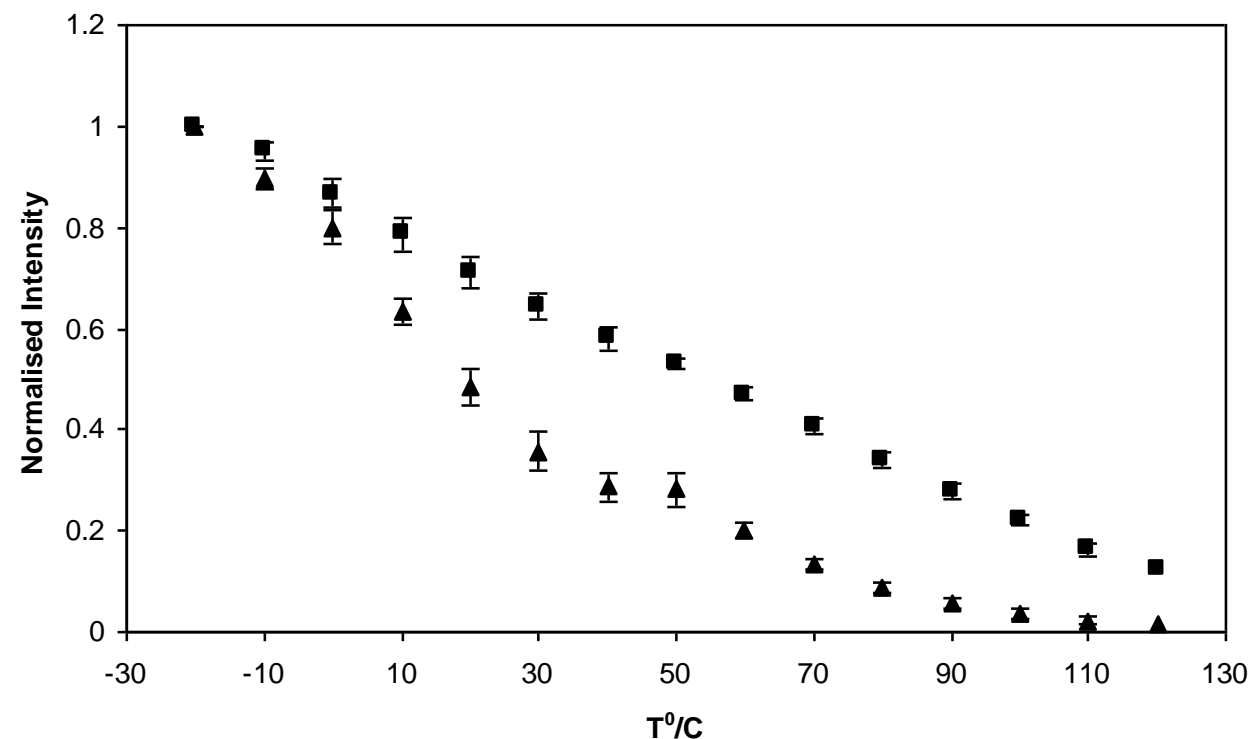

Figure II-3: The effect of temperature on the phosphorescence emission intensity of Erythrosin B in amorphous $\beta$-Lactoglobulin films equilibrated against nitrogen ( $\mathbf{-})$ and air $(\mathbf{\Lambda})$. Intensity was determined from analysis of the phosphorescence emission band using a log normal bandwidth function. 


\section{Figure II-4}
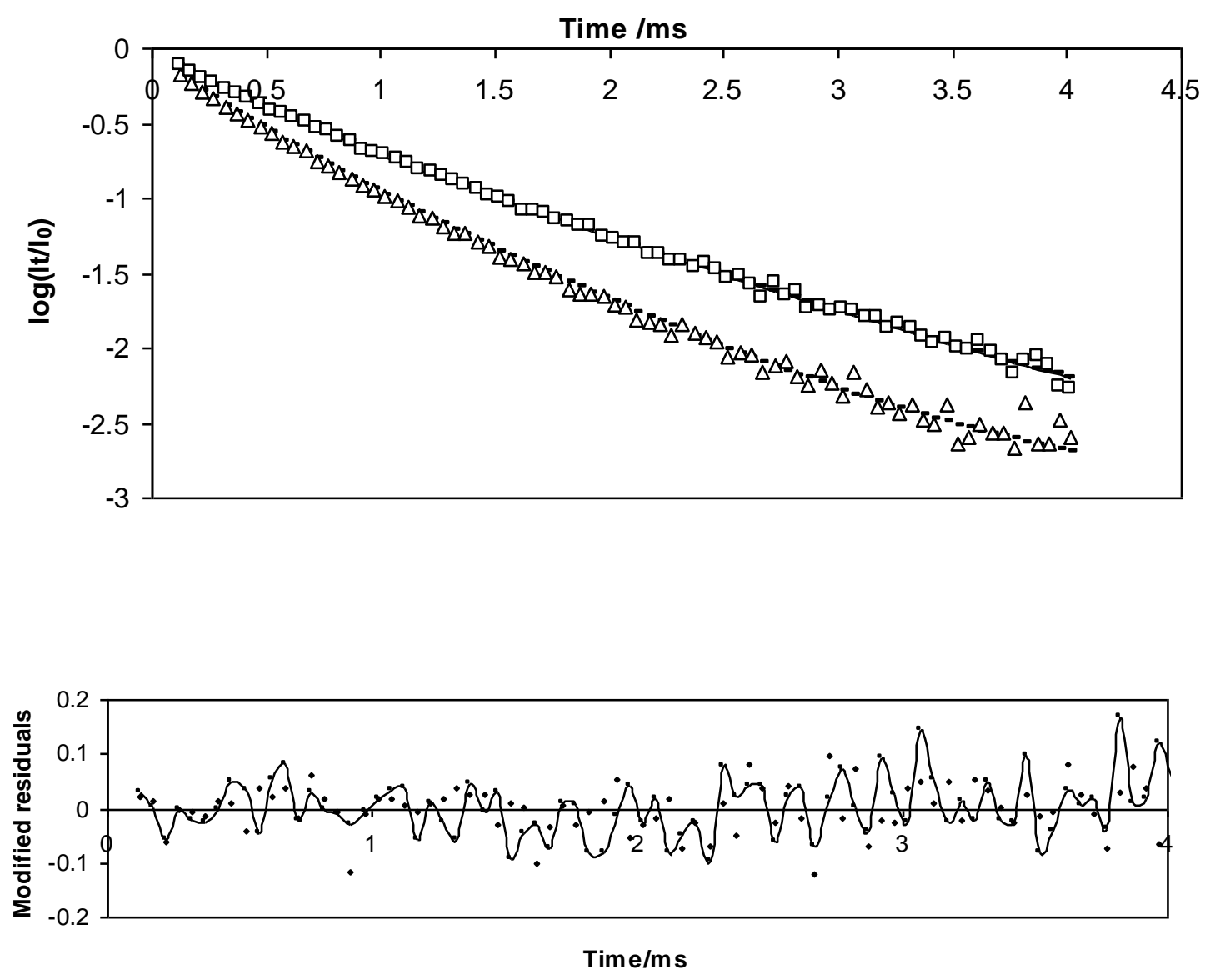

Figure II-4. Phosphorescence emission intensity decays of Erythrosin B dispersed in $\beta$-Lg films equilibrated against nitrogen $(\square)$ and air $(\Delta)$ at $20^{\circ} \mathrm{C}$. Solid lines show the results of a fit to the data using a stretched exponential decay function with $\tau=0.557 \mathrm{~ms}$ and $\beta=0.835$ for film in nitrogen and $\tau=0.369 \mathrm{~ms}$ and $\beta=0.810$ for film in air. The bottom graph is a plot of the modified residuals ((Data-Fit)/Data1/2) for the fit in nitrogen (solid line) and in air (dotted line). 
Figure II-5

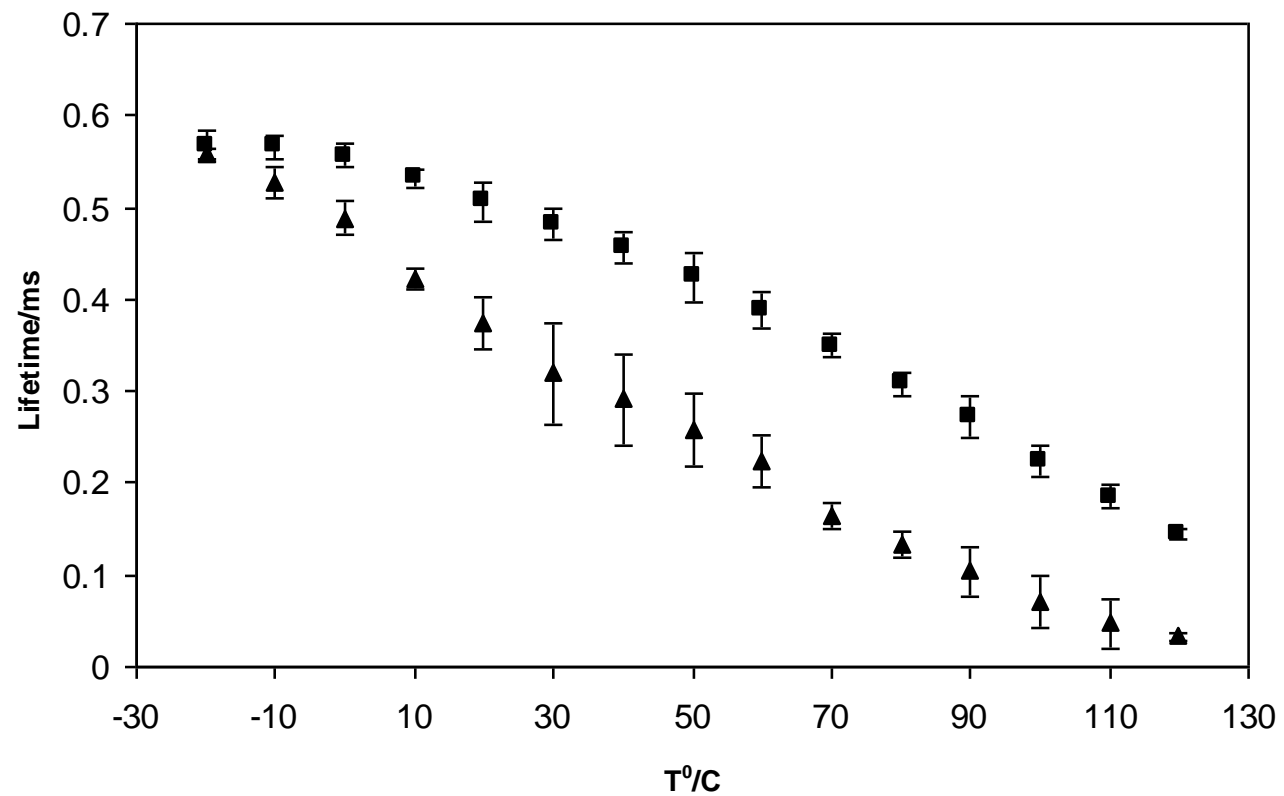

Figure II-5: The effect of temperature on the lifetime for Erythrosin B dispersed in $\beta$-Lactoglobulin films equilibrated against nitrogen ( $\boldsymbol{\square})$ and against air $(\boldsymbol{\Delta})$; the lifetime was determined from analysis using a stretched exponential decay function. 
Figure II-6

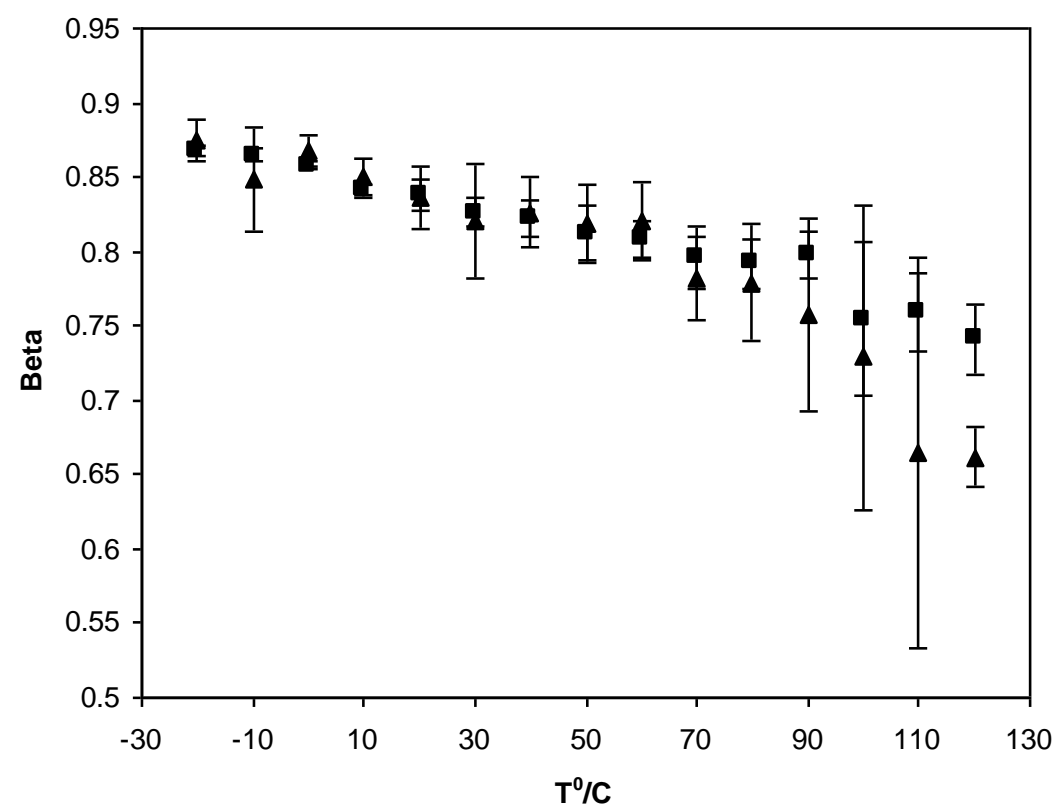

Figure II-6. The effect of temperature on the stretching exponent $\beta$ for Erythrosin B dispersed in $\beta$ Lactoglobulin films equilibrated against nitrogen ( $\bullet$ ) and against air $(\boldsymbol{\Lambda}) ; \beta$ was determined from analysis using a stretched exponential decay function. 
Figure II-7

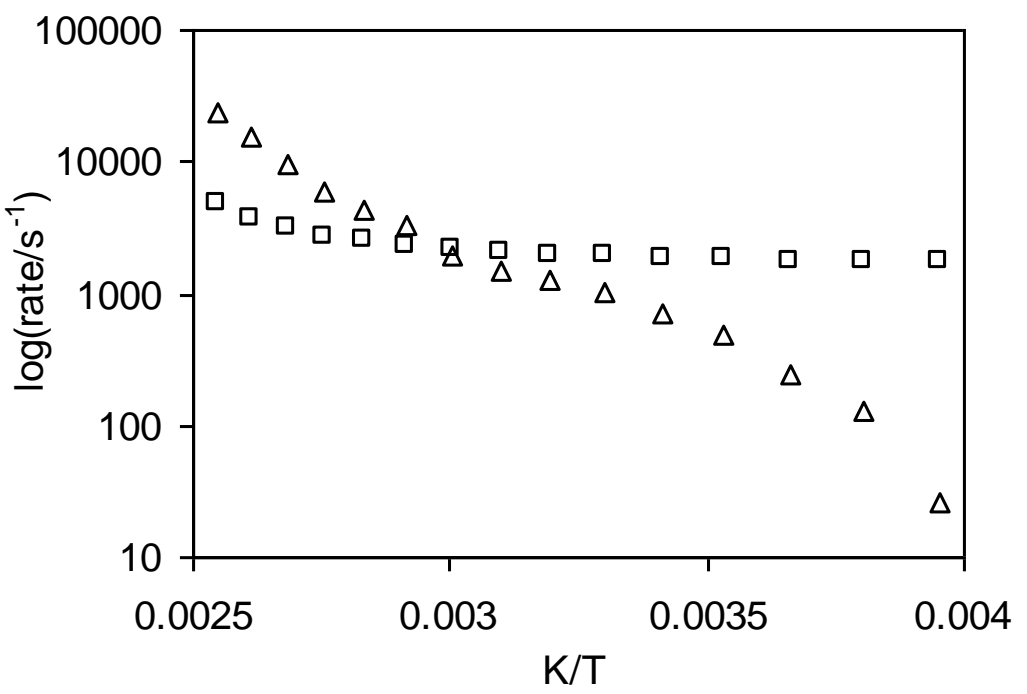

Figure II-7. Arrhenius plot of the effect of temperature on the triplet state deexcitation rates for intersystem crossing followed by vibrational relaxation to the ground state $\mathrm{k}_{\mathrm{TS} 0}(\omega)$ and for oxygen quenching $\mathrm{k}_{\mathrm{Q}}\left[\mathrm{O}_{2}\right]$ $(\Delta)$. 
Figure II-8

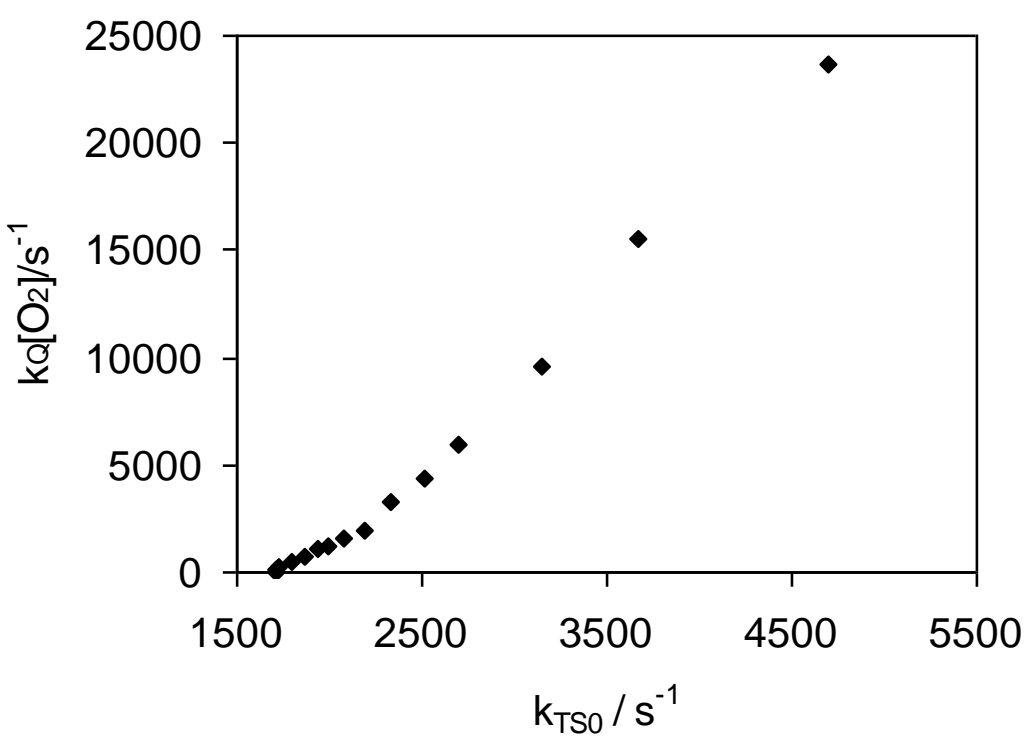

Figure II-8. Dependence of the oxygen quenching rate $\mathrm{k}_{\mathrm{Q}}\left[\mathrm{O}_{2}\right]$ on the rate of non-radiative quenching $\mathrm{k}_{\mathrm{TS} 0}$. 


\section{Chapter-III: Molecular Mobility and Oxygen Permeability in amorphous $\beta$ - Lactoglobulin $(\beta-\mathrm{Lg})$ Films with various sugars}

\section{Introduction}

Edible films are very promising systems for the improvement of food quality and preservation during processes and storage. Due to the recent increase in ecological consciousness considerable amount of research has been diverted towards the potential development and exploration of new frontiers in the field of edible film technology. The potential of whey proteins as mass-transfer barriers has recently generated interest due to their favorable nutritional characteristics and functional properties. $\beta$ - $\mathrm{Lg}$ is the most abundant protein in whey (almost 55\% of the total protein) (Dybing and Smith, 1991); therefore its properties are very important to the overall functionality of whey protein concentrates and isolates.

The transfer of oxygen to food from the environment has an important effect on food quality and shelf life. Oxygen causes food deterioration such as lipid and vitamin oxidation, leading to sensory and nutrient changes. Research by Sundaresan \& Ludescher (2007) has proven that pure $\beta$-Lg films are highly permeable to oxygen at room temperature. However, proper selection of additives can reduce the permeability of oxygen in edible films.

The addition of sugars to protein matrices can be very beneficial. Sugars can serve as plasticizers, stabilizers and protectants when added to protein matrices (Kaushik and Bhat, 2003). Bio-protectant media, such as polyol and particularly sugar-water matrices, show an outstanding ability in preserving structure and functionality of biomolecules, 
which has been often exploited in food, pharmaceutical, and biotechnology sciences to optimize lyophilization and long-term storage of biological samples (Hill et al., 2005). A number of studies have shown that freeze-drying in the presence of sugars aids in preserving the native confirmation of a protein. The specific sugar- protein interactions are generally thought to prevent conformational changes in the protein during dehydration and storage (Carpenter and Crowe, 1989; Constantino et al., 1998). Disaccharides are often used as additives in pharmaceutical, food and biomedical freezedrying processes to prepare glassy matrices for long term storage of biological materials Sucrose is a compatible osmolyte, belonging to a class of low molecular weight compounds produced by both prokaryotic and eukaryotic cells to protect proteins against the deleterious effects of harsh environmental conditions of water, salts, cold, and heat stress (Yancey et al., 1982; Borowitzka, 1985). Use of stabilizing excipients such as sucrose can prevent physical and chemical degradations of protein formulations by establishment of hydrogen bonds between protein and excipient and by forming an amorphous glassy matrix around proteins (Carpenter \& Crowe, 1989; Arakawa et al., 1993; Angel, 1995; Allison et al., 1999). Trehalose is another glass-forming material with specific interactions with proteins (Branca et al., 1999; Crowe et al., 1994, 1998; Green and Angell, 1989). Trehalose has a high hydrogen bonding affinity; it thus may replace the water molecules close to the protein surface. It has a rather high glass-transition temperature (Green \& Angell, 1989; Miller et al., 1999), and shows direct interaction with bilayers, thus stabilizing liposomes during freeze-drying (Crowe et al., 1985).

Although studies in the past have focused on the stabilizing interactions of sugars on proteins, but there is a constant need of studies that look into the molecular 
mechanisms underlying these interactions. Luminescence spectroscopy is a nondestructive method and has a wide range of research applications to monitor molecular dynamics in biological macromolecules such as proteins, nucleic acids and phospholipid bilayers. It shows a great deal of promise in elucidating the subtle micro-structural elements present in an amorphous matrix. Luminescence spectroscopy can generate detailed three-dimensional maps of the distribution of molecular mobility in complex heterogeneous foods. Such mobility contours can provide the molecular detail necessary to generate detailed physical-chemical models connecting food quality and stability to molecular structure and mobility (Ludescher, 2001). In this study, we employ a novel technique called phosphorescence spectroscopy as an indicator of the mobility and oxygen permeability of the sugar/protein films.

Erythrosin B (Ery B; tetraiodofluorescein) is perhaps the most widely used phosphorescent probe of the molecular mobility of water-soluble and membrane-bound proteins because of its large extinction coefficient and high phosphorescence quantum yield even in aqueous solution. We have successfully used Ery B phosphorescence to monitor molecular mobility in amorphous solid sugars and sugar alcohols (Pravinata et al., 2005; Shah \& Ludescher, 1995; Shirke \& Ludescher, 2005a, 2005b; Shirke, et al., 2005), in solid gelatin (Lukasik \& Ludescher, 2006a, 2006b; Lukasik \& Ludescher, 2004), and in amorphous solid bovine serum albumin (Nack \& Ludescher, 2006) and pure $\beta$-Lg films (Sundaresan \& Ludescher, 2006). The objective of this study was to utilize an integrated approach to evaluate the oxygen permeability and mobility of plasticized $\beta-\mathrm{Lg}$ films to compare the effectiveness of sucrose, trehalose and maltose as plasticizers in hampering the entry of atmospheric oxygen. This study aims at looking 
into the development of functional matrices that are less permeable to atmospheric oxygen.

\section{Materials and Methods}

\section{Sample preparation}

Solutions of $\beta$-Lg (90\% pure; crystallized and lyophilized from Sigma Chemical Co, St. Louis, MO, USA) were prepared in distilled de-ionized water at an approximate concentration of $10 \mathrm{mg} / \mathrm{ml}$. This concentration was selected based on the batch size to ensure maximum recovery after dialysis and to avoid long-term storage. An aqueous $\beta$ Lg solution was transferred to regenerated cellulose dialysis tubing having a $12-14 \mathrm{kDa}$ molecular weight cutoff (Spectrum, Houston, TX). In order to define and minimize the presence of counter ions the solution was extensively dialyzed against $0.1 \mathrm{M}$ potassium chloride for 24 hours with frequent change of buffer every 6 hours. The solution was then dialyzed against distilled de-ionized water for 24 hours with frequent change of water after every 6 hours. The proposed protocol was followed in a very stringent manner in order to control precipitation in the protein during dialysis. The dialyzed solution was then filtered through a $0.2-\mu \mathrm{m}$ membrane to remove particulates. In order to maximize the effectiveness of the dialysis process temperature of the solution was kept at $5^{\circ} \mathrm{C}$ to ensure that the protein remained folded. Several dilutions of a stock solution of dialyzed $\beta$-Lg were prepared. The dye to protein ratio was determined by measuring the absorbance of dilutions in a $1 \mathrm{~cm}$ path length quartz cuvette in a Bausch and Lomb Spectronic 2000 spectrophotometer (Milton Roy company, Analytical Products Division, Rochester, NY). The protein concentration was determined after dialysis by UV absorbance using an extinction coefficient of $0.96 \mathrm{~L} \mathrm{~g} \mathrm{~g}^{-1} \mathrm{~cm}^{-1}$. The recovered protein 
solution had an approximate concentration of $6-8 \mathrm{mg}$ of protein. ErythrosinB (EryB) (tetra iodofluorescein, FD\&C red\#3) from molecular probes (Eugene, OR), was dissolved in spectrophotometric grade N, N-dimethyl formamide (DMF)(Aldrich Chemical, Milwaukee, WI) to make $10 \mathrm{mM}$ and $100 \mathrm{mM}$ stock solutions. The solvent DMF was selected for probe stability during long time storage and the concentrations were selected in order to simplify the addition of probe to the protein matrix. After estimating the concentration protein solution was combined with aliquots of dye solution to make solutions with molar ratio of EryB: $\beta-\operatorname{Lg}$ of $1: 4$. This protein concentration was determined to produce tractable films under established conditions. The dye to protein concentration was sufficient to provide adequate signal/noise in spectroscopic measurements. Sucrose, maltose and trehalose were purchased from Sigma-Aldrich (St. Louis, MO) with minimum purity of $98 \%$ and were used without further purification. These components were dissolved to near saturation in de-ionized water at room temperature. Sucrose, maltose and trehalose were added in various molar ratios. The molar ratios ranged from 0.1:1 through 10,000:1 (sugar: $\beta$-Lg) for sucrose and ratios 0.1:1 through 1000:1 from trehalose and maltose. These ratios were selected in order to judge the threshold concentration of sugar required to control oxygen diffusion within the detectable limits of our instrumentation and to provide the best possible estimate of the permeability of oxygen in these functional matrices.

\section{Preparation of protein films}

To make glassy films $15 \mu \mathrm{l}$ of protein / sugar solution was spread on approximately one third of a quartz slide $(30 \times 13.5 \times 0.6 \mathrm{~mm}$; custom made by NSG Precision Cells, Farmingdale, NY). Before use the quartz slides were soaked in terg- 
enzyme soap solution overnight in order to remove the surface impurities and then washed with de-ionized water, soaked in ethanol for 1-2 hours and dried with acetone. Occasionally the slides were soaked in acid for thorough cleaning. The protein / sugar films approximately $(0.05 \mathrm{~mm}$ thick when dried) were visually checked for good spreading, uniformity, and consistency. The slides were stored at room temperature in a desiccator containing $\mathrm{P}_{2} \mathrm{O}_{5}$ and DrieRite for at least 7 days and protected from light to prevent any photobleaching of Erythrosin B prior to luminescence measurements. The slides were checked for crystallization under crossed polarizers using a Nikon Type 102 dissecting microscope (Nikon, Inc., Japan).

\section{Luminescence measurements}

All measurements were conducted using a CARY Eclipse spectrophotometer (Varian Instruments, Walnut Creek, CA). The temperature was controlled by using a TLC-50 thermoelectric heating/cooling system (Quantam Northwest, Spokane,WA). The TLC-50 sample compartment was fitted with a jacketed cover. For luminescence measurements necessitating the absence of oxygen (a luminescence quencher) the samples were flushed with nitrogen gas for at least 15 minutes to free the environment from oxygen. An oxygen free nitrogen stream was generated by passage of high purity nitrogen through a Supelco (Bellefonte, PA) carrier gas purifier.

Quartz slides were placed on end on the diagonal of a standard $1 \mathrm{~cm} \times 1 \mathrm{~cm}$ quartz fluorescence cuvette which was capped with a lid having inlet and outlet ports for gas lines, thus all experiments were conducted at constant total pressure. Dry air was flushed to prevent condensation on the faces of the cuvette at experimental conditions below room temperature. 
Delayed luminescence emission spectra were collected from 535-800 nm (10nm bandwidth) using excitation of $525 \mathrm{~nm}$ (10nm bandwidth) over the temperature range from $0^{\circ} \mathrm{C}$ to $100^{\circ} \mathrm{C}(10$ degree intervals) Each data point $(1 \mathrm{~nm}$ interval, $0.1 \mathrm{~s}$ averaging time) was collected from a single flash with a $0.2 \mathrm{~ms}$ delay, $0.5 \mathrm{~ms}$ gate time and $4.0 \mathrm{~ms}$ total decay time.

For lifetime measurements as a function of temperature samples were excited at $540 \mathrm{~nm}$ (20nm bandwidth) and emission transients collected at $690 \mathrm{~nm}$ (20nm bandwidth) at temperature ranging from $0^{\circ} \mathrm{C}-100^{\circ} \mathrm{C}$. Before collecting data samples were equilibrated for 15 minutes. The measurements were taken in the presence or absence of air (under a nitrogen purge). Each time-resolved decay transient was the average of 100 cycles and for each cycle data was collected from a single flash with a delay of $0.1 \mathrm{~ms}$, a 0.02 gate time and $4.0 \mathrm{~ms}$ total decay time. All experiments were done several times and the averages were used for data analysis and interpretation. In order to validate the repeatability the standard deviation in between experiments were calculated.

\section{Basic photophysics}

Luminescence is a general term used to describe what occurs when a probe molecule absorbs photons of higher energy (excitation) and subsequently releases photons of lower energy (emission). This phenomenon is a function of a molecule's structure, which in luminescent molecules is usually characterized by an extended delocalized $\pi$-orbital system. The Jablonski energy level diagram summarizes the various photophysical events occurring during luminescence. Following light absorption the ground state molecule makes an essentially instantaneous transition $\left(\sim 10^{-15} \mathrm{~s}\right)$ to one of the many vibrational levels of the first excited state $\left(S_{1}\right)$; excess vibrational energy is 
rapidly $\left(\sim 10^{-12} \mathrm{~s}\right)$ dissipated to the solvent matrix to relax to the lowest vibrational level of $\mathrm{S}_{1}$. Along with fluorescence (rate $\mathrm{k}_{\mathrm{RF}}$ ), the excited state may engage in several deexcitation processes including non-radiative decay to $S_{0}$ through exchange of vibrational energy to the matrix (rate constant $\mathrm{k}_{\mathrm{SS} 0}$ ), intersystem crossing from $\mathrm{S}_{1}$ to $\mathrm{T}_{1}$ (rate $\mathrm{k}_{\mathrm{ST}}$ ) and collisional quenching rate $\mathrm{k}_{\mathrm{q}}\left[\mathrm{O}_{2}\right]$. Along with phosphorescence (rate $\mathrm{k}_{\mathrm{RP}}$ ) occurs the deexcitation processes including non-radiative decay from $\mathrm{T}_{1}$ to $\mathrm{S}_{0}$ (rate $\mathrm{k}_{\mathrm{TS} 0}$ ), reverse intersystem crossing from $\mathrm{T}_{1}$ to $\mathrm{S}_{1}\left(\right.$ rate $\mathrm{k}_{\mathrm{TS} 1}$ ) and collisional quenching by oxygen (rate $\left.\mathrm{k}_{\mathrm{Q}}\left[\mathrm{O}_{2}\right]\right)$. The rate constants $\mathrm{k}_{\mathrm{RF}}, \mathrm{k}_{\mathrm{RP}}$ and $\mathrm{k}_{\mathrm{TS} 1}$ are fixed by the probe structure and largely unaffected by environment (Turro, 1991; Birks, 1970).

\section{Emission intensity}

Luminescence emission intensity is directly proportional to the emission quantum yield, $\Phi=$ photons emitted/photons absorbed. The emission quantum yield reflects the relative rates of the several de-excitation pathways available to the excited state of a chromophore. The quantum yield for fluorescence is given by the following ratio of rate constants (Lakowicz, 1999). The term $\mathrm{k}_{\mathrm{q}}\left[\mathrm{O}_{2}\right]$ describes the rate of collisional quenching of the excited state of the probe by oxygen.

$$
\Phi_{\mathrm{F}}=\mathrm{k}_{\mathrm{RF}} /\left(\mathrm{k}_{\mathrm{RF}}+\mathrm{k}_{\mathrm{SS} 0}+\mathrm{k}_{\mathrm{ST}}+\mathrm{k}_{\mathrm{q}}\left[\mathrm{O}_{2}\right]\right)
$$

In the absence of specific quenchers in the local environment, the typical situation for fluorescence, Eq. (1) simplifies to:

$$
\Phi_{\mathrm{F}}=\mathrm{k}_{\mathrm{RF}} /\left(\mathrm{k}_{\mathrm{RF}}+\mathrm{k}_{\mathrm{SS} 0}+\mathrm{k}_{\mathrm{ST}}\right)
$$

In the case of phosphorescence, the quantum yield for phosphorescence $\Phi_{\mathrm{P}}$ is the product of the quantum yield for $T_{1}$ formation $\left(\Phi_{T}\right)$ times the probability of emission from $T_{1}\left(q_{P}\right)$ (Hurtubise, 1990): 


$$
\Phi_{\mathrm{P}}=\Phi_{\mathrm{T}} \mathrm{q}_{\mathrm{P}}=\left\{\mathrm{k}_{\mathrm{ST}} /\left(\mathrm{k}_{\mathrm{RF}}+\mathrm{k}_{\mathrm{SS} 0}+\mathrm{k}_{\mathrm{ST}}+\mathrm{k}_{\mathrm{q}}\left[\mathrm{O}_{2}\right]\right)\right\}\left\{\mathrm{k}_{\mathrm{RP}} /\left(\mathrm{k}_{\mathrm{RP}}+\mathrm{k}_{\mathrm{TS} 0}+\mathrm{k}_{\mathrm{TS} 1}+\mathrm{k}_{\mathrm{Q}}\left[\mathrm{O}_{2}\right]\right)\right\}
$$

The quantum yield for delayed fluorescence in the absence of oxygen is the product of the quantum yield of fluorescence and the probabilities of intersystem crossing from $S_{1}$ to $\mathrm{T}_{1}$ and also from $\mathrm{T}_{1}$ to $\mathrm{S}_{1}$ (Duchowicz et al., 1998).

$$
\Phi_{\mathrm{DF}}=\Phi_{\mathrm{F}}\left\{\mathrm{k}_{\mathrm{ST}} /\left(\mathrm{k}_{\mathrm{RF}}+\mathrm{k}_{\mathrm{SS} 0}+\mathrm{k}_{\mathrm{ST}}\right)\right\}\left\{\mathrm{k}_{\mathrm{TS} 1} /\left(\mathrm{k}_{\mathrm{RP}}+\mathrm{k}_{\mathrm{TSO}}+\mathrm{k}_{\mathrm{TS} 1}\right)\right\}
$$

By comparing the emission intensities of delayed fluorescence and phosphorescence it is possible to obtain information about the rate constants for intersystem crossing $\mathrm{k}_{\mathrm{TS} 1}$.

$$
\mathrm{I}_{\mathrm{DF}} / \mathrm{I}_{\mathrm{P}}=\Phi_{\mathrm{F}}\left\{\mathrm{k}_{\mathrm{ST}} / \mathrm{k}_{\mathrm{F}}\right\}\left\{\mathrm{k}_{\mathrm{TS} 1} / \mathrm{k}_{\mathrm{P}}\right\} /\left\{\mathrm{k}_{\mathrm{ST}} / \mathrm{k}_{\mathrm{F}}\right\}\left\{\mathrm{k}_{\mathrm{RP}} / \mathrm{k}_{\mathrm{P}}\right\}=\Phi_{\mathrm{F}} \mathrm{k}_{\mathrm{TS} 1} / \mathrm{k}_{\mathrm{RP}}
$$

Where $\mathrm{k}_{\mathrm{F}}$ and $\mathrm{k}_{\mathrm{P}}$ are the total decay rates for the de-excitation of the singlet and triplet states respectively. This ratio is highly temperature dependent due to the additional energy required to go from $T_{1}$ to $S_{1}$ energy level and can be described by the following equation where $\Delta \mathrm{E}_{\mathrm{TS}}$ is the effective energy gap between the triplet and singlet states.

$$
\Phi_{\mathrm{F}} \mathrm{k}_{\mathrm{TS} 1} / \mathrm{k}_{\mathrm{R} P}=\left\{\Phi_{\mathrm{F} /} \mathrm{k}_{\mathrm{RP}}\right\} \mathrm{k}^{\mathrm{o}}{ }_{\mathrm{TS} 1} \exp \left(-\Delta \mathrm{E}_{\mathrm{TS}} / \mathrm{RT}\right)
$$

The slope of the Van't Hoff plot of the natural log of the ratio of delayed fluorescence $\left(\mathrm{I}_{\mathrm{DF}}\right)$ to phosphorescence $\left(\mathrm{I}_{\mathrm{P}}\right)$ intensity versus inverse temperature provides a measure of $\Delta \mathrm{E}_{\mathrm{TS}}$ (Duchowicz, et al., 1998).

Phosphorescence intensity decays are non-exponential and a stretched exponential decay function has been shown to be appropriate to describe the wide distribution of relaxation times (Champion et al., 2000; Richert, 2000). 


$$
\mathrm{I}(\mathrm{t})=\mathrm{I}_{0} \exp \left[-(\mathrm{t} / \tau)^{\beta}\right]+\mathrm{c}
$$

Where $I_{0}$ is the initial amplitude, $\tau$ is the stretched exponential lifetime, $\beta$ is an exponent varying from 0 to 1 and characterizing the distribution of lifetimes. The use of a stretched exponential model provides a direct measurement of a continuous distribution of lifetimes, which is appropriate for describing the decay in a complex glass possessing a distribution of relaxation times for the dynamic molecular processes. The phosphorescence lifetimes were used to calculate the rate constants associated with the various processes that depopulate the excited triplet state. In an amorphous solid in the absence of oxygen the temperature dependence of $\mathrm{k}_{\mathrm{P}}$ can be written as follows:-

$$
\mathrm{k}_{\mathrm{P}}(\mathrm{T})=\mathrm{k}_{\mathrm{RP}}+\mathrm{k}_{\mathrm{TS} 1}(\mathrm{~T})+\mathrm{k}_{\mathrm{TS} 0}(\mathrm{~T})
$$

But in the presence of oxygen the de-excitation of the triplet state is also influenced by the quencher species. The term $\mathrm{k}_{\mathrm{Q}}\left[\mathrm{O}_{2}\right]$ describes the rate of collisional quenching of the excited state of the probe by oxygen. Thus in the presence of oxygen $k_{P}(T)$ includes an additional term $\mathrm{k}_{\mathrm{Q}}\left[\mathrm{O}_{2}\right]$.

$$
\mathrm{k}_{\mathrm{P}}(\mathrm{T})=\mathrm{k}_{\mathrm{RP}}+\mathrm{k}_{\mathrm{TS} 1}(\mathrm{~T})+\mathrm{k}_{\mathrm{TS} 0}(\mathrm{~T})+\mathrm{k}_{\mathrm{Q}}\left[\mathrm{O}_{2}\right](\mathrm{T})
$$

Equation (8) can be used to calculate $\mathrm{k}_{\mathrm{TS} 0}$, the rate of collisional quenching to the ground state when $\mathrm{k}_{\mathrm{RP}}$ and $\mathrm{k}_{\mathrm{TS} 1}$ are known. $\mathrm{k}_{\mathrm{RP}}$, the radiative decay rate of the triplet state, is $41 \mathrm{~s}^{-1}$ for erythrosin B (Duchowicz et al., 1998; Lettinga et al., 2000).

$\mathrm{k}_{\mathrm{TS} 1}$ the rate of reverse intersystem crossing from the excited triplet state to the excited singlet state depends on $\Delta \mathrm{E}_{\mathrm{TS}}$, the energy gap between $\mathrm{S}_{1}$ and $\mathrm{T}_{1}$

$$
\mathrm{k}_{\mathrm{TS} 1}(\mathrm{~T})=\mathrm{k}^{\circ} \mathrm{TS} 1_{1} \exp \left(-\Delta \mathrm{E}_{\mathrm{TS}} / \mathrm{RT}\right)
$$


A plot of $\ln \left(\mathrm{I}_{\mathrm{DF}} / \mathrm{I}_{\mathrm{P}}\right)$ versus $1 / \mathrm{T}$ showed linearity and has slope of $-\mathrm{E}_{\mathrm{TS}} / \mathrm{R}$ and thus $\Delta \mathrm{E}$ for the $\beta$-Lg matrix was calculated . Unfortunately, literature values of $\mathrm{k}^{\circ}{ }_{\mathrm{TS} 1}$ for erythrosin $\mathrm{B}$ vary widely, from $0.3 \times 10^{7} \mathrm{~s}^{-1}$ in ethanol and $6.5 \times 10^{7} \mathrm{~s}^{-1}$ in water (Duchowicz et al., 1998) to $111 \times 10^{7} \mathrm{~s}^{-1}$ in solid polyvinyl alcohol (Lettinga et al., 2000), and thus provide little guidance. We estimated the maximum possible value for $\mathrm{k}^{\mathbf{o}}{ }_{\mathrm{TS} 1}$ in $\beta$-Lg sucrose films by assuming that $\mathrm{k}_{\mathrm{TS} 1}(\mathrm{~T})$ cannot result in values for $\mathrm{k}_{\mathrm{TS} 0}$ that decrease with temperature. This procedure thus estimated the minimum possible values of $\mathrm{k}_{\mathrm{TS} 0}(\mathrm{~T})$. Various values of $\mathrm{k}_{\text {TS1 }}^{\mathbf{o}}$ have been summarized up in Table III- $1 \mathrm{a}, \mathrm{b}, \mathrm{c}$.

In order to study the oxygen diffusion in the $\beta-\mathrm{Lg}$ matrix $\mathrm{k}_{\mathrm{Q}}\left[\mathrm{O}_{2}\right]$ can easily be calculated from the difference between the decay rate in the presence of oxygen and the absence of oxygen.

$$
\mathrm{k}_{\mathrm{Q}}\left[\mathrm{O}_{2}\right]=1 / \tau \text { (Air) }-1 / \tau \text { (Nitrogen) }
$$

Data analysis

Emission energy as a function of Temperature

Delayed luminescence spectra collected from $535 \mathrm{~nm}$ to $800 \mathrm{~nm}$ were fit using the program NFIT (Island Products) to a sum of distinct log-normal functions for delayed fluorescence $\left(\mathrm{I}_{\mathrm{DF}}(\mathrm{v})\right)$ and phosphorescence $\left(\mathrm{I}_{\mathrm{P}}(v)\right)$ in which all fit parameters were independent for each emission band. The energy of the emission maximum $\left(v_{\mathrm{P}}\right)$ and the full width at half maximum $(\Gamma)$ of the emission bands were determined from the function (Maroncelli and Fleming, 1987). Some of the data were fit using the program Igor (Wavemetrics, Inc., Lake Oswego, OR)

$$
\mathrm{I}(\mathrm{v})=\mathrm{I}_{0} \exp \left\{-\ln (2)\left[\ln \left(1+2 \mathrm{~b}\left(v-v_{\mathrm{P}}\right) / \Delta\right) / \mathrm{b}\right]^{2}\right\}
$$


In this equation $\mathrm{I}_{0}$ is the maximum emission intensity, $v_{\mathrm{P}}$ is the frequency $\left(\mathrm{cm}^{-1}\right)$ of the emission maximum, $\Delta$ is a line width parameter, and $\mathrm{b}$ is an asymmetry parameter The bandwidth $(\Gamma)$ of the emission band is related to $\mathrm{b}$ and $\Delta$ :

$$
\Gamma=\Delta\{\sinh (\mathrm{b}) / \mathrm{b}\}
$$

Phosphorescent intensity decay and rate calculations

Intensity decays were clearly non exponential and were analyzed using a stretched exponential function. Phosphorescence lifetimes were determined by nonlinear leastsquares analysis with the program NFIT (Island Products, Galveston, TX). Fits were judged acceptable if they had satisfactory fit errors and if data points were randomly distributed about the fit curve; most data sets had $\chi^{2} \leq 1.0$ and $\mathrm{R}^{2}$ in the range of $0.99-1.0$. Data were well analyzed with a stretched exponential decay model.

\section{$\underline{\text { Results }}$}

\section{Delayed emission spectra}

Delayed emission spectra of the triplet probe Erythrosin B, dispersed in optically clear amorphous films of dry $\beta-\operatorname{Lg}$ with 10 moles of sugar per protein (Ery: $\beta$ - $\operatorname{Lg} \quad 4: 1$ ), were collected over the temperature range from $0^{\circ} \mathrm{C}$ to $100^{\circ} \mathrm{C}$ (Figure III- $1 \mathrm{a}, \mathrm{b}, \mathrm{c}$ ). The long wavelength emission band reflects phosphorescence from the excited $\mathrm{T}_{1}$ state while the short wavelength band reflects delayed fluorescence from the excited $S_{1}$ state that has been repopulated by reverse intersystem crossing from $T_{1}$ (Parker, 1968). These spectra showed a decrease in the phosphorescence intensity $\left(\mathrm{I}_{\mathrm{P}}\right)$ and increase in the delayed fluorescence intensity $\left(\mathrm{I}_{\mathrm{DF}}\right)$ with increasing temperature as expected for a thermally stimulated process. Plots of $\ln \left(\mathrm{I}_{\mathrm{DF}} / \mathrm{I}_{\mathrm{P}}\right)$ versus $1 / \mathrm{T}$ (using the maximum intensity 
determined from fitting spectra to a log-normal function, Eq. 12 Materials and methods), were linear with $\mathrm{R}^{2}=0.9998$ and were used to calculate $\Delta \mathrm{E}_{\mathrm{TS}}$, the energy gap between $\mathrm{T}_{1}$ and $S_{1}$ (data not shown). The calculated energy gaps were further used for detailed analysis of matrix mobility. These values were significantly different from the pure $\beta$ - $\mathrm{Lg}$ matrix or the pure sugars by them self-suggesting that solvent (matrix) properties modulate the singlet-triplet energy gap (Table III- 2).

Peak frequencies of the phosphorescence of erythrosin B in $\beta$-Lg sugar films were plotted at various temperatures from $0^{\circ} \mathrm{C}$ to $100^{\circ} \mathrm{C}$. Peak frequencies were obtained by fitting the phosphorescence intensity data to a lognormal function (Material and Methods, $\mathrm{Eq} 12)$.

The phosphorescence emission peak frequency of erythrosin B varied systematically with temperature in the $\beta$-Lg sugar films (Figure III- $2 a, b, c$ ). (Due to complications inherent in the interpretation of relaxation events associated with the threestate $S_{0} \leftarrow S_{1} \leftarrow T_{1}$ transition involved in delayed fluorescence, these data are not analyzed here.). The emission peak frequency was constant at low temperatures but decreased monotonically at higher temperature in all the films. The peak frequency varied between $14900-14600 \mathrm{~cm}^{-1}$ at low temperatures in different sugars concentrations (10,000 sugars0.1 sugar) and between $14600-14300 \mathrm{~cm}^{-1}$ at high temperatures.

Over the temperature range from $0^{\circ} \mathrm{C}$ to $100^{\circ} \mathrm{C}$ the phosphorescence emission energy in sucrose / $\beta$-Lg films decreased in a linear fashion at all ratios except 1000:1 and 10,000:1. An interesting observation was made with respect to the addition of higher ratios of sucrose in the $\beta$-Lg films. It was seen that at ratios of 1000:1 and 10,000:1 (sucrose/ $\beta$-Lg) which are predominantly sugar matrices (92-99 wt\%), the 
phosphorescence emission energy remained constant at low temperatures and there was a noticeable decrease as the sucrose glass transition temperature was approached ( $\mathrm{Tg} \sim$ $65^{\circ} \mathrm{C}$ ). The decrease in emission energy reflects an increase in the average extent of matrix dipolar relaxation around the excited triplet state before emission (Lakowicz, 1999; Richert, 2000); the decrease in average emission energy reflects an increase in the rate of dipolar relaxation in the sucrose melt above $\mathrm{Tg}$.

It is clearly evident from the plots that addition of various ratios of sucrose brought an increase in the peak frequency. There was a small increase in the peak frequency at lower ratios of sucrose $/ \beta-\operatorname{Lg}(0.1: 1,1: 1,10: 1)$. At these ratios $v_{P}$ was essentially constant. However, there was a noticeable increase in the phosphorescence peak frequency at ratios of 100:1 and 1000:1. The emission energy increased further at a ratio of 10,000:1 at low temperatures, but after the glass transition there was a steep decline in the emission energy. Increases in peak frequency suggested that increased sucrose concentrations brought about a decrease in the dipolar relaxations.

The phosphorescence emission energy $v_{\mathrm{P}}$ of the trehalose / $\beta$-Lg films decreased linearly as a function of temperatures in all the ratios. As the Tg of trehalose is very high and due to inherent difficulties in analyzing the data at high temperatures the effect of Tg at higher concentration was not detectable in the trehalose / $\beta$ - $\mathrm{Lg}$ matrix. The peak frequency values increased with increasing trehalose concentration. However, the $v_{\mathrm{P}}$ values did not increase as a function of ratio of trehalose to $\beta$-Lg. From Figure III- $2 \mathrm{~b}$ it is clearly evident that even small amounts of trehalose (as low as 0.1:1 Treh: $\beta$ $\mathrm{Lg}$ ) had a great impact on the relaxation of the matrix as noticed by the significant increase in the emission energy. 
In maltose / $\beta$-Lg films the phosphorescence emission energy followed a similar trend with temperature as observed in trehalose / $\beta$-Lg films. Also in maltose / $\beta$ - $\mathrm{Lg}$ films due to the high glass transition temperature of maltose $\left(\mathrm{Tg} \sim 93^{\circ} \mathrm{C}\right.$ Roos, 1993) the effect of Tg was not evident.

Concomitant increase in maltose concentration led to an increase in peak frequency in maltose / $\beta$-Lg films, whereas in sucrose / $\beta$ - $\operatorname{Lg}$ and trehalose / $\beta$ - $\mathrm{Lg}$ although there was an increase in peak frequency with increasing sugar concentrations but $v_{\mathrm{P}}$ did not increase as a function of the sugar ratio. At concentrations as high as 100:1 we could see that the emission energy reached a threshold value above which there was no further increase with increase in sugar concentration indicating that the matrix had attained its maximum rigidity above which there were no further decreases in relaxation extent. Within the detectable limits of our instrumentation it can be seen that the threshold concentration for the attainment of maximum possible peak frequency is similar for all the sugars, which can be attributed to diminishing of the existing matrix relaxations in the presence of sugars.

Full width at half maximum is the width of the spectra at half maximum and is calculated using equation 13 (Materials and Methods). The plots of full width half maximum of erythrosin $\mathrm{B}$ in sugars protein films at various temperatures from $0^{\circ} \mathrm{C}$ to $100^{\circ} \mathrm{C}$ are shown in Figure III-3a, b, c. The emission bandwidth, a measure of the extent of inhomogeneous broadening due to the presence of probes in multiple sites with different emission energies, increased with increasing temperature. This increase was gradual at low temperature and dramatic at higher temperature, indicating that the increase in dipolar relaxation rate was accompanied by an increase in the width of the 
distribution of energetically distinct environments. Varying concentrations of maltose and trehalose had an insignificant impact on the bandwidth of the spectra. However, the bandwidth increased dramatically at temperatures approaching the sucrose glass transition in sucrose / $\beta$-Lg films at higher ratios of sucrose, clearly indicating that the distribution of site energies were significantly broadened due to the onset of $\alpha$ relaxations.

Phosphorescence Intensity Decays

Phosphorescence intensity decays of Ery B in sugar / $\beta$ - Lg films equilibrated against nitrogen $\left(-\mathrm{O}_{2}\right)$ and against air $\left(+\mathrm{O}_{2}\right)$ collected as a function of temperature from $0^{\circ} \mathrm{C}$ to $100^{\circ} \mathrm{C}$ were well analyzed using a stretched exponential decay model; the same model was used to fit Ery B decays in pure $\beta-\operatorname{Lg}$ (Sundaresan and Ludescher, 2007), BSA (Nack and Ludescher, 2006), gelatin (Lukasik and Ludescher, 2005), sucrose (Pravinata et al., 2005) and sugar alcohols (Shirke et al., 2005; 2006) (Eq. 7, Materials and Methods). The physically meaningful parameters in this decay model are the KWW lifetime and the stretching exponent. The stretched exponential function is considered appropriate for fitting the complex relaxation processes that depopulate the excited triplet state in the millisecond timeframe in amorphous material, (Richert, 1997). Lifetime is the average time a molecule spends in the excited triplet state before going back to ground state and can be an indicator of the rigidity of the matrix. In addition, because oxygen is a contact quencher of the excited triplet state, measurement of lifetime in the presence and absence of oxygen can also be used as a sensitive indicator of oxygen diffusion within the amorphous solid (Lukasik and Ludescher, 2006a, 
Lukasik and Ludescher, 2006b, Simon-Lukasik \& Ludescher, 2004, Nack and Ludescher, 2006).

Figures III- 4a, b, c, d, e, f depict the lifetime as a function of temperature in $\beta$ Lg films with varying concentrations of sucrose in the presence and absence of oxygen. The lifetime decreased linearly with increase temperature from $0^{\circ} \mathrm{C}$ to $100^{\circ} \mathrm{C}$ in all sucrose / $\beta$-Lg films.

Figure III- $4 \mathrm{~g}$ is a comparative plot of the lifetimes for Ery B in the sucrose/ $\beta$ Lg films under nitrogen conditions. Measured lifetimes can be indicative of the rigidity of the matrix. It is clear that lifetimes increased with increase in sucrose concentration indicating that sucrose increased the rigidity of the matrix. From Figure III- $4 \mathrm{~h}$, it is clearly evident that addition of sucrose brings about a lowering of the permeability to oxygen. In molar ratios of 10:1 and higher (Suc: $\beta-\mathrm{Lg})$ the lifetimes in the presence of nitrogen and air were identical. Ten moles of sucrose per mole $\beta$-Lg can be judged as the critical concentration of sucrose to decrease the permeability in the matrix. Above the critical ratio of 10:1 Suc / $\beta$-Lg oxygen quenching was undetectable in the films.

Figures III- $5 \mathrm{a}, \mathrm{b}, \mathrm{c}, \mathrm{d}$, e, f portray the lifetimes of $\beta$-Lg films with varying concentration of trehalose in the presence of nitrogen and air. Lifetimes decreased linearly with increase in temperature in all ratios of trehalose / $\beta$ - $\mathrm{Lg}$ and the shape of the curves were similar to the pure $\beta$-Lg films. Further comparison of the lifetimes in the presence of nitrogen and in air revealed that the lifetimes were identical at ratios of 60:1 and higher indicating that the molar ratio of $60: 1$ trehalose / $\beta$ - $\operatorname{Lg}$ was the critical concentration to restrict the entry of oxygen in the $\beta$-Lg matrix. Below the critical ratio, the lifetime values were lower due to the additional collisions of the probe with oxygen. 
Figures III $6 \mathrm{a}-\mathrm{h}$ are plots of the lifetime of $\beta-\mathrm{Lg}$ in various ratios of maltose. Maltose/ $\beta$-Lg films showed similar linear decreases with temperature as the other sugar/ $\beta$-Lg films. Lifetimes increased with increase in maltose in the presence of nitrogen. In the case of maltose, addition of 10 moles of maltose brought about a decline in the permeability but didn't act as a complete barrier. Oxygen quenching was undetectable at higher ratios. It is evident that, though these sugars had similar molecular weights, when incorporated in protein matrices they generate differences in permeability.

The stretching exponent $\beta$ is a measure of the dynamic heterogeneity in the matrix; values of $\beta$ close to 1 indicate that the matrix is dynamically homogenous while smaller values of $\beta$ indicate increasing dynamic heterogeneity (Lindsey \& Patterson, 1980). Values of $\beta$, a fit parameter in the stretched exponential function related to the distribution of decay times, followed a trend similar to that seen in lifetimes (Figures III 7-9). Values of $\beta$ were similar in the presence and absence of oxygen and were fairly constant at $\sim 0.86$ in the ratios of 100:1 to 10000:1 sugars: $\beta$-Lg. Below these concentrations $\beta$ differed for samples in air and those in nitrogen, and with significantly lower values in the presence of air. These trends suggest that the distribution of dynamic environments in sugar / $\beta$ - Lg films is fairly constant in the range from 10000100 but at lower sugar concentrations the distribution of dynamic environments broadened significantly. 
Photophysical rate constants

The non-radiative decay rate in air reflects the rate of intersystem crossing, $\mathrm{k}_{\mathrm{TS} 0}$, but also includes an additional term $\mathrm{k}_{\mathrm{Q}}\left[\mathrm{O}_{2}\right]$ describing oxygen quenching of the excited triplet state. The rate constant for quenching the triplet state by oxygen, $\mathrm{k}_{\mathrm{Q}}\left[\mathrm{O}_{2}\right]$, is calculated from the difference in inverse lifetime in air and in nitrogen (eq. 11; Materials and Methods). It's clearly evident from the study that addition of small amounts of sugar has a tremendous impact on the $\beta$ - $\mathrm{Lg}$ matrix. There is a great difference in the $\mathrm{k}_{\mathrm{Q}}\left[\mathrm{O}_{2}\right]$ values in the various $\beta$-Lg sugar matrices Although these sugars have similar molecular weights we see it requires varying amounts of different sugars to bring about a decrease in the permeability.

Addition of a small molecule like sucrose $(342 \mathrm{~g} / \mathrm{mol})$ brings about an enormous change in the oxygen quenching rate as depicted by the plot of $\mathrm{k}_{\mathrm{Q}}\left[\mathrm{O}_{2}\right]$ as a function of temperature(Figure III- 10a). Adding sucrose at molar ratios as low as 10 resulted in a 10fold decline in the oxygen-quenching rate. Even at high temperatures $\left(100^{\circ} \mathrm{C}\right)$ where the oxygen quenching constant $\mathrm{k}_{\mathrm{Q}}\left[\mathrm{O}_{2}\right]$ is almost $9580 \mathrm{~s}^{-1}$ in the pure $\beta$ - $\mathrm{Lg}$ matrix we see that addition of minute amounts of sucrose at a molar ratio of 10 lowers the $\mathrm{k}_{\mathrm{Q}}\left[\mathrm{O}_{2}\right]$ to $1647 \mathrm{~s}^{-1}$. Addition of higher amounts of sucrose further lowered the oxygen quenching constant (In our analysis negative oxygen collissional quenching constants have been ignored).

Studies have proven that trehalose offers better bio-protection when compared to sucrose and has a high glass transition $\left(\mathrm{T}_{\mathrm{g}} \sim 106^{\circ} \mathrm{C}\right)$, thus, we assumed that it might be a superior alternative to sucrose. But to our surprise we see that addition of trehalose at a 
molar ratio of 10 (critical concentration whereby we see a drastic lowering in $\mathrm{k}_{\mathrm{Q}}\left[\mathrm{O}_{2}\right]$ in sucrose) didn't hamper oxygen from entering into the matrix (Figure III-10b). At this ratio the $\mathrm{k}_{\mathrm{Q}}\left[\mathrm{O}_{2}\right]$ values were similar to the pure $\beta$-Lg film indicating that the oxygen permeability was unaffected at this ratio. A sharp decline in oxygen permeability was observed in between the ratios of 30:1 and 60:1. Oxygen permeability was negligible at higher ratios. The trends with respect to sucrose / $\beta$ - $\mathrm{Lg}$ and trehalose / $\beta$-Lg correlate with the results with another study conducted in amorphous BSA films (Nack, 2007). It is clear that sucrose offered superior protection against atmospheric oxygen when compared to trehalose.

It required varying amounts of maltose to bring about a decrease of the quenching by atmospheric oxygen. At ratios on par with the sucrose critical concentration (10 moles to 1) the oxygen quenching rate was reduced apppoximately by three folds. These results indicate that maltose addition at a ratio of 10:1 brought a decline in the permeability, but did not act as a complete barrier (as oxygen quenching was detectable). However, at higher ratios of maltose to $\beta-\operatorname{Lg}(100: 1$ and 1000:1), oxygen quenching was barely detectable. Future experiments should aim at studying intermediate concentrations in between the molar ratios 10:1 and 100:1, in order to reflect the exact breakpoint concentration for cessation of oxygen permeability in maltose $/ \beta$-Lg films.

The oxygen quenching rate constant $\mathrm{k}_{\mathrm{Q}}\left[\mathrm{O}_{2}\right]$ increased systematically with increase in temperature in all the sugar/ protein matrices at ratios below the detected critical concentrations. It is clear that temperature had a profound effect, as differences between ratios were noticeable at higher temperatures. However, no clear trend was observed at higher ratios of sugars, as oxygen quenching was almost negligible. 
The temperature effect on $\mathrm{k}_{\mathrm{Q}}\left[\mathrm{O}_{2}\right]$ was carried out by arrhenius analysis of $\mathrm{k}_{\mathrm{Q}}\left[\mathrm{O}_{2}\right]$ (Data not shown). It is clearly evident from Table III-3a that the $\mathrm{E}_{\mathrm{A}}$ values for sucrose / $\beta$-Lg films were fairly constant $\left(30-37 \mathrm{kJmol}^{-1}\right)$ at lower ratios of sucrose. However, at ratios of 1000:1 and 10000:1, $\mathrm{E}_{\mathrm{A}}$ reduced drastically.

In trehalose / $\beta$-Lg films the activation energy $\mathrm{E}_{\mathrm{A}}$ for $\mathrm{k}_{\mathrm{Q}}\left[\mathrm{O}_{2}\right]$ was constant up to a ratio of 30:1. At ratios higher than the critical concentration, whereby permeability became undetectable, activation energies were not measurable (Table III-3b).

In maltose/ $\beta$-Lg films $\mathrm{E}_{\mathrm{A}}$ for $\mathrm{k}_{\mathrm{Q}}\left[\mathrm{O}_{2}\right]$ decreased with increasing maltose ratio; the decrease was dramatic at a ratio of 10:1, and above that ratio due to a drastic lowering of the permeability, activation energies were not measurable (Table III-3c).

Analysis of lifetimes in terms of the underlying photophysical rate constants for de-excitation of the triplet state provides additional insight into the effect of temperature on matrix mobility $\left(\mathrm{k}_{\mathrm{TS} 0}\right)$ and oxygen quenching $\mathrm{k}_{\mathrm{Q}}\left[\mathrm{O}_{2}\right]$ in $\beta$-Lg films. In the absence of oxygen, the total rate of decay $\mathrm{k}_{\mathrm{P}}$ is the sum of the rates for radiative decay $\mathrm{k}_{\mathrm{RP}}$, for reverse intersystem crossing $\mathrm{k}_{\mathrm{TS} 1}$, and for vibrational relaxation due to collisional quenching $\mathrm{k}_{\mathrm{TS} 0}$. The radiative decay rate is $41 \mathrm{~s}^{-1}$ and constant for Ery B (Duchowicz et al., 1998; Lettinga et al., 2000). Thus, $\mathrm{k}_{\mathrm{TS} 0}$ acts as a direct indicator of mobility.

Figure III- $11 \mathrm{a}$ is a plot of $\mathrm{k}_{\mathrm{TS} 0}$ as a function of temperature in sucrose / $\beta$-Lg films . $\mathrm{k}_{\mathrm{TS} 0}$ increased with temperature for all ratios of sucrose to $\beta$-Lg owing to increase in matrix mobility at higher temperatures. A decline in the $\mathrm{k}_{\mathrm{TS} 0}$ values with increase in sucrose concentrations illustrates the fact that addition of small molecules like sucrose brought about a restriction in the matrix mobility. Upon addition of very high concentrations of sucrose (molar ratios of 1000, 10000) the $\mathrm{k}_{\mathrm{TS} 0}$ values remained constant 
at low temperatures, but as the temperature increased (while approaching the glass transition of sucrose) there was an increase in the $\mathrm{k}_{\mathrm{TS} 0}$ values as it mainly reflected the sucrose behavior in the glass and in the melt.

Trehalose is known as an exceptional stabilizer for protein matrices. Significantly lower $\mathrm{k}_{\mathrm{TS} 0}$ values in the presence of trehalose suggested that the interactions between trehalose and $\beta-\mathrm{Lg}$ lowered the mobility of the matrix. Among the studied disaccharides, trehalose caused significant reductions in the matrix mobility even at very small amounts (as low as 0.1:1) as illustrated in Figure III- 11b.

Figure III- 11c represents the plot of $\mathrm{k}_{\mathrm{TS} 0}$ as a function of temperature in maltose / $\beta$-Lg films. Addition of maltose brought a decline in the matrix mobility and the rate $\mathrm{k}_{\mathrm{TS} 0}$ decreased with concomitant increase in concentration.

In this study we noticed higher $\mathrm{k}_{\mathrm{TS} 0}$ values in sucrose / $\beta$ - $\mathrm{Lg}$ and maltose/ $\beta$ - $\mathrm{Lg}$ films at lower ratios of sugar / protein. This may be due to the fact that, while estimating these values the propagation of errors with respect to the rate constants were not taken into account. The trends in lifetimes clearly indicate that the differences in $\mathrm{k}_{\mathrm{TS} 0}$, between ratios actually fall within experimental error.

The apparent activation energy $\left(\mathrm{E}_{\mathrm{A}}\right)$ for $\mathrm{k}_{\mathrm{TS} 0}$ was calculated from the Arrhenius analysis of $\mathrm{k}_{\mathrm{TS} 0}$ (Data not shown). These values helped us gain a deeper understanding of the molecular processes within these sugar / $\beta$ - Lg matrices.

Table III $3 a, b, c$ is a summary of these activation energies in the sugar/ protein matrices. At lower ratios of sucrose it is clear that the matrix participates in two kinds of motions. The $\mathrm{E}_{\mathrm{A}}$ values for $\mathrm{k}_{\mathrm{TS} 0}$ at lower ratios were obtained from the analysis of the trendlines at high $\left(80-100^{\circ} \mathrm{C}\right)$ and low $\left(0-20^{\circ} \mathrm{C}\right)$ temperatures. It is clearly evident that the 
$\mathrm{E}_{\mathrm{A}}$ values in sucrose/ $\beta$ - $\mathrm{Lg}$ films (excluding the ratio $0.1: 1$ ) were considerably lower than the pure $\beta$-Lg matrix. At higher ratios of sucrose, $\mathrm{E}_{\mathrm{A}}$ became single valued. The apparent activation energies for $\mathrm{k}_{\mathrm{TS} 0}$ in trehalose / $\beta$-Lg films were lower in the presence of very small amounts of trehalose (The $\mathrm{E}_{\mathrm{A}}$ for $\mathrm{k}_{\mathrm{TS} 0}$ in the molar ratio 0.1:1 trehalose / $\beta$-Lg films

was $2.1 \mathrm{~kJ}$ mole $^{-1}$ at low temperatures $\left(0-20^{\circ} \mathrm{C}\right)$ and $6.2 \mathrm{~kJ}$ at high temperatures $(80-$ $\left.100^{\circ} \mathrm{C}\right)$ ). Maltose $/ \beta$-Lg films showed significantly lower activation energies for $\mathrm{k}_{\mathrm{TS} 0}$ at higher ratios of maltose (100:1 and 1000:1).

\section{$\underline{\text { Discussion }}$}

This research establishes a vital connection between molecular mobility and oxygen permeability in amorphous protein sugar films. Such connections are a necessary part of any physical-chemical model that allows us to predict, control and manipulate the quality of amorphous foods based on molecular principles. These mixed sugar matrices would be extremely important agents for the long-term stabilization of food matrices.

When protein molecules are embedded in an amorphous sugar matrix, the corresponding number of protein-protein and sugar-sugar interactions is replaced by protein-sugar interactions and these interactions can lead to dramatic changes in the matrix mobility and permeability. We present here an investigation of the effect of temperature and effect of change in composition by the addition of the sugars sucrose, maltose and trehalose on the matrix mobility and oxygen permeability of the amorphous $\beta$-Lg matrix. Phosphorescence from erythrosin $B$ in $\beta$-Lg provides information about important aspects of the matrix and provides us with a deep insight into the molecular mechanisms controlling oxygen permeability in amorphous protein sugar matrices. Effect of sugars on the matrix mobility 
Trends in matrix mobility essentially revealed that the disaccharides modulated the mobility of the $\beta$-Lg matrix in a complex concentration dependent fashion. The phosphorescence properties of Ery B in sugar / $\beta$ - $\mathrm{Lg}$ mixtures are consistent with the effect of two distinct modes of matrix molecular mobility: dipolar relaxation around the excited $\mathrm{T}_{1}$ state and molecular collision with the excited $\mathrm{T}_{1}$ state (Sundaresan and Ludescher, 2007; Nack and Ludescher, 2006; Pravinata et al., 2005; Lukasik et al., 2005; Ludescher et al., 2001).

Dipolar relaxation directly modulates the probe emission energy by stabilizing the excited triplet state (Richert, 2000). Molecular collision modulates the intensity and decay kinetics by modulating the rate of intersystem crossing to the ground state (Strambini and Gonnelli, 1985; Papp and Vanderkooi, 1989; Fisher et al., 2002). A change in emission energy gives us an indication of the change in local dipolar relaxations in the matrix.

Increase in emission energy with increase in sugar concentration suggested a decrease in the dipolar relaxation rate due to reduced mobility of the polar groups. The plots of emission energy versus molar ratio clearly illustrate these effects (Figure III 12 14). These plots mainly convey an important message that the intermolecular associations between sugar and protein result in a decrease in the local molecular mobility.

The emission lifetime provides a direct indicator of the rate of non-radiative coupling to the ground start due to matrix collisions with the probe. In the absence of $\mathrm{O}_{2}$ the lifetime of the excited state molecules is related to flexibility. The underlying scientific principle is phosphorescence lifetime is very long in rigid environments. In sugar / $\beta$-Lg films the increase in lifetime with increasing sugar concentration mainly 
reconfirmed the conclusion that sugars confer rigidity to the $\beta-\mathrm{Lg}$ matrix and restrict the flexibility.

The phosphorescence emission intensity $\left(\mathrm{I}_{\mathrm{P}}\right)$ and the lifetime are directly modulated by the rate of intersystem crossing $\mathrm{k}_{\mathrm{TS} 0}$; $\mathrm{k}_{\mathrm{TS} 0}$ in turn is modulated by the physical state of the amorphous matrix (Sundaresan and Ludescher, 2007; Nack and Ludescher, 2006; Pravinata et al., 2005; Lukasik and Ludescher, 2005). The magnitude of $\mathrm{k}_{\mathrm{TS} 0}$ reflects both internal factors related to the manner in which the excited $\mathrm{T}_{1}$ state of Ery $B$ is vibrational coupled to the $S_{0}$ ground state as well as external factors apparently related to the manner in which the ground-state vibrational energy can dissipate from the excited probe into the surrounding matrix (Fisher et al., 2002). Because the efficiency of this vibrational coupling is related to the overall mobility of the matrix; (Strambini and Gonnelli, 1985) the magnitude of $\mathrm{k}_{\mathrm{TS} 0}$ provides a direct measure of matrix mobility. Compared with $\beta$-Lg films, $\mathrm{k}_{\mathrm{TS} 0}$ decreased with increasing sugar content, indicating more restricted mobility in sugar $/ \beta$-Lg films.

It is clearly evident that these sugars have great similarities in the way they affect matrix mobility. Noticeable increase in emission energy (lower dipolar relaxations), decrease in $\mathrm{k}_{\mathrm{TS} 0}$ and longer lifetime (lower mobility) clearly indicate that sucrose (except at high concentrations), trehalose and maltose decrease the mobility of the $\beta$ - $\mathrm{Lg}$ matrix. Specific interactions between these disaccharides and $\beta$ - $\mathrm{Lg}$ constrained the local relaxations, thus causing a significant decline in the mobility. Trehalose was the most effective sugar in reducing the mobility of the matrix at very low concentrations (as low as $0.1: 1)$. The plots of emission energy and $\mathrm{k}_{\mathrm{TS} 0}$ as a function of molar ratio of trehalose / $\beta$-Lg clearly reveal these trends. However, at higher ratios trehalose / $\beta$ - $\mathrm{Lg}$ films and 
maltose / $\beta$-Lg films exhibited similarities in the manner in which they affected the mobility of the matrix (increases in emission energy, decreases in $\mathrm{k}_{\mathrm{TS} 0}$, increase in lifetime were very similar).

Emission energy and $\mathrm{k}_{\mathrm{TS} 0}$ in different sugar / $\beta$-Lg films showed similar trends as the pure $\beta$-Lg films with changes in temperature. Emission energy decreased and $\mathrm{k}_{\mathrm{TS} 0}$ increased with increase in temperature clearly showing that temperature enhanced the mobility of the matrix. But, the decrease in emission energy with temperature was considerably reduced in the presence of sugars (except sucrose at higher concentrations).

At higher ratios of sucrose (1000:1 and 10000:1), which are predominantly sucrose matrices, the magnitude of $\mathrm{k}_{\mathrm{TS} 0}$ increased slowly at low temperatures and dramatically above $65^{\circ} \mathrm{C}$. A dramatic lowering in the emission energy and a massive increase in $\mathrm{k}_{\mathrm{TS} 0}$ at this temperature necessarily reflects the activation of $\alpha$ relaxation at the glass transition of sucrose.

Decreases in the activation energies $\mathrm{E}_{\mathrm{A}}$ for $\mathrm{k}_{\mathrm{TS} 0}$ in the presence of sugars clearly indicate that sugars shift the motions from large-scale collective motions to more localized small scale vibrational and rotational motions in the $\beta-\operatorname{Lg}$ matrix.

Interactions between sugars and protein

Sugars for ages have been known to protect proteins from extreme dessication stress and confer increased stability. However, increased stability necessarily does not reflect a decrease in mobility. A look into the possible mechanisms by which sugars confer protection to proteins can help us gain a deeper insight into the manner in which they affect the mobility of the matrix. 
The preservative properties of sugar additives is attributed to sugar molecules being able to replace essential hydrogen-bonded water molecules which are important in stabilizing the protein and thus preservation is therefore determined by the hydrogen bond potential of the sugar (Lopez Diez and Bone, 2000). This theory has shown a direct relationship between the hydrogen bonding potential of the sugar and the preservation of the protein native conformation, in particular, the conservation of the native $\alpha$-helix content of the protein. It has been concluded that additives that hydrogen bond more extensively to proteins are better protectants (Imamura et al., 2000).

Lopiez diez and Bone (2000) compared the effects of the sugars trehalose and sucrose on the protein trypsin. In their study they illustrated that trehalose and sucrose are likely to be driven towards very different hydrogen bonding configurations under dehydration conditions and trehalose is more effective than sucrose simply because of the greater level of its interaction with the protein.

In the present study trehalose was the most effective in reducing the mobility of the $\beta$ - $\mathrm{Lg}$ matrix at lower ratios when compared to sucrose and maltose. Green and Angell, 1989 suggested that trehalose offered superior bio-preservation because of its high glass transition $\left(\mathrm{Tg} \sim 106^{\circ} \mathrm{C}\right.$ Roe and Labuza, 2005). On the basis of mole percent glucose rings, trehalose has the highest glass transition temperature among the tested sugars, followed by maltose and sucrose. In lieu of past literature glass transition theory does not seem to be a plausible explanation for this kind of occurrence. A recent study by You and Ludescher (2007) revealed that maltodextrins having a high glass transition enhanced the mobility of the sucrose matrix. Thus it is clear that a very high glass transition necessarily does not reflect a decrease in mobility. Carpenter and Crowe 
(1989) demonstrated that a direct interaction (hydrogen-bonding) between sugars and proteins are required for the stabilization of protein structures during drying. Studies have clearly indicated that trehalose when dried along with a protein is more likely to hydrogen bond with the protein rather than itself. However, sucrose in the presence of protein forms intra-molecular associations with itself rather than the protein (Lopiez diez and Bone, 2000). Stronger intermolecular hydrogen bonding interactions between the protein $\beta$ - Lg and trehalose may be attributed to the decrease in mobility at very low ratios of trehalose. Overlooking the effect of trehalose at lower ratios, it is clear that sugars affect the mobility of the $\beta$-Lg matrix in a similar manner with subtle differences. Minor differences in hydrogen bonding interactions between protein and the disaccharides can be attributed to these subtle differences in the mobility in the presence of sugars. However, looking at the big picture all these sugars with great similarities in structure introduce rigidity to the $\beta$ - $\mathrm{Lg}$ matrix, decrease the dipolar relaxations and thus decrease the mobility of the matrix. The manner in which these disaccharides affect the mobility of the matrix cannot be understood fully unless the dynamic interactions between various participating components are examined in detail with other complementary techniques. Oxygen permeability in $\beta$-Lg / sugar mixtures

Oxygen is a powerful quencher of both fluorescence and phosphorescence and quenching has been used as an effective method for probing the presence of molecular oxygen in polymeric media. The fundamental principle of the quenching action of atmospheric oxygen is explored in this study to detect the oxygen permeability in amorphous $\beta$-Lg / sugar mixtures. 
The oxygen quenching rate is the product of $\mathrm{k}_{\mathrm{Q}}$, which is proportional to the oxygen diffusion rate through the matrix, and $\left[\mathrm{O}_{2}\right]$, the concentration of oxygen in the film, it is proportional to the permeability of oxygen through the protein film (Nack \& Ludescher, 2006). Although, these disaccharides exhibit great similarities in the manner in which they affect the mobility of the $\beta-\operatorname{Lg}$ matrix, they exhibit clear differences in the way they affect the barrier properties.

In sucrose/ $\beta$-Lg films, a 10 fold decline in the collisional quenching constant for oxygen at a critical ratio of 10:1 (Fig III-15 a, b) clearly indicates that a small molecule like sucrose can have an enormous impact on the permeability of the matrix.

Sothornvit and Krochta (2000) studied the oxygen permeability of $\beta$-Lg films in the presence of sucrose and found that sucrose was the least effective plasticizer of the studied $\beta$-Lg films. They linked the oxygen barrier properties of sucrose to its bulky ring structure and its low water-attracting ability. The oxygen barrier properties of sucrose have been successfully used in edible films made from proteins to prevent nuts from oxidative rancidity (Krochta et al., 2005).

Studies have shown that trehalose offers superior bio-protection when compared to sucrose but the plots of $\mathrm{k}_{\mathrm{Q}}\left[\mathrm{O}_{2}\right]$ versus molar ratio (Fig III-16 a, b) clearly illustrate that it fails to act as superior oxygen barrier when compared to sucrose. Higher ratios of trehalose was required to lower the permeability of oxygen in the $\beta$-Lg films

The plots depicting the concentration dependence of maltose / $\beta-\mathrm{Lg}$ films also illustrate that although permeability is drastically reduced at ratios in par with the critical concentration of sucrose (10:1), but the permeability is not completely thwarted. (Fig III$17 \mathrm{a}, \mathrm{b})$. 
Free volume in an amorphous system is geometrically interpreted as a continuous network of 'lakes and channels', which permit the diffusive motion of its molecules (Miller et al., 1997). Although these sugars have similarities in structure, the intermolecular and intra molecular hydrogen bonding interactions in these sugars have been shown to differ considerably in various protein matrices (Lopiez diez and Bone, 2000). We hypothesize that these disaccharides exhibit differences in permeability due to their inherent differences in hydrogen bonding associations with the protein. The relatively low solubility of oxygen in sugars may also be attributed to decrease in the oxygen permeability in the presence of sugars (Whitcombe, 2005).

\section{Oxygen permeability and matrix mobility}

In $\beta$-Lg films, oxygen permeability was a biphasic linear function of $\mathrm{k}_{\mathrm{TS} 0}$ over nearly three orders of magnitude change in $\mathrm{k}_{\mathrm{Q}}\left[\mathrm{O}_{2}\right]$ (Sundaresan and Ludescher, 2007), showing there was a clear interdependence between $\mathrm{k}_{\mathrm{Q}}\left[\mathrm{O}_{2}\right]$ and $\mathrm{k}_{\mathrm{TS} 0}$. To investigate the relationship between mobility and permeability in sugar / protein matrices we generated plots of $\mathrm{k}_{\mathrm{TS} 0}$ versus $\mathrm{k}_{\mathrm{Q}}\left[\mathrm{O}_{2}\right]$ (Figure III 21-24). These results showed that these binary mixtures displayed linear and biphasic correlation between matrix mobility and permeability at lower ratios of sugars. However, at molar ratios higher than the critical concentration the correlation between $\mathrm{k}_{\mathrm{Q}}\left[\mathrm{O}_{2}\right]$ and $\mathrm{k}_{\mathrm{TS} 0}$ was not evident due to negligible oxygen permeability. So it becomes clearly evident that although pure $\beta$-Lg exhibited a linear relationship but in binary mixtures the complex association is missing.

Linear correlation at lower ratios of sugars clearly indicated that these fundamentally different processes were related to each other when the composition was 
altered minimally. However, at higher ratios of sugar this correlation was lost due to the drastic lowering in the permeability.

\section{$\underline{\text { Conclusion }}$}

Phosphorescence of erythrosin B in amorphous sugar/ $\beta$-Lg films clearly depicted the profound effect of sugars on the amorphous $\beta$-Lg matrix. Sugars modulated the mobility of the matrix by introducing rigidity to the matrix and lowered the permeability drastically. We hypothesize that strong hydrogen bonding interactions (protein- sugar and sugar-sugar) may be responsible for the reduction in the matrix mobility and permeability. This information is useful in generating edible films that are less permeable to atmospheric oxygen. 


\section{$\underline{\text { References }}$}

Allison, S. D., Chang, B., Randolph, T. W., and Carpenter, J. F. (1999). Hydrogen bonding between sugar and protein is responsible for inhibition of dehydration-induced protein unfolding. Archives of Biochemistry and Biophysics. 365, 289-298.

Angel, C. A. (1995). Formation of glasses from liquids and biopolymers, Science. 267, 1924 1935.

Arakawa, T., Prestrelski, S. J., Kenney, W. C., and Carpenter, J. F. (1993). Factors affecting short-term and long-term stabilities of proteins. Advanced Drug Delivery Reviews. 10, 128.

Bergquist, P., Zhu, Y., Jones, A. A., and Inglefield, P. T. (1999). Plasticization and Antiplasticization in Polycarbonates: The Role of Diluent Motion. Macromolecules. 32, 7925-7931.

Birks, J. B. (1970). Photophysics of Aromatic Molecules (Wiley Monographs in Chemical Physics). 704.

Borowitzka, L. J. R. and Gilles-Baillien, M. (1985.). Glycerol and other carbohydrate osmotic effectors. In Transport Processes, Iono- and Osmoregulation, Berlin.

Branca, C., Magazu, S., Maisano, G., and Migliardo, P. (1999). Anomalous cryoprotective effectiveness of trehalose: Raman scattering evidences. J. Chem. Phys. 111, 281-287.

Carpenter, J. F., Crowe, L. M., and Crowe, J. H. (1987). Stabilization of phosphofructokinase with sugars during freeze-drying: characterization of enhanced protection in the presence of divalent cations. Biochimica et biophysica acta. 923, 109-115.

Carpenter, J. F. and Crowe, J. H. (1989). An infrared spectroscopic study of the interactions of carbohydrates with dried proteins. Biochemistry. 28, 3916-3922.

Champion, D., Le Meste, M., and Simatos, D. (2000/2). Towards an improved understanding of glass transition and relaxations in foods: molecular mobility in the glass transition range. Trends in Food Science \& Technology. 11, 41-55.

Chang, L., Shepherd, D., Sun, J., Ouellette, D., Grant, K. L., Tang, X., and Pikal, M. J. (2005). Mechanism of protein stabilization by sugars during freeze-drying and storage: Native structure preservation, specific interaction, and/or immobilization in a glassy matrix? $J$. Pharm. Sci. 94, 1427-1444.

Constantino H.R. (1998). Int. J. Pharm. 166, 211-221.

Crowe, J. H., Carpenter, J. F., and Crowe, L. M. (1998). The role of vitrification in anhydrobiosis. Annual Review of Physiology. 60, 73-103. 
Crowe, J. H., Leslie, S. B., and Crowe, L. M. (1994). Is vitrification sufficient to preserve liposomes during freeze-drying? Cryobiology. 31, 355-366.

Crowe, J. H., Oliver, A. E., Hoekstra, F. A., and Crowe, L. M. (1997). Stabilization of Dry Membranes by Mixtures of Hydroxyethyl Starch and Glucose: The Role of Vitrification. Cryobiology. 35, 20-30.

Crowe, L. M., Crowe, J. H., Rudolph, A., Womersley, C., and Appel, L. (1985). Preservation of freeze-dried liposomes by trehalose. Arch. Biochem. Biophys. 242, 240-247.

Duchowicz, R., Ferrer, M. L., and Acuña, A. U. (1998). Kinetic Spectroscopy of Erythrosin Phosphorescence and Delayed Fluorescence in Aqueous Solution at Room Temperature. Photochemistry and Photobiology. 68, 494-501.

Dybing, S. T. and Smith, D. E. (1991). Relation of chemistry and processing procedures to whey protein functionality: a review. Cultured Dairy Products Journal. 26, 4-9, 11-12.

Einfeldt, J., Kwasniewski, A., Klemm, D., Dicke, R., and Einfeldt, L. (2000). Analysis of side group motion in O-acetyl starch using regioselective 2-O-acetyl starches by means of dielectric spectroscopy. Polymer. 41, 9273-9281.

Einfeldt, J., Meissner, D., Kwasniewski, A., and Einfeldt, L. (2001). Dielectric spectroscopic analysis of wet and well dried starches in comparison with other polysaccharides. Polymer. 42, 7049-7062.

Fischer, C. J., Gafni, A., Steel, D. G., and Schauerte, J. A. (2002). The triplet-state lifetime of indole in aqueous and viscous environments: significance to the interpretation of room temperature phosphorescence in proteins. J. Am. Chem. Soc. 124, 10359-10366.

Green, J. L. and Angell, C. A. (1989). Phase relations and vitrification in saccharide-water solutions and the trehalose anomaly. J. Phys. Chem. 93, 2880-2882.

Green, J. E., Sitaula, R., Fowler, A., Toner, M., and Bhowmick, S. (2007). Enthalpic relaxation of convective desiccated trehalose-water glasses. Thermochimica Acta. 453, 1-8.

Hill, J. J., Shalaev, E. Y., and Zografi, G. (2005). Thermodynamic and dynamic factors involved in the stability of native protein structure in amorphous solids in relation to levels of hydration. J. Pharm. Sci. 94, 1636-1667.

Hurtubise, R. J. (1990). Phosphorimetry:Theory,Instrumentation and applications. .

Imamura, K., Ogawa, T., Sakiyama, T., and Nakanishi, K. (2003). Effects of types of sugar on the stabilization of protein in the dried state. J. Pharm. Sci. 92, 266-274.

Jackson, W. J., Jr. and Caldwell, J. R. (1967). Antiplasticization. II. Characteristics of antiplasticizers. J Appl Polym Sci. 11, 211-226. 
Kaushik, J. K. and Bhat, R. (2003). Why Is Trehalose an Exceptional Protein Stabilizer?: AN ANALYSIS OF THE THERMAL STABILITY OF PROTEINS IN THE PRESENCE OF THE COMPATIBLE OSMOLYTE TREHALOSE. J. Biol. Chem. 278, 26458-26465.

Koreyoshi Imamura, Tomohiro Ogawa, Takaharu Sakiyama,Kazuhiro Nakanishi,. (2003). Effects of types of sugar on the stabilization of protein in the dried state. J. Pharm. Sci. 92, 266-274.

Krochta, J. M., Dangaran, K. L., and Lin, S. (2005). Protein-based formulations for providing gloss coatings to foods and for protecting nuts from rancidity. U.S.Pat.Appl.Publ. 2004791475, 20.

Lakowicz, J. R. (1999). Principles of fluorescence spectroscopyKluwer Academic/Plenum Press, Dordrecht/New York.

Lettinga, M. P., Zuilhof, H., and Van Zandvoort, M. A. M. J. (2000). Phosphorescence and fluorescence characterization of fluorescein derivatives immobilized in various polymer matrices. Physical Chemistry Chemical Physics. 2, 3697-3707.

Lindsey, C. P. and Patterson, G. D. (1980). Detailed comparison of the Williams-Watts and Cole-Davidson functions. J. Chem. Phys. 73, 3348-3357.

Lopez-Diez, E. C. and Bone, S. (2000). An investigation of the water-binding properties of protein + sugar systems. Phys. Med. Biol. 45, 3577-3588.

Lourdin, D., Ring, S. G., and Colonna, P. (1998). Study of plasticizer-oligomer and plasticizerpolymer interactions by dielectric analysis: maltose-glycerol and amylose-glycerol-water systems. Carbohydr. Res. 306, 551-558.

Ludescher, R. D., Shah, N. K., McCaul, C. P., and Simon, K. V. (2001). Beyond Tg: optical luminescence measurements of molecular mobility in amorphous solid foods. Food Hydrocolloids. 15, 331-339.

Lukasik, K. V. and Ludescher, R. D. (2006a). Effect of plasticizer on dynamic site heterogeneity in cold-cast gelatin films. Food Hydrocolloids. 20, 88-95.

Lukasik, K. V. and Ludescher, R. D. (2006b). Molecular mobility in water and glycerol plasticized cold- and hot-cast gelatin films. Food Hydrocolloids. 20, 96-105.

Maeda, Y. and Paul, D. R. (1987). Effect of antiplasticization on gas sorption and transport. I. Polysulfone. J. Polym. Sci. Part B. 25, 957-980.

. Maeda, Y. and Paul, D. R. (1987). Effect of antiplasticization on gas sorption and transport. III. Free volume interpretation. J. Polym. Sci. Part B. 25, 1005-1016. 
Maroncelli, M. and Fleming, G. R. (1987). Picosecond solvation dynamics of coumarin 153: the importance of molecular aspects of solvation. J. Chem. Phys. 86, 6221-6239.

McHugh, T. H. and Krochta, J. M. (1994). Milk-protein-based edible films and coatings. Food Technology (Chicago, IL, United States). 48, 97-103.

Miller, D. P., De Pablo, J. J., and Corti, H. R. (1999). Viscosity and Glass Transition Temperature of Aqueous Mixtures of Trehalose with Borax and Sodium Chloride. J Phys Chem B. 103, 10243-10249.

Miller, K. S. and Krochta, J. M. (1997). Oxygen and aroma barrier properties of edible films: a review. Trends Food Sci. Technol. 8, 228-237.

Nack, T. J. and Ludescher, R. D. (2006). Molecular Mobility and Oxygen Permeability in Amorphous Bovine Serum Albumin Films. Food Biophysics. 1557-1858.

Ngai, K. L., Rendell, R. W., Yee, A. F., and Plazek, D. J. (1991). Antiplasticization effects on a secondary relaxation in plasticized glassy polycarbonates. Macromolecules. 24, 61-67.

Noel, T. R., Ring, S. G., and Whittam, M. A. (1992). Dielectric relaxations of small carbohydrate molecules in the liquid and glassy states. J. Phys. Chem. 96, 5662-5667.

Papp, S. and Vanderkooi, J. M. (1989). Tryptophan phosphorescence at room temperature as a tool to study protein structure and dynamics. Photochem. Photobiol. 49, 775-784.

Parker, C. A., Ed. (1968.). Photoluminescence of SolutionsElsevier Publishing, Amsterdam, The Netherlands.

Pravinata, L. C., You, Y., and Ludescher, R. D. (2005). Erythrosin B Phosphorescence Monitors Molecular Mobility and Dynamic Site Heterogeneity in Amorphous Sucrose. Biophys. J. 88, 3551-3561.

Richert, R. (2000). Triplet state solvation dynamics: Basics and applications. J. Chem. Phys. 113, 8404-8429.

Richert, R. and Heuer, A. (1997). Rate-Memory and Dynamic Heterogeneity of First-Order Reactions in a Polymer Matrix. Macromolecules. 30, 4038-4041.

Robeson, L. M. (1969). Effect of antiplasticization on secondary loss transitions and permeability of polymers. Polym. Eng. Sci. 9, 277-281.

Roe, K. D. and Labuza, T. P. (2005). Glass Transition and Crystallization of Amorphous Trehalose-sucrose Mixtures. Int. J. Food Prop. 8, 559-574.

Shah, N. K. and Ludescher, R. D. (1995). Phosphorescence Probes of the Glassy State in Amorphous Sucrose. Biotechnol. Prog. 11, 540-544. 
Shah, N. K. and Ludescher, R. D. (1993). Influence of hydration on the internal dynamics of hen egg white lysozyme in the dry state. Photochem. Photobiol. 58, 169-174.

Shirke, S., Takhistov, P., and Ludescher, R. D. (2005). Molecular Mobility in Amorphous Maltose and Maltitol from Phosphorescence of Erythrosin B. J. Phys. Chem. B. 109, 16119-16126.

Shirke, S. and Ludescher, R. D. (2005/12/12). Molecular mobility and the glass transition in amorphous glucose, maltose, and maltotriose. Carbohydrate Research. 340, 2654-2660.

Shirke, S. and Ludescher, R. D. (2005a). Dynamic site heterogeneity in amorphous maltose and maltitol from spectral heterogeneity in erythrosin B phosphorescence. Carbohydr. Res. 340, 2661-2669.

Shirke, S. and Ludescher, R. D. (2005b). Molecular mobility and the glass transition in amorphous glucose, maltose, and maltotriose. Carbohydr. Res. 340, 2654-2660.

Shirke, S., Takhistov, P., and Ludescher, R. D. (2005). Molecular Mobility in Amorphous Maltose and Maltitol from Phosphorescence of Erythrosin B. J Phys Chem B. 109, 16119-16126.

Simon-Lukasik, K. V. and Ludescher, R. D. (2004/7). Erythrosin B phosphorescence as a probe of oxygen diffusion in amorphous gelatin films. Food Hydrocolloids. 18, 621-630.

Slavik, J. (1994). Fluorescent probes in cellular and molecular biologyCRC Press, Boca Raton, FL.

Sothornvit, R. and Krochta, J. M. (2000). Plasticizer effect on oxygen permeability of betalactoglobulin films. J. Agric. Food Chem. 48, 6298-6302.

Strambini, G. B. and Gonnelli, M. (1985). The indole nucleus triplet-state lifetime and its dependence on solvent microviscosity. Chemical Physics Letters. 115, 196-200.

Strasburg, G. M. and Ludescher, R. D. (1995). Theory and applications of fluorescence spectroscopy in food research. Trends Food Sci. Technol. 6, 69-75.

Sun, W. Q., Davidson, P., and Chan, H. S. O. (1998). Protein stability in the amorphous carbohydrate matrix: relevance to anhydrobiosis. Biochimica et Biophysica Acta General Subjects. 1425, 245-254.

Sundaresan, K. V. and Ludescher, R. D. (2007). Molecular mobility and oxygen permeability in amorphous beta -lactoglobulin films. Food Hydrocoll. 22, 403-413.

Turro, N., Ed. (1991). Modern molecular photochemistry, Sausalito,CA. 
Vanderkooi, J. M., Maniara, G., Green, T. J., and Wilson, D. F. (1987). An optical method for measurement of dioxygen concentration based upon quenching of phosphorescence. $J$. Biol. Chem. 262, 5476-5482.

Whitcombe, M. J., Parker, R., and Ring, S. G. (2005). Oxygen solubility and permeability of carbohydrates. Carbohydr. Res. 340, 1523-1527.

Yancey, P. H., Clark, M. E., Hand, S. C., Bowlus, R. D., and Somero, G. N. (1982). Living with water stress: evolution of osmolyte systems. Science (Washington, DC, United States). 217, 1214-1222.

Yoshioka, S., Miyazaki, T., and Aso, Y. (2006). b-Relaxation of Insulin Molecule in Lyophilized Formulations Containing Trehalose or Dextran as a Determinant of Chemical Reactivity. Pharm. Res. 23, 961-966. 


\section{Tables and Figures}

Table III-1a

The estimated $\mathrm{k}^{\circ}$ TS1 values for erythrosin B in sucrose / $\beta$-Lg films

\begin{tabular}{|l|l|}
\hline Ratio (sucrose: $\beta$-Lg) & Estimated $\mathrm{k}^{\circ}$ TS1 \\
\hline$\beta-\mathrm{Lg}$ & $6.5 \times 10^{7} \mathrm{~s}^{-1}$ \\
\hline $0.1: 1$ & $3.0 \times 10^{7} \mathrm{~s}^{-1}$ \\
\hline $1: 1$ & $6.5 \times 10^{7} \mathrm{~s}^{-1}$ \\
\hline $10: 1$ & $6.5 \times 10^{7} \mathrm{~s}^{-1}$ \\
\hline $100: 1$ & $3.5 \times 10^{7} \mathrm{~s}^{-1}$ \\
\hline $1000: 1$ & $6.5 \times 10^{7} \mathrm{~s}^{-1}$ \\
\hline $10000: 1$ & $3.0 \times 10^{7} \mathrm{~s}^{-1}$ \\
\hline
\end{tabular}

Table III- $1 b$

The estimated $\mathrm{k}^{\circ}$ TS1 values for erythrosin B in trehalose / $\beta$-Lg films

\begin{tabular}{|l|l|}
\hline $\begin{array}{l}\text { Ratio (Trehalose: } \beta- \\
\mathrm{Lg} \text { - }\end{array}$ & Estimated $\mathrm{k}^{\circ}$ TS1 \\
\hline$\beta-\mathrm{Lg}$ & $6.5 \times 10^{7} \mathrm{~s}^{-1}$ \\
\hline $0.1: 1$ & $3.0 \times 10^{7} \mathrm{~s}^{-1}$ \\
\hline $1: 1$ & $6.5 \times 10^{7} \mathrm{~s}^{-1}$ \\
\hline $10: 1$ & $6.5 \times 10^{7} \mathrm{~s}^{-1}$ \\
\hline $100: 1$ & $5.0 \times 10^{7} \mathrm{~s}^{-1}$ \\
\hline $1000: 1$ & $4.3 \times 10^{7} \mathrm{~s}^{-1}$ \\
\hline
\end{tabular}

Table III-1c

The estimated $\mathrm{k}^{\circ}$ TS1 values for erythrosin B in maltose / $\beta$-Lg films

\begin{tabular}{|l|l|}
\hline $\begin{array}{l}\text { Ratio (Trehalose: } \beta- \\
\text { Lg) }\end{array}$ & Estimated $\mathrm{k}^{\circ}$ TS1 \\
\hline$\beta-\mathrm{Lg}$ & $6.5 \times 10^{7} \mathrm{~s}^{-1}$ \\
\hline $0.1: 1$ & $4.5 \times 10^{7} \mathrm{~s}^{-1}$ \\
\hline $1: 1$ & $6.5 \times 10^{7} \mathrm{~s}^{-1}$ \\
\hline $10: 1$ & $3.5 \times 10^{7} \mathrm{~s}^{-1}$ \\
\hline $100: 1$ & $6.5 \times 10^{7} \mathrm{~s}^{-1}$ \\
\hline $1000: 1$ & $6.5 \times 10^{7} \mathrm{~s}^{-1}$ \\
\hline
\end{tabular}


Table III-2 Calculated $\Delta \mathrm{E}_{\mathrm{TS}}$, the energy gap between triplet and singlet state in sucrose/ $\beta$ - $\operatorname{Lg}$ (a) trehalose/ $\beta-\operatorname{Lg}$ (b) maltose/ $\beta$ - Lg (c) films.

Table III-2a

\begin{tabular}{|l|l|l|}
\hline Ratio (sucrose: $\beta$-Lg) & $\begin{array}{l}\Delta \mathrm{E}_{\mathrm{TS}}(\mathrm{kJ} \\
\left.\mathrm{mol}^{-1}\right)\end{array}$ & $\mathrm{STD}$ \\
\hline$\beta$-Lg & 33.6 & 0.8 \\
\hline $0.1: 1$ & 34.23 & 0.04 \\
\hline $1: 1$ & 35.52 & 0.029 \\
\hline $10: 1$ & 33.66 & 0.11 \\
\hline $100: 1$ & 30.66 & 0.08 \\
\hline $1000: 1$ & 34.77 & 0.027 \\
\hline $10000: 1$ & 33.99 & 0.33 \\
\hline
\end{tabular}

Table III-2b

\begin{tabular}{|l|l|l|}
\hline $\begin{array}{l}\text { Ratio (trehalose: } \beta- \\
\mathrm{Lg})\end{array}$ & $\begin{array}{l}\Delta \mathrm{E}_{\mathrm{TS}}(\mathrm{kJ} \\
\left.\mathrm{mol}^{-1}\right)\end{array}$ & STD \\
\hline$\beta$-Lg & 33.6 & 0.8 \\
\hline $0.1: 1$ & 34.63 & 0.58 \\
\hline $1: 1$ & 35.60 & 0.51 \\
\hline $10: 1$ & 34.62 & 0.31 \\
\hline $30: 1$ & 35.8 & 0.62 \\
\hline $60: 1$ & 34.17 & 0.49 \\
\hline $100: 1$ & 34.80 & 0.36 \\
\hline $1000: 1$ & 34.23 & 0.54 \\
\hline
\end{tabular}


Table III-2c

\begin{tabular}{|l|l|l|}
\hline Ratio (maltose: $\beta$-Lg) & $\begin{array}{l}\Delta \mathrm{E}_{\mathrm{TS}}(\mathrm{kJ} \\
\left.\mathrm{mol}^{-1}\right)\end{array}$ & STD \\
\hline$\beta$-Lg & 33.6 & 0.8 \\
\hline $0.1: 1$ & 32.27 & 0.74 \\
\hline $1: 1$ & 34.34 & 0.43 \\
\hline $10: 1$ & 34.99 & 0.77 \\
\hline $100: 1$ & 35.73 & 0.65 \\
\hline $1000: 1$ & 36.37 & 0.12 \\
\hline
\end{tabular}


Table III 3 Apparent activation energy $\left(\mathrm{E}_{\mathrm{A}}\right)$ for de-excitation of the triplet state, calculated from the slope of a $\ln \left(\mathrm{k}_{\mathrm{TS} 0}\right)$ versus $1 / \mathrm{T}$ and activation energy $\mathrm{E}_{\mathrm{A}}$ for oxygen permeability $\mathrm{k}_{\mathrm{Q}}\left[\mathrm{O}_{2}\right]$ in sucrose / $\beta$-Lg films(a) trehalose / $\beta$-Lg films (b) maltose / $\beta$ - $\mathrm{Lg}$ films(c).

Table III-3a

\begin{tabular}{|l|l|l|c|c|}
\hline \multirow{2}{*}{$\begin{array}{l}\text { Ratio } \\
\text { (sucrose: } \beta \text {-Lg) }\end{array}$} & \multicolumn{2}{|l|}{$\Delta \mathrm{E}_{\mathrm{A}}$ of $\mathrm{k}_{\mathrm{TS} 0}\left(\mathrm{~kJ} \mathrm{~mol}^{-1}\right)$} & \multicolumn{2}{c|}{$\Delta \mathrm{E}_{\mathrm{A}}$ of $\mathrm{k}_{\mathrm{Q}}\left[\mathrm{O}_{2}\right]\left(\mathrm{kJ} \mathrm{mol}^{-1}\right)$} \\
\cline { 2 - 5 } & $\mathrm{LT}$ & $\mathrm{HT}$ & $\mathrm{LT}$ & $\mathrm{HT}$ \\
\hline$\beta-\mathrm{Lg}$ & 2.35 & 21.4 & 20.8 & 54.8 \\
\hline $0.1: 1$ & 3.13 & 22.8 & \multicolumn{2}{|c|}{37.78} \\
\hline $1: 1$ & 2.09 & 12.54 & \multicolumn{2}{|c|}{32.88} \\
\hline $10: 1$ & 1.5 & 11.45 & \multicolumn{2}{|c|}{30.29} \\
\hline $100: 1$ & 1.6 & 8.0 & \multicolumn{2}{|c|}{12.48} \\
\hline $1000: 1$ & \multicolumn{3}{|c|}{3.8} & 10.02 \\
\hline $10000: 1$ & \multicolumn{3}{|c|}{6.4} & \multicolumn{3}{|c}{} \\
\hline
\end{tabular}

Table III-3b

\begin{tabular}{|l|l|l|c|c|}
\hline \multirow{2}{*}{$\begin{array}{l}\text { Ratio } \\
\text { trehalose: } \beta \text {-Lg) }\end{array}$} & \multicolumn{2}{|l|}{$\Delta \mathrm{E}_{\mathrm{A}}$ of $\mathrm{k}_{\mathrm{TS} 0}\left(\mathrm{~kJ} \mathrm{~mol}^{-1}\right)$} & \multicolumn{2}{c|}{$\Delta \mathrm{E}_{\mathrm{A}}$ of $\mathrm{k}_{\mathrm{Q}}\left[\mathrm{O}_{2}\right]\left(\mathrm{kJ} \mathrm{mol}^{-1}\right)$} \\
\cline { 2 - 5 } & $\mathrm{LT}$ & $\mathrm{HT}$ & $\mathrm{LT}$ & $\mathrm{HT}$ \\
\hline$\beta-\mathrm{Lg}$ & 2.35 & 21.4 & 20.8 & 54.8 \\
\hline $0.1: 1$ & 2.1 & 6.2 & 34.53 \\
\hline $1: 1$ & 1.6 & 10.6 & 35.35 \\
\hline $10: 1$ & 1.4 & 8.6 & 33.81 \\
\hline 30.1 & 1.5 & 12.2 & 30.82 \\
\hline $60: 1$ & \multicolumn{3}{|c|}{2.3} & - \\
\hline $100: 1$ & 2.4 & - \\
\hline $1000: 1$ & \multicolumn{3}{|c|}{2.4} & - \\
\hline
\end{tabular}


Table III-3c

\begin{tabular}{|l|l|l|c|c|}
\hline \multirow{2}{*}{$\begin{array}{l}\text { Ratio } \\
\text { (maltose: } \beta-\mathrm{Lg})\end{array}$} & \multicolumn{2}{|l|}{$\Delta \mathrm{E}_{\mathrm{A}}$ of $\mathrm{k}_{\mathrm{TS} 0}\left(\mathrm{~kJ} \mathrm{~mol}^{-1}\right)$} & \multicolumn{2}{|c|}{$\Delta \mathrm{E}_{\mathrm{A}}$ of $\mathrm{k}_{\mathrm{Q}}\left[\mathrm{O}_{2}\right]\left(\mathrm{kJ} \mathrm{mol}^{-1}\right)$} \\
\cline { 2 - 5 } & $\mathrm{LT}$ & $\mathrm{HT}$ & $\mathrm{LT}$ & $\mathrm{HT}$ \\
\hline$\beta-\mathrm{Lg}$ & 2.35 & 21.4 & 20.8 & 54.8 \\
\hline $0.1: 1$ & 2.2 & 18.07 & \multicolumn{2}{|c|}{38.29} \\
\hline $1: 1$ & 1.5 & 15.6 & \multicolumn{2}{|c|}{35.99} \\
\hline $10: 1$ & 1.9 & 21.9 & \multicolumn{2}{|l|}{28.75} \\
\hline $100: 1$ & 1.4 & 8.4 & \multicolumn{2}{|l|}{-} \\
\hline $1000: 1$ & 1.6 & 8.5 & \multicolumn{2}{|c|}{-} \\
\hline
\end{tabular}




\section{Figure III-1a}

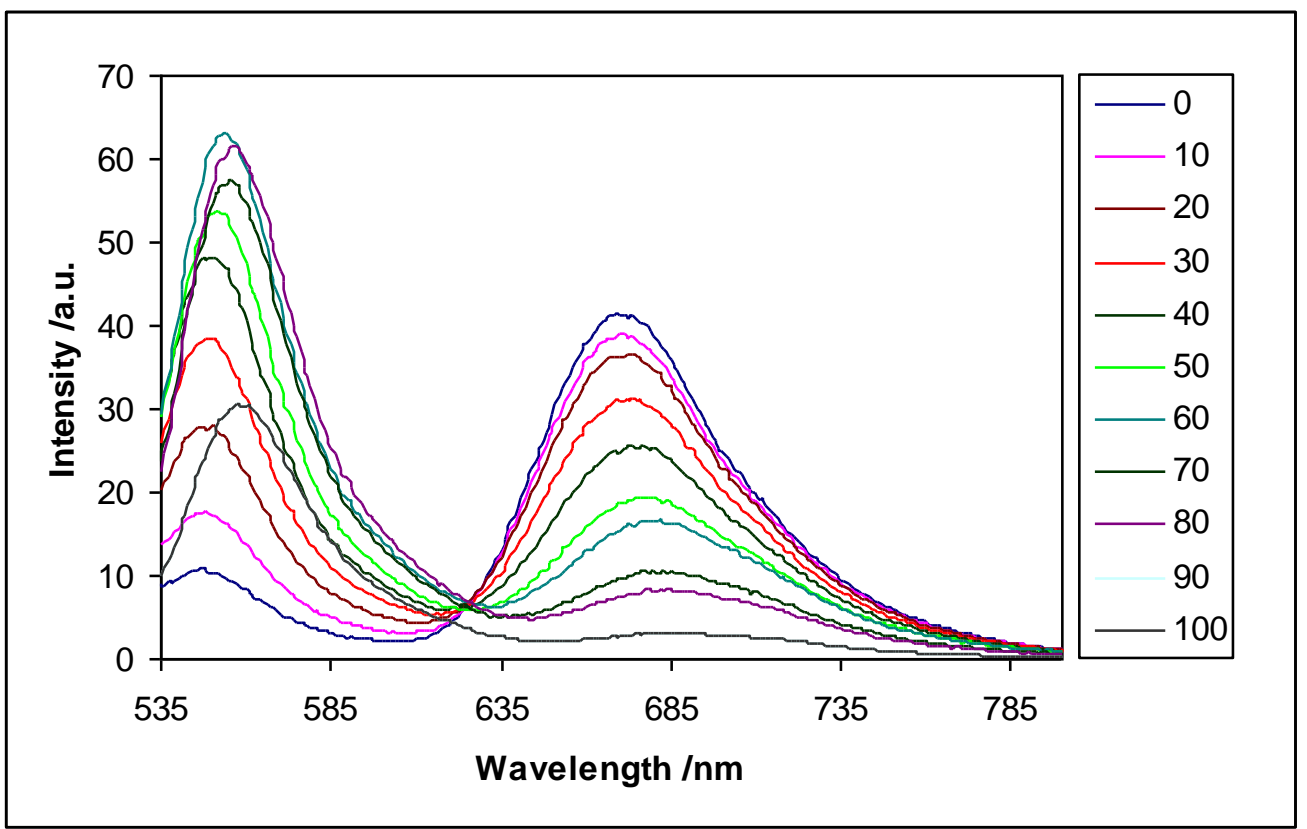

Figure III-1a: The effect of temperature on the delayed emission spectra of Erythrosin B dispersed in amorphous sucrose / $\beta$-Lg -films (sucrose: $\beta$-Lg 10:1).

\section{Figure III-1b}

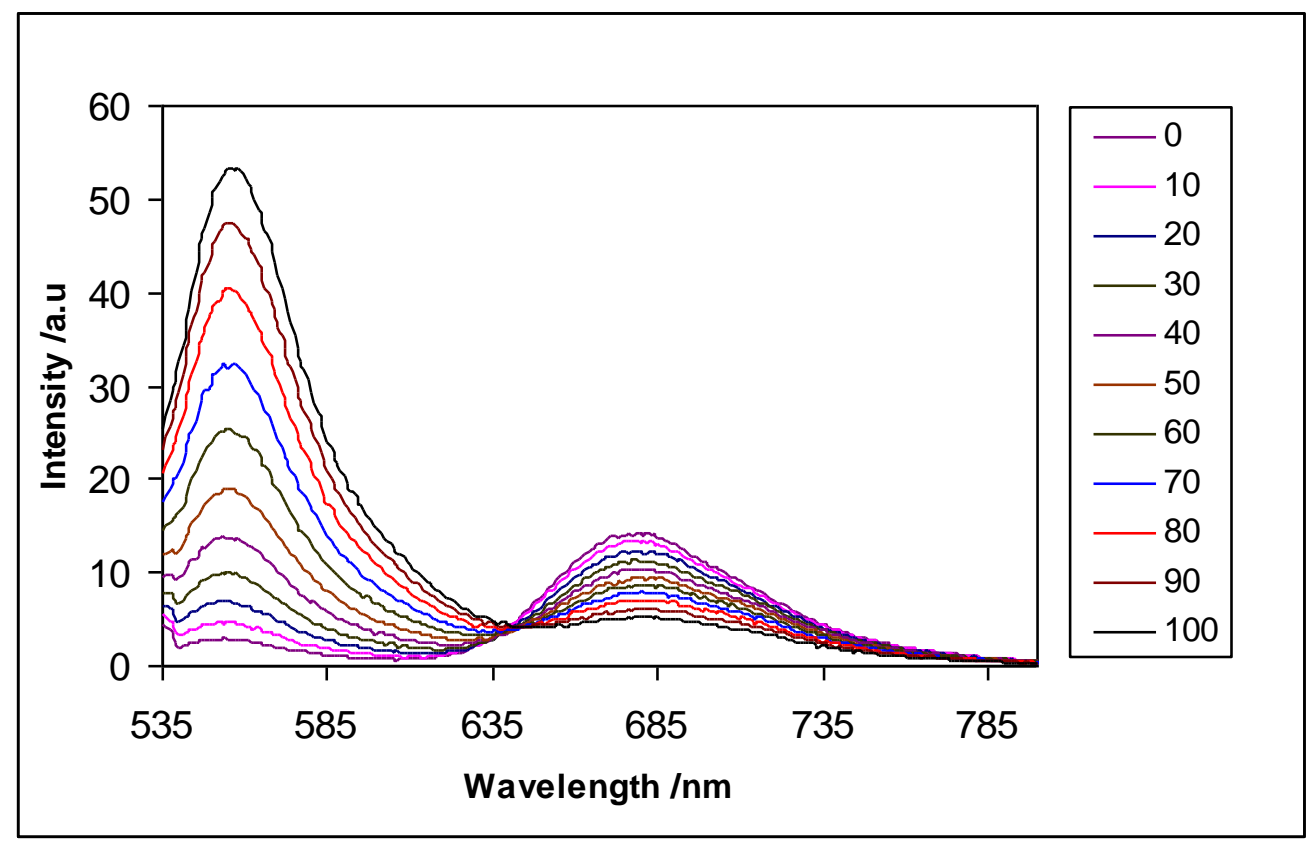

Figure III-1b: The effect of temperature on the delayed emission spectra of Erythrosin B dispersed in amorphous trehalose/ $\beta$-Lg films (trehalose: $\beta-\operatorname{Lg} 10: 1$ ) 
Figure III-1c

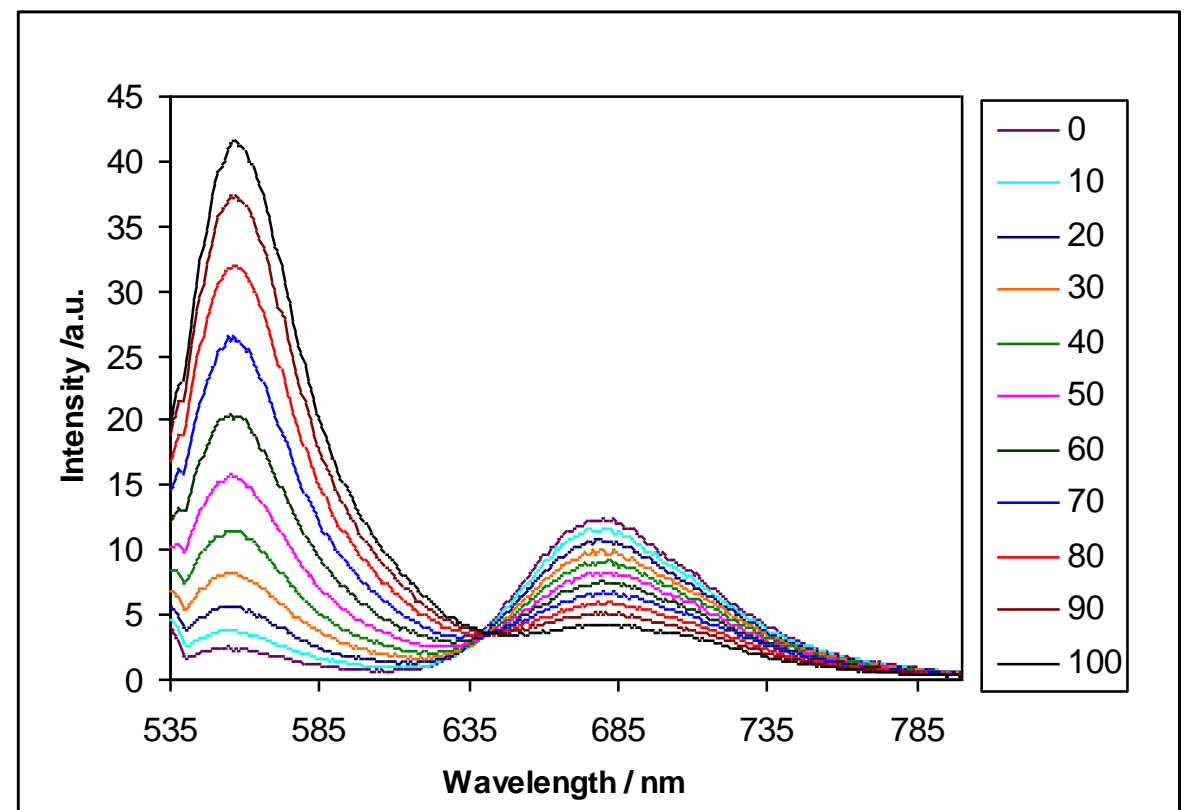

Figure III-1c: The effect of temperature on the delayed emission spectra of Erythrosin B dispersed in amorphous Maltose/ $\beta-\operatorname{Lg}$ films. (Maltose: $\beta-\operatorname{Lg} 10: 1$ ). 
Figure III-2a

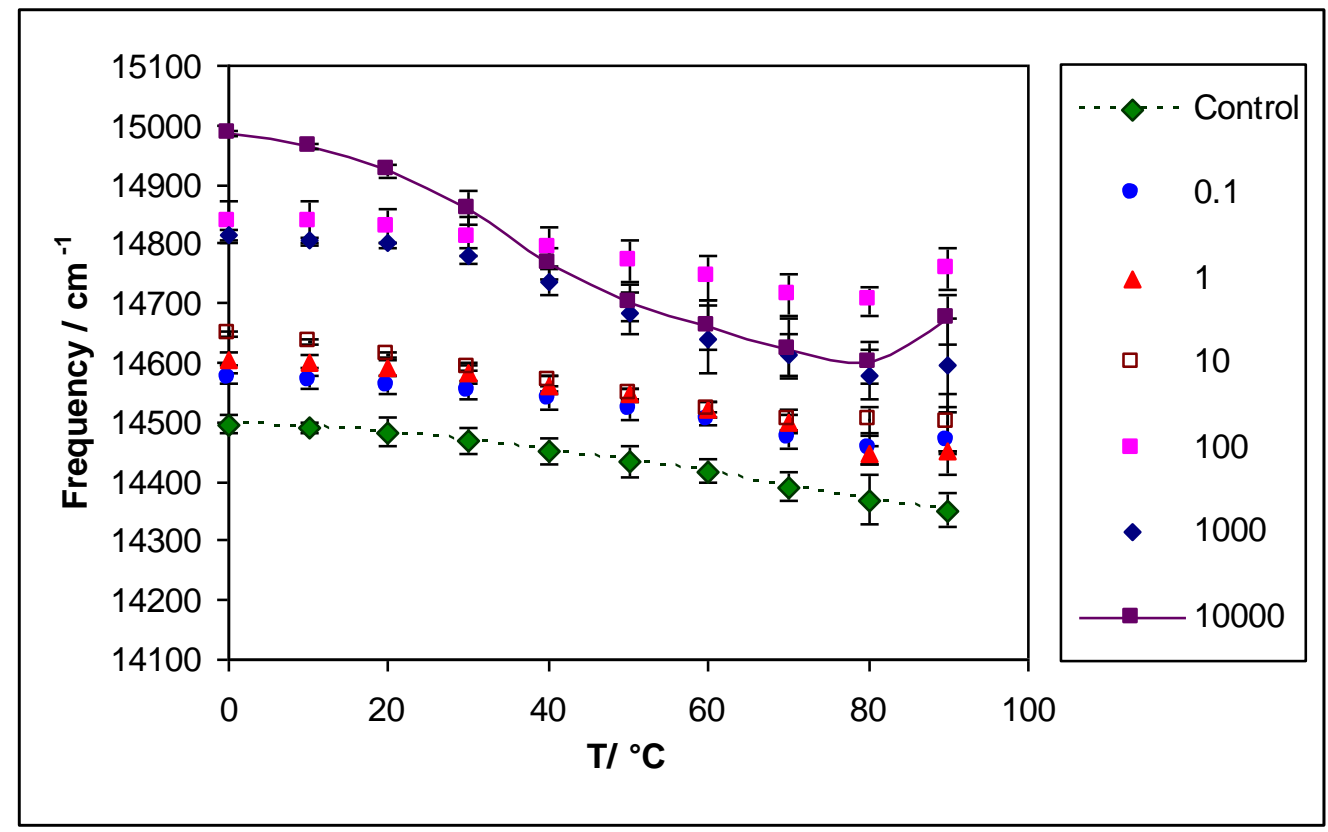

Figure III-2a: The effect of temperature on the emission maximum for phosphorescence from Erythrosin B in amorphous sucrose/ $\beta$ - Lg films.

Figure III-2b

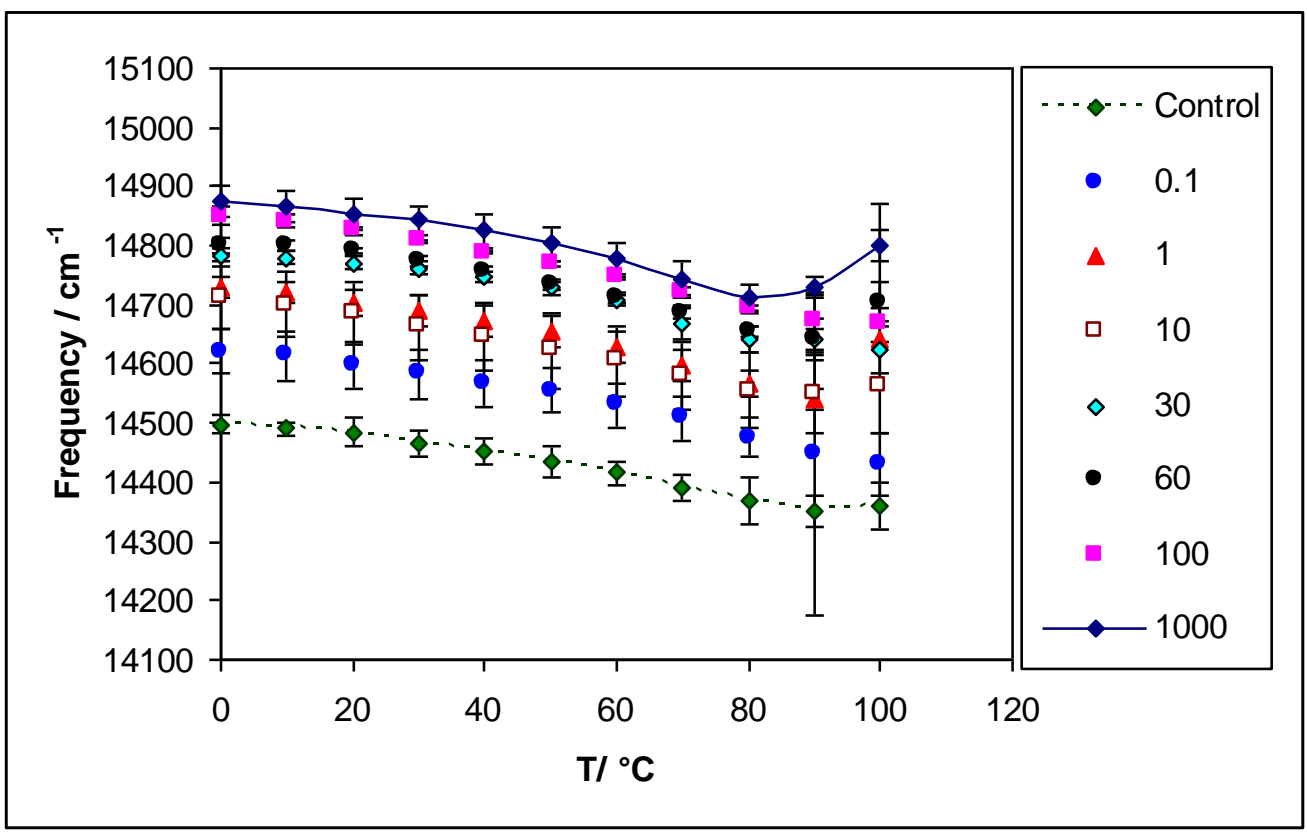

Figure III-2b: The effect of temperature on the emission maximum for phosphorescence from Erythrosin B in amorphous trehalose/ $\beta$-Lg films. 
Figure III-2c

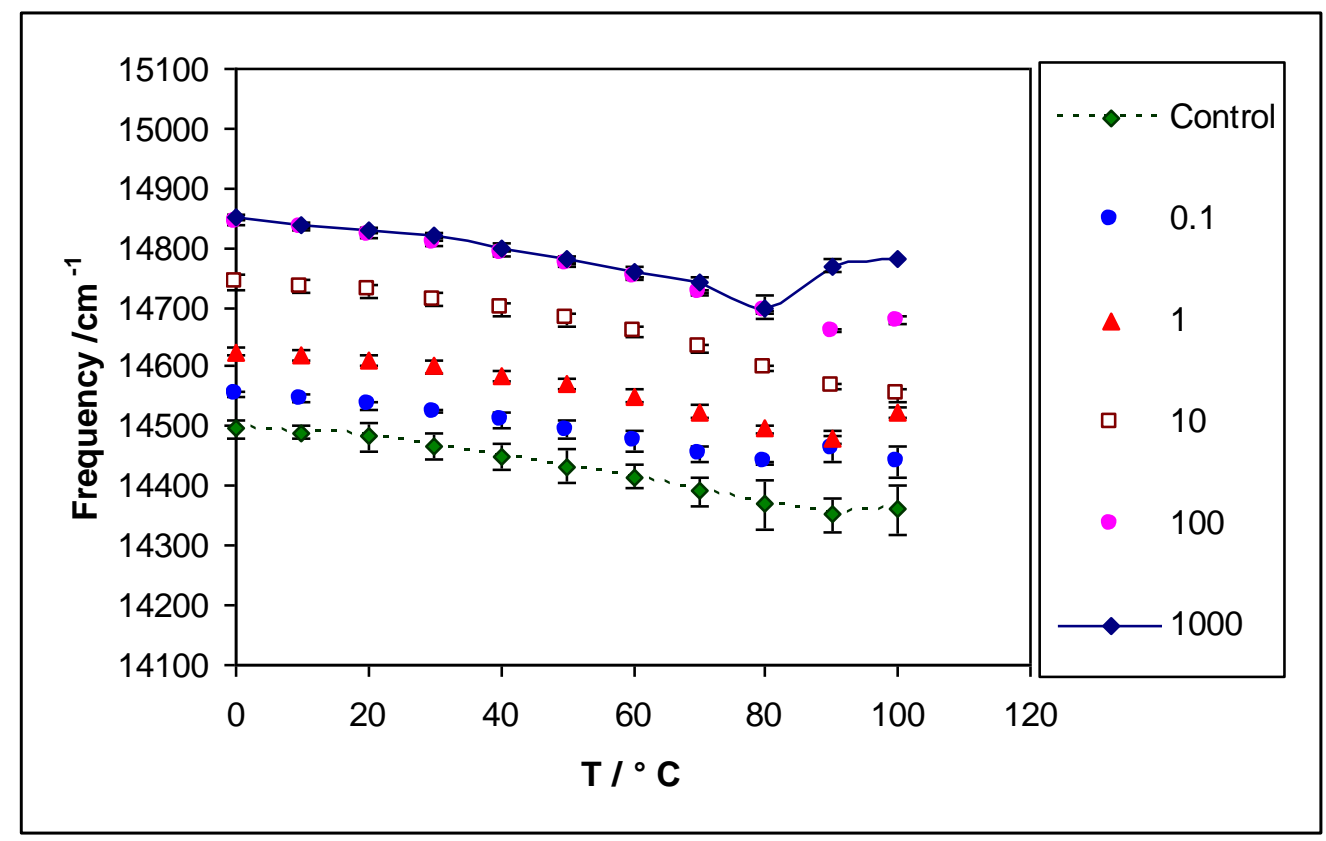

Figure III-2c: The effect of temperature on the emission maximum for phosphorescence from Erythrosin B in amorphous maltose/ $\beta$ - Lg films. 
Figure III-3a

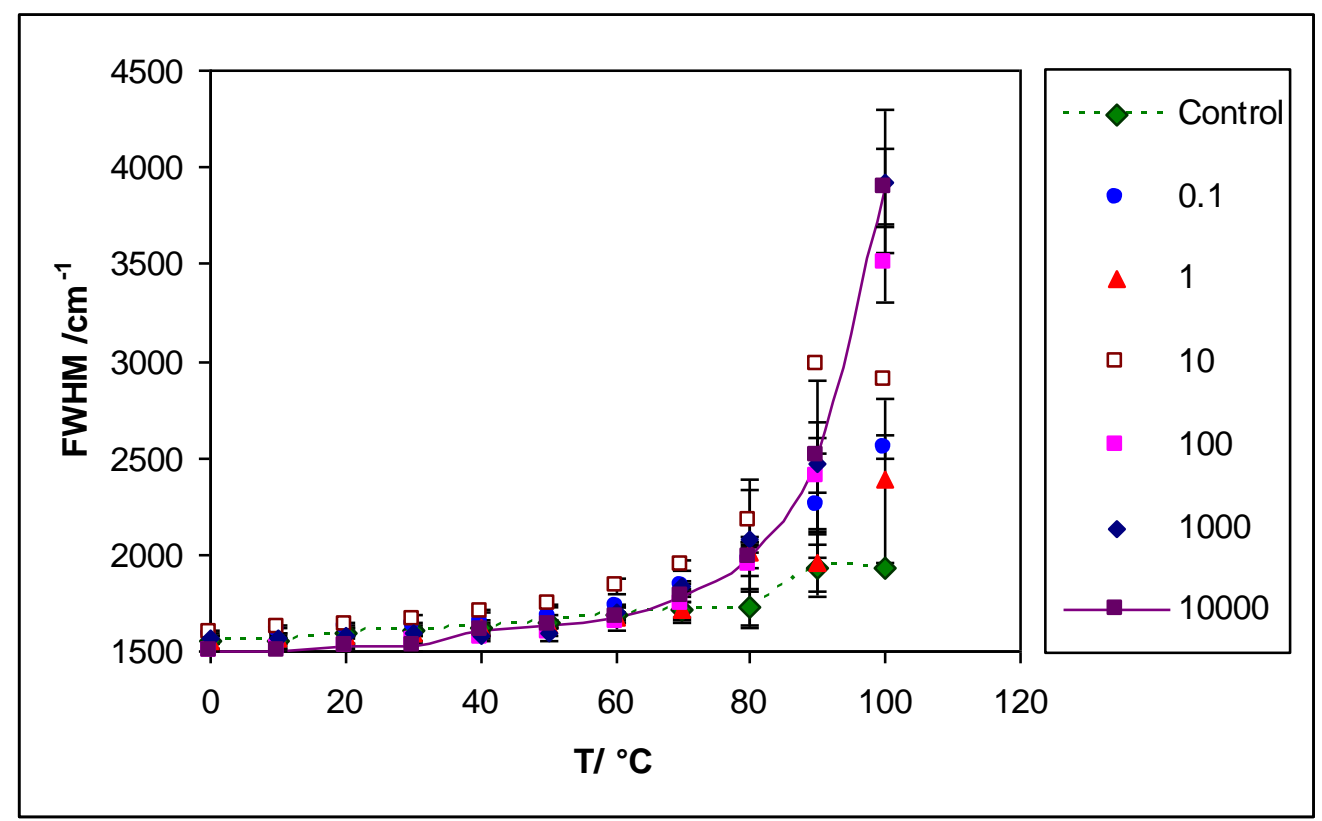

Figure III-3a: The effect of temperature on the bandwidth for phosphorescence from Erythrosin B in amorphous sucrose/ $\beta$-Lg films.

Figure III-3b

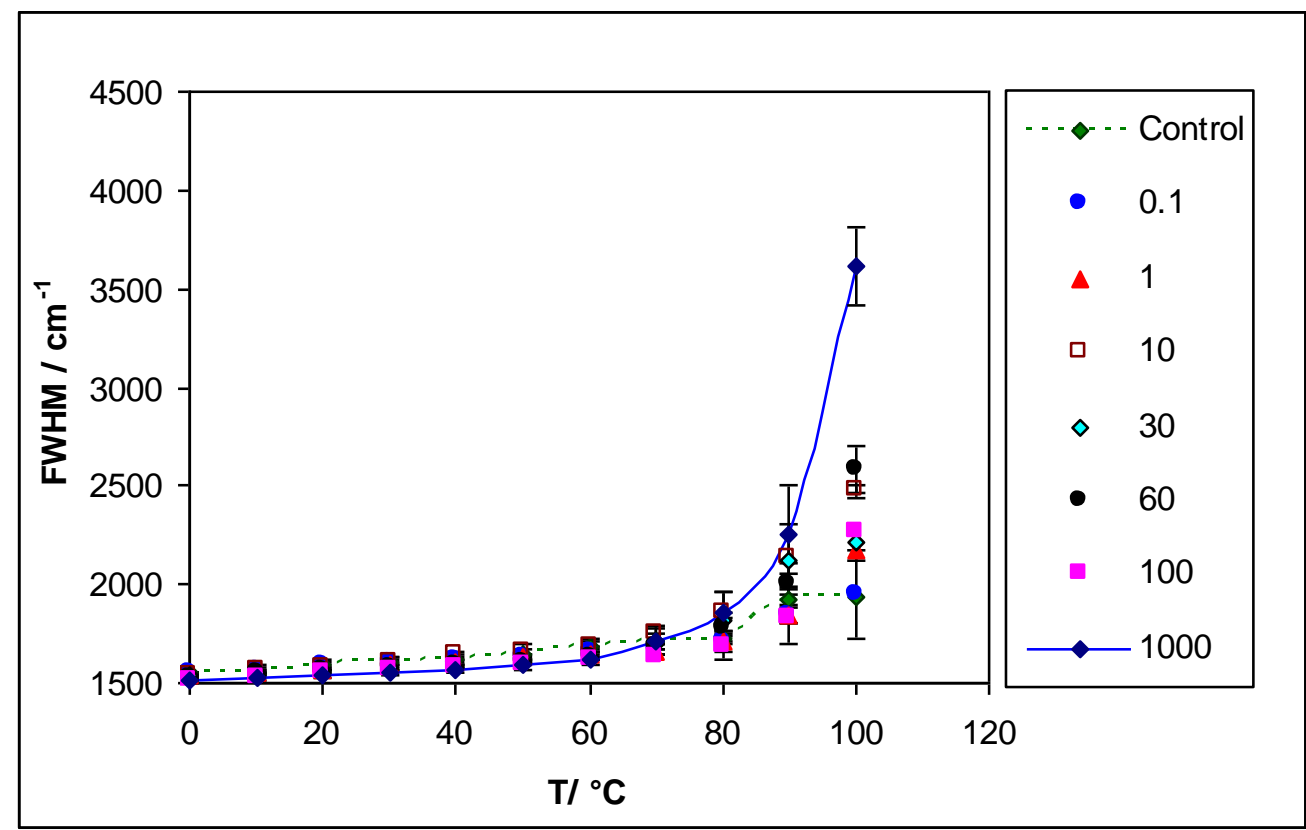

Figure III-3b: The effect of temperature on the bandwidth for phosphorescence from Erythrosin B in amorphous trehalose/ $\beta-\mathrm{Lg}$ films. 
Figure III-3c

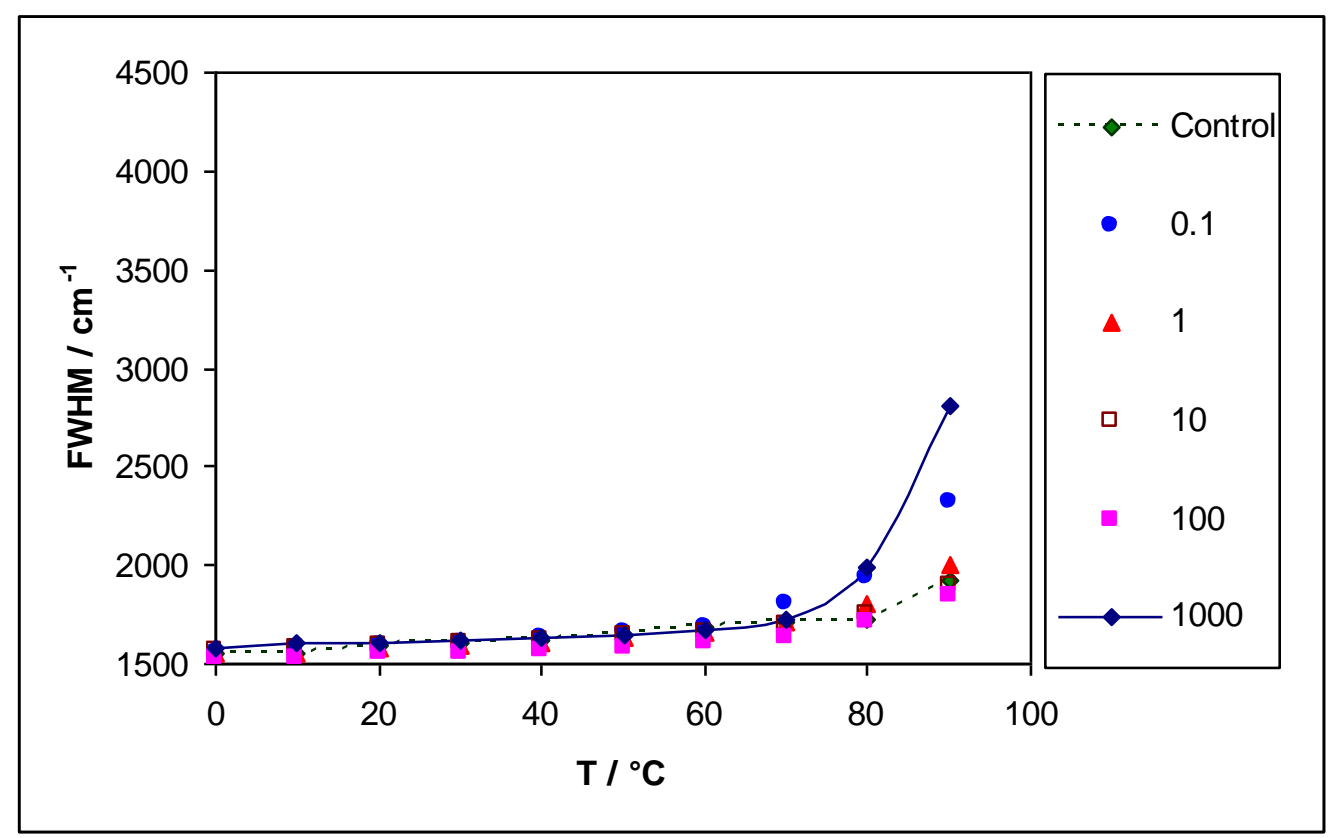

Figure III-3c: The effect of temperature on the bandwidth for phosphorescence from Erythrosin B in amorphous maltose / $\beta$-Lg films. 
Figure III-4a

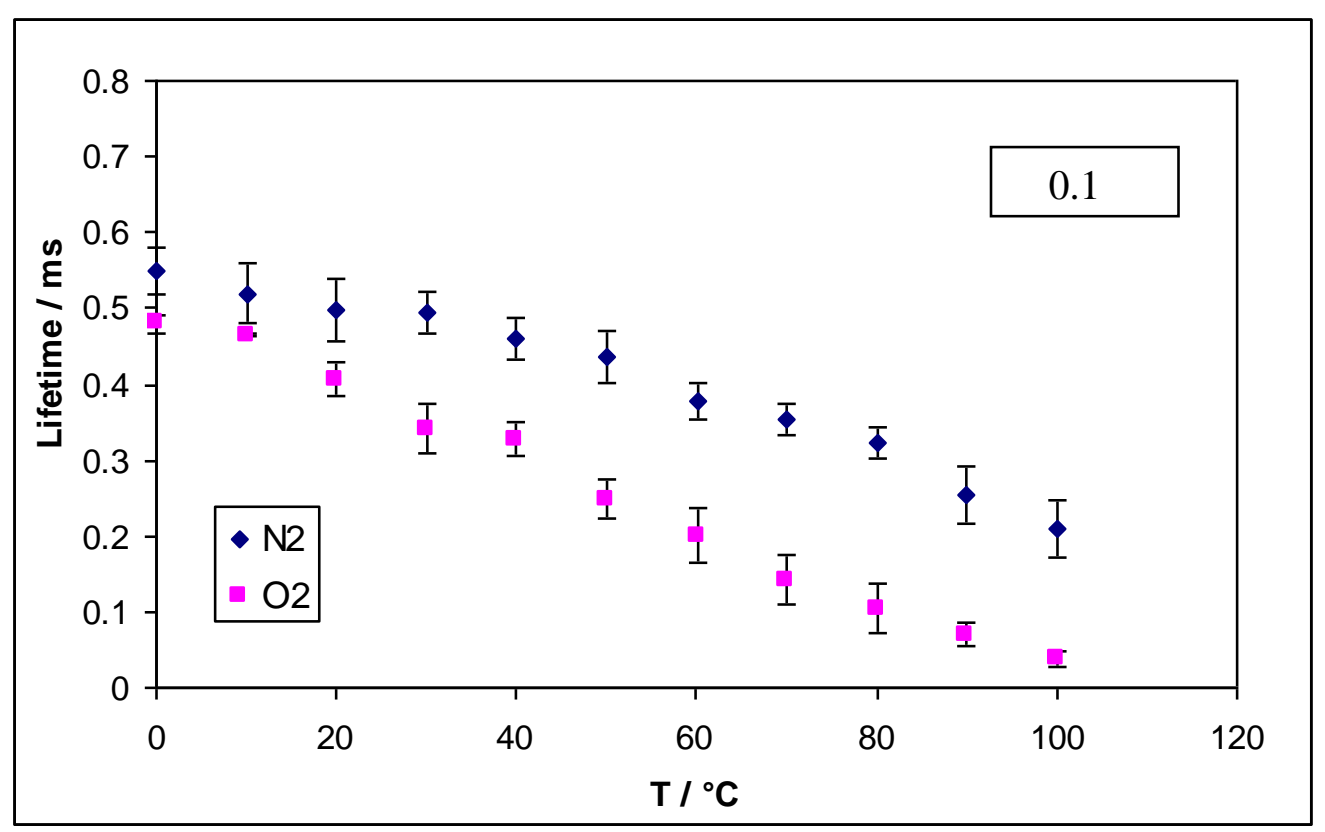

Figure III-4b

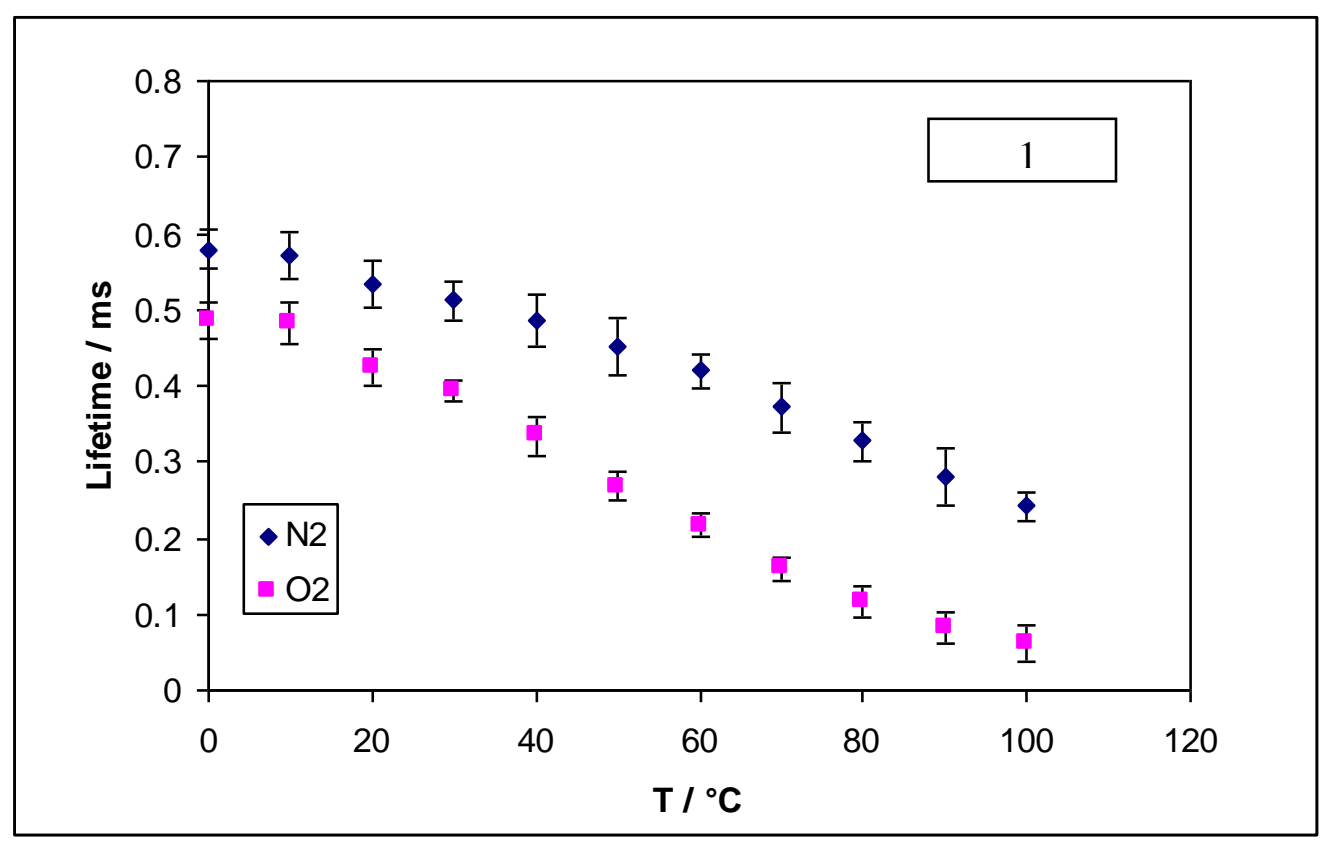


Figure III-4c

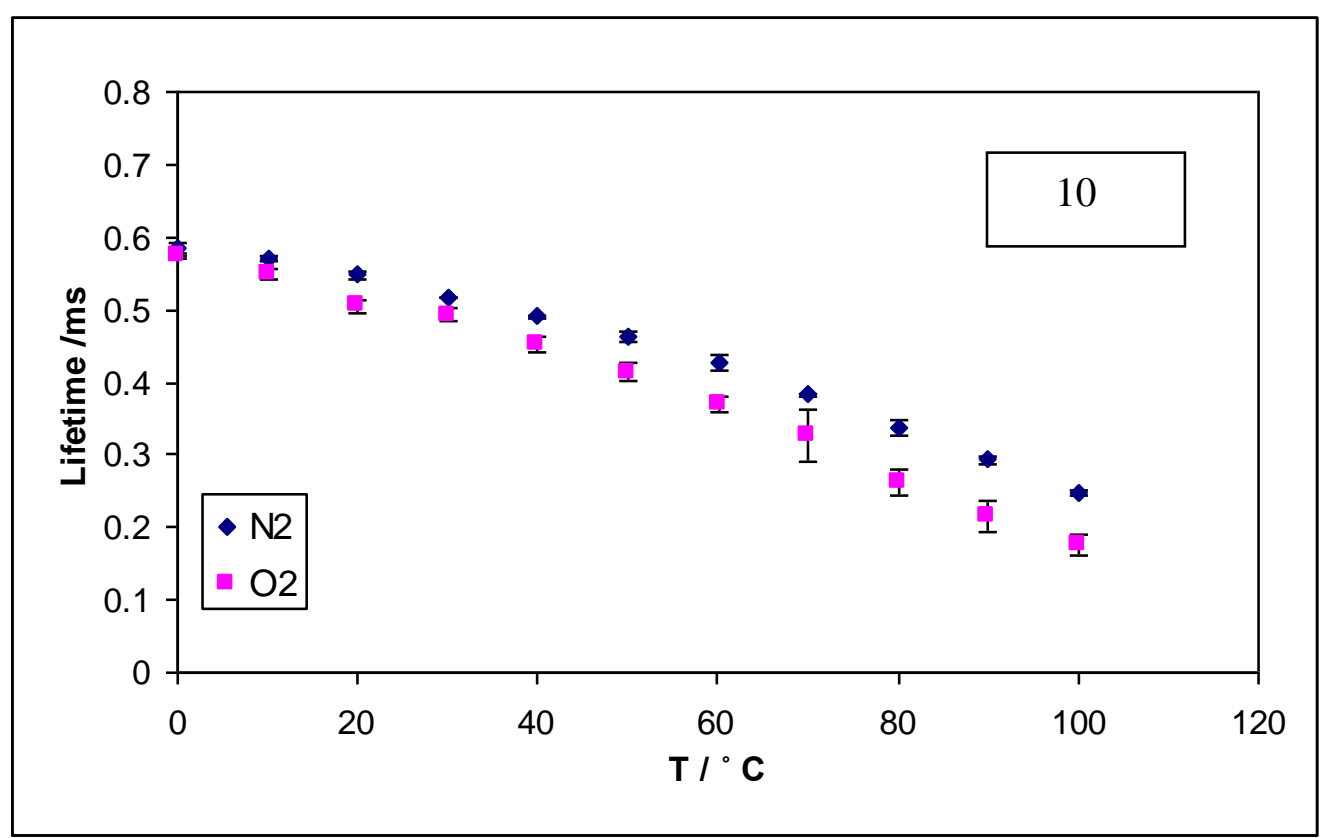

Figure III-4d

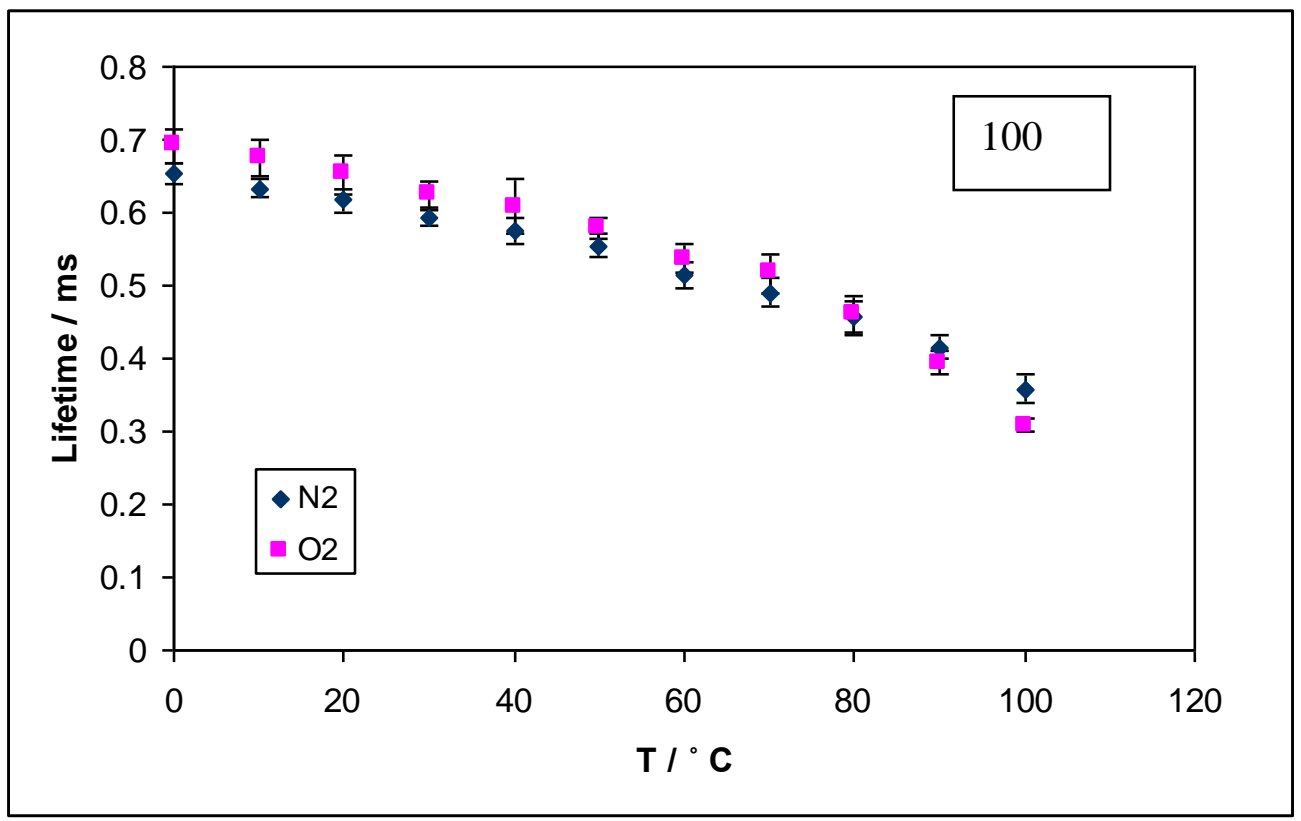


Figure III-4e

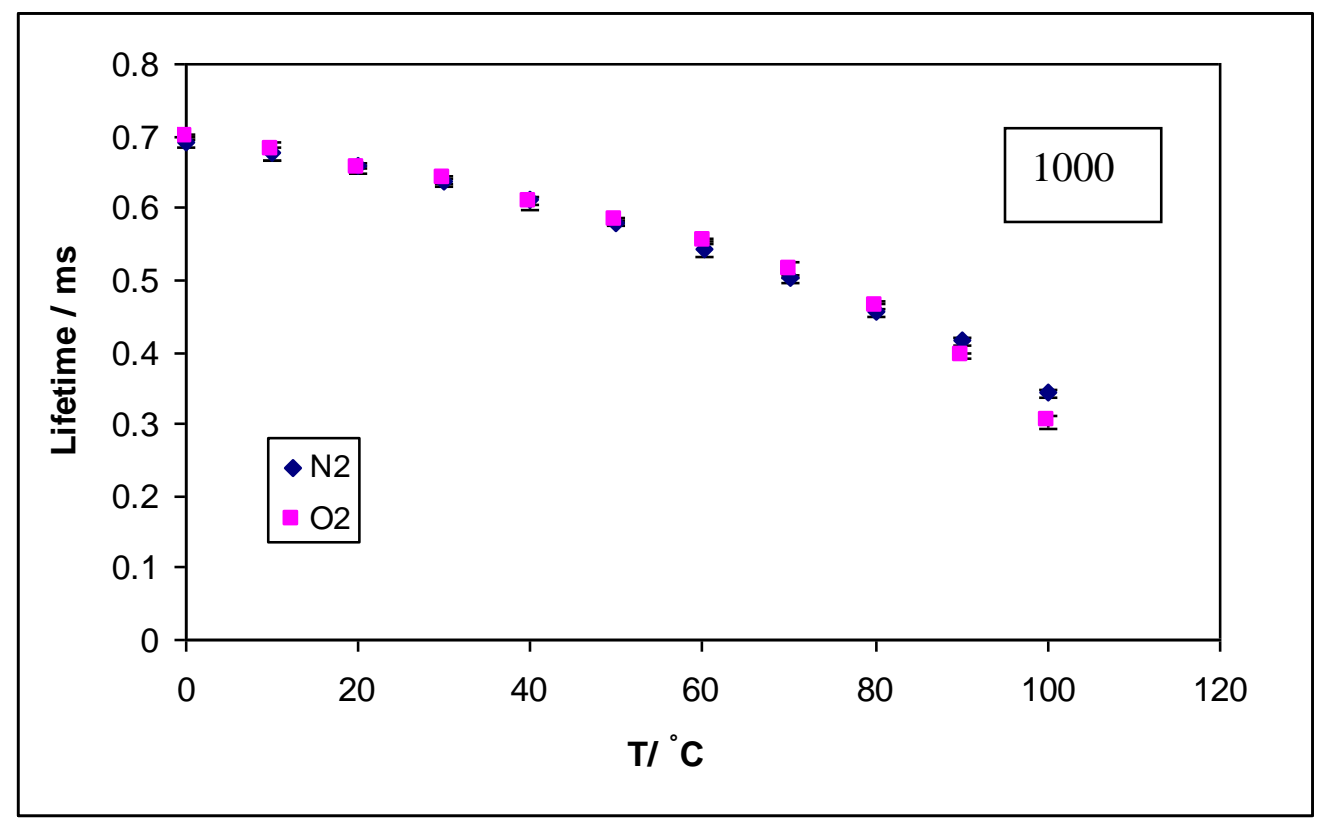

Figure III-4f

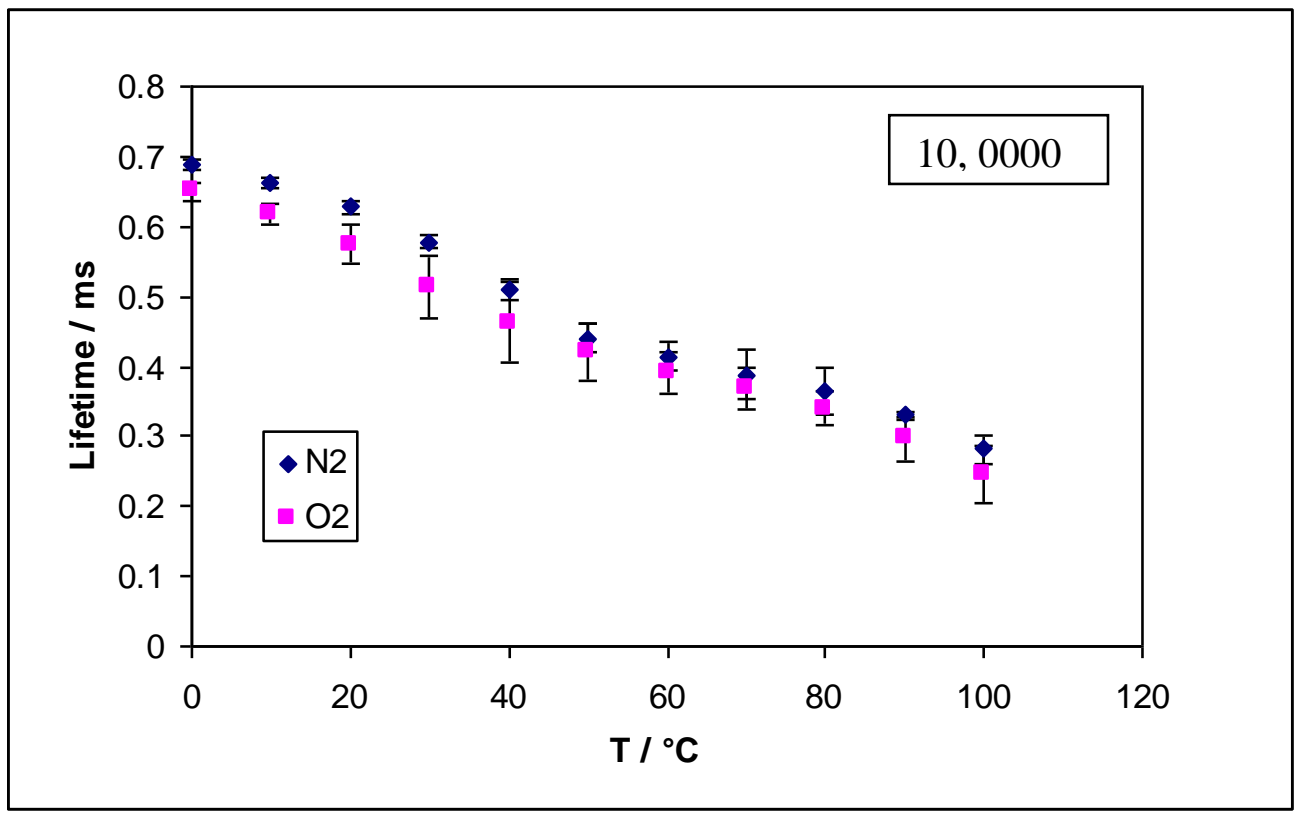

Figure III-4(a,b,c,d,e,f): The effect of temperature on the lifetime for Erythrosin B dispersed in $\beta$-Lg in various molar ratios of sucrose equilibrated against nitrogen $(\bullet)$ and against air $(\square)$; the lifetime was determined from analysis using a stretched exponential decay function. The text box in the graph refers to the molar ratio of sucrose: $\beta-\mathrm{Lg}$. 
Figure III-4g

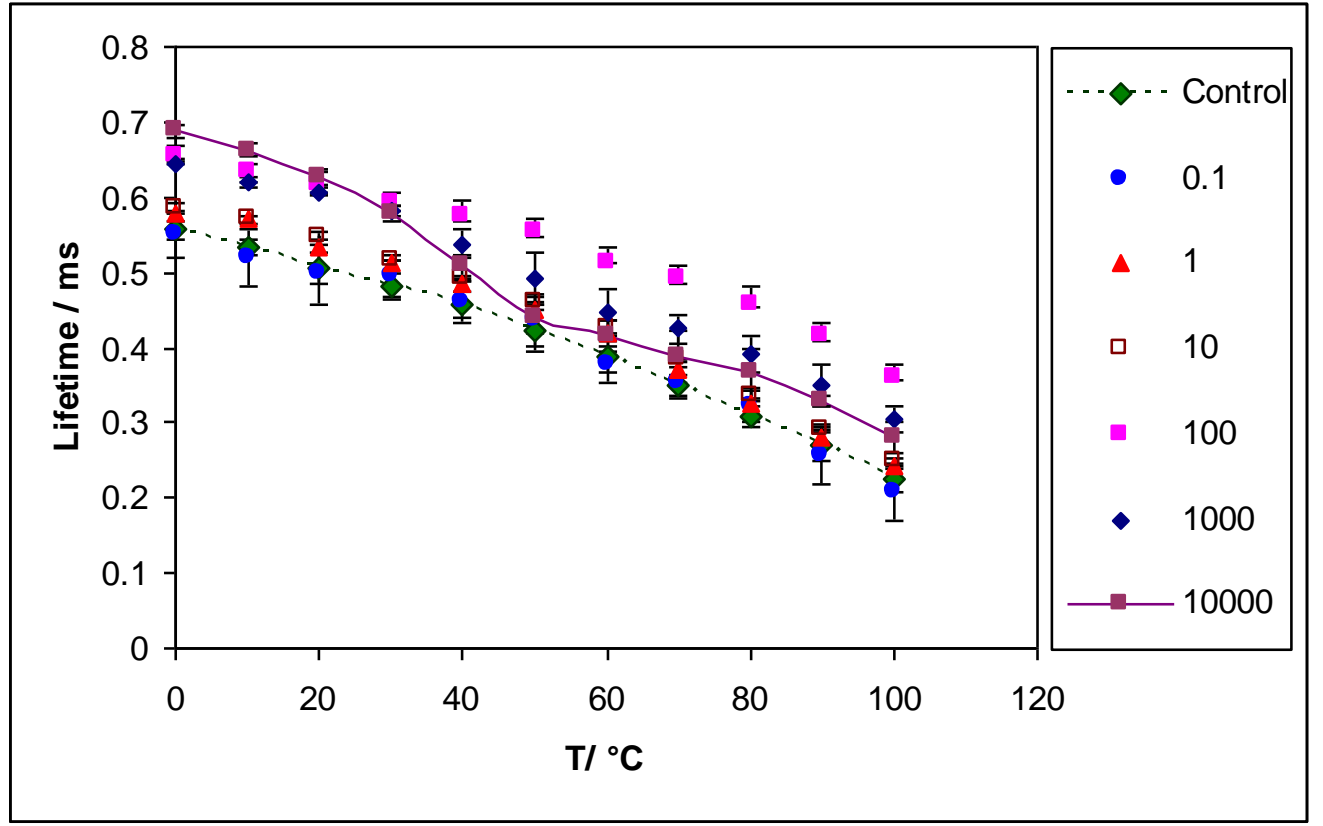

Figure III-4g: Comparison plot depicting the effect of temperature on the lifetime for Erythrosin B dispersed in $\beta$-Lg in various molar concentrations of sucrose equilibrated against nitrogen.

Figure III-4h

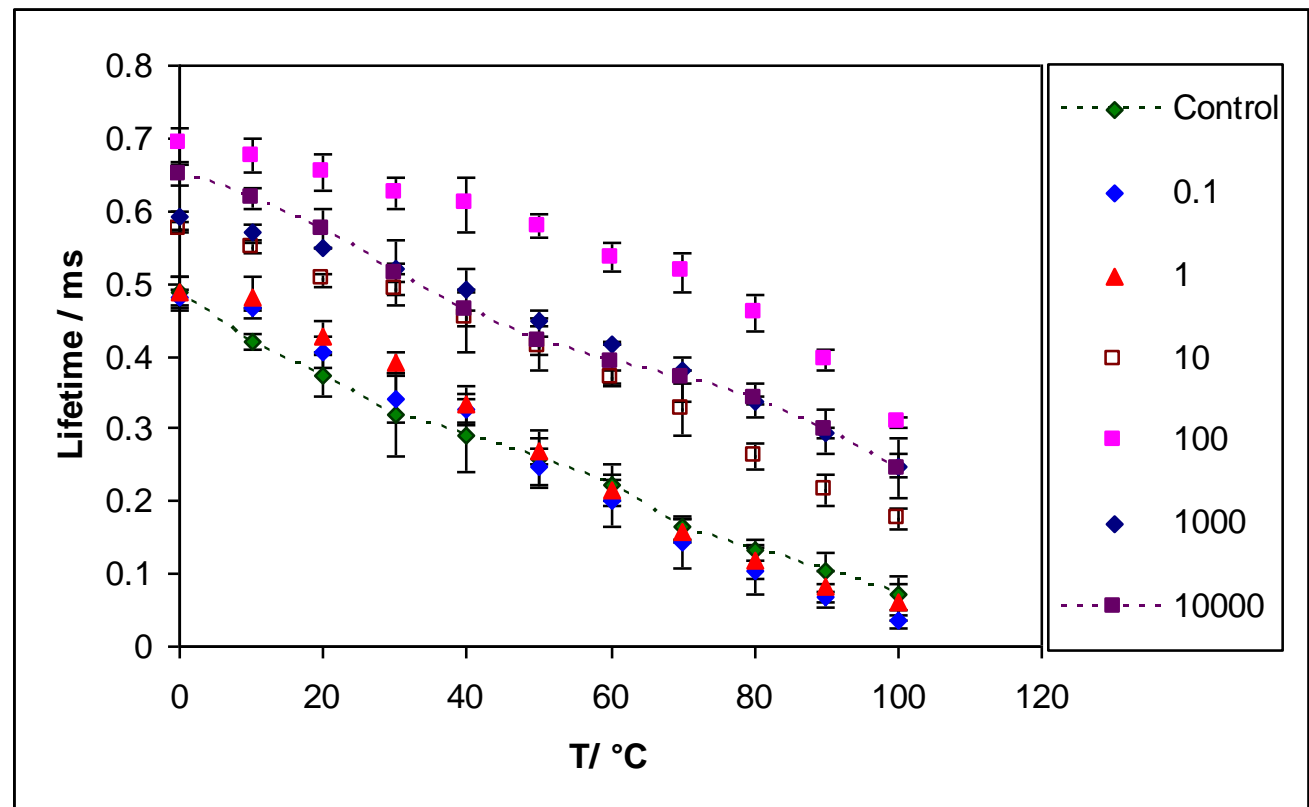

Figure III-4h: Comparison plot depicting the effect of temperature on the lifetime for Erythrosin B dispersed in $\beta$-Lg in various molar concentrations of sucrose equilibrated against air. 
Figure III-5a

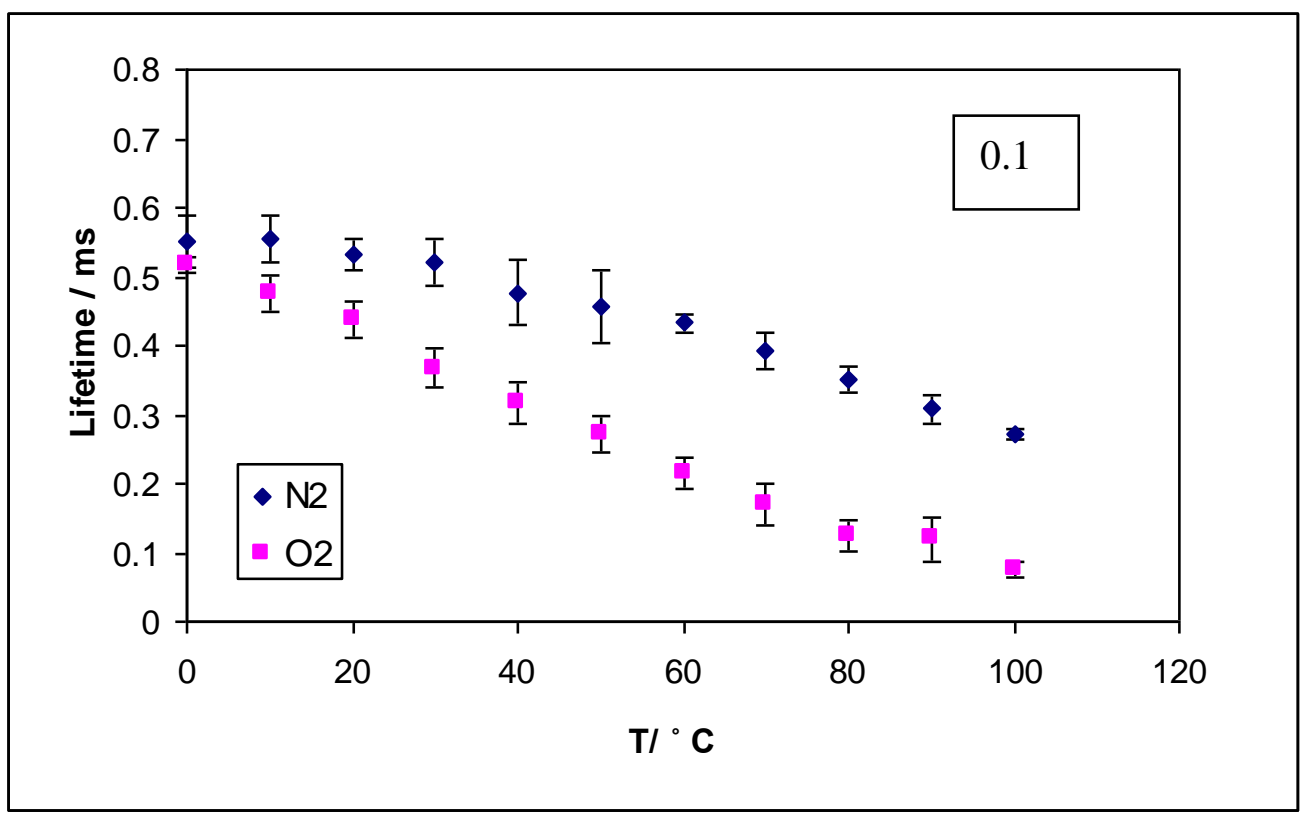

Figure III-5b

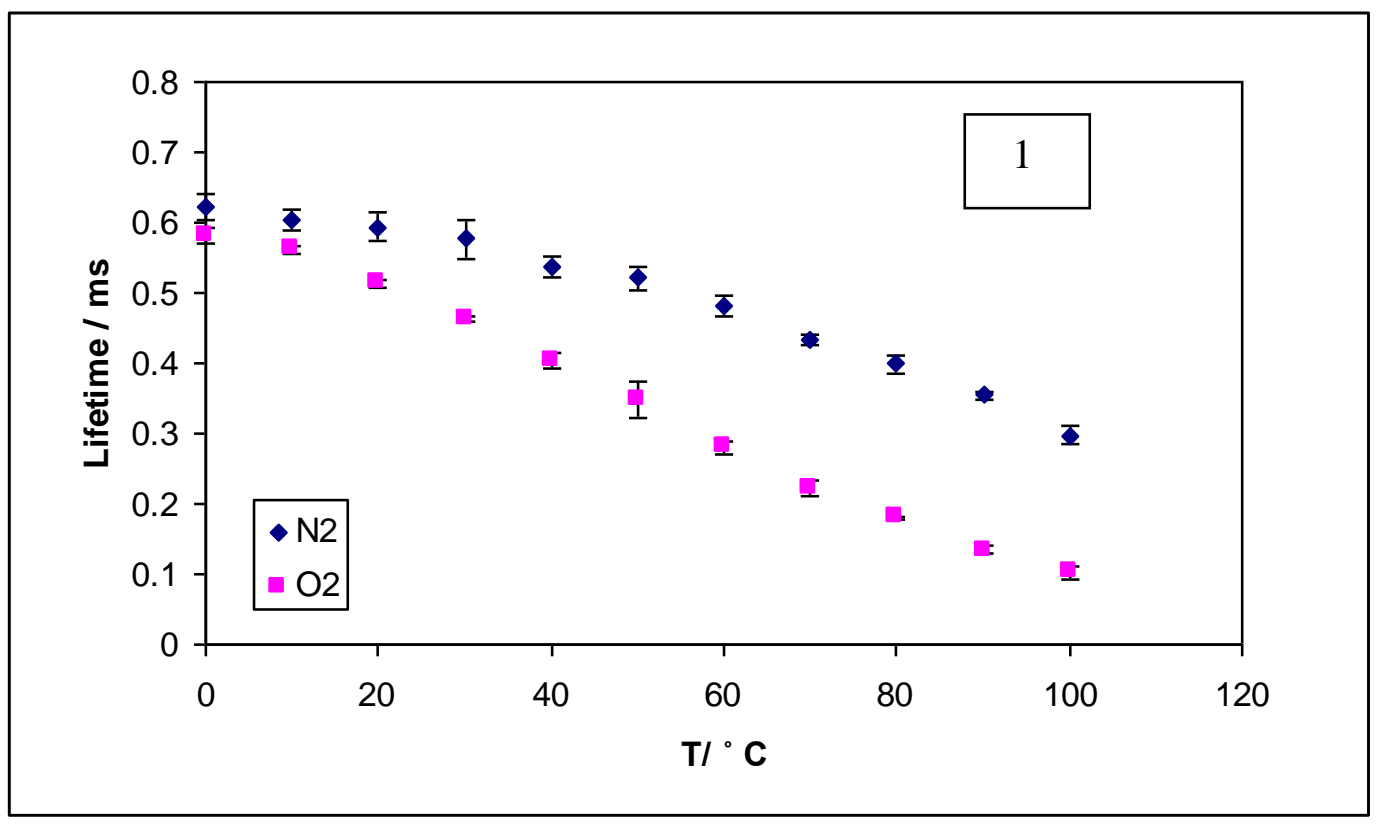


Figure III-5c

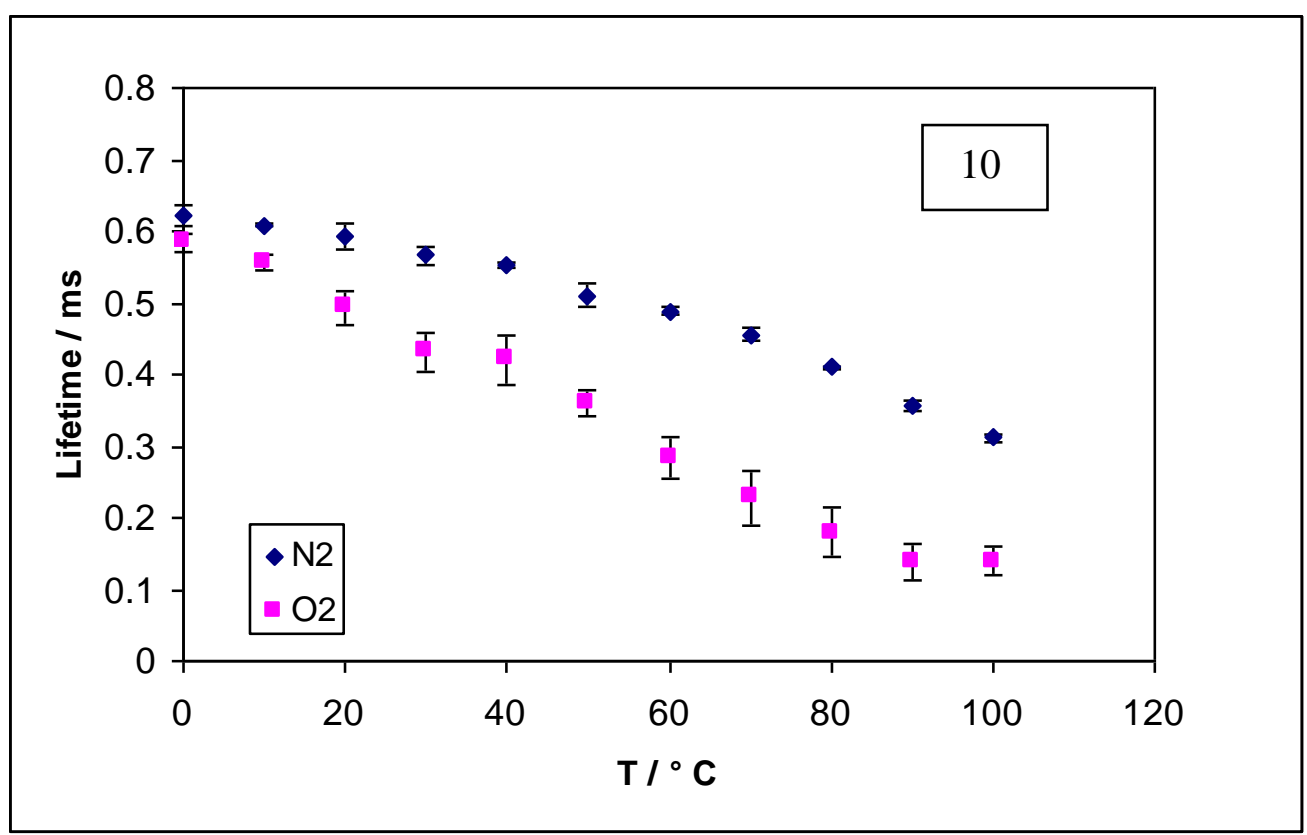

Figure III-5d

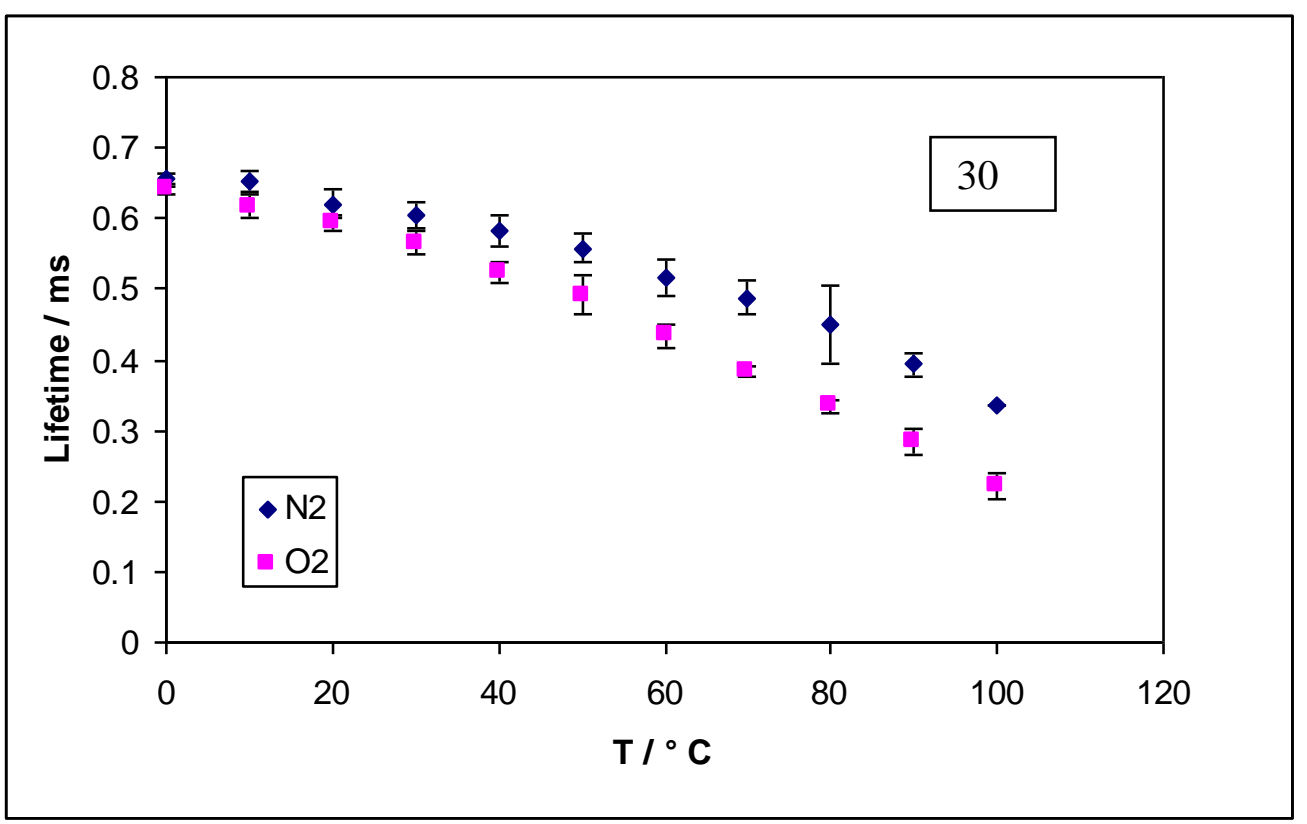


Figure III-5e

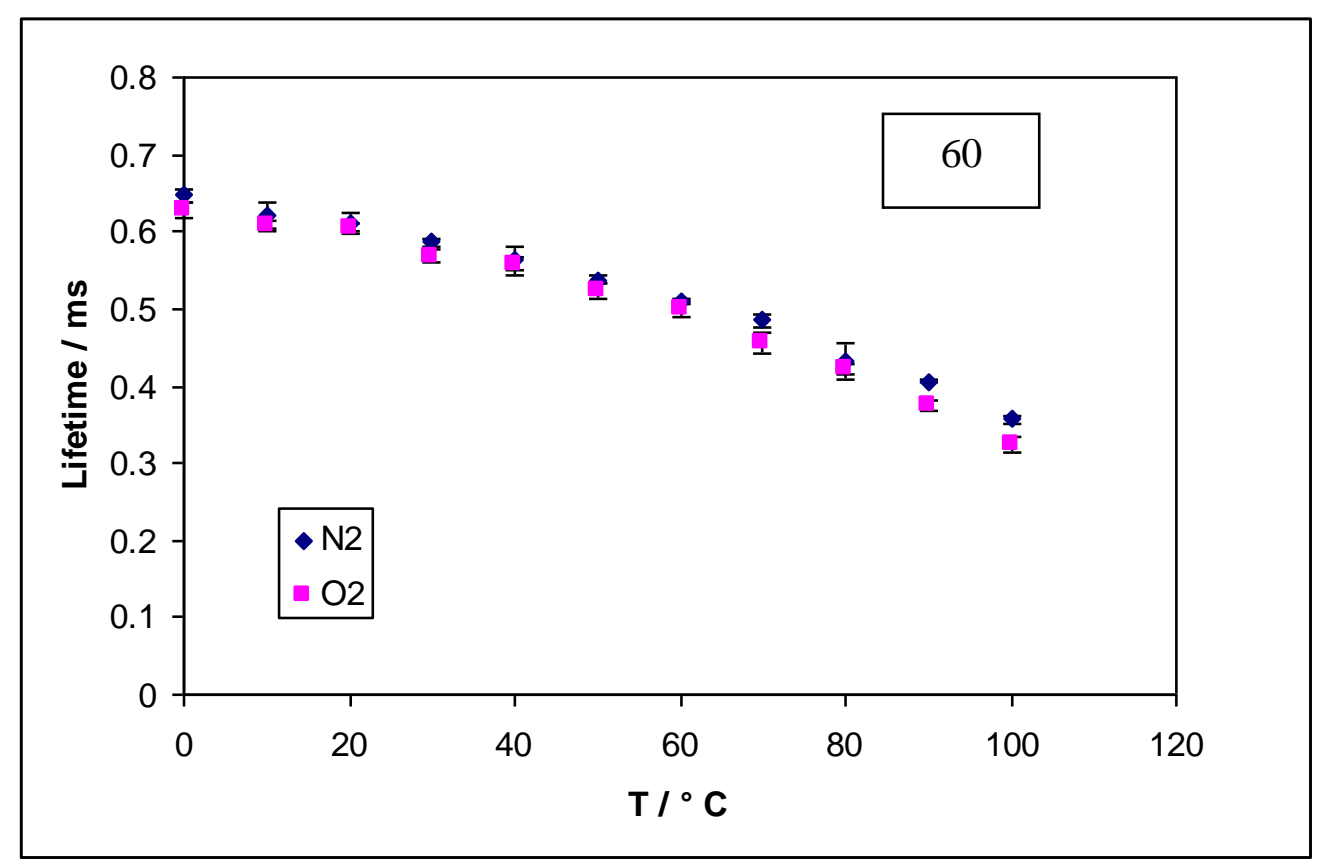

Figure III-5f

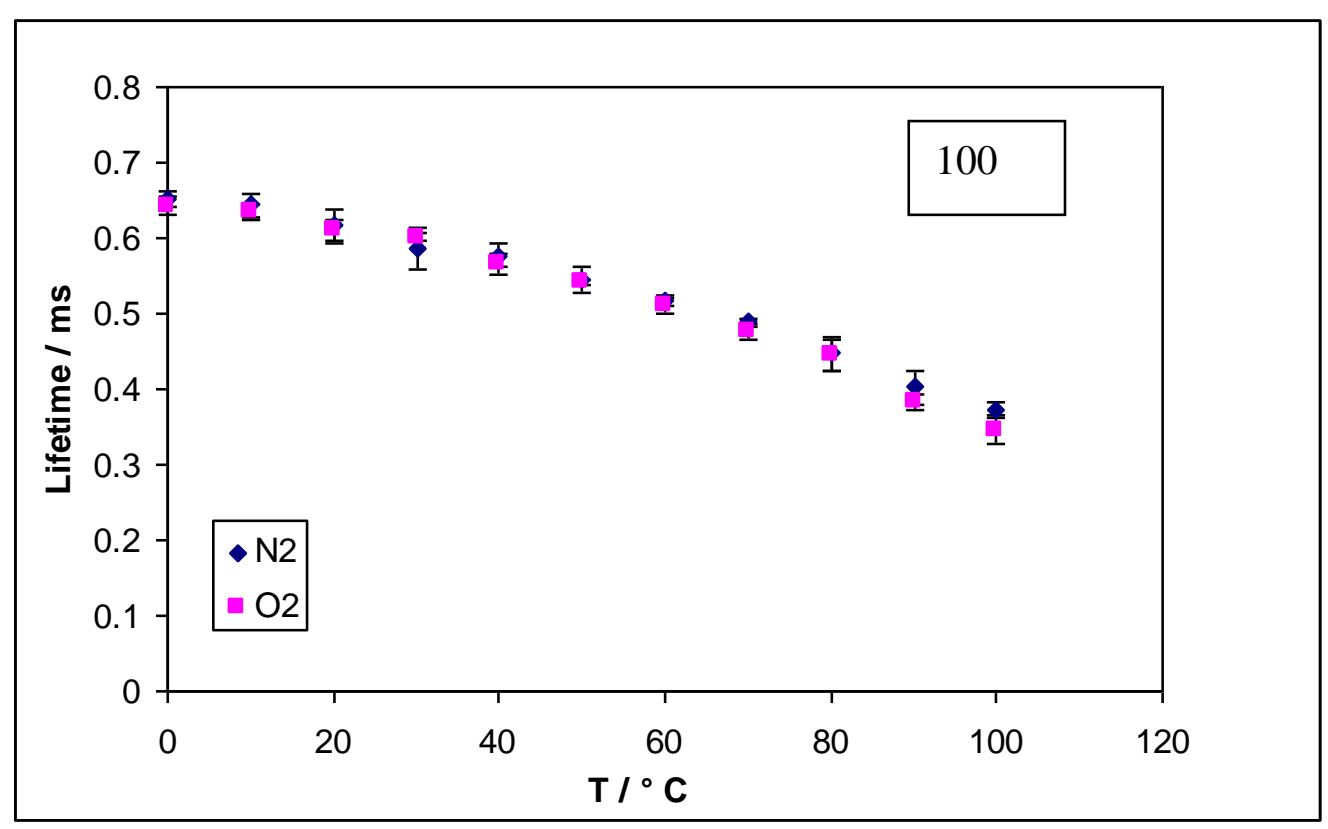




\section{Figure III-5g}

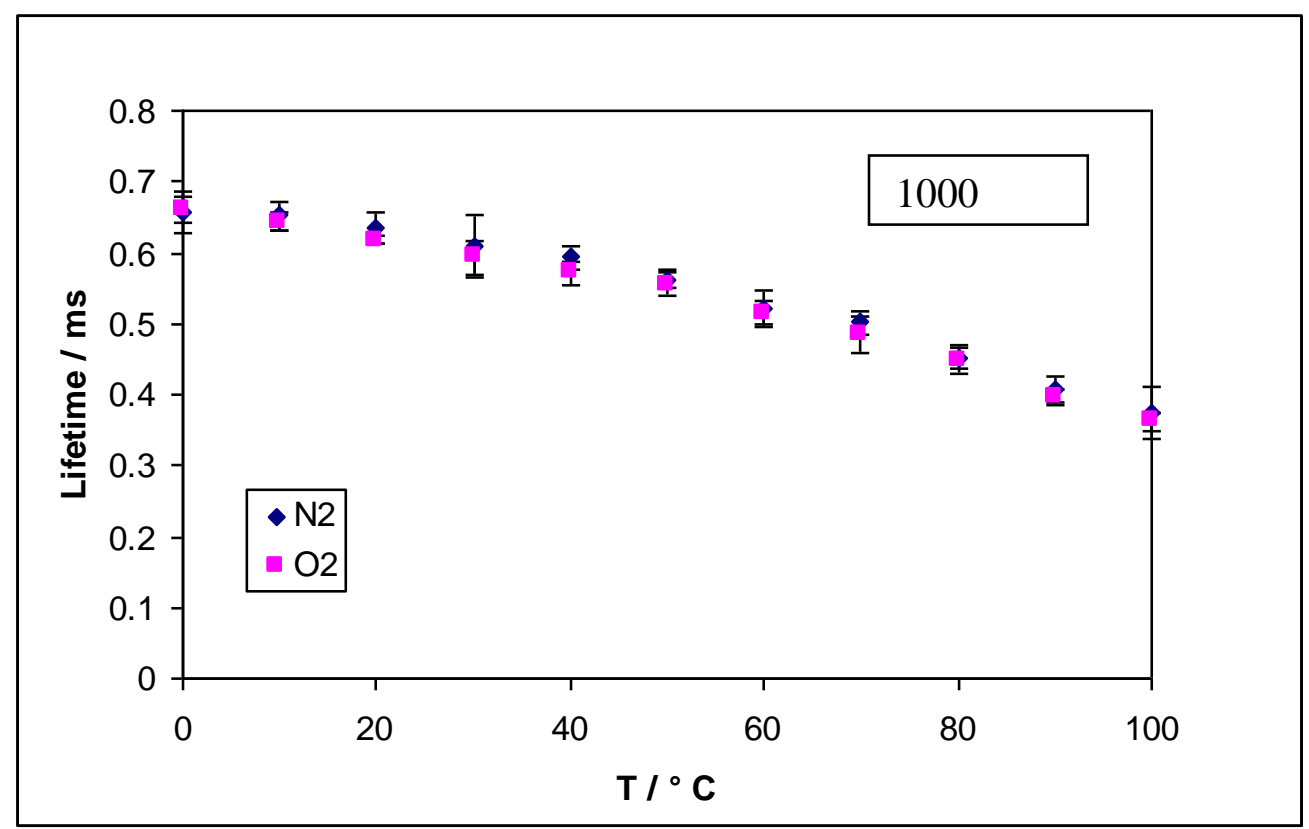

Figure III-5(a, b, c, d, e, f, g): The effect of temperature on the lifetime for Erythrosin B dispersed in $\beta$-Lg in various molar concentrations of trehalose equilibrated against nitrogen and against air. The lifetime was determined from analysis using a stretched exponential decay function. The text box in the graph refers to the molar ratio of trehalose: $\beta-\mathrm{Lg}$.

Figure III-5h

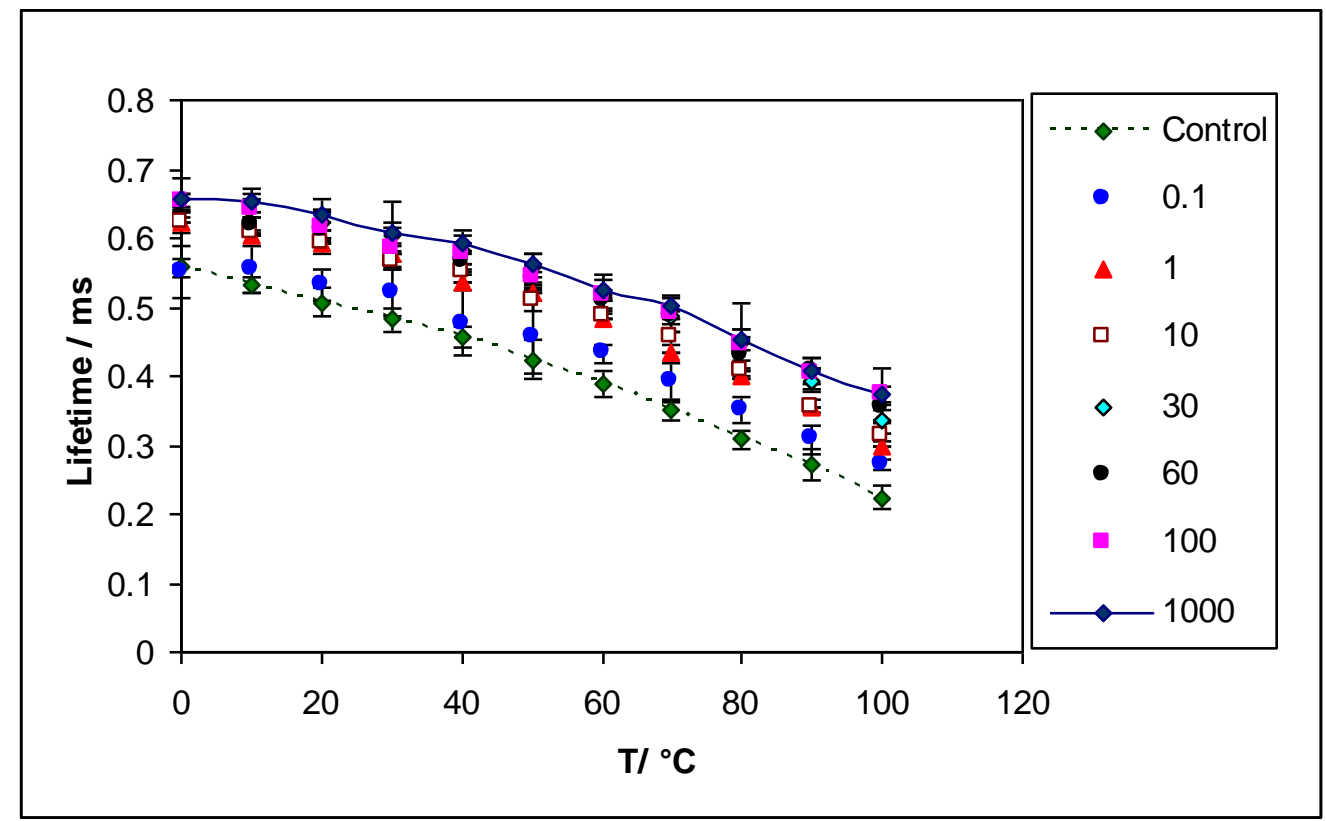

Figure III-5h: Comparison plot depicting the effect of temperature on the lifetime for Erythrosin B dispersed in $\beta-\mathrm{Lg}$ in various molar concentrations of trehalose equilibrated against nitrogen. 


\section{Figure III-5i}

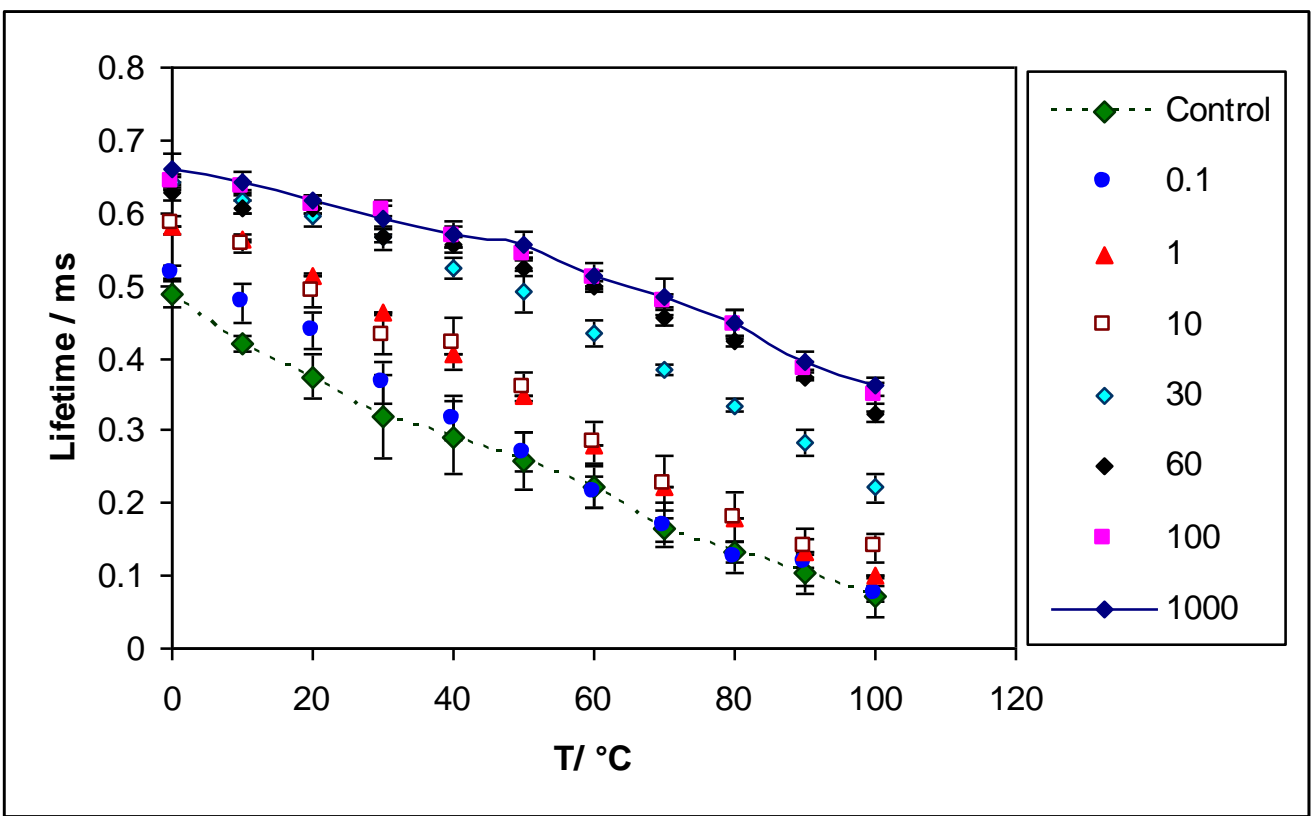

Figure III-5i: Comparison plot depicting the effect of temperature on the lifetime for Erythrosin B dispersed in $\beta$ - Lg in various molar concentrations of trehalose equilibrated against oxygen. 
Figure III-6a

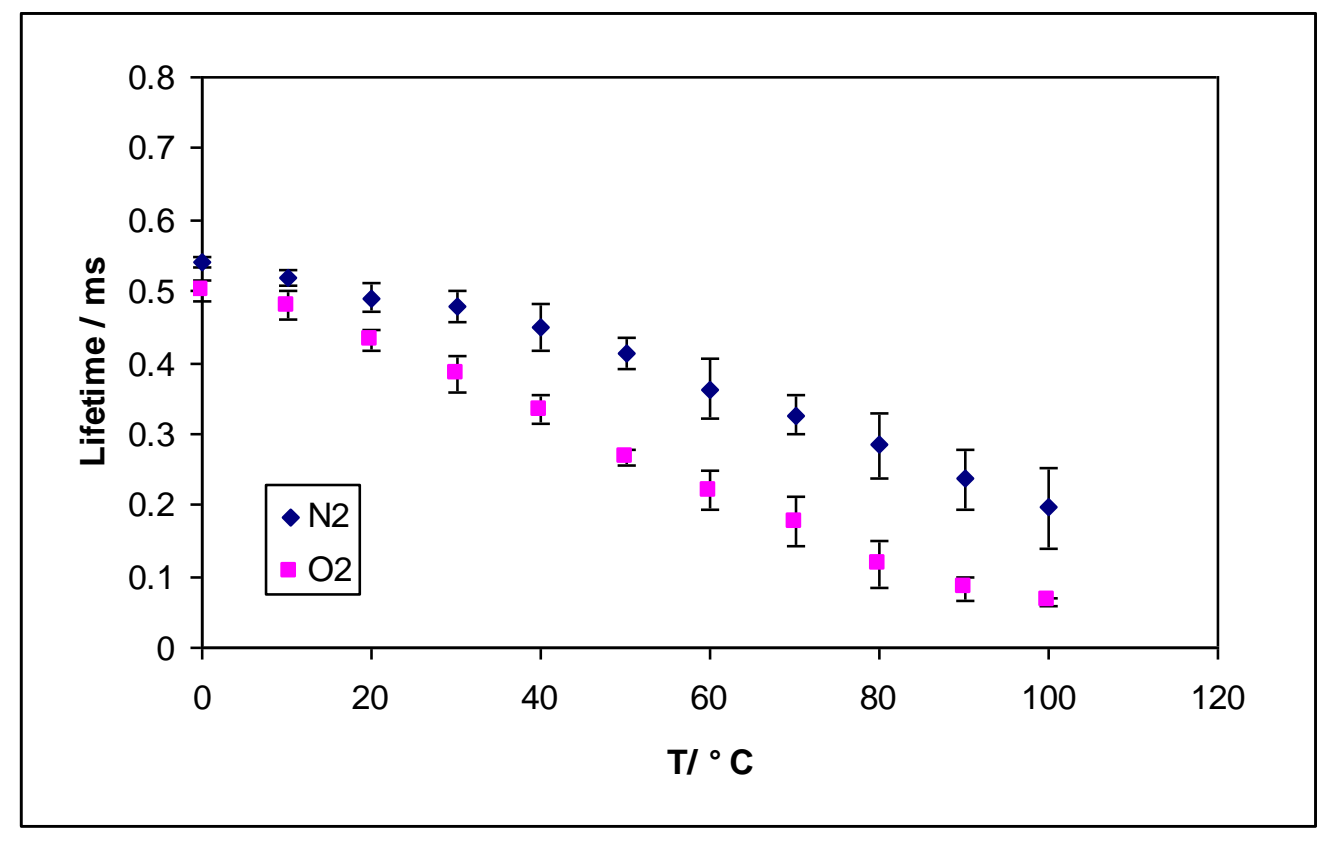

Figure III-6b

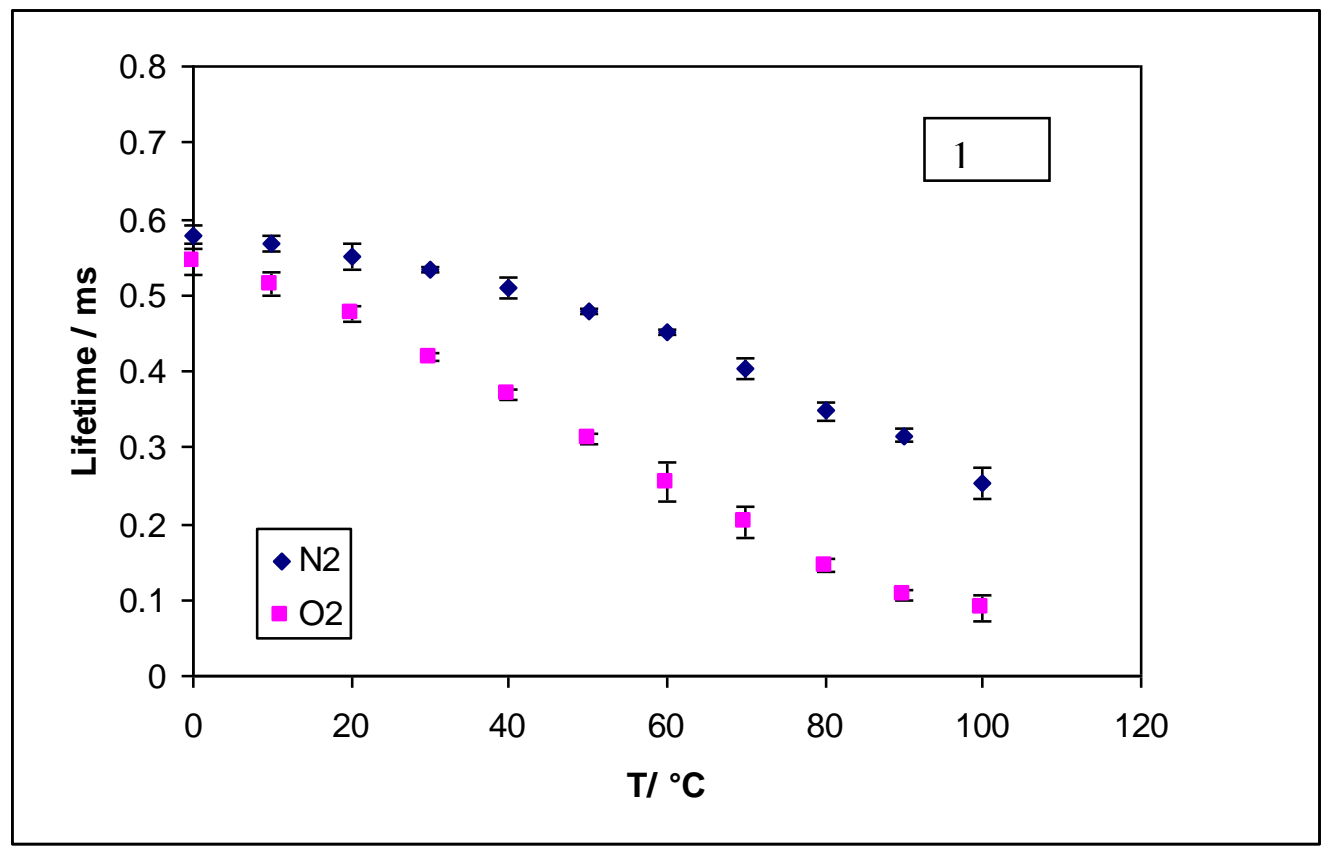


Figure III-6c

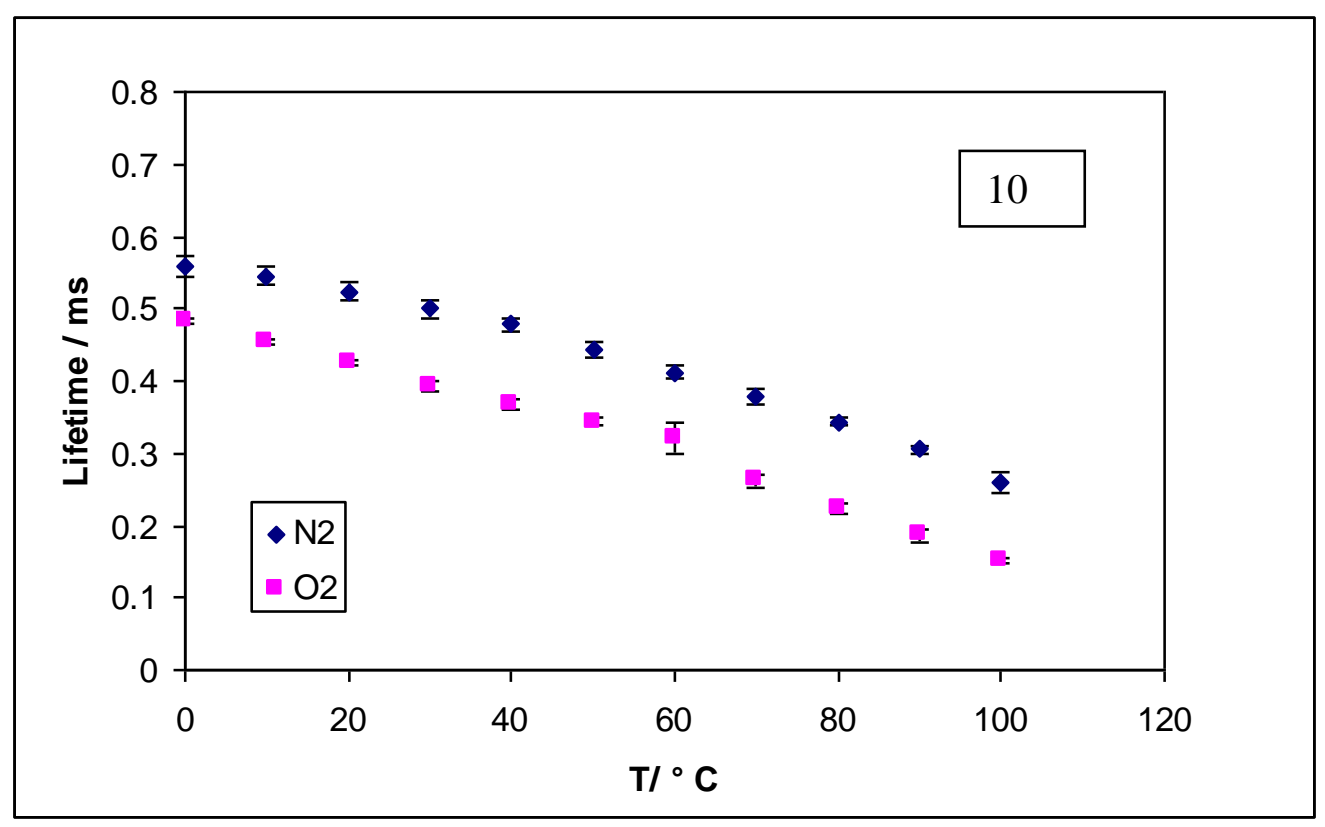

Figure III-6d

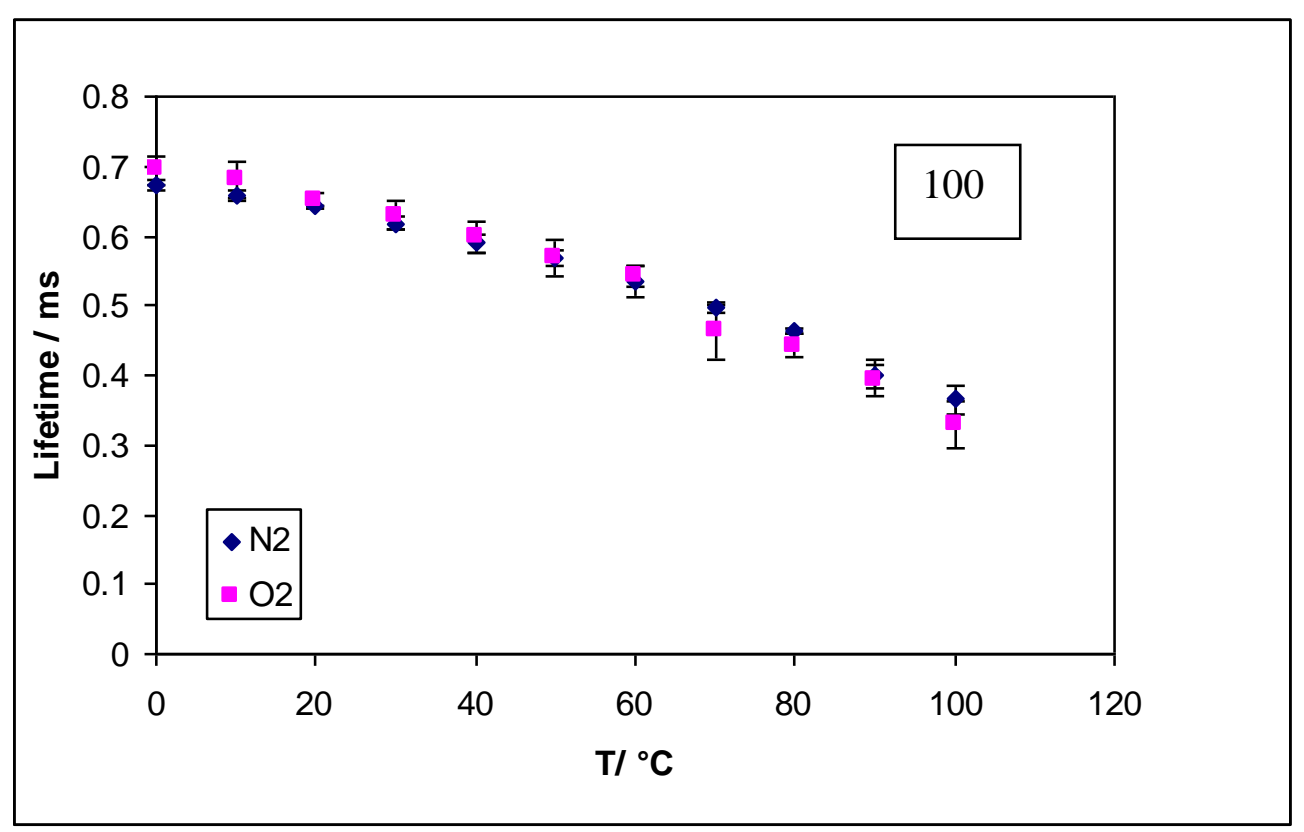




\section{Figure III-6e}

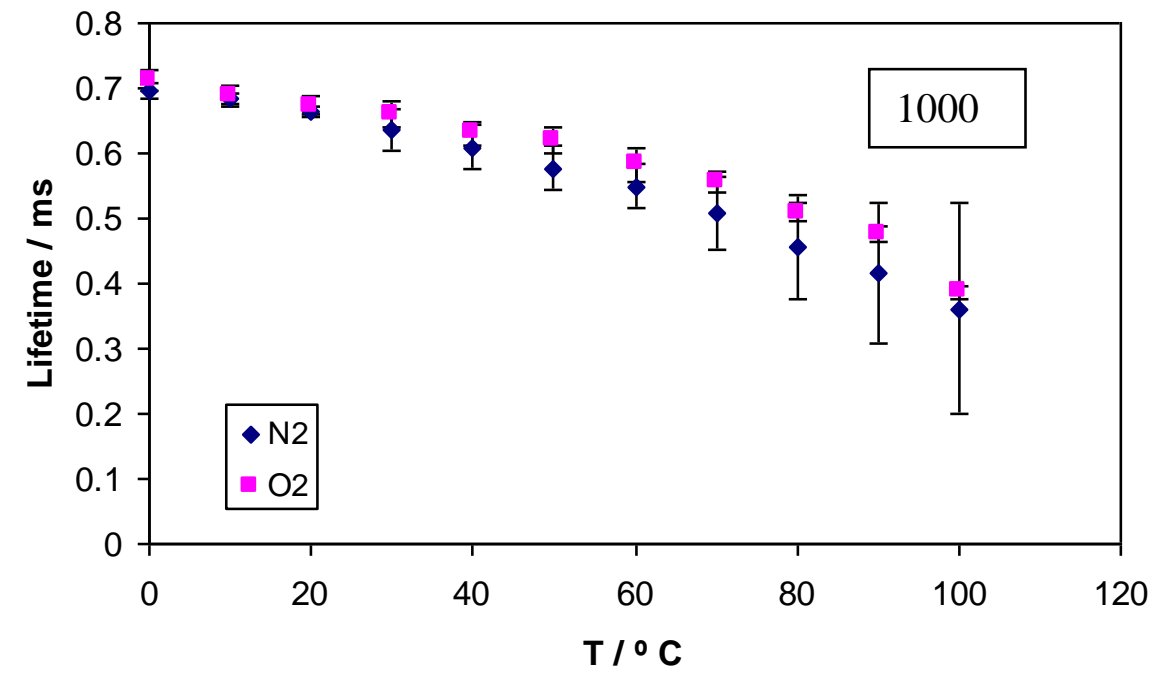

Figure III- $6(a, b, c, d, e)$ : The effect of temperature on the lifetime for Erythrosin B dispersed in $\beta$-Lg in various molar concentrations of maltose equilibrated against nitrogen and air. The lifetime was determined from analysis using a stretched exponential decay function. The text box in the graph refers to the molar ratio of maltose: $\beta-\mathrm{Lg}$.

Figure III-6f

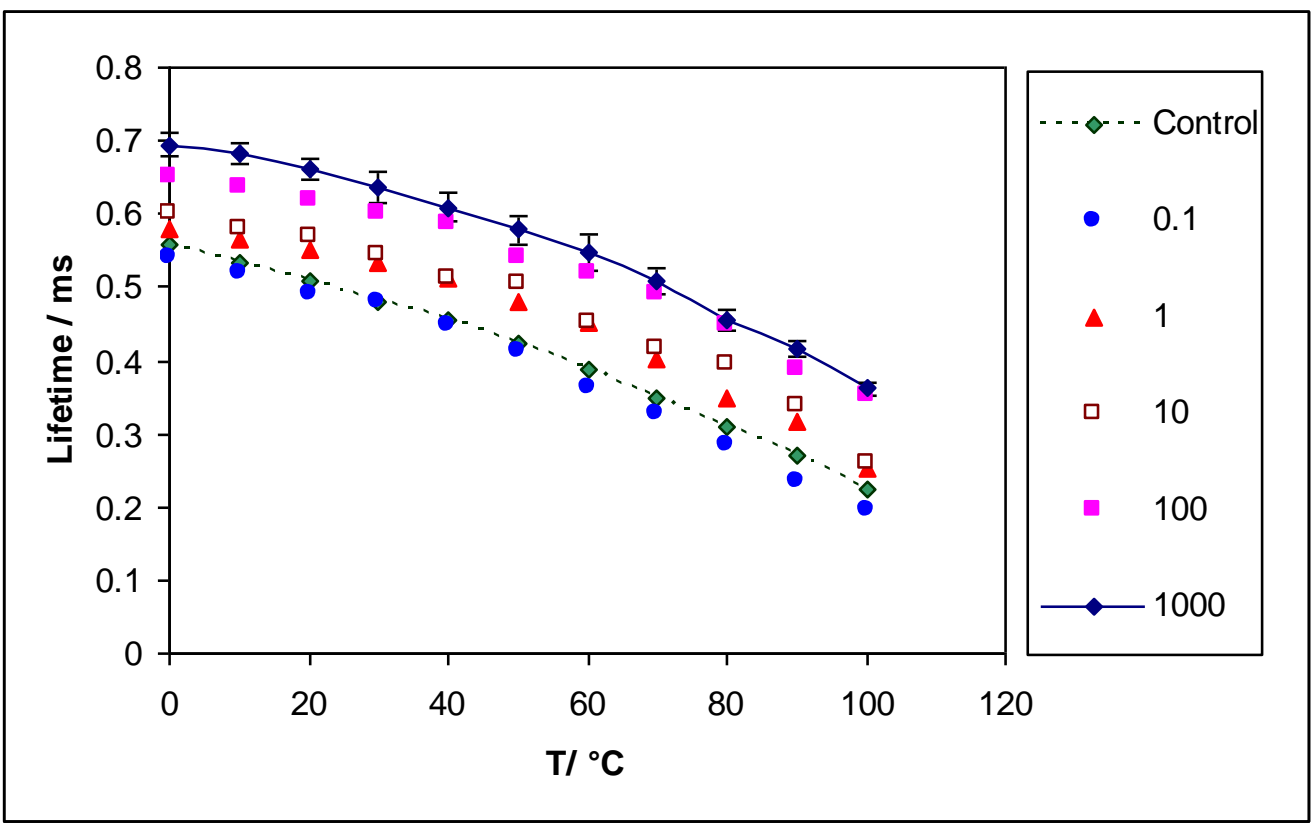

Figure III-6f: Comparison plot depicting the effect of temperature on the lifetime for Erythrosin $\mathrm{B}$ dispersed in $\beta-\mathrm{Lg}$ in various molar concentrations of maltose equilibrated against nitrogen 
Figure III-6g

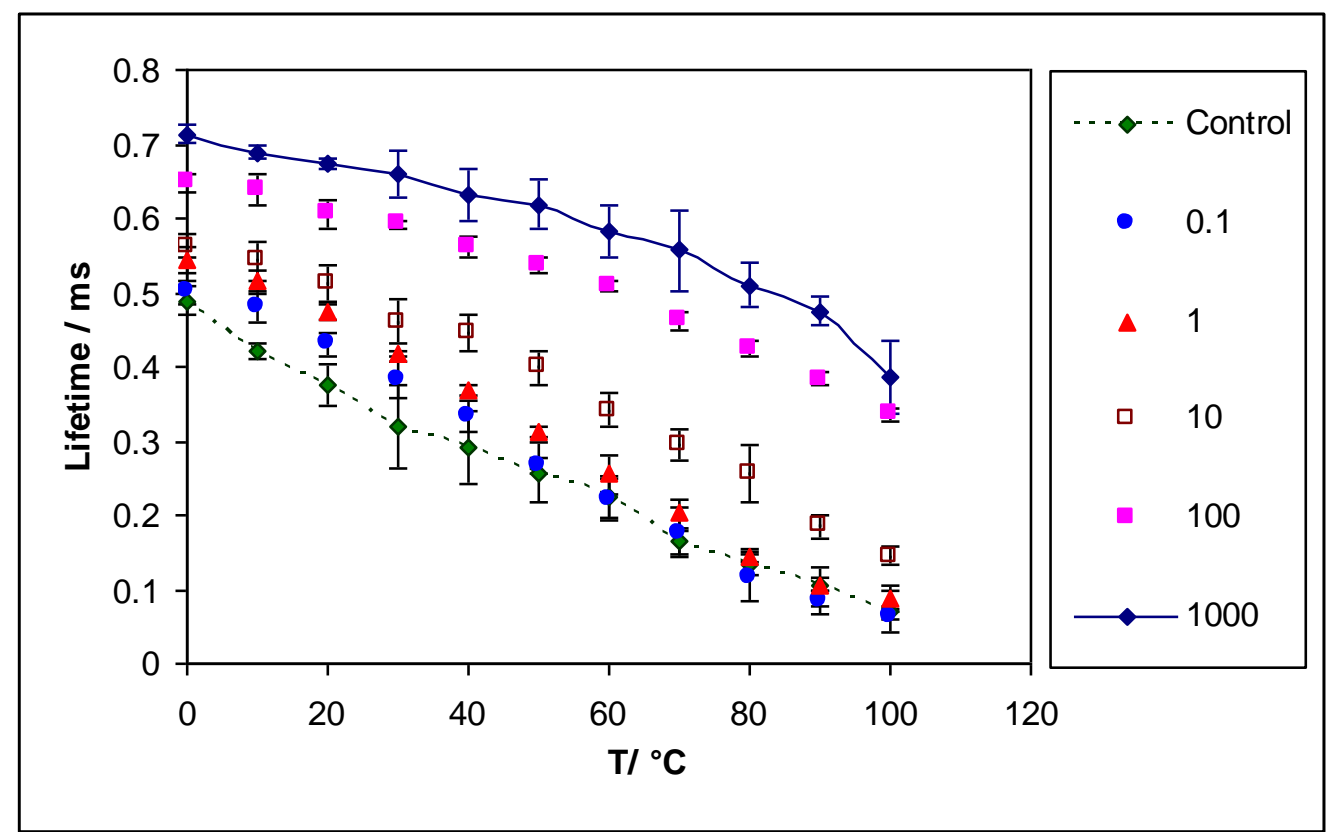

Figure III-6g: Comparison plot depicting the effect of temperature on the lifetime for Erythrosin B dispersed in $\beta-\mathrm{Lg}$ in various molar concentrations of maltose equilibrated against air. 
Figure III-7a

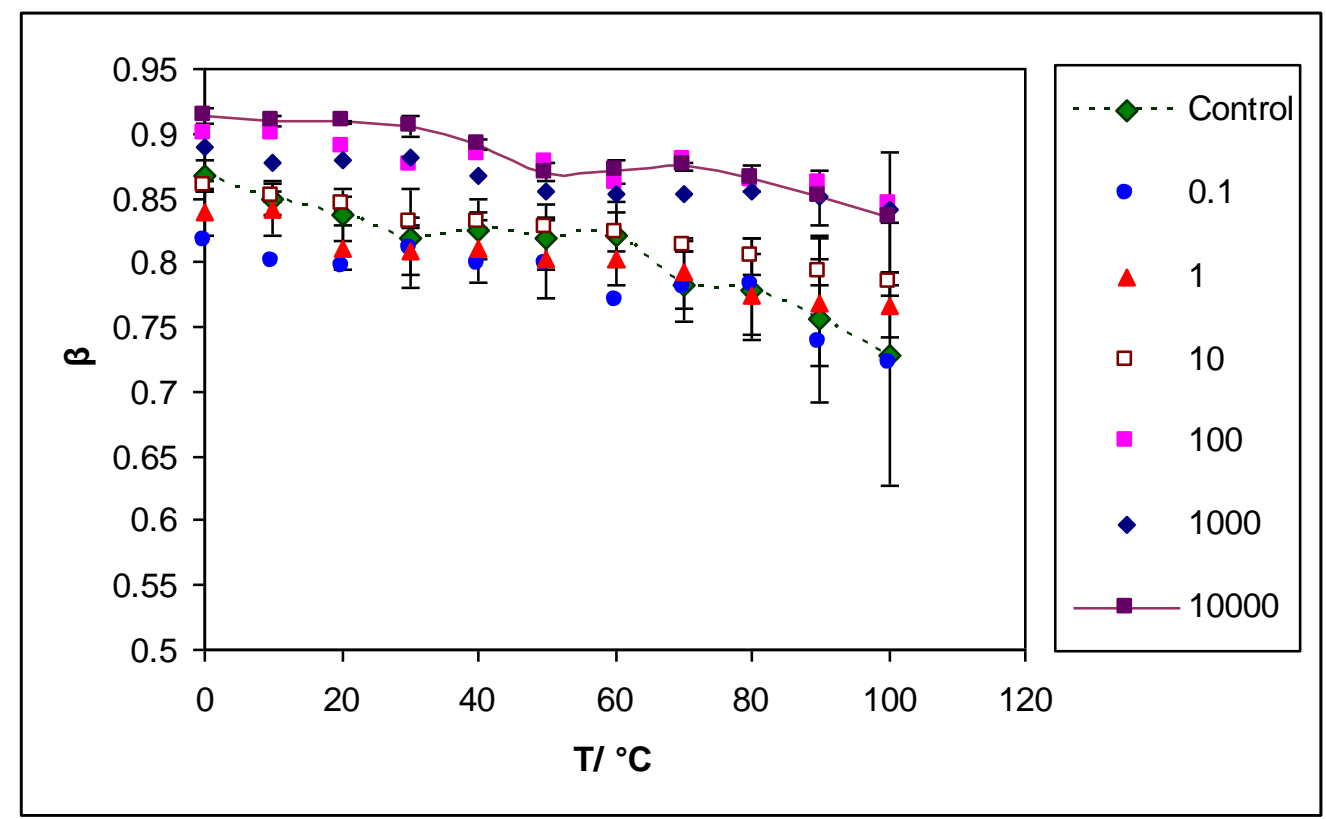

Figure III-7a: The effect of temperature on the stretching exponent $\beta$ for Erythrosin B dispersed in sucrose $\beta$-Lg films equilibrated against nitrogen. $\beta$ was determined from analysis using a stretched exponential decay function.

Figure III-7b

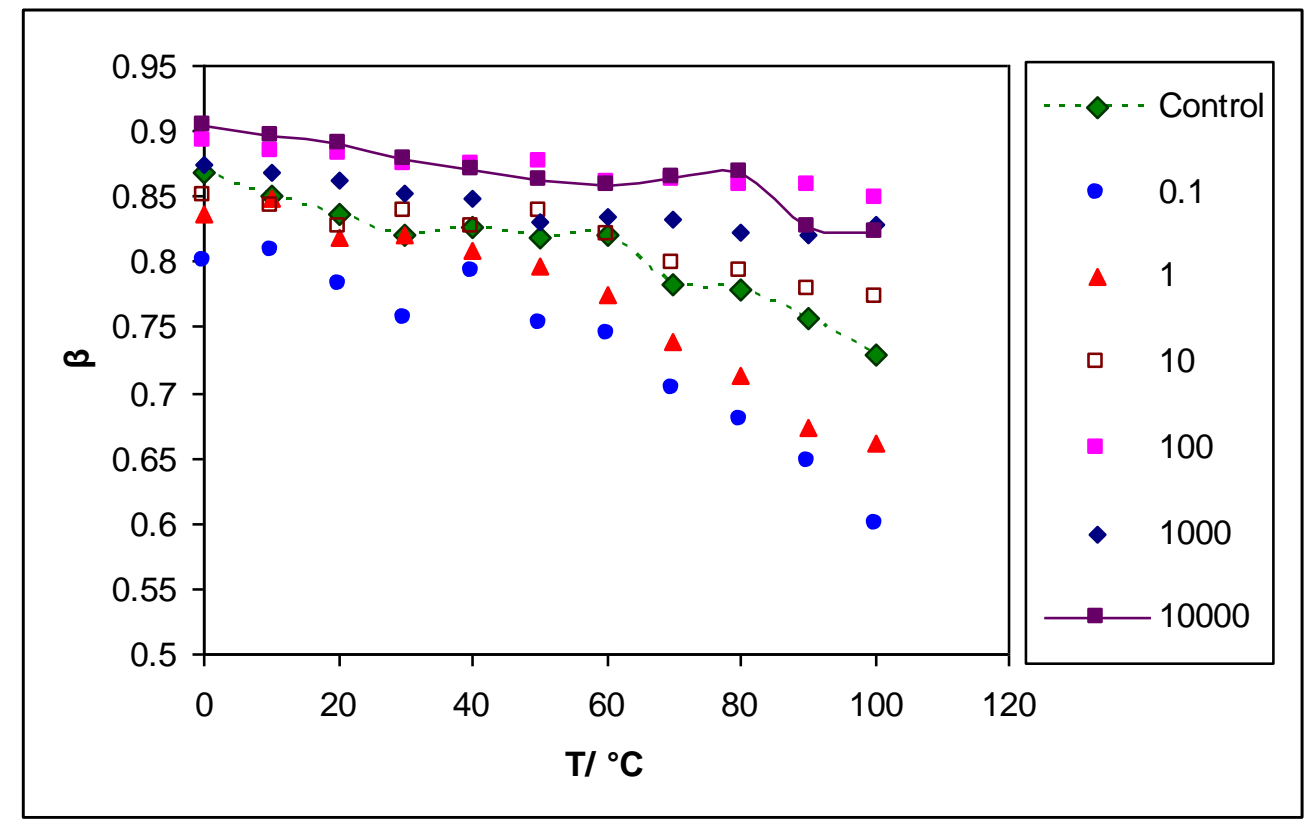

Figure III-7b: The effect of temperature on the stretching exponent $\beta$ for Erythrosin B dispersed in sucrose $\beta$-Lg films equilibrated against air. 
Figure III-8a

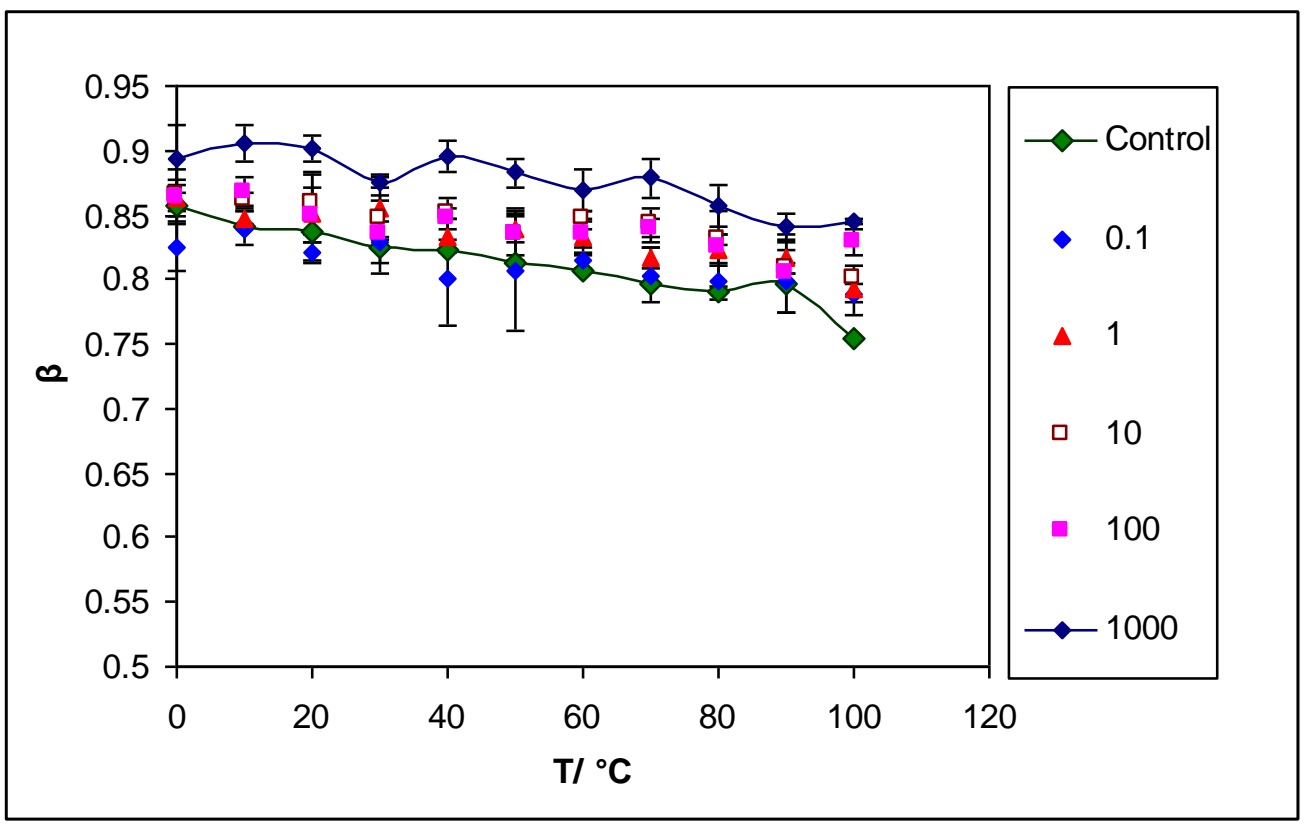

Figure III- $8 \mathrm{a}$ : The effect of temperature on the stretching exponent $\beta$ for Erythrosin B dispersed in trehalose/ $\beta-\mathrm{Lg}$ trehalose films equilibrated against nitrogen

Figure III-8b

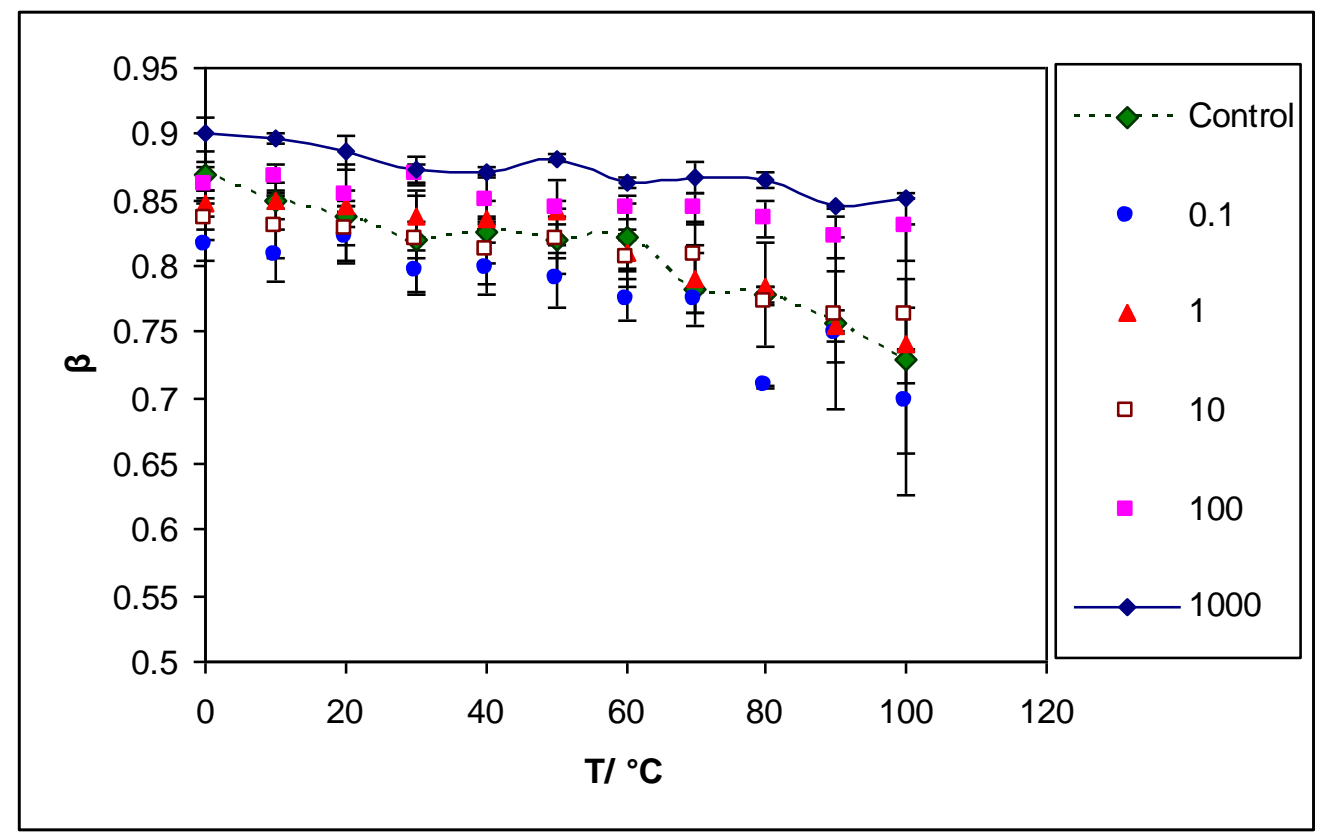

Figure III- $8 \mathrm{~b}$ : The effect of temperature on the stretching exponent $\beta$ for Erythrosin B dispersed in trehalose/ $\beta$-Lg films equilibrated against air. 
Figure III- $9 a$

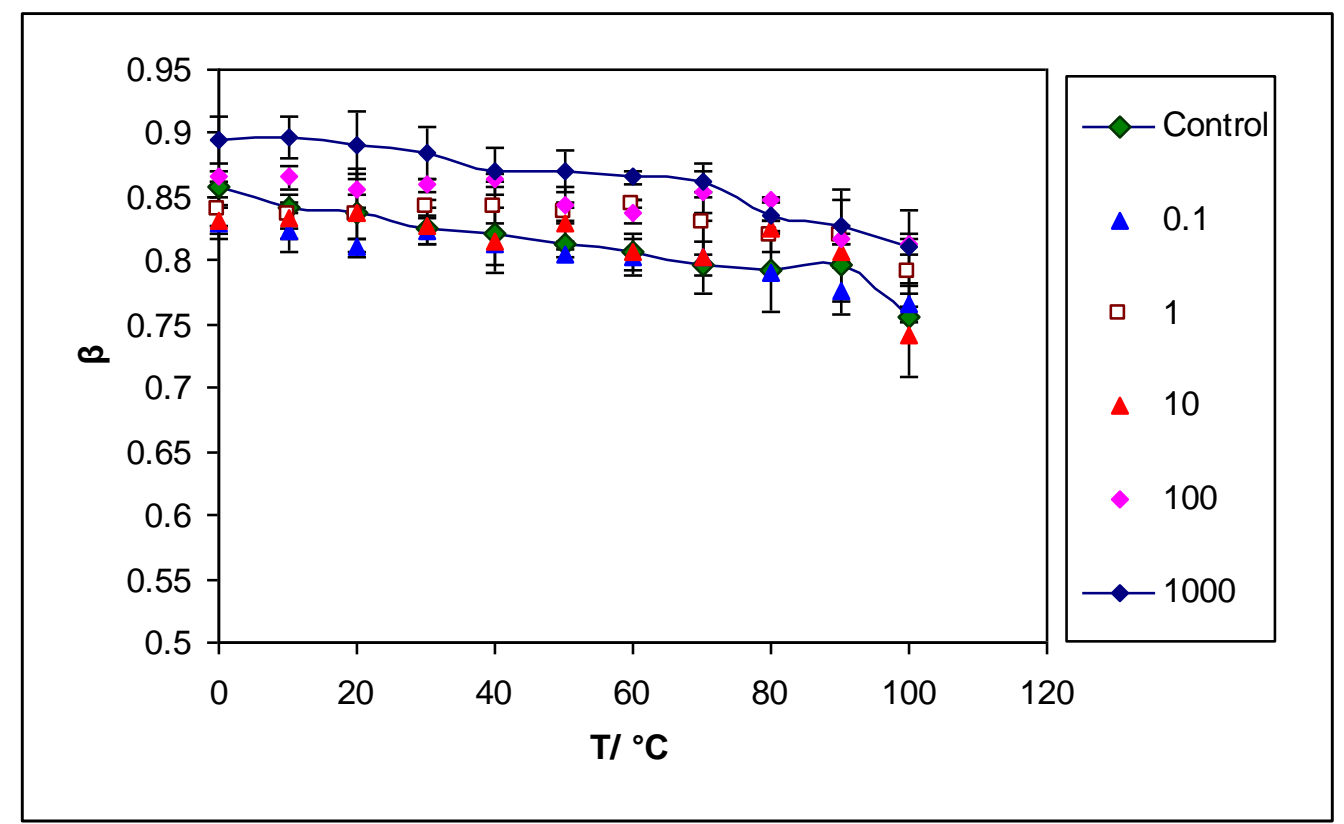

Figure III-9a: The effect of temperature on the stretching exponent $\beta$ for Erythrosin B dispersed in maltose/ $\beta$-Lg films equilibrated against nitrogen.

Figure III-9b

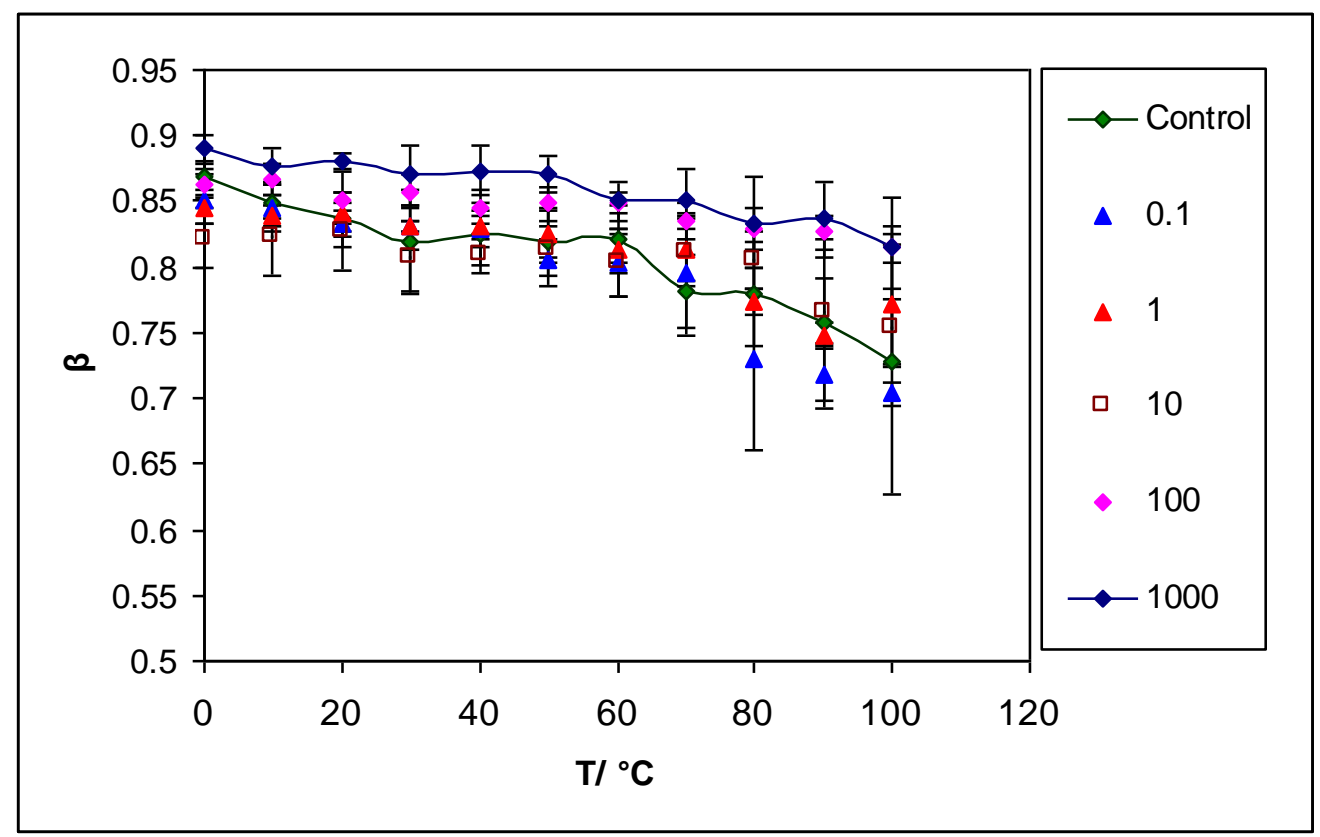

Figure III-9b: The effect of temperature on the stretching exponent $\beta$ for Erythrosin $\mathrm{B}$ dispersed in maltose $\beta$-Lg films equilibrated against nitrogen. 
Figure III-10a

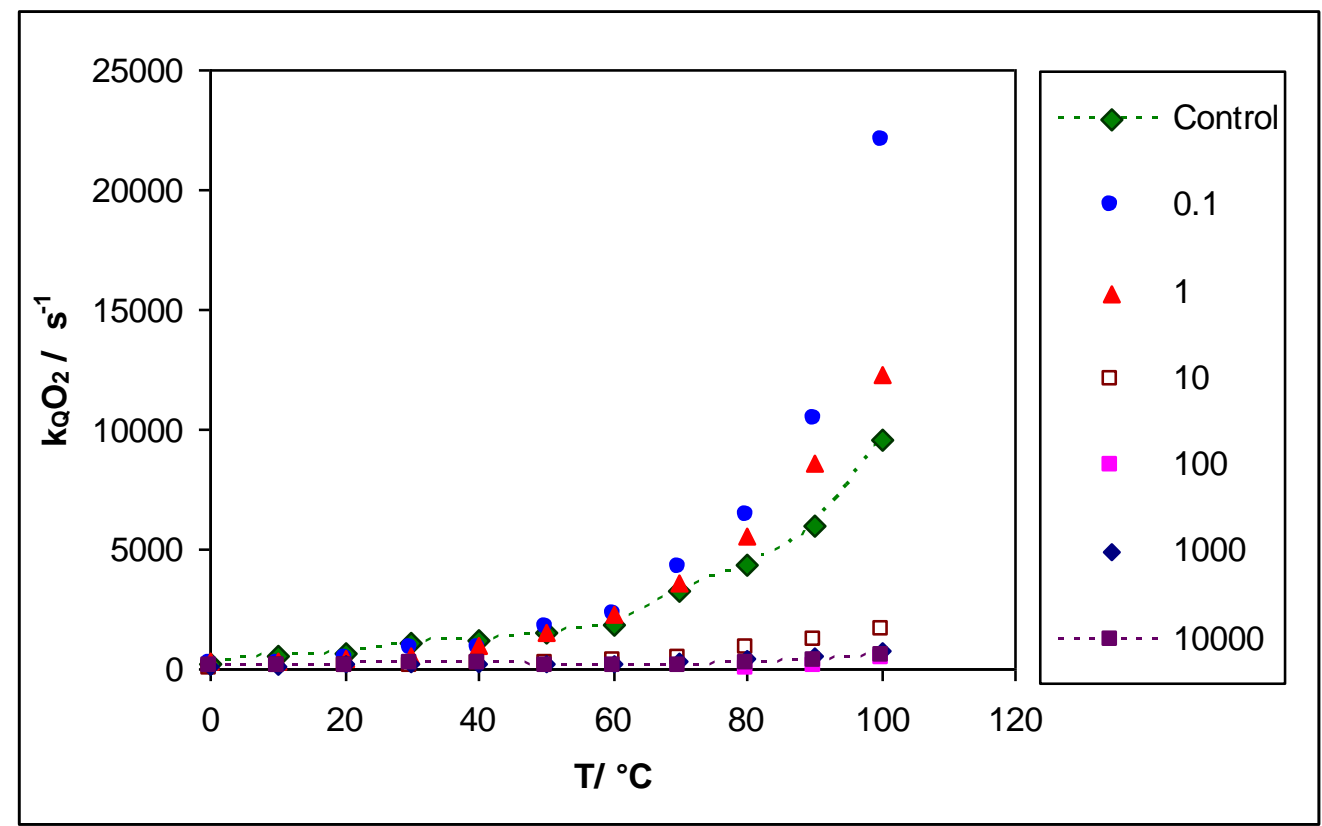

Figure III-10a: The oxygen quenching rate $\mathrm{k}_{\mathrm{Q}}\left[\mathrm{O}_{2}\right]$ as a function of temperature in the $\beta$-Lg and sucrose/ $\beta$-Lg films.

Figure III-10b

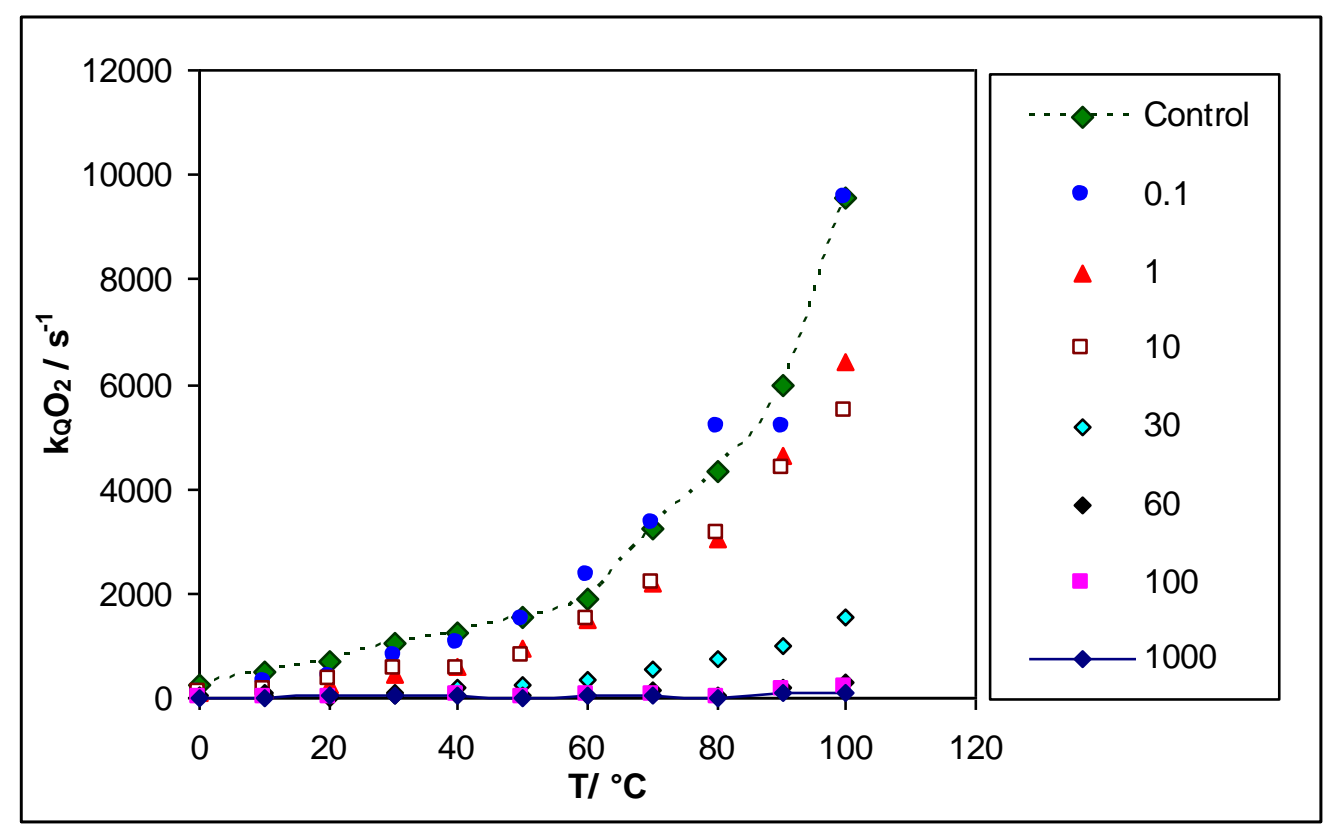

Figure III-10b: The oxygen quenching rate $\mathrm{k}_{\mathrm{Q}}\left[\mathrm{O}_{2}\right]$ as a function of temperature in the $\beta$-Lg and trehalose/ $\beta$ - $\mathrm{Lg}$ films 
Figure III-10c

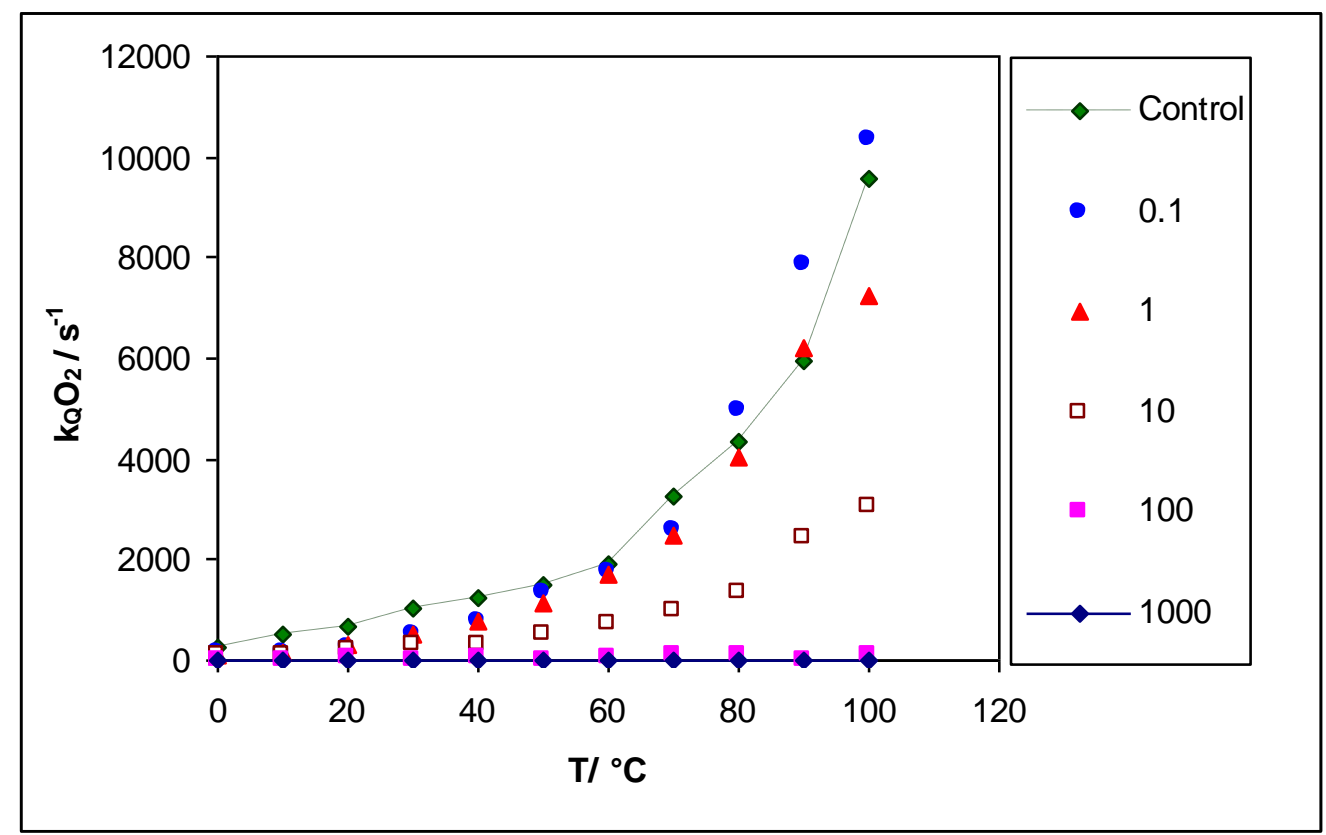

Figure III-10c: The oxygen quenching rate $\mathrm{k}_{\mathrm{Q}}\left[\mathrm{O}_{2}\right]$ as a function of temperature in the $\beta$-Lg and maltose/ $\beta$-Lg mixtures. 
Figure III-11a

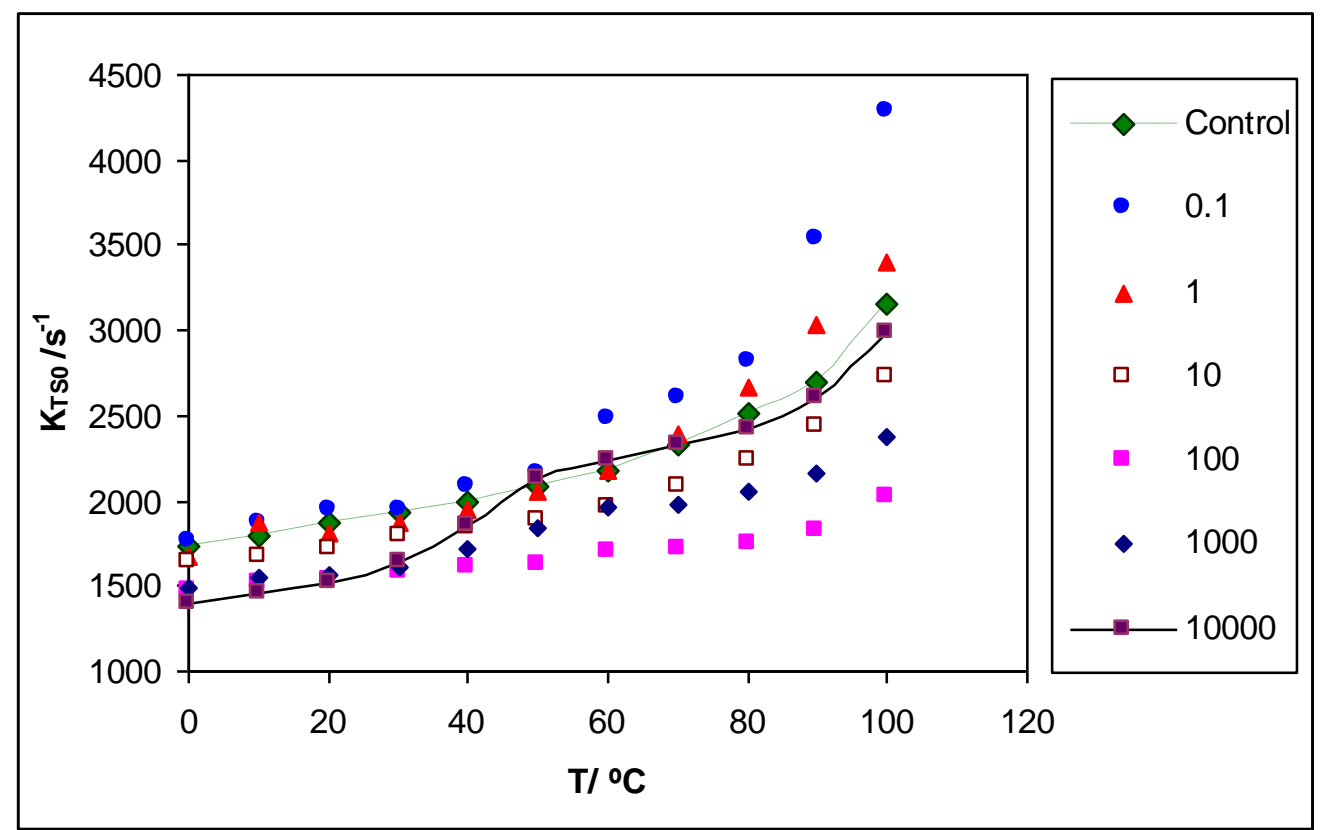

Figure III-11a: Plot of the effect of temperature on non-radiative decay from $\mathrm{T}_{1}$ to $\mathrm{S}_{0}\left(\mathrm{k}_{\mathrm{TS} 0}\right)$ in amorphous sucrose/ $\beta$-Lg films.

Figure III-11b

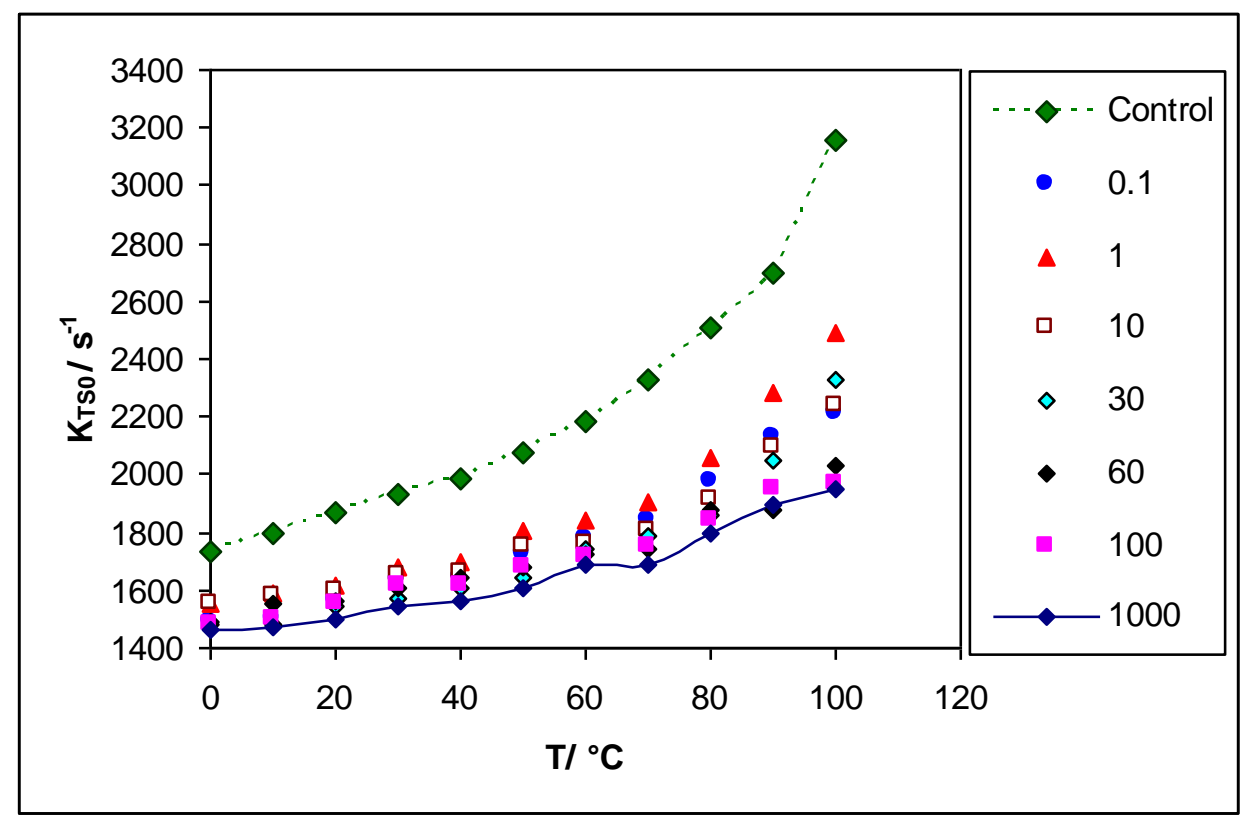

Figure III-11b: Plot of the effect of temperature on non-radiative decay from $\mathrm{T}_{1}$ to $\mathrm{S}_{0}\left(\mathrm{k}_{\mathrm{TS} 0}\right)$ in amorphous trehalose/ $\beta$-Lg films. 
Figure III-11c

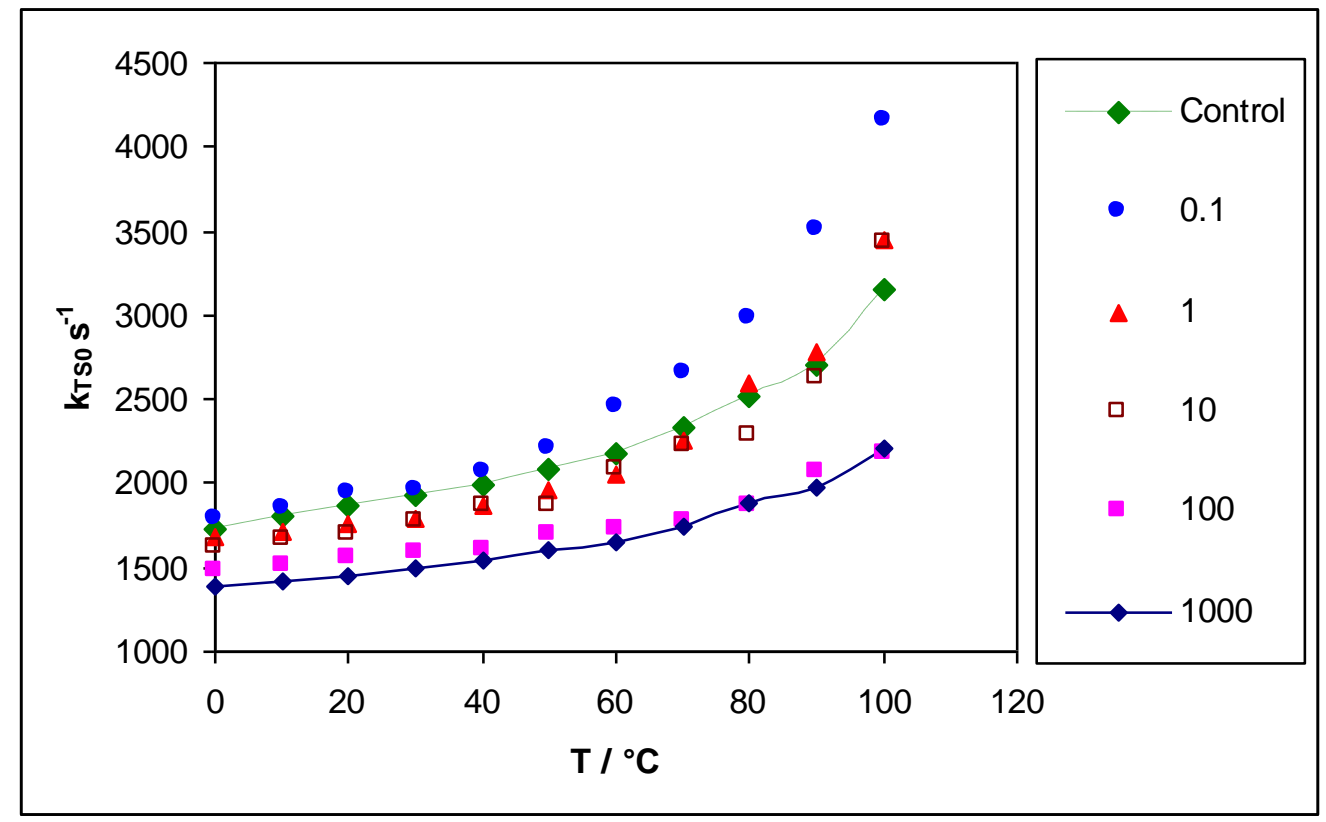

Figure III-11c: Plot of the effect of temperature on non-radiative decay from $\mathrm{T}_{1}$ to $\mathrm{S}_{0}\left(\mathrm{k}_{\mathrm{TS} 0}\right)$ in amorphous maltose/ $\beta$-Lg maltose films. 


\section{Figure III-12a}

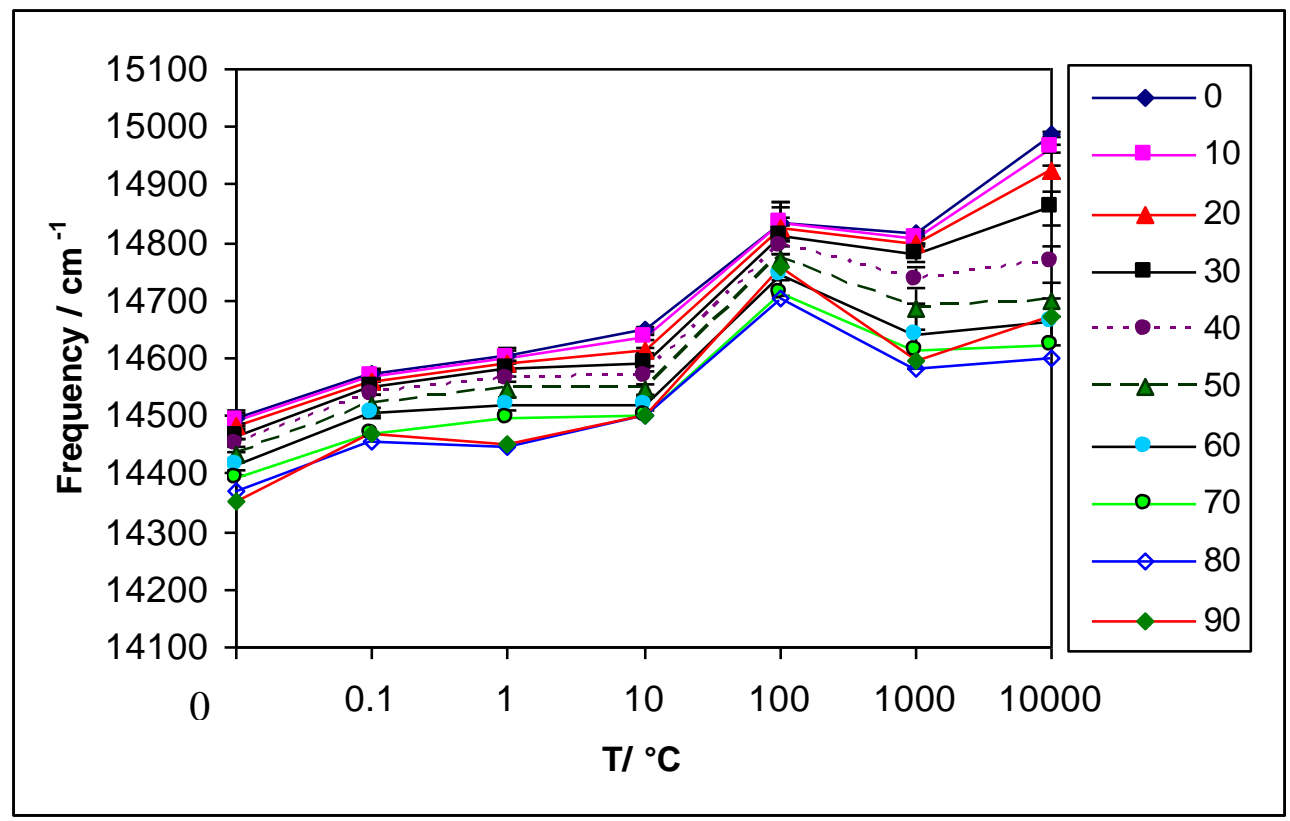

Figure III-12a: Emission energy as a function of concentration of sucrose at various temperatures in $\beta$-Lg films. Data from Figure III-2a replotted versus molar ratio.

Figure III-12b

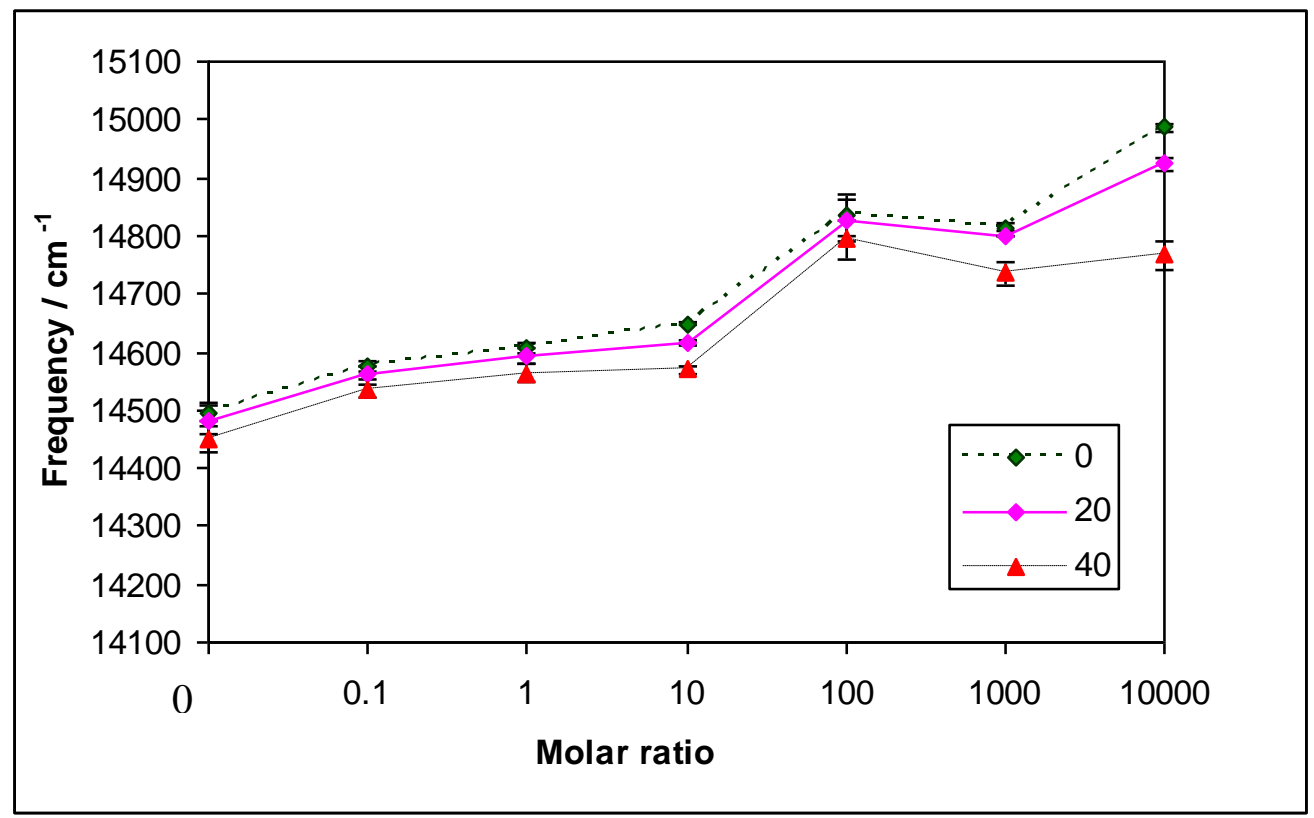

Figure III-12b: Zoom in effect of emission energy as a function of concentration of sucrose at 0,20 and $40^{\circ} \mathrm{C}$. Data from Figure III-2a replotted versus molar ratio. 


\section{Figure III-13a}

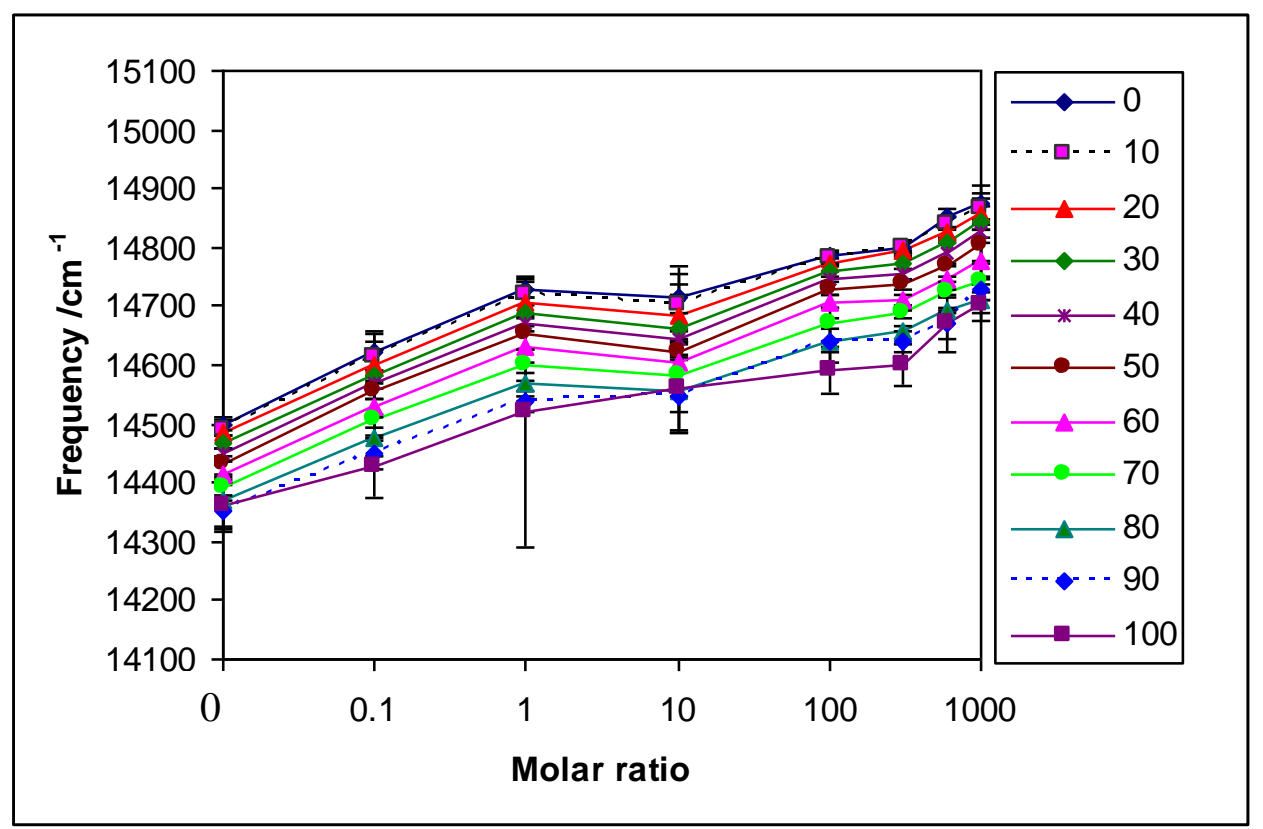

Figure III-13a: A plot of the effect of change in concentration of trehalose in emission energy at various temperatures. Data from Figure III-2b replotted versus molar ratio.

\section{Figure III-13b}

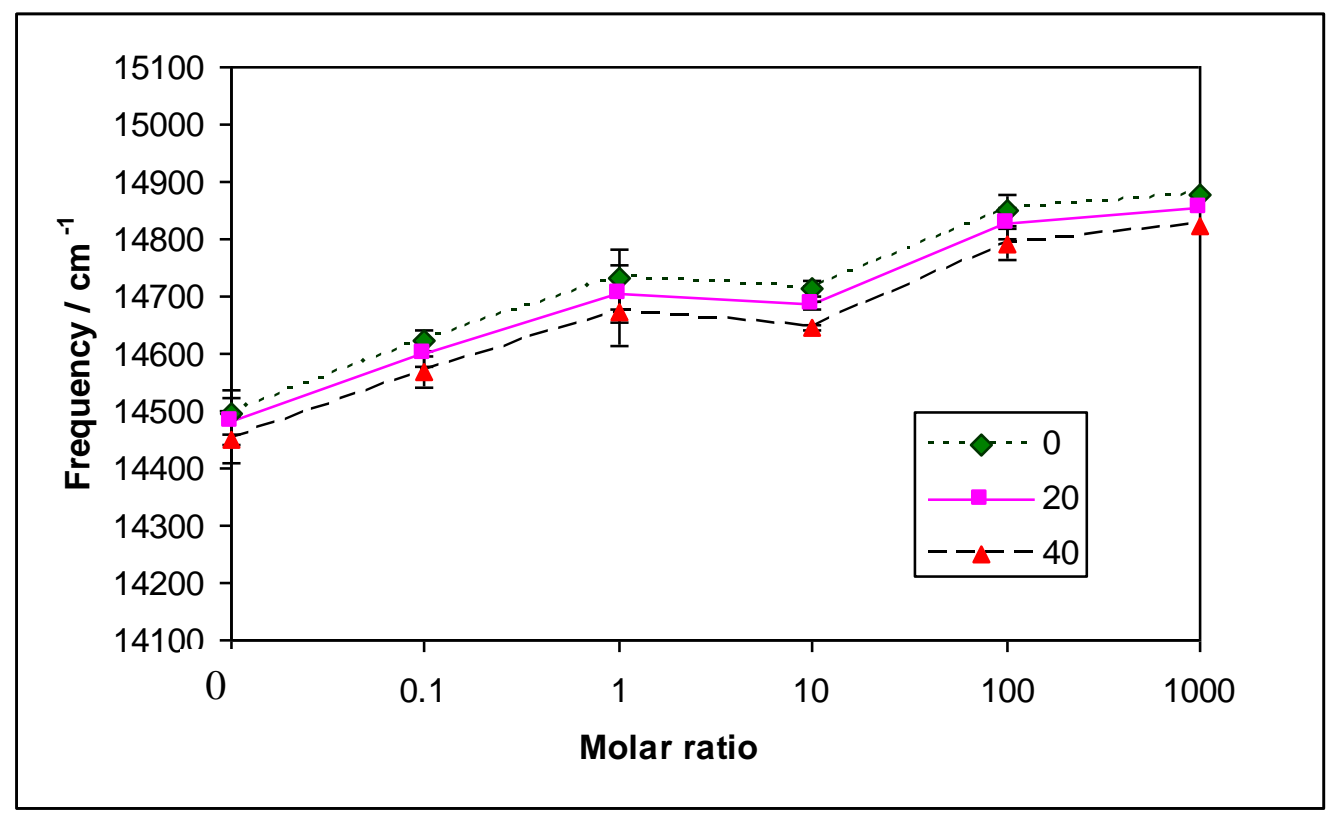

Figure III-13b: Zoom in effect of the change in concentration of trehalose in emission energy at 0,20 and $40^{\circ} \mathrm{C}$. Data from Figure III-2b replotted versus molar ratio. 
Figure III-14a

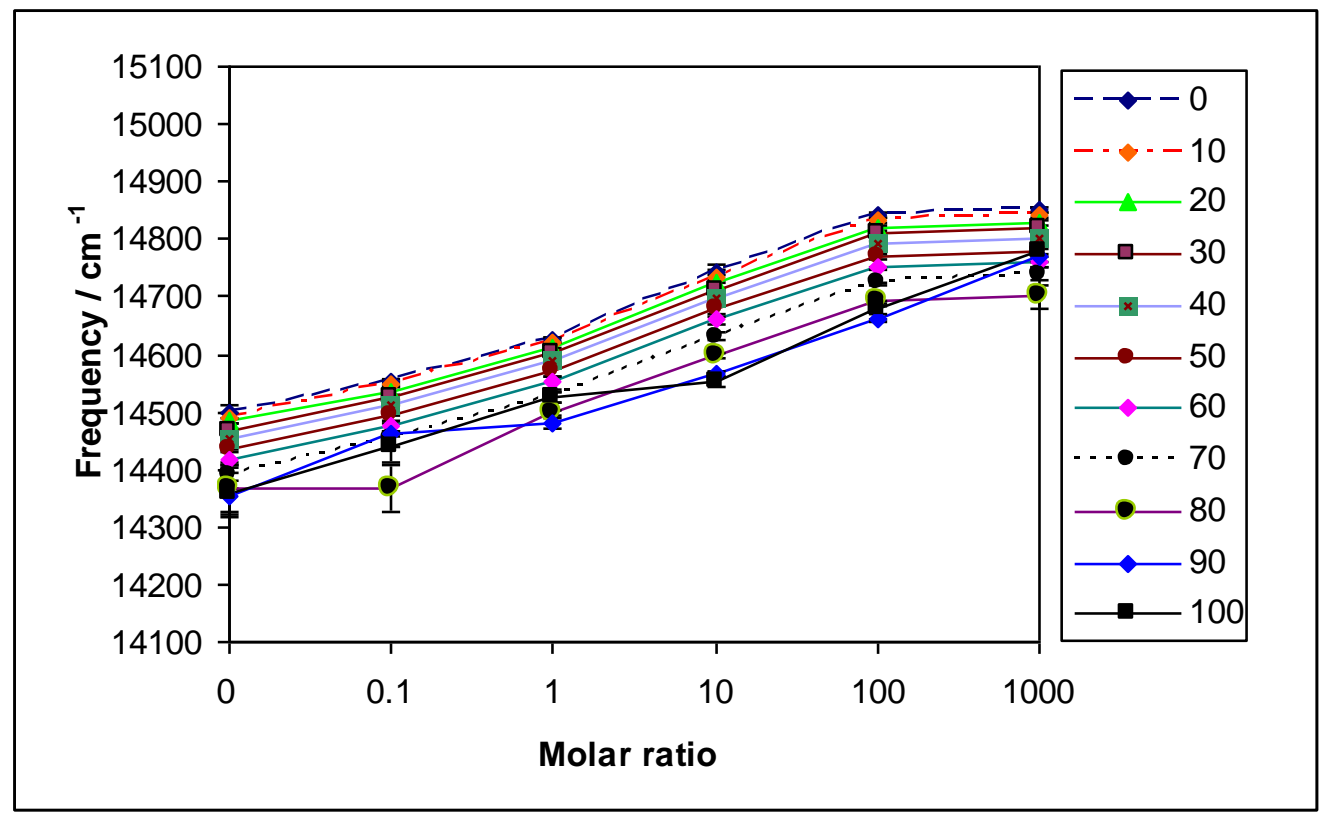

Figure III-14a: A plot of the effect of change in concentration of maltose in emission energy at various temperatures. Data replotted from Figure III-2c versus molar ratio.

Figure III-14b

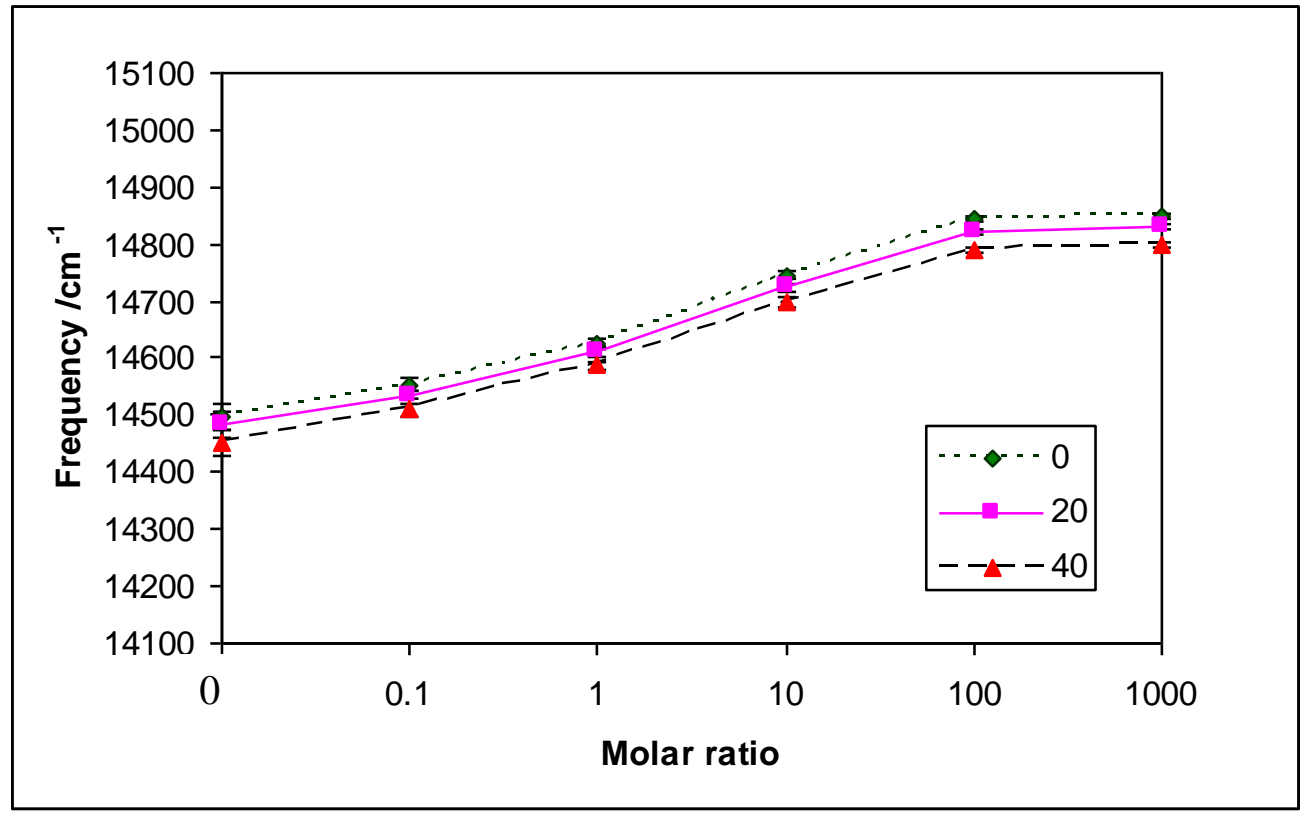

Figure III-14b: Zoom in effect of the change in concentration of maltose in emission energy at 0,20 and $40^{\circ} \mathrm{C}$. Data replotted from Figure III-2c versus molar ratio. 
Figure III-15a

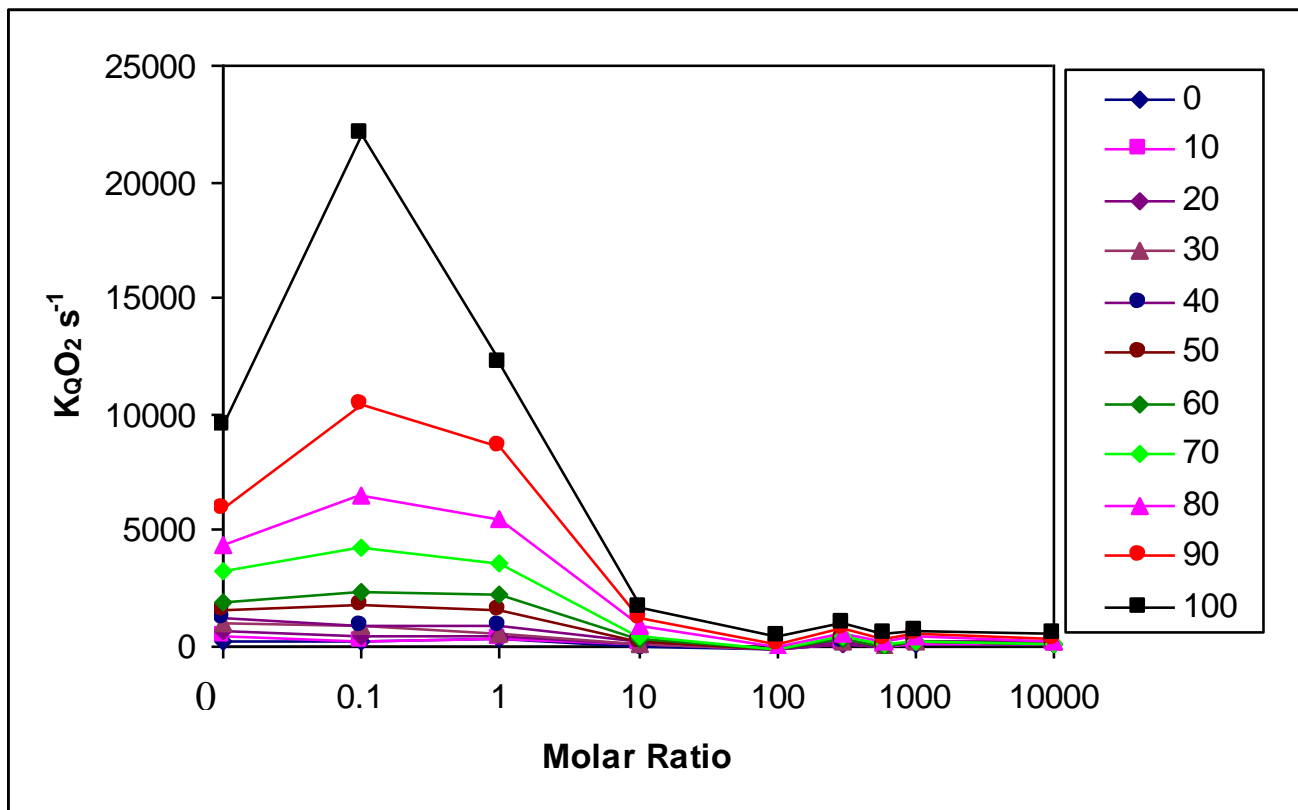

Figure III-15a: Effect of change in concentration of sucrose in $\mathrm{k}_{\mathrm{Q}}\left[\mathrm{O}_{2}\right]$ at various temperatures. Data replotted from Figure III-10a versus molar ratio.

Figure III-15b

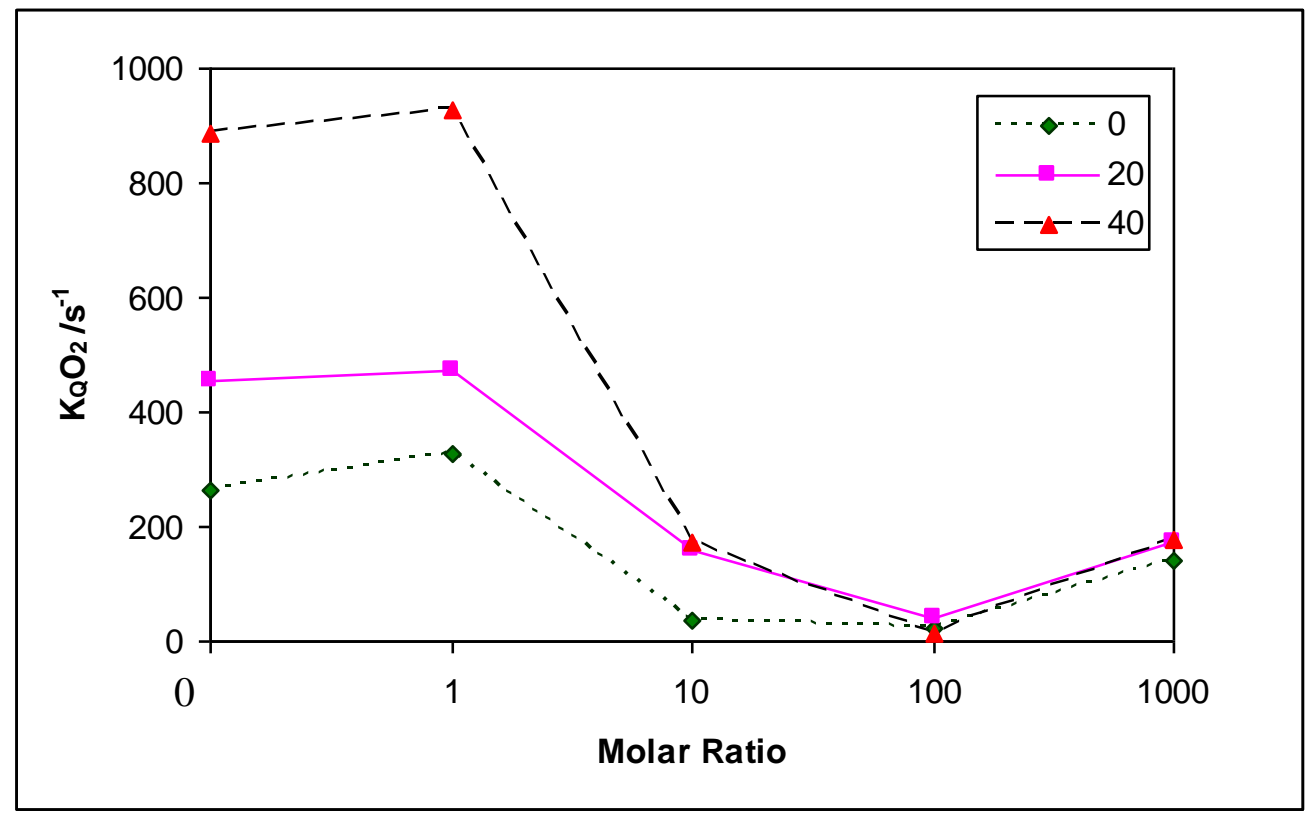

Figure III-15b: Zoom in effect of the change in concentration of sucrose in $\mathrm{k}_{\mathrm{Q}}\left[\mathrm{O}_{2}\right]$ at 0,20 and $40^{\circ}$ C. Data replotted from Figure III-10a versus molar ratio. 
Figure III-16a

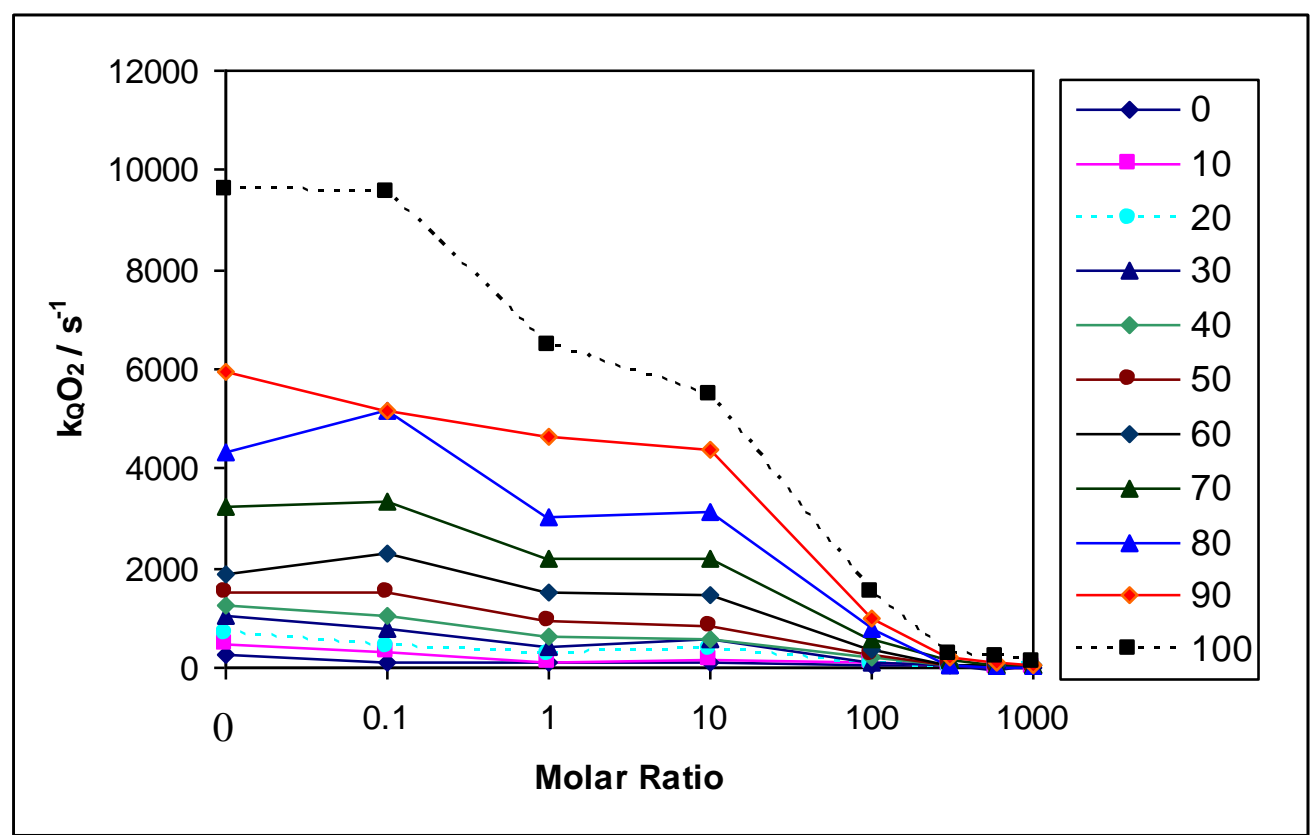

Figure III-16a: $\mathrm{k}_{\mathrm{Q}}\left[\mathrm{O}_{2}\right]$ as a function of concentration of trehalose at various temperatures. Data repotted from Figure III-10b.

Figure III-16b

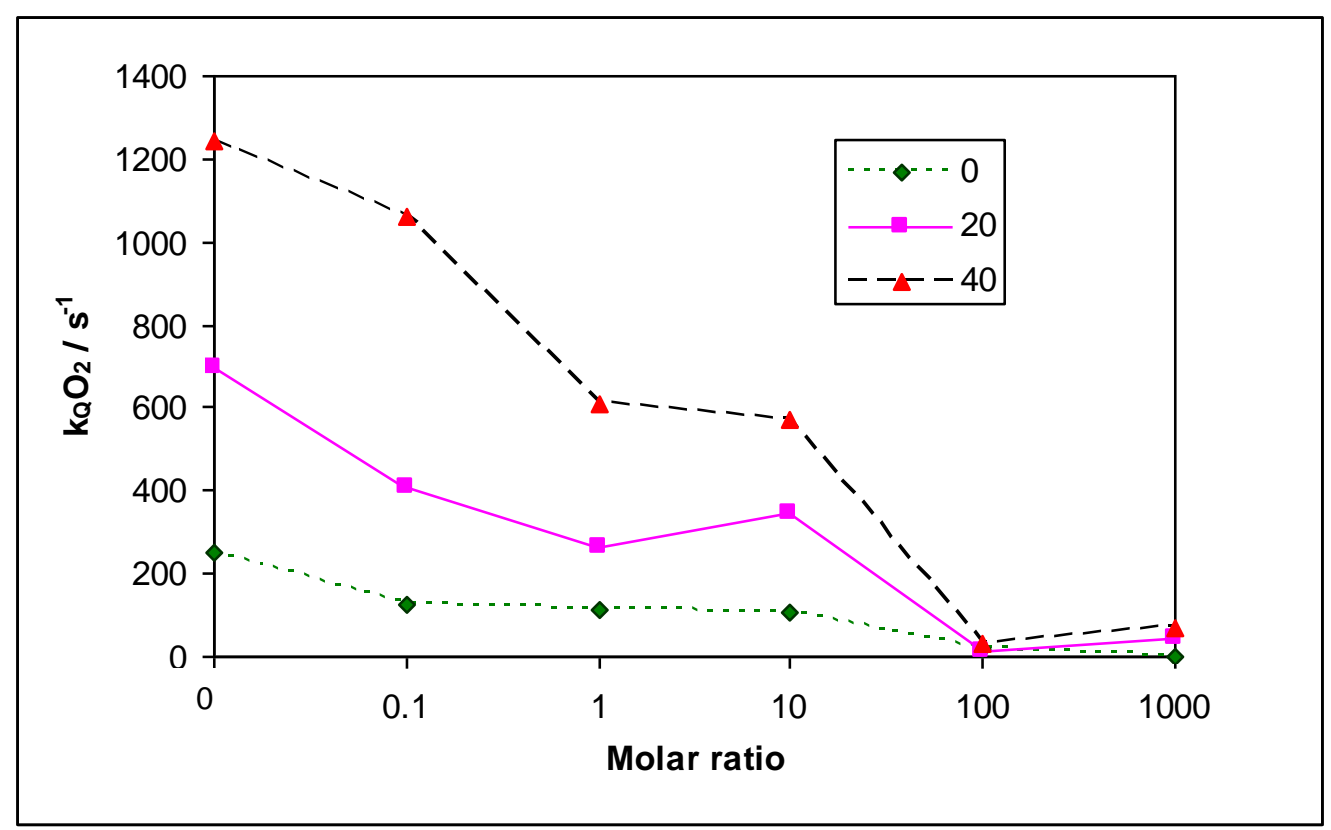

Figure III-16b: Zoom in effect of the change in concentration of trehalose in $\mathrm{k}_{\mathrm{Q}}\left[\mathrm{O}_{2}\right]$ at 0,20 and $40^{\circ} \mathrm{C}$. Data repotted from Figure III-10b. 
Figure III-17a

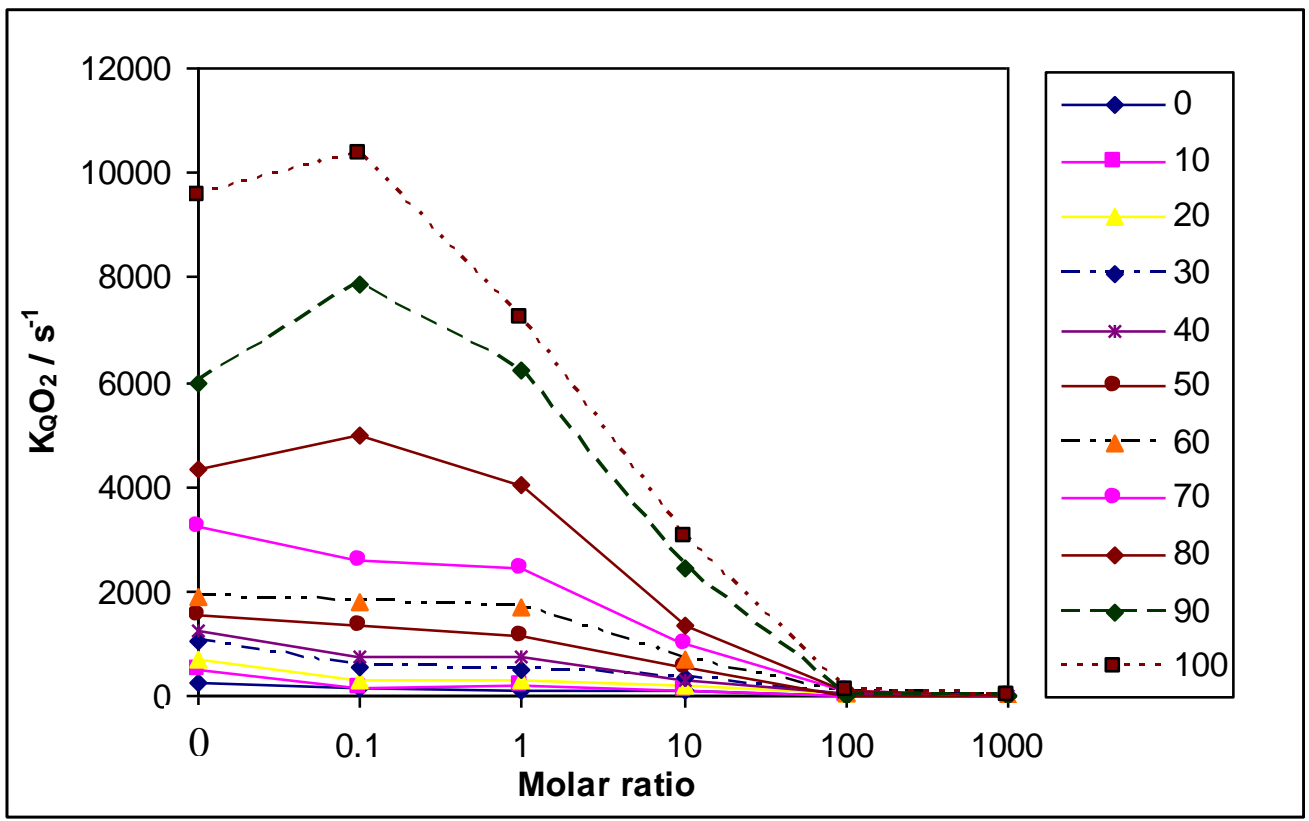

Figure III-17a: A plot of the effect of change in concentration of maltose in $\mathrm{k}_{\mathrm{Q}}\left[\mathrm{O}_{2}\right]$ at various temperatures. Data replotted from Figure 10c.

\section{Figure III-17b}

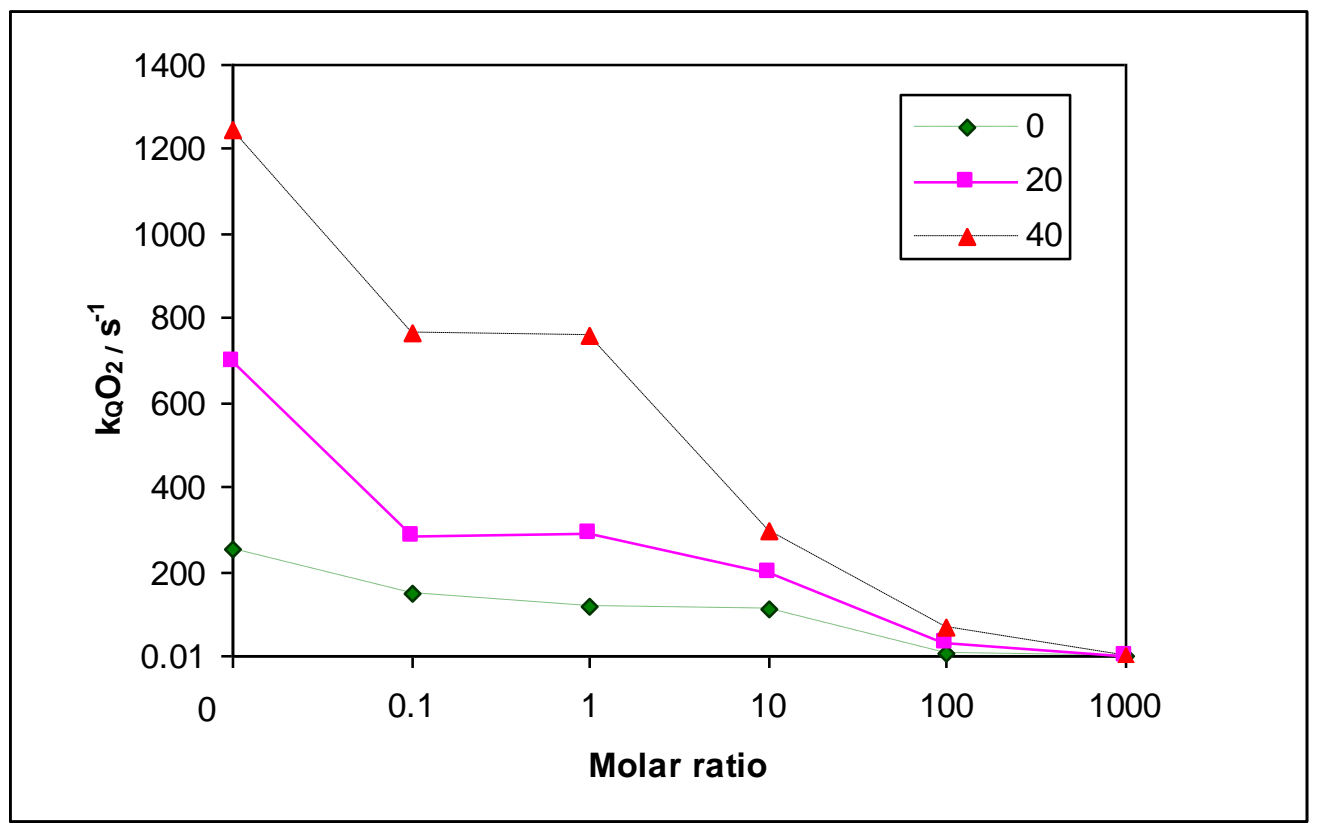

Figure III-17b: Zoom in effect of the change in concentration of maltose in $\mathrm{k}_{\mathrm{Q}}\left[\mathrm{O}_{2}\right]$ at 0,20 and $40^{\circ}$ C. Data repotted from Figure III-10c. 
Figure III-18a

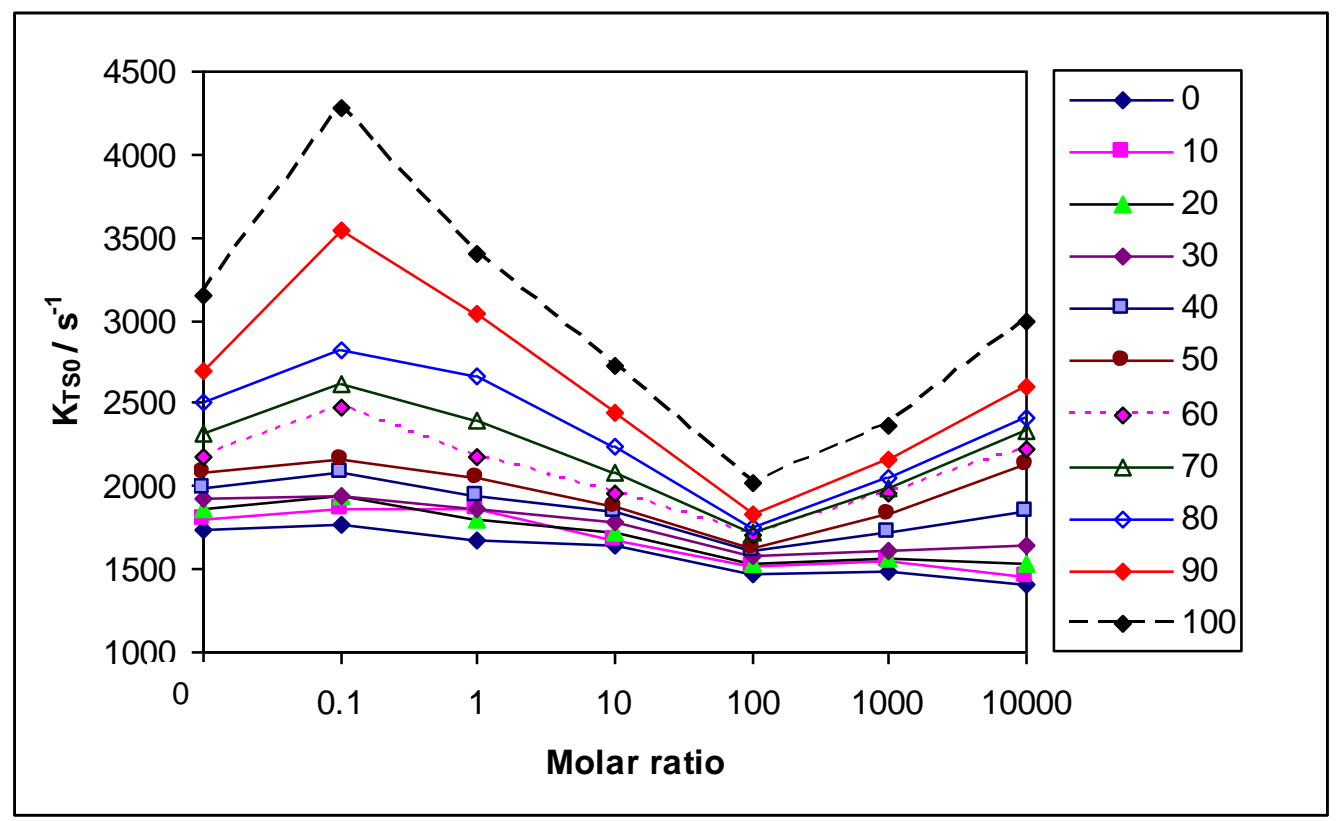

Figure III-18a: Effect of change in concentration of sucrose in $\mathrm{k}_{\mathrm{Ts} 0}$ at various temperatures. Data replotted from Figure III-11a.

Figure III-18b

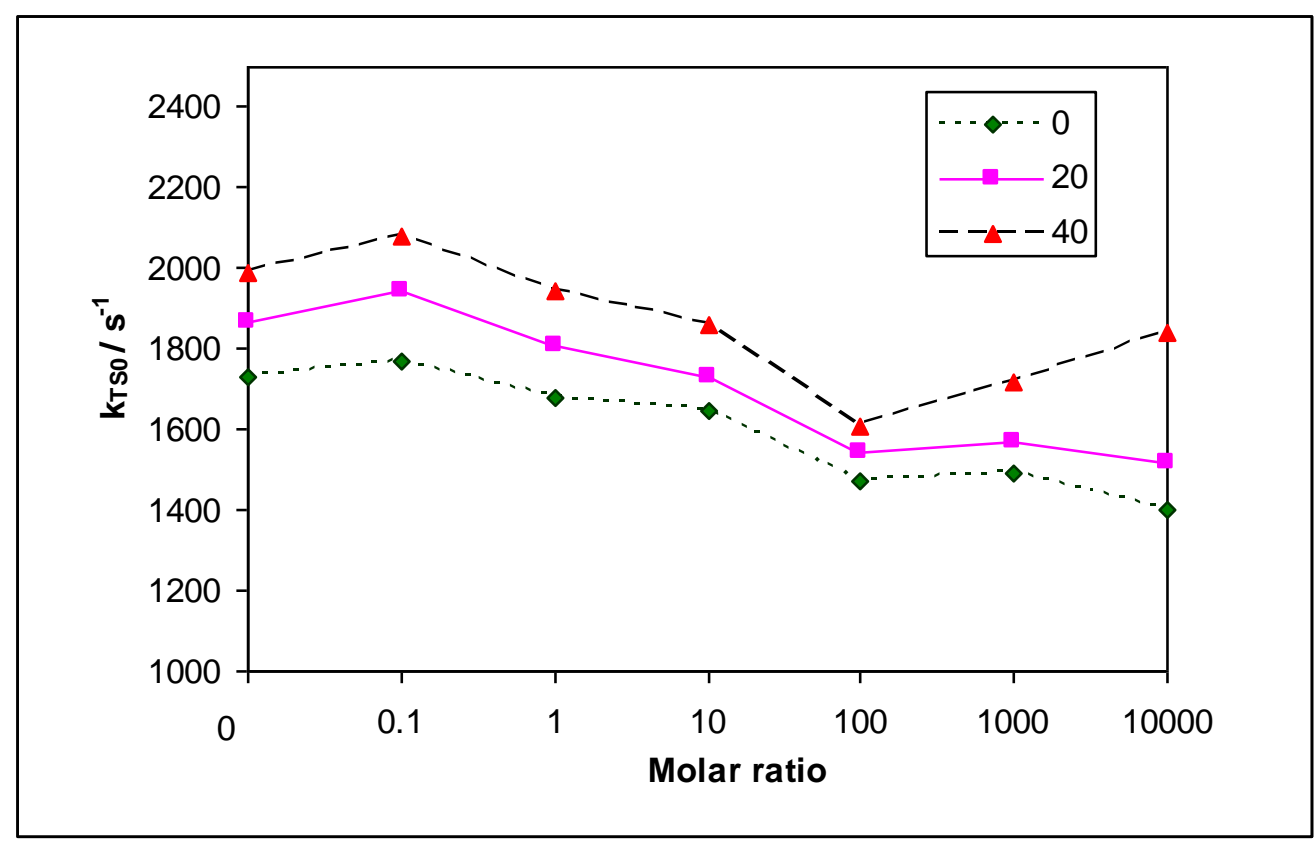

Figure III-18b: Zoom in effect of change in concentration of sucrose in $\mathrm{k}_{\mathrm{TS} 0}$ at 0,20 and $40^{\circ} \mathrm{C}$. Data replotted from Figure III-11a. 
Figure III-19a

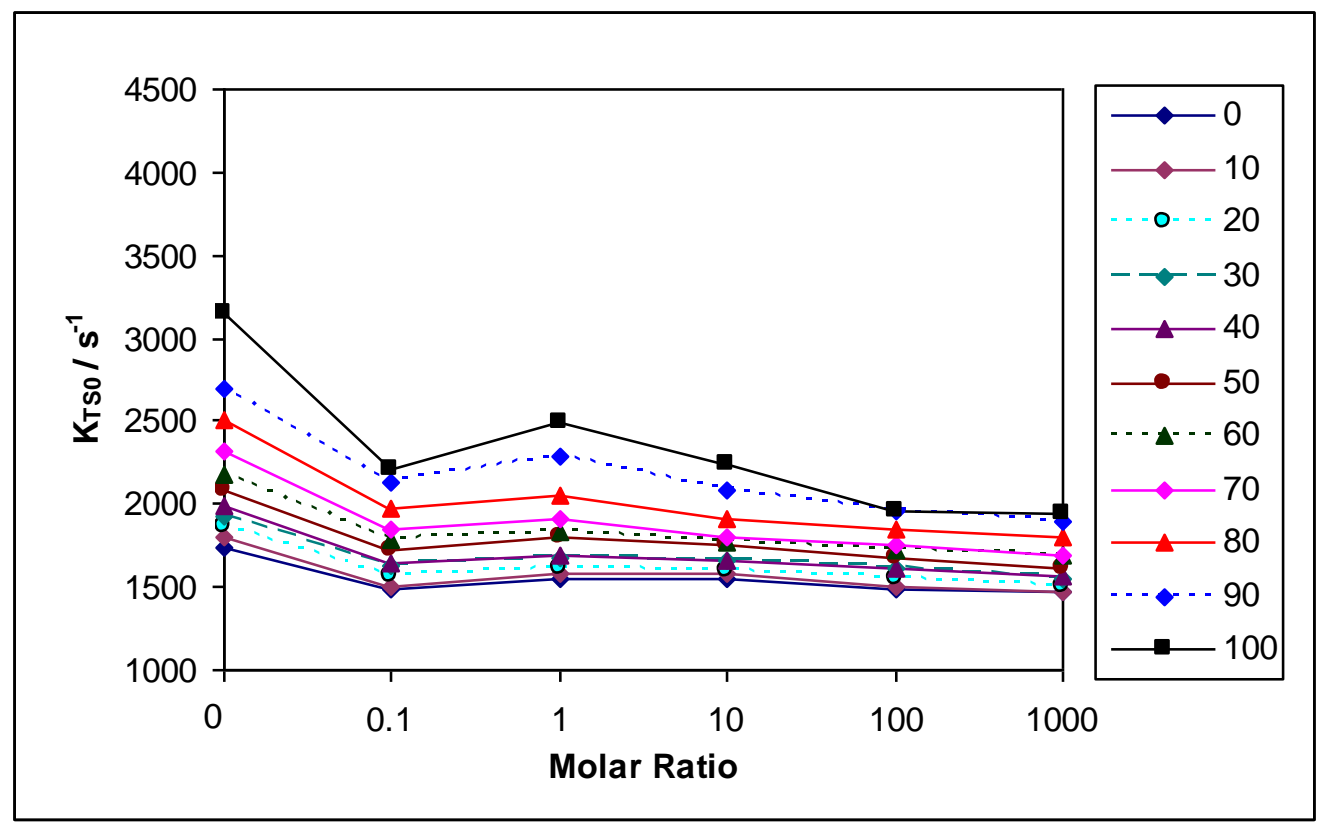

Figure III-19a: A plot of the effect of change in concentration of trehalose in $\mathrm{k}_{\mathrm{TS} 0}$ at various temperatures. Data replotted from Figure III-11b.

\section{Figure III-19b}

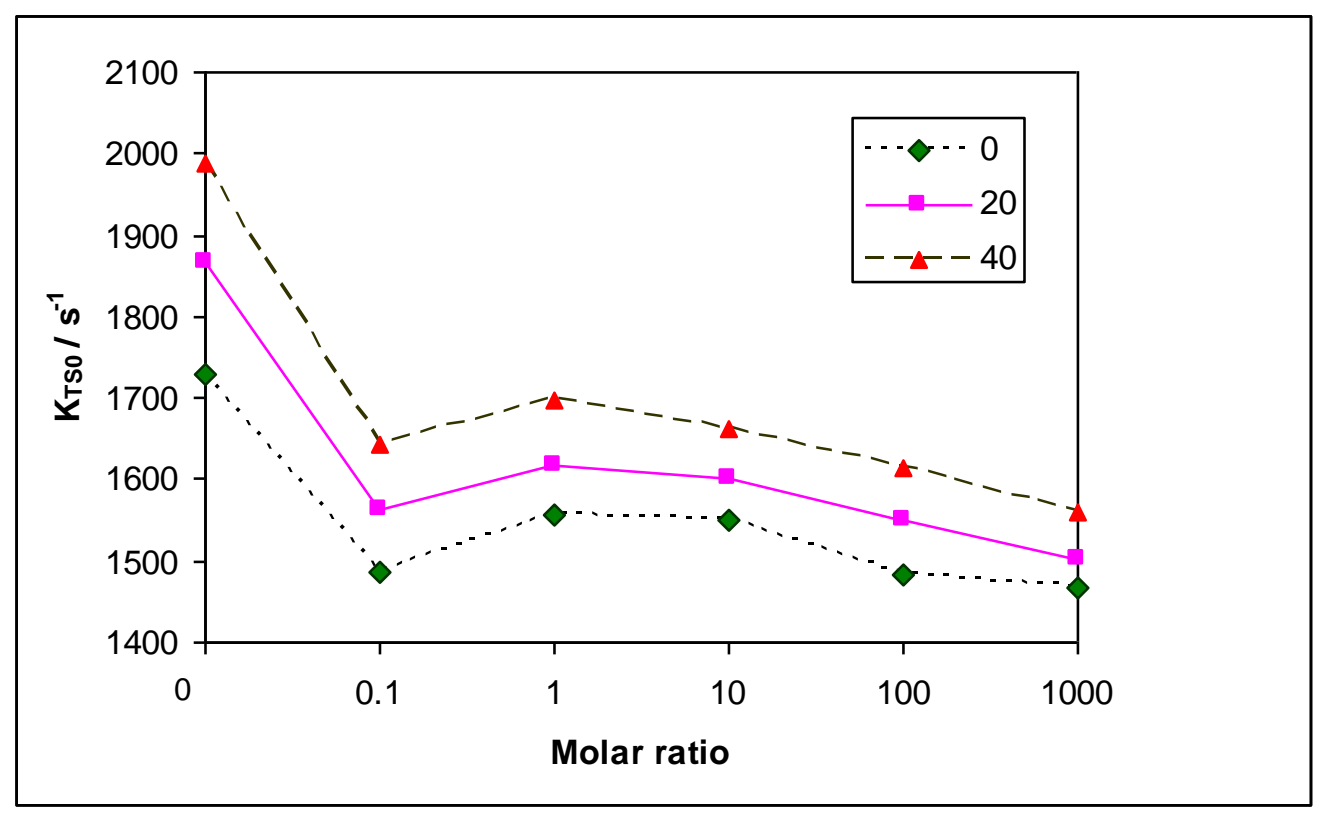

Figure III-19b: Zoom in effect of the change in concentration of trehalose in $\mathrm{k}_{\mathrm{TS} 0}$ at 0,20 and $40^{\circ} \mathrm{C}$. Data replotted from Figure III-11b. 
Figure III-20a

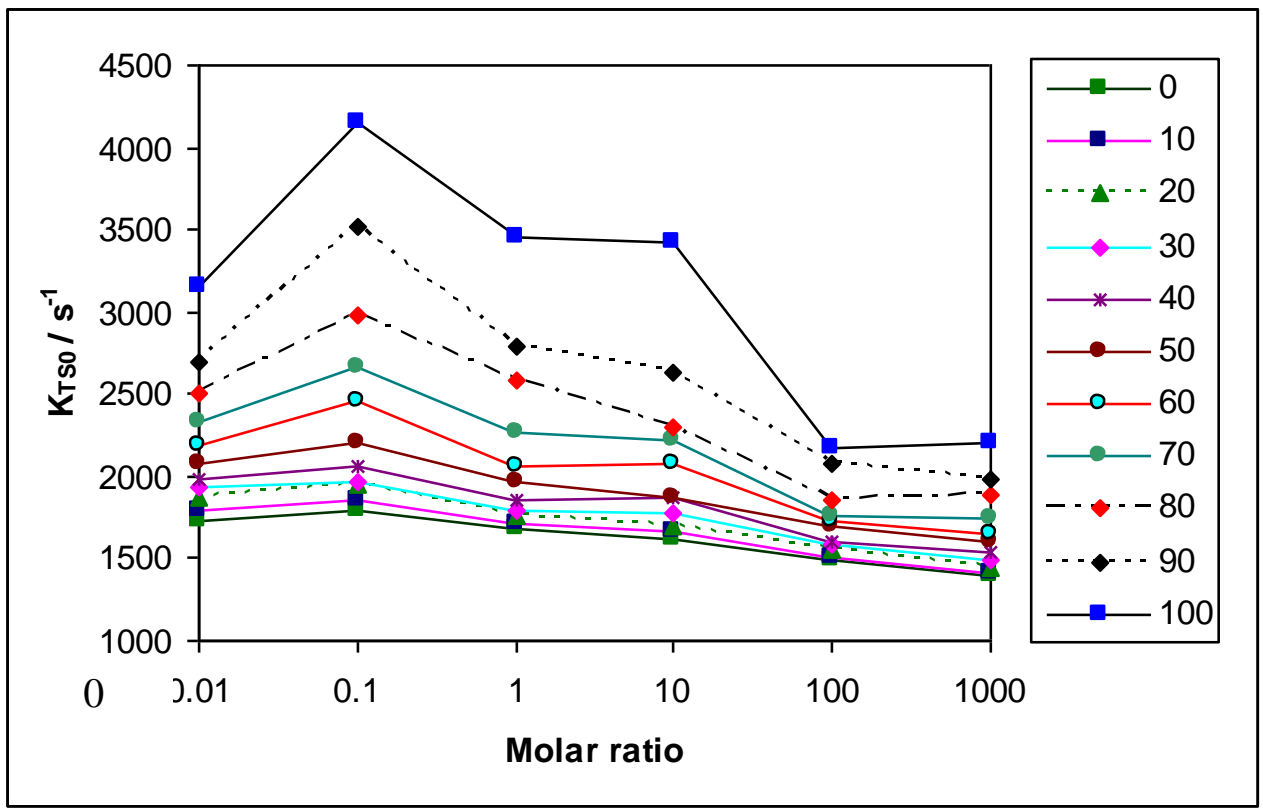

Figure III-20a: A plot of the effect of change in concentration of maltose in $\mathrm{k}_{\mathrm{TS} 0}$ at various temperatures. Data replotted from Figure III-11c.

Figure III-20b

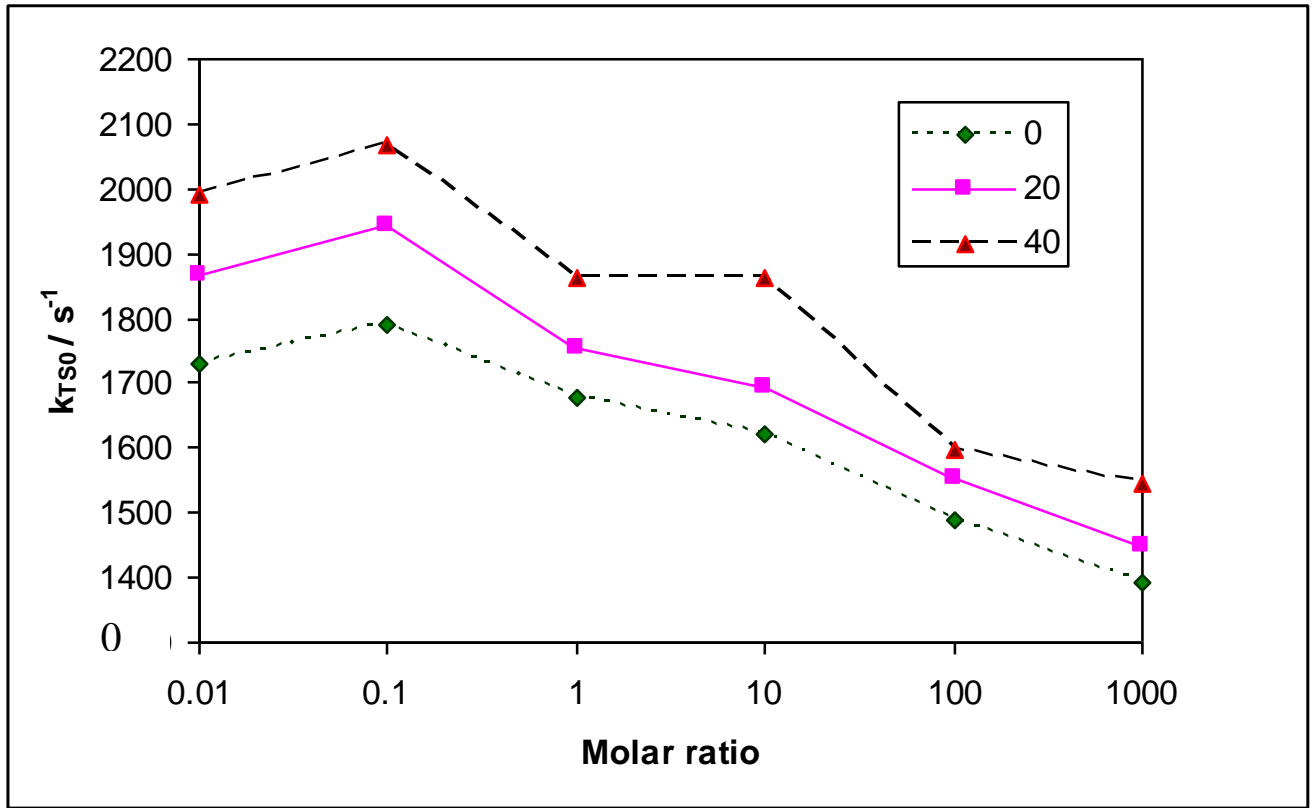

Figure III-20b: Zoom in effect of the change in concentration of maltose in $\mathrm{k}_{\mathrm{TS} 0}$ at 0,20 and $40^{\circ} \mathrm{C}$. Data replotted from Figure III-11c. 
Figure III-21a

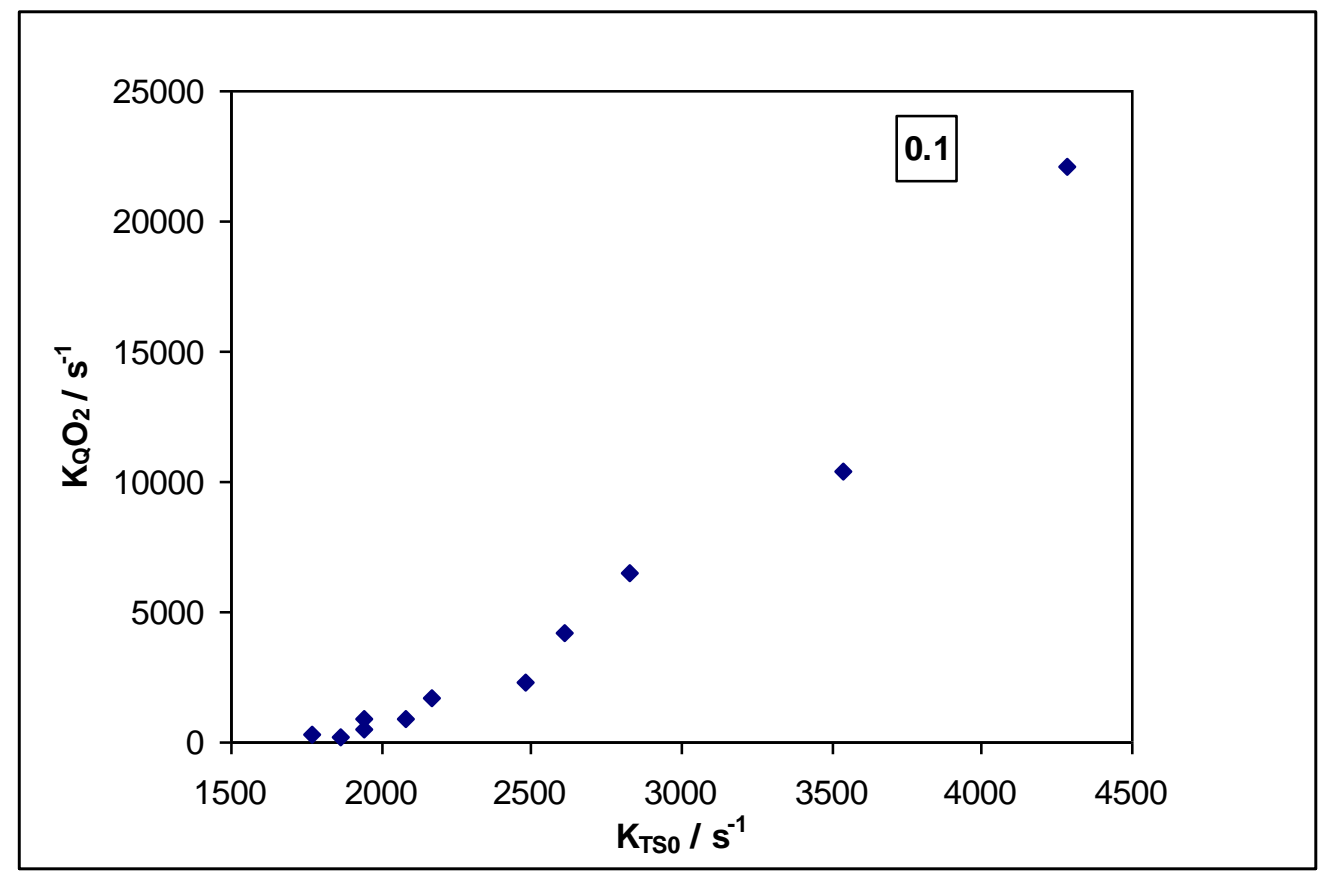

Figure III-21a: Dependence of the oxygen quenching rate $\mathrm{k}_{\mathrm{Q}}\left[\mathrm{O}_{2}\right]$ on the rate of non-radiative quenching $\mathrm{k}_{\mathrm{TS} 0} \mathrm{in}$ sucrose $\beta$-Lg films. The number in the text box refers to molar ratio of sucrose to $\beta-\mathrm{Lg}$.

Figure III-21b

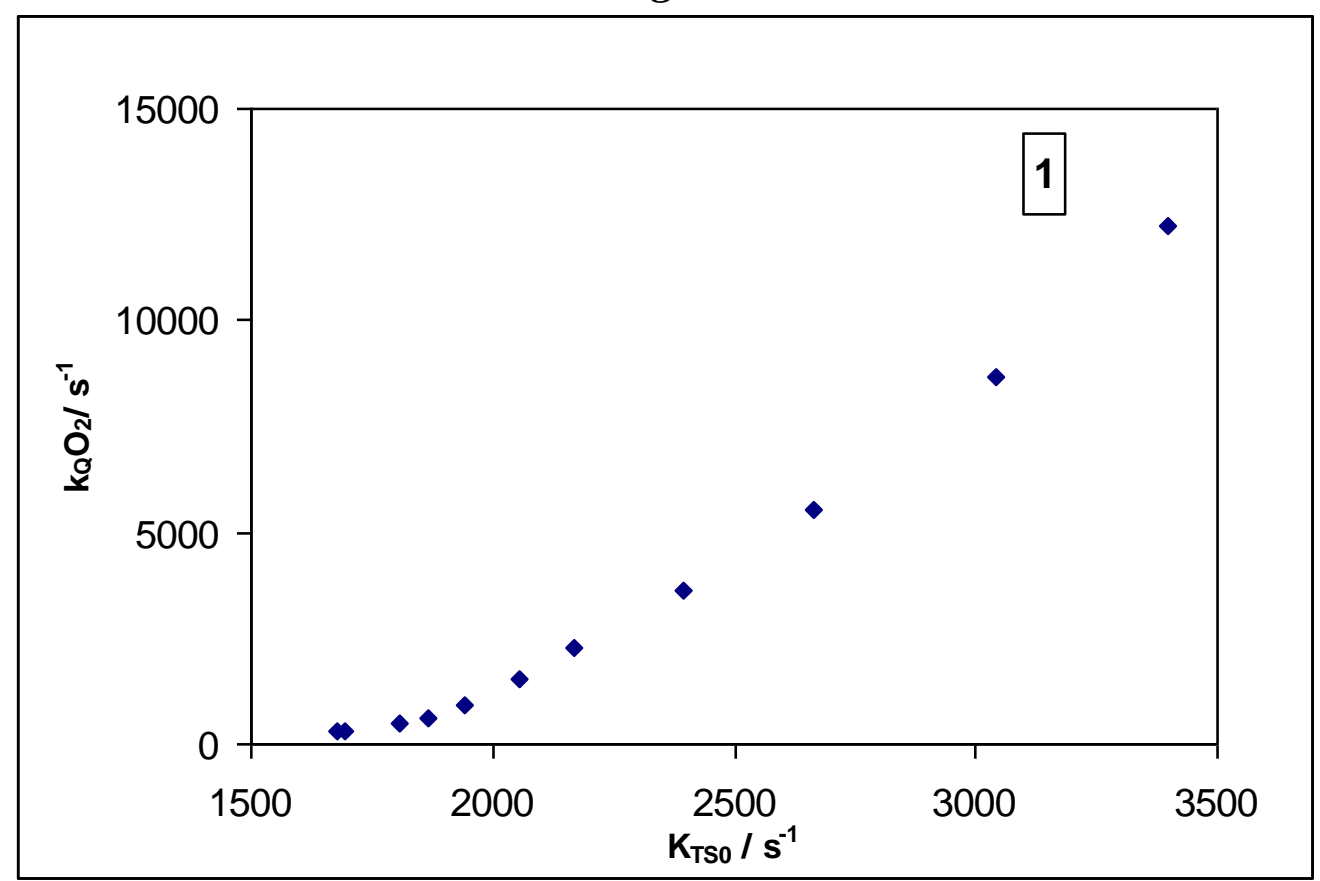

Figure III-21b: Dependence of the oxygen quenching rate $\mathrm{k}_{\mathrm{Q}}\left[\mathrm{O}_{2}\right]$ on the rate of non-radiative quenching $\mathrm{k}_{\mathrm{TS} 0}$ in sucrose $\beta$ - $\mathrm{Lg}$ films. The number in the text box refers to molar ratio of sucrose to $\beta$-Lg. 
Figure III-21c

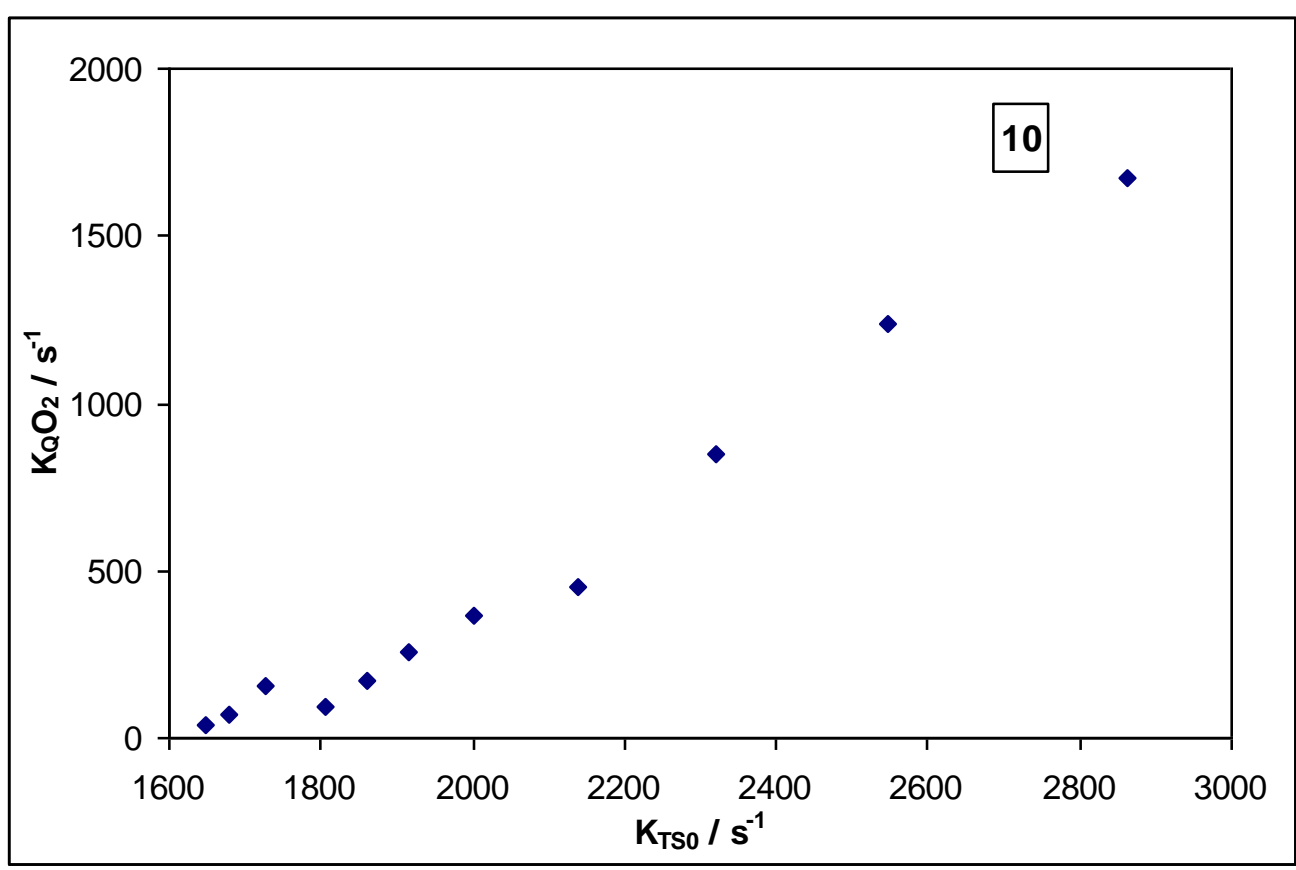

Figure III-21c: Dependence of the oxygen quenching rate $\mathrm{k}_{\mathrm{Q}}\left[\mathrm{O}_{2}\right]$ on the rate of non-radiative quenching $\mathrm{k}_{\mathrm{TS} 0}$ in sucrose $\beta-\mathrm{Lg}$ films. The number in the text box refers to molar ratio of sucrose to $\beta-\mathrm{Lg}$.

Figure III-21d

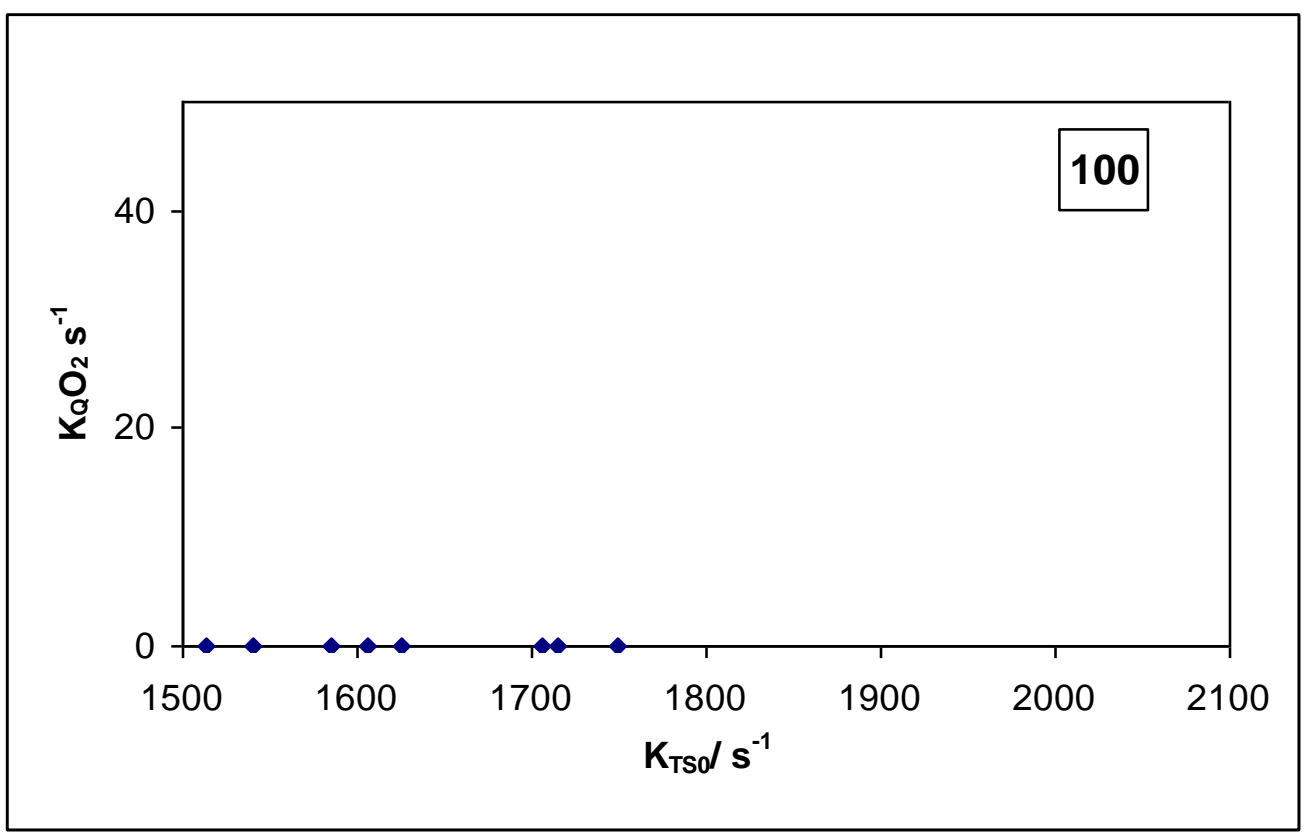

Figure III-21d: Dependence of the oxygen quenching rate $\mathrm{k}_{\mathrm{Q}}\left[\mathrm{O}_{2}\right]$ on the rate of non-radiative quenching $\mathrm{k}_{\mathrm{TS} 0}$ in sucrose $\beta-\mathrm{Lg}$ films. The number in the text box refers to molar ratio of sucrose to $\beta-\mathrm{Lg}$. 
Figure III-21e

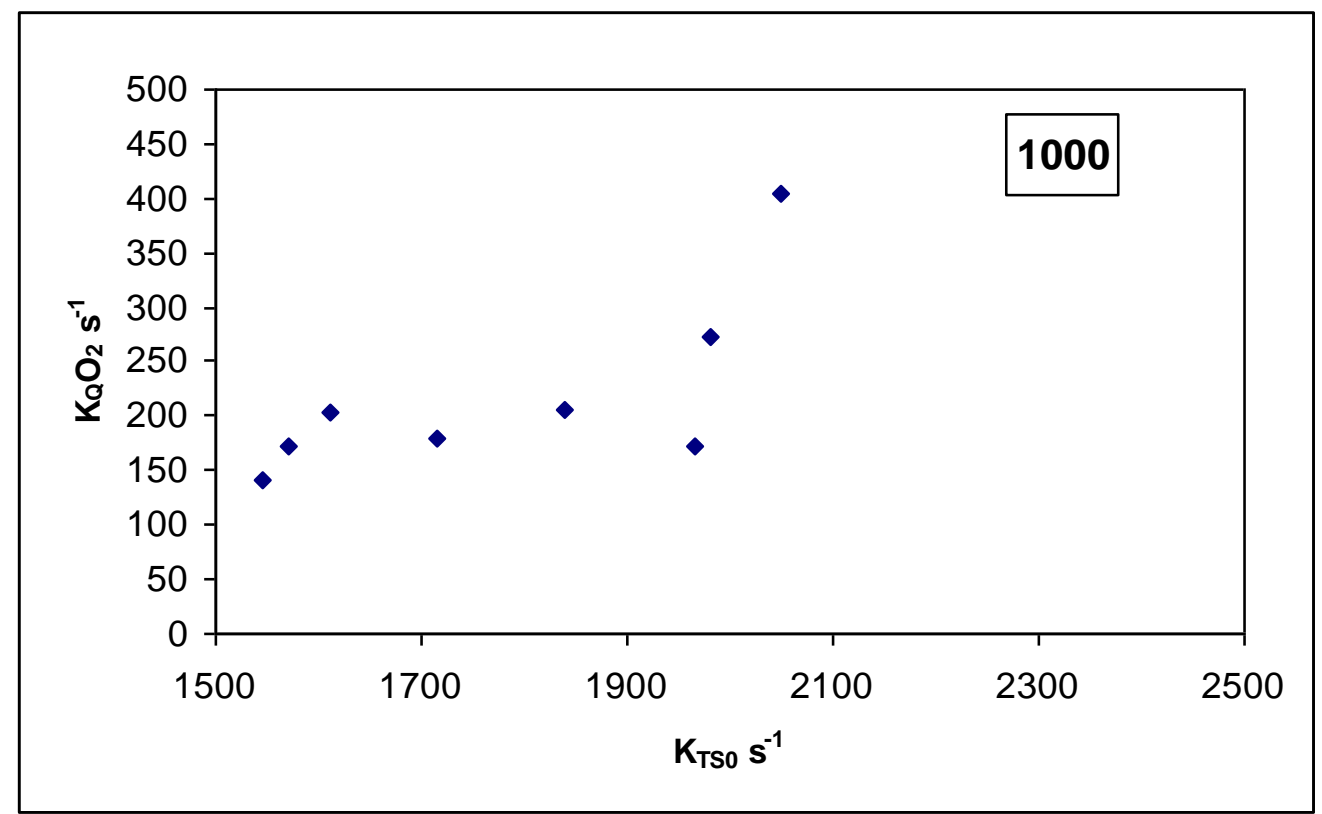

Figure III-21e: Dependence of the oxygen quenching rate $\mathrm{k}_{\mathrm{Q}}\left[\mathrm{O}_{2}\right]$ on the rate of non-radiative quenching $\mathrm{k}_{\mathrm{Ts} 0}$ in sucrose $\beta$-Lg films. The number in the text box refers to molar ratio of sucrose to $\beta$ - $\mathrm{Lg}$.

Figure III-21f

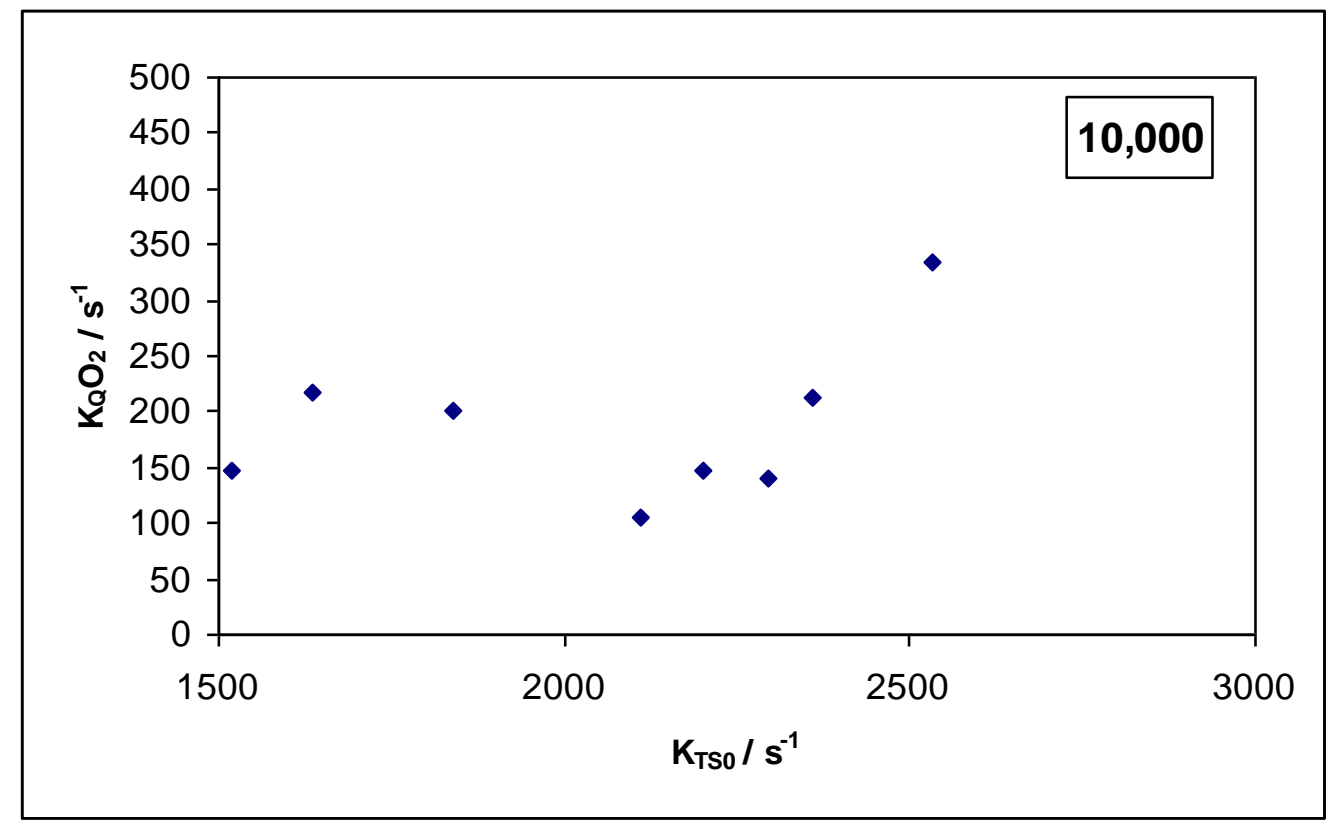

Figure III-21f: Dependence of the oxygen quenching rate $\mathrm{k}_{\mathrm{Q}}\left[\mathrm{O}_{2}\right]$ on the rate of non-radiative quenching $\mathrm{k}_{\mathrm{TS} 0}$ in sucrose $\beta$ - $\mathrm{Lg}$ films. The number in the text box refers to molar ratio of sucrose to $\beta-\mathrm{Lg}$. 


\section{Figure III-22a}

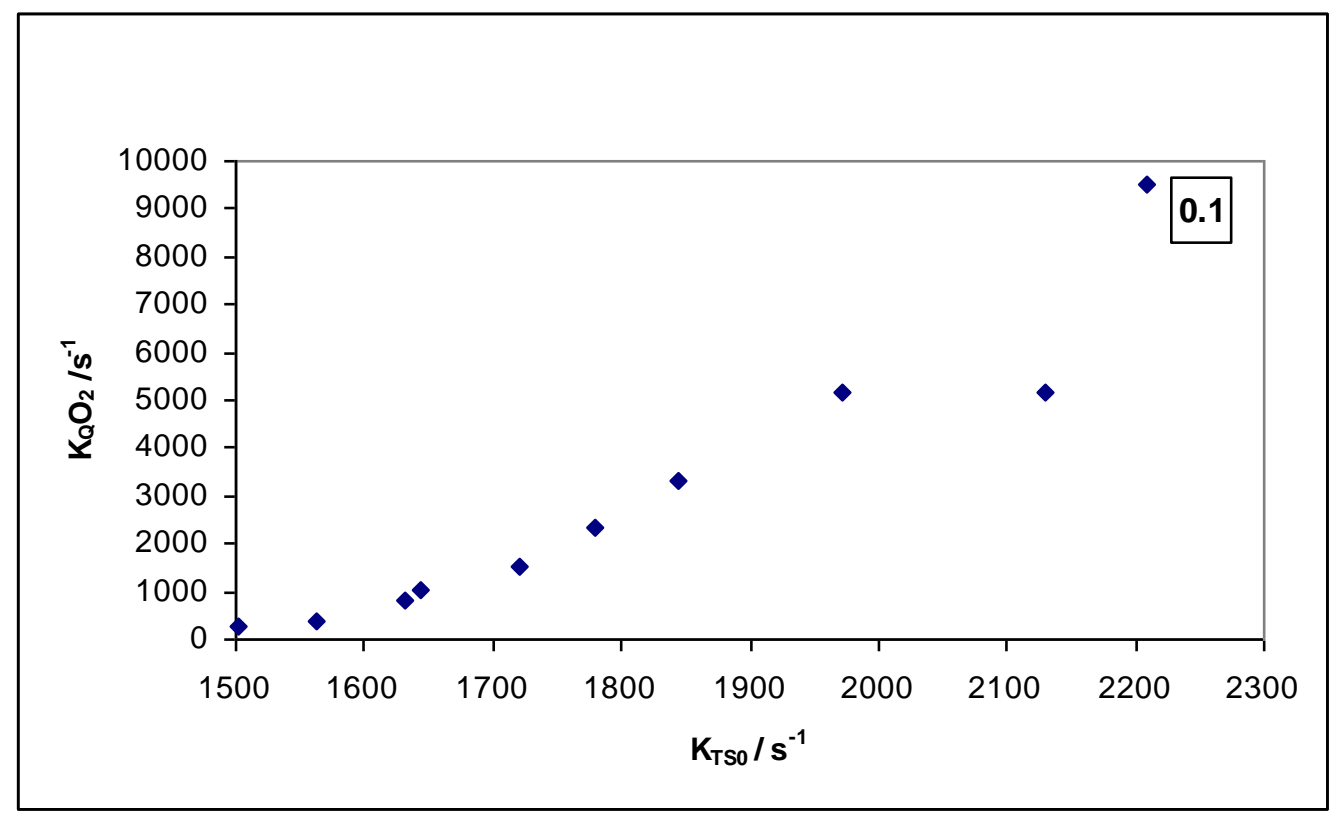

Figure III-22a: Dependence of the oxygen quenching rate $\mathrm{k}_{\mathrm{Q}}\left[\mathrm{O}_{2}\right]$ on the rate of non-radiative quenching $\mathrm{k}_{\mathrm{TS} 0}$ in trehalose $\beta$-Lg films. The number in the text box refers to molar ratio of trehalose to $\beta$-Lg.

\section{Figure III-22b}

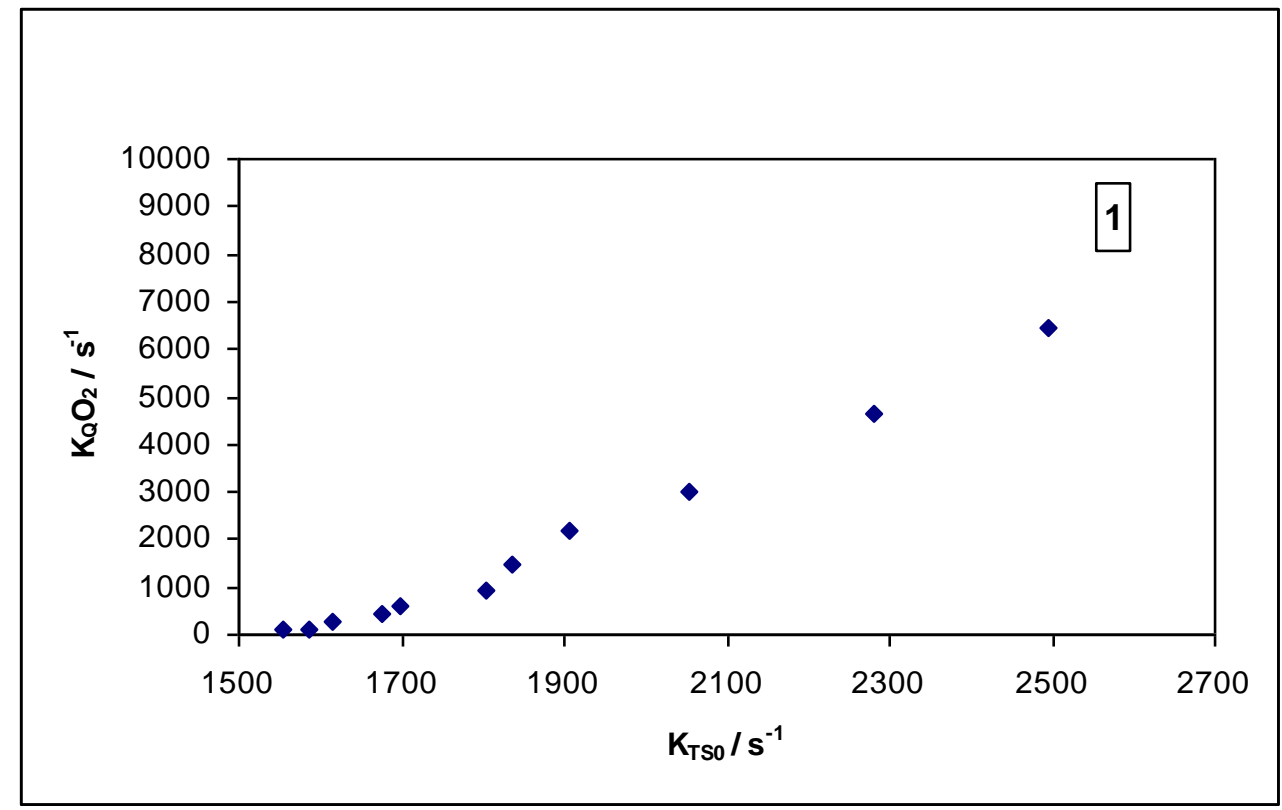

Figure III-22b: Dependence of the oxygen quenching rate $\mathrm{k}_{\mathrm{Q}}\left[\mathrm{O}_{2}\right]$ on the rate of non-radiative quenching $\mathrm{k}_{\mathrm{Ts} 0}$ in trehalose $\beta-\mathrm{Lg}$ films. The number in the text box refers to molar ratio of trehalose to $\beta-\mathrm{Lg}$. 
Figure III-22c

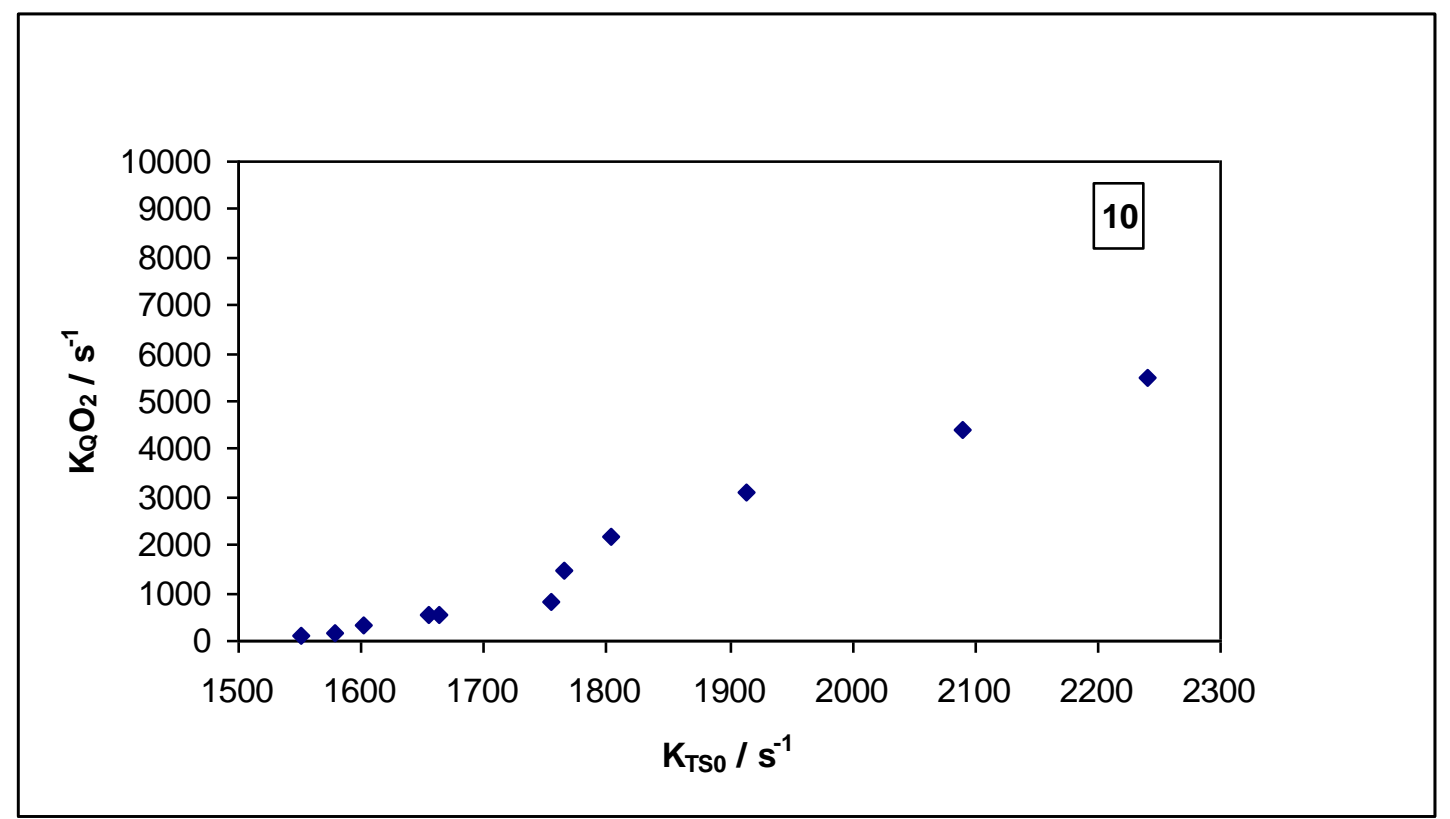

Figure III-22c: Dependence of the oxygen quenching rate $\mathrm{k}_{\mathrm{Q}}\left[\mathrm{O}_{2}\right]$ on the rate of non-radiative quenching $\mathrm{k}_{\mathrm{TS} 0}$ in trehalose $\beta$ - $\mathrm{Lg}$ films. The number in the text box refers to molar ratio of trehalose to $\beta$-Lg.

\section{Figure III-22d}

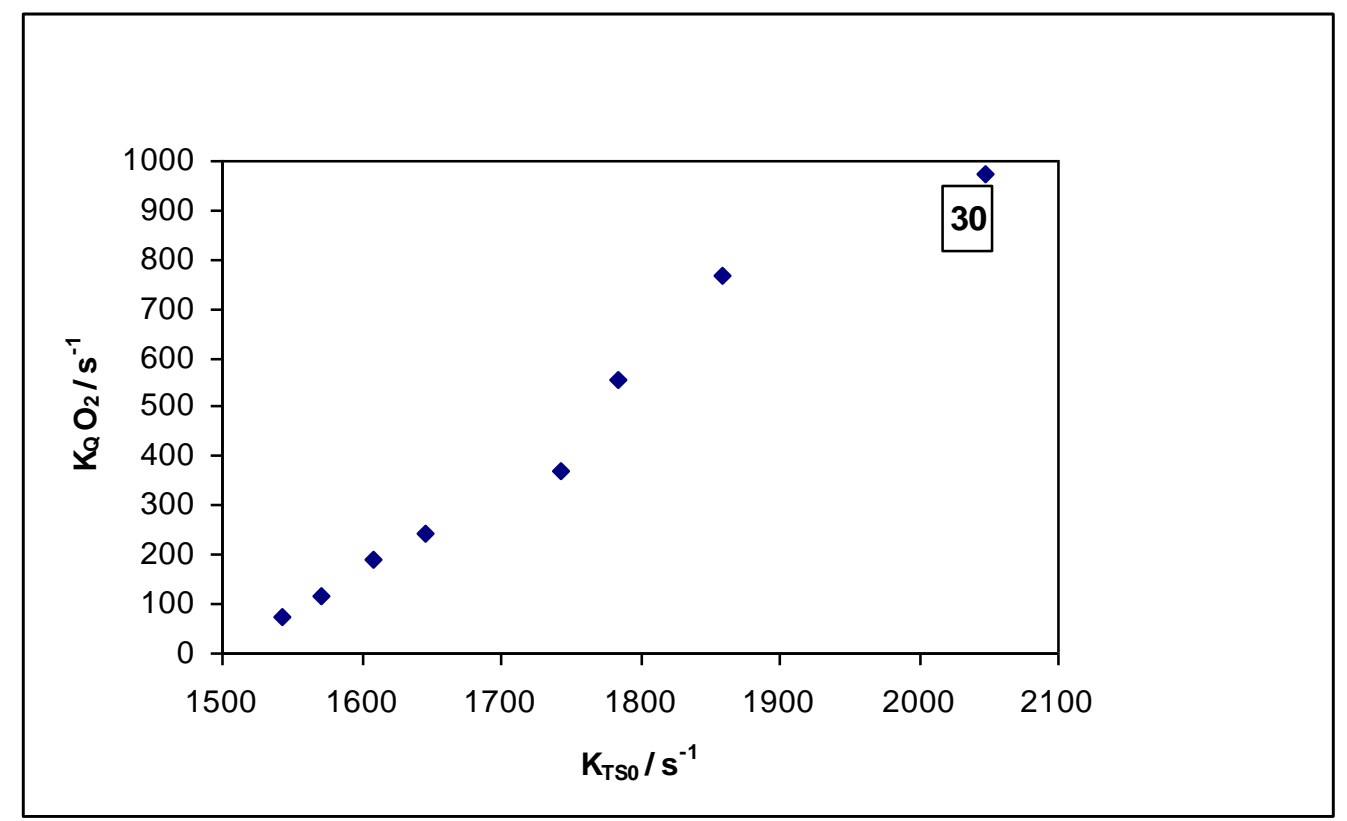

Figure III-22d: Dependence of the oxygen quenching rate $\mathrm{k}_{\mathrm{Q}}\left[\mathrm{O}_{2}\right]$ on the rate of non-radiative quenching $\mathrm{k}_{\mathrm{TS} 0}$ in trehalose $\beta$-Lg films. The number in the text box refers to molar ratio of trehalose to $\beta-\mathrm{Lg}$. 


\section{Figure III-22e}

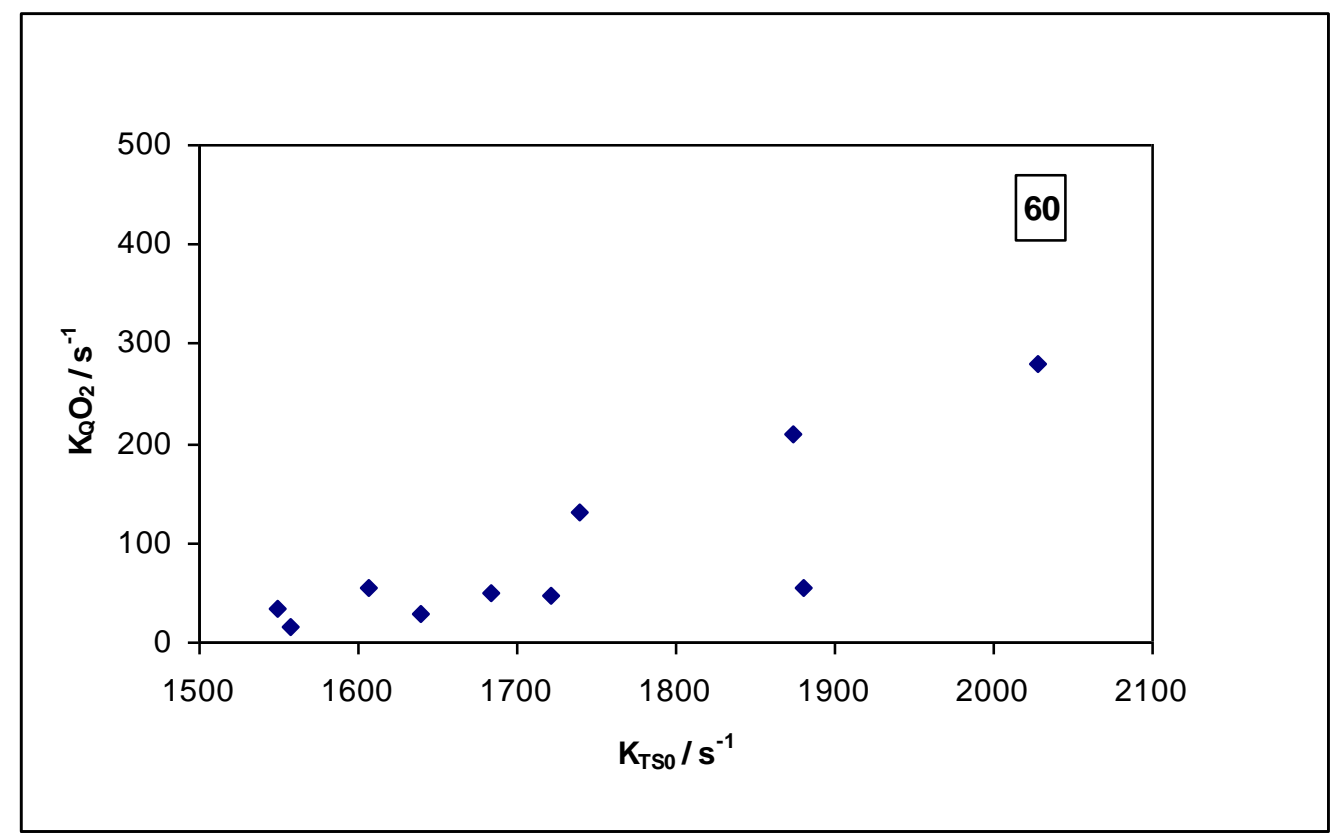

Figure III-22e: Dependence of the oxygen quenching rate $\mathrm{k}_{\mathrm{Q}}\left[\mathrm{O}_{2}\right]$ on the rate of non-radiative quenching $\mathrm{k}_{\mathrm{TS} 0}$ in trehalose/ $\beta$ - $\mathrm{Lg}$ films. The number in the text box refers to molar ratio of trehalose to $\beta$-Lg.

\section{Figure III-22f}

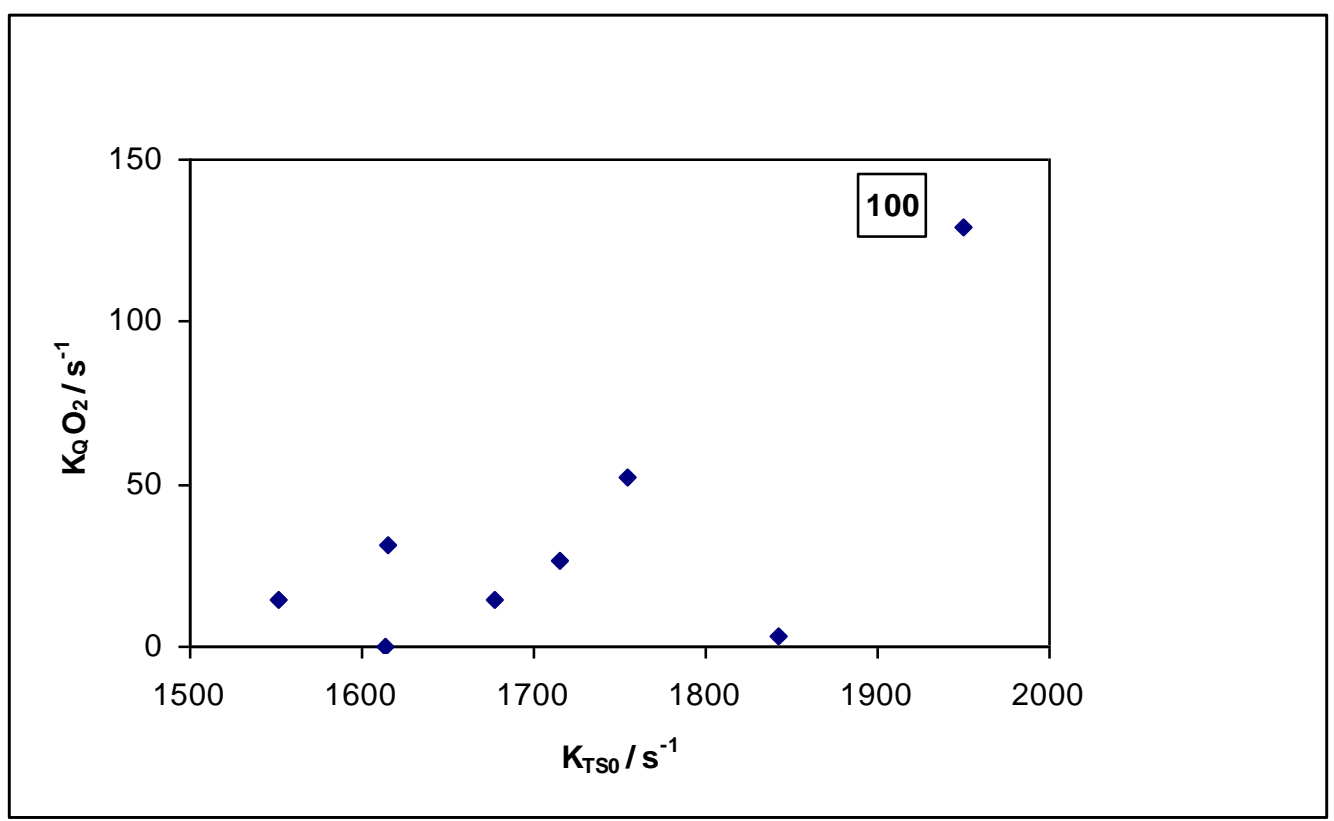

Figure III-22f: Dependence of the oxygen quenching rate $\mathrm{k}_{\mathrm{Q}}\left[\mathrm{O}_{2}\right]$ on the rate of non-radiative quenching $\mathrm{k}_{\mathrm{TS} 0}$ in trehalose/ $\beta$ - $\mathrm{Lg}$ films. The number in the text box refers to molar ratio of trehalose to $\beta-\mathrm{Lg}$. 


\section{Figure III-22g}

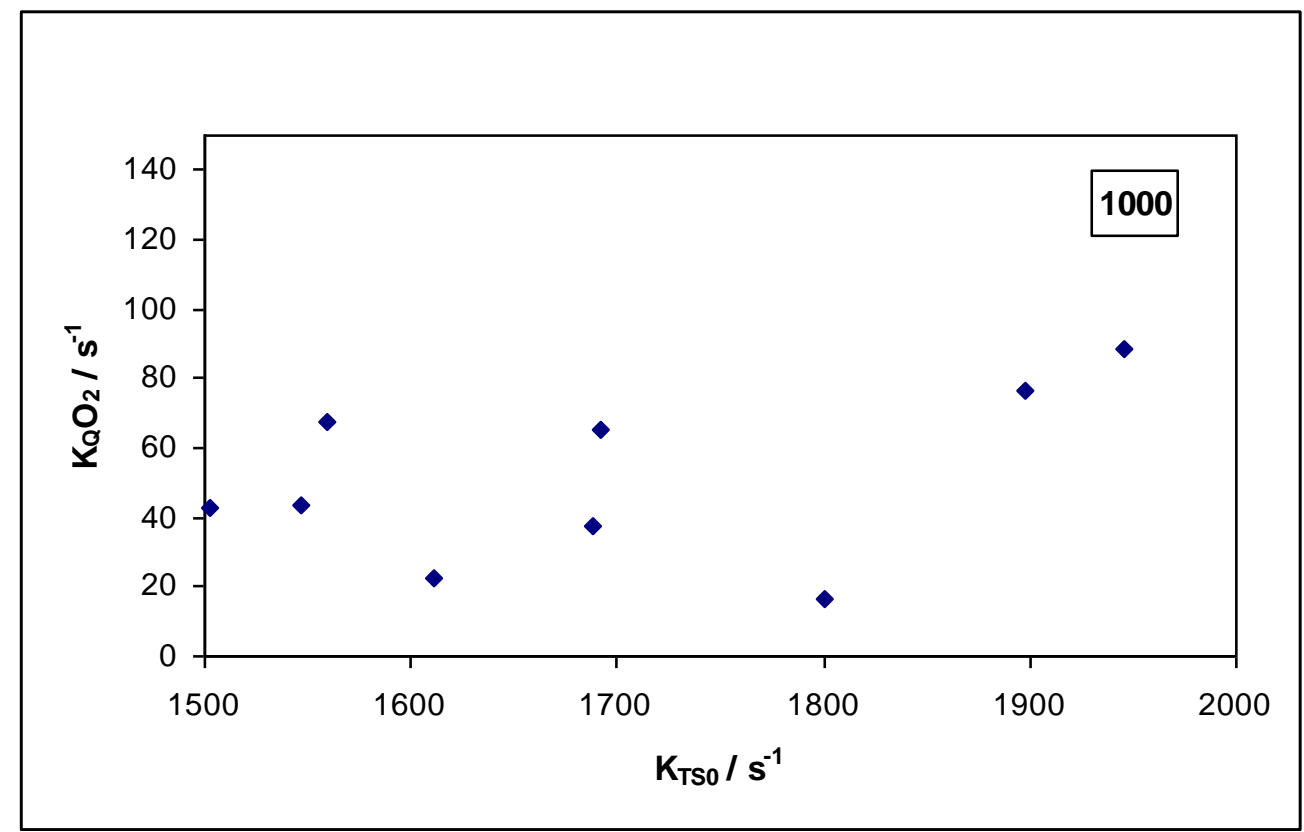

Figure III-22g: Dependence of the oxygen quenching rate $\mathrm{k}_{\mathrm{Q}}\left[\mathrm{O}_{2}\right]$ on the rate of non-radiative quenching $\mathrm{k}_{\mathrm{TS} 0}$ in trehalose/ $\beta$-Lg films. The number in the text box refers to molar ratio of trehalose to $\beta$ - $\mathrm{Lg}$. 
Figure III-23a

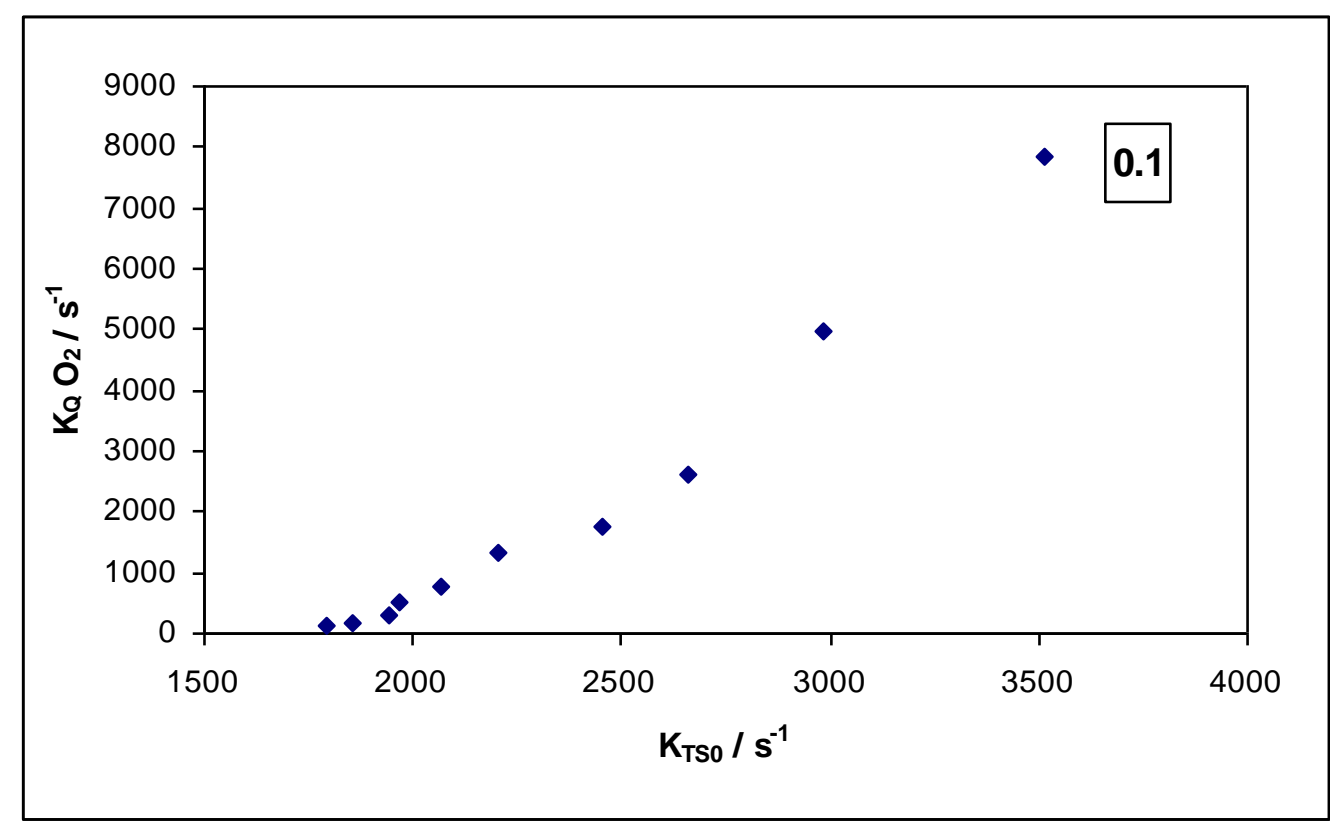

Figure III-23a: Dependence of the oxygen quenching rate $\mathrm{k}_{\mathrm{Q}}\left[\mathrm{O}_{2}\right]$ on the rate of non-radiative quenching $\mathrm{k}_{\mathrm{TS} 0}$ in maltose $\beta-\mathrm{Lg}$ films. The number in the text box refers to molar ratio of maltose to $\beta-\mathrm{Lg}$.

Figure III-23b

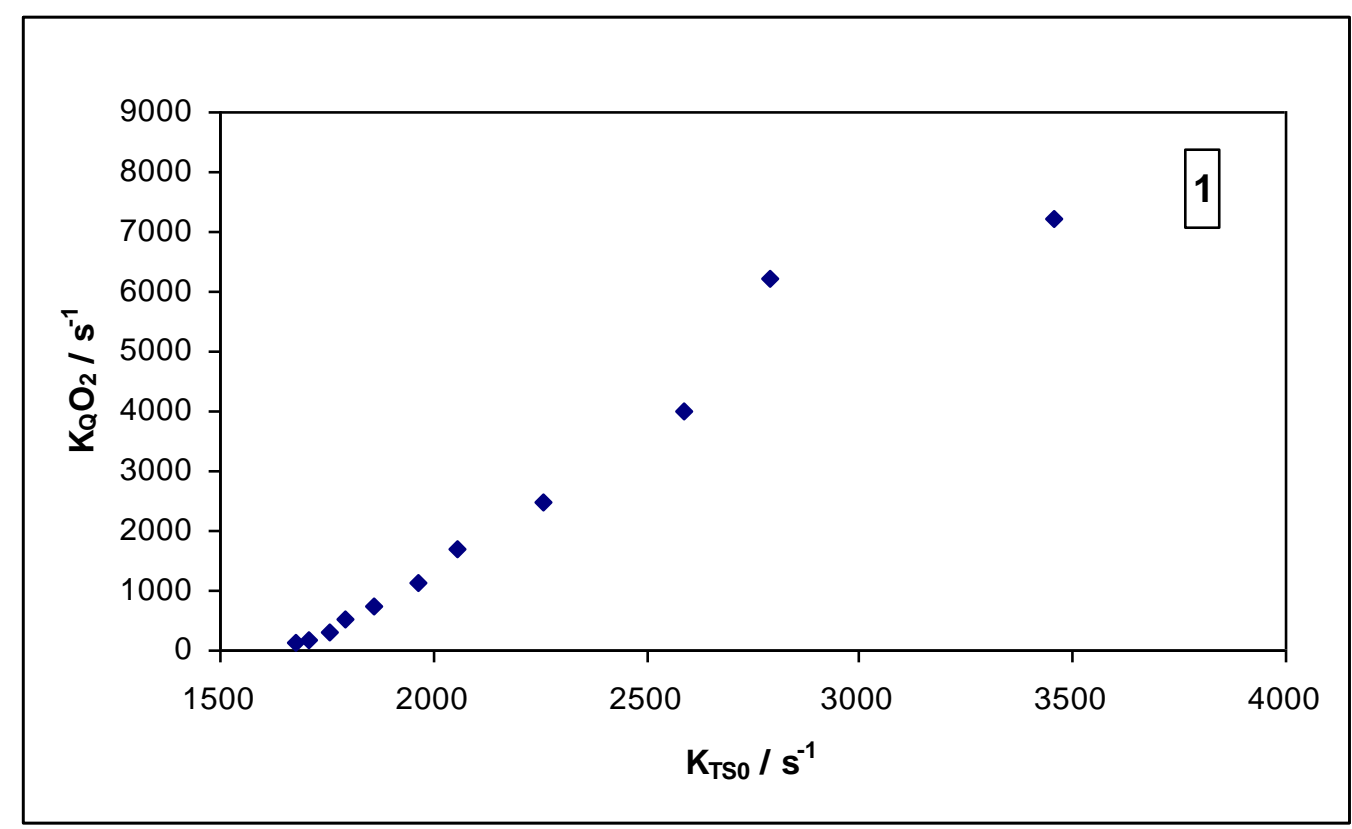

Figure III-23b: Dependence of the oxygen quenching rate $\mathrm{k}_{\mathrm{Q}}\left[\mathrm{O}_{2}\right]$ on the rate of non-radiative quenching $\mathrm{k}_{\mathrm{TS} 0}$ in maltose/ $\beta$-Lg films. The number in the text box refers to molar ratio of maltose to $\beta$ - $\mathrm{Lg}$. 
Figure III-23c

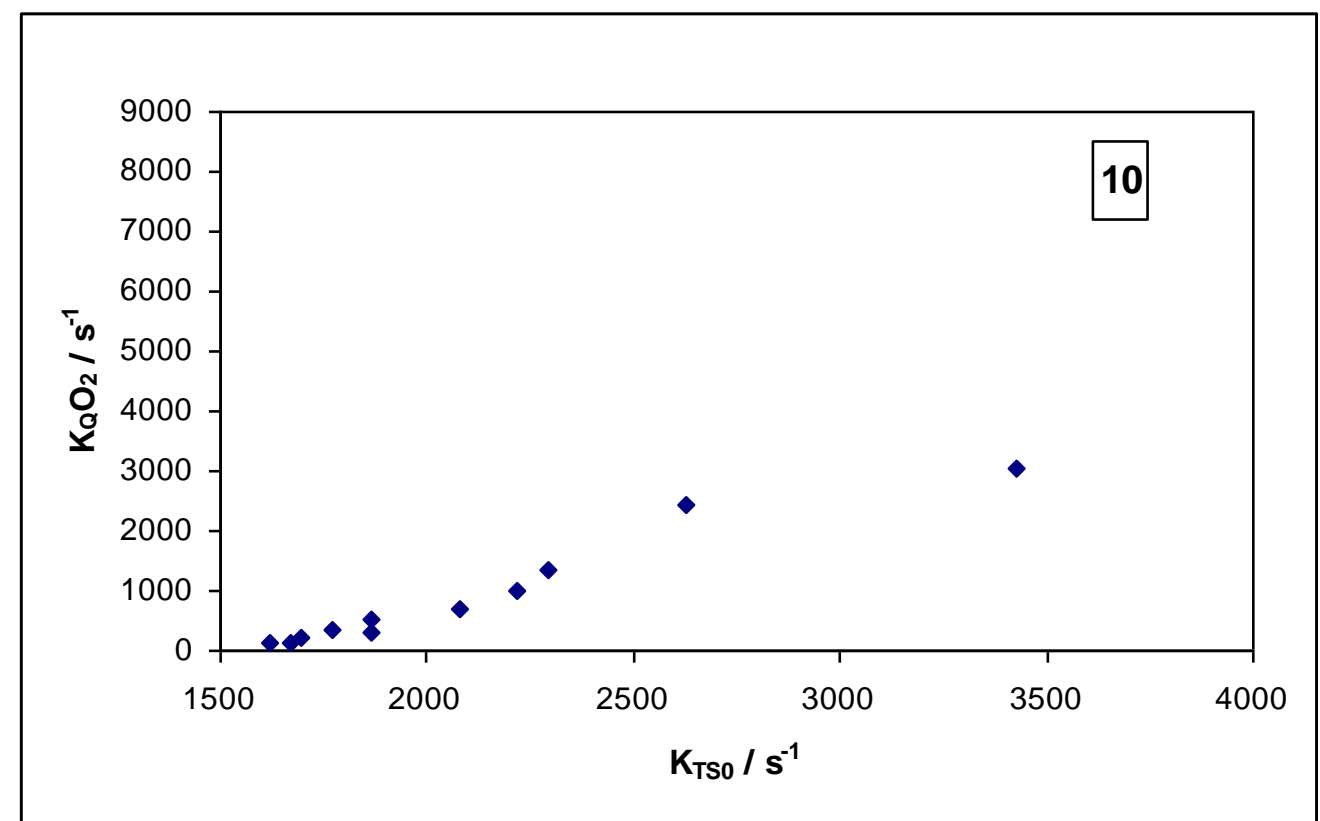

Figure III-23c: Dependence of the oxygen quenching rate $\mathrm{k}_{\mathrm{Q}}\left[\mathrm{O}_{2}\right]$ on the rate of non-radiative quenching $\mathrm{k}_{\mathrm{Ts} 0}$ in maltose/ $\beta$-Lg films. The number in the text box refers to molar ratio of maltose to $\beta$ - $\mathrm{Lg}$.

\section{Figure III-23d}

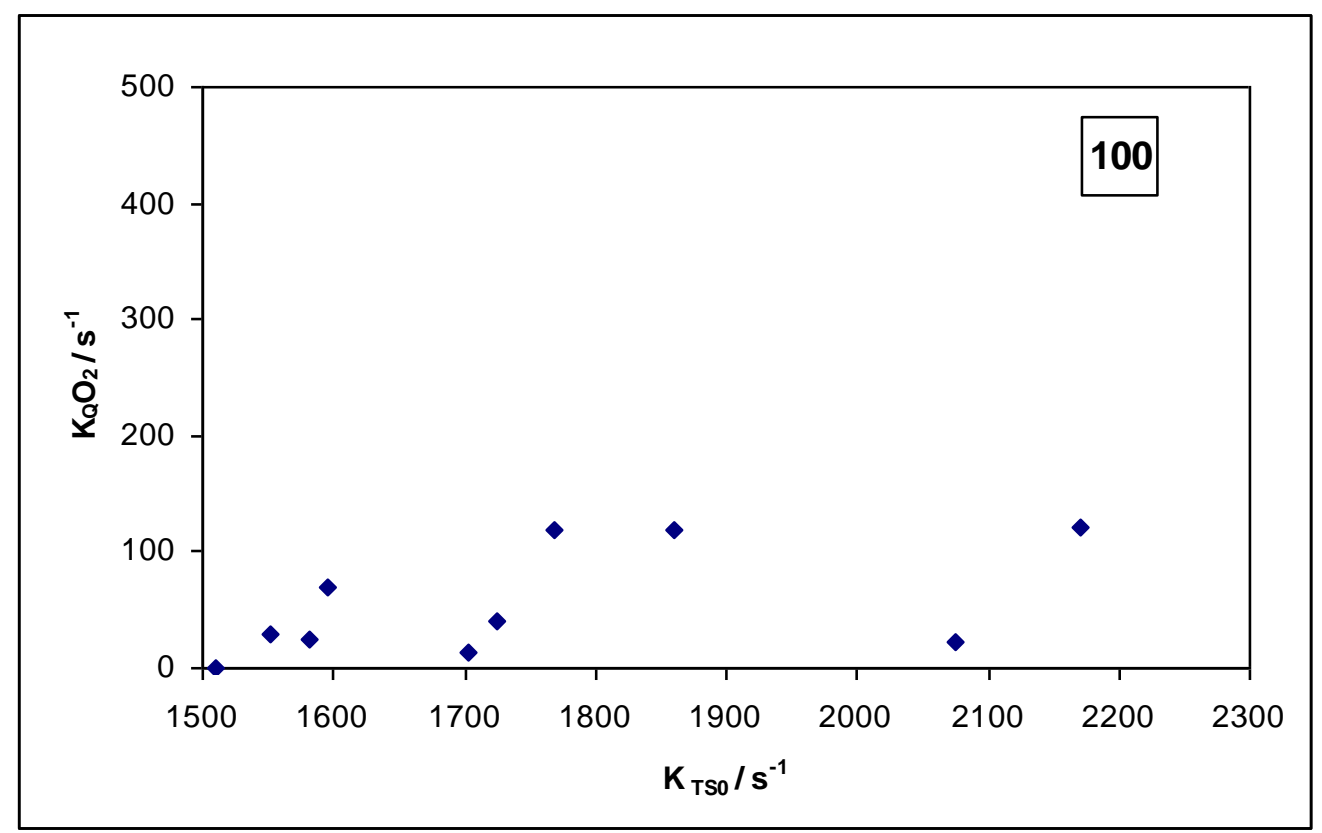

Figure III-23d: Dependence of the oxygen quenching rate $\mathrm{k}_{\mathrm{Q}}\left[\mathrm{O}_{2}\right]$ on the rate of non-radiative quenching $\mathrm{k}_{\mathrm{TS} 0}$ in $\beta$ - $\mathrm{Lg}$ maltose films. The number in the text box refers to molar ratio of maltose to $\beta-\mathrm{Lg}$. 
Figure III-23e

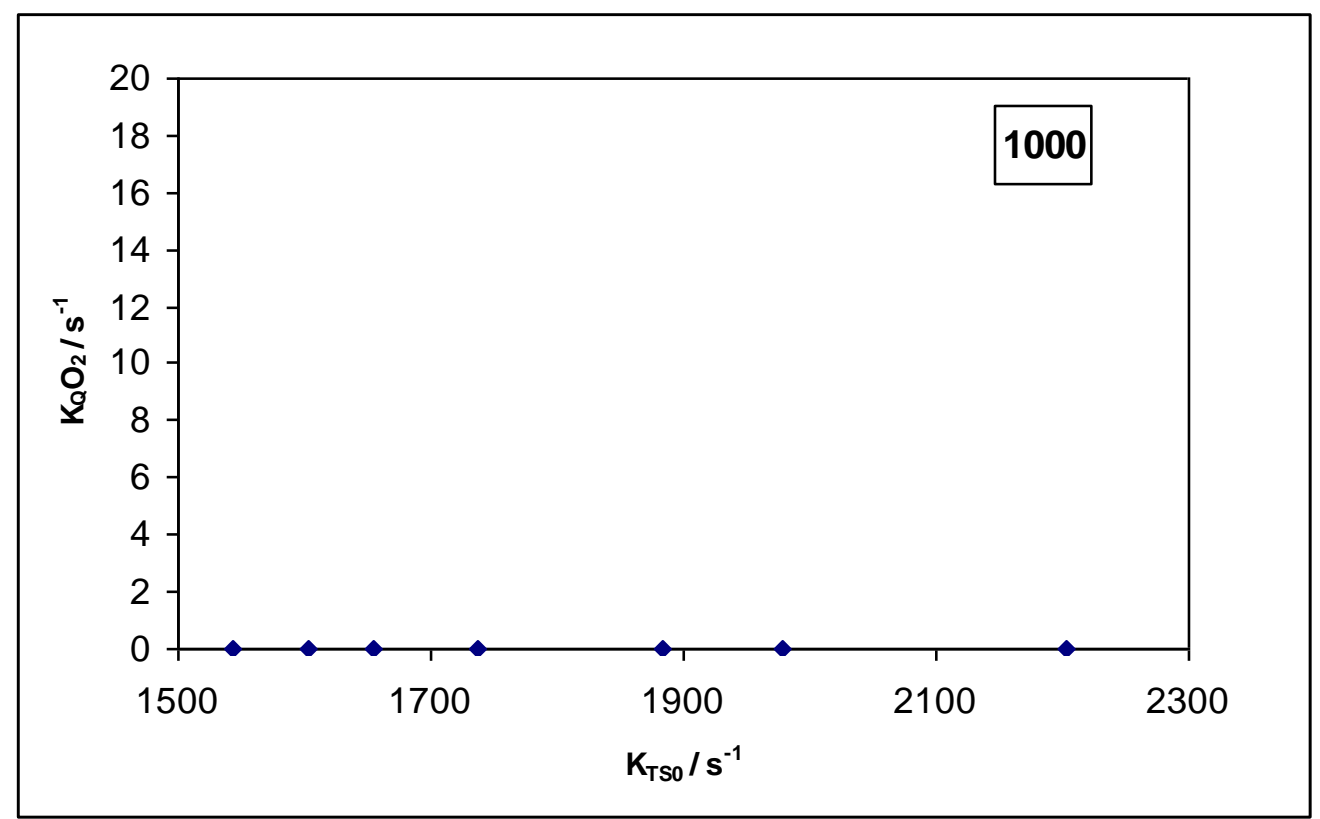

Figure III-23e: Dependence of the oxygen quenching rate $\mathrm{k}_{\mathrm{Q}}\left[\mathrm{O}_{2}\right]$ on the rate of non-radiative quenching $\mathrm{k}_{\mathrm{TS} 0}$ in maltose/ $\beta$-Lg films. The number in the text box refers to molar ratio of maltose to $\beta$ - $\mathrm{Lg}$. 
Chapter IV: Dynamic site heterogeneity in amorphous $\beta$-Lactoglobulin ( $\beta$-Lg) and sucrose / $\beta$-Lactoglobulin $(\beta$-Lg) from spectral heterogeneity in Erythrosin B phosphorescence

\section{Introduction}

Amorphous sugars, polyols and proteins appear to exhibit signs of dynamic heterogeneity. Studies previously done in our lab have shown that systematic variations in the phosphorescence lifetime across the emission band, as well as blue shifts in the emission spectra with time following excitation, provide a novel method of monitoring dynamic site heterogeneities in amorphous sugars (Pravinata et al., 2005; Shirke et al., 2005, 2006), sugar alcohols (Shirke et al., 2005) and proteins (Lukasik and Ludescher 2005; Nack and Ludescher, 2006).

The glass transition occurs over a temperature range, the width of which is controlled by the heterogeneity in the matrix. Tg has an important relationship with the macroscopic food quality (Fennema, 1996). But $\mathrm{Tg}$ is not an ultimate predictor of stability, as certain diffusion limited reactions occur at temperatures below $\mathrm{Tg}$. Sub $\mathrm{Tg}$ relaxations and physical ageing are processes that signify the presence of mobility below Tg. Yoshioka (1997) demonstrated that freeze dried protein stability is more closely related to molecular mobility than to $\mathrm{Tg}$ in amorphous pharmaceuticals. Dynamic transitions have been observed below $\mathrm{Tg}$ in synthetic polymers showing localized vibrational motions of molecules (McCrum et al., 1991). This dynamic heterogeneity is important in understanding the overall properties of the processes occurring in the glass.

Cicerone and Ediger (1995) first reported direct observation of spatially heterogeneous dynamics. They used a photo-bleaching technique to measure probe 
reorientation times in super cooled O-terphenyl (OTP). Near the glass transition there are regions with different dynamics; one region can be orders of magnitude faster than another region only a few nanometers apart. Schiener et al. (1997) successfully performed dielectric hole-burning experiments of propylene carbonate and glycerol near their glass transitions. This observation provides evidence for the existence of a distribution of relaxation times in these super-cooled liquids. Although these transitions have been observed in synthetic polymers very little research has been devoted to similar studies in more complex matrices such as food. These studies have the potential to provide information on the quality attributes pertinent to a food system (Ludescher et al., 2001).

Amorphous foods have structural complexity and have various structural elements of different length scales. Macroscopic heterogeneities in a food material can induce the presence of regions with higher or lower mobilities. Buera et al. (1998) investigated microbial stability in glassy white bread and maltodextrins. They showed that mold growth might be possible below $\mathrm{Tg}$ in a material broadly considered as glassy (only one $\mathrm{T}_{\mathrm{g}}$ determined by DSC), in some micro-regions supposed to be non- glassy.

According to Richert (2003), several relaxations can be observed for biopolymers. The secondary $\beta$ relaxation has been extensively studied. As described by Johary (1976) it corresponds to more localize molecular motions that persist in the glassy state (Chan et al., 1986). In sugar molecules, the $\beta$ process was firstly linked to the presence and the motions of hydroxyl groups (ref). More recently, it has been demonstrated that this relaxation is sensitive to the molecular structure of the carbohydrate and could be due to the rotation of the whole molecule in the more mobile regions in the glass ref. Dielectric 
hole burning experiments have clearly revealed the existence of dynamic heterogeneity in sorbitol glass whereby secondary process ( $\beta$ relaxation) was longer than the relaxation time of the melt state.

We have recently shown that phosphorescence from ErythrosinB can be used to monitor both the molecular mobility and oxygen diffusion in amorphous $\beta-\mathrm{Lg}$ and $\beta-\mathrm{Lg}$ sucrose mixtures (Sundaresan and Ludescher, 2007). We showed that the lifetime provides information about the rate of matrix collisions with the excited triplet state that quench the phosphorescence intensity. We report here that the phosphorescence lifetime of Ery $\mathrm{B}$ doped $\beta-\mathrm{Lg}$ and $\beta$ - $\mathrm{Lg}$ sucrose mixtures varies systematically with emission wavelength in a manner that provides valuable information about variations in the molecular mobility and about dynamic site heterogeneities within the matrix.

\section{Materials and Methods}

\section{Sample preparation}

Solutions of $\beta-\operatorname{Lg}$ (90\% pure; crystallized and lyophilized Sigma Chemical Co, St.Louis, MO, USA) were prepared in distilled de-ionized water at an approximate concentration of $10 \mathrm{mg} / \mathrm{ml}$. This concentration was selected based on the batch size to ensure maximum recovery after dialysis and to avoid long-term storage. An aqueous $\beta$ Lg solution was transferred to regenerated cellulose dialysis tubing having a $12-14 \mathrm{kDa}$ molecular weight cutoff (Spectrum, Houston, TX). In order to define and minimize the presence of counter ions the solution was extensively dialyzed against $0.1 \mathrm{M} \mathrm{kcl}$ and against distilled de-ionized water for 48 hours with frequent change of buffer every 6 hours. Several dilutions of a stock solution of dialyzed $\beta-\mathrm{Lg}$ were prepared. The dye to protein ratio was determined by measuring the absorbance of dilutions in a $1 \mathrm{~cm}$ path 
length quartz cuvette in a Bausch and Lomb Spectronic 2000 spectrophotometer. The protein concentration was determined after dialysis by UV absorbance using an extinction coefficient of $0.96 \mathrm{~L} \mathrm{~g}^{-1} \mathrm{~cm}^{-1}$.

Sucrose was purchased from Sigma-Aldrich (St. Louis, MO) with minimum purity of $98 \%$ and was used without further purification. These components were dissolved to near saturation in deionized water at room temperature. To study the site heterogeneity of protein/sucrose films a molar ratio of 10:1 (sucrose to $\beta$ - $\mathrm{Lg}$ ) was chosen. This was chosen as opposed to other sucrose concentrations because this was the desired ratio at which a noticeable change in permeability was observed.

Erythrosin B (Ery B) (tetra iodofluorescein, FD\&C red\#3) from molecular probes (Eugene, OR), was dissolved in spectrophotometric grade N,N-dimethyl formamide (DMF) (Aldrich Chemical, Milwaukee, WI) to make 10mM and 100mM stock solutions. After estimating the concentration protein solution was combined with aliquots of dye solution to make solutions with molar ratio of EryB: $\beta$-Lg of $1: 4$. The dye to protein concentration was sufficient to provide adequate signal /noise in spectroscopic measurements.

Preparation of films

To make glassy films $15 \mu \mathrm{l}$ of protein or protein/ sucrose solutions were spread on approximately one third of a quartz slide $(30 \times 13.5 \times 0.6 \mathrm{~mm}$; custom made by NSG Precision Cells, Farmingdale, NY). The films approximately (0.05 mm thick when dried) were visually checked for good spreading, uniformity, and consistency. The slides were stored at room temperature in a desiccator containing $\mathrm{P}_{2} \mathrm{O}_{5}$ and DrieRite for at least 7 days and protected from light to prevent any photo bleaching of Erythrosin B prior to 
luminescence measurements. The slides were checked for crystallization under crossed polarizers using a Nikon Type 102 dissecting microscope (Nikon, Inc., Japan).

\section{Luminescence measurements and analysis}

All luminescence measurements were made on a Cary Eclipse Fluorescence Spectrophotometer (Varian Instruments, Walnut Creek, CA). A slide was fitted diagonally into a standard fluorescence cuvette, which was flushed with oxygen-free $\mathrm{N}_{2}$ gas for at least 15 min prior to measurements. The temperature was controlled using a TLC 50 thermoelectric heating/cooling system (Quantum Northwest, Spokane, WA).

Phosphorescence emission spectra as a function of delay time following excitation at $525 \mathrm{~nm}$ (bandwidth $20 \mathrm{~nm}$ ) were collected from 640 to $740 \mathrm{~nm}$ (bandwidth $10 \mathrm{~nm}$ ). The emission intensity was collected from a single lamp flash over a 0.5 -ms gate window following a delay time that varied from 0.1 to $1.6 \mathrm{~ms} ; 10$ cycles of excitation were averaged. Phosphorescence spectra were converted to intensity versus frequency $\left(\mathrm{cm}^{-1}\right)$ and analyzed to obtain the peak frequency $\left(\mathrm{v}_{\mathrm{P}}\right)$ and spectral bandwidth $(\Gamma$, full width at half maxima) using a lognormal function. The emission spectra were analyzed by fitting both the delayed fluorescence and the phosphorescence to a lognormal function.

$$
\mathrm{I}(\mathrm{v})=\mathrm{I}_{0} \exp \left\{-\ln (2)\left[\ln \left(1+2 \mathrm{~b}\left(\mathrm{v}-\mathrm{v}_{\mathrm{p}}\right) / \Delta\right) / \mathrm{b}\right]^{2}\right\}
$$

In this equation $I_{0}$ is the maximum emission intensity, $v_{P}$ is the frequency $\left(\mathrm{cm}^{-1}\right)$ of the emission maximum, $\Delta$ is a linewidth parameter, and $\mathrm{b}$ is an asymmetry parameter The bandwidth $(\Gamma)$ of the emission band is related to $\mathrm{b}$ and $\Delta$ :

$$
\Gamma=\Delta\{\sinh (b) / b\}
$$

Intensity decays were collected as a function of emission wavelength over the emission range from $640 \mathrm{~nm}$ to $740 \mathrm{~nm}$ at $10 \mathrm{~nm}$ increments with an excitation wavelength of 
$540 \mathrm{~nm}$. The temperature range was $0-100^{\circ} \mathrm{C}$, and the excitation and emission bandwidths monochromators were both set at $20 \mathrm{~nm}$ band pass, with a delay time of $0.1 \mathrm{~ms}$, gate time of $0.02 \mathrm{~ms}$, and a total decay time of $4.0 \mathrm{~ms}$. The intensity transients $(\mathrm{I}(\mathrm{t}))$ were analyzed using a stretch-exponential decay function.

$$
\mathrm{I}(\mathrm{t})=\mathrm{I}(0) \exp [-(\mathrm{t} / \tau) \beta]+\mathrm{constant}
$$

The measured emission rate for phosphorescence $\left(\mathrm{k}_{\mathrm{P}}=1 / \tau\right)$ is the sum of all possible deexcitation rates for the triplet state $\mathrm{T}_{1}$

$$
\mathrm{k}_{\mathrm{P}}=\mathrm{k}_{\mathrm{RP}}+\mathrm{k}_{\mathrm{TS} 0}(\mathrm{~T})+\mathrm{k}_{\mathrm{TS} 1}(\mathrm{~T})
$$

$\mathrm{k}_{\mathrm{RP}}$ is the rate of radiative emission to the ground state $\mathrm{S}_{0}, \mathrm{k}_{\mathrm{TS} 0}$ is the rate of intersystem crossing to $\mathrm{S}_{0}$ (rate of collisional quenching), and $\mathrm{k}_{\mathrm{TS} 1}$ is the rate of reverse intersystem crossing to the excited singlet $\mathrm{S}_{1}$; only the latter two rate constants vary with temperature.

The rate of radiative emission $\left(\mathrm{k}_{\mathrm{RP}}\right)$ is $41 \mathrm{~s}^{-1}$ and constant. (Duchowicz et al., 1998; Lettinga et al., 2000), the rate of reverse intersystem crossing to $\mathrm{S}_{1}\left(\mathrm{k}_{\mathrm{TS} 1}\right)$ is a thermally activated process. $\mathrm{k}_{\mathrm{TS} 1}$ the rate of reverse intersystem crossing from the excited triplet state to the excited singlet state depends on $\Delta E_{T S}$, the energy gap between $S_{1}$ and $\mathrm{T}_{1}$

$$
\mathrm{k}_{\mathrm{TS} 1}(\mathrm{~T})=\mathrm{k}_{\mathrm{TS} 1}^{\circ} \exp \left(-\Delta \mathrm{E}_{\mathrm{TS}} / \mathrm{RT}\right)
$$

$\Delta \mathrm{E}_{\mathrm{TS}}$ is the energy gap between $\mathrm{S}_{1}$ and $\mathrm{T}_{1}, \mathrm{R}=8.314 \mathrm{~J} \mathrm{~K}^{-1} \mathrm{~mol}^{-1}$ and $\mathrm{T}$ is the temperature in Kelvin. The value of $\Delta \mathrm{E}_{\mathrm{TS}}$ is calculated from the slope of a Van't Hoff plot of the natural logarithm of the ratio of intensity of delayed fluorescence $\left(\mathrm{I}_{\mathrm{DF}}\right)$ to phosphorescence $\left(I_{P}\right)\left(\ln \left(I_{D F} / I_{P}\right)\right.$ vs $1 / T$.

Unfortunately, literature values of $\mathrm{k}^{\circ}{ }_{\mathrm{TS} 1}$ for Erythrosin B vary widely, from $0.3 \mathrm{x}$ $10^{7} \mathrm{~s}^{-1}$ in ethanol and $6.5 \times 10^{7} \mathrm{~s}^{-1}$ in water (Duchowicz et al., 1998) to $111 \times 10^{7} \mathrm{~s}^{-1}$ in 
solid polyvinyl alcohol, (Lettinga et al., 2000) and thus provide little guidance. We estimated the maximum possible value for $\mathrm{k}^{\mathbf{o}}{ }_{\mathrm{TS} 1}$ in films by assuming that $\mathrm{k}^{\mathbf{o}}{ }_{\mathrm{TS} 1}(\mathrm{~T})$ cannot result in values for $\mathrm{k}_{\mathrm{TS} 0}$ that decrease with temperature. This procedure thus estimated the minimum possible values of $\mathrm{k}_{\mathrm{Ts} 0}(\mathrm{~T})$. Emission spectra were fit using the program Igor (Wavemetrics, Inc., Lake Oswego, OR, USA). We also analyzed some data by fitting with the program Nfit (Island Products, Galveston, TX, USA) to make sure that the results agreed in both fitting programs.

\section{$\underline{\text { Results }}$}

Time- resolved phosphorescence emission spectra

Time-resolved emission spectra for Ery B in amorphous $\beta$ - $\mathrm{Lg}$ films as a function of delay time are depicted in Figure IV-1. The effect of delay time on the peak frequency of phosphorescence emission spectra of $\beta-\mathrm{Lg}$ films and sucrose / $\beta$ - $\mathrm{Lg}$ films at a ratio of 10:1 sucrose/ $\beta$-Lg are depicted in Figures IV-2, IV-3.

The phosphorescence emission of Erythrosin B in amorphous $\beta$-Lg films and $\beta$ $\mathrm{Lg} /$ sucrose films exhibited a blue shift to higher energy as a function of time which is unexpected and is not according to the homogenous relaxation model (Lakowicz, 1999; Richert, 2000) where emission energy is expected to decrease as the delay time after excitation increases. These shifts were approximately linear in time and of consistent magnitude $\left(\sim 50 \mathrm{~cm}^{-1}\right.$ for every $0.5 \mathrm{~ms}$ increase in delay time $)$ at all temperatures measured from -20 to $65^{\circ} \mathrm{C}$ in $\beta$-Lg films and from 0 to $65^{\circ} \mathrm{C}$ in $\beta$ - $\mathrm{Lg} /$ sucrose films. As already discussed in chapter III the measured emission energies in the presence of sugars were significantly higher when compared to the pure protein films at all temperatures, owing to decreased dipolar relaxation. 
Decreases in frequency with temperature reflected additional stabilization of the triplet state at higher temperature due to increasing dipolar relaxation rate. We observe that the magnitude of decrease in emission energy with temperature $\left(\Delta v_{\mathrm{P}}\right)$ was gradual and consistent in the pure $\beta$ - $\mathrm{Lg}$ films. $\Delta v_{\mathrm{P}}$ was $\sim 46 \mathrm{~cm}^{-1}$ from $-20^{\circ} \mathrm{C}$ to $0^{\circ} \mathrm{C}$ over the range from 0.1 to $2.5 \mathrm{~ms}$ delay time. But in sucrose / $\beta$-Lg films the decrease was gradual at low temperatures but as the $\mathrm{Tg}$ of sucrose $\left(62^{\circ} \mathrm{C}\right.$ ref $)$ was approached we noticed a larger decrease in the emission energy ( 0 to $25^{\circ} \mathrm{C}$ the decrease in the emission energy was $\sim 40$ $\mathrm{cm}^{-1}$ and from 25 to $65^{\circ} \mathrm{C} \Delta v_{\mathrm{P}} \sim 250 \mathrm{~cm}^{-1}$ over the range from 0.1 to $2.5 \mathrm{~ms}$ delay time) showing the effect of the sucrose glass transition in the $\beta$-Lg matrix.

The bandwidth $\left(\Gamma_{\mathrm{P}}\right)$, the FWHM provides a direct measure of the width of the distributions of energetically distinct matrix sites. The bandwidth $\left(\Gamma_{\mathrm{P}}\right)$, of the emission band in $\beta-\mathrm{Lg}$ films decreased slightly with change in delay time from $0.1 \mathrm{~ms}$ to $0.6 \mathrm{~ms}$ and then increased with increase in delay time at all temperatures in $\beta$ - $\mathrm{Lg}$ films. The change was gradual at low temperature $\left(12 \mathrm{~cm}^{-1}\right.$ at $-20^{\circ} \mathrm{C}$ to $\left.25^{\circ} \mathrm{C}\right)$ and greater at a temperature of $65^{\circ} \mathrm{C}\left(\sim 23 \mathrm{~cm}^{-1}\right)$ (Figure IV-4). Sucrose $/ \beta-\mathrm{Lg}$ films showed a small decrease in FWHM with increase in delay time from 0.1 to $2.1 \mathrm{~ms}$ (Figure IV-5). Although $\Gamma$, increased continuously with increasing temperatures in pure $\beta$ - $\mathrm{Lg}$ and sucrose/ $\beta$-Lg films but the increase was dramatic at higher temperatures suggesting that the extent of inhomogeneous broadening was larger at higher temperature

Systematic variations in the decay kinetics with emission wavelength provide a sensitive and novel indicator of the extent of dynamic site heterogeneity within the amorphous solid matrixes (Pravinata et al., 2005). The variation of $\tau$ with emission wavelength in pure $\beta$-Lg and sucrose/ $\beta-\mathrm{Lg}$ films is plotted in Figures IV-6 and IV-7. 
Phosphorescence intensity decays were measured as a function of emission wavelengths over the temperature range from $-20^{\circ} \mathrm{C}$ to $100^{\circ} \mathrm{C}$ in $\beta-\mathrm{Lg}$ and $0^{\circ} \mathrm{C}$ to $100^{\circ} \mathrm{C}$ in sucrose/ $\beta$ Lg films. In $\beta$ - $\mathrm{Lg}$ films, the lifetimes at $-20^{\circ} \mathrm{C}$ varied from a high of $0.69 \mathrm{~ms}$ at $640 \mathrm{~nm}$ to a low of $0.52 \mathrm{~ms}$ at $740 \mathrm{~nm}$; lifetimes also decreased monotonically with increasing wavelength at $-10^{\circ} \mathrm{C}$. However, at $0^{\circ} \mathrm{C}$ and above, lifetimes increased at the blue edge to a maximum at 660-670 $\mathrm{nm}$ and then decreased at higher wavelengths.

In sucrose/ $\beta$ - $\mathrm{Lg}$ films at 0,10 and $20^{\circ} \mathrm{C}$ the lifetimes decreased systematically with increase in emission wavelength up to $690 \mathrm{~nm}$ and was constant over the remaining emission wavelength range. However, at temperatures from $30^{\circ} \mathrm{C}$ and above, the lifetimes increased at intermediate wavelengths up to $660-680 \mathrm{~nm}$ and then remained constant. These trends in sucrose / $\beta$ - $\mathrm{Lg}$ films are similar to that observed in amorphous sucrose films (Pravinata et al., 2005). As expected, the lifetime values for all emission wavelengths decreased as temperature increased.

Variations of the stretching exponent with emission wavelength for both $\beta$-Lg and $\beta-\mathrm{Lg}$ sucrose films at several temperatures are plotted in Figure IV-8 and IV-9. The stretching exponent $\beta$ is a measure of the kinetic heterogeneity in the intensity decays. A value of 1 indicates a single exponent decay, while values less than 1 indicate increasing width of an asymmetric distribution. For Erythrosin $\mathrm{B}$ in $\beta-\mathrm{Lg}$ at $-20^{\circ} \mathrm{C}$, there was a very narrow distribution of lifetimes over the entire temperature range. At $-10^{\circ} \mathrm{C}, \beta$ increased with increasing wavelength from 0.80 at $640 \mathrm{~nm}$ to a high of $0.88 \mathrm{up}$ at $690 \mathrm{~nm}$ and then decreased. This trend was consistent over the entire temperature range. The $\beta$ values were consistently lower at the red and the blue edges of the spectrum. $\beta$ values in sucrose/ $\beta$ - $\mathrm{Lg}$ 
films varied greatly at the blue edge $(640-670 \mathrm{~nm})$, but were essentially constant at higher wavelengths.

The variation in lifetime across the emission bands serves as an indicator of the dynamic heterogeneity. Studies in the past have shown that amorphous proteins and sugars (Nack and Ludescher, 2006; Pravinata et al., 2005; Shirke and Ludescher, 2005) have shown that the variation in lifetimes as a function of wavelength is a reflection of the variation of $\mathrm{k}_{\mathrm{TS} 0}$, with probes in high energy sites having smaller values of $\mathrm{k}_{\mathrm{TS} 0}$ and probes in low energy sites having higher $\mathrm{k}_{\mathrm{TS} 0}$ values.

Trends in $\mathrm{k}_{\mathrm{TS} 0}$ are reflective of the trends in lifetime in the emission wavelength range. The calculated quenching rate $\mathrm{k}_{\mathrm{TS} 0}$ varied with emission wavelength in $\beta$-Lg and $\beta$-Lg sucrose films.

Figure IV-10 is a plot of the wavelength dependence of $\mathrm{k}_{\mathrm{TS} 0}$ in $\beta$-Lg films. The calculated quenching rate $\mathrm{k}_{\mathrm{TS} 0}$, increased with increasing emission wavelength at $-20^{\circ} \mathrm{C}$. At $-10^{\circ} \mathrm{C}$ and above, the $\mathrm{k}_{\mathrm{TS} 0}$ values decreased at the blue edge to a minimum at $660-670$ $\mathrm{nm}$ and then increased linearly at higher wavelengths. However, at higher temperatures $\left(80-100^{\circ} \mathrm{C}\right)$ no clear trend in $\mathrm{k}_{\mathrm{TS} 0}$ was observed

In $\beta$ - $\mathrm{Lg}$ films the variation in $\mathrm{k}_{\mathrm{TS} 0}$ was lower at the temperature range from $-20^{\circ} \mathrm{C}$ to $20^{\circ} \mathrm{C}$ across the emission band suggesting that the spectral heterogeneity was minimal at low temperatures. However, the variation in $\mathrm{k}_{\mathrm{Ts} 0}$ enhanced greatly after the softening temperature of $\sim 80^{\circ} \mathrm{C}$. Since the variation of $\mathrm{k}_{\mathrm{TS} 0}$ with wavelength reflects the presence of a broad continuum of local matrix sites that vary in terms of their overall molecular mobility, increase in temperature appears to increase the variation across this continuum in the $\beta$ - $\mathrm{Lg}$ matrix. $\mathrm{k}_{\mathrm{TS} 0}$ was also the highest at the blue edge and the red edges. 
The measured $\mathrm{k}_{\mathrm{TS} 0}$ values of sucrose / $\beta$ - $\mathrm{Lg}$ films were significantly lower than the $\beta$-Lg films at all temperatures (Figure IV-11). In sucrose / $\beta$ - $\mathrm{Lg}$ films $\mathrm{k}_{\mathrm{TS} 0}$ values at a temperature range of $0-40^{\circ} \mathrm{C}$, increased with increase in wavelength peaked at $690 \mathrm{~nm}$ and then remained constant. However at $50-100^{\circ} \mathrm{C}, \mathrm{k}_{\mathrm{TS} 0}$ decreased at intermediate wavelengths up to $660-680 \mathrm{~nm}$ and then was constant for the remaining emission wavelength range. Variation of $\mathrm{k}_{\mathrm{TS} 0}$ was lower in sucrose / $\beta$ - $\mathrm{Lg}$ films when compared to pure $\beta$ - $\mathrm{Lg}$ films, indicating lesser spectral heterogeneity in $\beta-\mathrm{Lg}$ in the presence of sucrose. With increase in temperature there was an increase in $\mathrm{k}_{\mathrm{TS} 0}$ in the entire temperature range, the increase being more pronounced at temperatures approaching the glass transition.

Arrhenius analysis of the temperature dependence of the rate constant for collisional quenching $\mathrm{k}_{\mathrm{TS} 0}$ at each wavelength provides the activation energy $\mathrm{E}_{\mathrm{A}}$. The values for activation energy in $\beta-\mathrm{Lg}$ and $\beta-\mathrm{Lg}$ sucrose films are plotted as function of wavelength in Figure IV-12 and IV-13 respectively. The activation energy $\mathrm{E}_{\mathrm{A}}$ for nonradiative quenching was significantly greater at high temperatures (three fold higher), than at low temperatures in the $\beta$ - $\mathrm{Lg}$ matrix. Activation energy $\mathrm{E}_{\mathrm{A}}$ varied from $3.3 \mathrm{~kJ}$ $\mathrm{mol}^{-1}$ to $0.3 \mathrm{kJmol}^{-1}$ at low temperatures $\left(0-20^{\circ} \mathrm{C}\right)$ and from 12.1 to $4.1 \mathrm{kJmol}^{-1}$ at high temperatures $\left(60-80^{\circ} \mathrm{C}\right)$ from $640 \mathrm{~nm}$ to $740 \mathrm{~nm}$. The observed trend, in which activation energy decreased with emission wavelength in $\beta$-Lg films is similar to that seen for Erythrosin B in amorphous sucrose, sugar alcohols and BSA (Pravinata et al., 2005; Shirke et al., 2005a: Shirke et al., 2005b: Nack and Ludescher., 2006)

In $\beta$-Lg sucrose films activation energy $\mathrm{E}_{\mathrm{A}}\left(0-100^{\circ} \mathrm{C}\right)$ decreased with increase in wavelength from $10.8 \mathrm{~kJ} \mathrm{~mol}^{-1}$ at $640 \mathrm{~nm}$ to $8.6 \mathrm{~kJ} \mathrm{~mol}^{-1}$ at $740 \mathrm{~nm}$. 


\section{Discussion}

Spectral heterogeneity in Ery B phosphorescence is observed in amorphous sugars and sugar alcohols (Pravinata et al., 2005; Shirke et al., 2005, 2006) and proteins (Nack and Ludescher, 2006; Lukasik and Ludescher, 2005) indicating that dynamic site heterogeneity may be a characteristic feature of amorphous food and biomaterials.

Ery B phosphorescence emission bandwidth, variations in lifetime (because of variations in $\mathrm{k}_{\mathrm{TS} 0}$ ) across the emission band and the emission decay stretching exponent $\beta$ all provide direct information about matrix heterogeneity. A study by Ediger (2000) on the dynamics of super-cooled liquids revealed that near the glass transition, dynamics in one region of a super-cooled liquid can be orders of magnitude faster than dynamics in another region only a few nanometers away. This heterogeneity in dynamics has important consequences for understanding transport properties and the kinetics of chemical reactions in such materials.

Matrix heterogeneity can arise from changes in composition. The dynamic site heterogeneity is associated with the variations in packing density of the regions in the matrix. In the case of a protein sugar matrix, specific hydrogen bonding interactions will affect the packing and thus will have an overall impact on the stability and heterogeneity of the matrix. So the present study investigated the differences in heterogeneity between pure $\beta$ - Lg and sucrose / $\beta$-Lg films. The triplet state photo-physics within amorphous $\beta$ $\operatorname{Lg}$ and $\beta-\mathrm{Lg}$ sucrose films are modulated primarily by two dynamic properties of the matrix; dipolar relaxations that lower the energy of the excited triplet state and molecular motions that quench the excited triplet state. 
The spectral features reported here for Erythrosin B in amorphous $\beta$-Lg matrix are very similar to those reported for the same probe in other proteins and sugars. In this chapter we looked into heterogeneities in the $\beta$-Lg matrix in two different ways: (1) The effect of temperature (2) The effect of change in composition.

\section{Dynamic site heterogeneity in the pure $\beta$-Lg matrix}

In the pure $\beta$-Lg matrix, the decrease in emission energy across the emission band is not consistent with the standard relaxation model, in which longer lifetime probes have red shifted emission due to greater dipolar relaxations around the longer lived excited states (Pravinata et al., 2005). However, this decrease is consistent with the dynamic site heterogeneity model in which probes are distributed in sites, which differ in mobility.

The local environments within the $\beta$-Lg matrix behave differently, whereby the blue sites have lower molecular mobility (lower $\mathrm{k}_{\mathrm{TS} 0}$ ), and the red shifted sites have faster modes of mobility (higher $\mathrm{k}_{\mathrm{TS} 0}$ ). A study by Pravinata et al. (2005) explained this phenomenon effectively in amorphous sucrose by the dynamic site heterogeneity model in which probes are distributed among a continuum of matrix environments ranging from more rigid (blue-shifted emission) to more mobile (red-shifted emission) sites.

Rigid sites have slower dipolar relaxation rates and slower collisional quenching rates; these are sites of lower overall molecular mobility. Mobile sites, on the other hand, have faster dipolar relaxation rates and faster collisional quenching rates; these sites of higher overall molecular mobility may be physical regions of the amorphous matrix that are organized into smaller aggregates of weakly interacting molecules, probably due to less extensive hydrogen bonding. 
The $\mathrm{E}_{\mathrm{A}}$ values of $\mathrm{k}_{\mathrm{TS} 0}$ at various wavelengths provide insight on the variation of matrix mobility in the $\beta$-Lg matrix. It is clear from this study that the blue shifted sites have higher apparent activation energies then red shifted sites. Similar trends were noticed in amorphous BSA (Nack and Ludescher, 2006). Higher values of $\mathrm{E}_{\mathrm{A}}$ at blue shifted sites provide evidence that the molecular collisions that activate non-radiative quenching involve collective motions of the larger regions of the protein matrix.

Change in temperature brought about differences in spectral heterogeneity in the $\beta$-Lg matrix. At $-20^{\circ} \mathrm{C}$ there was a slight linear decrease in the lifetimes across the emission band, indicating that the probe molecules resided in a rigid atmosphere, whereby changes in heterogeneity were minimal. At $0^{\circ} \mathrm{C}$ and above the lifetimes were highest at 660-670 $\mathrm{nm}$ and decreased linearly with further increase in wavelength. This observation is indicative of the presence of distinct regions of mobility in the pure $\beta$ - $\mathrm{Lg}$ matrix where there are pockets of high and low mobility. Further increase in temperature $\left(80-100^{\circ} \mathrm{C}\right)$ however seemed to obliterate any possible heterogeneity, as we see the flattening of the curves. $\mathrm{k}_{\mathrm{TS} 0}$ was low at lower temperatures and enhanced greatly after the softening temperature of $\beta$ - $\operatorname{Lg}\left(\sim 81^{\circ} \mathrm{C}\right)$ (Sundaresan and Ludescher, 2007).

The stretching exponent $\beta$ provides a non linear measure of the width of the lifetime distribution required to fit emission decay (Lindsey and Paterson, 1980), a value of 1 indicating an infinitely narrow distribution and lower values indicating significantly broader lifetime distributions. $\beta$ values varied significantly as a function of emission wavelength indicating that the probe molecules are distributed in different local environments in the $\beta$ - $\mathrm{Lg}$ matrix. 
Temperature had a great effect in varying the distribution of these local mobilities. The stretching exponent was high and didn't change significantly, indicating lower matrix heterogeneity at low temperatures $\left(-20^{\circ} \mathrm{C}\right)$. There was a decrease in $\beta$ with further increase in temperature indicating an increase in the degree of dynamic heterogeneity. A broader lifetime distribution is due to the probe sensing multiple mobility sites or more diverse local environments, which indicates higher range of mobility at higher temperatures. An increase in temperature increases the heterogeneity ( $\beta$ decreases) but eliminates coupling (lifetime variation at wavelength is lost).

Measurements of emission energy as a function of delay time showed that as the delay time increased the peak frequency increased. The short delay time reflects the average emission energy from all the chromophores distributed and the long delay time is indicative of the emission energies due to high-energy sites that have long lived chromophores.

These findings suggest the existence of a coupling between the rates of dipolar relaxations and molecular collisions within the amorphous $\beta-\mathrm{Lg}$ matrix, which gives, rise to dynamic site heterogeneity. Such heterogeneities may be especially important giving rise to local mobile regions, where the rates of reaction are significantly higher than the matrix average (Ediger, 2000; Shirke and Ludescher, 2005). Identification of such regions can be of great use to food technologists.

\section{Effect of sucrose on the dynamic site heterogeneity of the matrix}

Previous studies on the effect of sucrose on the $\beta$-Lg matrix have clearly shown that sucrose had a significant effect on the overall mobility of the matrix. We attribute this to sucrose affecting the packing density in the polymer molecule by different 
hydrogen bonding interactions. Studies by You and Ludescher (2007) and Lukasik and Ludescher (2005) have clearly showed that addition of a small molecule can have a significant impact on the heterogeneity of a polymer matrix. This study wanted to analyze the effect of change in composition by adding sucrose to the $\beta$ - $\mathrm{Lg}$ matrix and studies the changes in heterogeneity of the matrix. This study can answer important questions related to stability of edible films and encapsulated materials made with a combination of proteins and sugars.

The variation in lifetime (and thus $\mathrm{k}_{\mathrm{TS} 0}$ ) across the emission band provides an indicator of the dynamic heterogeneity. Ery B molecules in sucrose $\beta$ - $\mathrm{Lg}$ films appear to be distributed among the continuum of matrix sites that differ in mobility. A wide variation of lifetimes (thus $\mathrm{k}_{\mathrm{Ts} 0}$ ) was essentially observed at the blue edge and the lifetimes were essentially constant at higher wavelengths $(690-740 \mathrm{~nm})$ in the sucrose / $\beta$ Lg matrix. It thus appears that although blue shifted sites have greater rigidity and lower average molecular mobility but have a wide variation in the $\mathrm{k}_{\mathrm{TS} 0}$.

A comparative plot of the effect of temperature on the lifetime variation with wavelength reveals that the lifetimes in $\beta$-Lg sucrose films (Figure IV-14) were higher than the $\beta-\mathrm{Lg}$ films at $25^{\circ} \mathrm{C}$. In comparison with $\beta-\mathrm{Lg}$, variation in lifetime with wavelength (thus reflecting variations in $\mathrm{k}_{\mathrm{TS} 0}$ ) at $25^{\circ} \mathrm{C}$ in sucrose $/ \beta$-Lg films were smaller indicating that in the presence of sucrose spectral heterogeneity decreased in the $\beta-\mathrm{Lg}$ matrix. In this temperature sucrose $-\beta-\operatorname{Lg}$ interactions throughout the matrix resulted in the formation of a strong hydrogen- bonded network making the matrix more homogenous. Small molecules like sucrose confer enough rigidity that significantly 
affects the packing density of the local pockets in which the probe resides and thus lowers the site heterogeneity.

The variation in lifetime with wavelength decreased at $65^{\circ} \mathrm{C}$ as noticed by the super-imposable curves. This trend clearly indicates that as the glass transition of sucrose was approached differences in heterogeneities were reduced.

The stretching exponent $\beta$ provides a non linear measure of the width of the lifetime distribution required to fit an emission decay transient, a value of 1 indicating an infinitely narrow distribution and lower values indicating significantly broader lifetime distributions (Lindsey and Paterson, 1980). The magnitude $\beta$ of provides another indicator of dynamic heterogeneity. Significantly lower $\beta$ values at the blue edge clearly indicate that the blue shifted sites exhibit a broader distribution of decay times, and thus a broader distribution of $\mathrm{k}_{\mathrm{TS} 0}$ values.

In $\beta-\mathrm{Lg} /$ sucrose matrix the graph depicting the variation in the lifetime with wavelength clearly indicates that lifetime is clearly higher in the blue edges when compared to the red edges. It thus appears that although the blue shifted sites have higher rigidity, they also display broader distribution of dynamic environments. Thus in the $\beta-$ $\operatorname{Lg} /$ sucrose matrix the physical coupling between $\beta$ and $\tau$ is not evident. Similar observations were made by Shirke et al. (2005) in amorphous maltose and maltitol. They proposed an appropriate photo-physical model to describe these trends effectively. Ery B probes are distributed among a continuum of matrix environments ranging from blue shifted to red shifted sites. Blue sites have greater rigidity characterized by lower dipolar relaxation rates, lower collisional quenching rates and higher activation energies for collisional quenching. However, they exhibit a great variability in the rates of $\mathrm{k}_{\mathrm{TS} 0}$. Red 
sites, on the other hand, have greater mobility marked by faster dipolar relaxations, faster collisional quenching rates, lower activation energies for collisional quenching and lower variability in the rates of collisional quenching.

The stretching exponent $\beta$ was significantly higher in sucrose / $\beta$-Lg films than the pure $\beta$-Lg films showing that addition of sucrose homogenized the dynamic environments in the $\beta$ - $\operatorname{Lg}$ matrix, within the measured time frame of the probe. At $25^{\circ} \mathrm{C}$, $\beta$ was essentially higher in sucrose $\beta$ - $\operatorname{Lg}$ film when compared to $\beta$ - $\operatorname{Lg}$ films and was constant over the entire wavelength (Figure IV-15). With increase in temperature $\beta$ decreased with increase in temperature indicating a high degree of dynamic heterogeneity within the melt. At $65^{\circ} \mathrm{C}$, in sucrose $\beta$ - $\mathrm{Lg}$ film, trends in $\beta$ were less uniform at the blue edge but were essentially constant at higher wavelengths (above $690 \mathrm{~nm}$ ).

Increase in temperature showed two contrasting trends in spectral heterogeneity in $\beta$ - $\operatorname{Lg}$ and sucrose/ $\beta$ - $\operatorname{Lg}$ films, decrease in $\beta$ (increase in the distribution heterogeneity) and decrease in the variations in lifetime. The salient effect of temperature can be explained as follows: At low temperatures high $\beta$ values and a greater variability in lifetimes, shows the existence of a physical coupling between $\beta$ and lifetime.

However, increase in temperature marked a significant decrease in $\beta$ (an increase in the distribution heterogeneity) but also resulted in a significant decrease in the variation of lifetime (thus $\mathrm{k}_{\mathrm{TS} 0}$ ) suggesting that at high temperatures the coupling between $\beta$ and lifetimes was lost. .

The phosphorescence spectrum can be acquired as a function of time, this experiment is sensitive not only to the ensemble-average relaxation but also to the distribution of relaxation rates around different chromophores; changes in the width of 
the spectrum with time indicating heterogeneity in the local relaxation rates. In the sucrose / $\beta$-Lg matrix the measured emission energies were clearly higher than the pure $\beta-\mathrm{Lg}$ matrix over the entire temperature range (Figure IV-16). The spectra blue shifted with increase in delay time. The shifts towards higher wavelengths were similar in magnitude in both $\beta$ - $\mathrm{Lg}$ and $\beta-\mathrm{Lg} /$ sucrose films despite the dramatic differences in spectral heterogeneities in both matrices (decreased heterogeneity in sucrose / $\beta$-Lg films marked by reduced variation in the distribution of lifetimes). The decreased spectral heterogeneity reported by the probe Ery B in sucrose / $\beta$ - $\mathrm{Lg}$ films when compared to $\beta$ Lg films may be a limitation of this model and complementary techniques may offer a better overall picture of the trends in heterogeneity. Probes with longer lifetimes may offer a better solution to report this kind of phenomenon.

The emission bandwidth in $\beta$-Lg sucrose was significantly lower when compared to the pure $\beta$-Lg films over the entire wavelength range with the addition of sucrose (Figure IV-17). The decrease in bandwidth indicates that the distribution of site energies were narrowed upon addition of sucrose, probably as a result of formation of hydrogen bonding interactions between sucrose and protein leading to uniform allocation of site energies. The emission bandwidth increased with increase in temperature and the increase was higher as the glass transition was approached, which reflected the effect of $\mathrm{Tg}$. This particular phenomenon may be attributed to weakening of the interactions between sucrose and $\beta-\mathrm{Lg}$ at the glass transition owing to the onset of $\alpha$ relaxation in sucrose. Possible phase separation and crystallization of the local regions cannot be ruled out.

All the above findings show strong evidence of decreased dynamic heterogeneity in $\beta-\mathrm{Lg}$ in the presence of sucrose. The dynamical slowing down of the $\beta-\mathrm{Lg}$ matrix is 
suggested to be controlled by the percolation of the sugar hydrogen bonds networks. Introduction of sucrose molecules in the $\beta-\operatorname{Lg}$ matrix narrows the distribution of local regions with differences in packing density. Intermolecular association between $\beta$-Lg and sucrose make the matrix more homogenous with similar local environments.

A study on the effect of glycerol on gelatin revealed that glycerol made the gelatin matrix more homogenous (Lukasik and Ludescher, 2005). But this effect was attributed to glycerol decreasing the size of the cooperative units within the gelatin matrix, perhaps by decreasing the strength or the extent of molecular interactions within the matrix and reducing spectral heterogeneity. Glycerol effectively eliminated any coupling between the rates of matrix relaxation and collisional quenching for the Erythrosin probe. Similar observations were made by You and Ludescher, (2007). They found that glycerol effectively decreased the spectral heterogeneity in amorphous sucrose. So the effect of glycerol was similar to the effect of high temperature in the $\beta-\operatorname{Lg}$ matrix whereby increases in matrix mobility diminished the variation and the heterogeneity in the matrix. The effect of sucrose however was distinct in the sense it caused a decrease in the packing density and thus affected the mobility of the local regions in which the probe resided. The inference drawn from this study is differences in packing density (increases or decreases) can have a significant impact on the spectral heterogeneity.

\section{Conclusions}

Spectral heterogeneity in Ery B phosphorescence provides direct evidence to support the physical model of dynamic site heterogeneity. Studies on site heterogeneity can potentially address the issue of shelf stability in amorphous solids. They can potentially answer important questions related to shelf stability, which will have long- 
term implications to food science. Our data provide direct evidence indicating that amorphous solid $\beta-\mathrm{Lg}$, is organized into distinct molecular regions with characteristic rates of mobility and that this dynamic heterogeneity is reduced in the presence of sucrose. Temperature has profound effect in modulating the heterogeneity in the matrix. 


\section{$\underline{\text { References }}$}

Buera, M. P., Jouppila, K., Roos, Y. H., and Chirife, J. (1998). Differential scanning calorimetry glass transition temperatures of white bread and mold growth in the putative glassy state. Cereal Chem. 75, 64-69.

Chan, R. K., Pathmanathan, K., and Johari, G. P. (1986). Dielectric relaxations in the liquid and glassy states of glucose and its water mixtures. J. Phys. Chem. 90, 6358-6362.

Cicerone, M. T., Blackburn, F. R., and Ediger, M. D. (1995). How do molecules move near Tg? Molecular rotation of six probes in o-terphenyl across 14 decades in time. J. Chem. Phys. 102, 471-479.

Cicerone, M. T. and Ediger, M. D. (1995). Relaxation of spatially heterogeneous dynamic domains in supercooled ortho-terphenyl. J. Chem. Phys. 103, 5684-5692.

Duchowicz, R., Ferrer, M. L., and Acuña, A. U. (1998). Kinetic Spectroscopy of Erythrosin Phosphorescence and Delayed Fluorescence in Aqueous Solution at Room Temperature. Photochemistry and Photobiology. 68, 494-501.

Ediger, M. D. (2000). Spatially heterogeneous dynamics in supercooled liquids. Annu. Rev. Phys. Chem. 51, 99-128.

Fennema, O. R. and Editor. (1996). Food Chemistry, Third Edition. [In: Food Sci. Technol. (N. Y.), 1996; 76]. 1089.

Johari, G. P. (1976). GLASS TRANSITION AND SECONDARY RELAXATIONS IN MOLECULAR LIQUIDS AND CRYSTALS. Ann. N. Y. Acad. Sci. 279, 117-140.

Lakowicz, J. R. (1999). Principles of fluorescence spectroscopyKluwer Academic/Plenum Press, Dordrecht/New York.

Lettinga, M. P., Zuilhof, H., and Van Zandvoort, M. A. M. J. (2000). Phosphorescence and fluorescence characterization of fluorescein derivatives immobilized in various polymer matrices. Physical Chemistry Chemical Physics. 2, 3697-3707.

Lindsey, C. P. and Patterson, G. D. (1980). Detailed comparison of the Williams-Watts and Cole-Davidson functions. J. Chem. Phys. 73, 3348-3357.

Ludescher, R. D., Shah, N. K., McCaul, C. P., and Simon, K. V. (2001). Beyond Tg: optical luminescence measurements of molecular mobility in amorphous solid foods. Food Hydrocolloids. 15, 331-339.

Lukasik, K. V. and Ludescher, R. D. (2006a). Effect of plasticizer on dynamic site heterogeneity in cold-cast gelatin films. Food Hydrocolloids. 20, 88-95. 
Lukasik, K. V. and Ludescher, R. D. (2006b). Molecular mobility in water and glycerol plasticized cold- and hot-cast gelatin films. Food Hydrocolloids. 20, 96-105.

Maroncelli, M. and Fleming, G. R. (1987). Picosecond solvation dynamics of coumarin 153: the importance of molecular aspects of solvation. J. Chem. Phys. 86, 62216239.

McCrum, N. G. (1984). The kinetics of the alpha -relaxation in an amorphous polymer at temperatures close to the glass transition. Polymer. 25, 309-317.

Nack, T. J. and Ludescher, R. D. (2006). Molecular Mobility and Oxygen Permeability in Amorphous Bovine Serum Albumin Films. Food Biophysics. 1557-1858.

Pravinata, L. C., You, Y., and Ludescher, R. D. (2005). Erythrosin B Phosphorescence Monitors Molecular Mobility and Dynamic Site Heterogeneity in Amorphous Sucrose. Biophys. J. 88, 3551-3561.

Richert, R. (2003). Solvation dynamics in the plastic crystal and supercooled liquid state of ethanol. J.Phys.: Condens.Matter. 15, 5429-5438.

Richert, R. (2000). Triplet state solvation dynamics: Basics and applications. J. Chem. Phys. 113, 8404-8429.

Schiener, B., Chamberlin, R. V., Diezemann, G., and Bohmer, R. (1997). Nonresonant dielectric hole burning spectroscopy of supercooled liquids. J. Chem. Phys. 107, 7746-7761.

Shirke, S. and Ludescher, R. D. (2005a). Dynamic site heterogeneity in amorphous maltose and maltitol from spectral heterogeneity in erythrosin B phosphorescence. Carbohydr. Res. 340, 2661-2669.

Shirke, S. and Ludescher, R. D. (2005b). Molecular mobility and the glass transition in amorphous glucose, maltose, and maltotriose. Carbohydr. Res. 340, 2654-2660.

Shirke, S., Takhistov, P., and Ludescher, R. D. (2005). Molecular Mobility in Amorphous Maltose and Maltitol from Phosphorescence of Erythrosin B. J Phys Chem B. 109, 16119-16126.

Shirke, S., You, Y., and Ludescher, R. D. (2006). Molecular mobility and dynamic site heterogeneity in amorphous lactose and lactitol from erythrosin B phosphorescence. Biophys. Chem. 123, 122-133.

Simon-Lukasik, K. V. and Ludescher, R. D. (2004/7). Erythrosin B phosphorescence as a probe of oxygen diffusion in amorphous gelatin films. Food Hydrocolloids. 18, 621-630. 
Sundaresan, K. V. and Ludescher, R. D. (2007). Molecular mobility and oxygen permeability in amorphous beta -lactoglobulin films. Food Hydrocoll. 22, 403413.

Yoshioka, S., Aso, Y., and Kojima, S. (1997). Dependence of the molecular mobility and protein stability of freeze-dried gamma-globulin formulations on the molecular weight of dextran. Pharm. Res. 14, 736-741.

You Yumin and Ludescher. R. D. (December, 2007). The Effect of Glycerol on Molecular Mobility in Amorphous Sucrose Detected by Phosphorescence of Erythrosin B. Journal Food Biophysics. 2, 133-145. 


\section{Figure IV-1}

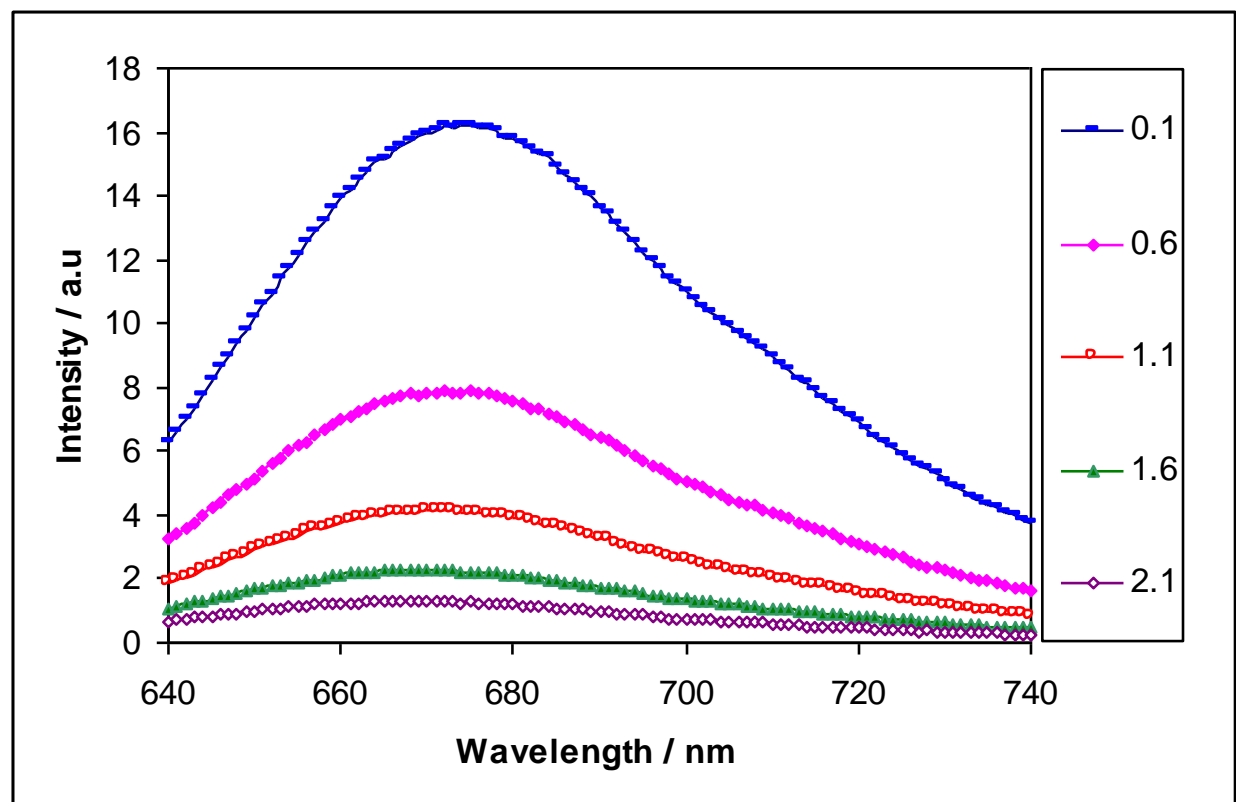

Figure IV-1: Phosphorescence emission spectra for Erythrosin B in amorphous $\beta-$ Lg films collected as a function of delay time in ms. 
Figure IV-2

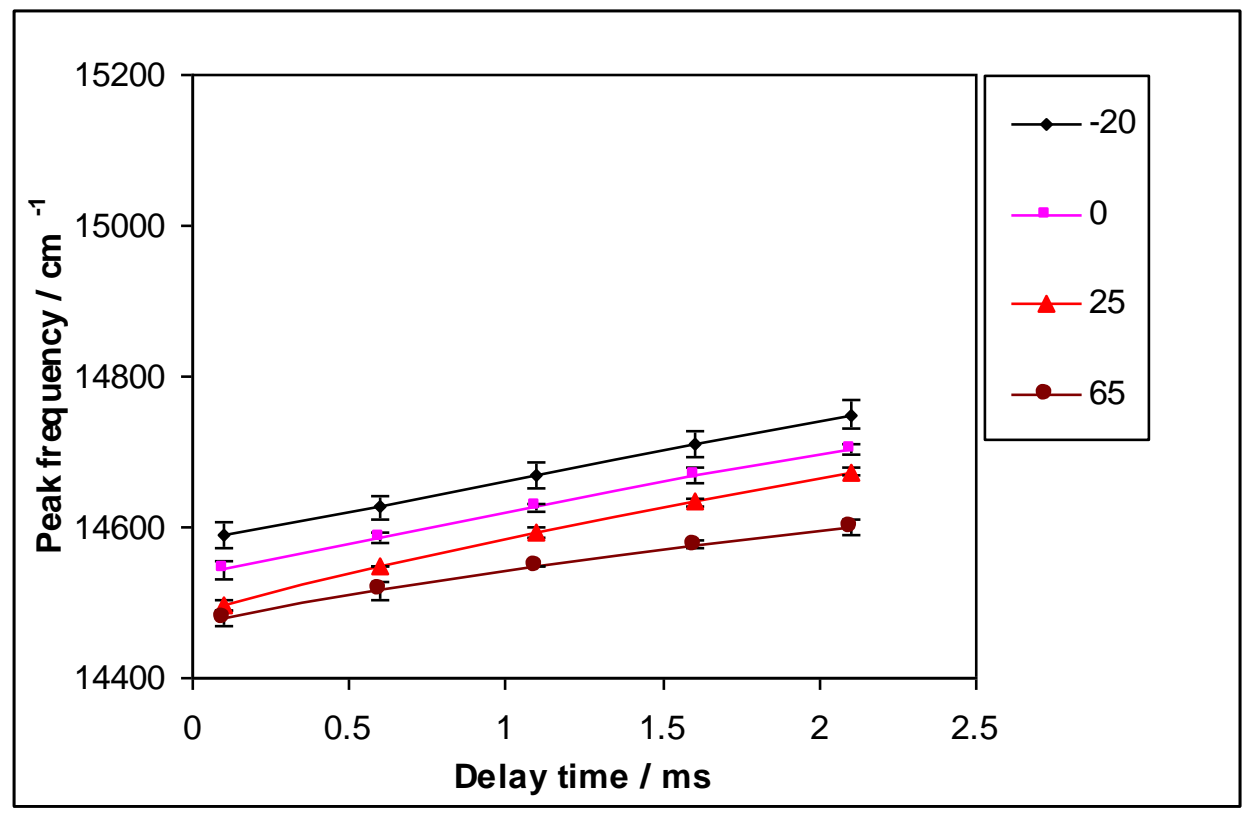

Figure IV-2: Evolution of phosphorescence emission peak frequency with time following excitation in amorphous $\beta$ - Lg film.

Figure IV-3

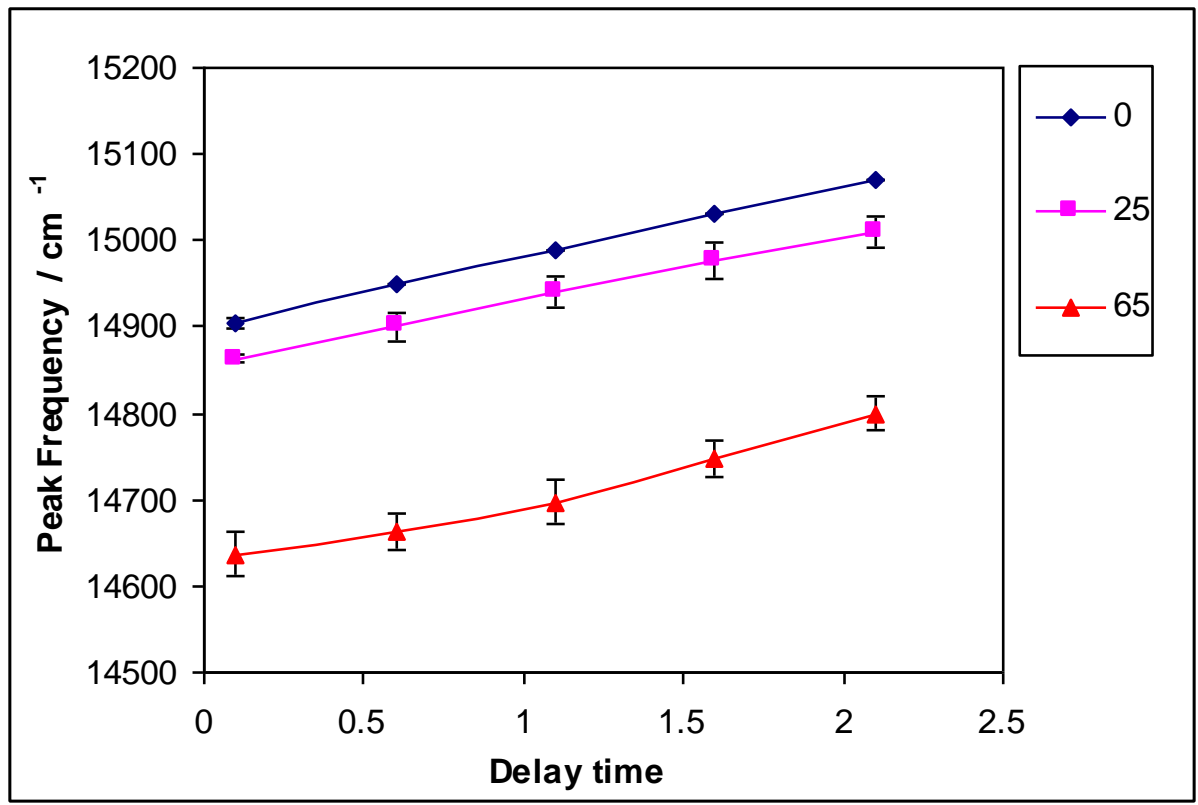

Figure IV-3: Evolution of phosphorescence emission peak frequency with time following excitation in amorphous sucrose/ $\beta-\operatorname{Lg}$ film (Ratio of 10:1). 
Figure IV-4

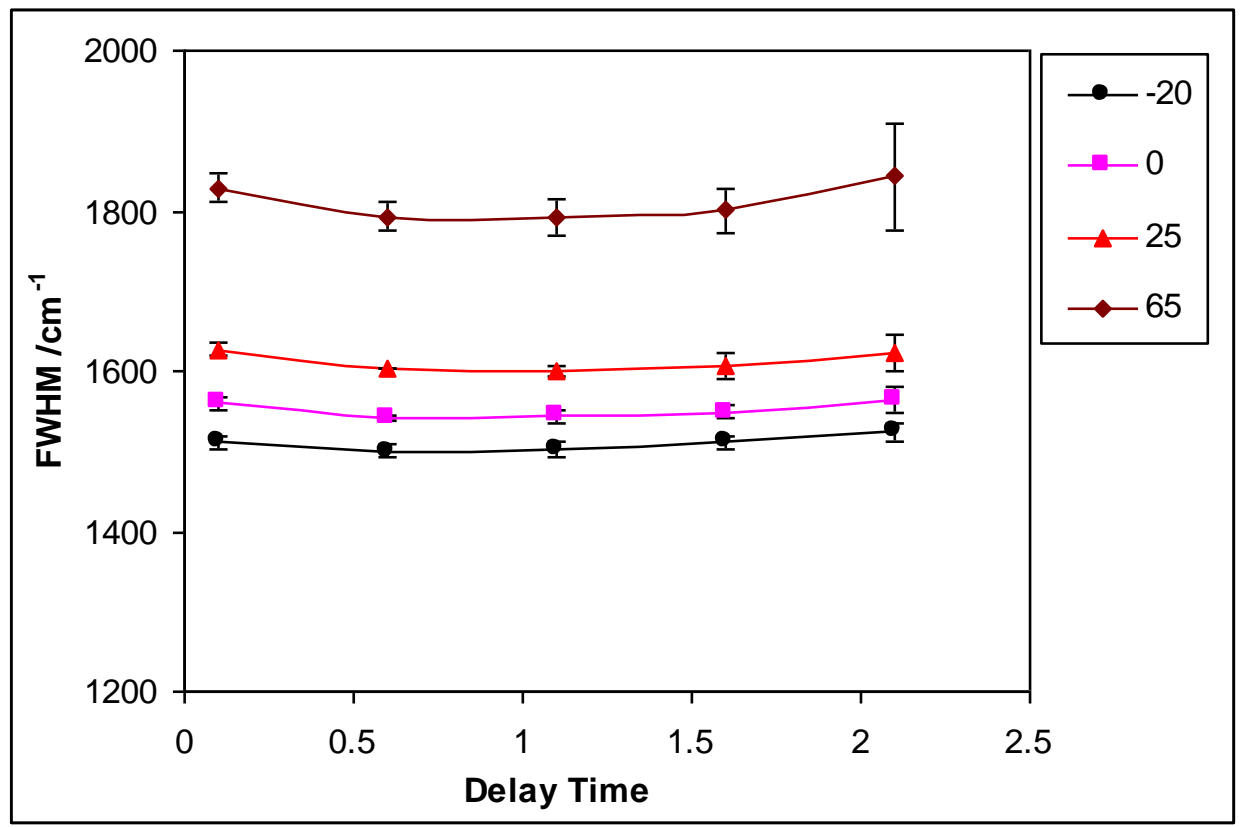

Figure IV-4: Evolution of phosphorescence emission bandwidth with time following excitation in amorphous $\beta-\mathrm{Lg}$ films.

Figure IV-5

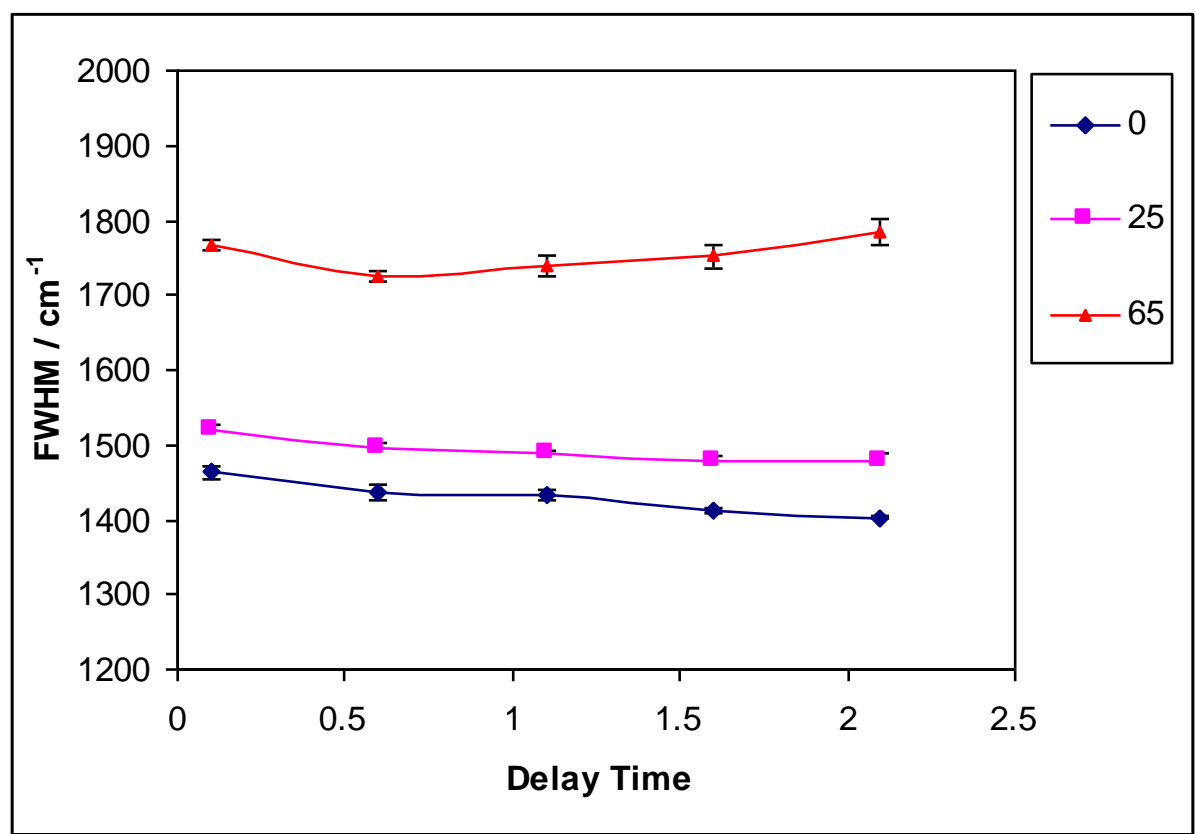

Figure IV-5: Evolution of phosphorescence emission bandwidth with time in amorphous sucrose/ $\beta$-Lg films (Ratio 10:1). 
Figure IV-6

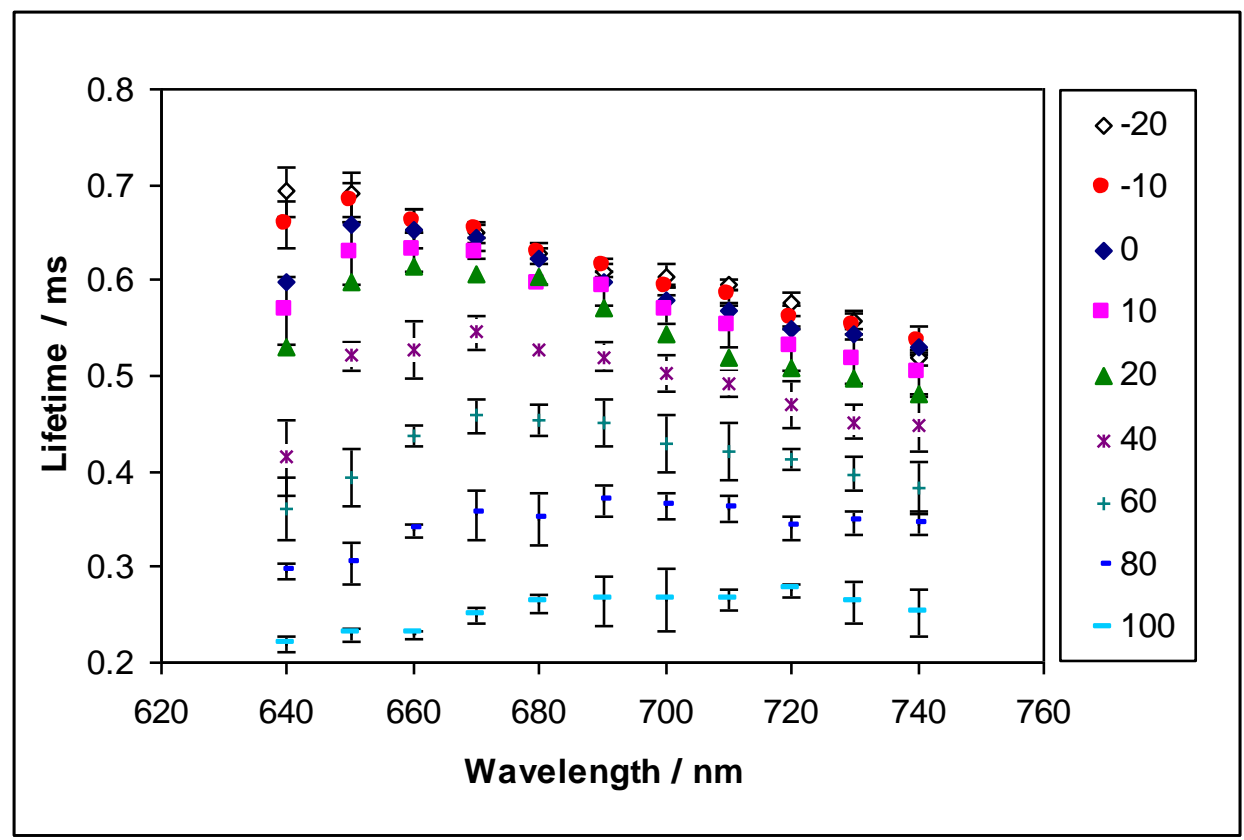

Figure IV-6: Effect of emission wavelength on the Erythrosin B phosphorescence lifetimes in amorphous $\beta$-Lg films determined from analysis using a stretched exponential model.

Figure IV-7

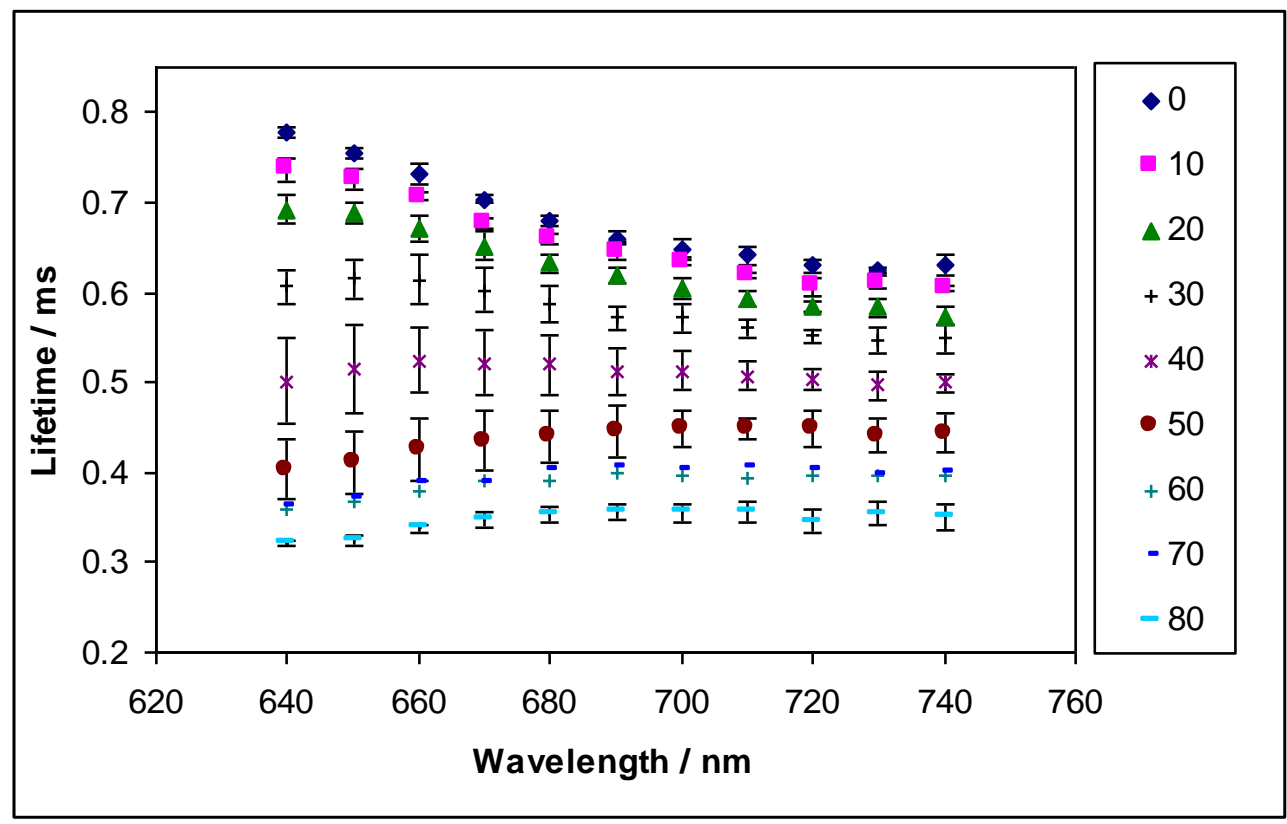

Figure IV-7: Effect of emission wavelength on the Erythrosin B phosphorescence lifetimes in amorphous sucrose/ $\beta$-Lg film determined from analysis using a stretched exponential model. 
Figure IV-8

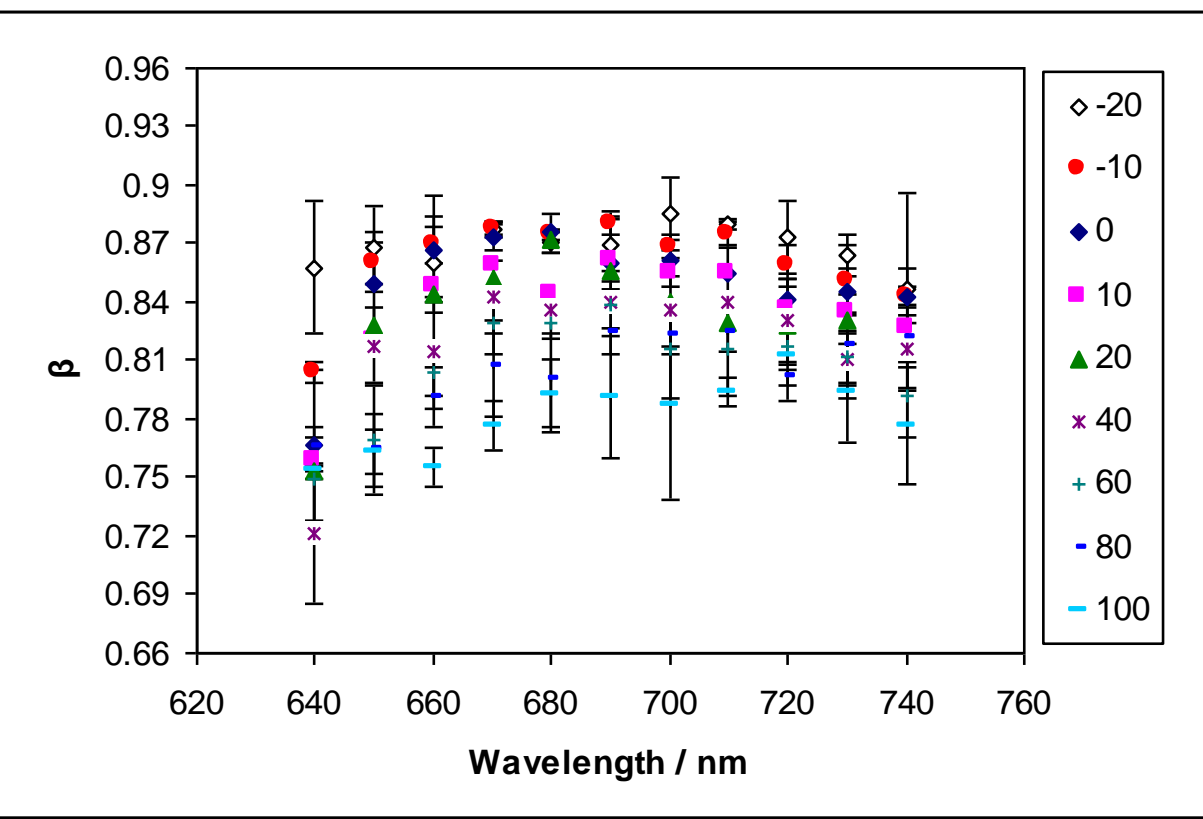

Figure IV-8: Effect of emission wavelength on the stretching exponent $(\beta)$ characterizing the intensity decay transients of Erythrosin B phosphorescence in amorphous $\beta$ - Lg films determined from analysis using a stretched exponential model.

Figure IV-9

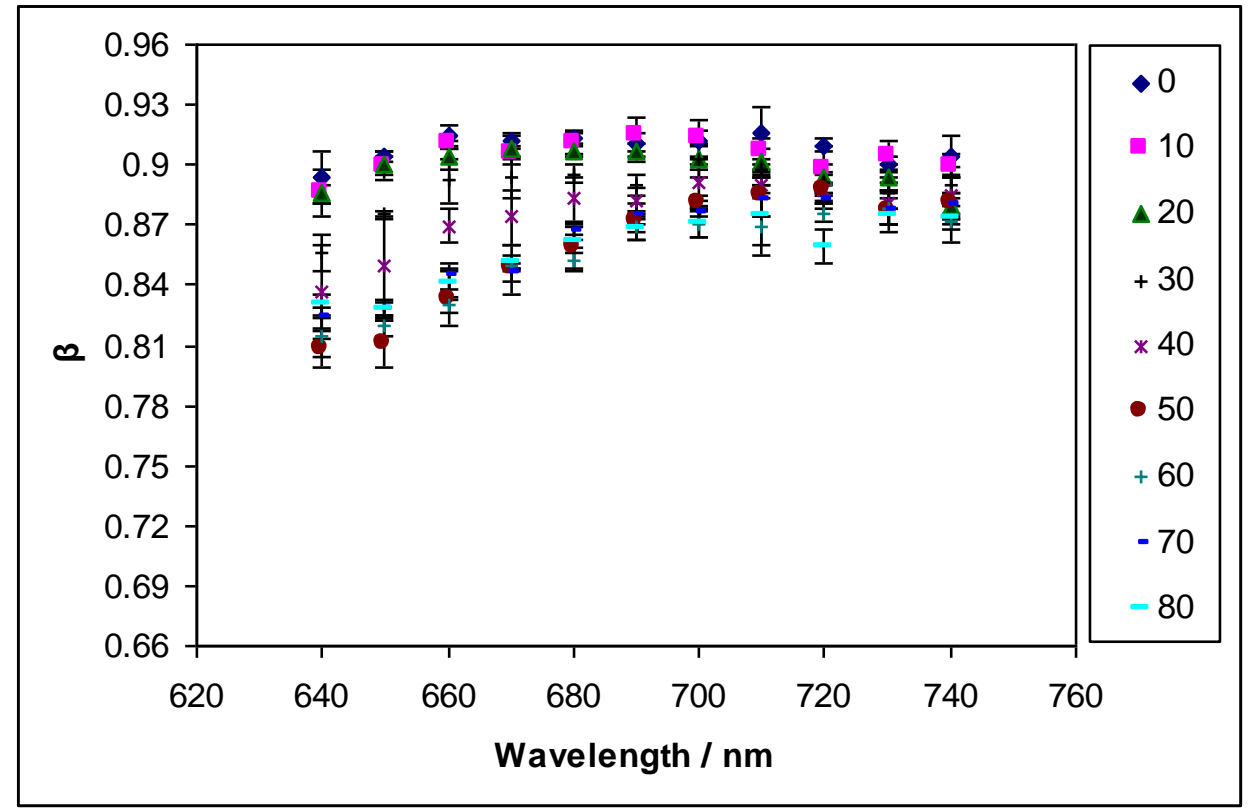

Figure IV-9: Effect of emission wavelength on the stretching exponent $(\beta)$ characterizing the intensity decay transients of Erythrosin B phosphorescence in amorphous sucrose $\beta$ Lg film determined from analysis using a stretched exponential model. 
Figure IV-10

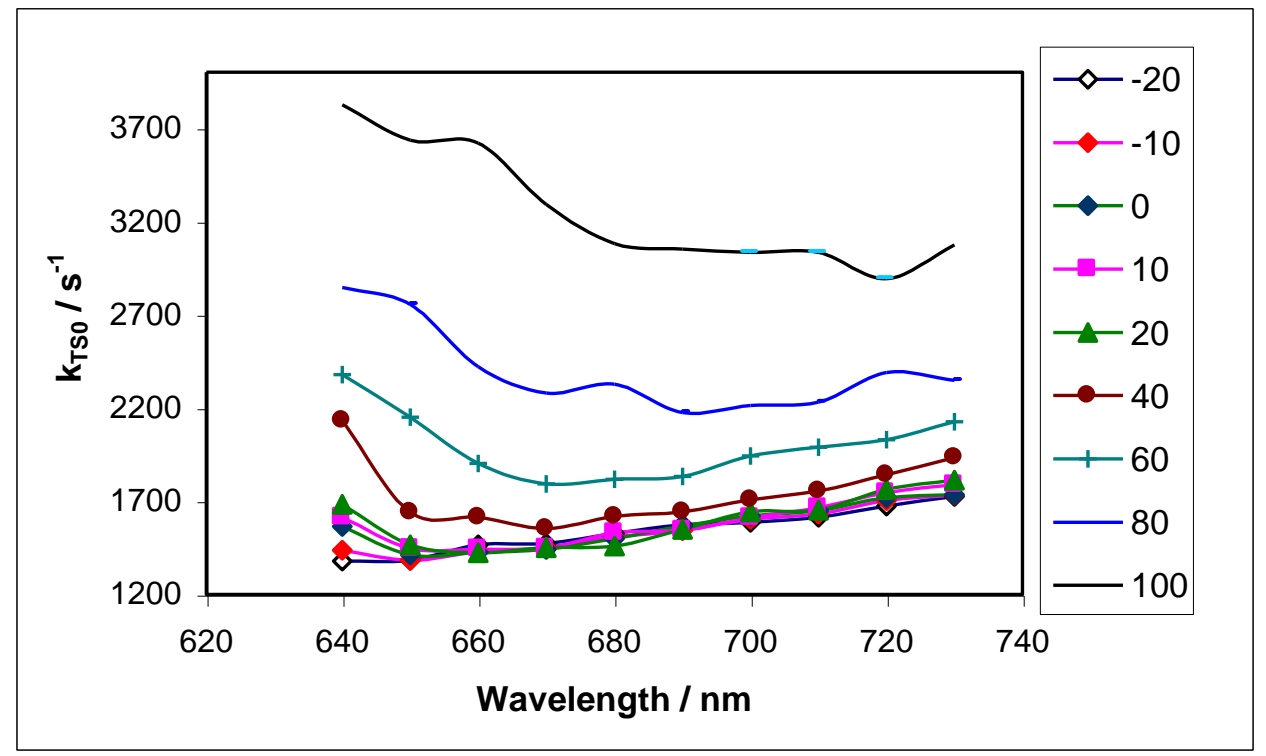

Figure IV-10: The rate constant for non-radiative decay of the triplet state to $\mathrm{S}_{0}\left(\mathrm{k}_{\mathrm{TS} 0}\right)$ plotted as a function of emission wavelength from Erythrosin B in $\beta$-Lg film.

Figure IV-11

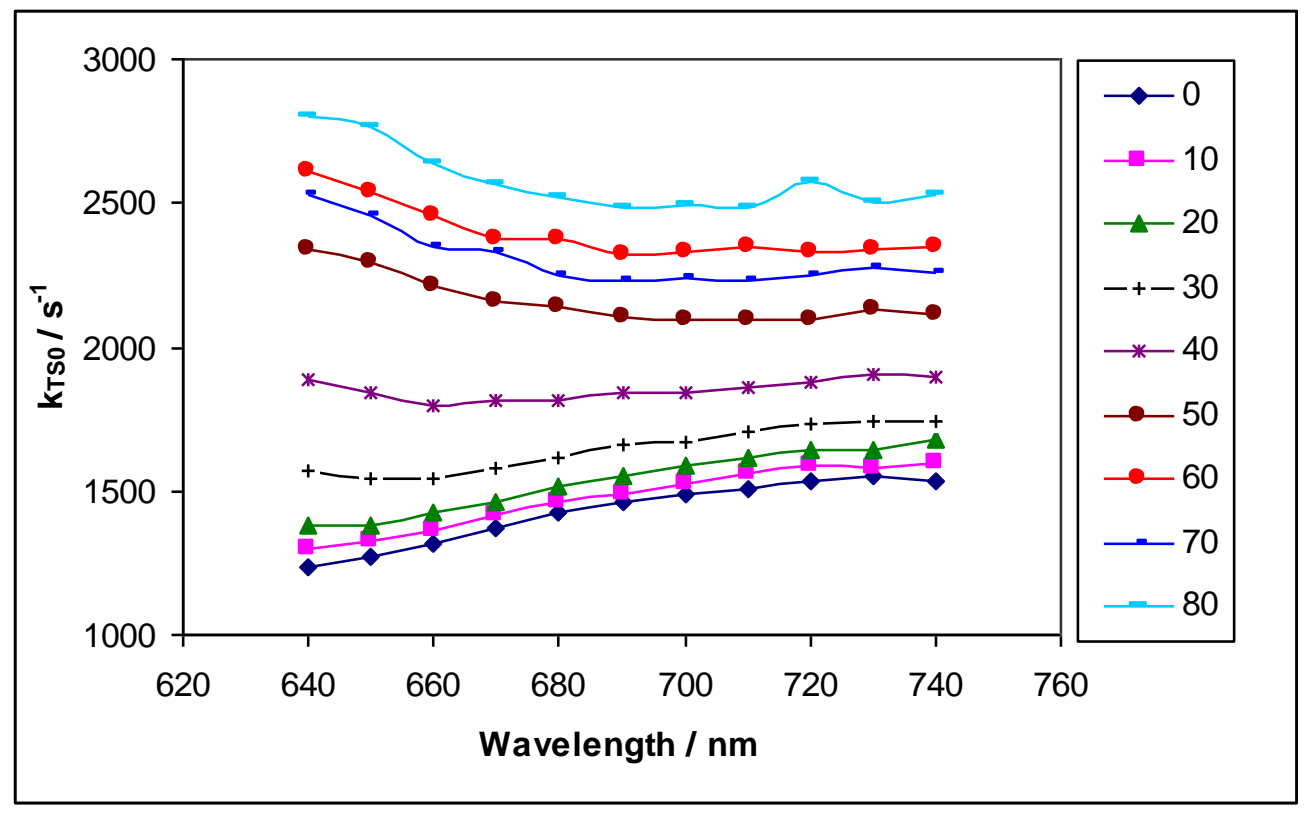

Figure IV-11: The rate constant for non- radiative decay of the triplet state to $S_{0}\left(k_{T S 0}\right)$ plotted as a function of emission wavelength from Erythrosin B in sucrose/ $\beta$-Lg films. 
Figure IV-12

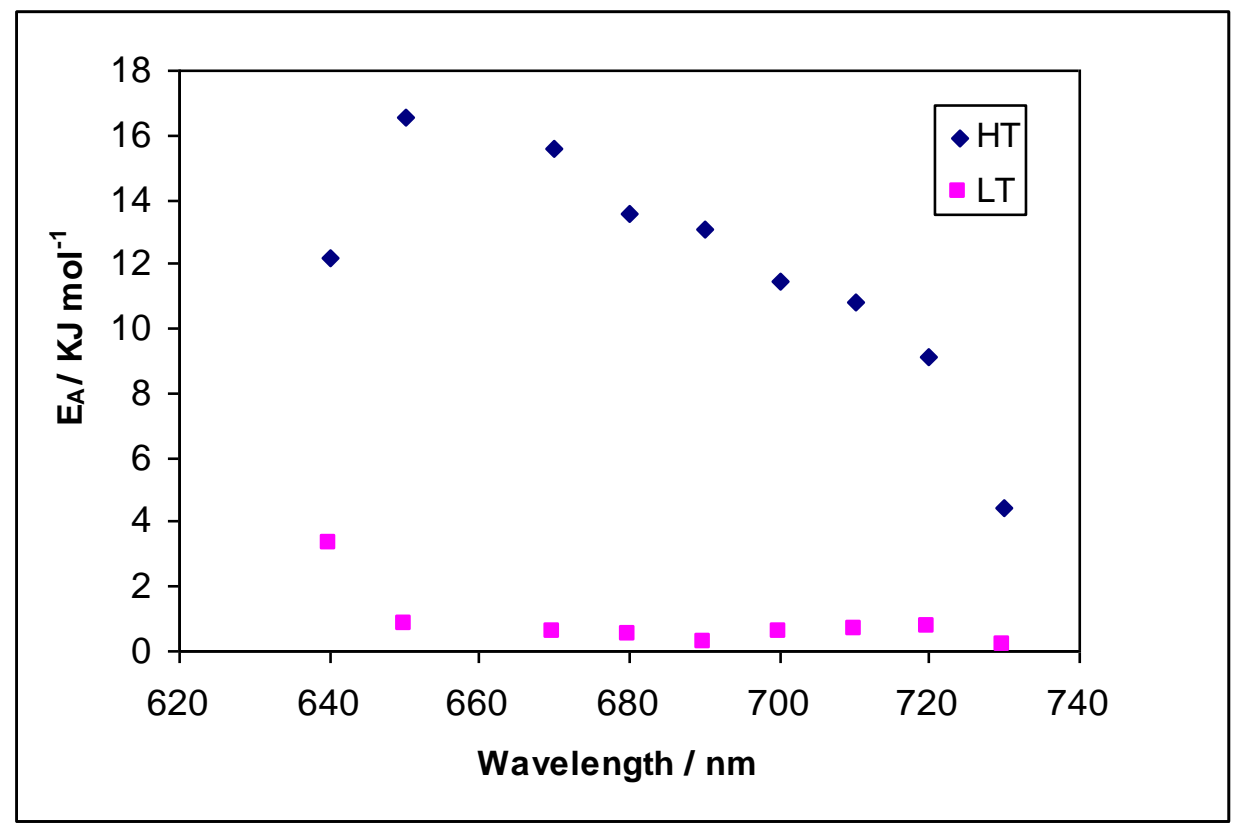

Figure IV-12: Apparent activation energy $\mathrm{E}_{\mathrm{A}}$ for non- radiative decay rate of Erythrosin $\mathrm{B}$ in amorphous $\beta-\mathrm{Lg}$ films as a function of emission wavelength (calculated from an Arrhenius analysis of the data from Figure 10 using the three lowest and highest temperatures respectively). 
Figure IV-13

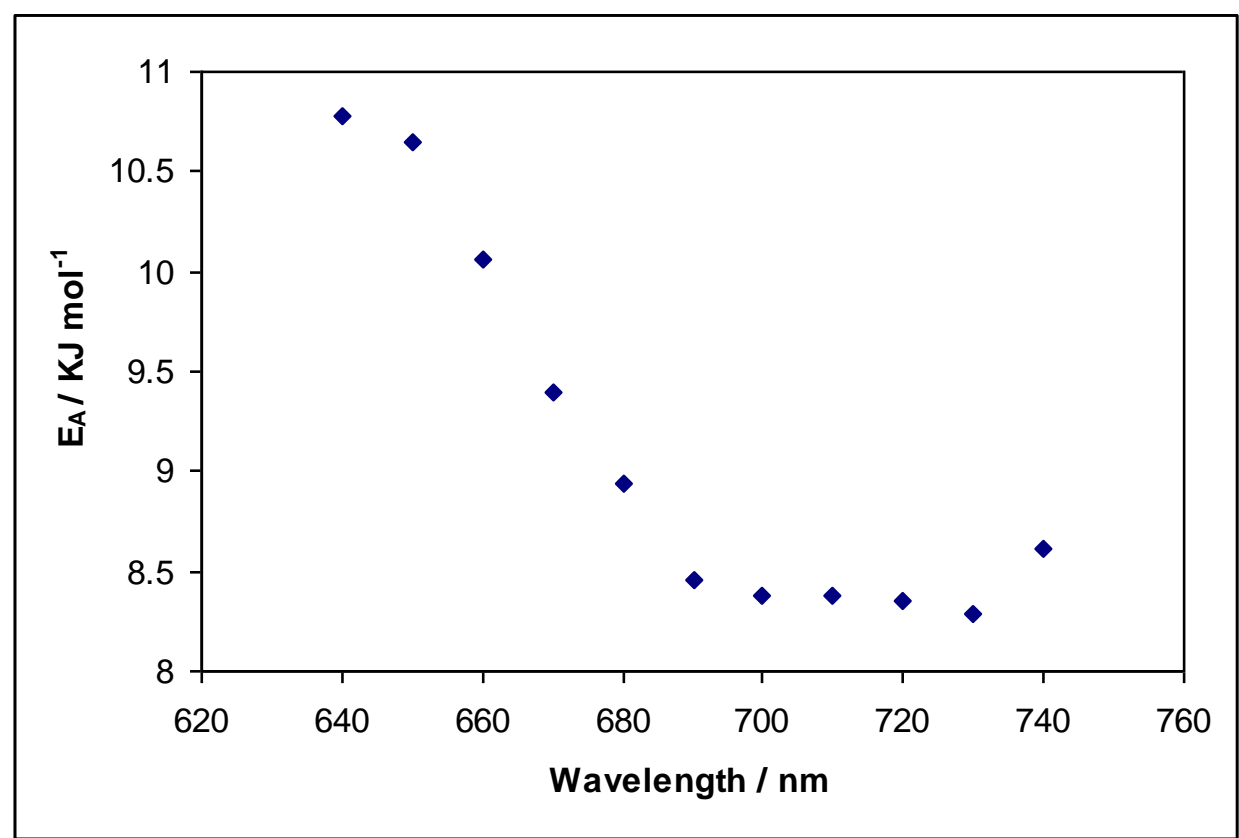

Figure IV-13: Apparent activation energy $\mathrm{E}_{\mathrm{A}}$ for non- radiative decay rate of Erythrosin $\mathrm{B}$ in amorphous sucrose/ $\beta$ - $\mathrm{Lg}$ films as a function of emission wavelength (calculated from an Arrhenius analysis of the data from figure 11). 
Figure IV-14

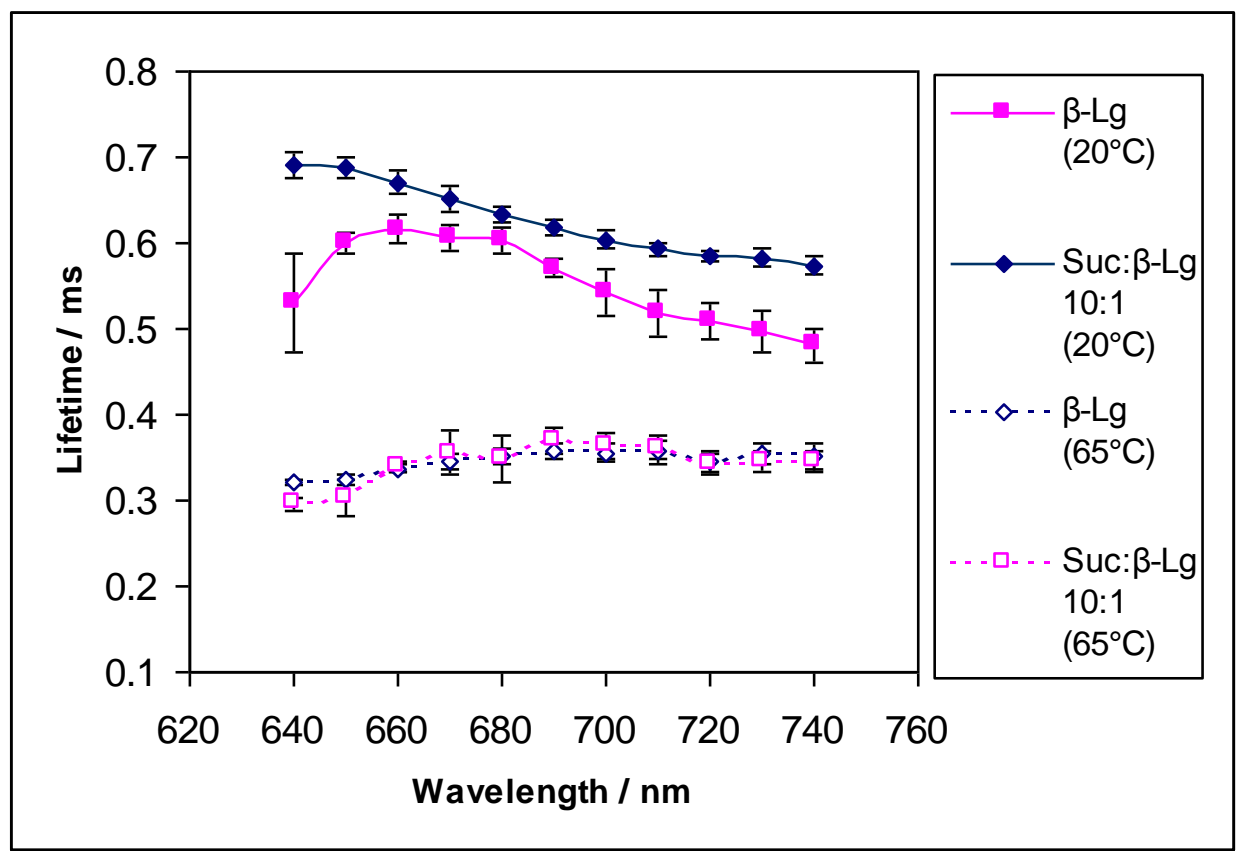

Figure IV-14: Comparative plot of lifetime as a function of emission wavelength following excitation in amorphous $\beta-\mathrm{Lg}$ and $\beta$ - $\mathrm{Lg}$ sucrose films at $20^{\circ} \mathrm{C}$ and $65^{\circ} \mathrm{C}$.

Figure IV-15

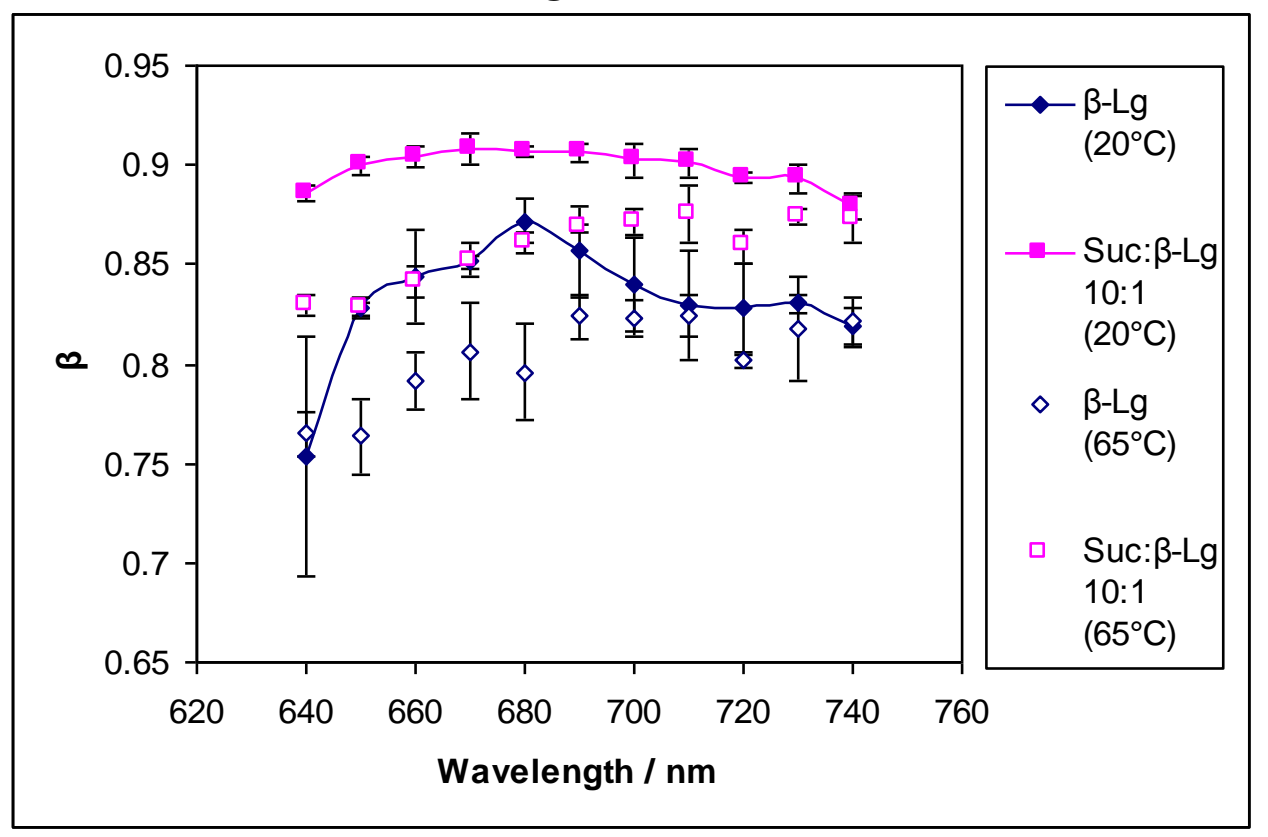

Figure IV-15: Comparative plot of $\beta$ as a function of emission wavelength following excitation in amorphous $\beta-\mathrm{Lg}$ and $\beta-\mathrm{Lg}$ sucrose films at $20^{\circ} \mathrm{C}$ and $65^{\circ} \mathrm{C}$. 
Figure IV-16

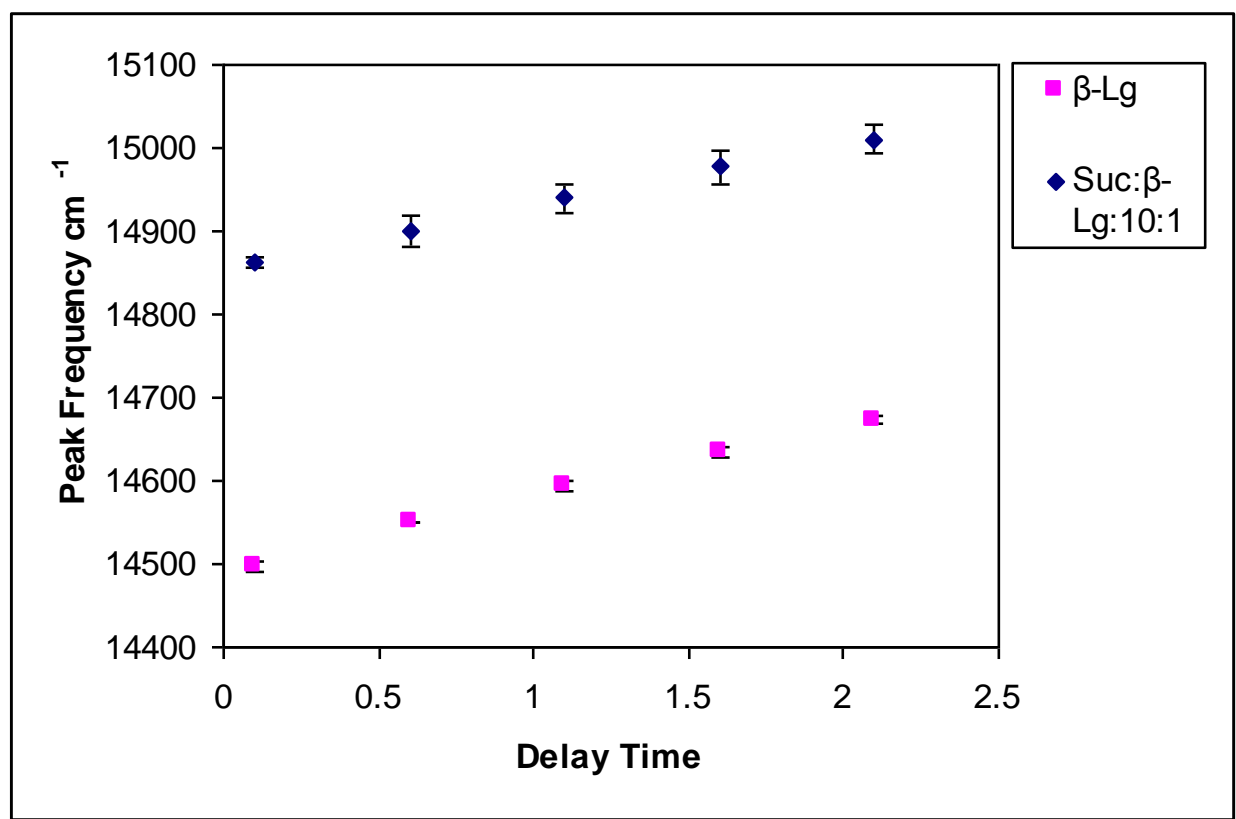

Figure IV-16: Comparative plot of peak frequency with delay time following excitation in amorphous $\beta-\operatorname{Lg}$ and $\beta-\operatorname{Lg}$ sucrose films at $25^{\circ} \mathrm{C}$.

Figure IV-17

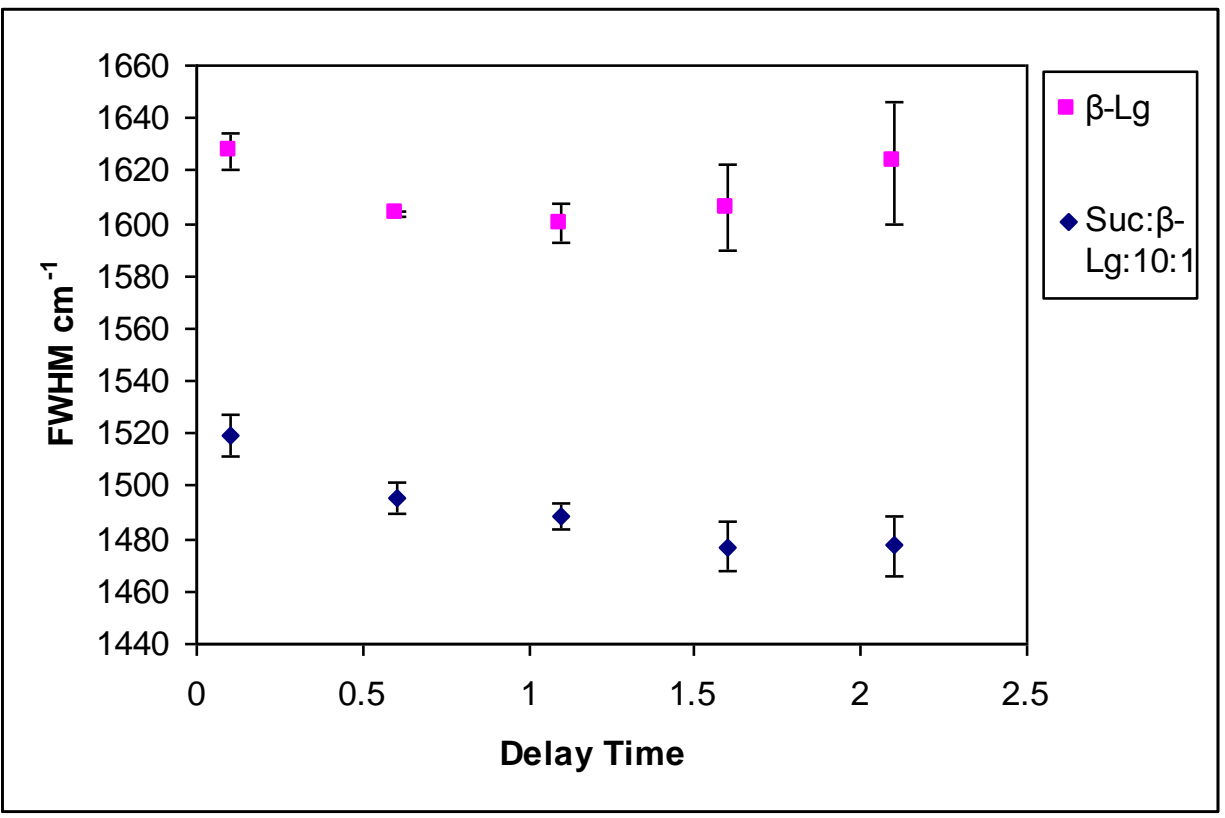

Figure IV-17: Comparative plot of FWHM with time in amorphous $\beta$-Lg and $\beta$-Lg sucrose films at $25^{\circ} \mathrm{C}$. 


\section{Chapter V: Molecular Mobility and Oxygen Permeability in amorphous $\beta$ - Lactoglobulin Films with various plasticizers}

\section{$\underline{\text { Introduction }}$}

Edible films can improve food quality, extend shelf life, add some beneficial properties to foods and reduce the use of synthetic packaging. The formation of edible films essentially requires two ingredients: a film forming material and a plasticizer to reduce film brittleness. "A "Plasticizer" is defined as a substantially nonvolatile, high boiling, non-separating substance, which when added to another material changes the physical and /or mechanical properties of that material" (Banker, 1966). According to Sothornvit and Krochta (2000), plasticizer selection is normally based on the compatibility between plasticizer and protein, permanence in the film and amount necessary for plasticization.

The addition of a plasticizing agent to edible films is required to overcome film brittleness and thus breakage caused by extensive intermolecular forces. Incorporation of a plasticizer results in an increase in the mobility of polymer chains and leads to the formation of more flexible films (Banker, 1966). Since edible films often contain a plasticizer, the macroscopic functional properties of edible films are controlled by molecular mobility in the rubbery state (Anker et al., 1998).

The ability of a plasticizer to disrupt protein-chain hydrogen bonding is influenced by the plasticizer's composition, size, and shape (Gueguen et al., 1998). Plasticizers which can interfere with protein chain-to-chain hydrogen bonding, such as glycerol (gly), polyethylene glycol (PEG), sorbitol (sor), and water, are generally used in protein films. 
Edible films from $\beta$ - $\mathrm{Lg}$ have received much attention because of their ability to act as good oxygen, aroma and water vapor barriers. However, $\beta$ - Lg films by themselves are very brittle and difficult to handle and generally require plasticizers to reduce protein chain to chain interactions in the films. But plasticizers not only improve the mechanical properties of films but also increase the film permeability (Gontard et al., 1993). Gas permeability is an important characteristic of an edible film as oxygen is responsible for many of the degradative reactions in food. A study by McHugh and Krochta (1994) in whey proteins revealed that increasing concentrations of glycerol and sorbitol significantly decreased tensile strength and increased elongation but also enhanced oxygen permeability. Enhanced permeability is undesirable for food quality so fundamental studies are still needed to investigate the effect of composition and to relate permeability to underlying molecular mobility as a critical step towards food application.

Luminescence spectroscopy is a simple, non-invasive technique and has been successfully used in a wide range of research applications. Luminescence probes have been refereed to as "molecular reporters". From the fluorescence or phosphorescence signals these probes are ideally suited to provide detailed information about the environment (Slavik, 1994). As compared to other, more traditional analytical methods these luminescence studies provide a unique perspective on edible food systems, which will help optimize its use in future, applications.

Phosphorescence is especially useful for probing molecular mobility on the millisecond and longer time scale, which corresponds to the modes of local and segmental motion seen in solid phase proteins (Shah and Ludescher, 1993), due to the long lifetime of the triplet state $\left(10^{-4}-10 \mathrm{~s}\right)$. Erythrosin B (Ery B; tetraiodofluorescein) is 
perhaps the most widely used phosphorescent probe of the molecular mobility of watersoluble and membrane-bound proteins because of its large extinction coefficient and high phosphorescence quantum yield even in aqueous solution. We have successfully used Ery B phosphorescence to monitor molecular mobility in amorphous solid sugars and sugar alcohols (Shah and Ludescher, 1995; Pravinata et al., 2005; Shirke et al., 2005, 2006; Shirke and Ludescher, 2005), in solid gelatin (Simon-Lukasik and Ludescher, 2004; Lukasik \& Ludescher, 2006a,b), in amorphous bovine serum albumin (Nack \& Ludescher, 2006) and $\beta-\mathrm{Lg}$ (Sundaresan and Ludescher, 2007). In addition, phosphorescence is effectively and efficiently quenched by collision with molecular oxygen. It is thus possible to probe both the presence of oxygen and specific molecular properties of a matrix using an appropriate phosphorescent probe molecule.

In a previous study we reported how phosphorescence emission from the triplet probe Erythrosin B can be used to monitor molecular mobility and oxygen permeability in amorphous $\beta$-Lg films and sugar / $\beta$-Lg films (Sundaresan and Ludescher, 2007). We report here how phosphorescence can provide insight into the effects of the plasticizers glycerol, sorbitol, maltitol and polyethylene glycol on the molecular mobility of $\beta$ - $\mathrm{Lg}$ films. The investigations aim at looking into the effect of these plasticizers as a function of temperature, and establish vital connections with oxygen permeability and molecular mobility. The temperature dependence of mobility will be analyzed at different plasticizer concentration generating families of mobility versus temperature curves. Comparisons should provide us with information into the mechanisms by which these small molecules influence the matrix mobility. The effect of plasticizer concentration on oxygen permeability and molecular mobility will be of substantial interest to food scientists. 
These results provide insight into the molecular mechanisms that underlie the macroscopic effect of these modifications of amorphous $\beta$ - $\mathrm{Lg}$ films.

\section{Materials and methods}

\section{Sample preparation}

Solutions of $\beta-\operatorname{Lg}$ (90\% pure; crystallized and lyophilized Sigma Chemical Co, St.Louis, MO, USA) were prepared in distilled de-ionized water at an approximate concentration of $10 \mathrm{mg} / \mathrm{ml}$. This concentration was selected based on the batch size to ensure maximum recovery after dialysis and to avoid long-term storage. An aqueous $\beta$ Lg solution was transferred to regenerated cellulose dialysis tubing having a $12-14 \mathrm{kDa}$ molecular weight cutoff (Spectrum, Houston, TX). In order to define and minimize the presence of counter ions the solution was extensively dialyzed against $0.1 \mathrm{M}$ potassium chloride for 24 hours with frequent change of buffer every 6 hours. The solution was then dialyzed against distilled de-ionized water for 24 hours with frequent change of water after every 6 hours. The proposed protocol was followed in a very stringent manner in order to control precipitation in the protein during dialysis. The dialyzed solution was then filtered through a $0.2-\mu \mathrm{m}$ membrane to remove particulates. In order to maximize the effectiveness of the dialysis process temperature of the solution was kept at $5^{\circ} \mathrm{C}$ to ensure that the protein remained folded. Several dilutions of a stock solution of dialyzed $\beta$-Lg were prepared. The dye to protein ratio was determined by measuring the absorbance of dilutions in a $1 \mathrm{~cm}$ path length quartz cuvette in a Bausch and Lomb Spectronic 2000 spectrophotometer (Milton Roy Company, Analytical Products Division, Rochester, NY). The protein concentration was determined after dialysis by UV absorbance using an extinction coefficient of $0.96 \mathrm{~L} \mathrm{~g} \mathrm{~g}^{-1} \mathrm{~cm}^{-1}$. Erythrosin B (EryB) (tetra 
iodofluorescein,FD\&C red\#3)from molecular probes(Eugene, OR), was dissolved in spectrophotometric grade N,N-dimethyl formamide (DMF)(Aldrich Chemical, Milwaukee, WI) to make $10 \mathrm{mM}$ and $100 \mathrm{mM}$ stock solutions. The solvent DMF was selected for probe stability during long time storage and the concentrations were selected in order to simplify the addition of probe to the protein matrix. After estimating the concentration protein solution was combined with aliquots of dye solution to make solutions with molar ratio of EryB: $\beta$-Lg of 1:4. The dye to protein concentration was sufficient to provide adequate signal /noise in spectroscopic measurements.

\section{$\beta$-Lg films with plasticizers}

Glycerol, sorbitol, maltitol and PEG 400 were purchased from Sigma-Aldrich (St. Louis, MO) with minimum purity of $98 \%$ and were used without further purification. Maltitol and sorbitol were dissolved to near saturation in deionized water at room temperature and then added to $\beta$-Lg solutions. Glycerol and polyethylene glycol 400 (PEG 400) were added as such in various molar ratios. The ratios were selected in order to judge the critical concentration of plasticizer required to control oxygen diffusion within the detectable limits of our instrumentation and to provide the best possible estimate of the permeability of oxygen in these functional matrices. In the case of glycerol, sorbitol and PEG 400 phase separation was also a major problem in the films. The concentrations of plasticizers were determined based on the compatibility limits of the plasticizers on the $\beta$ - $\mathrm{Lg}$ films. Concentrations higher than the threshold concentration resulted in white spotting on the films whereby excess amounts of sorbitol, glycerol or PEG 400 (a precipitate) were excluded from the surface of the protein films. A similar phenomenon was observed in sorbitol plasticized pea starch films whereby high 
concentrations of sorbitol resulted in the occurrence of white residues (sorbitol precipitate) on the surface of the films after 72 hours in $50 \%$ humidity (Zhang and Han, 2006)

Jangchud and Chinnan, (1999) and Aulton et al. (1981) assumed that when the plasticizer concentration was more than its compatability limit in the polymer, phase separation and plasticizer physical exclusion occurred.

\section{Preparation of protein films}

To make glassy films, $15 \mu \mathrm{l}$ of protein / plasticizer solution was spread on approximately one third of a quartz slide $(30 \times 13.5 \times 0.6 \mathrm{~mm}$; custom made by NSG Precision Cells, Farmingdale, NY). The protein / sugar films approximately $(0.05 \mathrm{~mm}$ thick when dried) were visually checked for good spreading, uniformity, and consistency. The slides were stored at room temperature in a desiccator containing $\mathrm{P}_{2} \mathrm{O}_{5}$ and DrieRite for at least 7 days and protected from light to prevent any photobleaching of Erythrosin B prior to luminescence measurements. The slides were checked for crystallization under crossed polarizers using a Nikon Type 102 dissecting microscope (Nikon, Inc., Japan).

\section{Luminescence measurements}

All measurements were conducted using a CARY Eclipse spectrophotometer (Varian Instruments, Walnut Creek, CA). The temperature was controlled by using a TLC 50 thermoelectric heating /cooling system (Quantam Northwest, Spokane,WA). The TLC-50 sample compartment was fitted with a jacketed cover. For luminescence measurements necessitating the absence of oxygen (a luminescence quencher) the samples were flushed with nitrogen gas for at least 15 minutes to free the environment 
from oxygen. An oxygen free nitrogen stream was generated by passage of high purity nitrogen through a Supelco (Bellefonte, PA) carrier gas purifier.

Quartz slides were placed on end on the diagonal of a standard $1 \mathrm{~cm} \times 1 \mathrm{~cm}$ quartz fluorescence cuvette which was capped with a lid having inlet and outlet ports for gas lines, thus all experiments were conducted at constant total pressure. Dry air was flushed to prevent condensation on the faces of the cuvette at experimental conditions below room temperature.

Delayed luminescence emission spectra were collected from 535-800 nm (10nm bandwidth) using excitation of $525 \mathrm{~nm}$ (10nm bandwidth) over the temperature range from $0^{\circ} \mathrm{C}$ to $100^{\circ} \mathrm{C}$ (at 10 degree intervals). Each data point ( $1 \mathrm{~nm}$ interval, $0.1 \mathrm{~s}$ averaging time) was collected from a single flash with a $0.2 \mathrm{~ms}$ delay, $0.5 \mathrm{~ms}$ gate time and $4.0 \mathrm{~ms}$ total decay time.

For lifetime measurements as a function of temperature samples were excited at $540 \mathrm{~nm}$ (20nm bandwidth) and emission transients collected at 690nm (20nm bandwidth) at temperature ranging from $0^{\circ} \mathrm{C}-100^{\circ} \mathrm{C}$. Before collecting data samples were equilibrated for 15 minutes. The measurements were taken in the presence or absence of air (under a nitrogen purge). Each time- resolved decay transient was the average of 100 cycles and for each cycle data was collected from a single flash with a delay of $0.1 \mathrm{~ms}$, a 0.02 gate time and $4.0 \mathrm{~ms}$ total decay time. All experiments were done several times and the averages were used for data analysis and interpretation. In order to validate the repeatability the standard deviation between experiments was calculated. 


\section{Data analysis}

The emission spectra were analyzed by fitting both the delayed fluorescence and the phosphorescence to a lognormal function.

$$
\mathrm{I}(v)=\mathrm{I}_{0} \exp \left\{-\ln (2)\left[\ln \left(1+2 \mathrm{~b}\left(v-v_{\mathrm{P}}\right) / \Delta\right) / \mathrm{b}\right]^{2}\right\}
$$

In this equation $\mathrm{I}_{0}$ is the maximum emission intensity, $v_{\mathrm{P}}$ is the frequency $\left(\mathrm{cm}^{-1}\right)$ of the emission maximum, $\Delta$ is a linewidth parameter, and $\mathrm{b}$ is an asymmetry parameter The bandwidth $(\Gamma)$ of the emission band is related to $\mathrm{b}$ and $\Delta$ :

$$
\Gamma=\Delta\{\sinh (\mathrm{b}) / \mathrm{b}\}
$$

Emission spectra were fit using the program Igor (Wavemetrics, Inc., Lake Oswego, OR, USA). We also analyzed some data by fitting with the program Nfit (Island Products, Galveston, TX, USA) to make sure that the results agreed in both fitting programs.

Phosphorescence lifetimes were determined by nonlinear least-squares analysis with the statistical programs Igor and Nfit. Fits were judged satisfactory if the $\mathrm{R}^{2}$ values were in the range of 0.995-1.0. Data were analyzed using a stretched exponential, or Kohlrausch-Williams-Watts (KWW), decay model; this has been shown to be appropriate to describe the wide distribution of relaxation times for the molecular processes that depopulate excited states (Champion et al, 2000; Richert, 2000)

$$
\mathrm{I}(\mathrm{t})=\mathrm{I}_{0} \exp \left[-(\mathrm{t} / \tau)^{\beta}\right]+\mathrm{c}
$$

Where $I_{0}$ is the initial amplitude, $\tau$ is the stretched exponential lifetime, $\beta$ is an exponent varying from 0 to 1 and characterizing the distribution of lifetimes. The phosphorescence lifetimes were used to calculate the rate constants associated with the various processes 
that depopulate the excited triplet state. In an amorphous solid in the absence of oxygen the temperature dependence of $k_{P}$ can be written as follows:

$$
\mathrm{k}_{\mathrm{P}}(\mathrm{T})=\mathrm{k}_{\mathrm{RP}}+\mathrm{k}_{\mathrm{TS} 1}(\mathrm{~T})+\mathrm{k}_{\mathrm{TS} 0}(\mathrm{~T})
$$

But in the presence of oxygen the de-excitation of the triplet state is also influenced by the quencher species. The term $\mathrm{k}_{\mathrm{Q}}\left[\mathrm{O}_{2}\right]$ describes the rate of collisional quenching of the excited state of the probe by oxygen. Thus in the presence of oxygen $k_{P}(T)$ includes an additional term $\mathrm{k}_{\mathrm{Q}}\left[\mathrm{O}_{2}\right]$.

$$
\mathrm{k}_{\mathrm{P}}(\mathrm{T})=\mathrm{k}_{\mathrm{RP}}+\mathrm{k}_{\mathrm{TS} 1}(\mathrm{~T})+\mathrm{k}_{\mathrm{TS} 0}(\mathrm{~T})+\mathrm{k}_{\mathrm{Q}}\left[\mathrm{O}_{2}\right](\mathrm{T})
$$

Equation 5 can be used to calculate $\mathrm{k}_{\mathrm{TS} 0}$, the rate of collisional quenching to the ground state, when $\mathrm{k}_{\mathrm{RP}}$ and $\mathrm{k}_{\mathrm{TS} 1}$ are known. Under anoxic conditions, $\mathrm{k}_{\mathrm{Q}}\left[\mathrm{O}_{2}\right]$ the rate of oxygen quenching, is zero; $\mathrm{k}_{\mathrm{RP}}$, the radiative decay rate of the triplet state, is $41 \mathrm{~s}^{-1}$ for Ery $\mathrm{B}$ (Duchowicz et al.,1998; Lettinga et al., 2000).

$\mathrm{k}_{\mathrm{TS} 1}$ the rate of reverse intersystem crossing from the excited triplet state to the excited singlet state depends on $\Delta E_{T S}$, the energy gap between $S_{1}$ and $T_{1}$

$$
\mathrm{k}_{\mathrm{TS} 1}(\mathrm{~T})=\mathrm{k}^{\circ} \mathrm{TS} 1 \exp \left(-\Delta \mathrm{E}_{\mathrm{TS}} / \mathrm{RT}\right)
$$

A plot of $\ln \left(\mathrm{I}_{\mathrm{DF}} / \mathrm{I}_{\mathrm{P}}\right)$ versus $1 / \mathrm{T}$ showed linearity and has slope of $-\mathrm{E}_{\mathrm{TS}} / \mathrm{R}$ and thus $\Delta \mathrm{E}$ for the $\beta$-lg matrix was calculated. Unfortunately, literature values of $\mathrm{k}^{\circ} \mathrm{TS} 1$ for Erythrosin $\mathrm{B}$ vary widely, from $0.3 \times 10^{7} \mathrm{~s}^{-1}$ in ethanol and $6.5 \times 10^{7} \mathrm{~s}^{-1}$ in water (Duchowicz et al., 1998) to $111 \times 10^{7} \mathrm{~s}^{-1}$ in solid polyvinyl alcohol (Lettinga et al., 2000), and thus provide little guidance. We estimated the maximum possible value for $\mathrm{k}_{\mathrm{TS} 1}^{\mathrm{o}}$ in $\beta$ - $\mathrm{Lg}$ - plasticizer films by assuming that $\mathrm{k}_{\mathrm{TS} 1}(\mathrm{~T})$ cannot result in values for $\mathrm{k}_{\mathrm{TS} 0}$ that decrease with temperature. This procedure thus estimated the minimum possible values of $\mathrm{k}_{\mathrm{TS} 0}(\mathrm{~T})$. Various values of $\mathrm{k}^{\mathbf{o}}$ TS1 have been summed up in Table V 1a, b, c, $\mathrm{d}$ 
In order to study the oxygen diffusion in the $\beta$ - $\mathrm{Lg}$ matrix $\mathrm{k}_{\mathrm{Q}}\left[\mathrm{O}_{2}\right]$ can easily be calculated from the difference between the decay rate in the presence of oxygen and the absence of oxygen

$$
\mathrm{k}_{\mathrm{Q}}\left[\mathrm{O}_{2}\right]=1 / \tau(\text { Air })-1 / \tau(\text { Nitrogen })
$$

\section{$\underline{\text { Results }}$}

Plasticization of edible films is believed to disrupt intermolecular interactions between polymer molecules with the effect of decreasing brittleness and increasing film flexibility (Lieberman and Gilbert 1973; Sears and Darby 1982). The plasticizing effect of polyols, particularly, can be attributed to their ability to locate between polymer molecules, bind water, and disrupt intermolecular polymer associations (Gontard et al., 1993). The present study throws light on the effect of the plasticizers glycerol, sorbitol, maltitol and PEG 400 on the molecular mobility and oxygen permeability of the amorphous $\beta$-Lg matrix.

\section{Delayed Emission Spectra}

Figures V-1a, b, c, d depict the emission spectra of Erythrosin B in $\beta$-Lg with various plasticizers. The long wavelength emission band reflects phosphorescence from the triplet state whereas the short wavelength emission band reflects delayed fluorescence from the singlet state that has been repopulated by reverse intersystem crossing from the triplet state (Parker, 1968). Delayed emission spectra collected over the temperature range from $0^{\circ} \mathrm{C}$ to $100^{\circ} \mathrm{C}$ showed a decrease in phosphorescence $\left(\mathrm{I}_{\mathrm{P}}\right)$ and increase in delayed fluorescence $\left(\mathrm{I}_{\mathrm{DF}}\right)$ intensity at higher temperatures as expected for a thermally stimulated process (Parker, 1968). 
The value for, $\Delta \mathrm{E}_{\mathrm{TS}}$, the energy gap between the $T_{1}$ and $S_{1}$ states, was calculated from the slope of a Van't Hoff plot of $\ln \left(\mathrm{I}_{\mathrm{DF}} / \mathrm{I}_{\mathrm{P}}\right)$ vs. 1/T (Eq. 6, Materials and Methods) using values for the intensity of delayed fluorescence $\left(\mathrm{I}_{\mathrm{DF}}\right)$ and phosphorescence $\left(\mathrm{I}_{\mathrm{P}}\right)$ determined from a fit of the spectra to a sum of two lognormal functions (Eq. 1 in Materials and Methods, data not shown). $\Delta \mathrm{E}_{\mathrm{TS}}$ followed the acceptable trend of the probe Erythrosin B and was linear over the entire range of measured temperatures with no systematic deviations. The values for $\Delta \mathrm{E}_{\mathrm{TS}}$ varied throughout the ratios of plasticizers to proteins. The average values of $\Delta \mathrm{E}_{\mathrm{TS}}$ were $34.7 \mathrm{~kJ} \pm 0.5 \mathrm{~kJ} \mathrm{~mol}^{-1}$ for the glycerol $/ \beta-\mathrm{Lg}$ films, $33.3 \pm 1.2 \mathrm{~kJ} \mathrm{~mol}^{-1}$ for sorbitol $/ \beta-\mathrm{Lg}$ films and $34.0 \pm 0.5 \mathrm{~kJ} \mathrm{~mol}^{-1}$ for maltitol $/ \beta-$ Lg films. These values were significantly smaller than in water $\left(36.9 \pm 0.6 \mathrm{~kJ} \mathrm{~mol}^{-1}\right)$ or $66 \mathrm{wt} . \%$ aqueous sucrose $\left(36.9 \pm 0.1 \mathrm{~kJ} \mathrm{~mol}^{-1}\right)$ and in polyvinyl alcohol $(41.2 \pm 0.4 \mathrm{~kJ}$ $\left.\mathrm{mol}^{-1}\right)$, and larger than the value in ethanol $\left(28.5 \pm 2.5 \mathrm{~kJ} \mathrm{~mol}^{-1}\right)$. PEG $\beta$-Lg films had higher $\Delta \mathrm{E}_{\mathrm{TS}}$ values than other plasticizers / $\beta$-Lg films $\left(35.6 \pm 0.5 \mathrm{~kJ} \mathrm{~mol}^{-1}\right)$. The $\Delta \mathrm{E}_{\mathrm{TS}}$ values as a function of plasticizer concentration are summed up in Table V-2a, b, c, d. These trends suggest that solvent (matrix) properties modulate the singlet-triplet energy gap of Erythrosin B

The phosphorescence emission band shifted to longer wavelength (lower energy) with increasing temperature. The peak emission energy $v_{P}$ was calculated using the lognormal function (Eq. 1, Materials and Methods); these parameters are plotted vs. temperature in Figures V 2 a, b, c, d. The phosphorescence emission energy decreased with temperature over the range from 0 to $90^{\circ} \mathrm{C}$ in all the $\beta-\mathrm{Lg}$ plasticizer films. The decrease in emission energy reflects an increase with temperature in the extent of matrix dipolar relaxation around the excited triplet state prior to emission. 
Figure V-2a represents the emission energy as a function of temperature in glycerol $\beta$-Lg films. The phosphorescence emission energy followed a similar trend with temperature as observed in $\beta$ - $\mathrm{Lg}$ films with no evidence of a breakpoint. There was a slight variation in the peak frequency $\left(\Delta v_{\mathrm{P}}\right)$ in between $\beta$-Lg and the various ratios of glycerol $\beta-\operatorname{Lg}\left(\Delta v_{\mathrm{P}} \sim 74 \mathrm{~cm}^{-1}\right)$ at the measured temperature range. Within the scope of the error the effect of glycerol seemed relatively insignificant on the $\beta$ - $\mathrm{Lg}$ matrix.

Sorbitol had a concentration dependent effect on the $\beta$-Lg matrix. Figure V-2b represents the emission energy as a function of temperature in sorbitol plasticized $\beta$ - $\mathrm{Lg}$ films. Various concentrations of sorbitol exhibited differences in behavior with respect to trends in emission energy. Over the tested temperature range $0-100^{\circ} \mathrm{C}$ the phosphorescence emission energy decreased in a linear manner at all ratios except 300:1 whereby there was a biphasic decline. At a molar ratio of 10: 1 sorbitol to $\beta$-Lg the emission energy reached the maximum, which was also judged as the critical concentration for massive decline in the permeability (discussed in the later part of this chapter). At this ratio of sorbitol to $\beta$ - $\mathrm{Lg}$ the matrix became rigid and probe molecules could not relax easily. However at higher concentrations (100: 1 sorbitol to $\beta$-Lg) there was a steep decline in the emission energy owing to greater dipolar relaxations. At a molar ratio of 300: 1sorbitol/ $\beta$-Lg, emission energies were higher at low temperatures but after a certain temperature a transition similar to the glass transition was observed whereby there was an abrupt drop in the emission energy. Although studies show that the glass transition temperature of sorbitol is $-4^{\circ} \mathrm{C}$ (Matveev, 2006; Slade and Levine, 1995), in the present study at higher ratios $(300: 1)$ we see that the transition temperature is in 
between $30^{\circ} \mathrm{C}-40^{\circ} \mathrm{C}$. This decrease in emission energy at higher temperatures might be related to phase separation or possible formation of sorbitol clusters.

Maltitol is a polyol used as a sugar substitute. Maltitol is obtained by highpressure hydrogenation of maltose syrup using a nickel catalyst. Many of its physicochemical properties are comparable to those of sucrose. Figure V $2 \mathrm{c}$ depicts the plot of emission energy versus temperature in maltitol $\beta$-Lg films. Over the temperature range of $0-100^{\circ} \mathrm{C}$, emission energy decreased linearly at low concentrations and biphasically at high concentrations (100:1 and 1000:1 maltitol: $\beta$-Lg). At these higher ratios of maltitol after the glass transition temperature of maltitol, a significant decline in the emission energy was observed. The glass transition of maltitol is approximately $39^{\circ} \mathrm{C}$ (Roos, 1995).

In maltitol / $\beta-\mathrm{Lg}$ films, the peak frequency increased with an increase in maltitol concentration. A slight increase was observed in the phosphorescence emission peak frequency at lower ratios $(0.1: 1,1: 1)$ of maltitol / $\beta$-Lg. However there was a significant increase in the peak frequency at a ratio of 10:1 over the temperature range from 0 $100^{\circ} \mathrm{C}$. This increase is attributed to the decline in the vibrational relaxations around the excited state prior to emission (Lakowicz, 1999; Pravinata et al., 2005). At ratios of 100:1 and 1000:1, maltitol : $\beta$-Lg, addition of maltitol brought about a concomitant increase in the emission energy. Maltitol displayed anti-plasticization characteristics similar to the sugars on the $\beta$-Lg matrix (chapter III).

Polyethylene glycol 400 is another common plasticizer used in the food industry. From Figure V-2d, it is evident that addition of PEG 400 increased the emission energy of $\beta-\mathrm{Lg}$ films with no clear concentration dependence. At ratios 0.1:1 and 1:1 PEG / $\beta$ - 
Lg there was an increase in the emission energy with subsequent addition of PEG, showing an increment of $164.33 \mathrm{~cm}^{-1}$ at $0^{\circ} \mathrm{C}$ and $75 \mathrm{~cm}^{-1}$ at $100^{\circ} \mathrm{C}$. When the PEG / $\beta-\mathrm{Lg}$ mole ratio increased to 10:1 the peak frequency decreased but the values were still greater than the pure $\beta$-Lg matrix. Clearly PEG 400 had significant effect on the peak frequency of the $\beta$-Lg matrix.

The emission bandwidth, $\Gamma$, or full width at half maximum, increased with increasing temperature. This increase was linear and gradual at low temperatures and quite dramatic at high temperatures $\left(>80^{\circ} \mathrm{C}\right)$, indicating that the increase in dipolar relaxation rate was accompanied by an increase in the extent of in-homogenous broadening of the emission spectra. The emission bandwidth $(\Gamma)$ for the plasticizer/ $\beta$ - $L g$ films did not exhibit any significant differences between the different ratios of plasticizers to $\beta$ - $\mathrm{Lg}$ until $80^{\circ} \mathrm{C}$. Although there was a dramatic increase at $80^{\circ} \mathrm{C}$ and above no clear trend was observed in between various plasticizers (Figure V 3 a, b, c, d). An increase in the bandwidth at high temperatures is related to an increase in the distribution of energetically distinct matrix environments. These trends were quite similar to that seen in the sugar $/ \beta$-Lg films (chapter III).

\section{Phosphorescence Decay kinetics}

The stretched exponential function is appropriate for fitting complex relaxation mechanisms that depopulate the excited triplet state (Richert, 1997). The physically meaningful parameters KWW lifetime (Williams and Watts, 1970) and $\beta$ (Lindsey and Patterson 1980) are highly sensitive to the molecular environment of the matrix and can be indicative of complex processes occurring in the matrix. 
Lifetime is an average time a probe spends in the excited triplet state. A study by Buettner, (1964) has shown that comparison of the probe emission lifetimes in presence and absence of oxygen is a facile indicator of the quenching of oxygen in the matrix

Lifetimes were measured by exciting Erythrosin B at $540 \mathrm{~nm}$ over a temperature range from $0^{\circ} \mathrm{C}$ to $100^{\circ} \mathrm{C}$ and the lifetime decays were fit using a stretched exponential function (equation 3 Materials and Methods). Figures V- 4 a, b, c, d, e are plots of lifetime as a function of temperature in glycerol plasticized $\beta$-Lg films. The text box refers to the molar ratio of glycerol in the matrix. The lifetime ranged from $0.56 \pm 0.032$ at $0^{\circ} \mathrm{C}$ to $0.25 \pm 0.0209$ at $100^{\circ} \mathrm{C}$ in the presence of $\mathrm{N}_{2}$ at varying ratios of glycerol plasticized $\beta$-Lg films. It is evident that addition of glycerol didn't have a major impact, as the measured lifetimes in the presence of glycerol were very similar to the control (Figure V4f).

The lifetimes in the presence of air were significantly different from those in the presence of nitrogen indicating that the glycerol/ $\beta$-Lg matrix was highly permeable to atmospheric oxygen. The lifetimes in the presence of nitrogen and presence of air were nearly identical at the concentration of 100:1 (glycerol: $\beta$-Lg) indicating a reduction in oxygen quenching at this concentration. However, at a ratio of $300: 1$ Glycerol/ $\beta-\mathrm{Lg}$ (a predominant glycerol matrix) there was a great divergence in the lifetime in air and lifetime in nitrogen at high temperatures $\left(70^{\circ} \mathrm{C}\right.$ and above) whereby the lifetime in air almost reduced to zero. This indicates that the oxygen molecules completely quench Erythrosin B phosphorescence in this matrix, at high temperatures. Predominant glycerol -glycerol interactions and specific hydrogen bonding interactions are thought to be responsible for these changes. 
Addition of sorbitol to the $\beta$ - $\mathrm{Lg}$ matrix had a concentration dependent effect as depicted in Figures V 5 a, b, c, d, e, f, g. We see that the lifetimes of Erythrosin B changed over the temperature range $0^{\circ} \mathrm{C}$ to $100^{\circ} \mathrm{C}$ from $0.65 \mathrm{~ms}$ to $0.167 \mathrm{~ms}$ in 300:1(sorbitol: $\beta-\mathrm{Lg}$ ), from $0.53 \mathrm{~ms}$ to $0.11 \mathrm{~ms}$ in $100: 1$ (sorbitol: $\beta-\mathrm{Lg}$ ), from $0.56 \mathrm{~ms}$ to $0.21 \mathrm{~ms}$ in $10: 1$ ( sorbitol: $\beta$ - $\mathrm{Lg}$ ), from $0.56 \mathrm{~ms}$ to $0.24 \mathrm{~ms}$ in $1: 1$ (sorbitol: $\beta-\mathrm{Lg}$ ) and from $0.6 \mathrm{~ms}$ to $0.2 \mathrm{~ms}$ in $0.1: 1$ (sorbitol: $\beta-\mathrm{Lg}$ ). Both in the presence of nitrogen and in the presence of air, no clear trends in lifetimes were observed.

Lifetimes are indicative of complex interactions occurring in the matrix and provide information about the various quenching mechanisms. The lifetimes decreased linearly under nitrogen conditions. The decrease in lifetime was due to an increase in the rate of non-radiative decay of the excited triplet state $T_{1}$ more specifically, increases in the rate of intersystem crossing back to the $\mathrm{S}_{1}\left(\mathrm{k}_{\mathrm{TS} 1}\right)$ and the rate of non radiative decay of the $\mathrm{T}_{1}$ state, or collisional quenching $\left(\mathrm{k}_{\mathrm{TS} 0}\right)$. However at a ratio of $0.1: 1$ sorbitol $/ \beta-\mathrm{Lg}$ the lifetime was higher than the control showing the origins of anti-plasticization. Above that ratio (> 0.1:1) addition of sorbitol brought about a reduction in the lifetime. At a molar ratio of 300: 1 Sorbitol/ $\beta$-Lg (Figure V-5e) we see that at a particular transition temperature $\left(30-40^{\circ} \mathrm{C}\right)$ there was a rapid decline in the lifetimes both in air and nitrogen, following a trend very similar to that seen in emission energy.

Upon comparing the lifetimes in air and nitrogen we see that at a critical concentration of 10:1 sorbitol: $\beta$-Lg the lifetimes in the presence of nitrogen and presence of air remain the same indicating that as low as 10 moles of sorbitol is sufficient to control the permeability of molecules in the matrix. At higher concentrations the divergence in between lifetimes in air and nitrogen was minimal. 
Figures V- 6 a, b, c, d, e, f, g depict the lifetimes in maltitol/ $\beta$-Lg films. Addition of maltitol to the $\beta$-Lg matrix had a similar effect as that of the addition of sugars to the matrix. We see that addition of maltitol brought about a significant increase in the lifetime indicating that maltitol brought about a reduction in the collisional quenching by conferring rigidity to the matrix. The range of lifetimes $\left(0^{\circ} \mathrm{C}\right.$ to $\left.100^{\circ} \mathrm{C}\right)$ were $0.54 \mathrm{~ms}$ to $0.22 \mathrm{~ms}$ at a ratio of $0.1: 1 \mathrm{maltitol} / \beta-\mathrm{Lg}$, which increased to $0.71 \mathrm{~ms}$ to $0.30 \mathrm{~ms}$ at $1000: 1$ maltitol $/ \beta$-Lg. Lifetimes values are a measure of rigidity of the matrix and it is clear that addition of maltitol conferred rigidity to the matrix. At ratios of 10:1 and higher, the lifetimes in the presence of nitrogen and presence of air remained the same, within error (Figures V 6 c, d, e) suggesting that oxygen could not penetrate into the matrix at these ratios. Small concentrations of maltitol as low as 10:1 were sufficient to control the permeability of oxygen in the films.

Polyethylene glycol is another plasticizer, which is widely used in edible films and has a very high plasticizing efficiency. Lifetimes at low concentrations of PEG (ratios of $0.1: 1$ and 1:1) were essentially similar to the control. But the measured lifetimes were significantly greater in the ratio $10: 1 \mathrm{PEG} / \beta-\mathrm{Lg}\left(0.66 \mathrm{~ms}\right.$ at $0^{\circ} \mathrm{C}$ to $0.26 \mathrm{~ms}$ at $\left.100^{\circ} \mathrm{C}\right)$. Lifetimes in the presence of air were much lower than those in nitrogen in all the concentrations indicating that the gas permeability was very high in this functional matrix. The increase in lifetime with an increase in PEG 400 is ostensibly related to an increase in local rigidity but it is clearly evident that the rigidity conferred is insufficient to hamper the permeant molecules from entering the matrix (Figure 7a, b, c, d, e).

The stretching exponent $\beta$ is a measure of the dynamic heterogeneity of the matrix (Eq 3-Materials and Methods). Trends in $\beta$ act as a quantitative index of the non - 
exponentiality of the probe decay behavior. The stretching factor followed similar trends in regards to the ratio of plasticizer to protein, as observed in lifetime values.

In the presence of nitrogen in varying concentrations of glycerol, $\beta$ was $0.85 \pm$ 0.0162 at $0^{\circ} \mathrm{C}$ and decreased insignificantly within error to $0.78945 \pm 0.0162$ at $100^{\circ} \mathrm{C}$. A small decrease in $\beta$ corresponds to a very large increase in the distribution of lifetimes (Lindsey and Paterson, 1980). It is clearly evident from Fig V- 8a that increasing glycerol concentration in the $\beta-\operatorname{Lg}$ matrix brings about an increase in the dynamic heterogeneity. A subsequent comparison of $\beta$ between air and $\mathrm{N}_{2}$, showed that at a ratio of 300: 1 glycerol $/ \beta-\mathrm{Lg}$ there was a significant difference between the $\beta$ in the presence of air and nitrogen. The large decrease in $\beta$ values in the presence of air at high glycerol concentrations is attributed to the enhanced mobility in the matrix due to softening. Glycerol softens the $\beta$-Lg matrix and results in an enhancement in the permeability. Thus, in the presence of oxygen there is a broader distribution of lifetimes

Figure V-9a, b depict $\beta$ as a function of temperature in sorbitol plasticized $\beta$-Lg films in the presence and absence of $\mathrm{O}_{2}$. The values of $\beta$ decreased gradually at low temperatures and dramatically at high temperatures in all the ratios. Addition of sorbitol had little effect on the $\beta$ of the matrix. $\beta$ values essentially remained constant within the scope of error in the presence of nitrogen $\left(0.8482 \pm 0.02\right.$ at $0^{\circ} \mathrm{C}$ and $0.76 \pm 0.02$ at $\left.100^{\circ} \mathrm{C}\right)$ and presence of air $\left(0.851137 \pm 0.058\right.$ at $0^{\circ} \mathrm{C}$ and $0.75 \pm 0.058$ at $\left.100^{\circ} \mathrm{C}\right)$ at various concentrations of sorbitol.

Maltitol had a concentration dependent effect on $\beta$ as portrayed in Figures V 10a and V 10b. The stretching factor $(\beta)$, followed similar trends as observed in the lifetime values. Addition of maltitol brought about an increase in the $\beta$ suggesting that in the 
presence of maltitol the distribution of dynamically different environments was decreased (Increase in homogeneity).

At a ratio of 10:1 the $\beta$ values in the presence of nitrogen and air were essentially similar which probably envisaged the dynamic synergy between $\beta$ and lifetime in judging the critical concentration for permeability. In the presence of oxygen, the $\beta$ values below the critical concentration were significantly lower in the presence of oxygen compared to the nitrogen conditions. At very high maltitol concentrations (100:1 and 1000:1) the distribution of environments with increase in maltitol concentration remained constant. The breakpoints in lifetime values correlate strongly with the oxygen permeability data.

Exponent $\beta$ in Peg 400 plasticized $\beta$-Lg essentially remained constant and showed similar behavior over the temperature range from $0^{\circ} \mathrm{C}-100^{\circ} \mathrm{C}$ in the nitrogen atmosphere (Figure11a). The values of $\beta$ in Peg 400 plasticized $\beta$-Lg films decreased gradually over the entire temperature range. Under oxygen conditions there was a precipitous drop in the $\beta$ at a concentration of $10: 1 \mathrm{Peg} / \beta-\mathrm{Lg}$ suggesting that the distribution of environments significantly broadened at this concentration. (Figure11b) The large decrease in $\beta$ at high PEG ratios is related to the oxygen molecules able to penetrate the matrix. When oxygen is present, there is a broader distribution of lifetimes as various sites with different mobilities.

Phosphorescence Decay Rate constants

Oxygen permeability

The term $k_{\mathrm{Q}}\left[\mathrm{O}_{2}\right]$ describes the rate of quenching of the excited triplet state of the probe by oxygen. Permeability is equal to the rate of diffusion multiplied by the solubility of the gas $(\mathrm{P}=\mathrm{DS}) . k_{\mathrm{Q}}\left[\mathrm{O}_{2}\right]$ is proportional to both the rate of oxygen diffusion $\left(\mathrm{k}_{\mathrm{Q}}\right)$ and the 
thermodynamics of oxygen solubility $\left(\mathrm{O}_{2}\right)$. (Nack and Ludescher, 2005; Simon Lukasik and Ludescher, 2004). This rate constant is calculated from the difference in inverse lifetime in air and in nitrogen (Eq 7 Materials and Methods). It is clearly evident from the study that additions of small molecule plasticizers have a tremendous impact on the $\beta$ - $\mathrm{Lg}$ matrix. There is a great difference in the $\mathrm{k}_{\mathrm{Q}}\left[\mathrm{O}_{2}\right]$ values in the various plasticizer/ $\beta$ - $\mathrm{Lg}$ matrices . Values of $\mathrm{k}_{\mathrm{Q}}\left[\mathrm{O}_{2}\right]$ are depicted in Figures V $12 \mathrm{a}, \mathrm{b}, \mathrm{c}, \mathrm{d}$

Figure $\mathrm{V}-12 \mathrm{a}$ depicts the collisional quenching constant $\mathrm{k}_{\mathrm{Q}}\left[\mathrm{O}_{2}\right]$ for the $\beta$ - $\mathrm{Lg}$ matrix with varying concentrations of glycerol. Addition of glycerol at ratios lower than 300:1 had an insignificant impact on the $\beta$ - $\mathrm{Lg}$ matrix, as $k_{\mathrm{Q}}\left[\mathrm{O}_{2}\right]$ was essentially similar to the control (pure $\beta$-Lg matrix) and displayed active oxygen quenching. At higher concentrations of glycerol $/ \beta-\operatorname{Lg}(300: 1)$, there was a dramatic increase in the $\mathrm{k}_{\mathrm{Q}}\left[\mathrm{O}_{2}\right]$.

Figure $\mathrm{V}-12 \mathrm{~b}$ is a plot of $\mathrm{k}_{\mathrm{Q}}\left[\mathrm{O}_{2}\right]$ as a function of temperature in sorbitol plasticized $\beta$-Lg films. Different concentrations of sorbitol affected the permeability of the $\beta$-Lg matrix in a distinct way. An optimum concentration was required to control the oxygen diffusion in the matrix. We could see that the oxygen quenching was negligible at a ratio of 10:1(sorbitol to $\beta$-Lg). At higher concentrations of sorbitol/ $\beta$ - $\operatorname{Lg}(100: 1$ and 300:1) the quenching was drastically reduced (It was lower than the control (pure $\beta$-Lg), but still higher than the determined breakpoint concentration). Addition of sorbitol greatly reduced the oxygen permeability in the $\beta$ - Lg matrix, which has been substantiated with other studies on edible films (MC Hugh and Krochta, 1993).

Maltitol is a disaccharide and has a ring structure. Figure V $12 \mathrm{c}$ is a plot of $\mathrm{k}_{\mathrm{Q}}\left[\mathrm{O}_{2}\right]$ in maltitol plasticized $\beta-\mathrm{Lg}$ as a function of temperature. It is evident from this plot that the effects of maltitol are quite similar to those of the sugars (chapter III). The 
$\mathrm{k}_{\mathrm{Q}}\left[\mathrm{O}_{2}\right]$ values were greatly diminished upon addition of maltitol. Maltitol addition at ratios as low as 10 moles of maltitol to 1 mole of $\beta$-Lg brought about a ten fold decline in $\mathrm{k}_{\mathrm{Q}}\left[\mathrm{O}_{2}\right]$ values in the tested temperature range from $0^{\circ} \mathrm{C}$ to $100^{\circ} \mathrm{C}\left(\mathrm{k}_{\mathrm{Q}}\left[\mathrm{O}_{2}\right]\right.$ at $0^{\circ} \mathrm{C}$ decreased from $250.3 \mathrm{~s}^{-1}$ to $57 \mathrm{~s}^{-1}$ and from $9577 \mathrm{~s}^{-1}$ to $1454 \mathrm{~s}^{-1}$ at $100^{\circ} \mathrm{C}$ ). Higher concentrations of maltitol brought about a further decline in the collisional quenching rate constant for oxygen permeability.

Figure V-12d depicts the plot of $\mathrm{k}_{\mathrm{Q}}\left[\mathrm{O}_{2}\right]$ in PEG 400 plasticized $\beta$ - $\mathrm{Lg}$ films. This plot is quite a contrast from what is seen in maltitol / $\beta$-Lg films. It is evident that PEG 400 enhances the permeability of the matrix. At a PEG to $\beta$-Lg ratio of 10:1 there was an approximate 5-6-fold increase in the permeability of the matrix.

\section{Matrix mobility}

Analysis of the Erythrosin B lifetime in terms of the underlying photophysical rate constants gives an indication of the mobility of the matrix. Depopulation of the excited triplet state in nitrogen is primarily due to non- radiative processes associated with intersystem crossing (rate $\mathrm{k}_{\mathrm{TS} 0}$ ), and this term is a direct indicator of the mobility in the matrix. The values of $\mathrm{k}_{\mathrm{TS} 0}$ were calculated based on $\mathrm{k}_{\mathrm{RP}}$ and $\mathrm{k}_{\mathrm{TS} 1}$ measured by Duchowicz et al., 1998 (Eq 4. Materials and Methods). $\beta$-Lg films had different trends in mobility with respect to different plasticizers and these trends are depicted in Figures V $13 \mathrm{a}, \mathrm{b}, \mathrm{c}$ and $\mathrm{d}$.

Figure $\mathrm{V}-13 \mathrm{a}$ shows a plot of $\mathrm{k}_{\mathrm{TS} 0}$ as a function of temperature in glycerol plasticized $\beta$-Lg films. $\mathrm{k}_{\text {TS0 }}$ increased with temperature for all ratios of glycerol $/ \beta-\mathrm{Lg}$. At low temperatures an increase in glycerol concentration did not significantly alter the mobility of the matrix. However differences in matrix mobility were clearly apparent at 
higher temperatures (increase in glycerol brought about increases in matrix mobility). An interesting trend was observed at $1: 1$ glycerol/ $\beta$ - Lg. A noticeable decrease in the matrix mobility was observed at this concentration. This can be compared to the phenomenon of anti-plasticization, were there is decrease of local molecular mobility at certain concentrations and there is induction of rigidification. This kind of behavior was recently observed in starch containing low glycerol (Loudin et al., 1997) and sorbitol (Gaudin et al., 1999). However, glycerol at high concentration brings about an increase in the matrix mobility (due to plasticization).

An effect of temperature on the mobility parameter $\mathrm{k}_{\mathrm{TS} 0}$ in sorbitol plasticized $\beta$ $\mathrm{Lg}$ films is depicted in Figure V $13 \mathrm{~b}$. It is clearly evident that at low temperatures, $\mathrm{k}_{\mathrm{TS} 0}$ essentially remained constant and there was no significant change in between concentrations. However at high temperatures above a certain concentration (>1:1) $\mathrm{k}_{\mathrm{TS} 0}$ increased with increase in sorbitol concentration. At a molar ratio of 300:1 Sorbitol/ $\beta$-Lg a dramatic increase in $\mathrm{k}_{\mathrm{Ts} 0}$ at a temperature range of $30 \mathrm{C}-40^{\circ} \mathrm{C}$ was observed. This trend in mobility essentially correlates with the other findings (emission energy and lifetimes). Sharp increase in $\mathrm{k}_{\mathrm{TS} 0}$ may be indicative of important changes occuring in the matrix, as all the parameters essentially reflect the occurance of a phenomenon. This may also be attributed to phase separation in the matrix.

Figure V-13 c shows $\mathrm{k}_{\mathrm{TS} 0}$ as a function of temperature in maltitol/ $\beta$ - $\mathrm{Lg}$ films. The collisional quenching rate $\mathrm{k}_{\mathrm{TS} 0}$ increased systematically with temperature in all maltitol/ $\beta$ Lg films. Addition of maltitol brought about a lowering in the $\mathrm{k}_{\mathrm{TS} 0}$, indicating maltitol brings rigidity into the matrix in a concentration dependent fashion. Maltitol at high concentrations $(100: 1,1000: 1)$ drastically reduced the mobility of the matrix. At a ratio 
of $1000: 1$ the $\mathrm{k}_{\mathrm{TS} 0}$ decreased by $22 \%$ at $0^{\circ} \mathrm{C}$ and $30 \%$ at $100^{\circ} \mathrm{C}$. Trends in $\mathrm{k}_{\mathrm{TS} 0}$ essentially substantiate the fact that maltitol follows maltose like behavior (Chapter III with respect to matrix mobility.

The $\mathrm{k}_{\mathrm{TS} 0}$ of PEG 400 plasticized films are depicted as a function of temperature in Figure $\mathrm{V}-13 \mathrm{~d}$. The $\mathrm{k}_{\mathrm{TS} 0}$ essentially remained constant upon addition of PEG at low concentrations. Unexpectedly Peg 400 plasticized $\beta$-Lg films had a decline in the mobility at a ratio of 10:1. This decrease in mobility is mainly attributed to the fact that increase in concentration of PEG changes the local mobility of the matrix.

\section{Relationship between Oxygen permeability and Molecular mobility}

Despite the dramatically different trends with respect to mobility and permeability, there was a direct correlation between oxygen permeability and molecular mobility in plasticizer/ $\beta$-Lg films. The plots for $\mathrm{k}_{\mathrm{TS} 0}$ and $\mathrm{k}_{\mathrm{Q}} \mathrm{O}_{2}$ clearly illustrates the interdependence between mobility amd permeability.

In glycerol (Figure V-14a-d) and sorbitol plasticized $\beta$-Lg films (FigureV 15ae), $\mathrm{k}_{\mathrm{TS} 0}$ and $\mathrm{k}_{\mathrm{Q}}\left[\mathrm{O}_{2}\right]$ had a biphasic correlation with minor differences. At low temperatures there was a gradual increase in permeability $\mathrm{k}_{\mathrm{Q}}\left[\mathrm{O}_{2}\right]$ with increase in mobility. However at higher temperatures there was a massive increase in permeability. In all $\beta$-Lg plasticized films certain basal level of mobility was required to initiate permeability.

In Maltitol/ $\beta-\mathrm{Lg}$ films at low ratios of maltitol, oxygen permeability was a biphasic linear function of $\mathrm{k}_{\mathrm{TS} 0}$ (Figure V-16a-e). At ratios of 100:1 and 1000:1 Maltitol/ $\beta-\mathrm{Lg}$, there was no longer a direct correlation, as the oxygen permeability reduced drastically. 
In PEG plasticized $\beta$-Lg films permeability was a linear function of kTS0 (overlooking the effect at $100^{\circ} \mathrm{C}$ ) at all ratios (Figure $17 \mathrm{a}-\mathrm{c}$ ).

\section{$\underline{\text { Discussion }}$}

Edible films can improve food quality and increase food product shelf life by serving as selective barriers to moisture transfer, oxygen uptake, aroma loss and lipid oxidation (Donhowe and Fennema, 1993; Krochta, 1997). Proteins have been known for their excellent film forming capabilities. However, proteins by themselves form very brittle films, which are useless in application. Usually plasticizers, which are low molecular weight non- volatile compounds, are essentially added to film formulations to increase flexibility (Banker, 1966). The macroscopic functional properties of edible films are modulated by mobility in the rubbery state (Anker et al., 2001; Zhang et al., 2000).

Oxygen permeability is the most commonly studied transport property of edible polymer films. Permeability, defined as the product of the coefficients of solubility and diffusion, involves a kinetic factor affected by molecular mobilities (diffusion). Permeability depends on the availability of free interstitial space for the permeant molecules to move. The chemical structure of the polymer backbone, the degree of crystallinity, orientation of molecular chains and the nature of plasticizer added affect the barrier properties (Salame, 1986). Hydrophilic compounds such as polyols (glycerol, sorbitol and polyethylene glycol) are commonly used as plasticizers in hydrophilic film formation.

In the present study we used phosphorescence emission from the triplet probe erythrosin B to monitor the molecular mobility and oxygen permeability in amorphous $\beta$ - 
Lg plasticizer films. Phosphorescence from erythrosin B provides information about three different aspects of molecular mobility within the $\beta$-Lg plasticized film. Firstly, emission energy senses the changes in dipolar relaxation around the excited triplet state (Demchenko, 2002; Ludescher et al., 2001; Pravinata et al., 2005; Richert, 2000). Dipolar relaxations lower the energy of the excited triplet state and thus changes in emission energy can be attributed to changes in relaxation rates. Secondly, the emission lifetime $(\tau)$ provides a direct measure of the rate of non-radiative coupling to the ground state $\left(\mathrm{k}_{\mathrm{TS} 0}\right)$ due to matrix collisions with the probe (Pravinata et al., 2005). Thirdly, quenching by molecular oxygen, which is a potent diffusional quencher of the triplet state, can provide a direct measure of the rate of oxygen permeability through the matrix (Simon-Lukasik and Ludescher, 2004). Such studies provide a deeper understanding of the molecular mechanisms controlling oxygen permeability. There was a wide variation in between the various plasticizers with respect to trends in mobility and permeability. To depict an overview of the changes occurring we present here a summary, which are a complete representation of the mobility and permeability of the $\beta$ - Lg matrix in the presence of various plasticizers.

Effect of glycerol on the matrix mobility of $\beta$ - Lg films

This study looks into the effect of glycerol in two main ways: Plasticization and anti- plasticization. The matrix mobility in amorphous glycerol plasticized films was modulated in a complex concentration dependent manner. Plots of emission energy versus molar concentration and matrix mobility parameter $\mathrm{k}_{\mathrm{TS} 0}$ versus molar concentration were generated to investigate the concentration dependence of matrix mobility in glycerol plasticized $\beta$ - Lg films. 
Anti-plasticization effects of glycerol on the $\beta$-Lg matrix.

Plasticization by polyols affects the $\mathrm{Tg}$ of amorphous or partially amorphous matrices such as gelatin (Cruz et al., 2005), wheat gluten films (Pouplin et al., 1999), and potato starch films (Riku, 2007) resulting in a drop of Tg. But glycerol at a ratio of 1:1 has an anti-plasticizing effect on the $\beta$-Lg matrix marked by a decline in the mobility (decrease in $\mathrm{k}_{\mathrm{TS} 0}$ ) and dipolar relaxations (increase in emission energy). Strong interactions between glycerol and $\beta$ - $\operatorname{Lg}$ are apparently more dominant than the plasticizing capabilities of glycerol at this particular concentration. These effects appear to be manifestations of the phenomenon of anti-plasticization widely seen in synthetic polymer systems with low concentrations of small molecule plasticizers (Loudin et al., 2003). Anti-plasticization may reflect the ability of these small molecules to form a tightly coupled hydrogen-bonded network within the amorphous matrix that lowers overall molecular mobility. A recent study by You and Ludescher (2007) has shown that glycerol can decrease the molecular mobility of amorphous sucrose glasses at low concentrations. The presence of strong hydrogen bonded glycerol-sugar interactions may constrain the local motions of a sugar matrix dominated by sugar-sugar interactions. Similar effects of glycerol have been studied by dielectric relaxation spectroscopy on the mobility of trehalose (Anopchenko et al., 2006) and maltose (Lourdin et al, 2003). It is clear that the effect of anti-plasticization occurs over a concentration range below the plasticization threshold as noticed in other studies (You and Ludescher, 2007; Sears and Darby, 1982; Vrentas et al., 1988; Shuster et al., 1994; Anderson et al., 1995; Seow et al., 1999). At this concentration due to presence of plasticizer molecules adjacent to polar 
groups, polymer-plasticizer interactions create steric-hindrance and decrease segmental mobility (Anderson et al., 1995). This anti- plasticization is also clearly illustrated in the plot of emission energy as a function of molar ratio in glycerol plasticized $\beta$ - Lg films (Figure V-18a, b) and plots of $\mathrm{k}_{\mathrm{TS} 0}$ as a function of molar ratio (Figure V-19a,b).

Plasticization effects of glycerol on the $\beta$-Lg matrix

At higher concentrations $(>1: 1)$ of glycerol the plasticizing effects of glycerol are dominant, marked by a clear increase in matrix mobility $\left(\mathrm{k}_{\mathrm{TS} 0}\right)$ and noticeable decrease in emission energy. The possible reasons for this phenomenon are as follows: Glycerol with a molecular weight of $92 \mathrm{~g} / \mathrm{mol}$ can be easily inserted between the side chains (Gontard et al., 1993) and therefore has a high probability of hydrogen bonding at all hydrophilic locations. Thus the interactions between proteins are reduced and inter-molecular spacing is increased resulting in its plasticizing action. Noticeable fluctuation in emission energy in $\beta$ - $\mathrm{Lg}$ films in the presence of high concentrations of glycerol may be due to the possible formation of glycerol clusters. Possible phase separation also cannot be ruled out.

In glycerol/ $\beta$-Lg films, mobility can be characterized into three regions based on concentration dependence: At a critical concentration of 1:1 the resultant interactions between glycerol / $\beta$-Lg lead to reductions in mobility (anti-plasticization effects). At concentrations lower than the critical concentrations the matrix is predominantly a $\beta$ - $\mathrm{Lg}$ matrix and the mobility is unaffected. At ratios higher than the critical concentration it is a glycerol dominated matrix leading to increases in mobility (plasticization). 


\section{Oxygen permeability of glycerol plasticized $\beta$-Lg films}

Oxygen is the key factor that might cause oxidation, which initiates several food changes such as odor, color, flavor and nutrients deterioration, so obtaining films with proper oxygen barrier can help improving food quality and extending food shelf life. The oxygen quenching constant $\mathrm{k}_{\mathrm{Q}}\left[\mathrm{O}_{2}\right]$ is the product of terms proportional to both the rate of oxygen diffusion $\mathrm{k}_{\mathrm{Q}}$ and the thermodynamics of oxygen solubility $\left[\mathrm{O}_{2}\right]$; it reflects the permeability of the matrix ( Simon-Lukasik and Ludescher, 2004).

Glycerol had no noticeable effect on the permeability of the $\beta$ - $\operatorname{Lg}$ matrix at concentrations up to $100: 1$ (glycerol: $\beta$-Lg). It is also seen that there was an insignificant change in the permeability at the "critical concentration" whereby we saw origins of antiplasticization (as reflected by increases in emission energy and decreases in $\mathrm{k}_{\mathrm{TS} 0}$ ). But addition of glycerol as high as 300 moles per mole of $\beta$-Lg brought about dramatic increases in the permeability of the matrix (Figure V-20 a, b).

A study by Gounga et al. (2005) examined the effect of glycerol on the oxygen permeability of edible films made from whey protein isolates. A slight, yet significant increase in oxygen permeability was observed, when the glycerol amount was increased in the film solution. Glycerol may compete with water for the active sites on the polymer, thus promoting water clustering and increased free volume in the polymers at low moisture levels. The effect of glycerol concentration on diffusivity was greater than that on solubility and resulted in increased permeability values (Lieberman \& Gilbert, 1973). Addition of linear molecules like glycerol, lessen the intermolecular interactions, maximize free volume, reduces the viscosity of the solution and increase the intermolecular distance thus making more holes for the permeant molecules to jump 
(Gontard et al., 1993) The decreases in intermolecular interactions may be attributed to the enhancement of the permeability in high glycerol concentration.

It is evident that at higher ratios of glycerol the effect of glycerol plasticization dominates, which results in an increase in molecular mobility of the matrix and as a consequence diffusive processes within the matrix become much more rapid.

The plasticizing effect of glycerol is more complex with respect to modulation of barrier properties. Lack of a consistent trend in permeability at high concentrations of glycerol may be due to phase separation, formation of glycerol clusters, etc. Permeability is a product of solubility and diffusivity; low solubility of oxygen in glycerol may also be attributed to differences in permeability.

Despite exhibiting differences in permeability and matrix mobility the plots of $\mathrm{k}_{\mathrm{Q}}\left[\mathrm{O}_{2}\right]$ versus $\mathrm{k}_{\mathrm{TS} 0}$ clearly illustrated their inter-dependence in glycerol plasticized $\beta$ - $\mathrm{Lg}$ films. The similarities in the relationship between oxygen permeability and molecular mobility between $\beta-\mathrm{Lg}$ and glycerol/ $\beta$ - Lg films suggest that the basic mechanisms controlling oxygen permeability are similar in the two matrixes.

Effect of sorbitol on the matrix mobility of $\beta$-Lg films

Sorbitol is another plasticizer commonly used in the food industry. The present study looked into the effect of this conventional plasticizer on the molecular mobility and permeability of $\beta$-Lg films.

Emission energy decreased linearly with increase in temperature in sorbitol plasticized $\beta$ - Lg films at most sorbitol ratios owing to increased dipolar relaxations. But, at a ratio of 300:1 sorbitol / $\beta$ - Lg a sharp decline in the emission energy was observed at a temperature range between $30-40^{\circ} \mathrm{C}$. This temperature may be reflective of important 
changes occurring in the matrix. We attribute this transition to a phenomenon similar to softening transition. This trend may also be attributed to phase separation at high sorbitol concentration which may result in drastic fluctuations in the mobility.

Phase separations can lead to dramatic changes in the composition of the matrix resulting in higher local moisture contents which may also lead to increases in mobility (Sun and Davidson, 1998)

It is clear that addition of sorbitol affected emission energy in a complex concentration dependent manner. The plots of emission energy as a function of molar ratio clearly illustrate these effects (Figure V-21a). Sorbitol at a critical concentration of 10:1 sorbitol / $\beta$ - Lg displayed a significant increase in emission energy. The probable reason for this kind of phenomenon is at this ratio intermolecular interactions between sorbitol and $\beta$ - Lg bring about a decrease in the local relaxation mechanisms in the matrix. However, at a ratio of 100:1 there was a phenomenal decline in the emission energy. We speculate that the increase in sorbitol concentration (approx 50wt \% in the film) is probably responsible for the decrease. However, an unexpected increase in the emission energy at higher concentrations (300:1, 74\%wt) was observed. Complex hydrogen bonding interactions owing to the matrix changing from a predominant protein matrix to a plasticizer matrix and the resultant change in hydrogen bonding interactions may be responsible for these changes. Possible formation of clusters of sorbitol may also lead to regions of high and low mobility, which may be evident in these results.

Similar findings have been reported with respect to variation in mechanical properties in sorbitol plasticized starch films (Gaudin et al. (2000)). The possible occurrence of phase separation also cannot be ruled out. These trends need to be further 
investigated with detailed analysis of the mechanical properties, which may throw light on important relevant mechanisms.

Figure V-21b is the zoom in version of emission energy as a function of molar ratio of sorbitol at functional temperatures that affect edible films and it essentially conveys the same message.

The rate $\mathrm{k}_{\mathrm{TS} 0}$ provides a measure of the molecular mobility because it monitors the interactions between the probe and the environment (the energy dissipated from the excited state to the surrounding matrix). To clearly illustrate the matrix mobility trends, $\mathrm{k}_{\mathrm{TS} 0}$ as a function of sorbitol concentration is depicted in Figure V-22a. It is evident that the addition of sorbitol had no effect on the matrix mobility until a certain concentration was approached. Surprisingly, the critical concentration (10:1) at which there was a decline in permeability there was no noticeable change in the mobility parameter $\mathrm{k}_{\mathrm{TS} 0}$. However there was a phenomenal increase in the mobility $\left(\mathrm{k}_{\mathrm{TS} 0}\right)$ at a concentration of 100:1 Sorbitol / $\beta-\mathrm{Lg}$, followed by a decline in $\mathrm{k}_{\mathrm{TS} 0}$ at a higher concentration of 300:1. In the sorbitol matrix these differences in mobility may be attributed to the presence of sorbitol clusters which are characterized by regions of low and high mobility and the probe may be sensing these differences in mobilities. Further detailed investigations by the use of other instruments like AFM, FTIR, NMR etc may throw light on the complex changes occurring in the matrix and give us a detailed picture of the exact hydrogen bonding interactions.

Effects of sorbitol on the Oxygen permeability of the matrix

Figure V-23a shows $\left(\mathrm{k}_{\mathrm{Q}}\left[\mathrm{O}_{2}\right]\right)$ as a function of molar concentrations in sorbitol plasticized $\beta$-Lg films at different temperatures. This graph gives us a clear picture of the 
permeability of sorbitol plasticized $\beta-\mathrm{Lg}$ films as affected by concentration. Sorbitol concentration was a determining factor in these films. At very low concentrations of sorbitol (0.1:1 and 1:1) the permeability was unaffected by change in plasticizer concentration. However, at a critical concentration of 10:1 (approximately 9 wt\% sorbitol) there was a phenomenal decline in the permeability. This concentration can be a very useful index for food scientists as this particular concentration can be referred to as the "critical concentration" for optimum control of oxygen permeability in this functional matrix. Oxygen permeability even though showing a reversal of tendency at a ratio of 100:1 did not change abruptly. The increase in $\mathrm{k}_{\mathrm{Q}}\left[\mathrm{O}_{2}\right]$ at higher ratios may be attributed to the increase in the error in lifetime measurements at higher concentrations. However, the present analysis of the calculation of rate constants for oxygen permeability did not take those errors into account. A look into the plots of lifetimes in the presence of air and $\mathrm{N}_{2}$ (Figure V-5d and 5-e) clearly reveal that the permeability is unaffected within error at high ratios of sorbitol. So in sorbitol plasticized $\beta$ - Lg films permeability could be divided into two regions based on concentration dependence. 1. Below the critical concentration range the permeability was unaffected. 2 . Permeability greatly declined at the threshold concentration and at higher ratios.

This phenomenon is very similar to what is observed in sorbitol plasticized starch films. Increase in permeability at high concentrations was less pronounced at low temperatures as evident in the zoom in plot (Figure V-23b) Indeed, non-classical behaviour has been reported by Gaudin et al. (2000) who have studied oxygen permeability of sorbitol plasticised starch at low plasticiser and water contents. They reported a decrease in oxygen flux (10-fold drop) from 0 to $8.8 \%$ plasticizer and related 
to the increase of the starch secondary relaxation for starch/sorbitol systems. Sorbitol can develop strong interactions with starch, which could in turn affect its macromolecular motion and limit oxygen permeability. As a result, permeability decrease is explained by diffusivity decrease. A study by Mc High and Krochta (1994) revealed that the oxygen permeability of sorbitol was significantly lower than that of glycerol plasticized

In $\beta$-Lg without added sorbitol, local mobilities favored oxygen diffusion whereas the loss of these mobilities attributable to the creation of bonds between $\beta$-Lg and sorbitol decreased oxygen permeability in the polymer.

\section{Matrix mobility in maltitol / $\beta$ - Lg films}

Maltitol is a polyol and has recently been used a lot in edible films. Maltitol affected the mobility in a simple concentration dependent manner. Small amounts of maltitol (10:1) resulted in a massive increase in the emission energy, and decrease in $\mathrm{k}_{\mathrm{TS} 0}$ in $\beta \mathrm{Lg}$ films. The graphs for concentration dependence for emission energy (Figure $\mathrm{V}$ $24 \mathrm{a}, \mathrm{b}$ ) and $\mathrm{k}_{\mathrm{TS} 0}$ (Figure V- $25 \mathrm{a}, \mathrm{b}$ ) clearly illustrate these effects.

Higher lifetime, increase in emission energy and decreases in $\mathrm{k}_{\mathrm{TS} 0}$ clearly indicated that maltitol had a "rigidification" effect on the $\beta$ - Lg matrix.

Maltitol addition had an antagonistic effect on the permeability of the matrix as evident in the decreasing collisional quenching rates for oxygen permeability $\left(\mathrm{k}_{\mathrm{Q}}\left[\mathrm{O}_{2}\right]\right)$ (Figure 26a, b). Whitcombe et al. (2005) studied the oxygen solubility of maltitol and concluded that low-oxygen solubility is a major factor contributing to the barrier properties of maltitol. A study by Zhang and Han (2006) studied the effect of maltitol on pea starch films and found that maltitol was the least effective plasticizer among the 
studied plasticizers. They attributed the barrier properties of maltitol to its bulky ring structure and increased resistance to moisture permeation.

It is evident that Maltitol has a profound effect on the permeability and mobility of the $\beta$-Lg matrix, and exhibits anti-plasticization characteristics similar to that observed in the disaccharides (Chapter III).

The concentration dependence of emission energy and $\mathrm{k}_{\mathrm{TS} 0}$ in PEG plasticized $\beta$ $\lg$ films are depicted in Figures V-27a, b and 28a, b respectively. Addition of PEG brought about an enhancement in the emission energy. However at a concentration of 10:1 PEG: $\beta$ - Lg there was a precipitous decline in the emission energy and a decrease in the $\mathrm{k}_{\mathrm{TS} 0}$ with addition of PEG. The anomolous trends in PEG with respect to mobility in $\beta$ - Lg films may be attributed to the following explanations. A study on the effect of PEG on protein lysozyme has clearly indicated that PEG does not hydrogen bond with proteins and is preferentially excluded. So there is a possibility that there may specific PEG clusters and $\beta$ - Lg cluster and the probe may be sensing these differences in mobility. This is just hypothetical explanation and further analysis needs to be done with complementary techniques to derive a suitable explanation. Another possible explanation is phase separation is also a great possibility in PEG plasticized $\beta$ - $\mathrm{Lg}$ films.

Figure V-29a shows $\mathrm{k}_{\mathrm{Q}}\left[\mathrm{O}_{2}\right]$ as a function of molar ratio of in PEG plasticized Lg films. It is evident that PEG 400 enhances the permeability of the $\beta$ - $\mathrm{Lg}$ matrix. A study on the effect of plasticizer of PEG 400 showed that addition of PEG 400 resulted in a decrease in the EM and TS and concomitant increase in percentage elongation (Sothornvit and Krochta, 2000). It was also found that PEG 400 had the largest effect on tensile strength (decrease in tensile strength) when compared to other polyols like 
glycerol and sorbitol. The possible explanation for this phenomenon is as more amount of plasticizer is inserted into the protein matrix the protein - weaker plasticizer bonds replace protein bonds. This results in an increase in the intermolecular spacing (Siew et al., 1999). In PEG plasticized $\beta$-Lg films phenomenal increases in permeability at high plasticizer concentrations can be attributed to the loss of cohesiveness in the film network due to increase in the number of plasticizer - plasticizer associations

Previous studies have shown that PEG is an excellent plasticizer of polymers. In a study of Hydrogen Bonding in methylcellulose-based edible films plasticized by polyethylene glycol revealed that as the concentration of PEG 400 increased there was a significant increase in the hydrogen bond formation (Turhan, 2001). This result was in accordance with hypothesis that the plasticizer "solvates" polar sites on the polymer chains, especially at high plasticizer levels, thereby reducing intermolecular attraction (Martin-Polo, 1995) and increasing intermolecular spacing. This hypothesis may serve as a possible explanation of the enhancement of the permeability in the presence of PEG 400 .

It is clear that all these plasticizers affected mobility and permeability in a unique way. These distinct trends with respect to mobility may be attributed to different set of interactions with the $\beta$ - $\operatorname{Lg}$ matrix. Despite exhibiting differences in the trends in mobility and permeability all these plasticizers had a linear correlation between mobility and permeability. The similarities in the relationship between oxygen permeability and molecular mobility between $\beta$ - Lg and plasticizer/ $\beta$-Lg suggest that the basic mechanisms controlling oxygen permeability are similar in these matrices. 


\section{$\underline{\text { Conclusions }}$}

The molecular dynamics and the oxygen permeability of $\beta$ - $\mathrm{Lg}$ films were found to be altered by plasticizer concentration. Glycerol at a ratio of 1:1 showed a decrease in mobility and increase in emission energy showing clear signs of the phenomenon of antiplasticization at that ratio. Glycerol has a stabilization effect on the $\beta$-Lg matrix in that ratio and specific interactions between glycerol and $\beta-\mathrm{Lg}$ at this ratio is responsible for this phenomenon. Sorbitol on the other hand had no significant change in mobility but at a critical concentration of 1:10 exhibited a drastic decrease in permeability. It is clear that sorbitol proved as a superior oxygen barrier when compared to glycerol. Maltitol owing to its ring structure exhibits a "rigidification" effect and showed a drastic decrease in permeability and mobility. PEG led to drastic increases in permeability at very low ratios clearly indicating that it was the least effective plasticizer in terms of barrier properties. Each plasticizer had a different set of interactions with the $\beta$-Lg matrix and led to generation of different kinds of mobility contours. Such mobility contours can enable man to characterize the role of plasticizers in the formulation of edible films and successfully manipulate the functionality of edible films. 


\section{$\underline{\text { References }}$}

Anderson, S. L., Grulke, E. A., DeLassus, P. T., Smith, P. B., Kocher, C. W., and Landes, B. G. (1995). A Model for Antiplasticization in Polystyrene. Macromolecules. 28, 2944-2954.

Anker, M., Stading, M., and Hermansson, A. (1998). Mechanical Properties, Water Vapor Permeability, and Moisture Contents of b-Lactoglobulin and Whey Protein Films Using Multivariate Analysis. J. Agric. Food Chem. 46, 1820-1829.

Anopchenko, A., Psurek, T., VanderHart, D., Douglas, J. F., and Obrzut, J. (2006). Dielectric study of the antiplasticization of trehalose by glycerol. Physical Review E: Statistical, Nonlinear, and Soft Matter Physics. 74, 031501/1-031501/10.

Arvanitoyannis, I. S., Nakayama, A., and Aiba, S. (1998). Chitosan and gelatin based edible films: state diagrams, mechanical and permeation properties. Carbohydr. Polym. 37, 371382.

Aulton, M. E., Abdul-Razzak, M. H., and Hogan, J. E. (1981). The mechanical properties of hydroxypropylmethylcellulose films derived from aqueous systems. Part 1: The influence of plasticizers. Drug Dev. Ind. Pharm. 7, 649-668.

Banker, G. S. (1966). Film coating theory and practice. J. Pharm. Sci. 55, 81-89.

Buettner, A. V. (1964). Flash photolysis in thin films of gelatin and other polymers. Journal of Physical Chemistry. 68, 3253-3259.

Champion, D., Le Meste, M., and Simatos, D. (2000). Towards an improved understanding of glass transition and relaxations in foods: molecular mobility in the glass transition range. Trends Food Sci. Technol. 11, 41-55.

Cuq, B., Gontard, N., Cuq, J., and Guilbert, S. (1997). Selected Functional Properties of Fish Myofibrillar Protein-Based Films As Affected by Hydrophilic Plasticizers. J. Agric. Food Chem. 45, 622-626.

D'Cruz, N. M. and Bell, L. N. (2005). Thermal unfolding of gelatin in solids as affected by the glass transition. J. Food Sci. 70, E64-E68.

Demchenko, A. P. (2002). The red-edge effects: 30 years of exploration. Luminescence. 17, 1942.

Donhowe, I. G. and Fennema, O. (1993). The effects of plasticizers on crystallinity, permeability, and mechanical properties of methylcellulose films. J. Food Process. Preserv. 17, 247-257. 
Duchowicz, R., Ferrer, M. L., and Acuña, A. U. (1998). Kinetic Spectroscopy of Erythrosin Phosphorescence and Delayed Fluorescence in Aqueous Solution at Room Temperature. Photochemistry and Photobiology. 68, 494-501.

Gaudin, S., Lourdin, D., Forssell, P. M., and Colonna, P. (2000). Antiplasticization and oxygen permeability of starch-sorbitol films. Carbohydr. Polym. 43, 33-37.

Gaudin, S., Lourdin, D., Le Botlan, D., Ilari, J. L., and Colonna, P. (1999). Plasticisation and mobility in starch-sorbitol films. Journal of Cereal Science. 29, 273-284.

Gennadios, A., Weller, C. L., and Testin, R. F. (1993). Temperature effect on oxygen permeability of edible protein-based films. J. Food Sci. 58, 212-14, 219.

Gontard, N., Guilbert, S., and Cuq, J. L. (1993). Water and glycerol as plasticizers affect mechanical and water vapor barrier properties of an edible wheat gluten film. J. Food Sci. 58, 206-211.

Gounga, M. E., Xu, S., and Wang, Z. (2007). Whey protein isolate-based edible films as affected by protein concentration, glycerol ratio and pullulan addition in film formation. Journal of Food Engineering,. 83, 521-530.

Gueguen, J., Viroben, G., Barbot, J., and Subirade, M. (1998). Comparative properties of pea protein and wheat gluten films. Influence of various plasticizers and aging. Plant Proteins from European Crops. 319-323.

Hernandez-Munoz, P., Lopez-Rubio, A., Lagaron, J. M., and Gavara, R. (2004). Gliadins Polymerized with Cysteine: Effects on the Physical and Water Barrier Properties of Derived Films. Biomacromolecules. 5, 1503-1510.

Jangchud, A. and Chinnan, M. S. (1999). Properties of peanut protein film: sorption isotherm and plasticizer effect. Lebensmittel-Wissenschaft und -Technologie. 32, 89-94.

Kalichevsky, M. T., Blanshard, J. M. V., and Tokarczuk, P. F. (1993). Effect of water content and sugars on the glass transition of casein and sodium caseinate. Int.J.Food Sci.Technol. 28, 139-151.

Karbowiak, T., Hervet, H., Leger, L., Champion, D., Debeaufort, F., and Voilley, A. (2006). Effect of Plasticizers (Water and Glycerol) on the Diffusion of a Small Molecule in IotaCarrageenan Biopolymer Films for Edible Coating Application. Biomacromolecules. 7, 2011-2019.

Krochta, J. M. (1997). Edible protein films and coatings. Food Sci.Technol.(N.Y.). 80, 529-549.

Lakowicz, J. R. (1999). Principles of fluorescence spectroscopyKluwer Academic/Plenum Press, Dordrecht/New York. 
Lettinga, M. P., Zuilhof, H., and Van Zandvoort, M. A. M. J. (2000). Phosphorescence and fluorescence characterization of fluorescein derivatives immobilized in various polymer matrices. Physical Chemistry Chemical Physics. 2, 3697-3707.

Lieberman, E. R. and Gilbert, S. G. (1973). Gas permeation of collagen films as affected by crosslinkage, mositure, and plasticizer content. J.Polym.Sci., Polym.Symp. No. 41, 33-43.

Lindsey, C. P. and Patterson, G. D. (1980). Detailed comparison of the Williams-Watts and Cole-Davidson functions. J. Chem. Phys. 73, 3348-3357.

Lourdin, D., Bizot, H., and Colonna, P. (1997). I"Antiplasticizationl" in starch-glycerol films? J Appl Polym Sci. 63, 1047-1053.

Lourdin, D., Coignard, L., Bizot, H., and Colonna, P. (1997). Influence of equilibrium relative humidity and plasticizer concentration on the water content and glass transition of starch materials. Polymer. 38, 5401-5406.

Lourdin, D., Colonna, P., and Ring, S. G. (2003). Volumetric behaviour of maltose-water, maltose-glycerol and starch-sorbitol-water systems mixtures in relation to structural relaxation. Carbohydr. Res. 338, 2883-2887.

Ludescher, R. D., Shah, N. K., McCaul, C. P., and Simon, K. V. (2001). Beyond Tg: optical luminescence measurements of molecular mobility in amorphous solid foods. Food Hydrocolloids. 15, 331-339.

Lukasik, K. V. and Ludescher, R. D. (2005 a). Effect of plasticizer on dynamic site heterogeneity in cold-cast gelatin films. Food Hydrocoll. 20, 88-95.

Lukasik, K. V. and Ludescher, R. D. (2005 b). Molecular mobility in water and glycerol plasticized cold- and hot-cast gelatin films. Food Hydrocoll. 20, 96-105.

Martin-Polo, M. O. (1995). Biopolymers in the fabrication of edible and biodegradable materials for food preservation. Food Preserv.Moisture Control, ISOPOW Pract.II. 849-868.

Matveev, Y. I. (2006). Influence of tautomerism of mono- and disaccharides on their glass transition temperatures. Food Hydrocoll. 20, 663-668.

McHugh, T. H. and Krochta, J. M. (1994). Milk-protein-based edible films and coatings. Food Technology (Chicago, IL, United States). 48, 97-103.

McHugh, T. and Krochta, J. (1994). Sorbitol- vs Glycerol-Plasticized Whey Protein Edible Films: Integrated Oxygen Permeability and Tensile Property Evaluation. J. Agric. Food Chem. 42, 841-845.

Nack, T. J. and Ludescher, R. D. (2006). Molecular Mobility and Oxygen Permeability in Amorphous Bovine Serum Albumin Films. Food Biophysics. 1557-1858. 
Park, H. J., Weller, C. L., Vergano, P. J., and Testin, R. F. (1993). Permeability and mechanical properties of cellulose-based edible films. J. Food Sci. 58, 1361-4, 1370.

Parker, C. A., Ed. (1968.). Photoluminescence of SolutionsElsevier Publishing, Amsterdam, The Netherlands.

Poirier-Brulez, F., Roudaut, G., Champion, D., Tanguy, M., and Simatos, D. (2006). Influence of sucrose and water content on molecular mobility in starch-based glasses as assessed through structure and secondary relaxation. Biopolymers. 81, 63-73.

Pouplin, M., Redl, A., and Gontard, N. (1999). Glass Transition of Wheat Gluten Plasticized with Water, Glycerol, or Sorbitol. J. Agric. Food Chem. 47, 538-543.

Pravinata, L. C., You, Y., and Ludescher, R. D. (2005). Erythrosin B Phosphorescence Monitors Molecular Mobility and Dynamic Site Heterogeneity in Amorphous Sucrose. Biophys. J. 88, 3551-3561.

Richert, R. (2000). Triplet state solvation dynamics: Basics and applications. J. Chem. Phys. 113, 8404-8429.

Riku A. Talja. (2007). Preparation and characterization of potato starch films plasticized with polyols. Ph.D dissertation.

Roos, Y. H. (1995). Phase Transitions in Foods.

Salame, M. (1986). The use of barrier polymers in food and beverage packaging. J.Plast.Film Sheeting. 2, 321-334.

Sears, J. K. and Darby, J. R. (1982). The Technology of Plasticizers. 1166.

Seow, C. C., Cheah, P. B., and Chang, Y. P. (1999). Antiplasticization by water in reducedmoisture food systems. J. Food Sci. 64, 576-581.

Shah, N. K. and Ludescher, R. D. (1993). Influence of hydration on the internal dynamics of hen egg white lysozyme in the dry state. Photochem. Photobiol. 58, 169-174.

Shirke, S., Takhistov, P., and Ludescher, R. D. (2005a). Molecular Mobility in Amorphous Maltose and Maltitol from Phosphorescence of Erythrosin B. J. Phys. Chem. B. 109, 16119-16126.

Shirke, S. and Ludescher, R. D. (2005b). Molecular mobility and the glass transition in amorphous glucose, maltose, and maltotriose. Carbohydr. Res. 340, 2654-2660. 
Shirke, S. and Ludescher, R. D. (2005). Dynamic site heterogeneity in amorphous maltose and maltitol from spectral heterogeneity in erythrosin B phosphorescence. Carbohydr. Res. 340, 2661-2669.

Shirke, S., Takhistov, P., and Ludescher, R. D. (2005). Molecular Mobility in Amorphous Maltose and Maltitol from Phosphorescence of Erythrosin B. J Phys Chem B. 109, 16119-16126.

Shuster. M. (1994). Polymer Engin Sci. 34(2), 1613.

Siew, D. C., Heilmann, C., Easteal, A. J., and Cooney, R. P. (1999). Solution and film properties of sodium caseinate/glycerol and sodium caseinate/polyethylene glycol edible coating systems. J. Agric. Food Chem. 47, 3432-3440.

Simon-Lukasik, K. V. and Ludescher, R. D. (2004). Erythrosin B phosphorescence as a probe of oxygen diffusion in amorphous gelatin films. Food Hydrocoll. 18, 621-630.

Slade, L. and Levine, H. (1995). Glass transitions and water-food structure interactions. $A d v$. Food Nutr. Res. 38, 103-269.

Slavik, J. (1994). Fluorescent probes in cellular and molecular biologyCRC Press, Boca Raton, FL.

Sothornvit, R. and Krochta, J. M. (2000). Plasticizer effect on oxygen permeability of betalactoglobulin films. J. Agric. Food Chem. 48, 6298-6302.

Sothornvit, R., Reid, D. S., and Krochta, J. M. (2002). Plasticizer effect on the glass transition temperature of beta-lactoglobulin films. Trans. ASAE. 45, 1479-1484.

Sundaresan, K. V. and Ludescher, R. D. (2007). Molecular mobility and oxygen permeability in amorphous beta -lactoglobulin films. Food Hydrocoll. 22, 403-413.

Talja, R. A., Helen, H., Roos, Y. H., and Jouppila, K. (2007). Effect of various polyols and polyol contents on physical and mechanical properties of potato starch-based films. Carbohydr. Polym. 67, 288-295.

Turhan, K. N., Sahbaz, F., and Guner, A. (2001). A spectrophotometric study of hydrogen bonding in methylcellulose-based edible films plasticized by polyethylene glycol. J. Food Sci. 66, 59-62.

van den Bogaart, G., Hermans, N., Krasnikov, V., de Vries, A. H., and Poolman, B. (2007). On the decrease in lateral mobility of phospholipids by sugars. Biophys. J. 92, 1598-1605.

Vrentas, J. S., Duda, J. L., and Ling, H. C. (1988). Antiplasticization and volumetric behavior in glassy polymers. Macromolecules. 21, 1470-1475. 
Vyazovkin, S. and Dranca, I. (2006). Comparative Relaxation Dynamics of Glucose and Maltitol. Pharm. Res. 23, 2158-2164.

Whitcombe, M. J., Parker, R., and Ring, S. G. (2005). Oxygen solubility and permeability of carbohydrates. Carbohydr. Res. 340, 1523-1527.

Williams, G. and Watts, D. C. (1970). Non-symmetrical dielectric relaxation behavior arising from a simple empirical decay function. Trans.Faraday Soc. 66, 80-85.

You Yumin and Ludescher. R. D. (December, 2007). The Effect of Glycerol on Molecular Mobility in Amorphous Sucrose Detected by Phosphorescence of Erythrosin B. Journal Food Biophysics. 2, 133-145.

Zhang, J., Mungara, P., and Jane, J. (2000). Mechanical and thermal properties of extruded soy protein sheets. Polymer. 42, 2569-2578.

Zhang, Y. and Han, J. H. (2006). Plasticization of pea starch films with monosaccharides and polyols. J. Food Sci. 71, E253-E261. 


\section{Tables and Figures}

Table V-1a

The estimated maximum $\mathrm{k}^{\circ}$ TS1 values in Glycerol / $\beta$-lg films

\begin{tabular}{|l|l|}
\hline Ratio (glycerol: $\beta$-Lg) & Estimated $\mathrm{k}^{\circ}$ TS1 \\
\hline$\beta-\mathrm{Lg}$ & $6.5 \times 10^{7} \mathrm{~s}^{-1}$ \\
\hline $0.1: 1$ & $6.5 \times 10^{7} \mathrm{~s}^{-1}$ \\
\hline $1: 1$ & $4.5 \times 10^{7} \mathrm{~s}^{-1}$ \\
\hline $10: 1$ & $3.0 \times 10^{7} \mathrm{~s}^{-1}$ \\
\hline $100: 1$ & $2.5 \times 10^{7} \mathrm{~s}^{-1}$ \\
\hline
\end{tabular}

Table V-1b

The estimated maximum $\mathrm{k}^{\circ}$ TS1 values in Sorbitol / $\beta$-lg films

\begin{tabular}{|l|l|}
\hline Ratio (Sorbitol: $\beta$-Lg) & Estimated $\mathrm{k}^{\circ}$ TS1 \\
\hline$\beta-\mathrm{Lg}$ & $6.5 \times 10^{7} \mathrm{~s}^{-1}$ \\
\hline $0.1: 1$ & $6.5 \times 10^{7} \mathrm{~s}^{-1}$ \\
\hline $1: 1$ & $6.5 \times 10^{7} \mathrm{~s}^{-1}$ \\
\hline $10: 1$ & $2.0 \times 10^{7} \mathrm{~s}^{-1}$ \\
\hline $100: 1$ & $6.5 \times 10^{7} \mathrm{~s}^{-1}$ \\
\hline $300: 1$ & $4.5 \times 10^{7} \mathrm{~s}^{-1}$ \\
\hline
\end{tabular}

Table V-1c

The estimated maximum $\mathrm{k}^{\circ}$ TS1 values for Maltitol / $\beta$-lg films

\begin{tabular}{|l|l|}
\hline Ratio (Maltitol: $\beta$-Lg) & Estimated $\mathrm{k}^{\circ}$ TS1 \\
\hline$\beta-\mathrm{Lg}$ & $6.5 \times 10^{7} \mathrm{~s}^{-1}$ \\
\hline $0.1: 1$ & $6.5 \times 10^{7} \mathrm{~s}^{-1}$ \\
\hline $1: 1$ & $4.0 \times 10^{7} \mathrm{~s}^{-1}$ \\
\hline $10: 1$ & $3.0 \times 10^{7} \mathrm{~s}^{-1}$ \\
\hline $100: 1$ & $6.5 \times 10^{7} \mathrm{~s}^{-1}$ \\
\hline $1000: 1$ & $6.5 \times 10^{7} \mathrm{~s}^{-1}$ \\
\hline
\end{tabular}


Table V-1d

The estimated maximum $\mathrm{k}^{\circ}$ TS1 values for PEG / $\beta$-lg films

\begin{tabular}{|l|l|}
\hline Ratio (PEG: $\beta$-Lg) & Estimated k ${ }^{\circ}$ TS1 \\
\hline$\beta-\operatorname{Lg}$ & $6.5 \times 10^{7} \mathrm{~s}^{-1}$ \\
\hline $0.1: 1$ & $6.5 \times 10^{7} \mathrm{~s}^{-1}$ \\
\hline $1: 1$ & $4.0 \times 10^{7} \mathrm{~s}^{-1}$ \\
\hline $10: 1$ & $6.5 \times 10^{7} \mathrm{~s}^{-1}$ \\
\hline & \\
\hline
\end{tabular}

Table V-2 Calculated $\Delta \mathrm{E}_{\mathrm{TS}}$, the energy gap between triplet and singlet state in glycerol/ $\beta$ $\operatorname{Lg}$ (a) sorbitol/ $\beta$ - Lg (b) maltitol/ $\beta$ - Lg (c) PEG / $\beta$-Lg (d)films.

Table V-2a

\begin{tabular}{|l|l|l|}
\hline Ratio (Glycerol: $\beta$-Lg) & $\Delta \mathrm{E}_{\mathrm{TS}}(\mathrm{kJ})$ & $\mathrm{STD}$ \\
\hline$\beta$-Lg & 33.6 & 0.8 \\
\hline $0.1: 1$ & 34.38 & 0.46 \\
\hline $1: 1$ & 34.61 & 0.26 \\
\hline $10: 1$ & 35.56 & 0.10 \\
\hline $100: 1$ & 34.69 & 0.02 \\
\hline
\end{tabular}

Table V-2b

\begin{tabular}{|l|l|l|}
\hline Ratio (Sorbitol: $\beta$-Lg) & $\Delta \mathrm{E}_{\mathrm{TS}}(\mathrm{kJ})$ & $\mathrm{STD}$ \\
\hline$\beta$-Lg & 33.6 & 0.8 \\
\hline $0.1: 1$ & 34.77 & 1.55 \\
\hline $1: 1$ & 34.33 & 0.36 \\
\hline $10: 1$ & 32.09 & 0.57 \\
\hline $100: 1$ & 32.45 & 0.52 \\
\hline $300: 1$ & 32.60 & 0.76 \\
\hline
\end{tabular}


Table V-2c

\begin{tabular}{|l|l|l|}
\hline Ratio (Maltitol: $\beta$-Lg) & $\Delta \mathrm{E}_{\mathrm{TS}}(\mathrm{kJ})$ & $\mathrm{STD}$ \\
\hline$\beta-\mathrm{Lg}$ & 33.6 & 0.8 \\
\hline $0.1: 1$ & 34.25 & 0.00 \\
\hline $1: 1$ & 33.02 & 0.59 \\
\hline $10: 1$ & 34.18 & 0.29 \\
\hline $100: 1$ & 34.41 & 0.62 \\
\hline $1000: 1$ & 34.43 & 0.00 \\
\hline
\end{tabular}

Table V-2d

\begin{tabular}{|l|l|l|}
\hline Ratio (PEG-Lg) & $\Delta \mathrm{E}_{\mathrm{TS}}(\mathrm{kJ})$ & $\mathrm{STD}$ \\
\hline$\beta-\mathrm{Lg}$ & 33.6 & 0.8 \\
\hline $0.1: 1$ & 35.24 & 0.01 \\
\hline $1: 1$ & 35.24 & 0.37 \\
\hline $10: 1$ & 36.19 & 0.34 \\
\hline
\end{tabular}


Figure V-1a

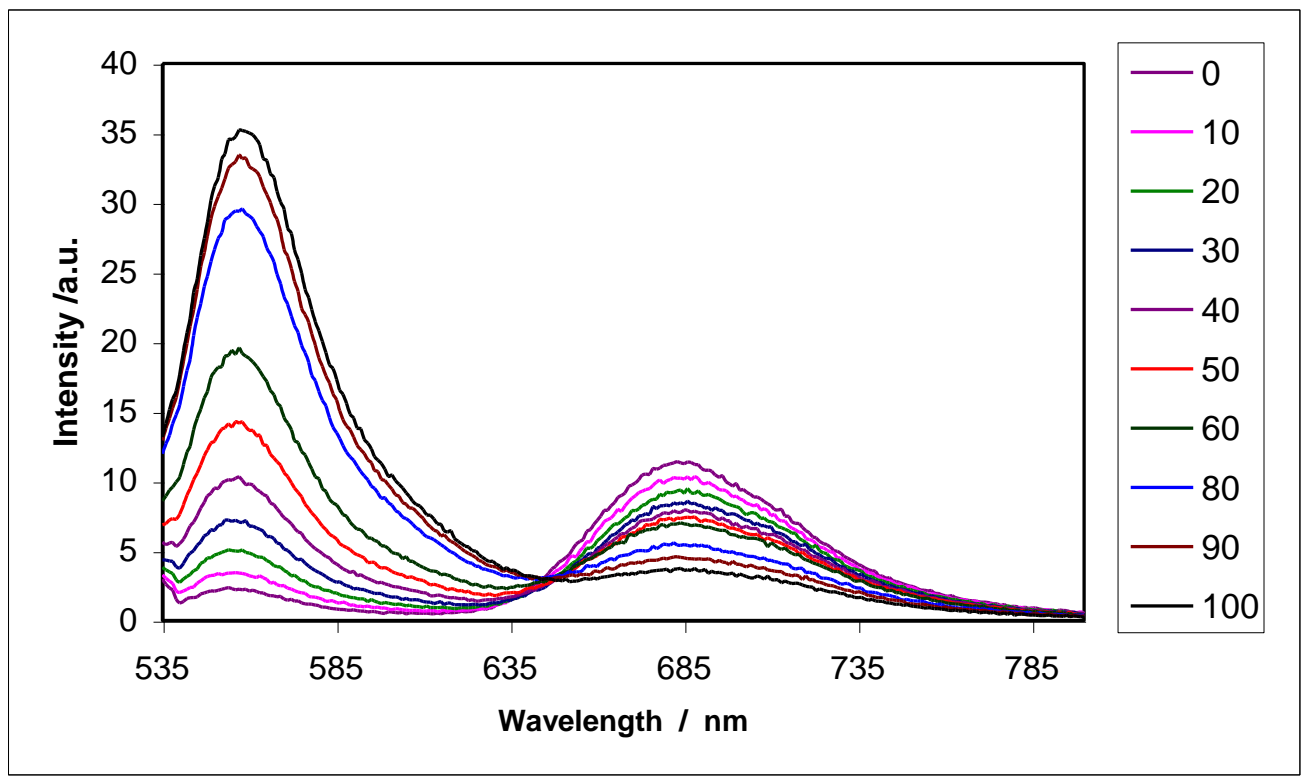

Figure V-1a: The effect of temperature on the delayed emission spectra of Erythrosin B dispersed in amorphous $\beta$ - $\mathrm{Lg}$ - glycerol films (Glycerol: $\beta$ - $\operatorname{Lg} 10: 1$ )

\section{Figure V-1b}

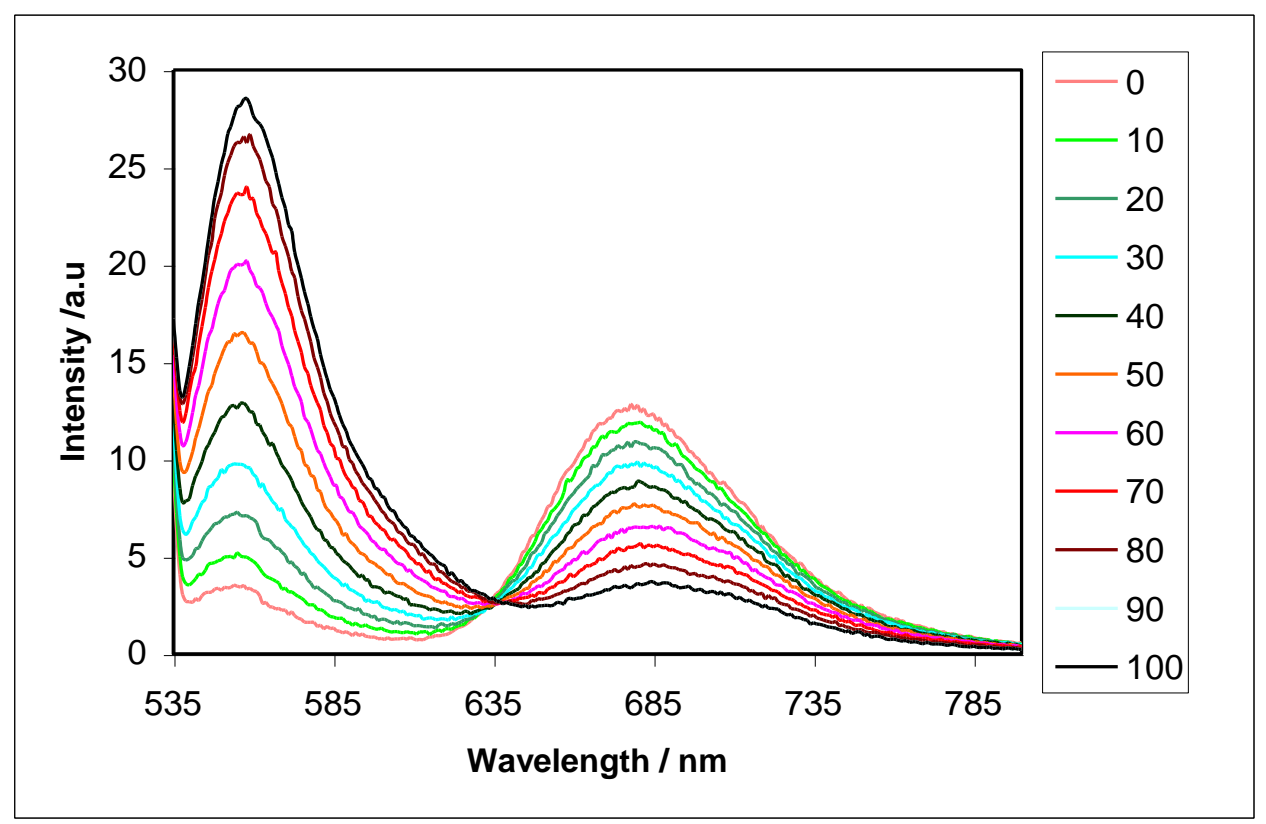

Figure V-1b: The effect of temperature on the delayed emission spectra of Erythrosin B dispersed in amorphous $\beta-\mathrm{Lg}$ - sorbitol films (Sorbitol: $\beta-\operatorname{Lg} 10: 1$ ). 
Figure $V-1 c$

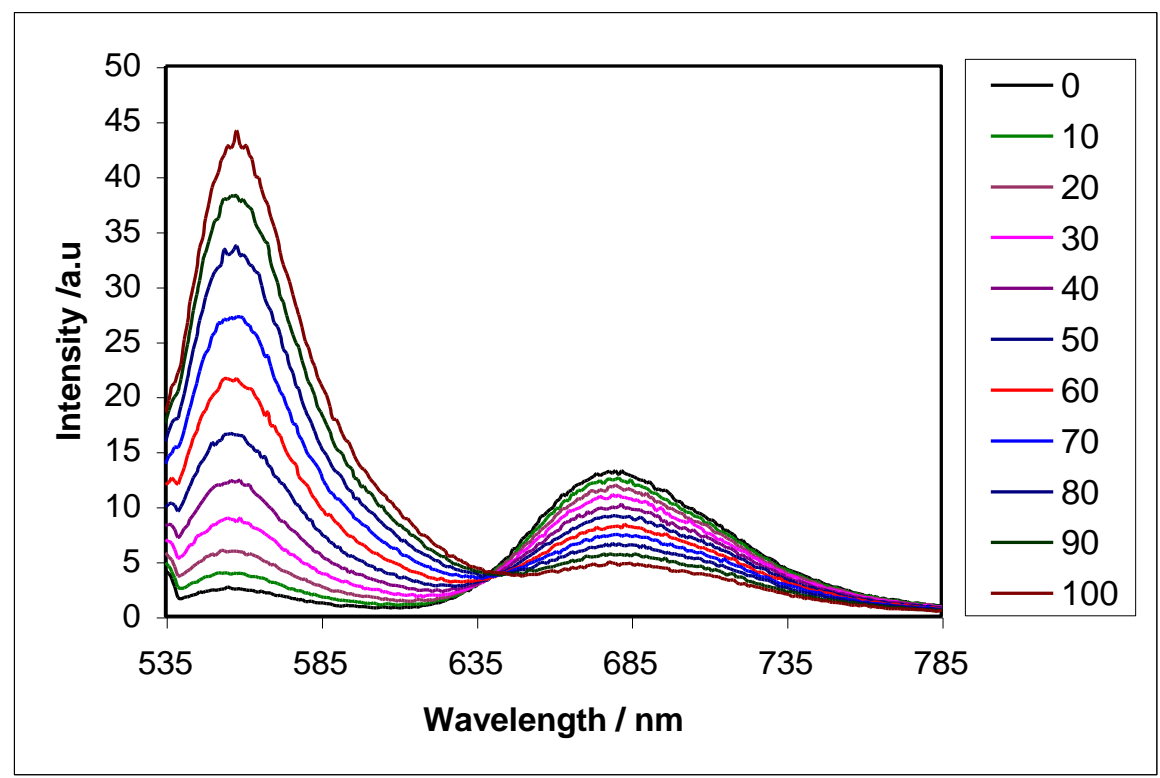

Figure V-1c: The effect of temperature on the delayed emission spectra of Erythrosin B dispersed in amorphous $\beta$-Lg - Maltitol films (Maltitol: $\beta$-Lg 10:1).

Figure V-1d

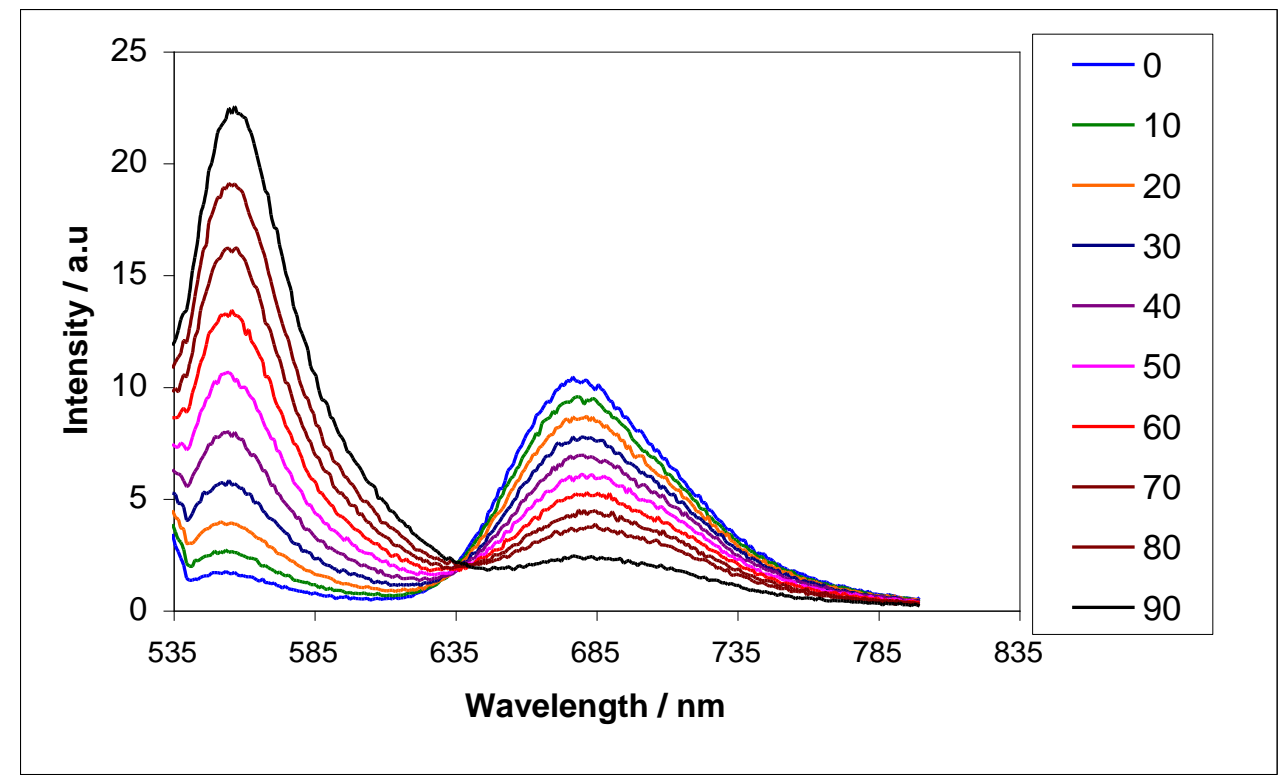

Figure V-1d: The effect of temperature on the delayed emission spectra of Erythrosin B dispersed in amorphous $\beta$-Lg - PEG films (PEG 400: $\beta$-Lg 10:1). 
Figure $V$-2a

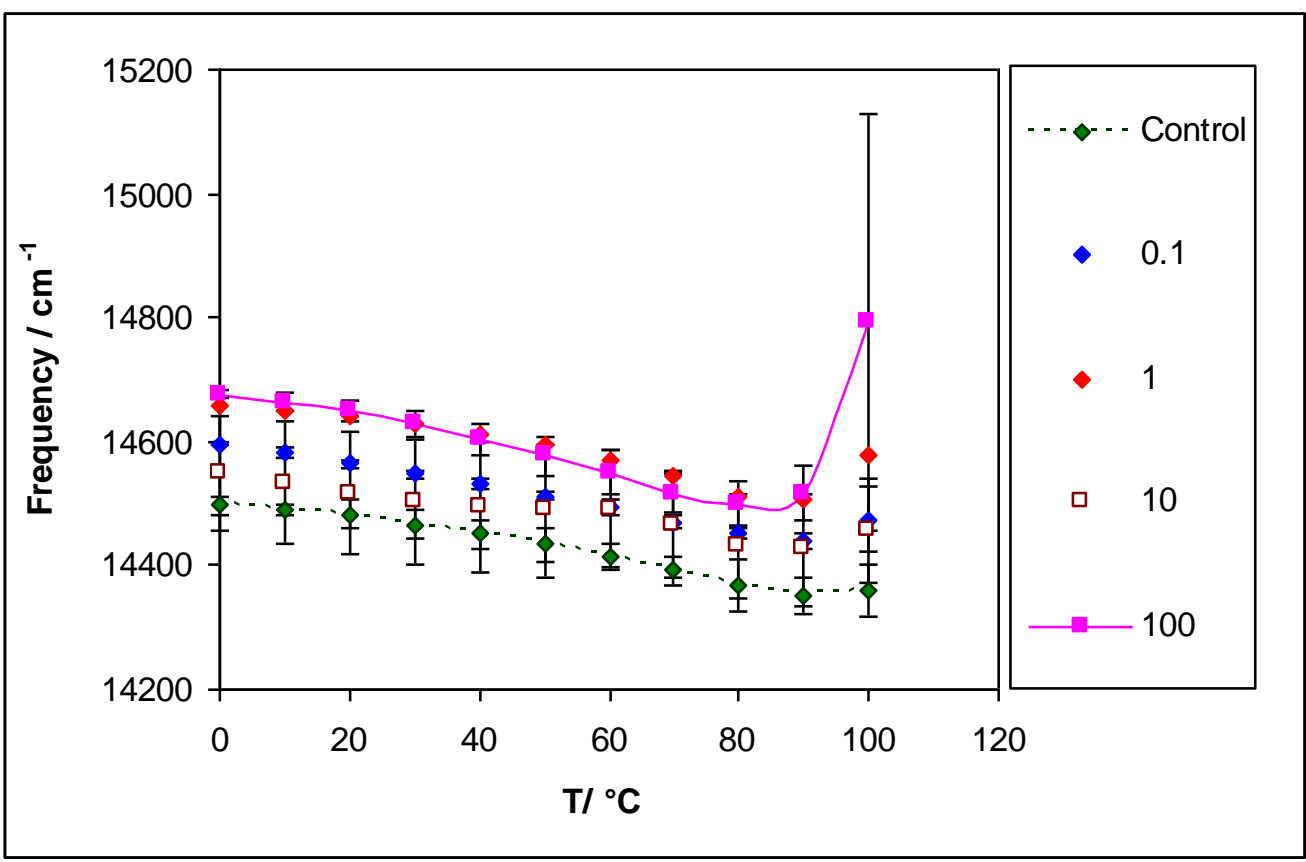

Figure V-2a: The effect of temperature on the emission maximum for phosphorescence from Erythrosin B in amorphous glycerol/ $\beta$-Lg films.

Figure $V-2 b$

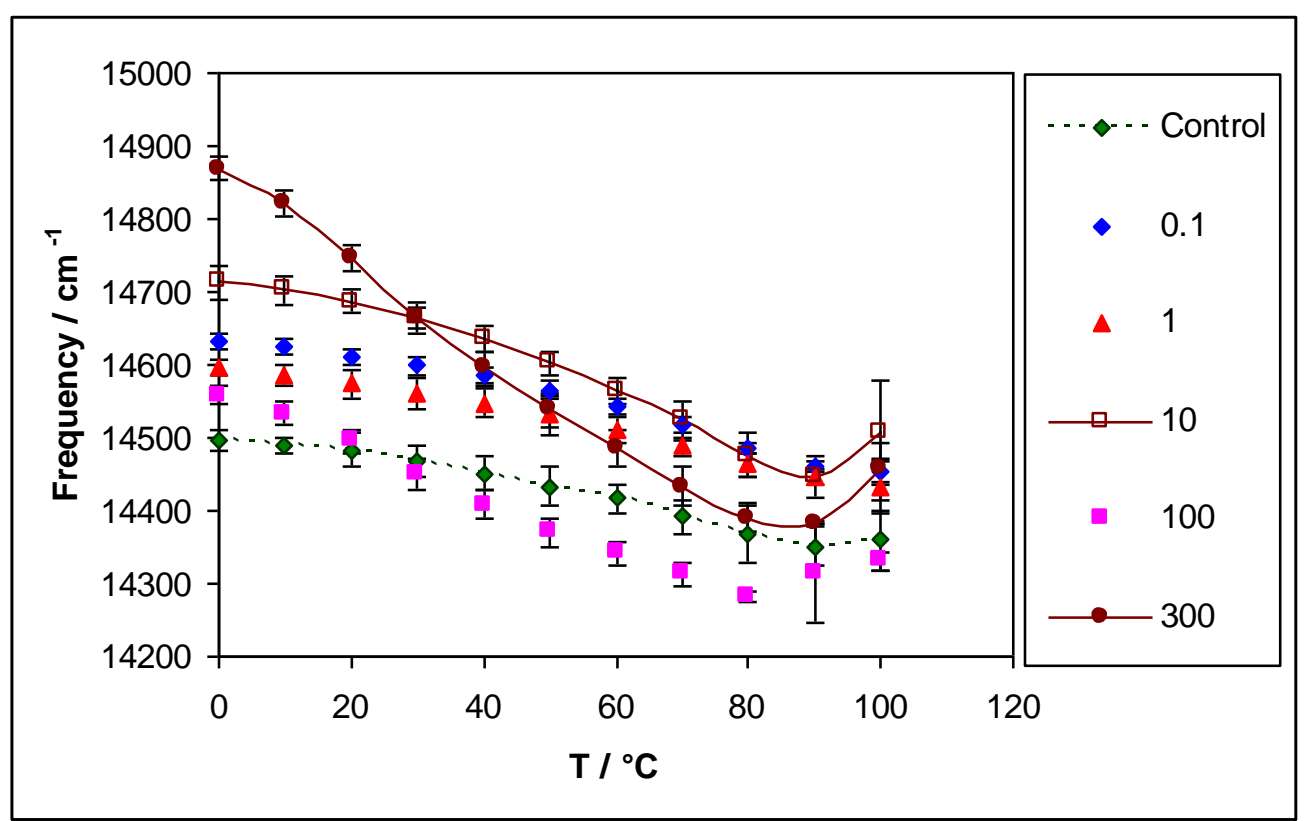

Figure V-2b: The effect of temperature on the emission maximum for phosphorescence from Erythrosin B in amorphous sorbitol/ $\beta$-Lg films. 
Figure $V-2 c$

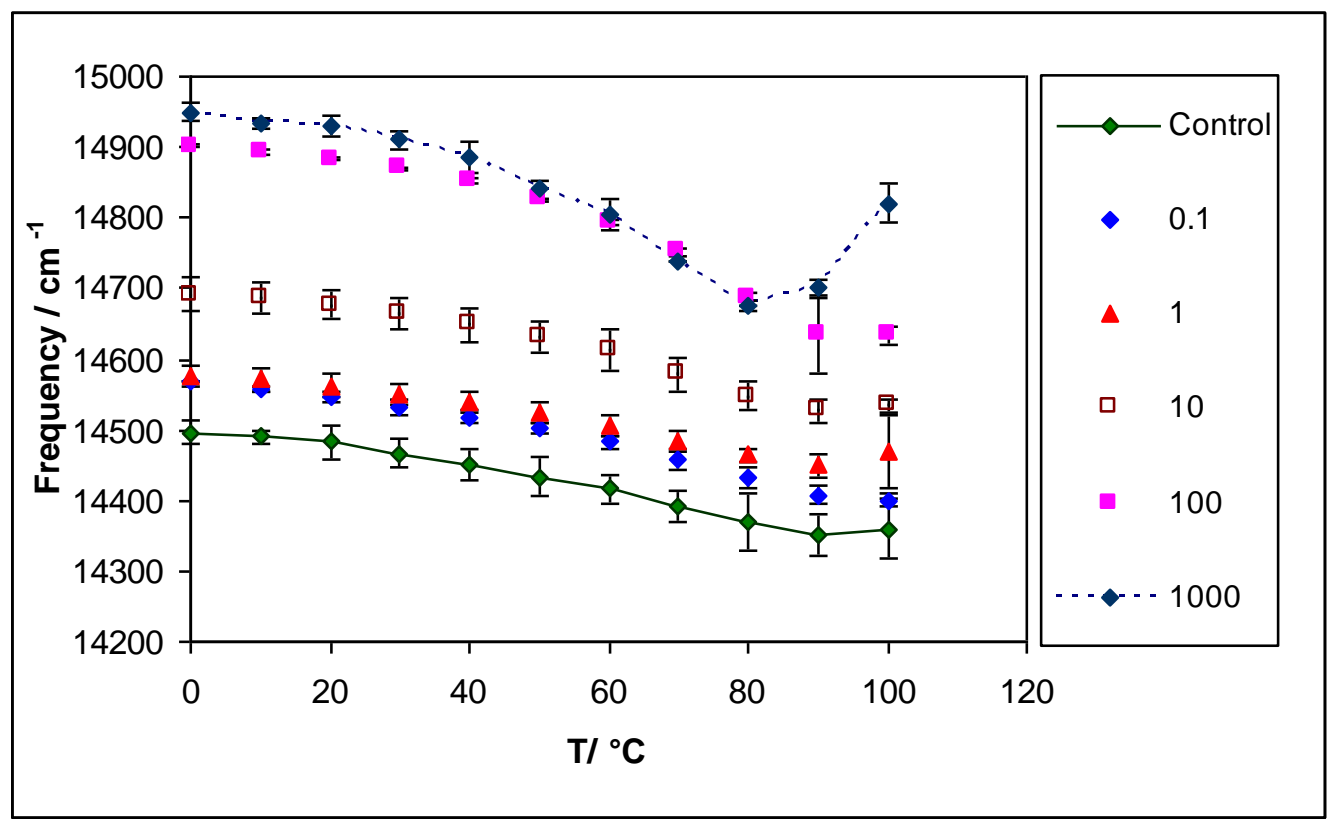

Figure V-2c: The effect of temperature on the emission maximum for phosphorescence from Erythrosin B in amorphous maltitol/ $\beta$-Lg films.

Figure $V$-2d

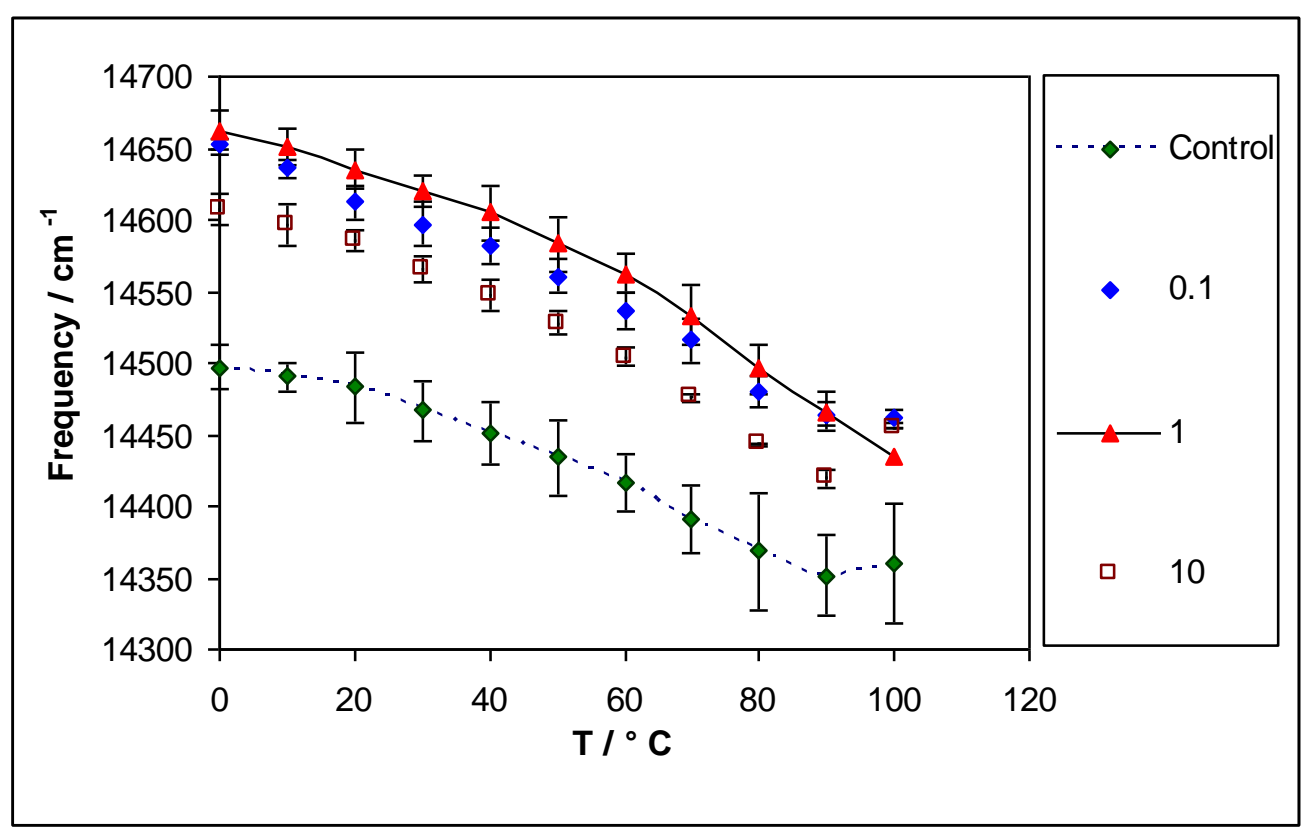

FigureV-2d: The effect of temperature on the emission maximum for phosphorescence from Erythrosin B in amorphous PEG 400/ $\beta$-Lg films. 
Figure $V-3 a$

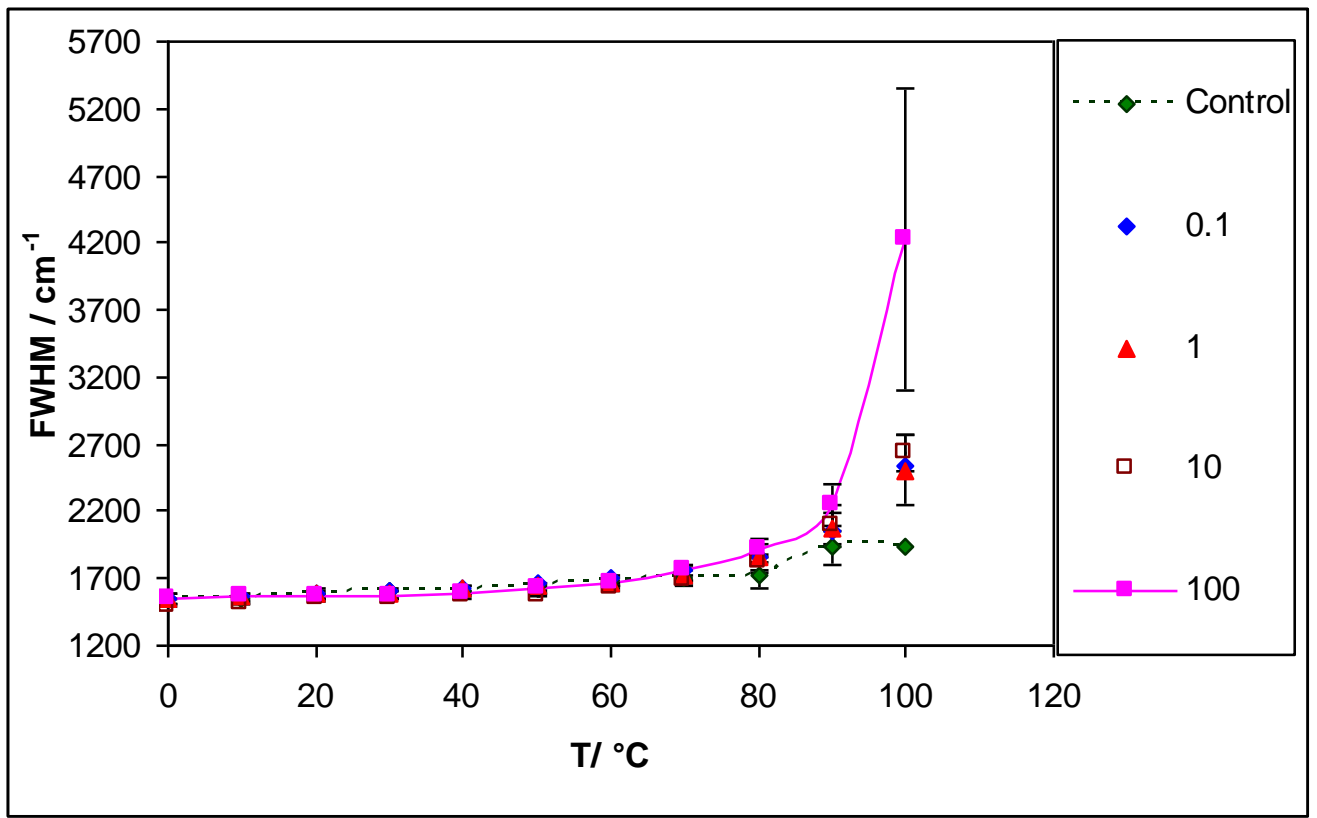

Figure V-3a: The effect of temperature on the bandwidth for phosphorescence from Erythrosin B in amorphous glycerol/ $\beta-\mathrm{Lg}$ films.

Figure $V-3 b$

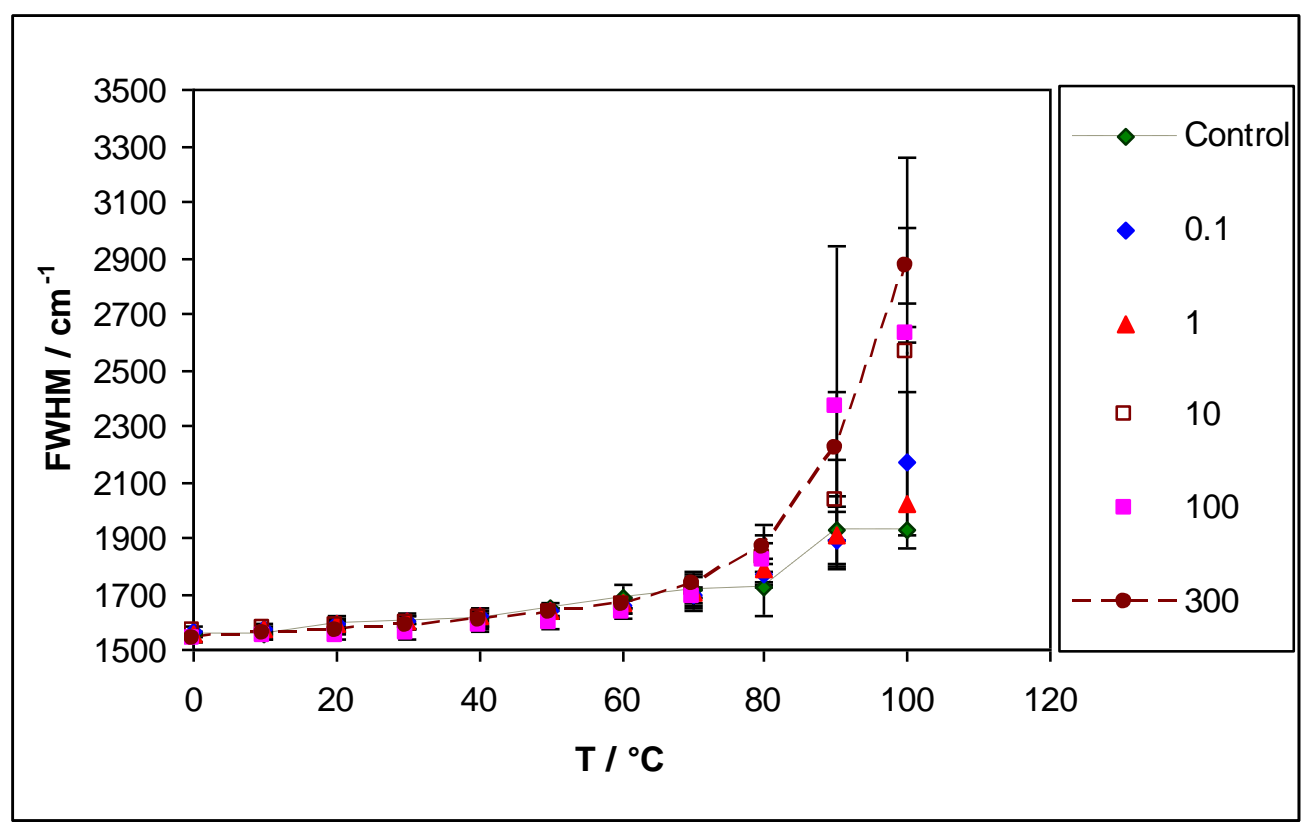

Figure V-3b: The effect of temperature on the bandwidth for phosphorescence from Erythrosin B in amorphous sorbitol $/ \beta-\mathrm{Lg}$ films. 
Figure $V-3 c$

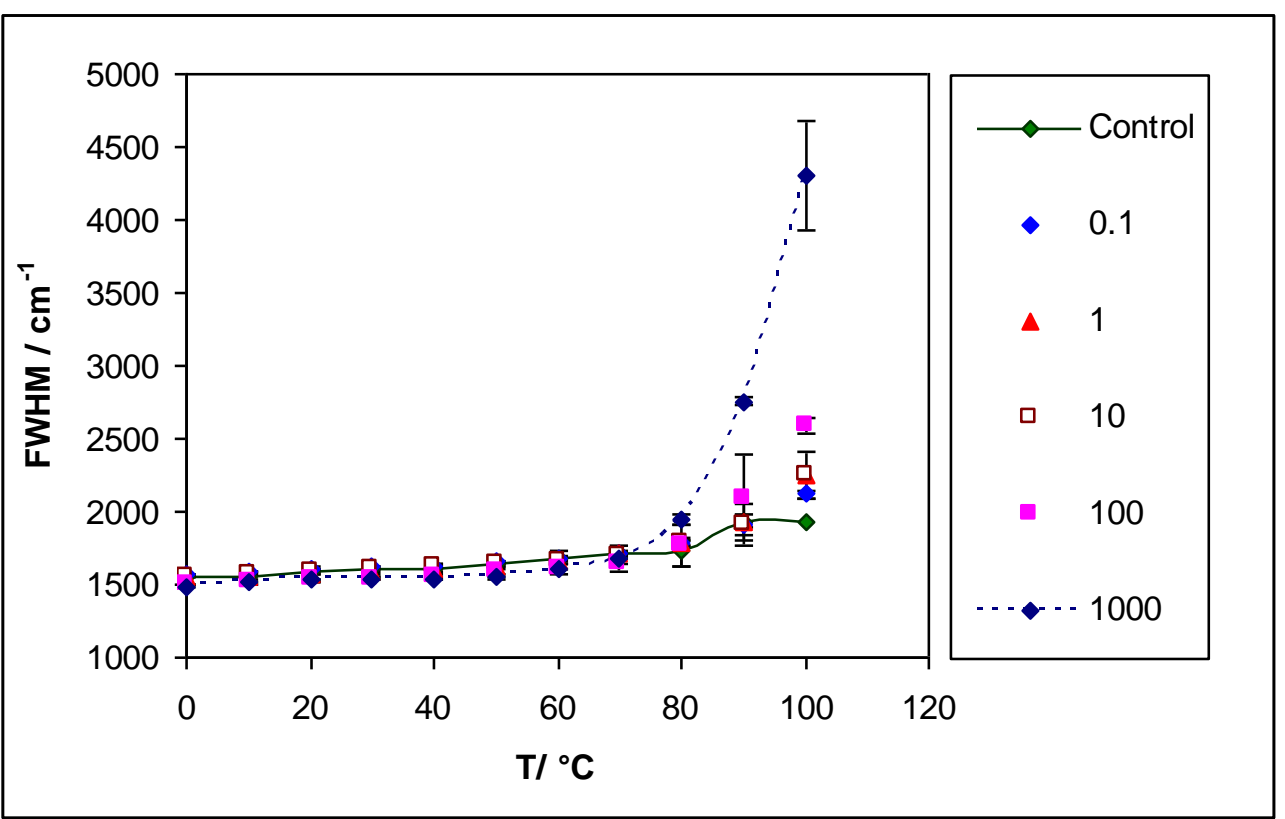

Figure V-3c: The effect of temperature on the bandwidth for phosphorescence from Erythrosin B in amorphous maltitol/ $\beta$-Lg films.

Figure $V-3 d$

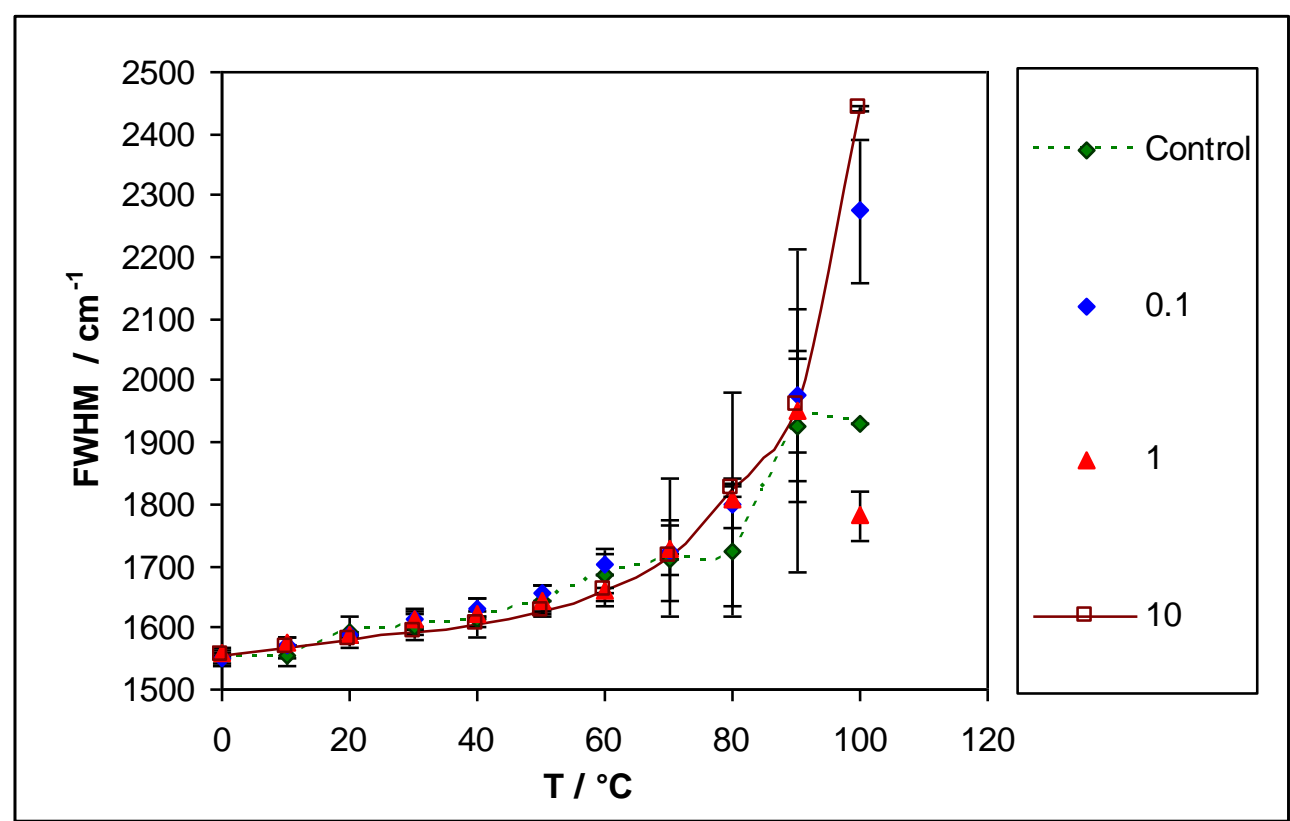

Figure V-3d: The effect of temperature on the bandwidth for phosphorescence from Erythrosin B in amorphous PEG/ $\beta$-Lg films. 
Figure $\mathrm{V}-4 a$

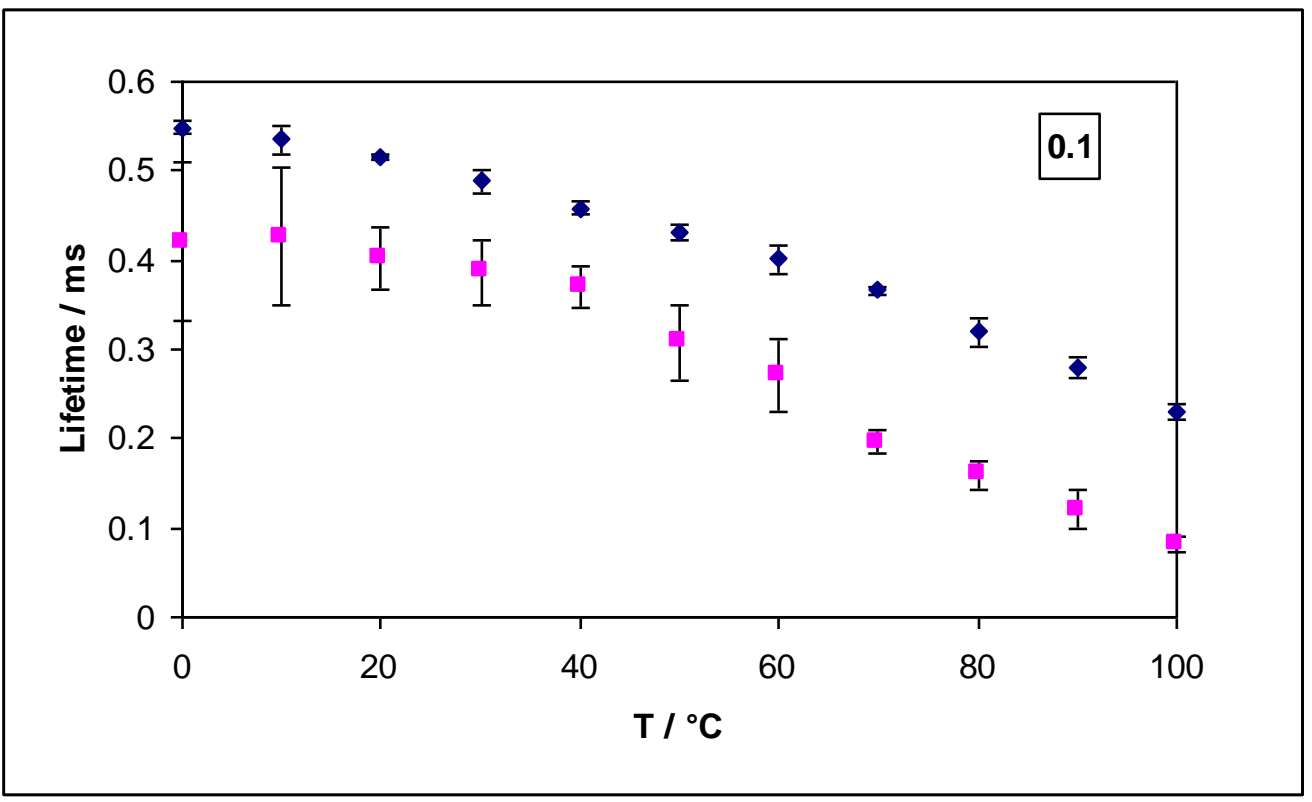

Figure $V-4 b$

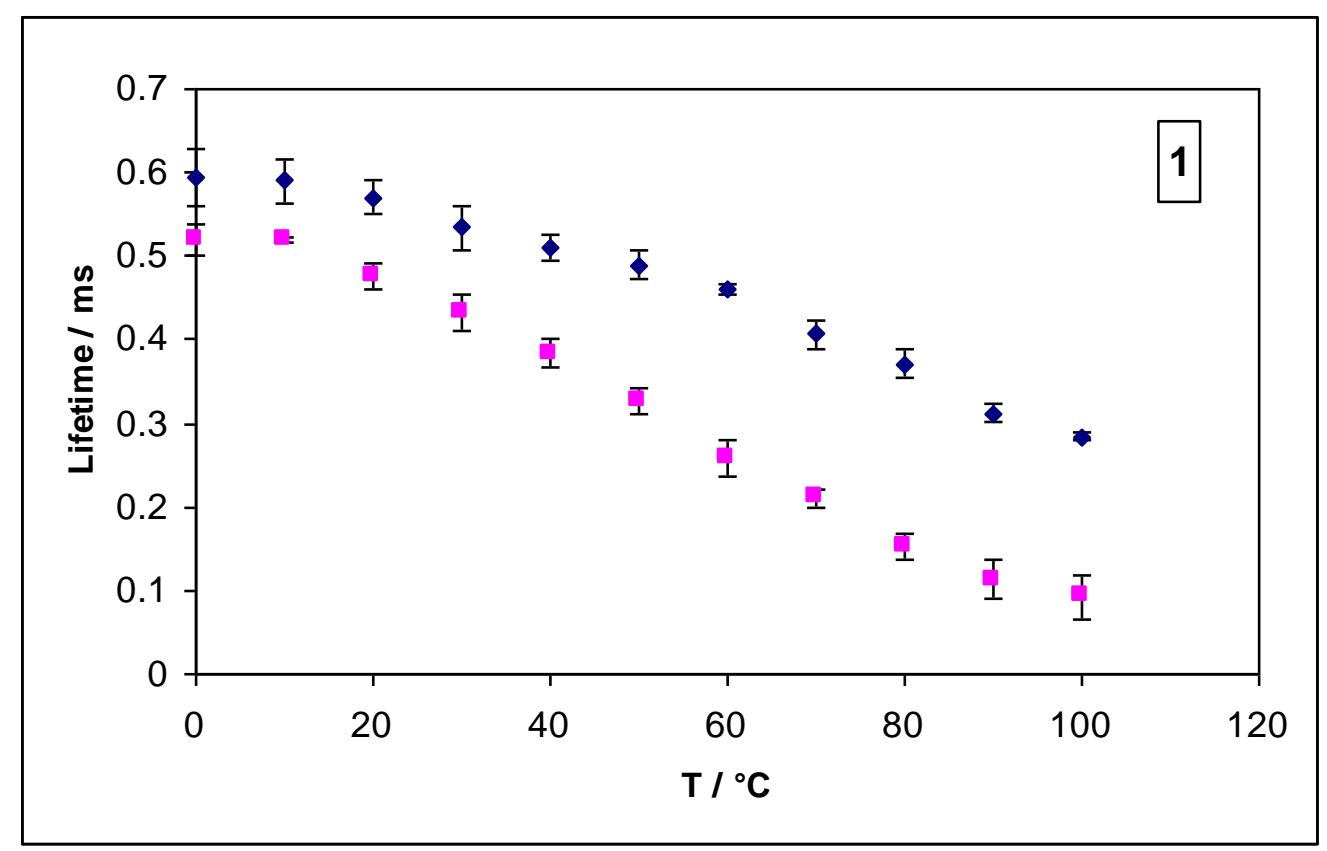


Figure $V-4 c$

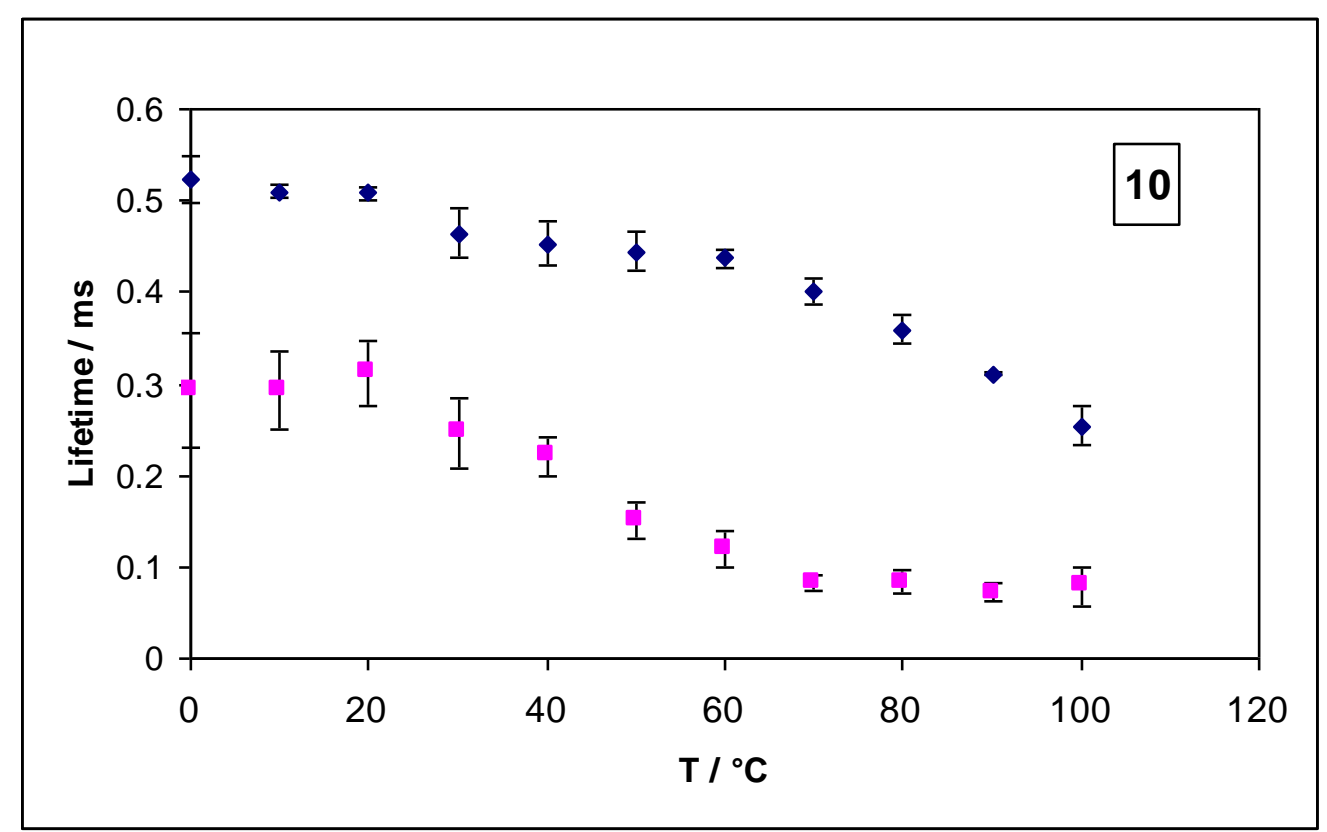

Figure V-4d

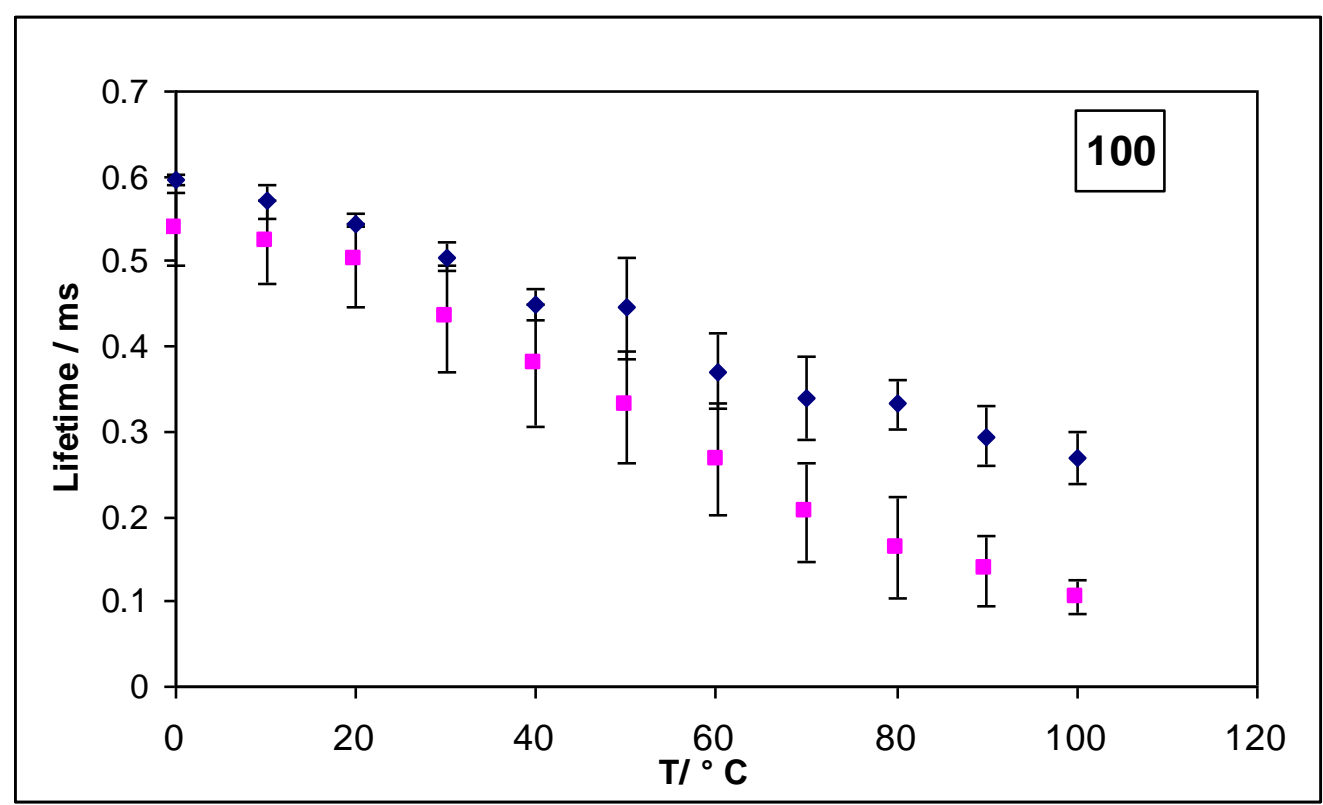


Figure $V-4 e$

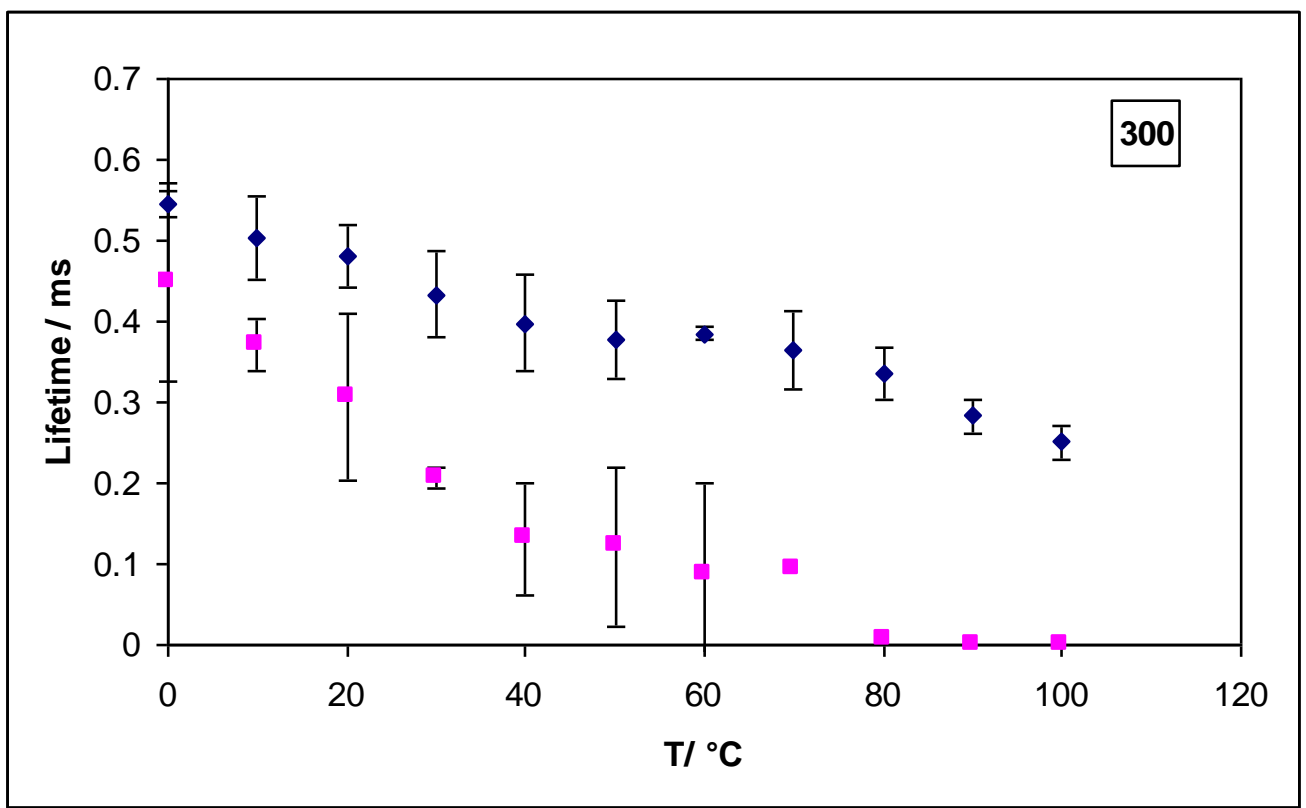

Figure V-4 a, b, c d, e: The effect of temperature on the lifetime for Erythrosin B dispersed in $\beta$ $\mathrm{Lg}$ in various molar concentrations of glycerol equilibrated against nitrogen $(\bullet)$ and against air $(\square)$; the lifetime was determined from analysis using a stretched exponential decay function. The text box in the graph refers to the molar ratio of Glycerol: $\beta-\mathrm{Lg}$. 
Figure $V-4 f$

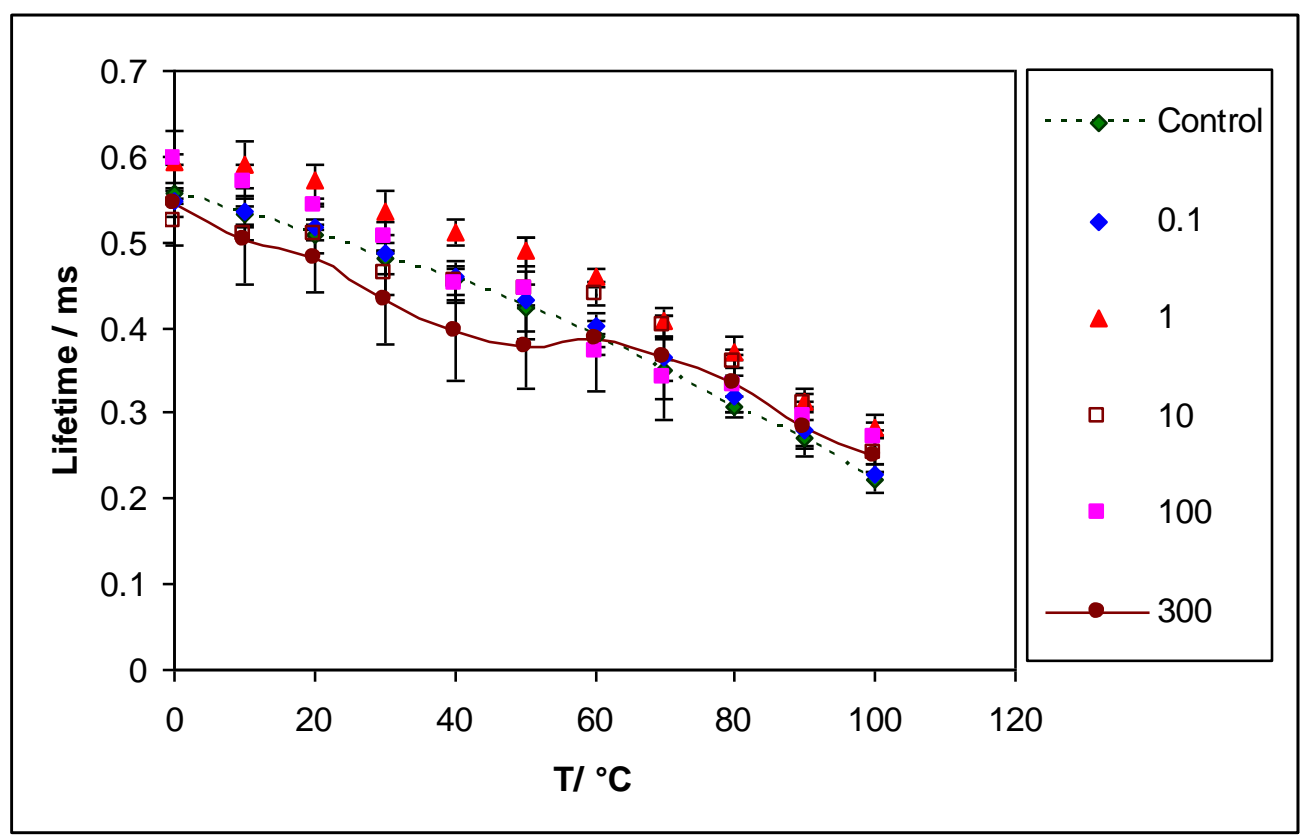

Figure V-4f: Comparison plot depicting the effect of temperature on the lifetime for Erythrosin $\mathrm{B}$ dispersed in $\beta$ - $\mathrm{Lg}$ in various molar concentrations of glycerol equilibrated against nitrogen.

Figure $\mathrm{V}-4 \mathrm{~g}$

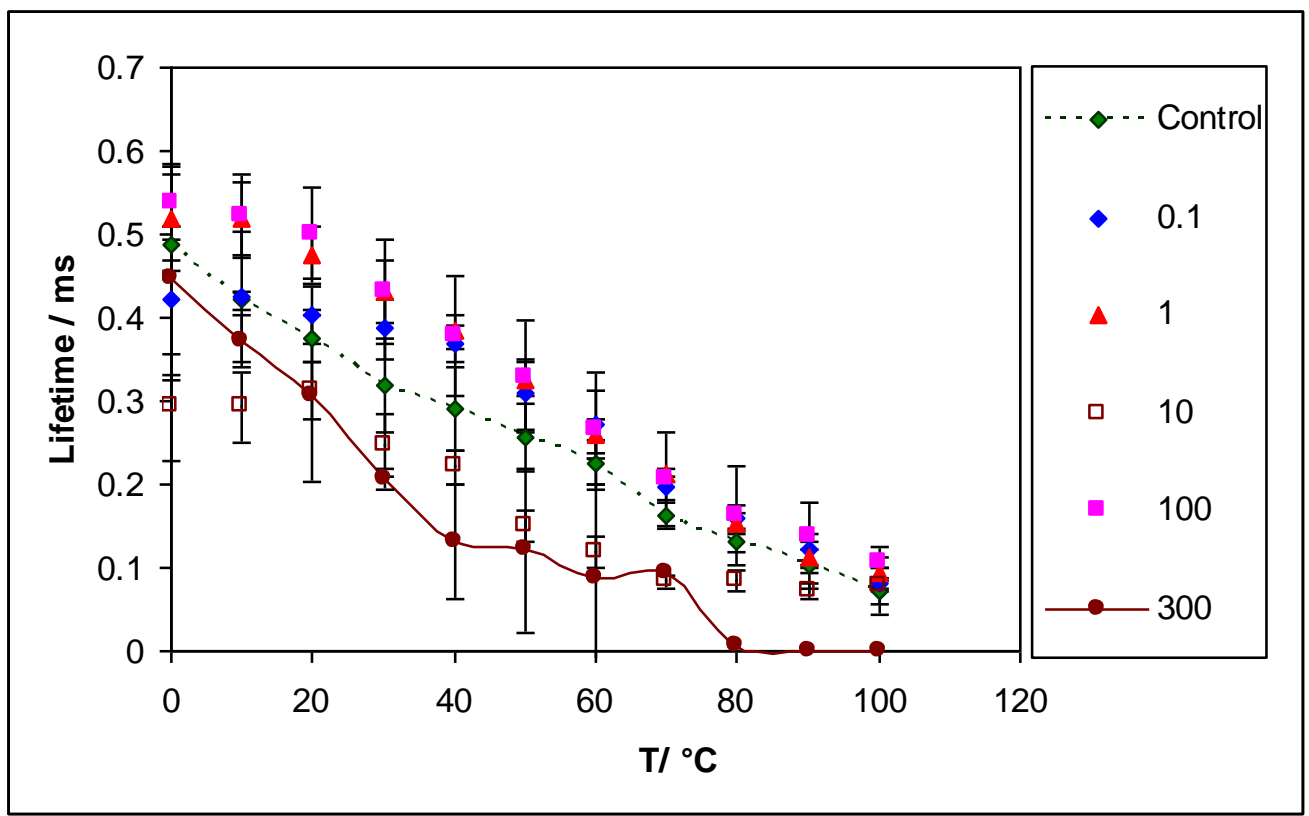

Figure V-4g: Comparison plot depicting the effect of temperature on the lifetime for Erythrosin $\mathrm{B}$ dispersed in $\beta$-Lg in various molar concentrations of glycerol equilibrated against oxygen. 
Figure V-5a

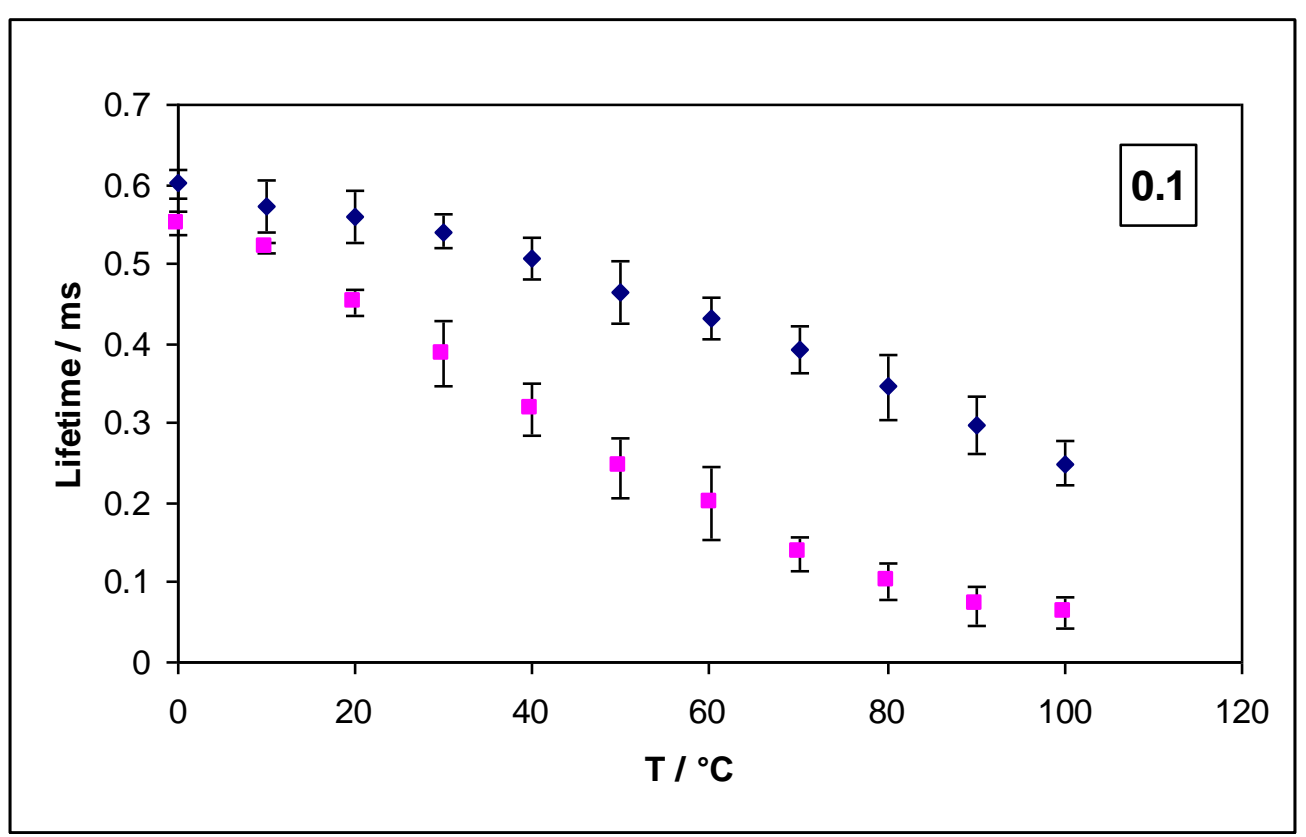

Figure $V-5 b$

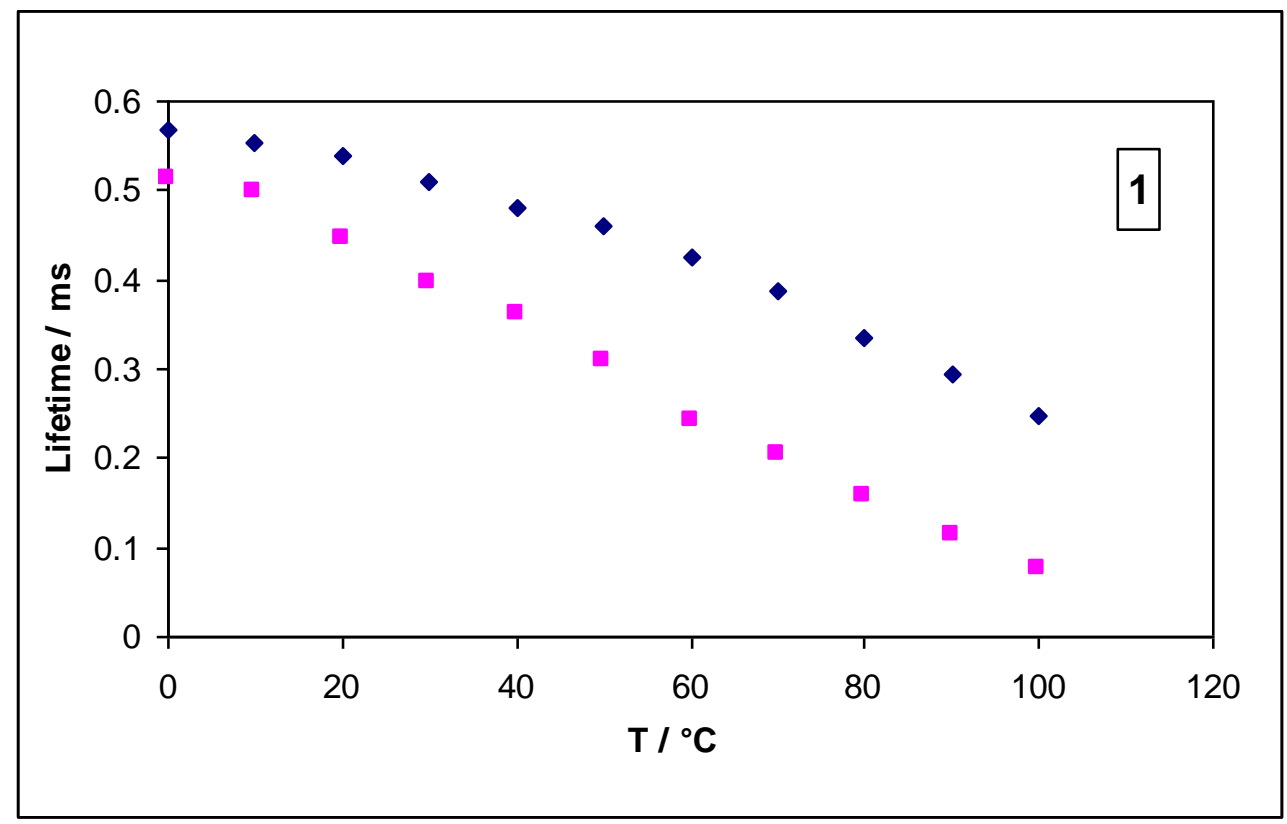


Figure V-5c

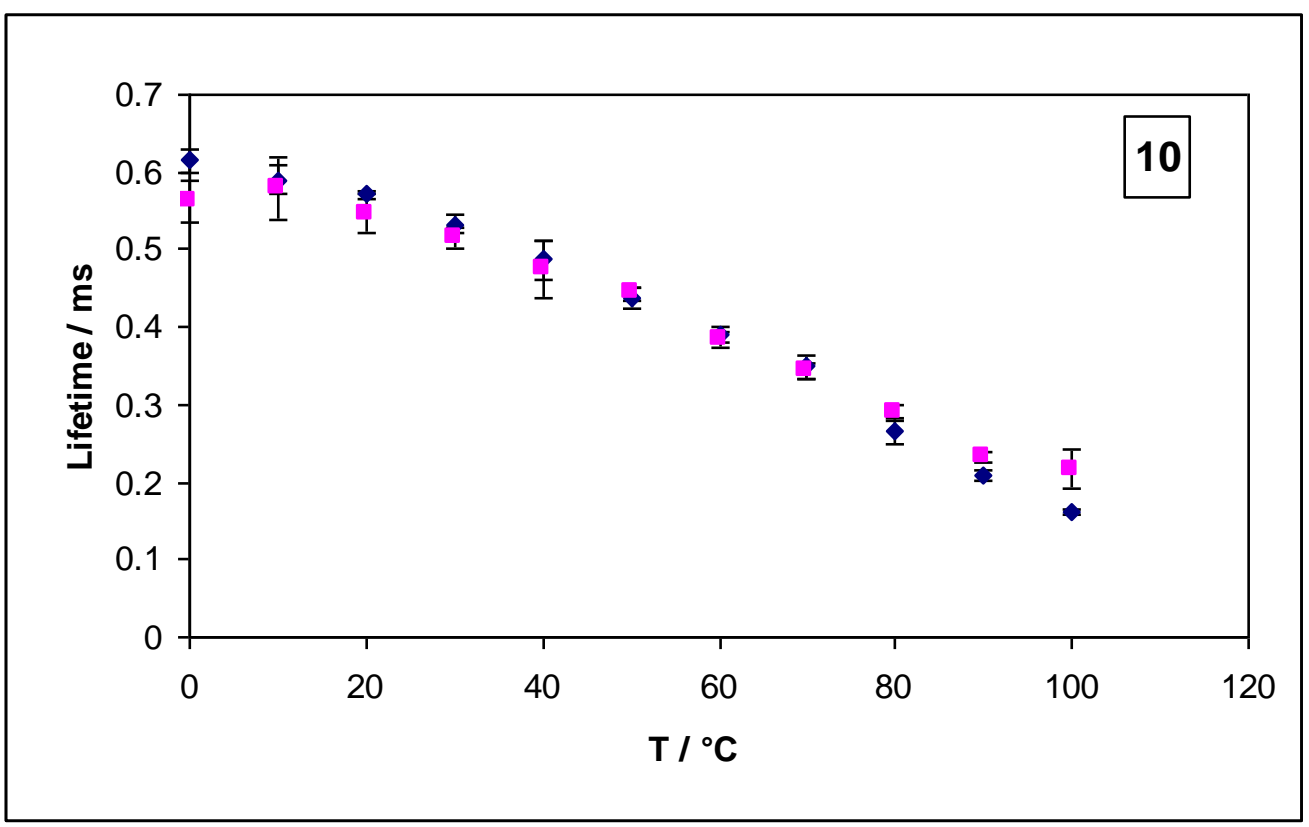

Figure V-5d

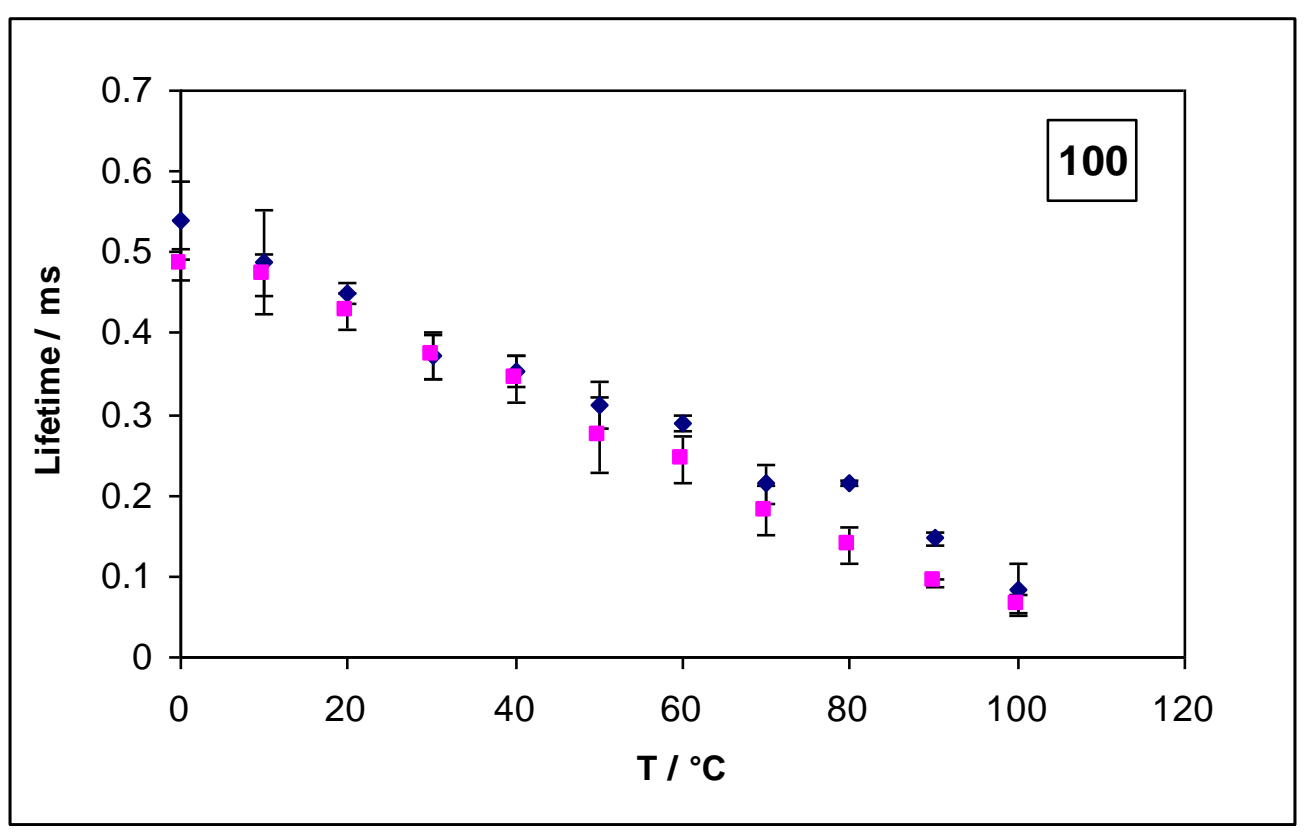


Figure V-5e

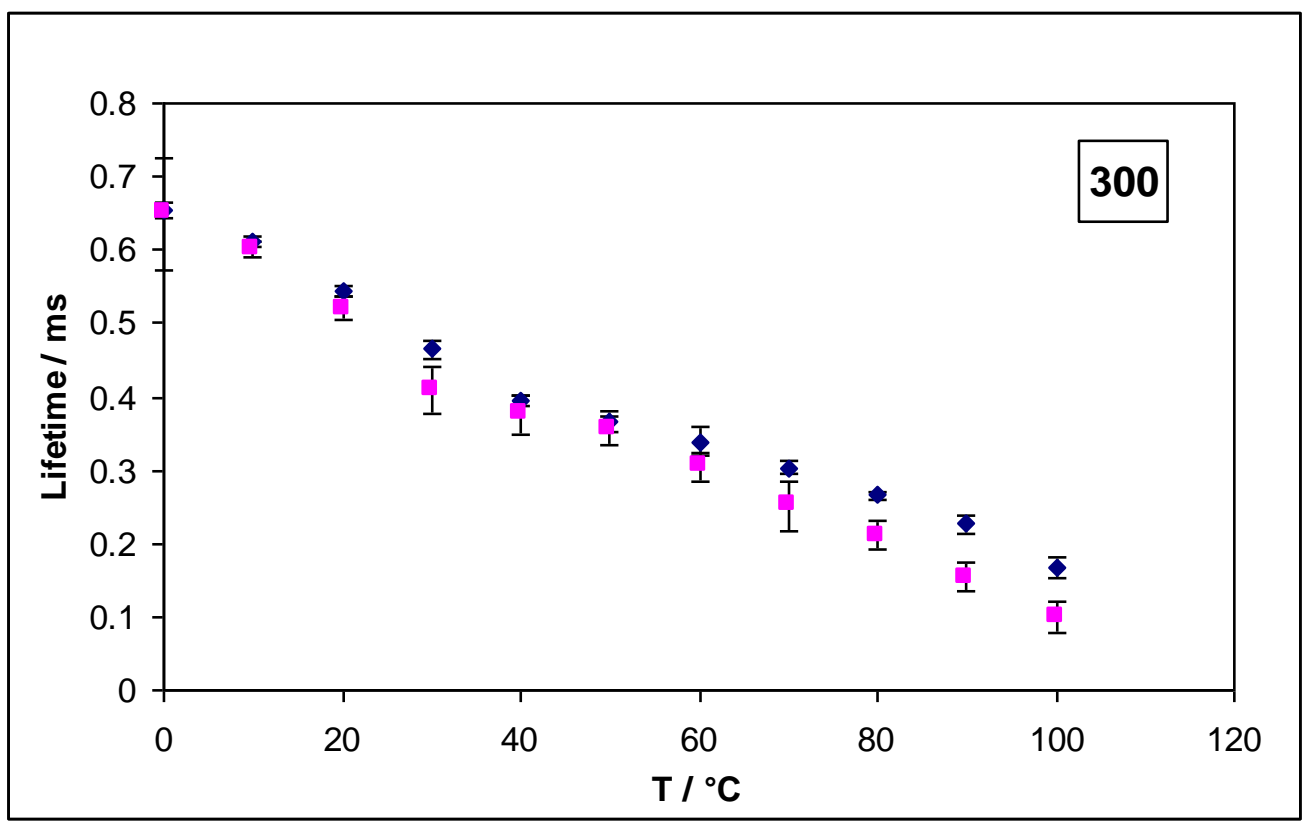

Figure V-5 a,b,c,d,e: The effect of temperature on the lifetime for Erythrosin B dispersed in $\beta$ $\mathrm{Lg}$ in various molar concentrations of sorbitol equilibrated against nitrogen ( $\bullet$ ) and against air ( $\square$ ); the lifetime was determined from analysis using a stretched exponential decay function. The text box in the graph refers to the molar ratio of Sorbitol: $\beta-\mathrm{Lg}$.

Figure $V-5 f$

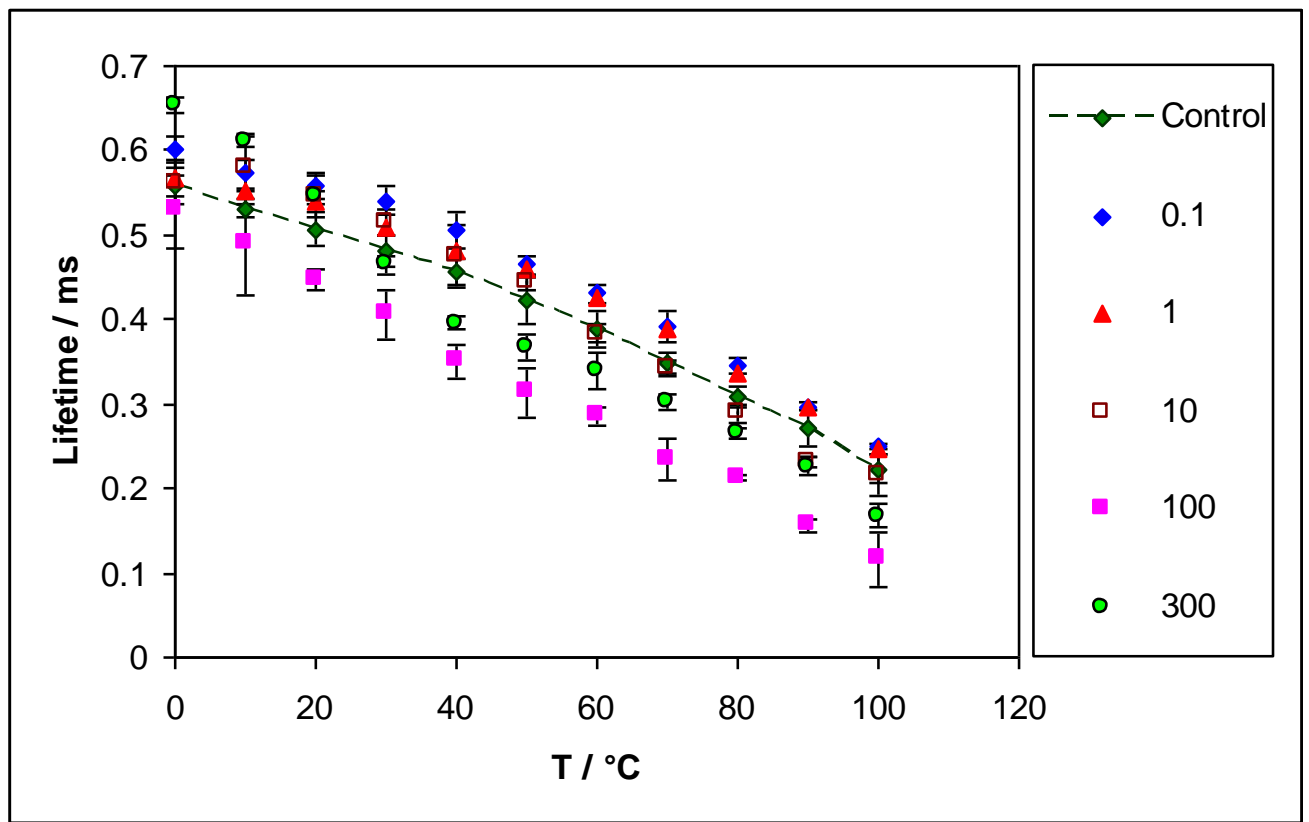

Figure V-5f: Comparison plot depicting the effect of temperature on the lifetime for Erythrosin B dispersed in $\beta-\mathrm{Lg}$ in various molar concentrations of sorbitol equilibrated against nitrogen. 
Figure V-5g

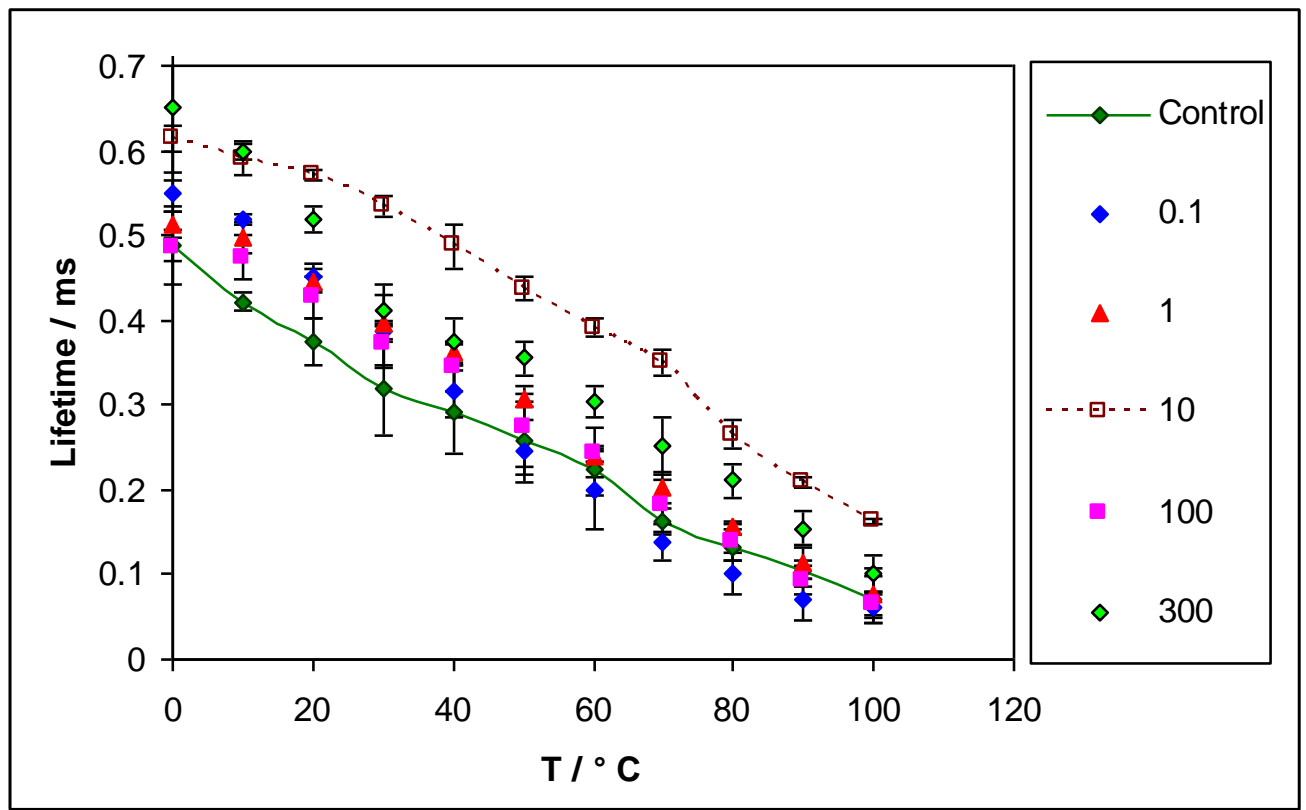

Figure V-5g: Comparison plot depicting the effect of temperature on the lifetime for Erythrosin B dispersed in $\beta-\mathrm{Lg}$ in various molar concentrations of sorbitol equilibrated against oxygen. 
Figure V-6a

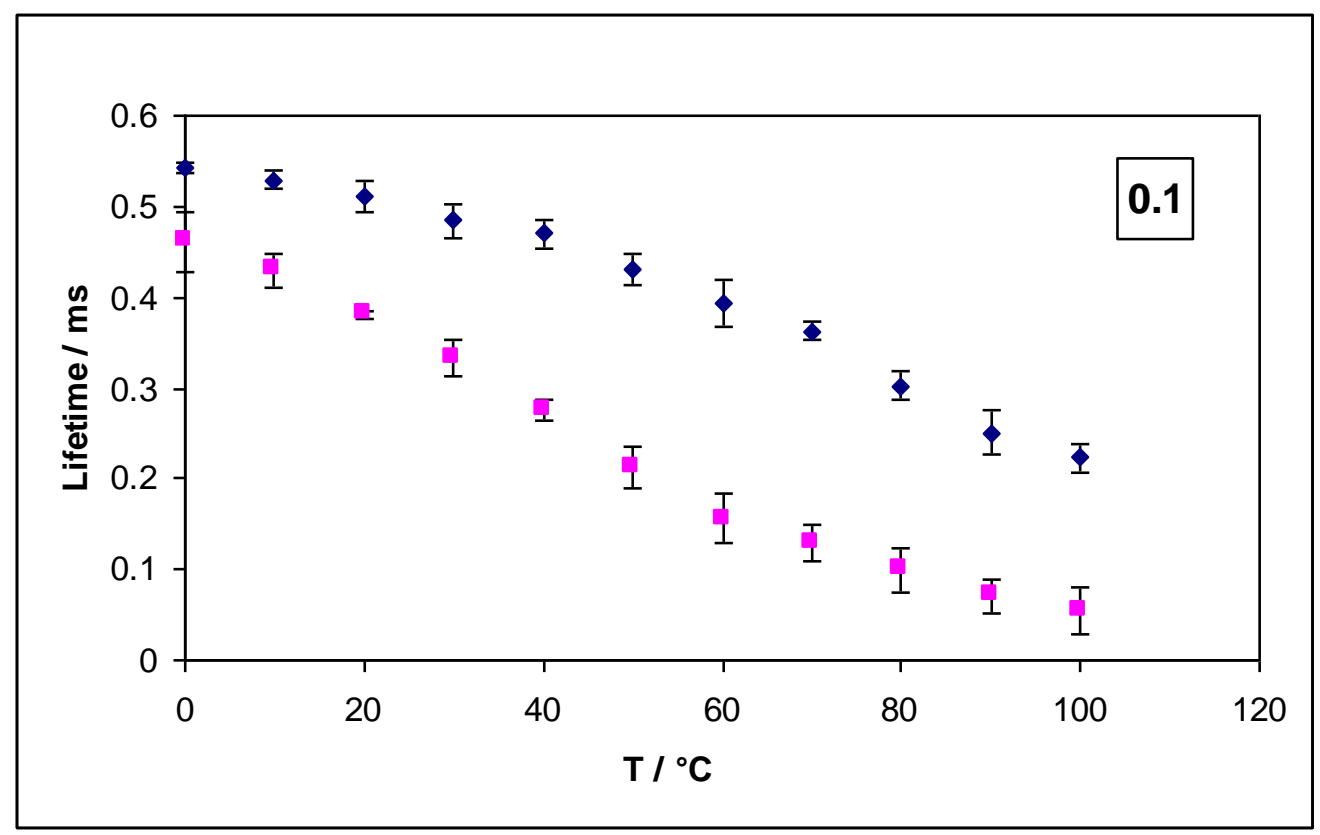

Figure V-6b

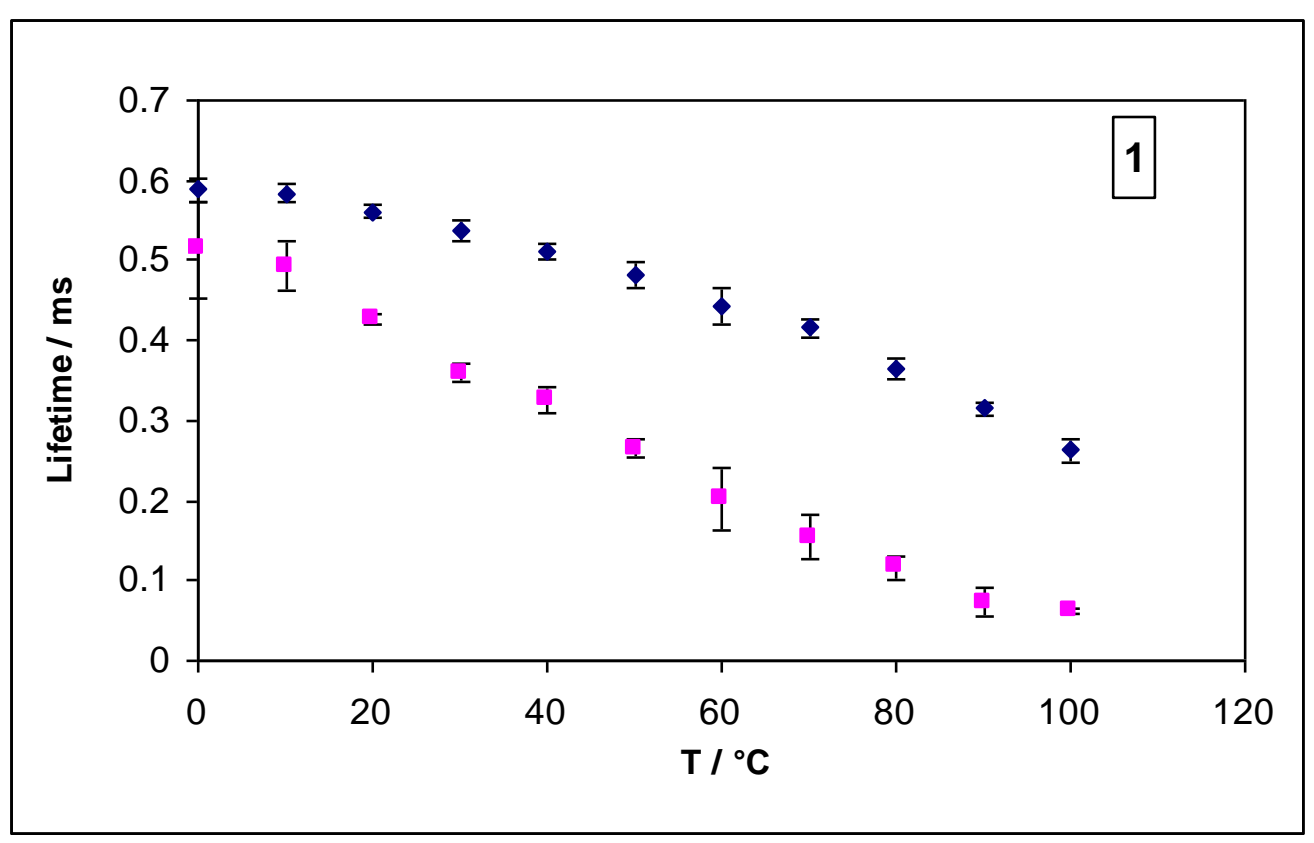


Figure V-6c

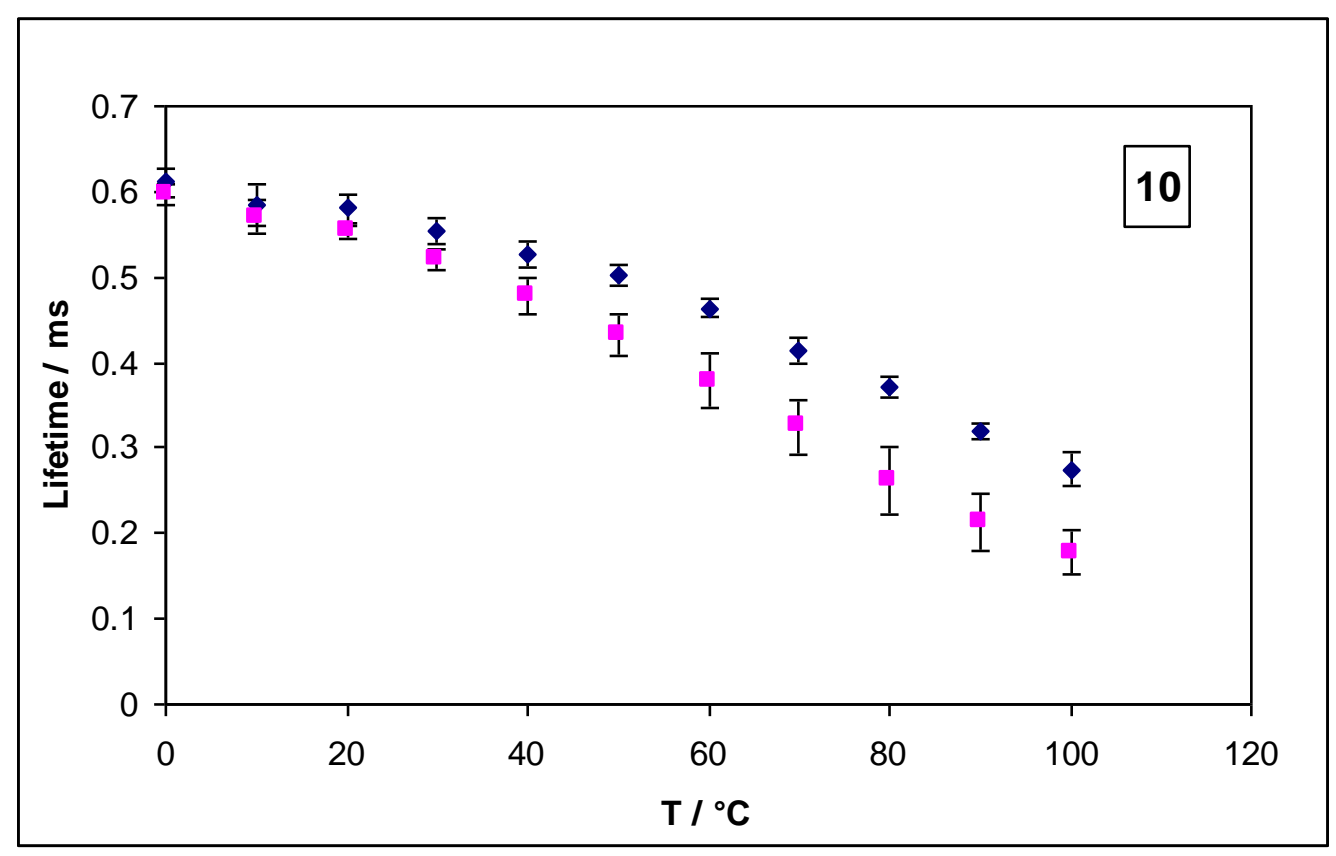

Figure V-6d

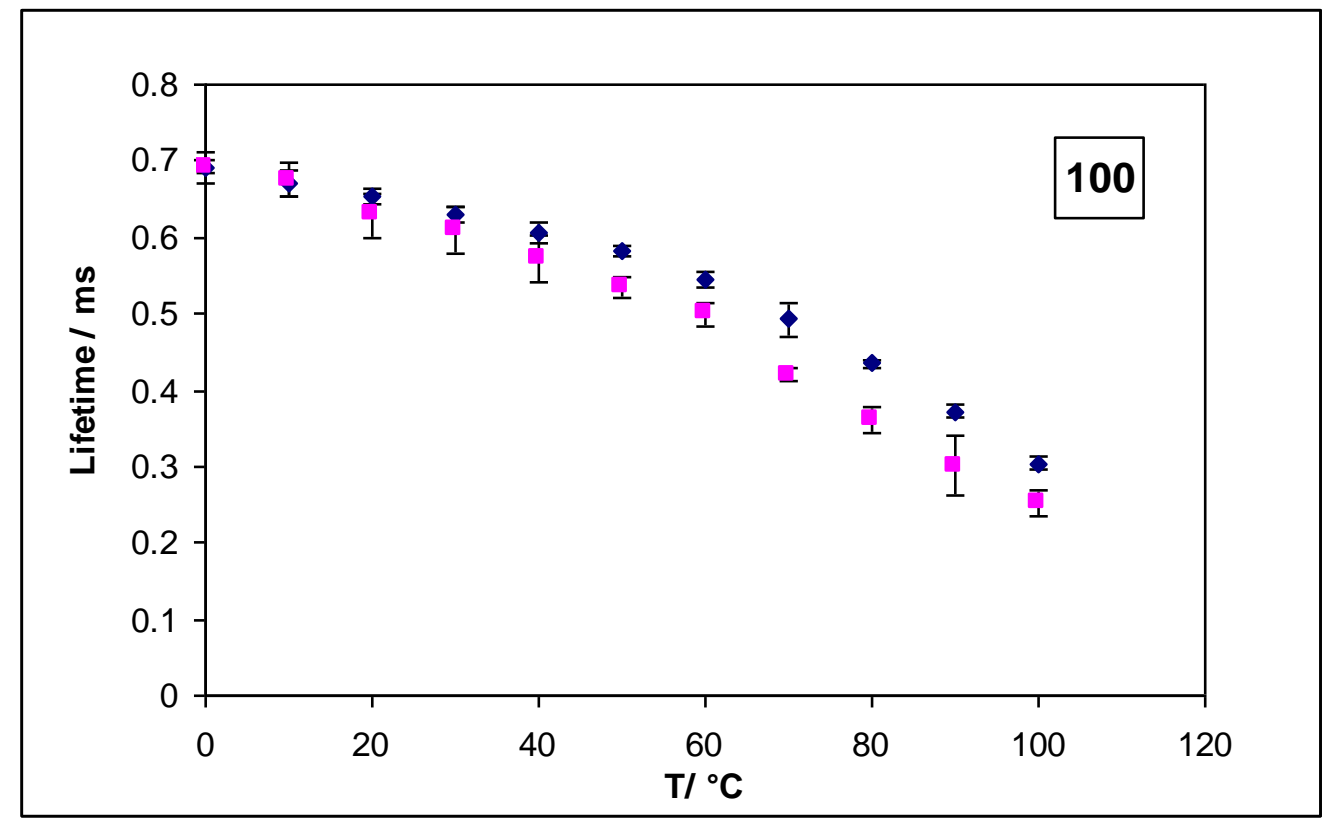


Figure V-6e

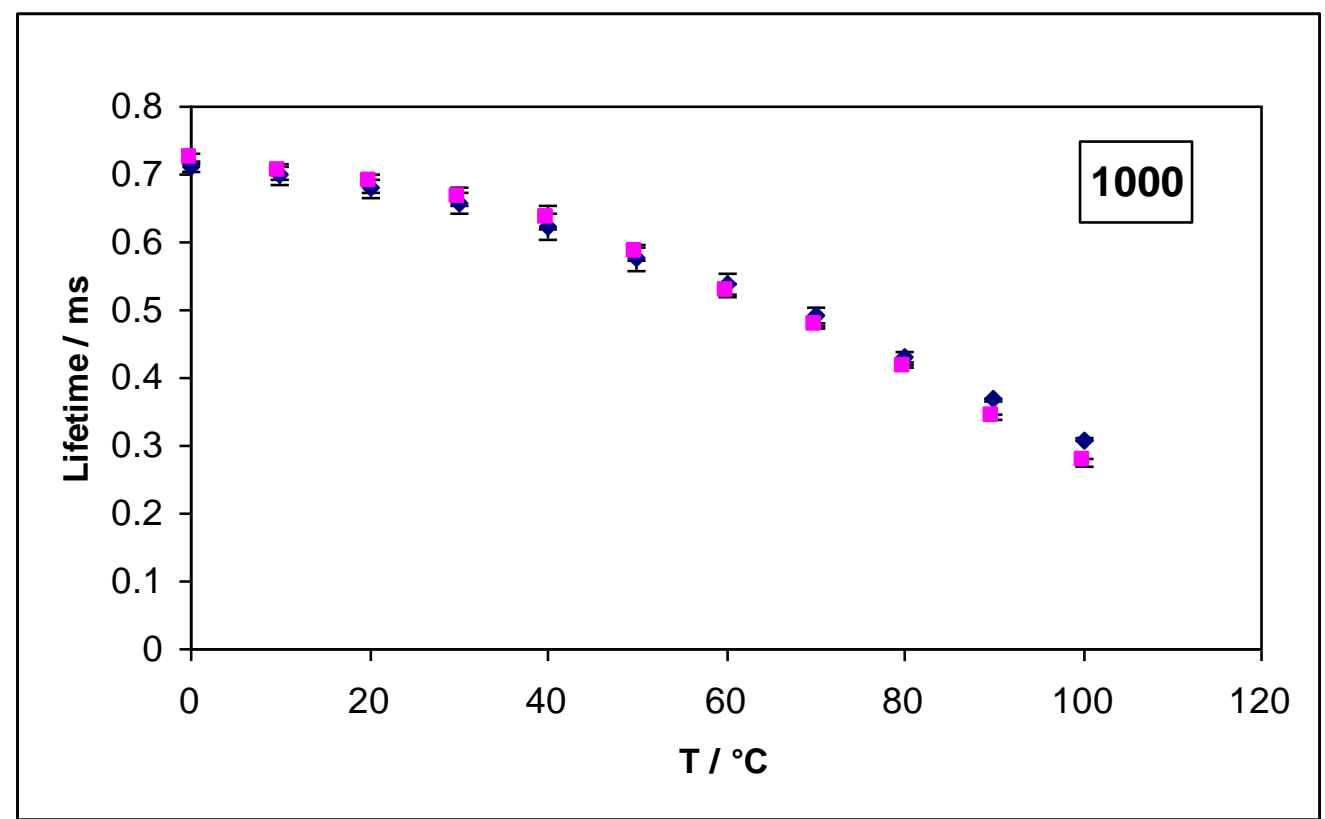

Figure V-6 a, b, c, d, e: The effect of temperature on the lifetime for Erythrosin B dispersed in $\beta-\mathrm{Lg}$ in various molar concentrations of maltitol equilibrated against nitrogen $(\bullet)$ and against air ( $\square$ ); the lifetime was determined from analysis using a stretched exponential decay function. The text box in the graph refers to the molar ratio of Maltitol: $\beta-\mathrm{Lg}$. 
Figure V-6f

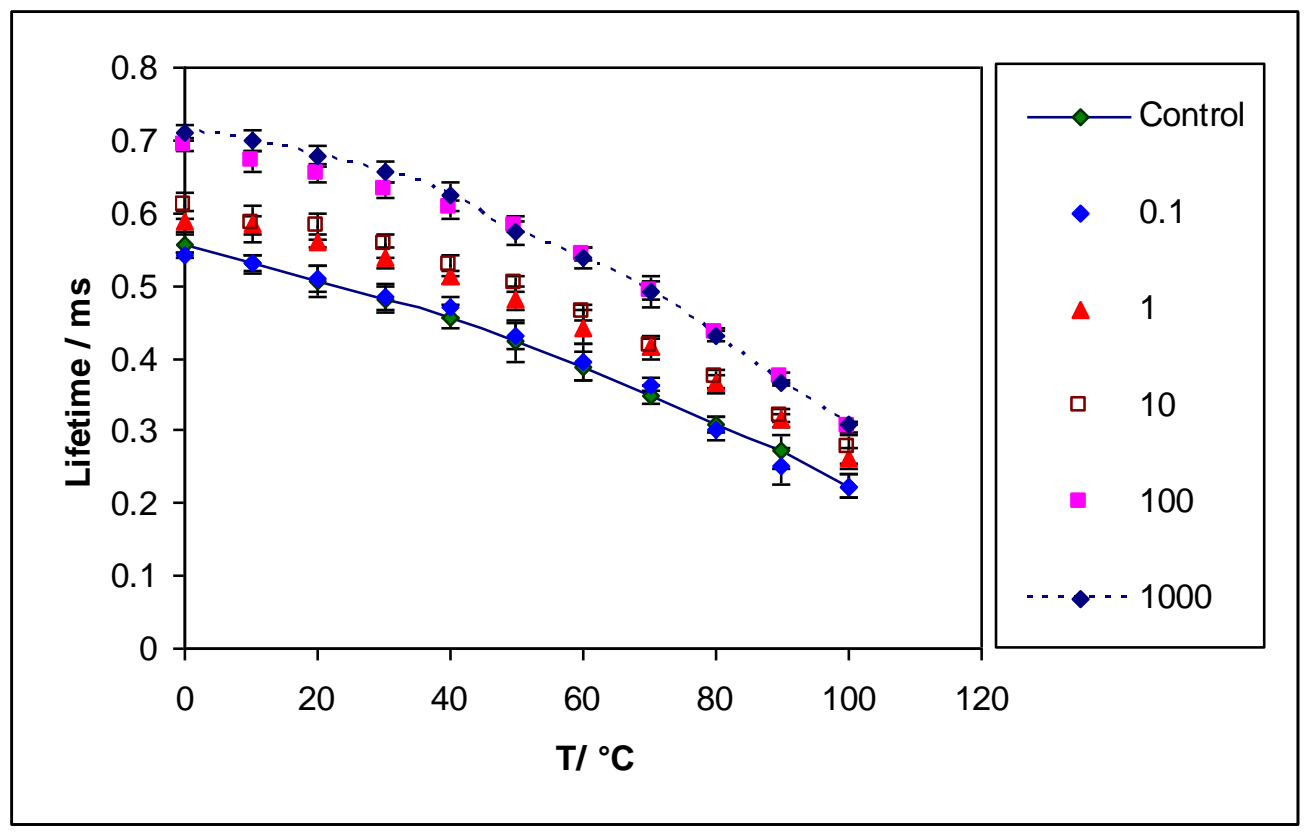

Figure V-6f: Comparison plot depicting the effect of temperature on the lifetime for Erythrosin B dispersed in $\beta$-Lg in various molar concentrations of maltitol equilibrated against nitrogen.

Figure V- 6 g

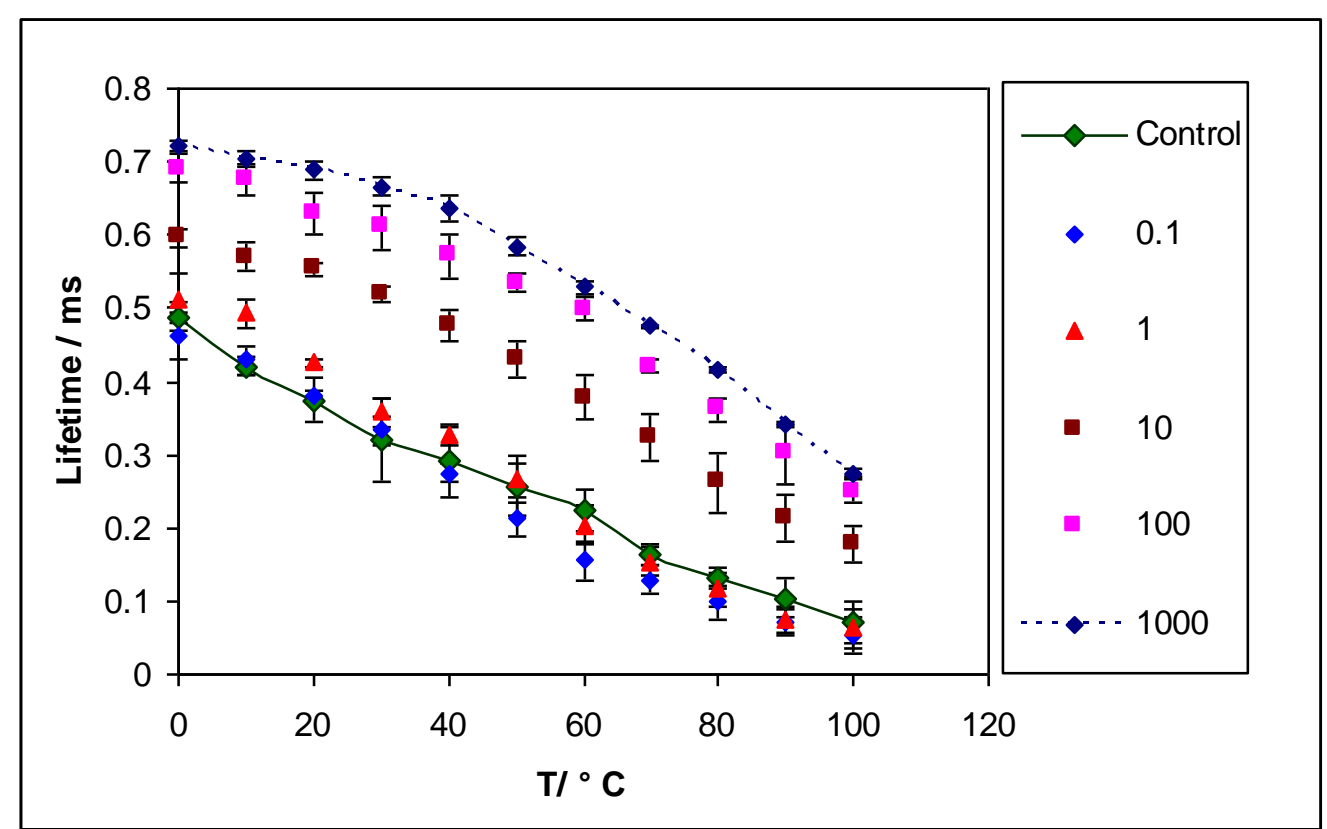

Figure V-6g: Comparison plot depicting the effect of temperature on the lifetime for Erythrosin B dispersed in $\beta$-Lg in various molar concentrations of maltitol equilibrated against oxygen. 
Figure V-7a

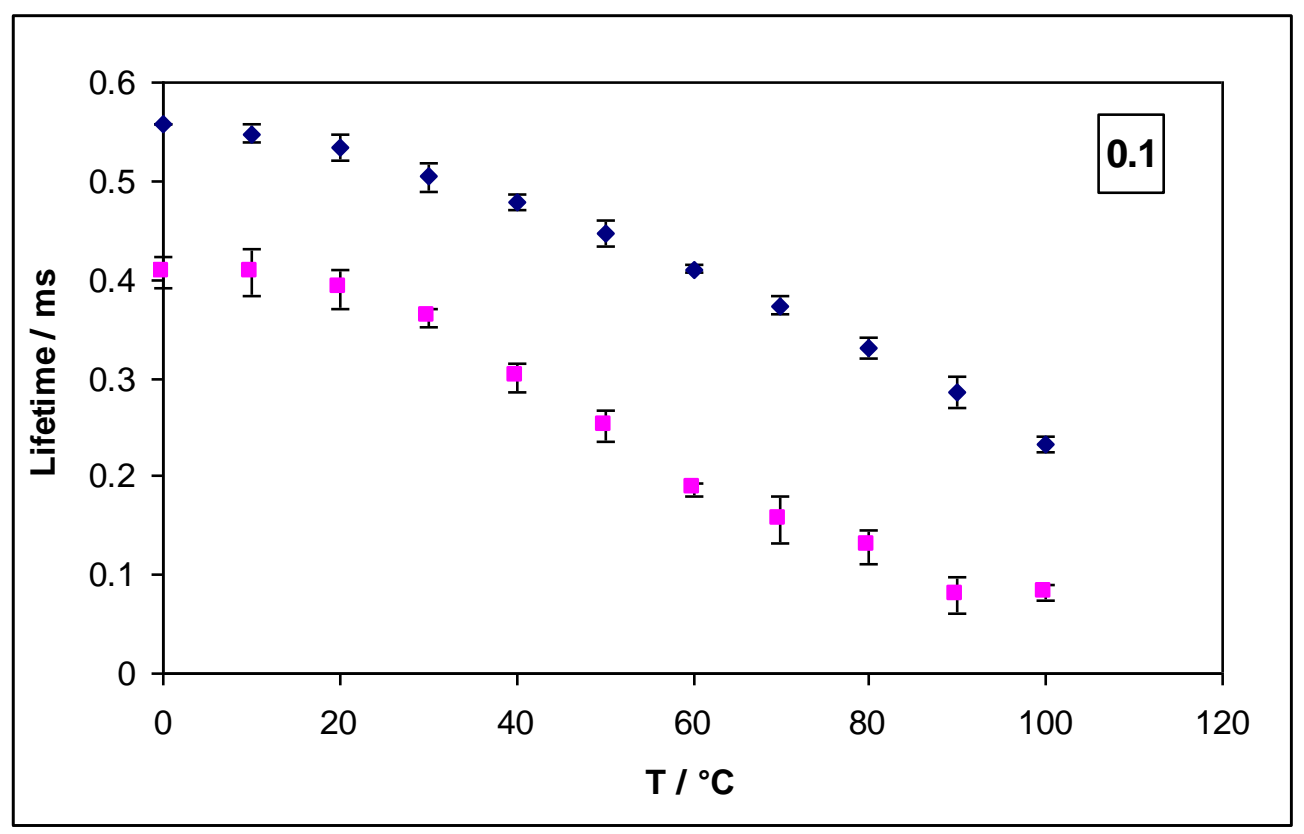

Figure V-7b

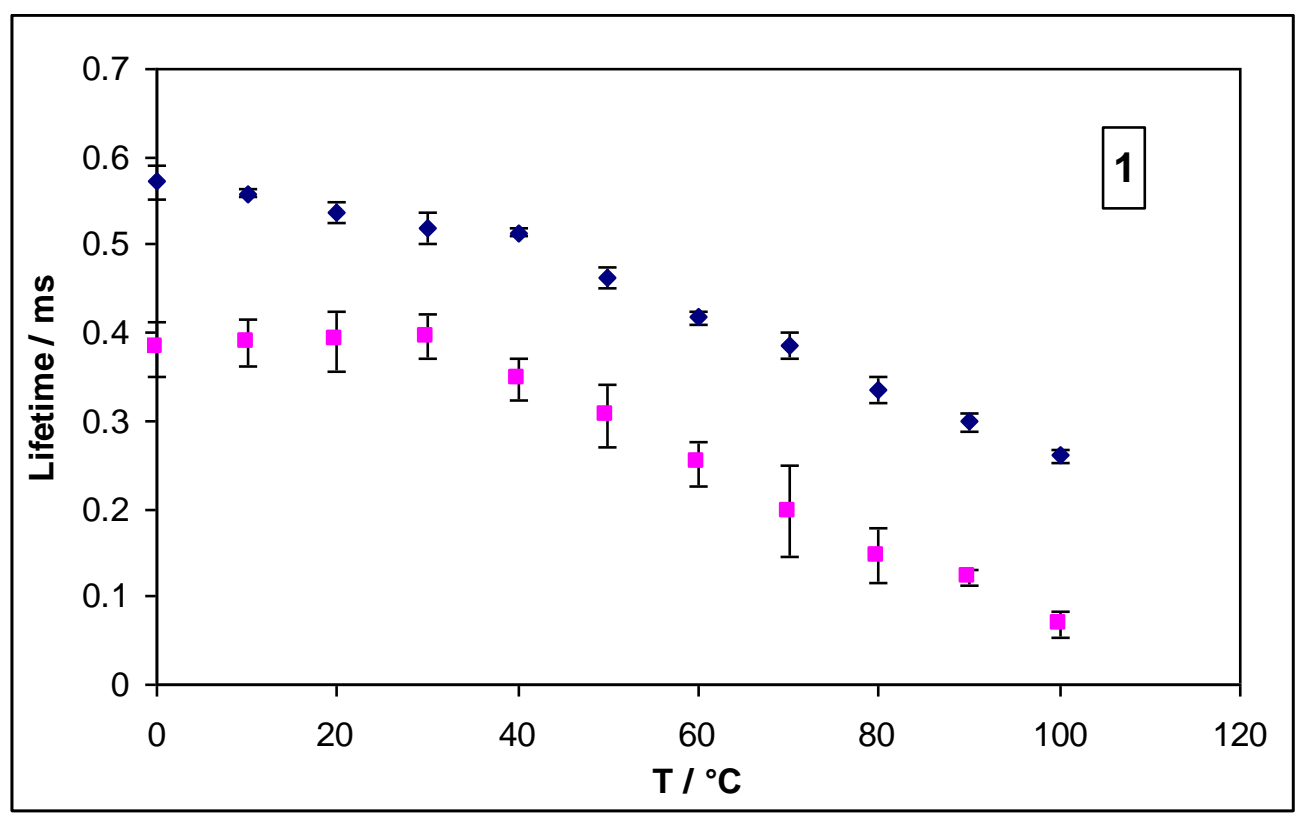


Figure $V-7 c$

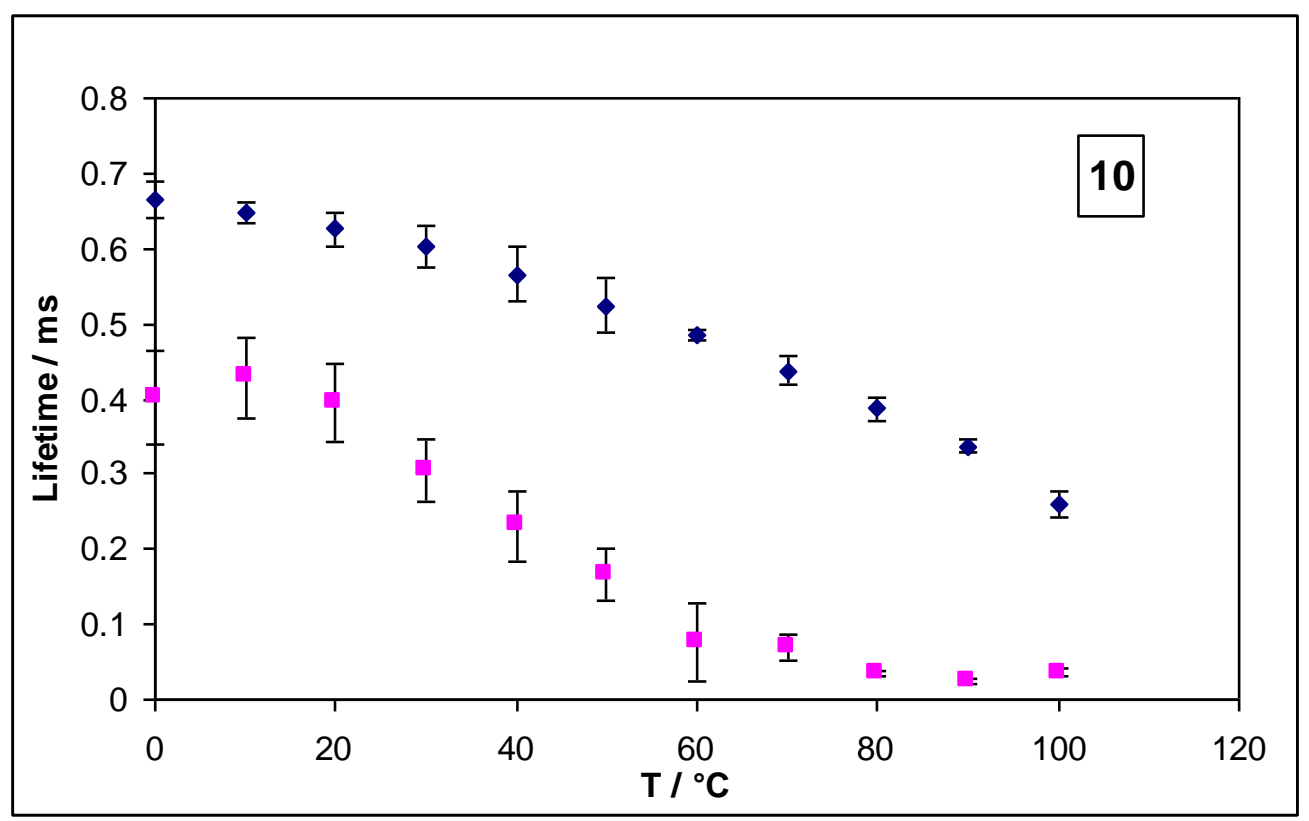

Figure V -7a, b, c The effect of temperature on the lifetime for Erythrosin B dispersed in $\beta$ - $\mathrm{Lg}$ in various molar concentrations of PEG 400 equilibrated against nitrogen $(\downarrow)$ and against air $(\square)$; the lifetime was determined from analysis using a stretched exponential decay function. The text box in the graph refers to the molar ratio of PEG 400: $\beta$-Lg.

\section{Figure V-7d}

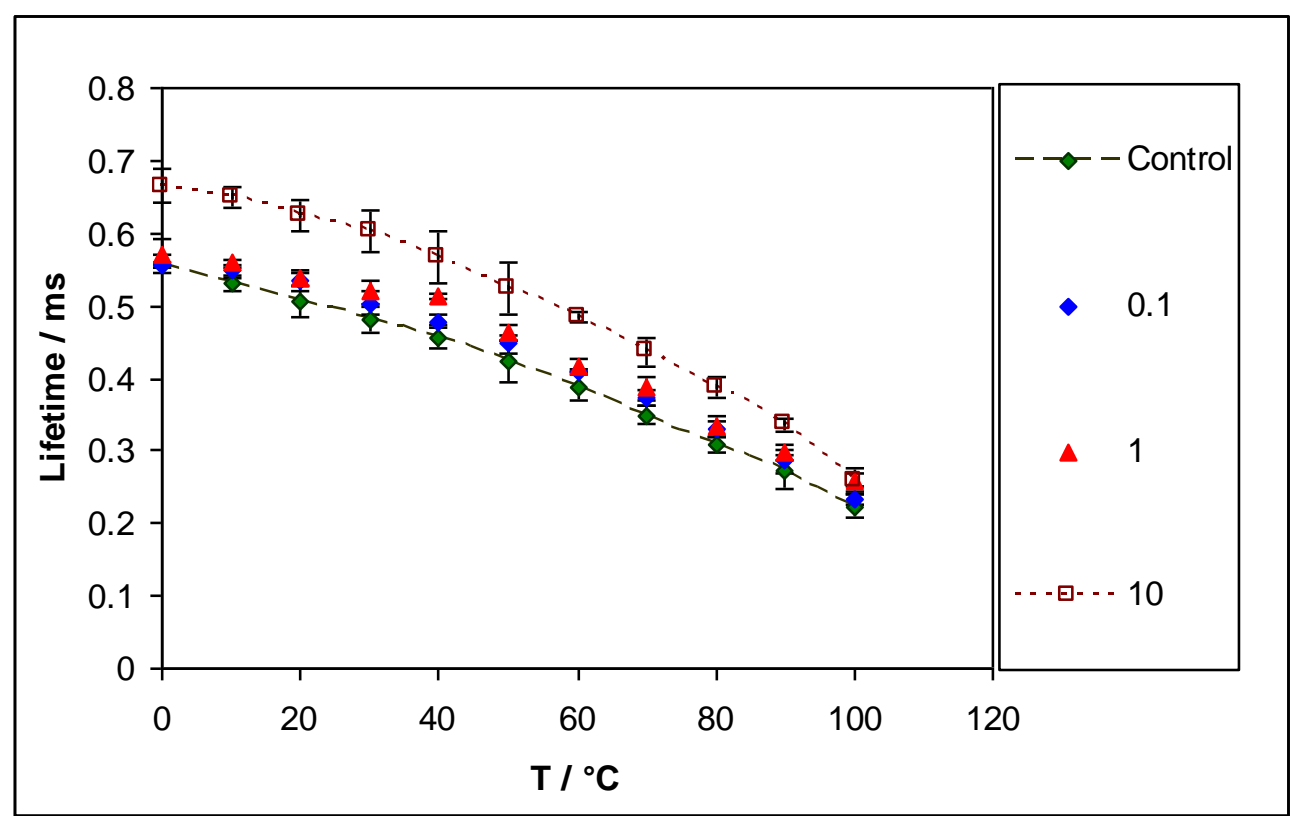

Figure V-7d: Comparison plot depicting the effect of temperature on the lifetime for Erythrosin B dispersed in $\beta-\mathrm{Lg}$ in various molar concentrations of PEG 400 equilibrated against oxygen. 
Figure V-7e

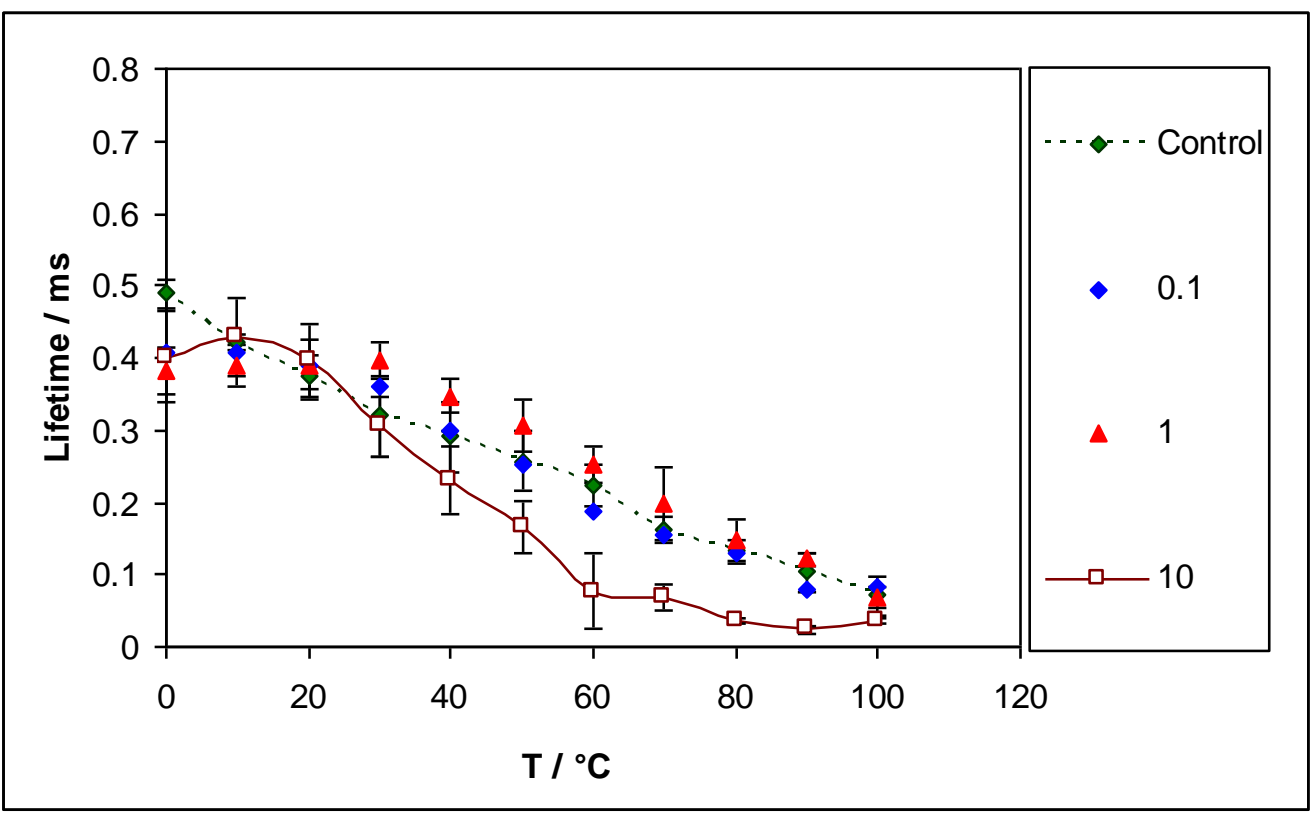

Figure V-7e: Comparison plot depicting the effect of temperature on the lifetime for Erythrosin B dispersed in $\beta-\mathrm{Lg}$ in various molar concentrations of PEG 400 equilibrated against oxygen. 
Figure $V-8 a$

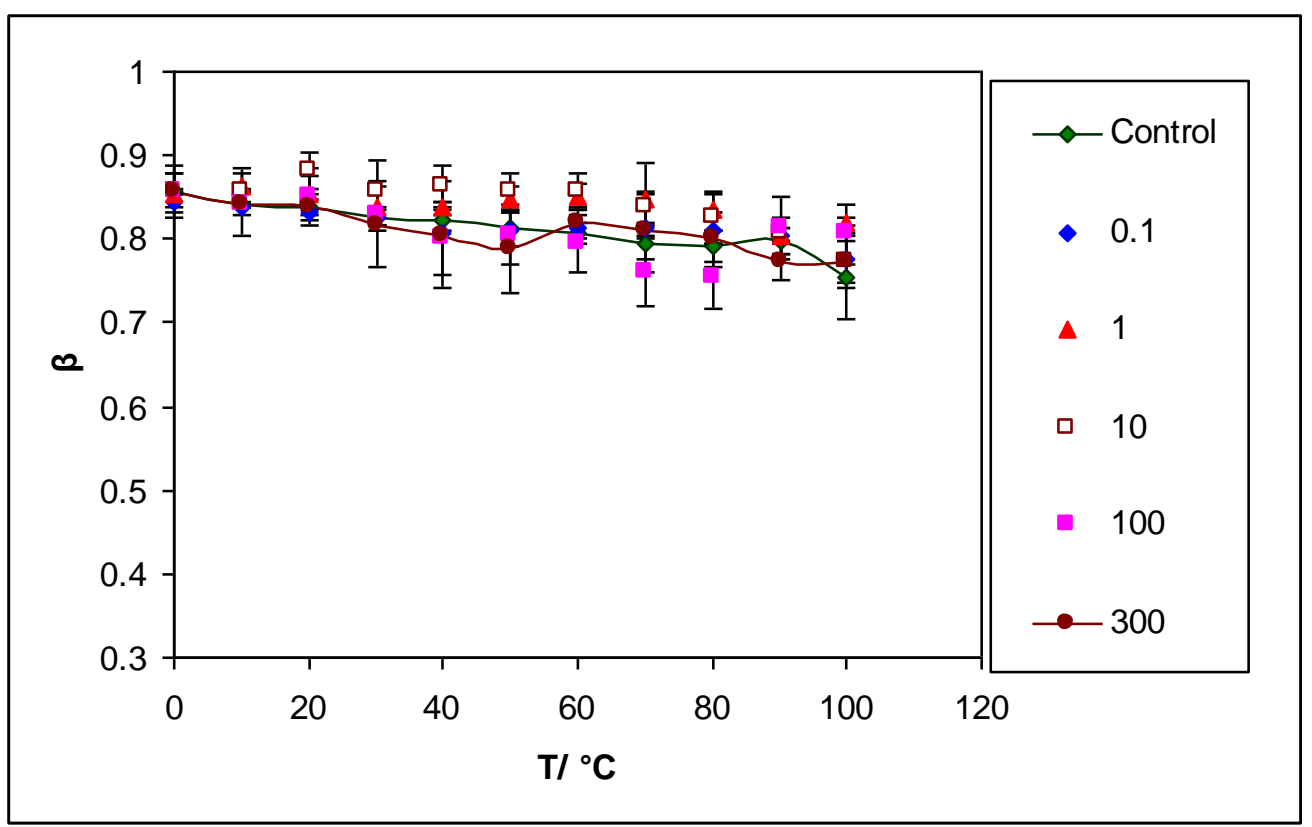

Figure V-8a: Beta as a function of temperature in glycerol $/ \beta$ - $\mathrm{Lg}$ films equilibrated against nitrogen.

Figure $V-8 b$

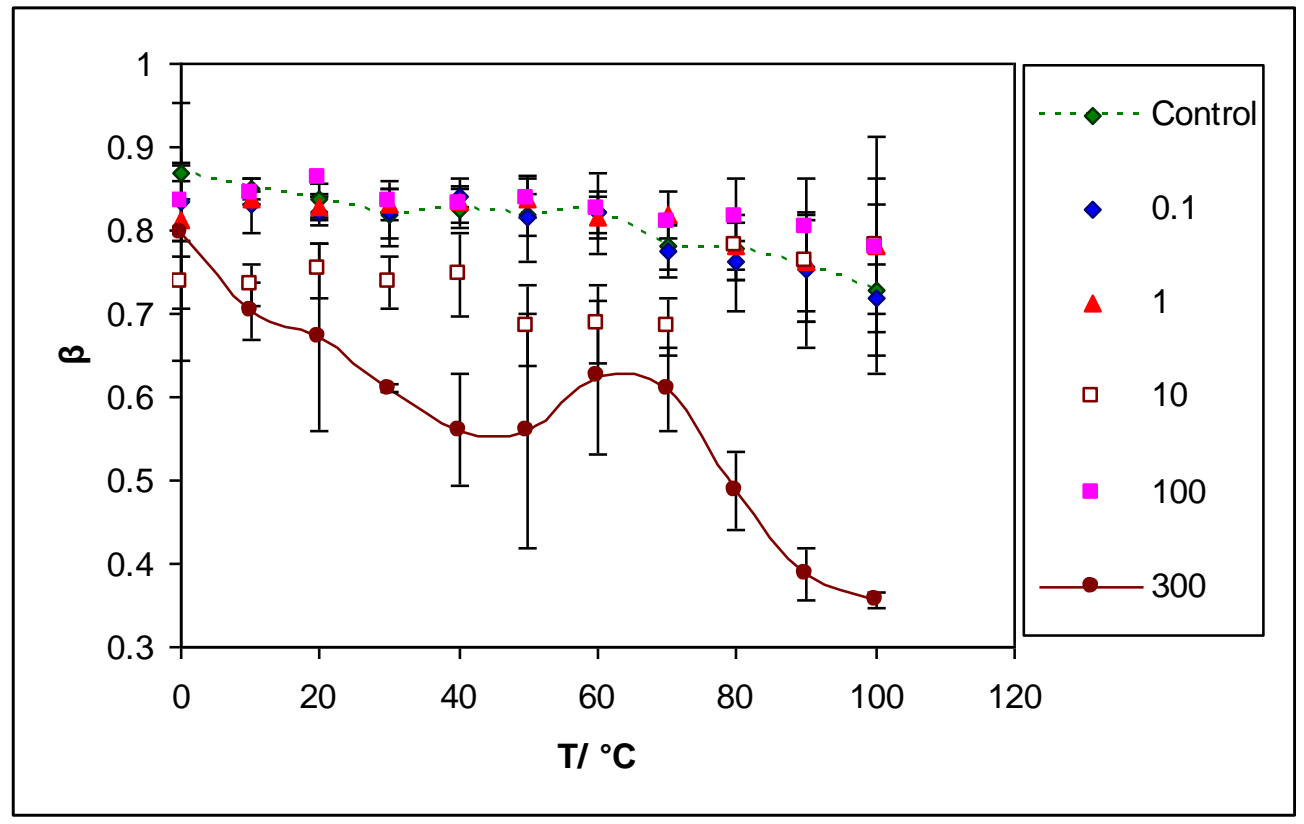

Figure V-8b: Beta as a function of temperature in glycerol $\beta-\mathrm{Lg}$ films equilibrated against oxygen. 
Figure V-9a

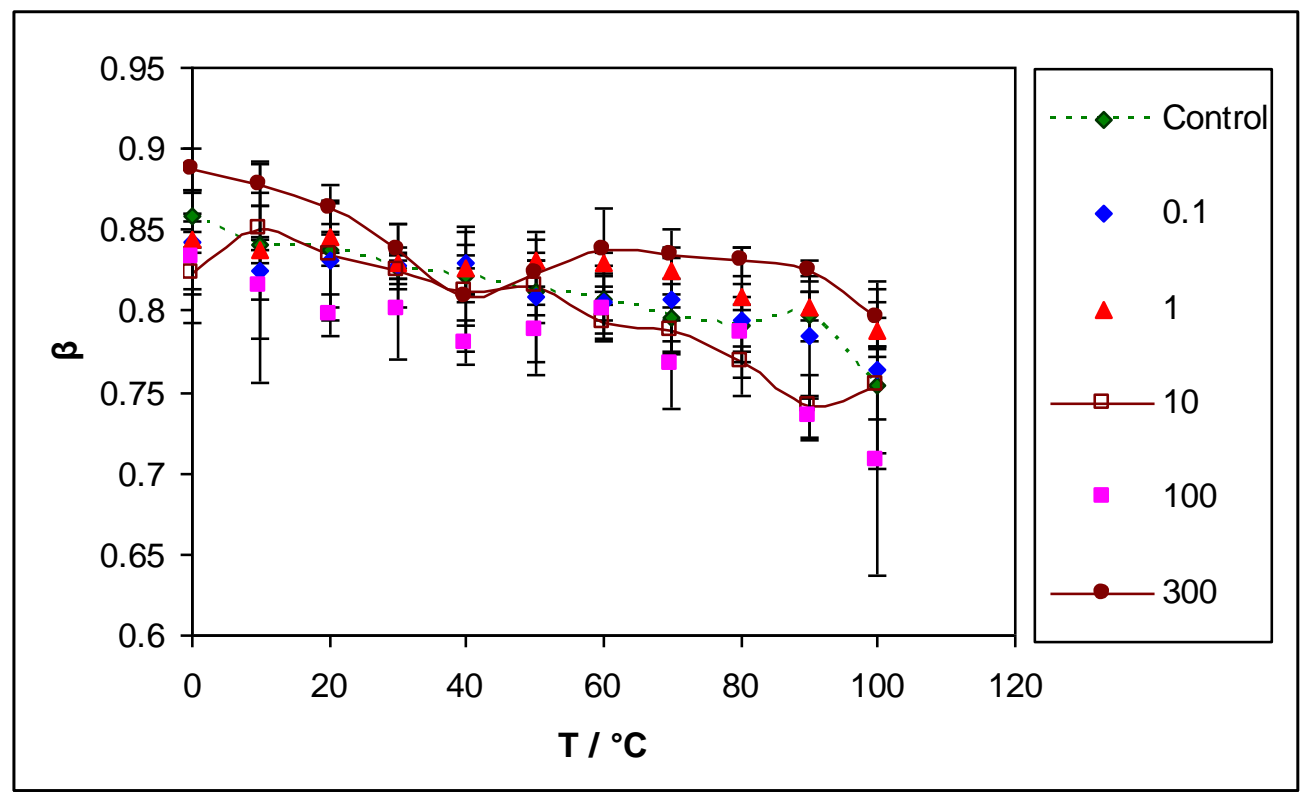

Figure V-9a: Beta as a function of temperature in sorbitol / $\beta-\mathrm{Lg}$ films equilibrated against nitrogen.

Figure V-9b

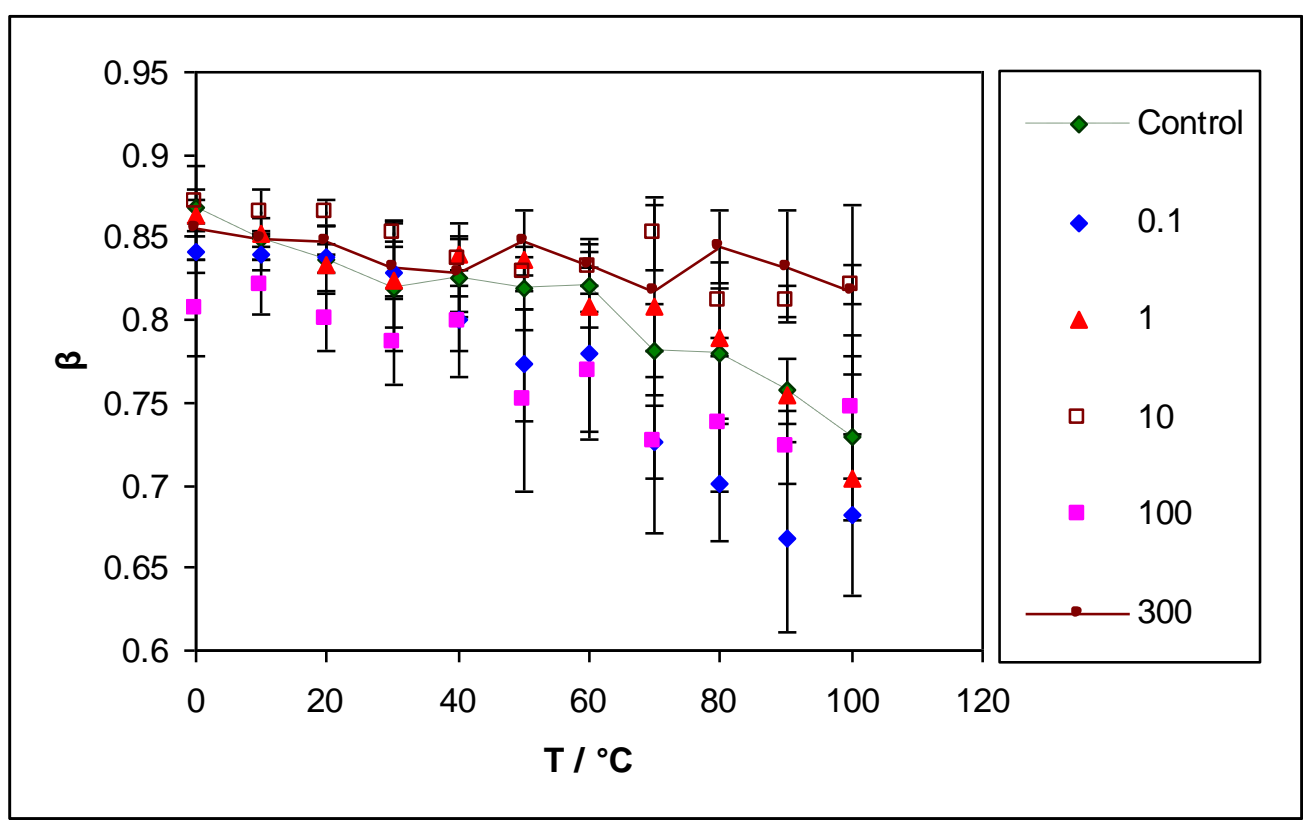

Figure V-9b: Beta as a function of temperature in sorbitol/ $\beta$-Lg films equilibrated against oxygen. 
Figure V-10a

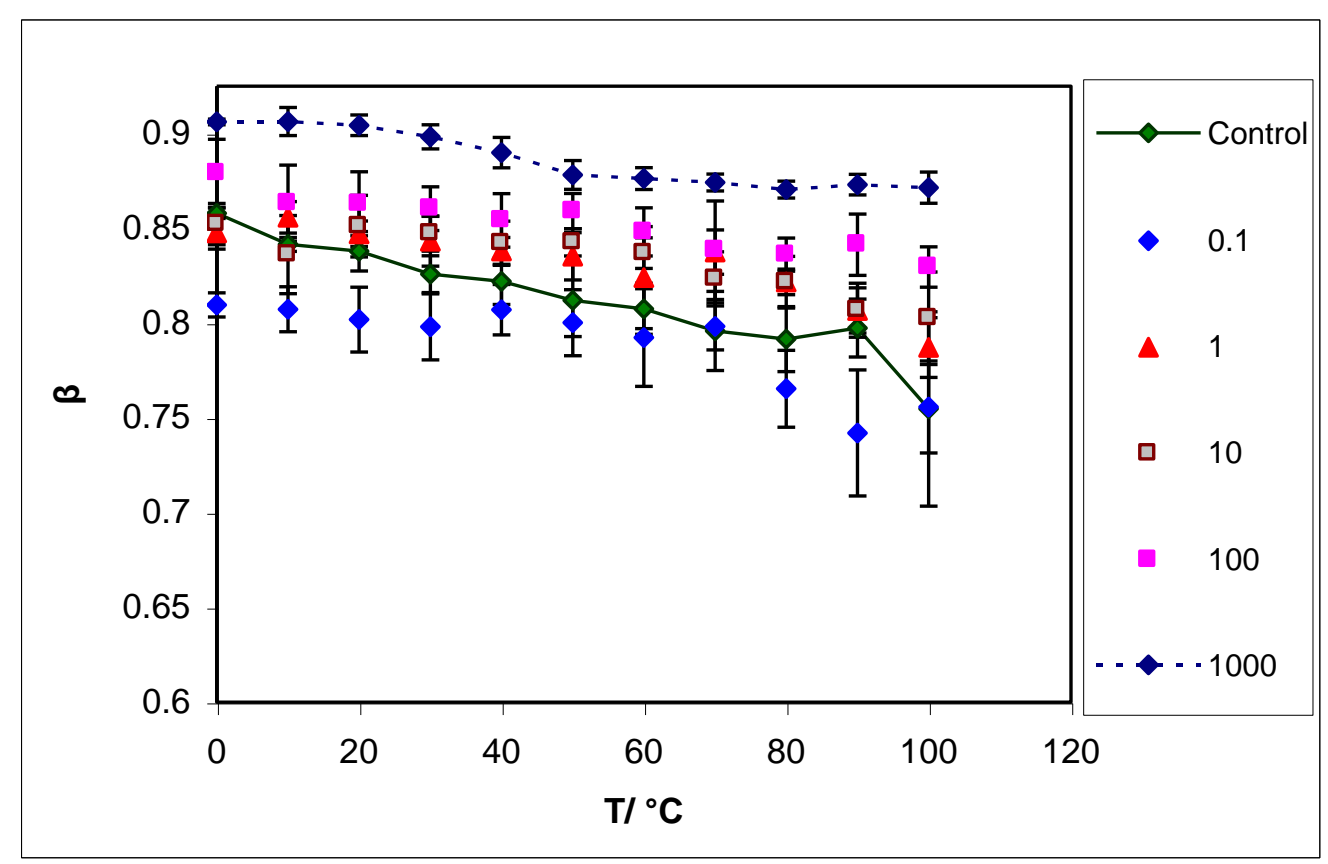

Figure V-10a: Beta as a function of temperature in maltitol/ $\beta$-Lg films equilibrated against nitrogen.

Figure $V-10 b$

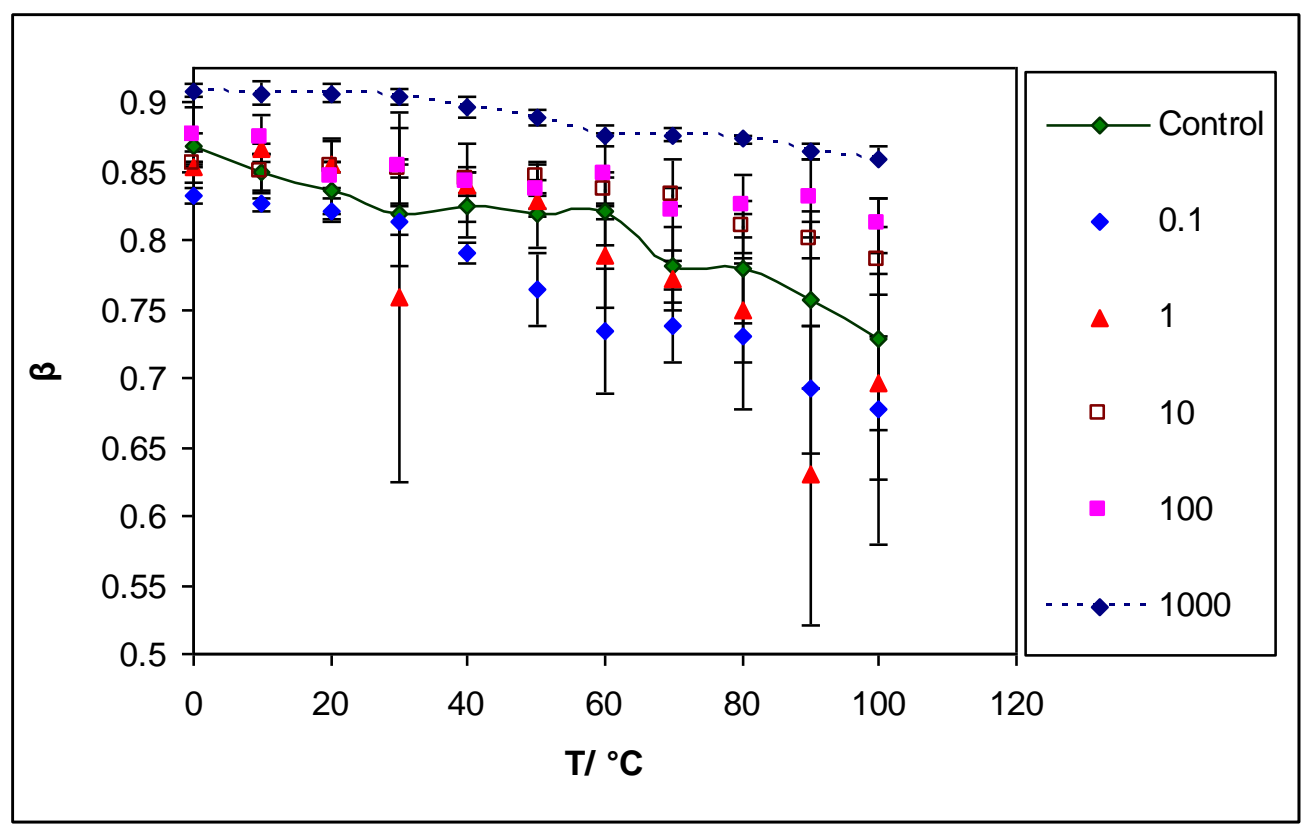

Figure V-10b: Beta as a function of temperature in maltitol/ $\beta$-Lg films equilibrated against oxygen. 
Figure V-11a

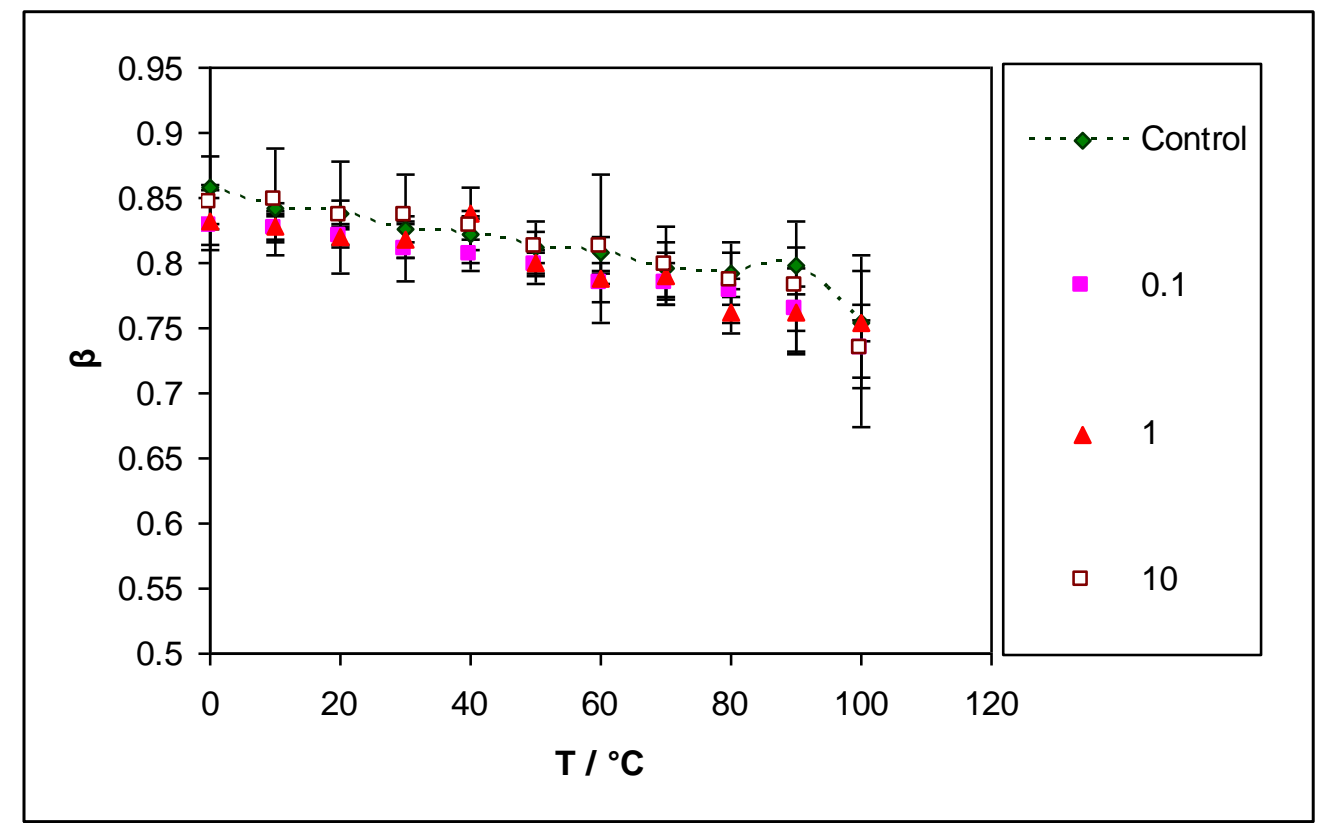

Figure V-11a: Beta as a function of temperature in PEG/ $\beta$-Lg films equilibrated against nitrogen.

Figure V-11b

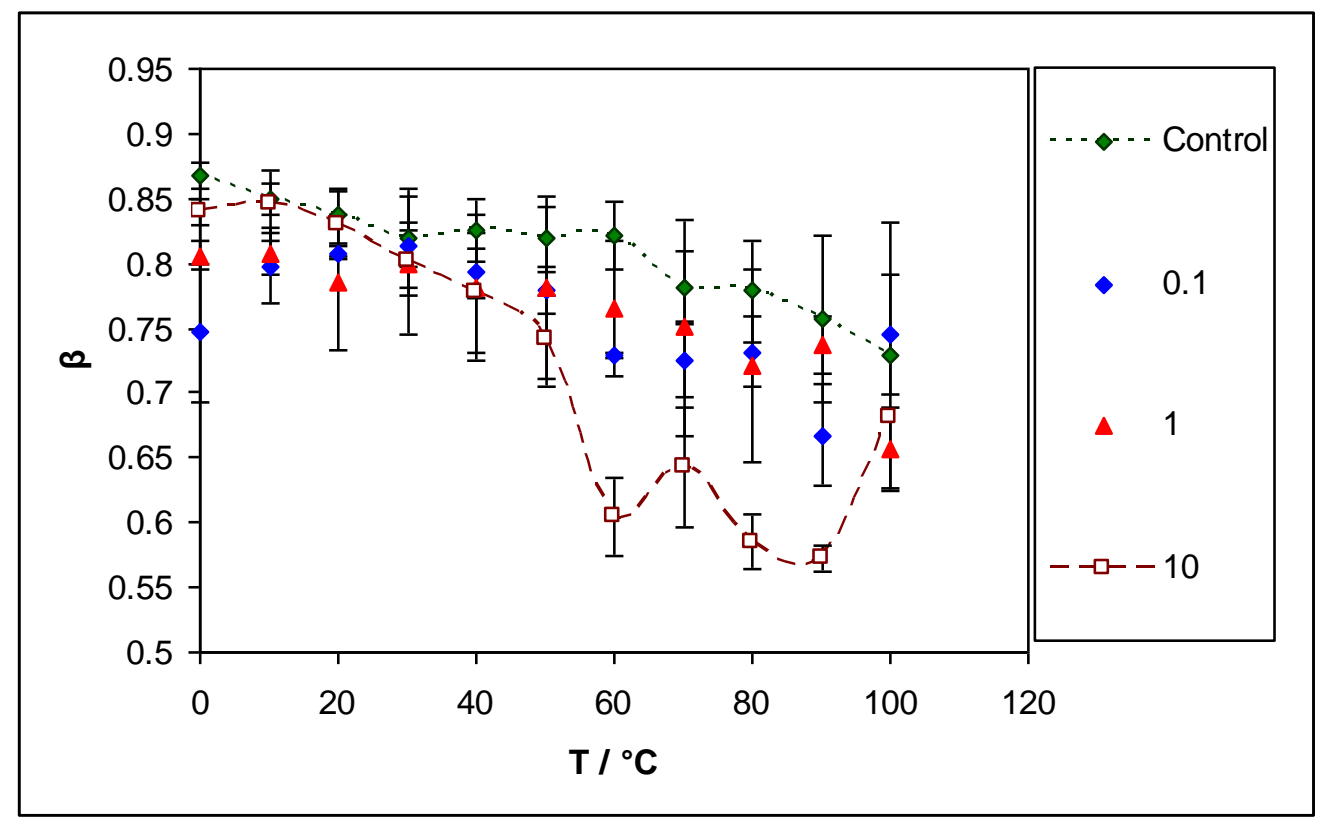

Figure V-11b: Beta as a function of temperature in PEG/ $\beta$-Lg films equilibrated against oxygen. 
Figure V-12a

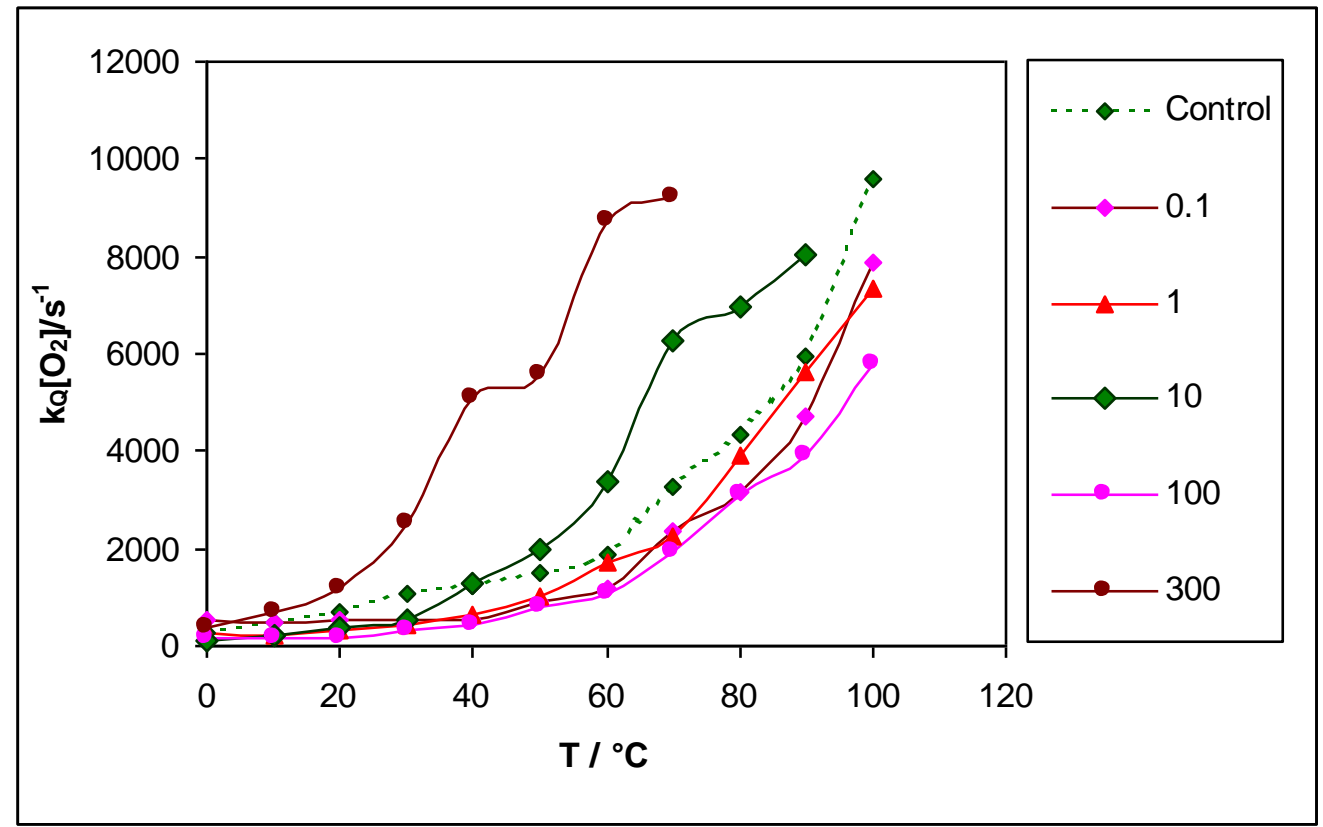

Figure $\mathrm{V}-12 \mathrm{a}$ : The oxygen quenching rate $\mathrm{k}_{\mathrm{Q}}\left[\mathrm{O}_{2}\right]$ as a function of temperature in glycerol/ $\beta-\mathrm{Lg}$ mixtures.

Figure $V-12 b$

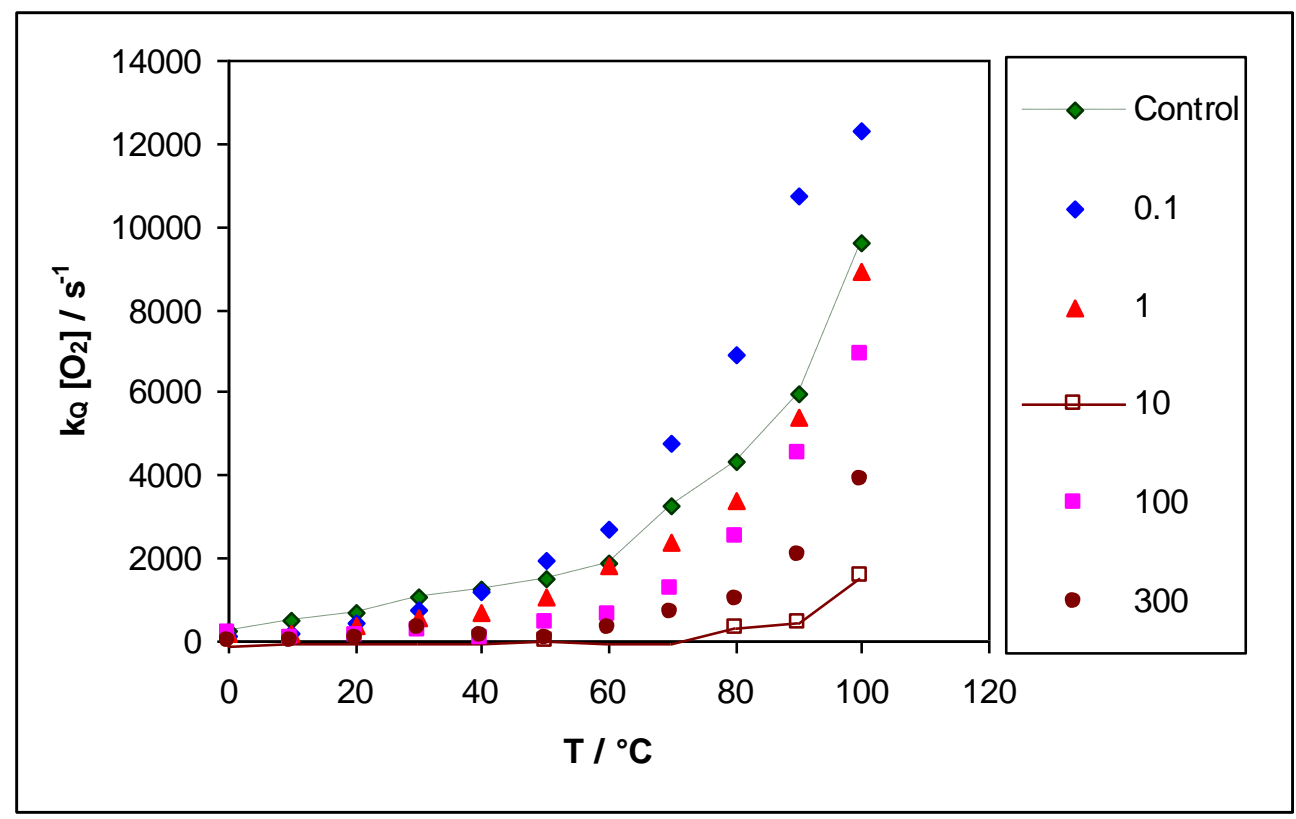

Figure $\mathrm{V}-12 \mathrm{~b}$ : The oxygen quenching rate $\mathrm{k}_{\mathrm{Q}}\left[\mathrm{O}_{2}\right]$ as a function of temperature in the $\beta$ - $\mathrm{Lg}$ / sorbitol mixtures. 
Figure V-12c

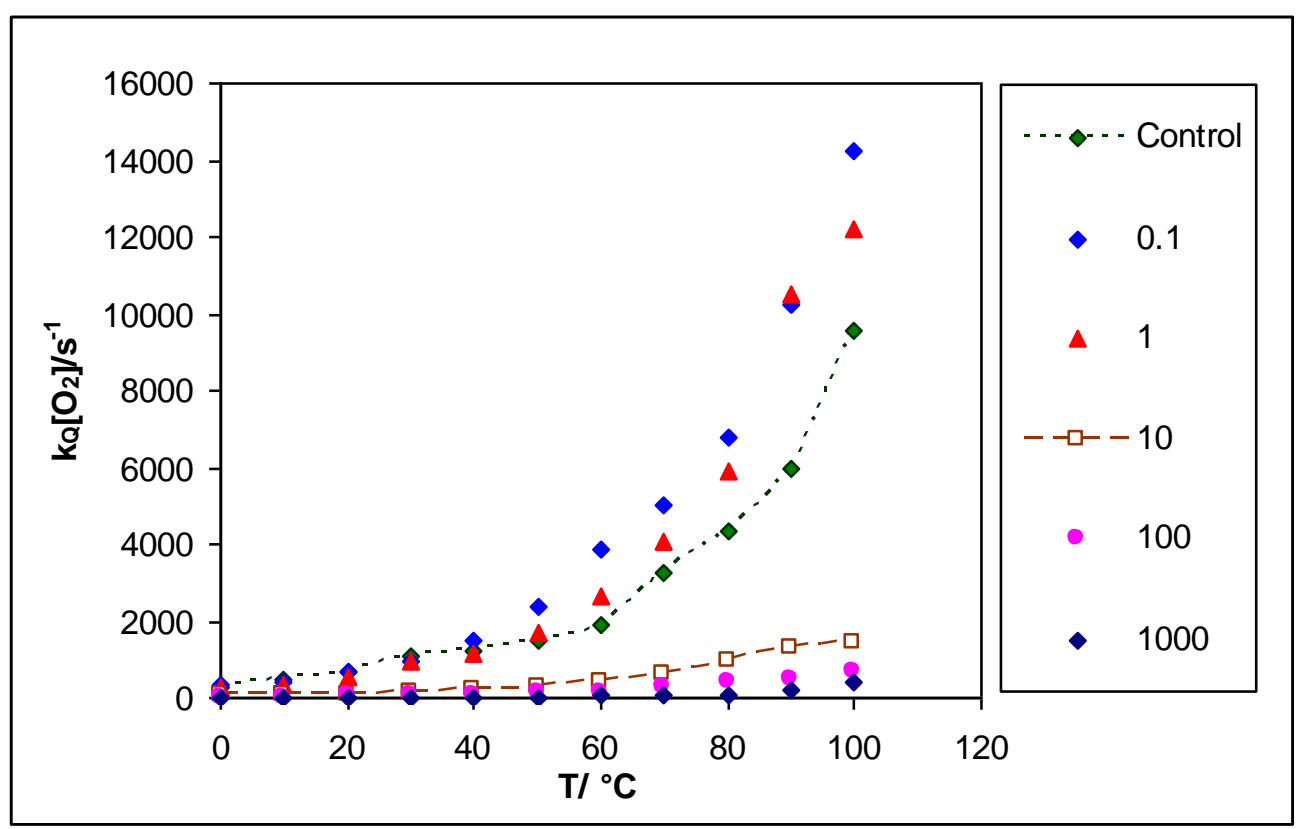

FigureV-12c: The oxygen quenching rate $\mathrm{k}_{\mathrm{Q}}\left[\mathrm{O}_{2}\right]$ as a function of temperature in maltitol/ $\beta$ - $\mathrm{Lg}$ films.

Figure $V-12 d$

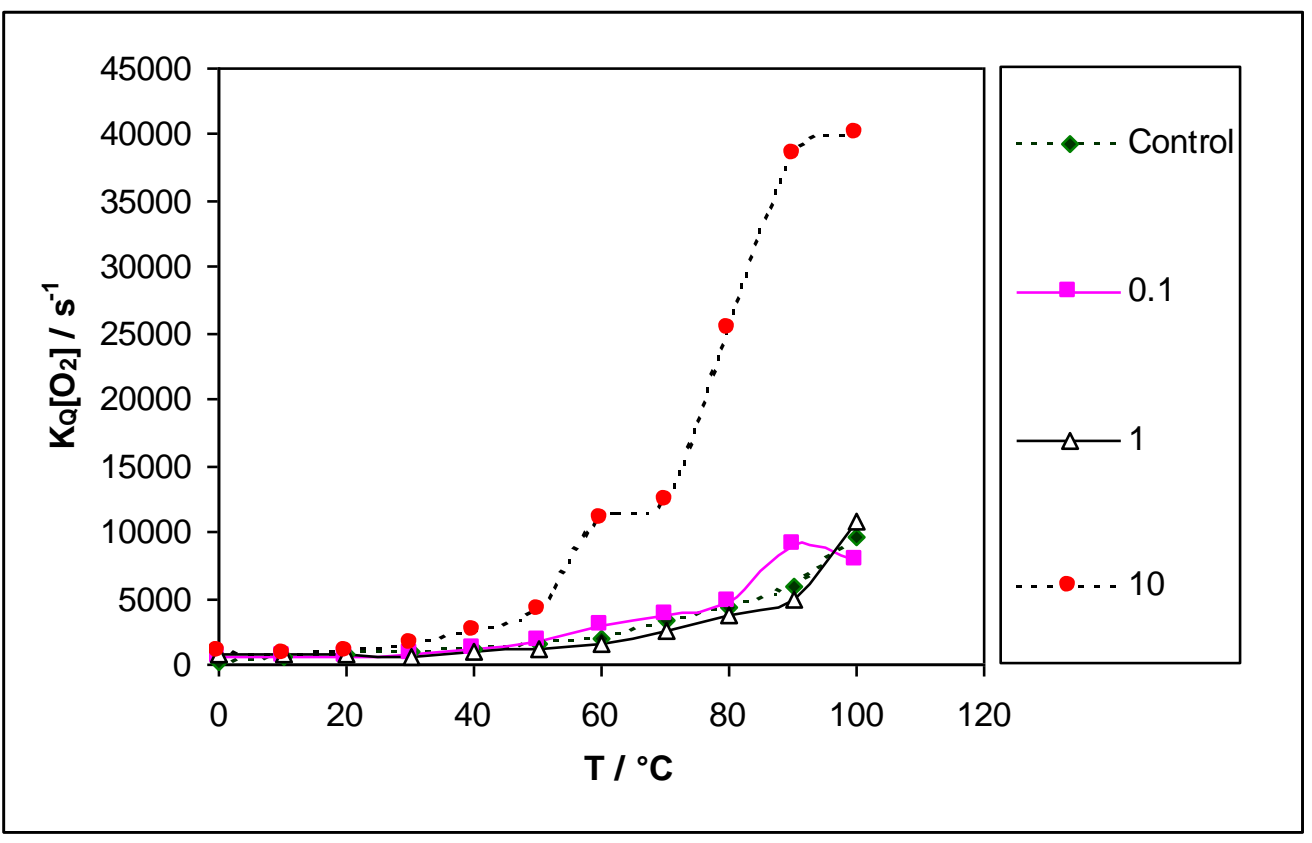

Figure V-12d: The oxygen quenching rate $\mathrm{k}_{\mathrm{Q}}\left[\mathrm{O}_{2}\right]$ as a function of temperature in the $\beta$-Lg and PEG/ $\beta$-Lg mixtures. 
Figure V-13a

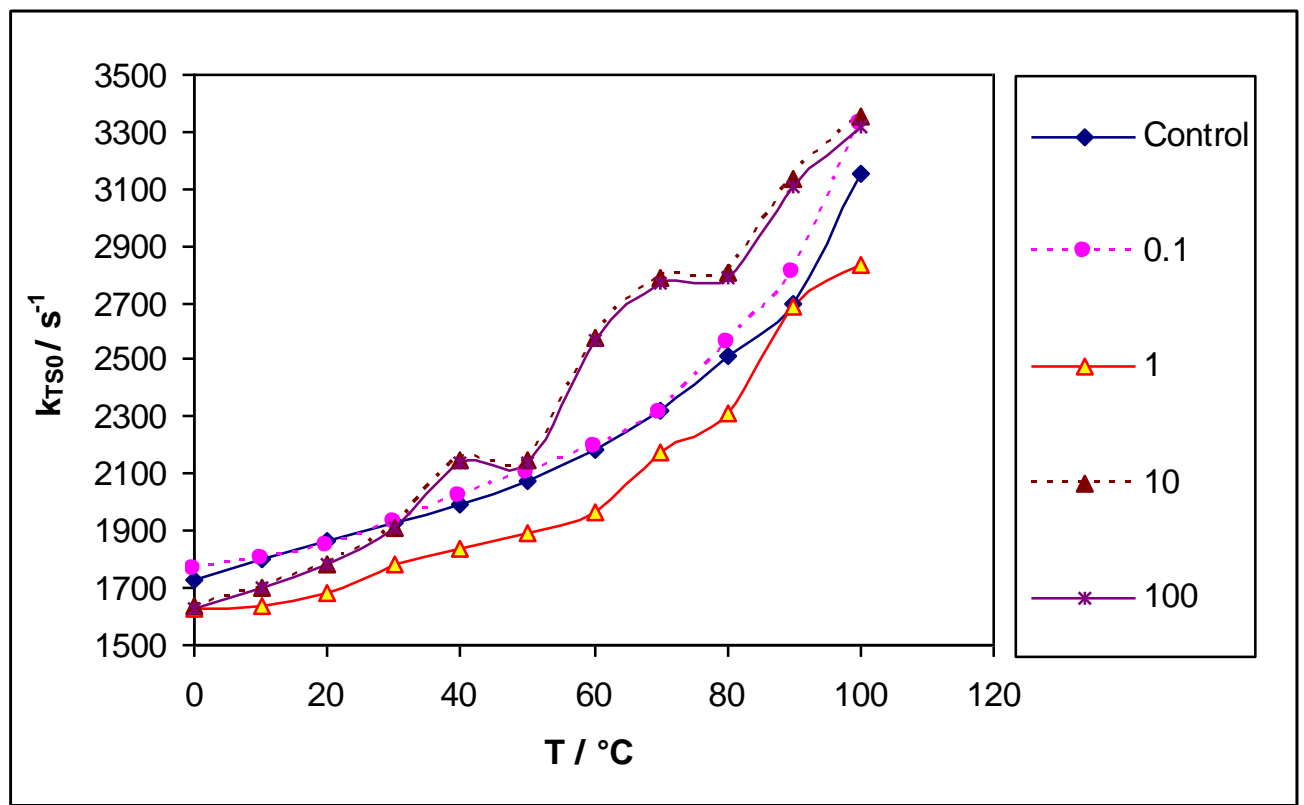

Figure V-13a: Plot of the effect of temperature on non-radiative decay from $\mathrm{T}_{1}$ to $\mathrm{S}_{0}\left(\mathrm{k}_{\mathrm{TS} 0}\right)$ in amorphous glycerol/ $\beta$-Lg films.

Figure $V-13 b$

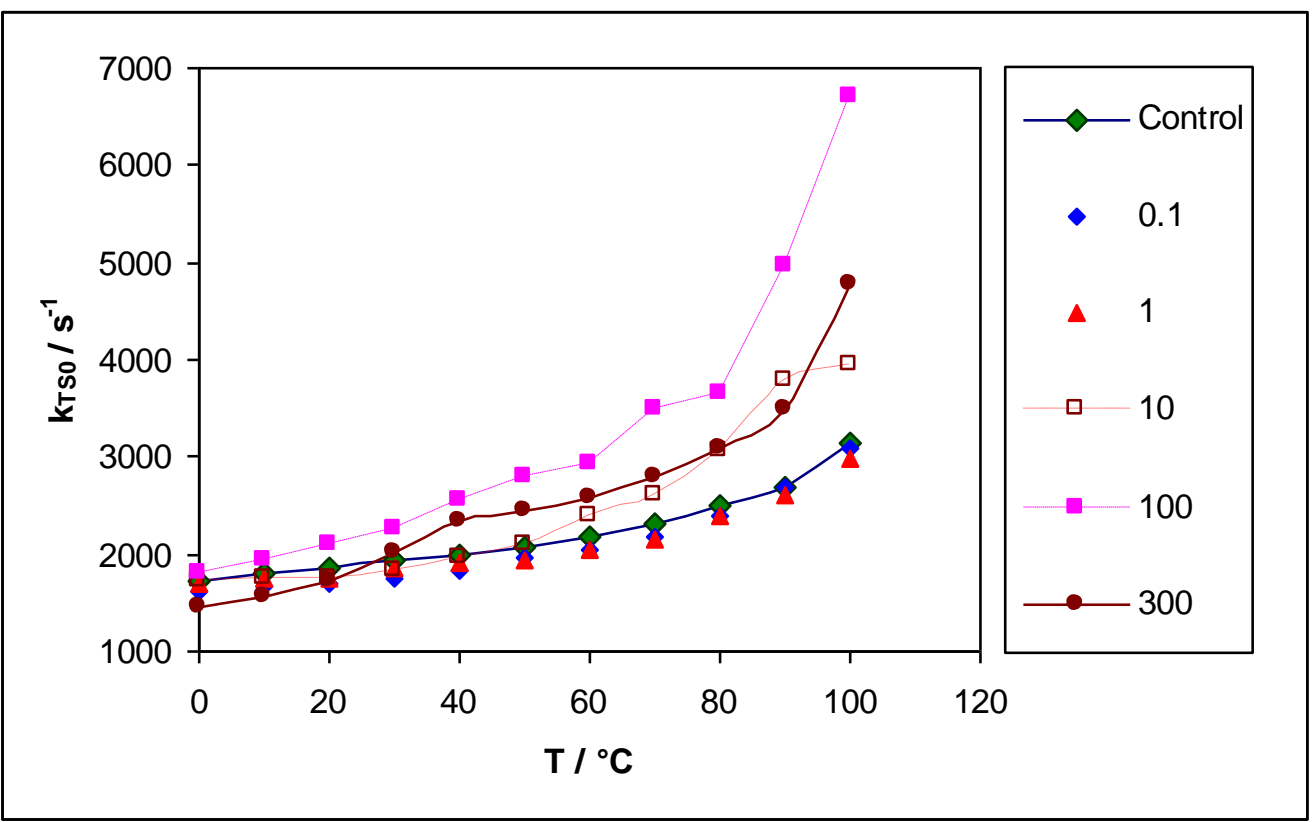

Figure V-13b: Plot of the effect of temperature on non-radiative decay from $T_{1}$ to $S_{0}\left(k_{T S 0}\right)$ in amorphous sorbitol/ $\beta$-Lg films. 


\section{Figure V-13c}

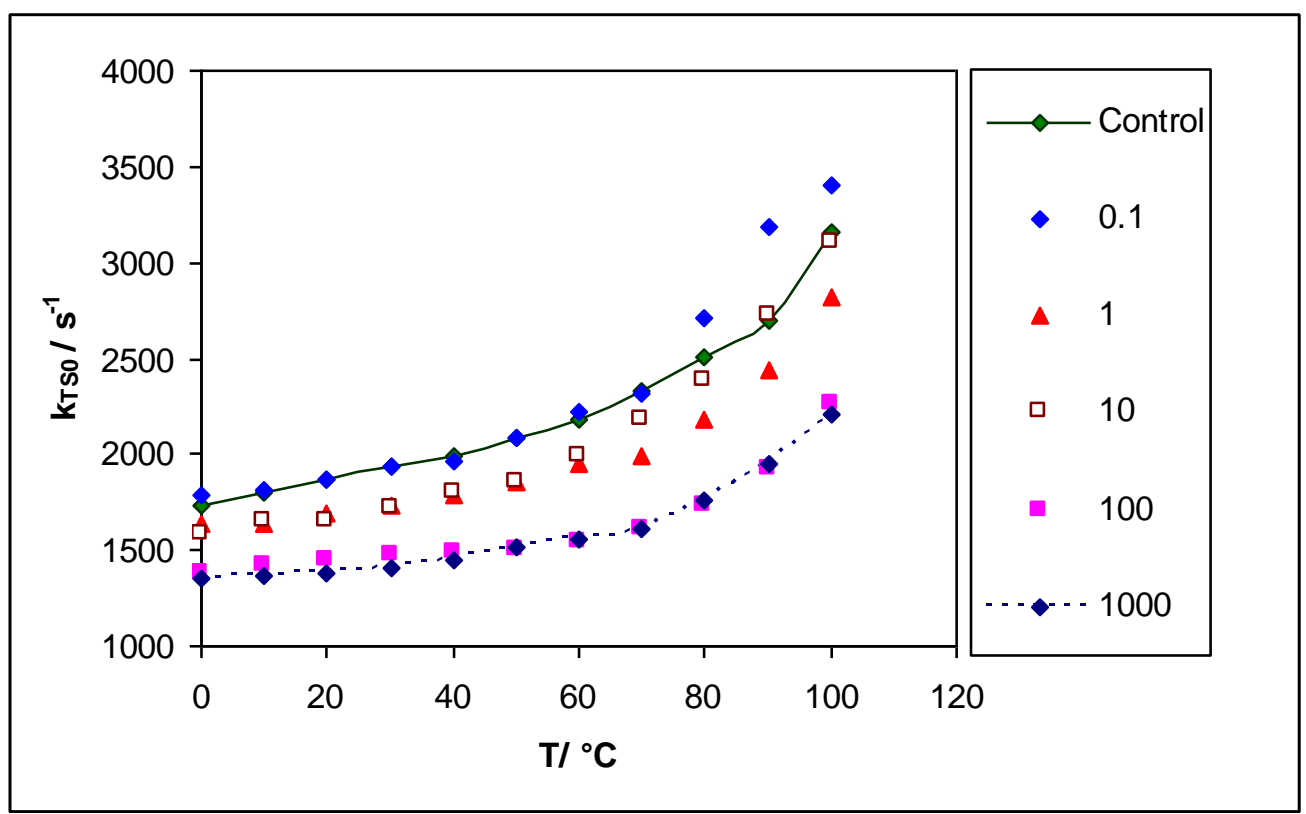

Figure V-13c: Plot of the effect of temperature on non-radiative decay from $T_{1}$ to $S_{0}\left(k_{T S 0}\right)$ in amorphous maltitol/ $\beta$-Lg films.

Figure V-13d

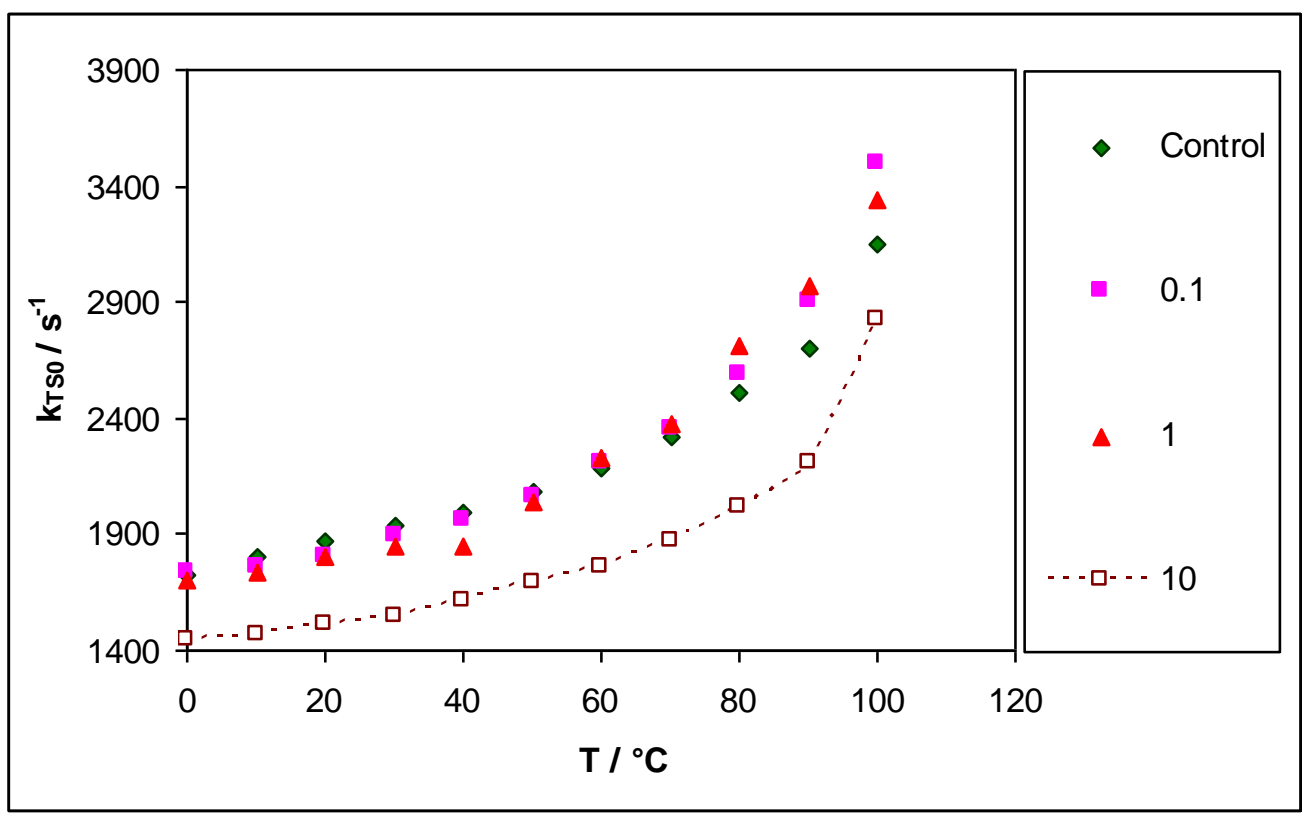

Figure V-13d: Plot of the effect of temperature on non-radiative decay from $\mathrm{T}_{1}$ to $\mathrm{S}_{0}\left(\mathrm{k}_{\mathrm{TS} 0}\right)$ in amorphous PEG 400/ $\beta$-Lg films. 
Figure V-14a

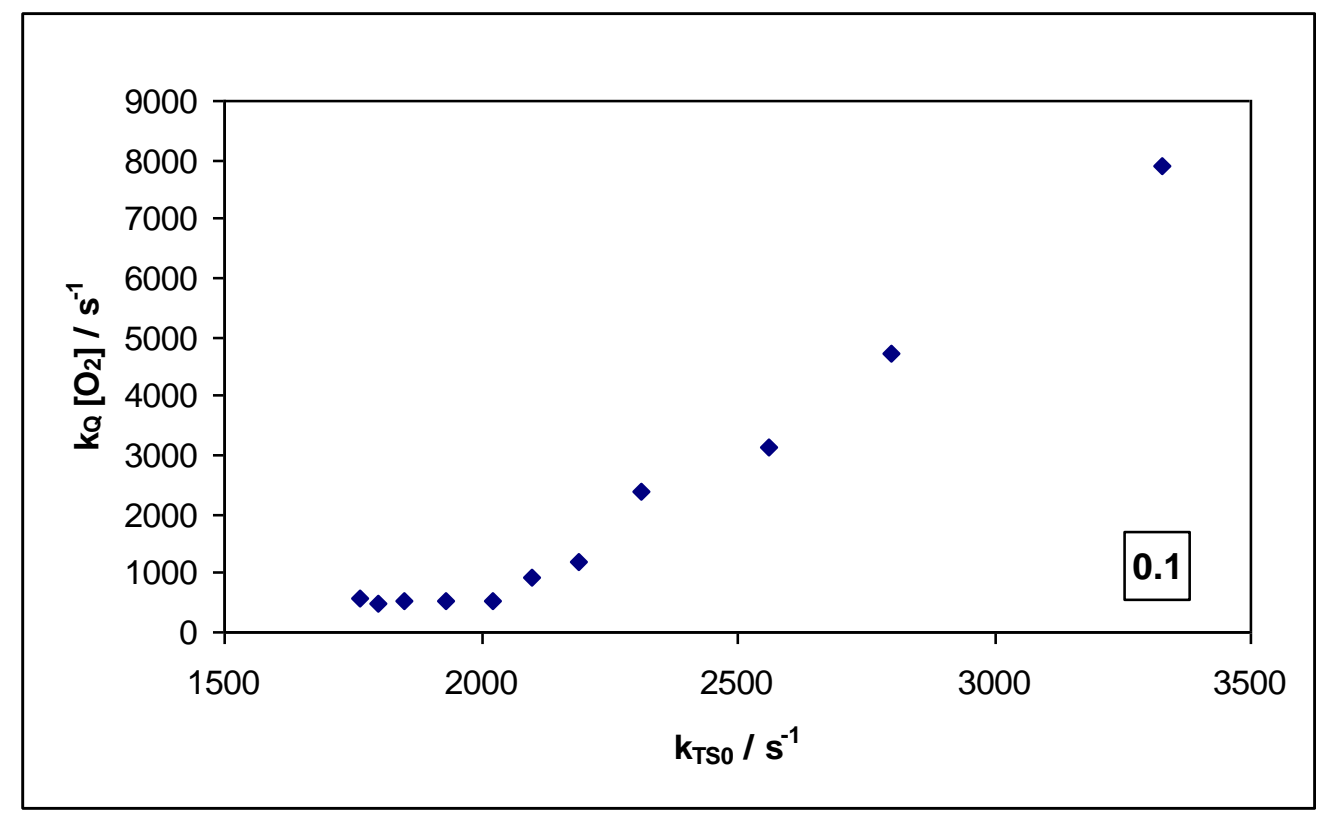

Figure V-14b

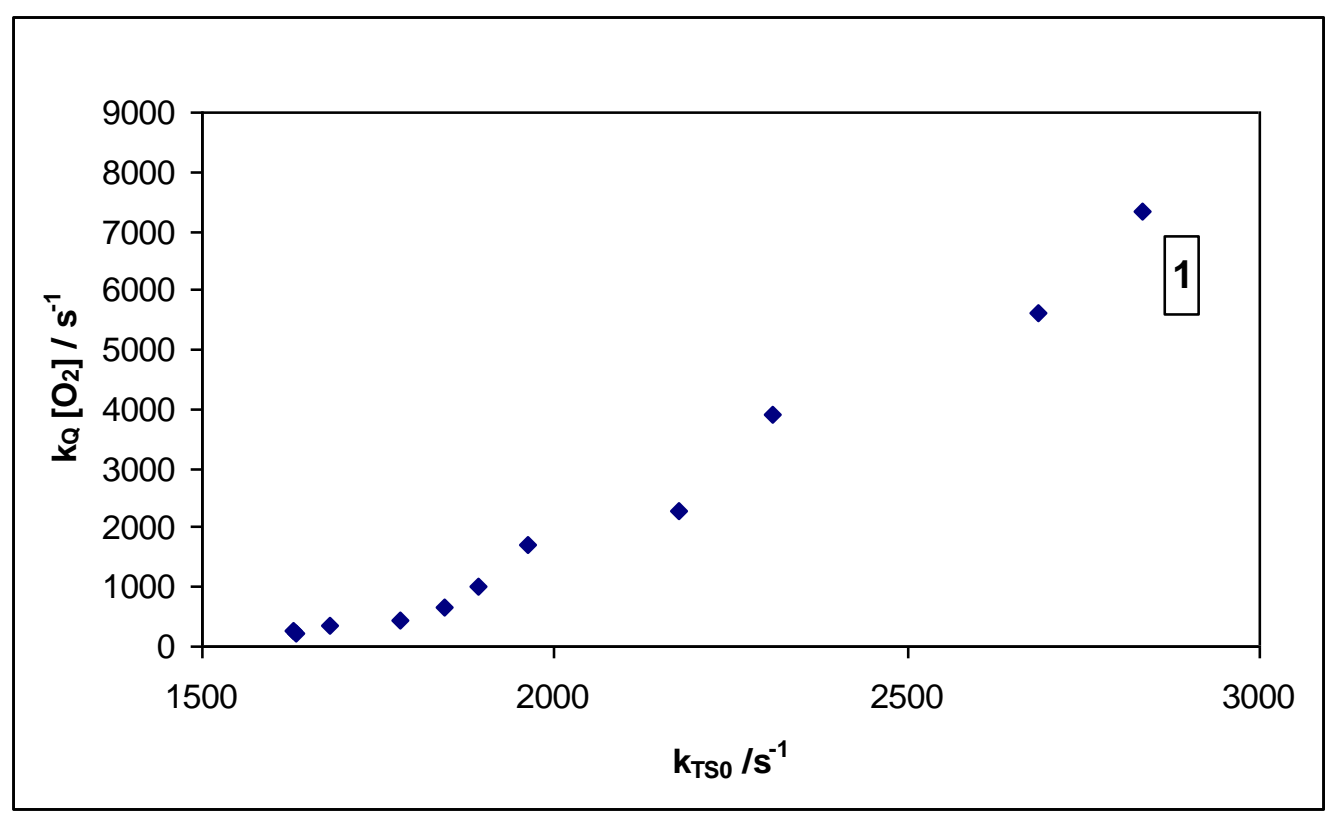


Figure $V-14 c$

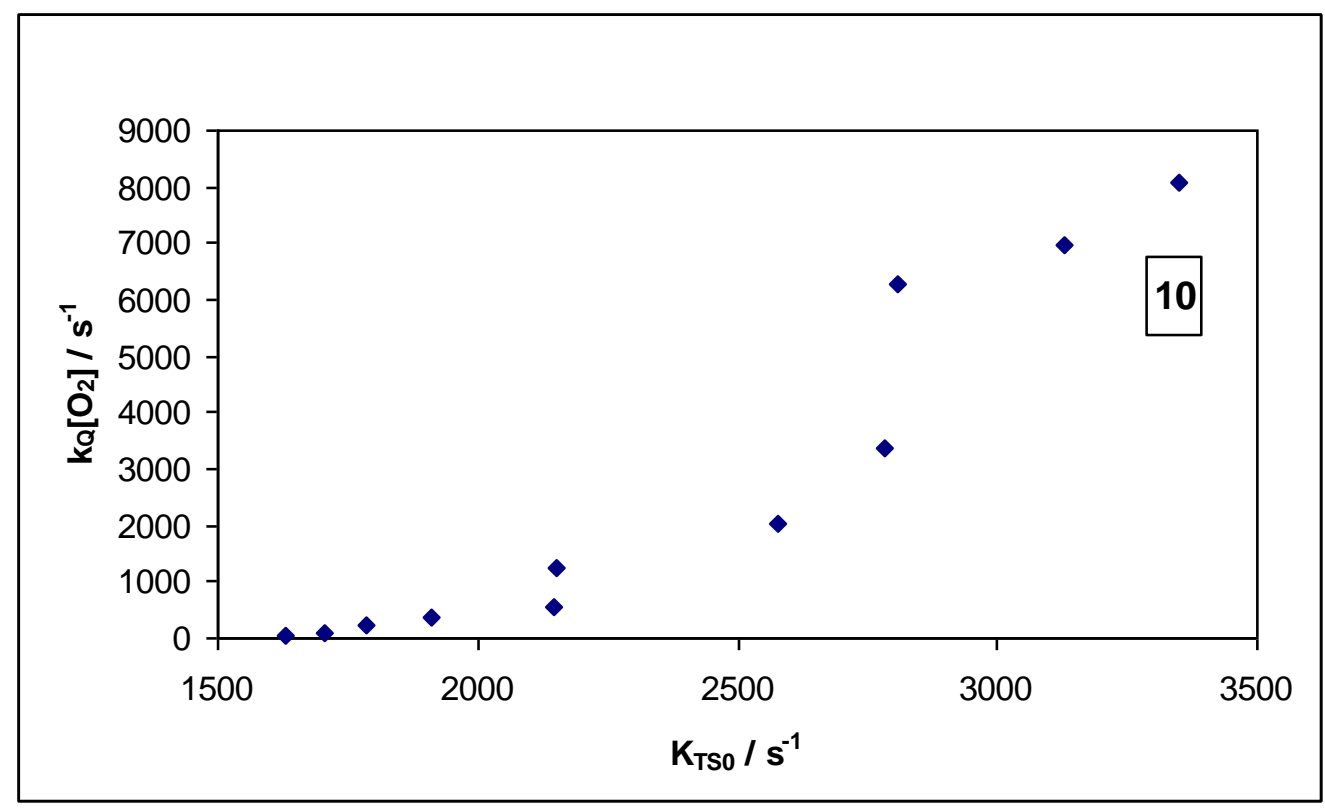

Figure $V-14 d$

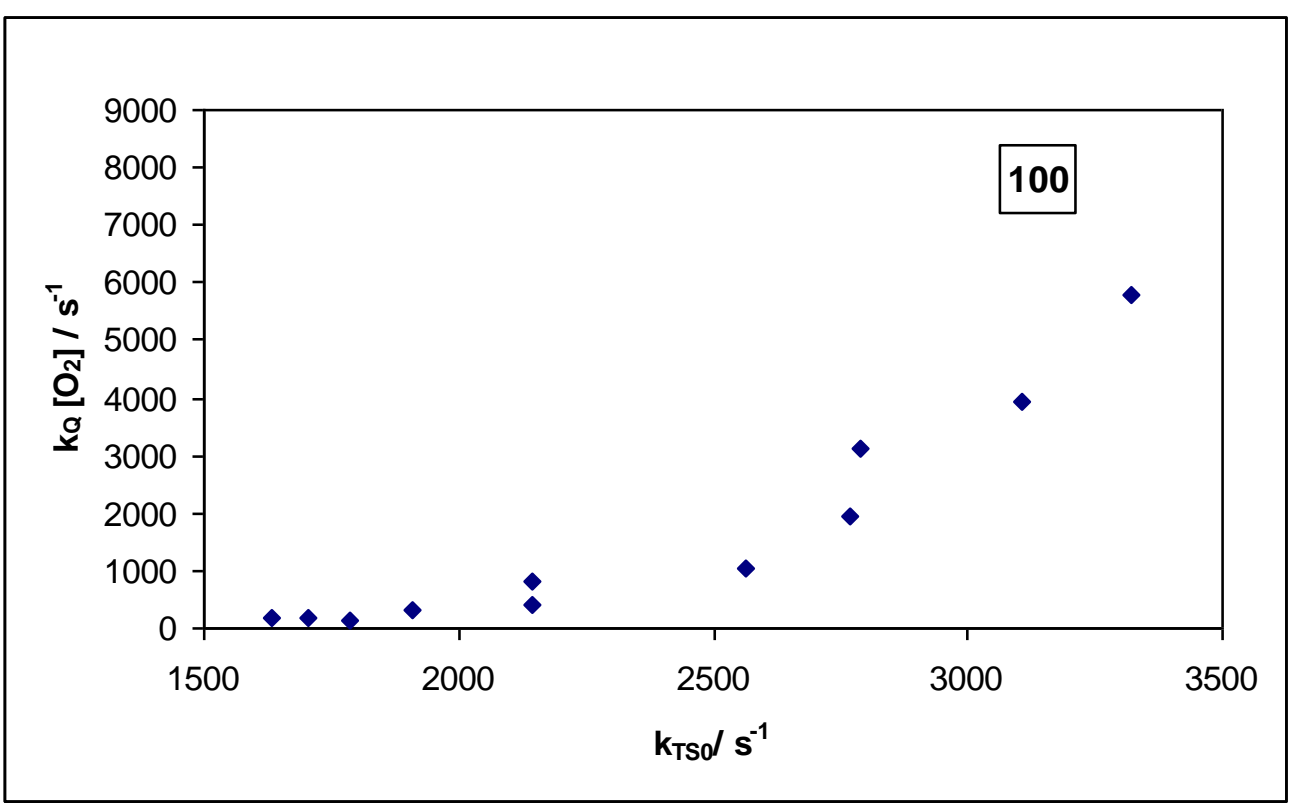

Figure V-14 a, b, c, d: Dependence of the oxygen quenching rate $\mathrm{k}_{\mathrm{Q}}\left[\mathrm{O}_{2}\right]$ on the rate of nonradiative quenching $\mathrm{k}_{\mathrm{TS} 0} \mathrm{In} \beta$-Lg glycerol films. The number in the text box refers to molar ratio of glycerol to $\beta$-Lg. 
Figure V-15a

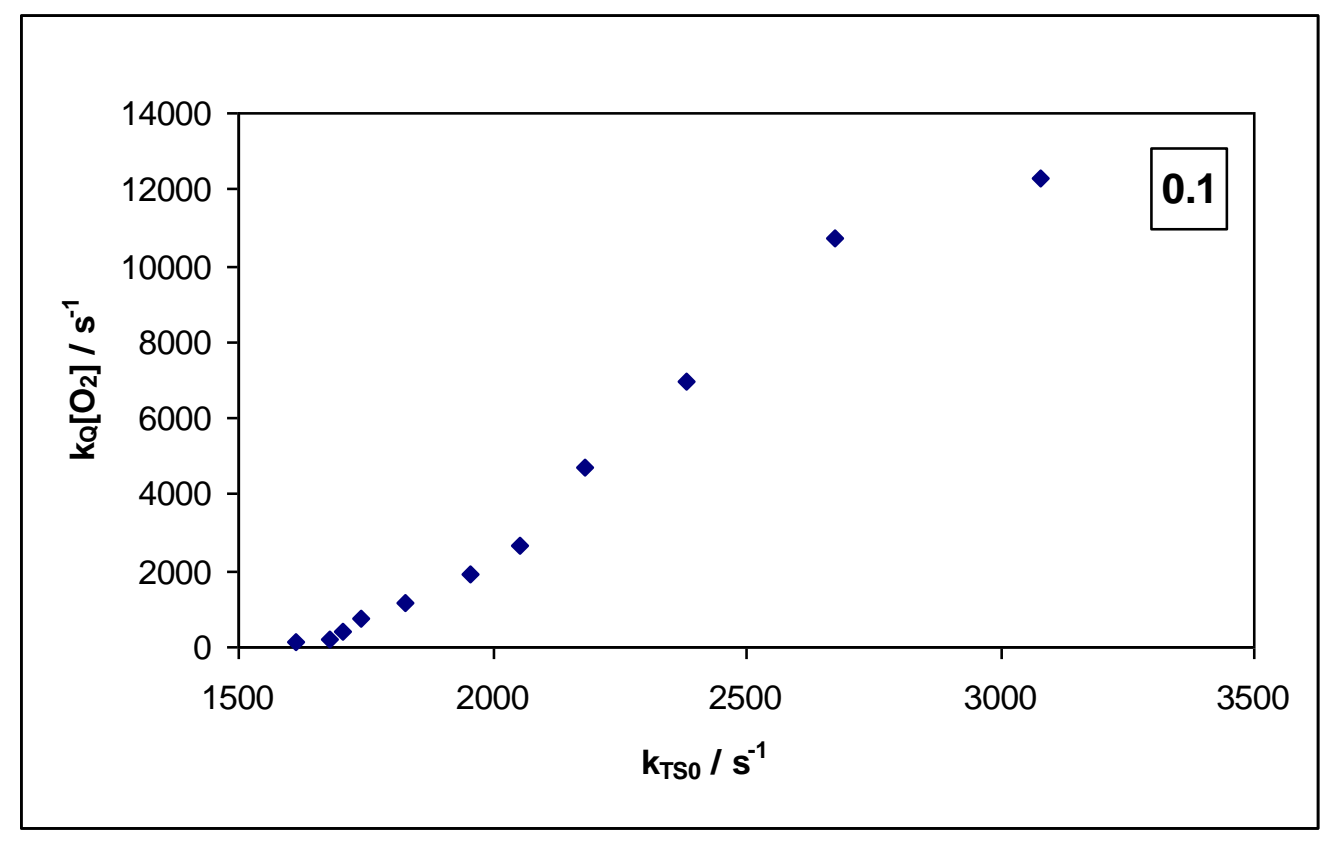

Figure V-15b

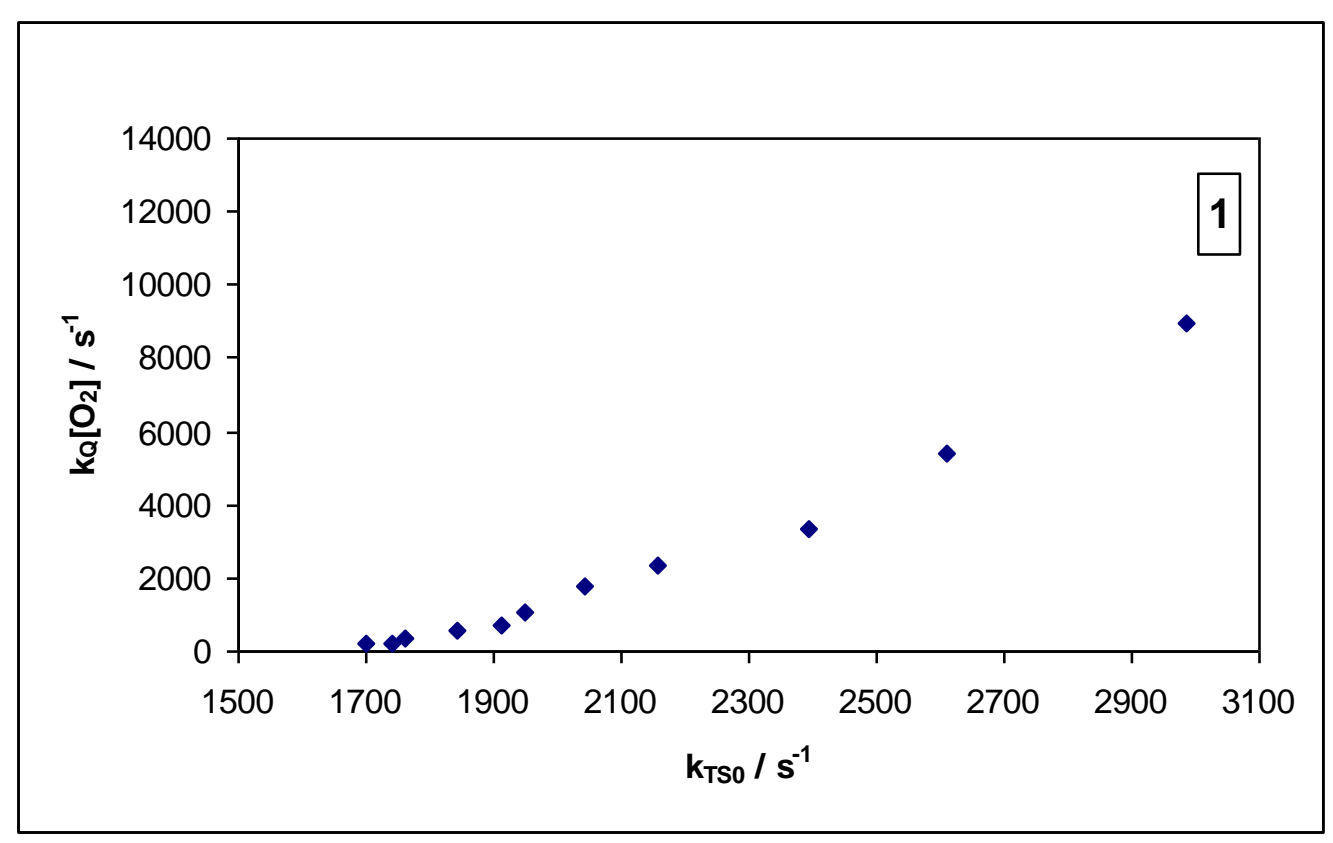


Figure V-15c

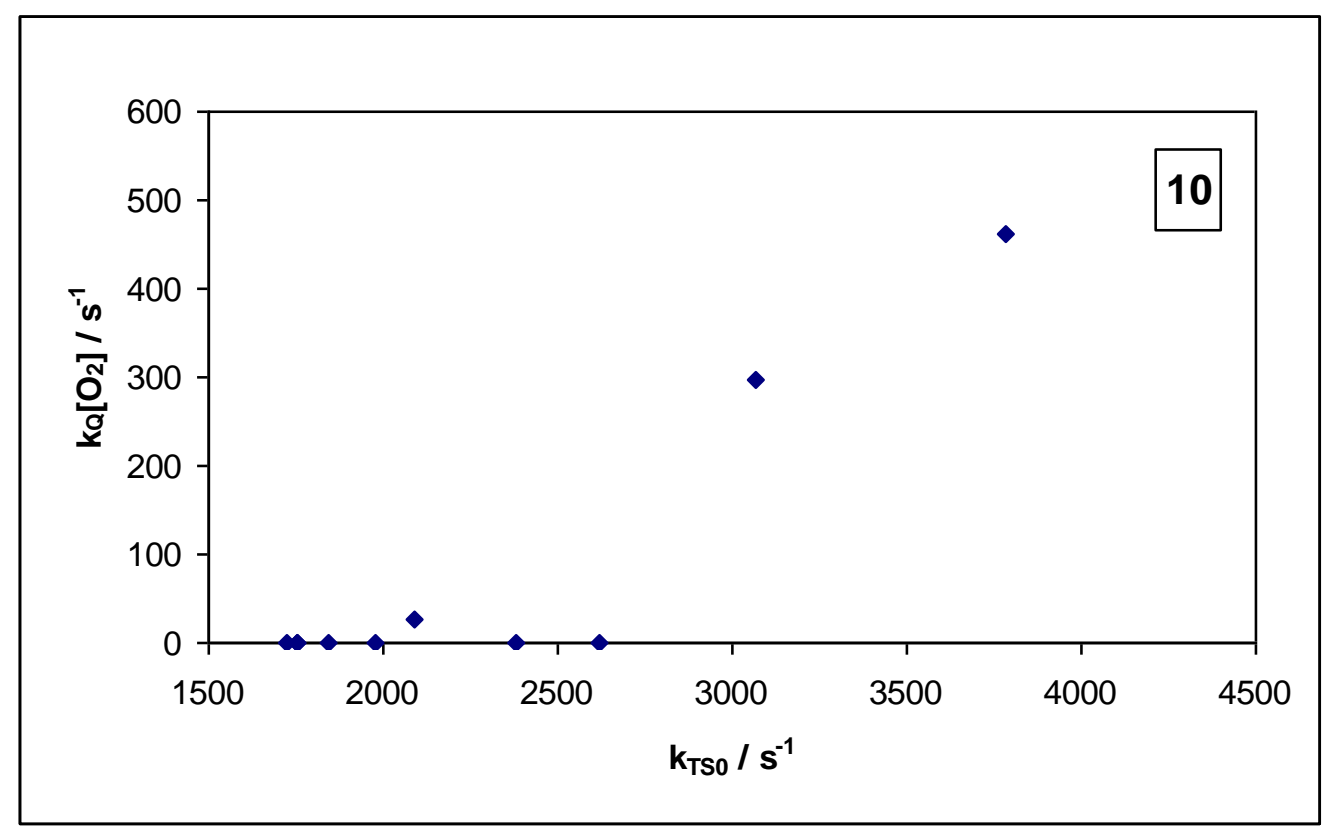

Figure V-15d

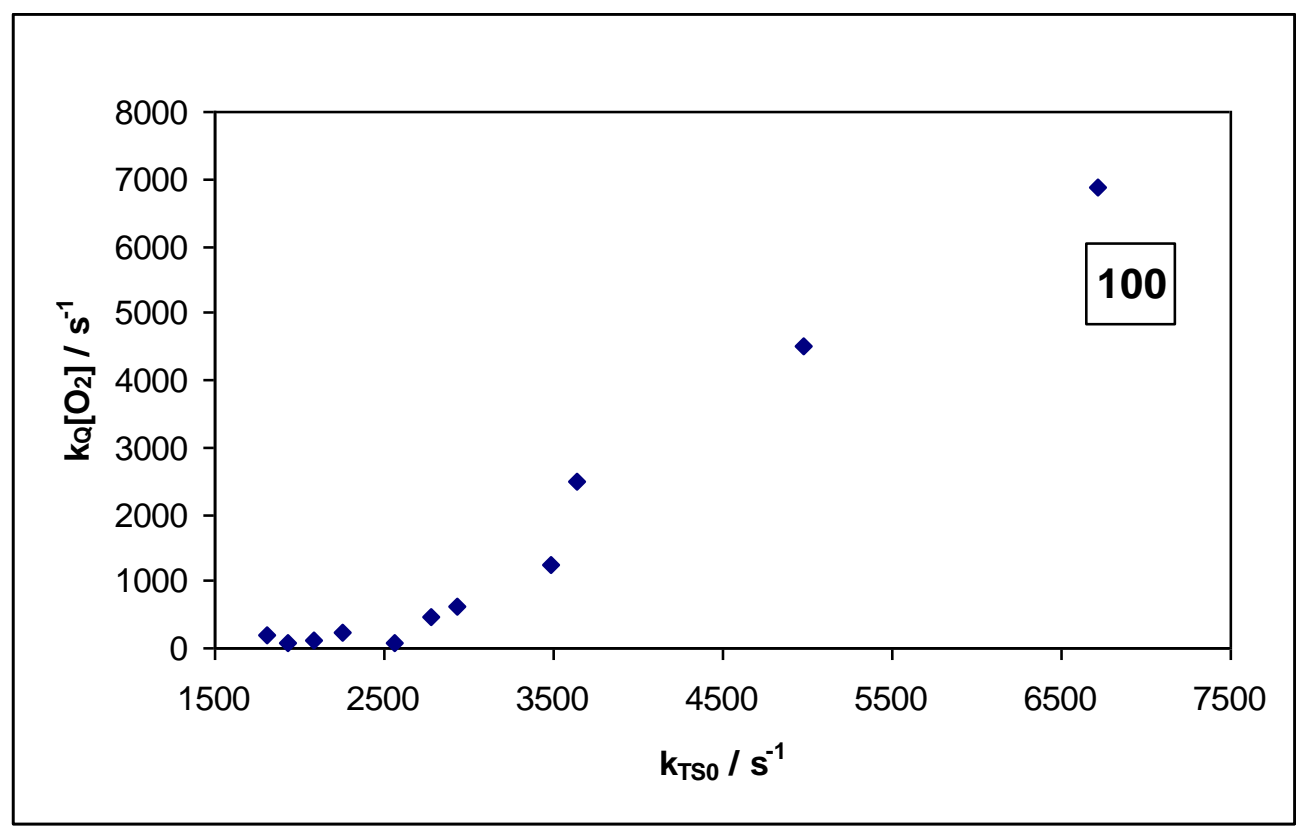




\section{Figure V-15e}

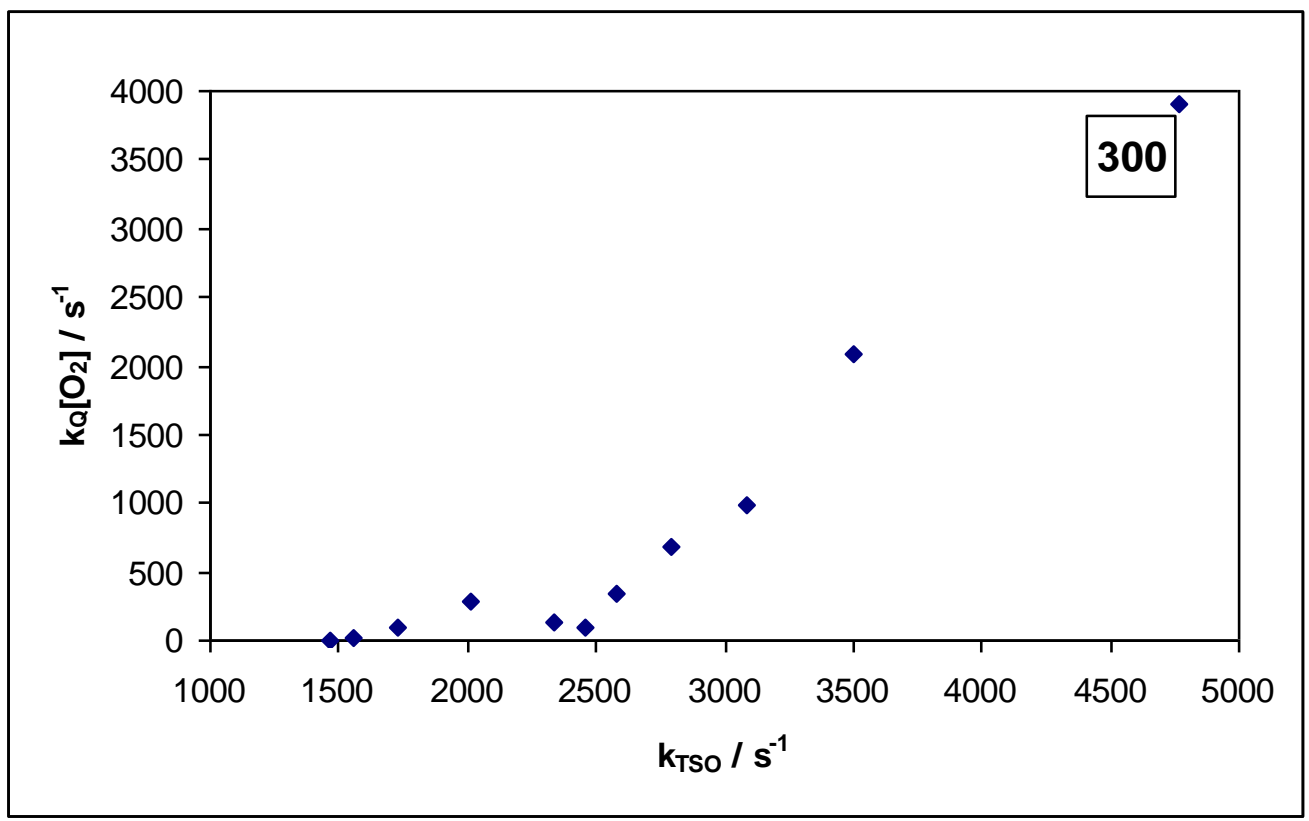

Figure $\mathrm{V}-15 \mathrm{a}, \mathrm{b}, \mathrm{c}, \mathrm{d}$, e: Dependence of the oxygen quenching rate $\mathrm{k}_{\mathrm{Q}}\left[\mathrm{O}_{2}\right]$ on the rate of nonradiative quenching $\mathrm{k}_{\mathrm{TS} 0}$ in sorbitol/ $\beta-\mathrm{Lg}$ films. The number in the text box refers to molar ratio of sorbitol to $\beta$ - $\mathrm{Lg}$.

Figure V-16a

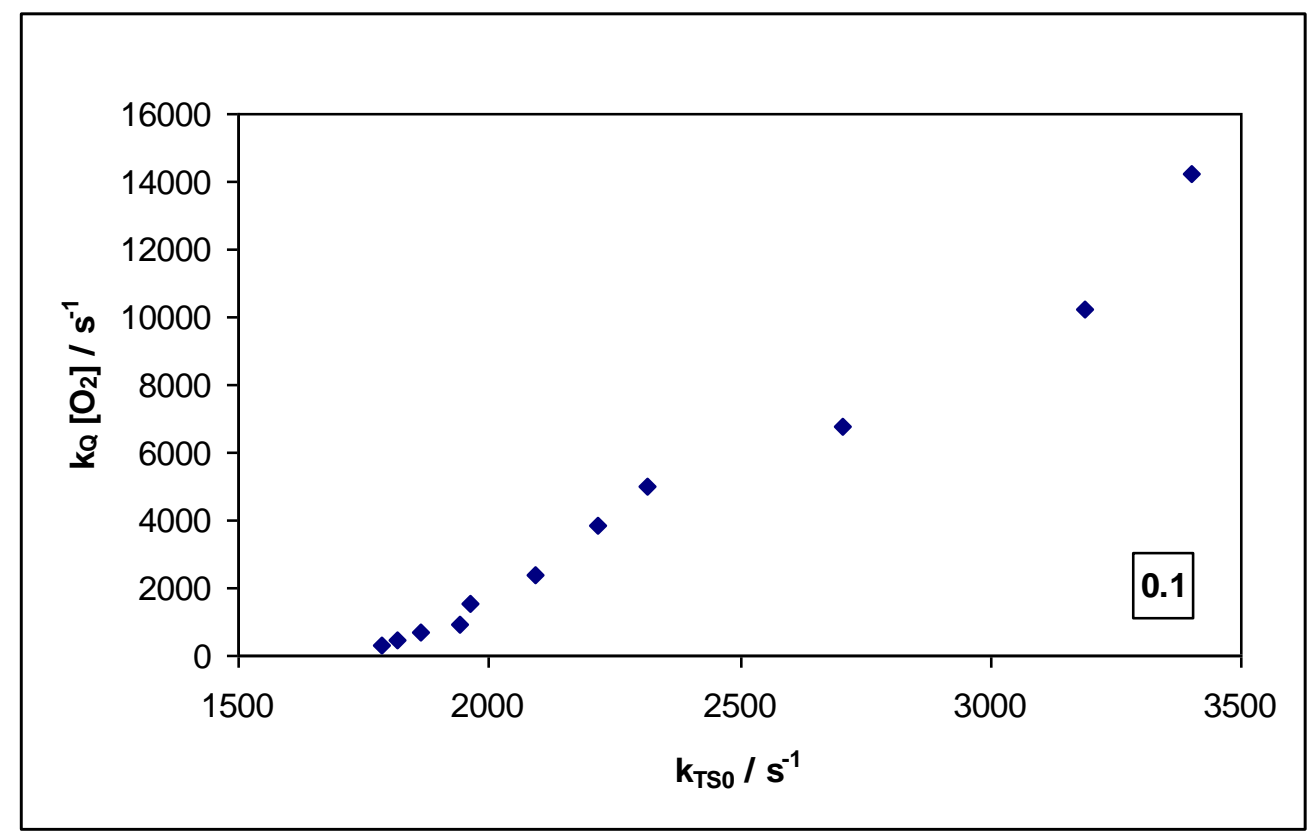


Figure V-16b

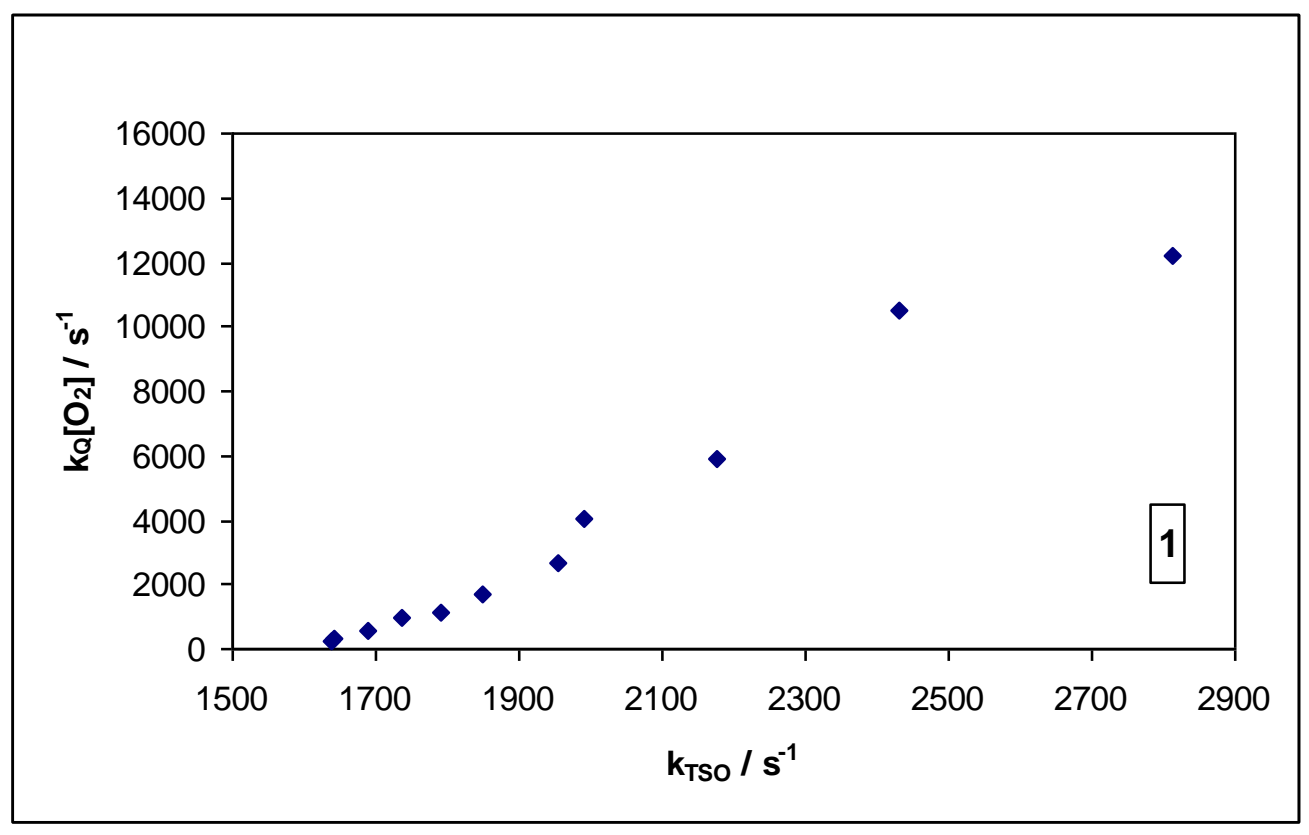

Figure V-16c

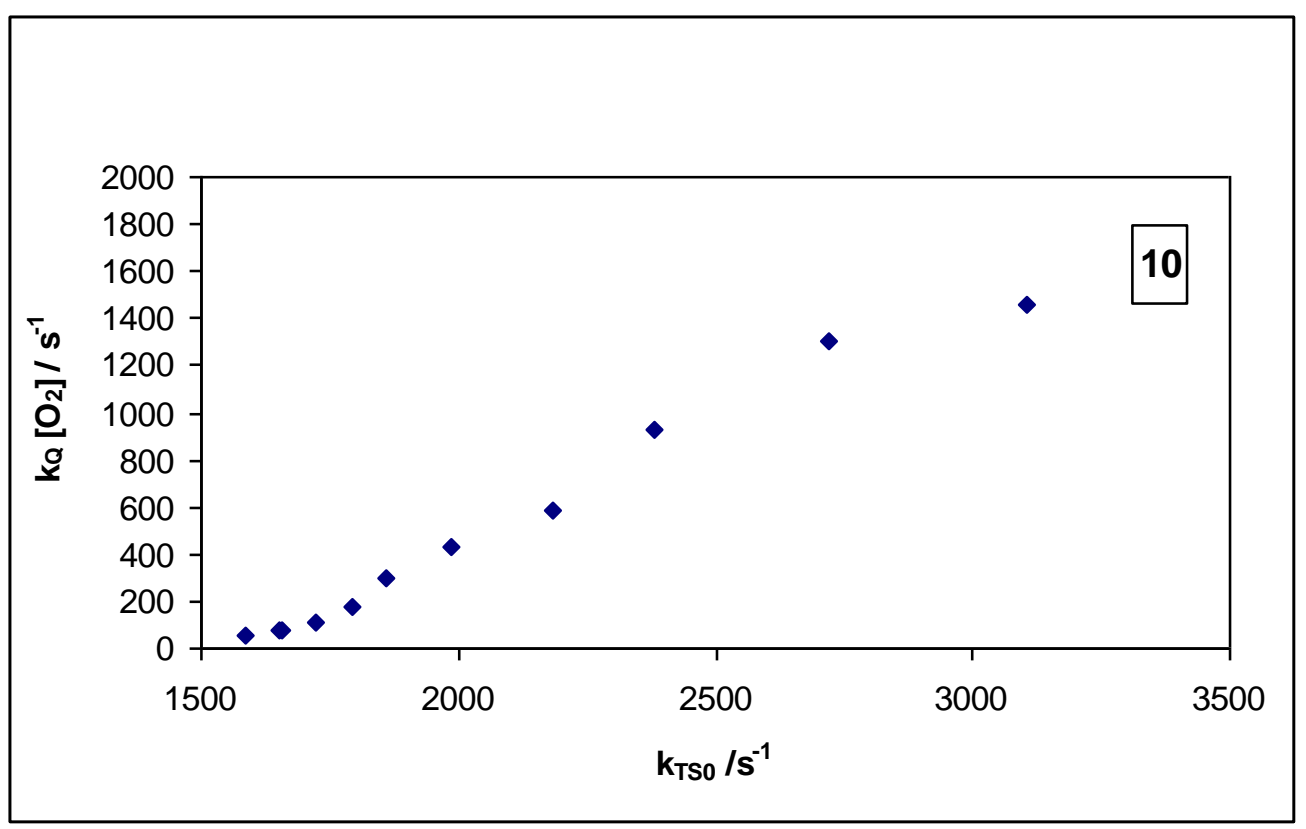




\section{Figure V-16d}

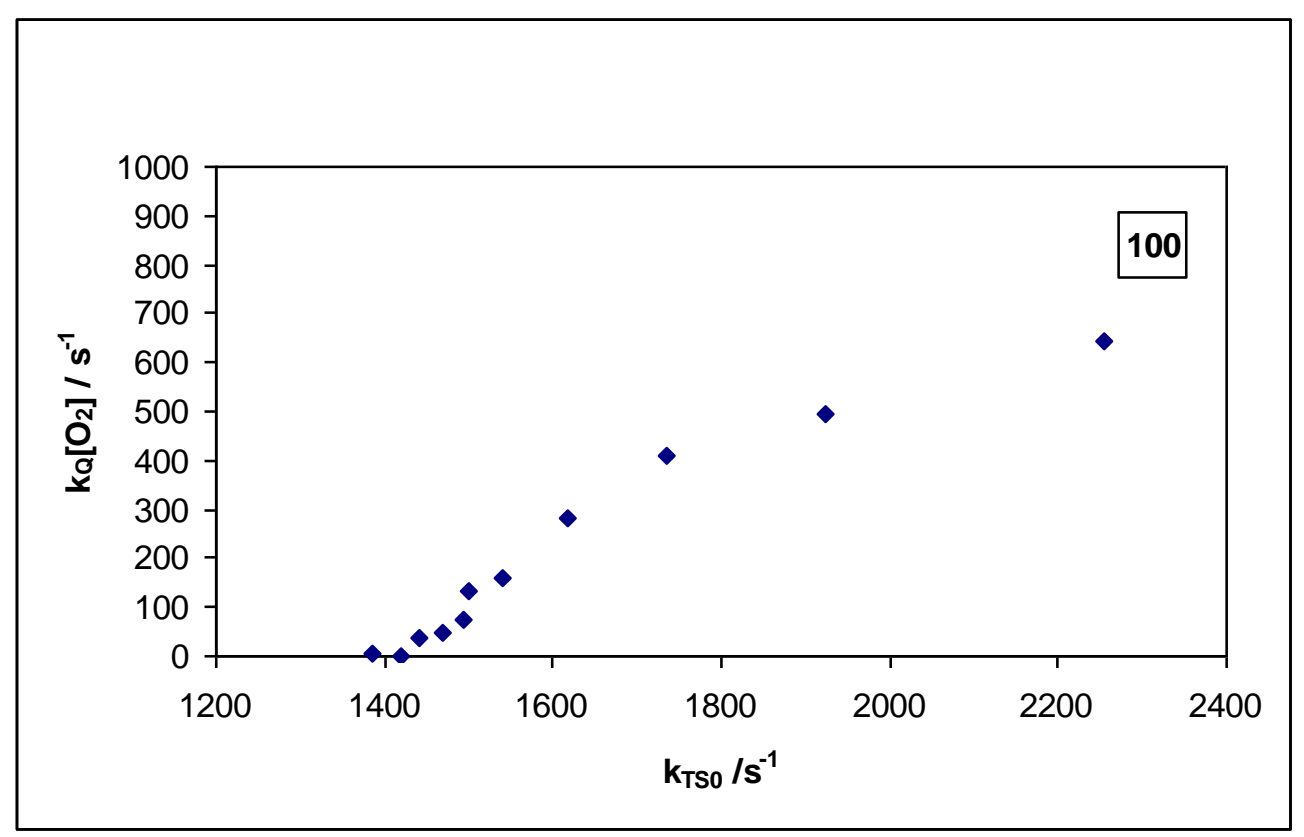

Figure V-16e

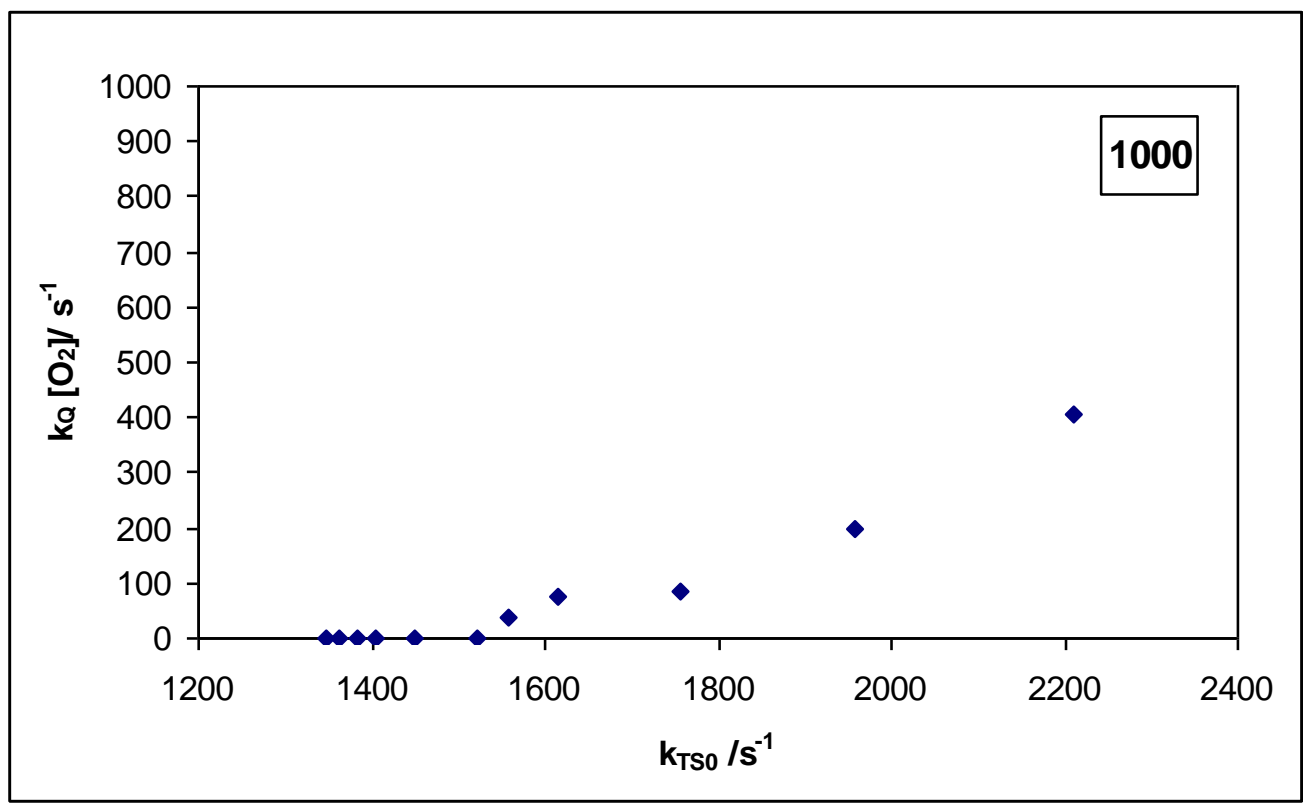

Figure V-16 a, b, c, d, e: Dependence of the oxygen quenching rate $\mathrm{k}_{\mathrm{Q}}\left[\mathrm{O}_{2}\right]$ on the rate of nonradiative quenching $\mathrm{k}_{\mathrm{TS} 0}$ in maltitol/ $\beta$-Lg films. The number in the text box refers to molar ratio of maltitol to $\beta$ - $\mathrm{Lg}$. 
Figure V-17a

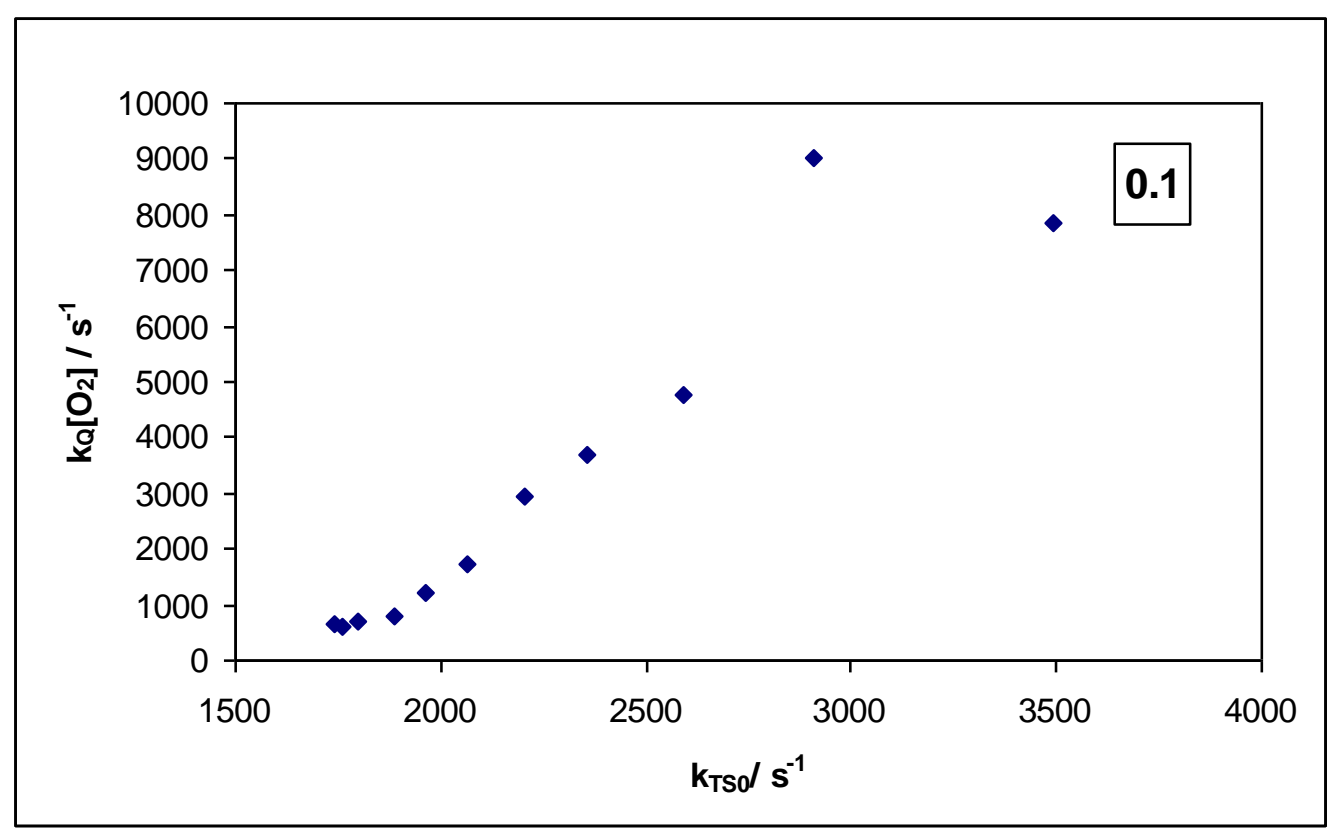

Figure V-17b

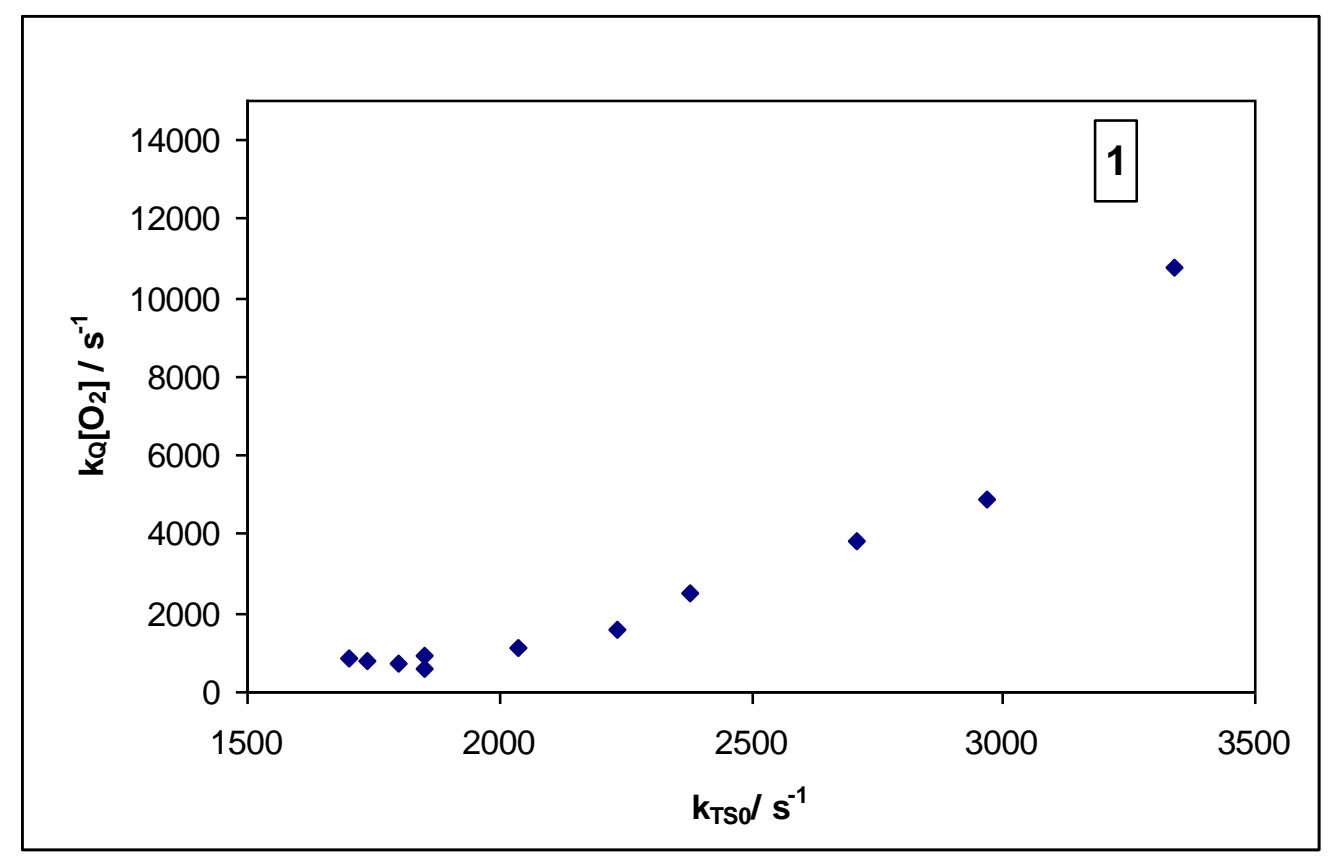


Figure $V-17 c$

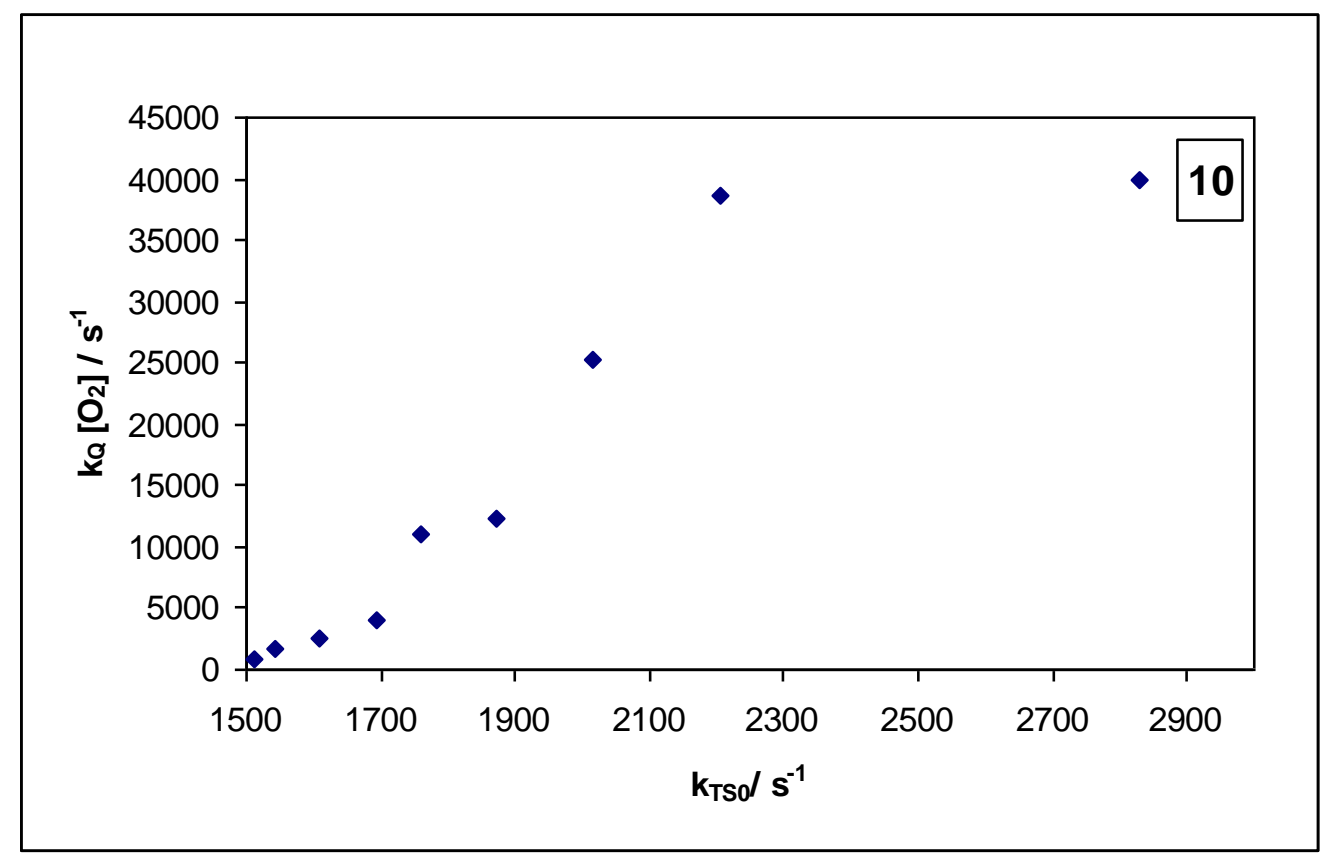

Figure V-17a, b, c: Dependence of the oxygen quenching rate $\mathrm{k}_{\mathrm{Q}}\left[\mathrm{O}_{2}\right]$ on the rate of nonradiative quenching $\mathrm{k}_{\mathrm{TS} 0}$ in $\mathrm{PEG} / \beta$-Lg films. The number in the text box refers to molar ratio of PEG 400 to $\beta$ - Lg. 
Figure $V-18 a$

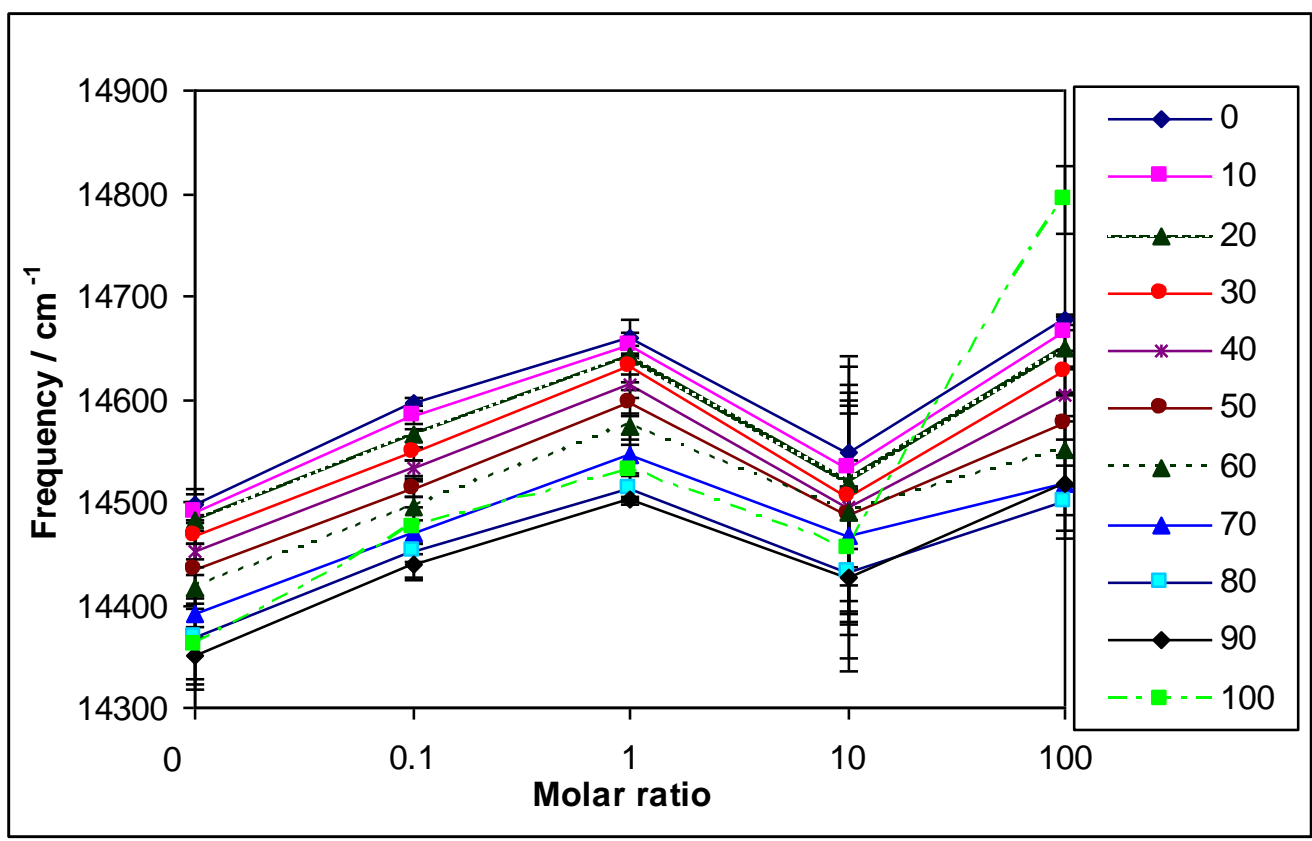

Figure V-18a: Emission energy as a function of glycerol concentration at various temperatures in $\beta$ Lg films. Data replotted from Figure V-2a.

Figure $V-18 b$

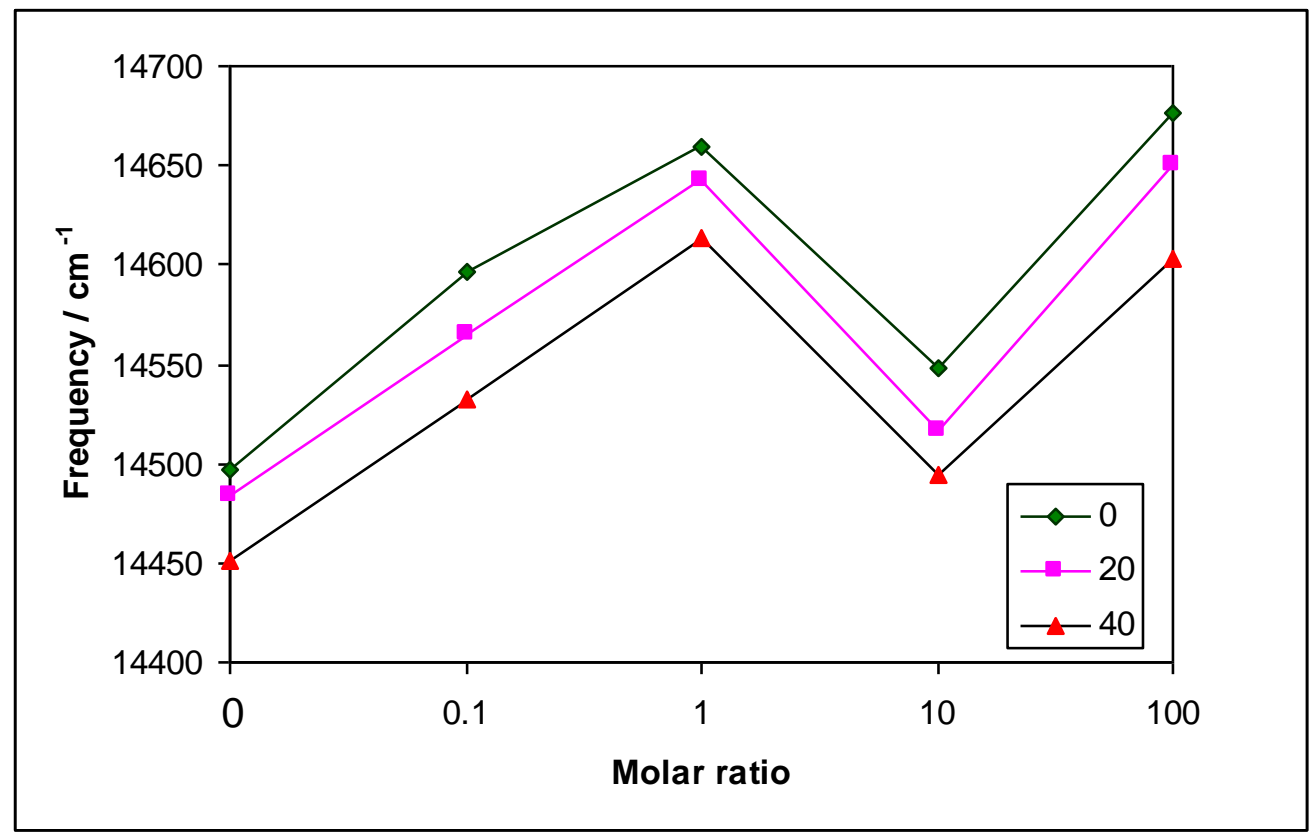

FigureV-18b: Zoom in plot of emission energy as a function of glycerol concentration at various temperatures in $\beta$-Lg films. Data replotted from Figure V-2a. 
Figure V-19a

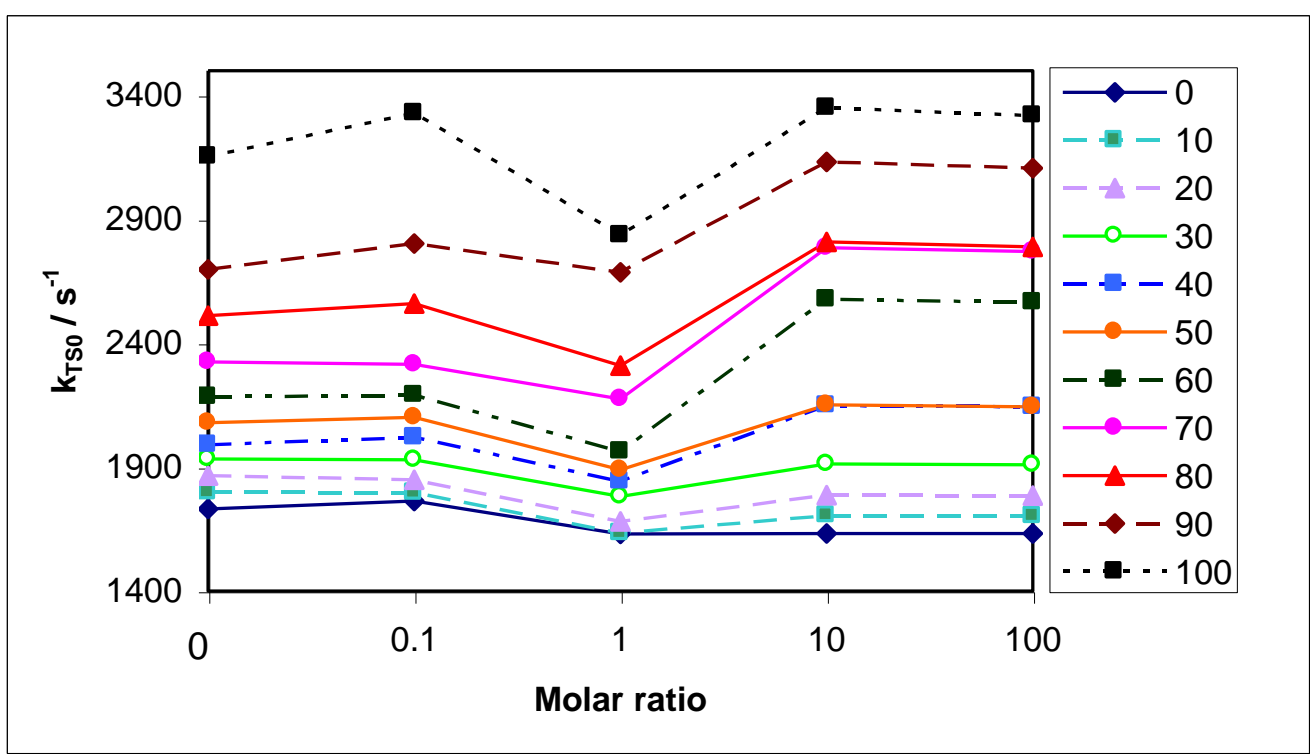

Figure V-19a: $\mathrm{k}_{\mathrm{Ts} 0}$ as a function of glycerol concentration at various temperatures in $\beta$-Lg films. Data replotted from Figure V-13a.

Figure $V-19 b$

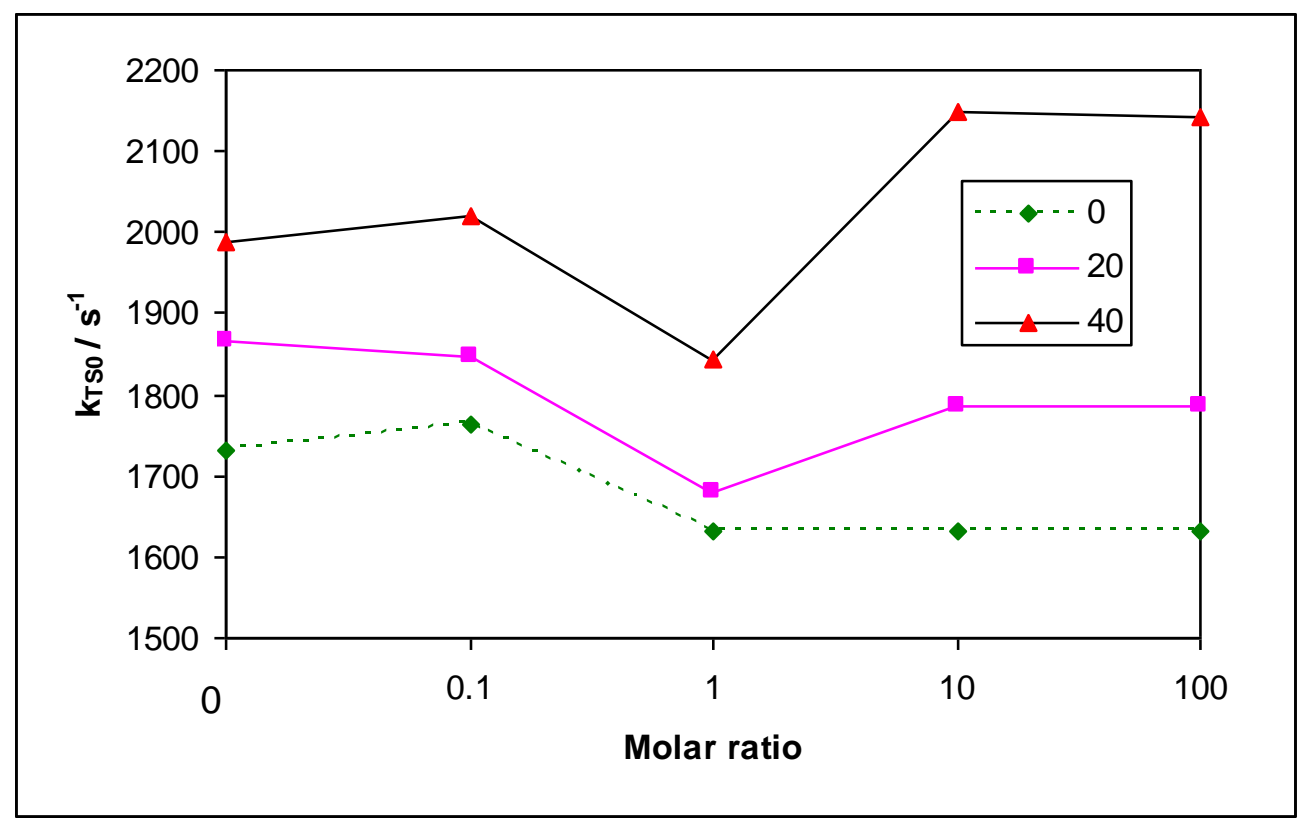

Figure V-19b: Zoom in plot of $\mathrm{k}_{\mathrm{TS} 0}$ as a function of glycerol concentration at various temperatures in $\beta$-Lg films. Data replotted from Figure V-13a. 
Figure V-20a

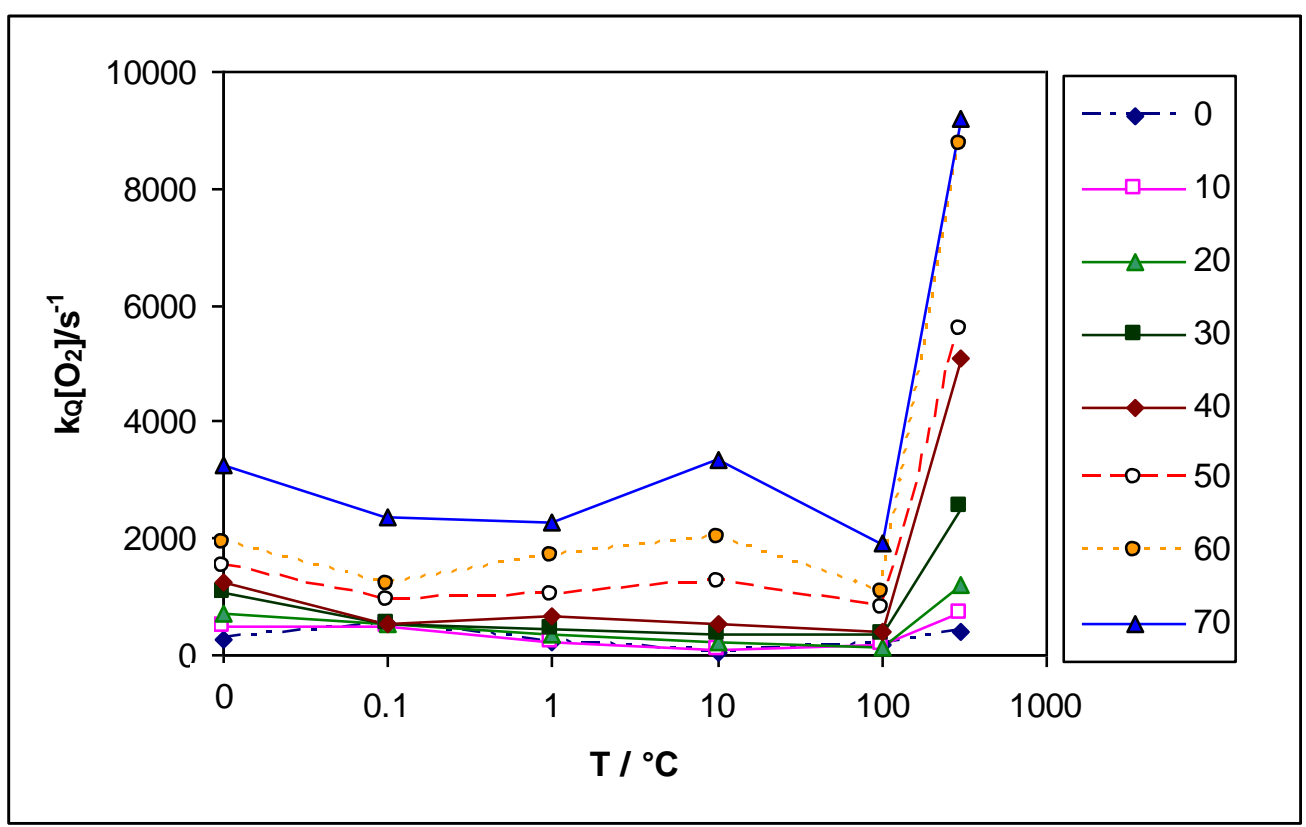

Figure V-20a: $\mathrm{k}_{\mathrm{Q}}\left[\mathrm{O}_{2}\right]$ as a function of glycerol concentration at various temperatures in $\beta$-Lg films. Data replotted from Figure V-12a.

Figure V-20b

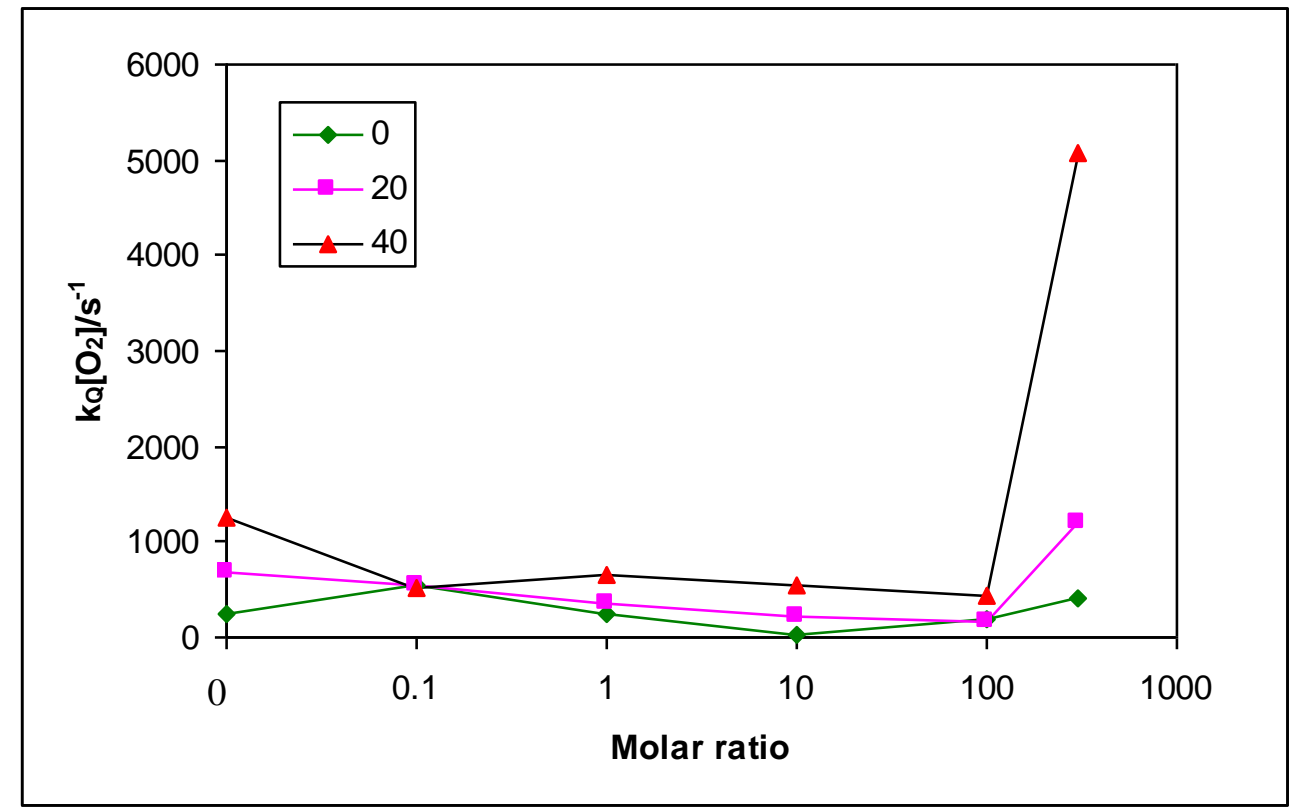

Figure V-20b: Zoom in plot of $\mathrm{k}_{\mathrm{Q}}\left[\mathrm{O}_{2}\right]$ as a function of glycerol concentration at various temperatures in $\beta$-Lg films. Data replotted from Figure V-12a. 
Figure V-21a

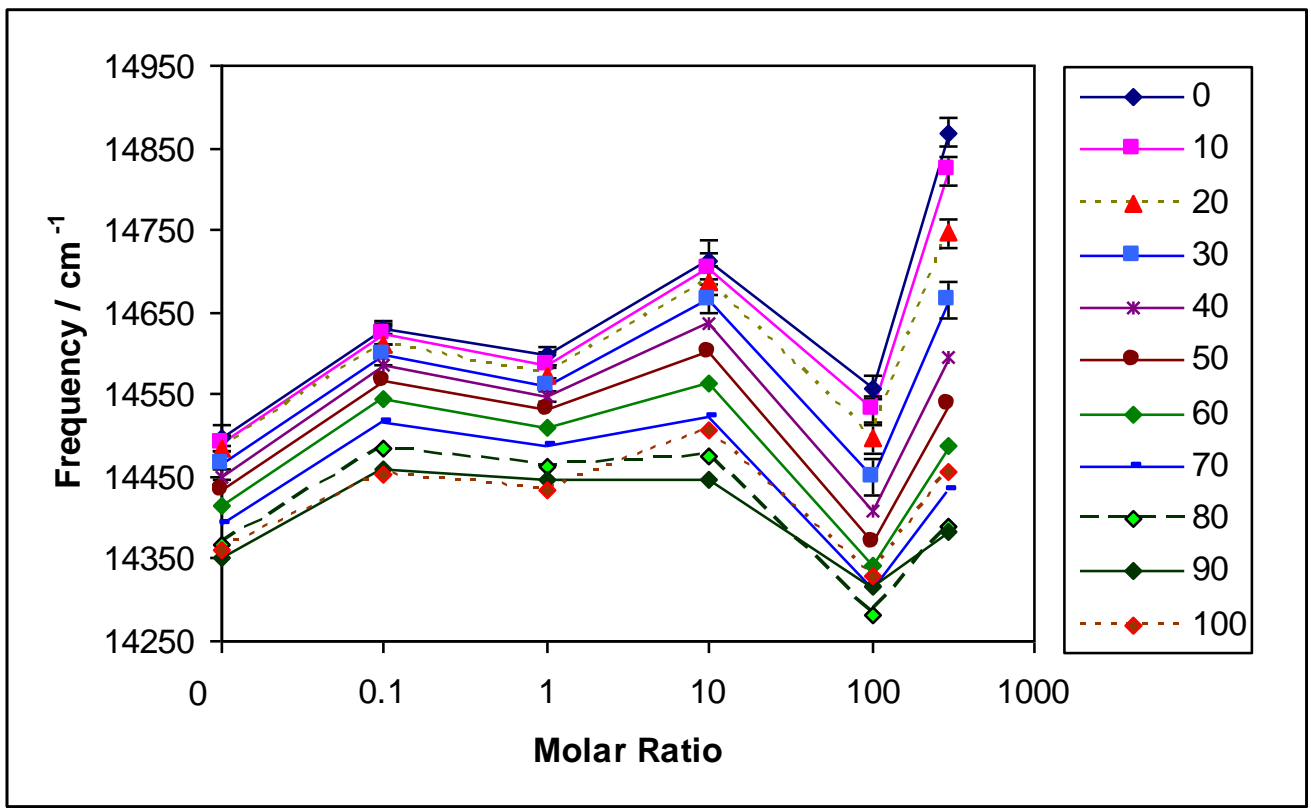

Figure V-21a: Emission energy as a function of concentration of sorbitol at various temperatures in $\beta$-Lg films. Data replotted from Figure V-2b.

Figure V-21b

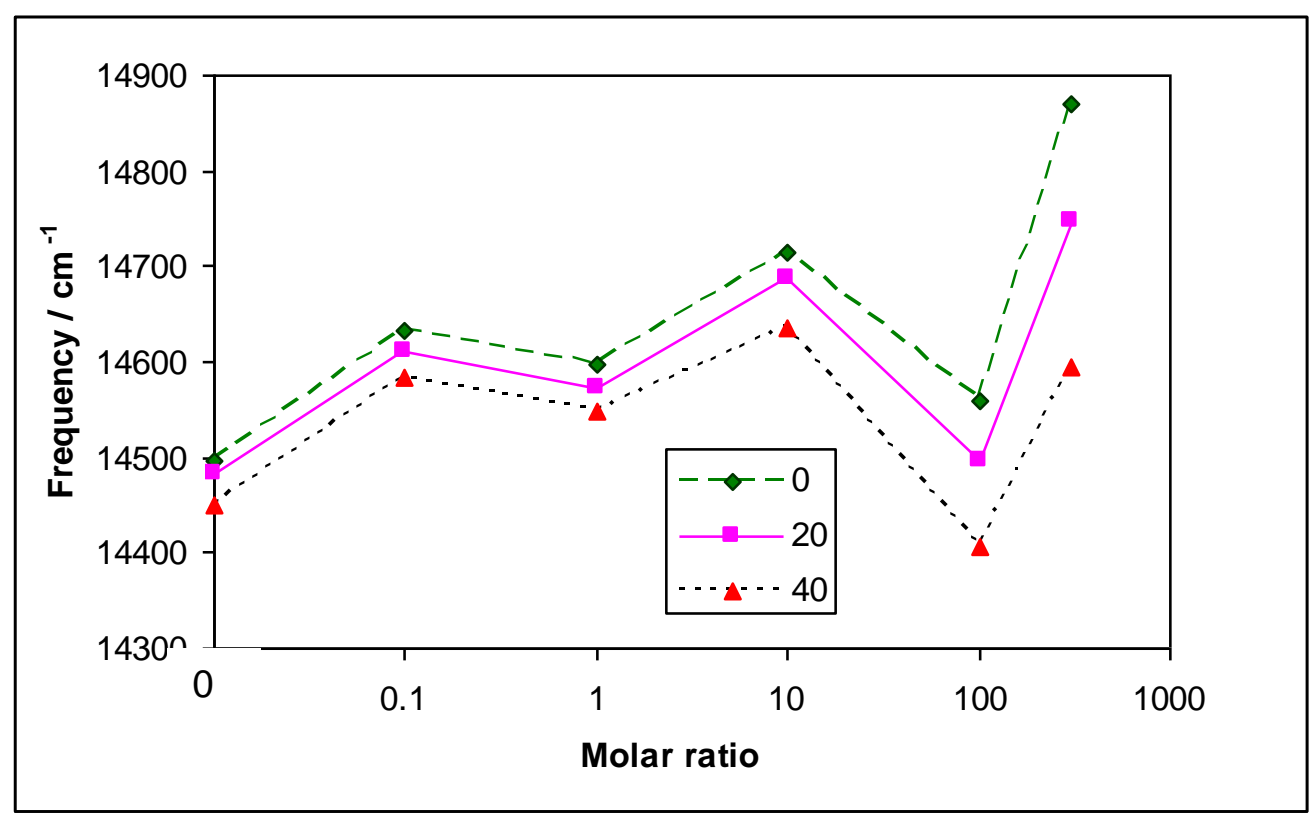

Figure V-21b: Zoom in plot of emission energy as a function of sorbitol concentration at various temperatures in $\beta$-Lg films. Data replotted from Figure V-2b. 


\section{Figure V-22a}

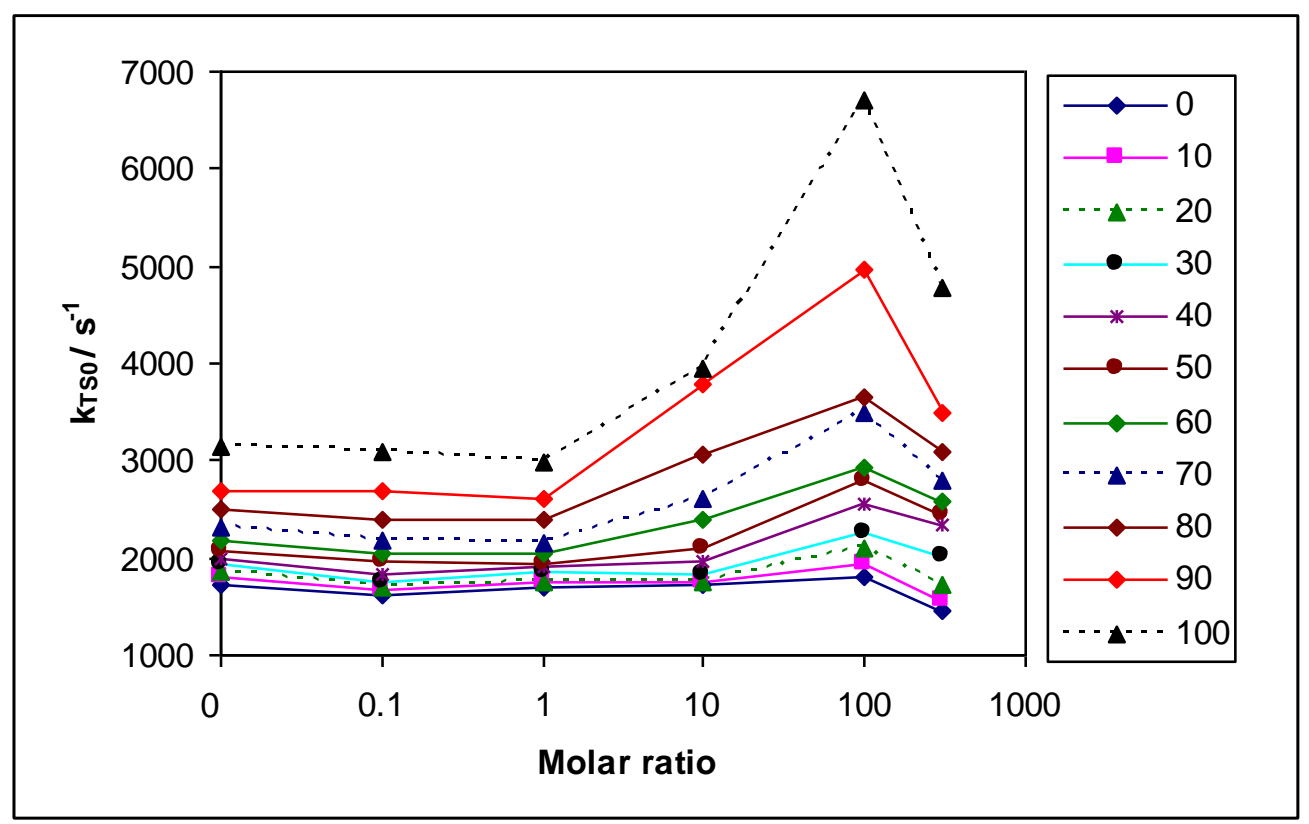

Figure V-22a: A plot of $\mathrm{k}_{\mathrm{TS} 0}$ as a function of sorbitol concentration at various temperatures in $\beta$ - $\mathrm{Lg}$ films. Data replotted from Figure V-13b.

Figure V-22b

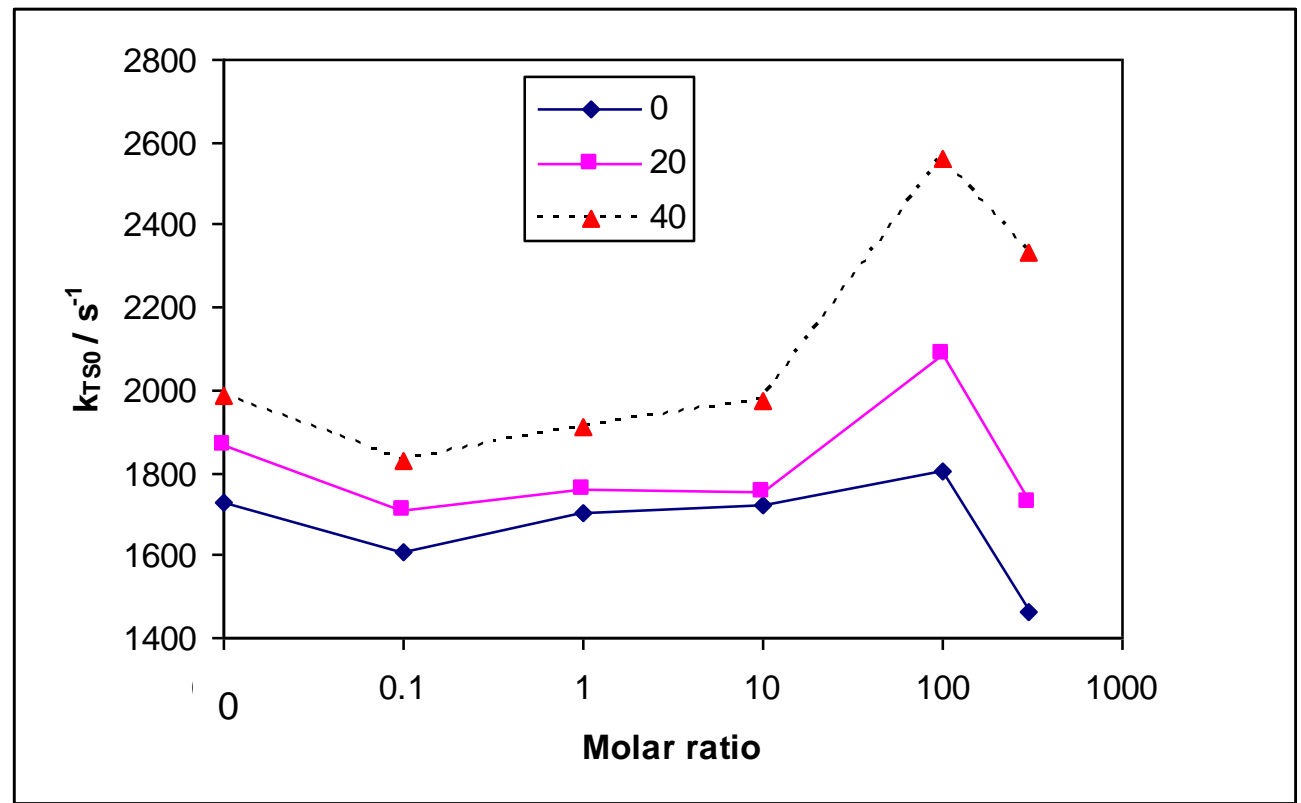

Figure V-22b: Zoom in plot of $\mathrm{k}_{\mathrm{TS} 0}$ as a function of sorbitol concentration at various temperatures in $\beta$-Lg films. Data replotted from Figure V-13b. 


\section{Figure V-23a}

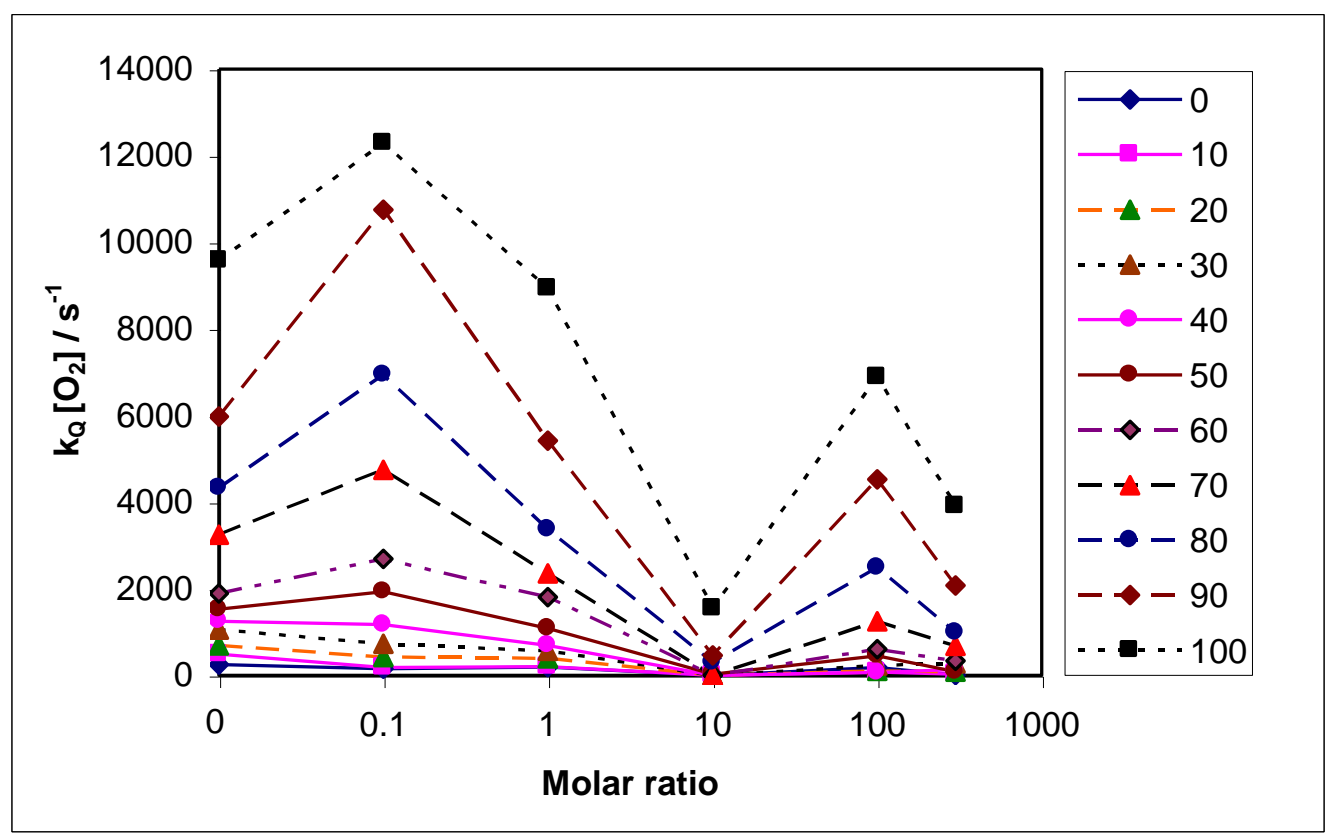

Figure V-23a: $\mathrm{k}_{\mathrm{Q}}\left[\mathrm{O}_{2}\right]$ as a function of sorbitol concentration at various temperatures in $\beta$ - $\mathrm{Lg}$ films. Data replotted from Figure $\mathrm{V}-12 \mathrm{~b}$.

Figure V-23b

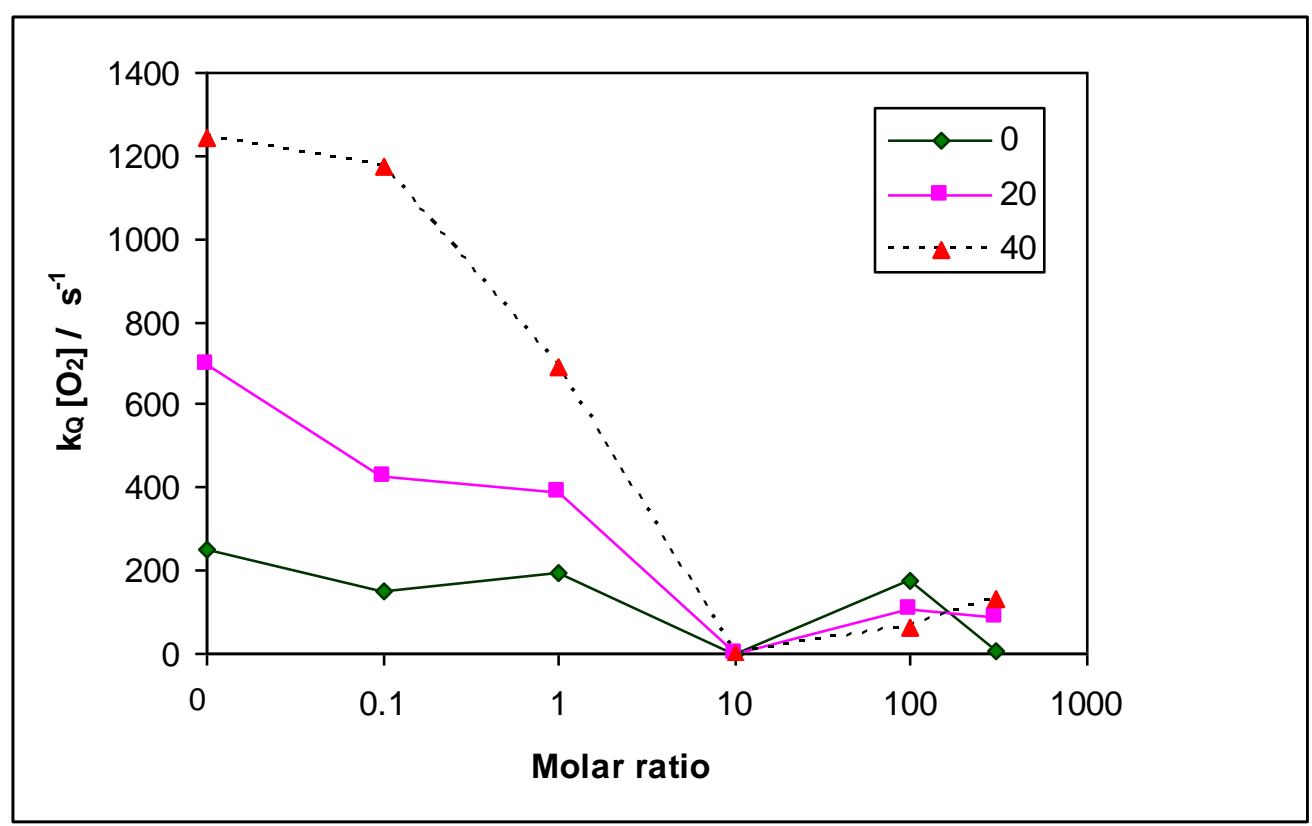

Figure V-23b: Zoom in plot of $\mathrm{k}_{\mathrm{Q}}\left[\mathrm{O}_{2}\right]$ as a function of sorbitol concentration at various temperatures in $\beta$-Lg films. Data replotted from Figure V-12b. 


\section{Figure V-24a}

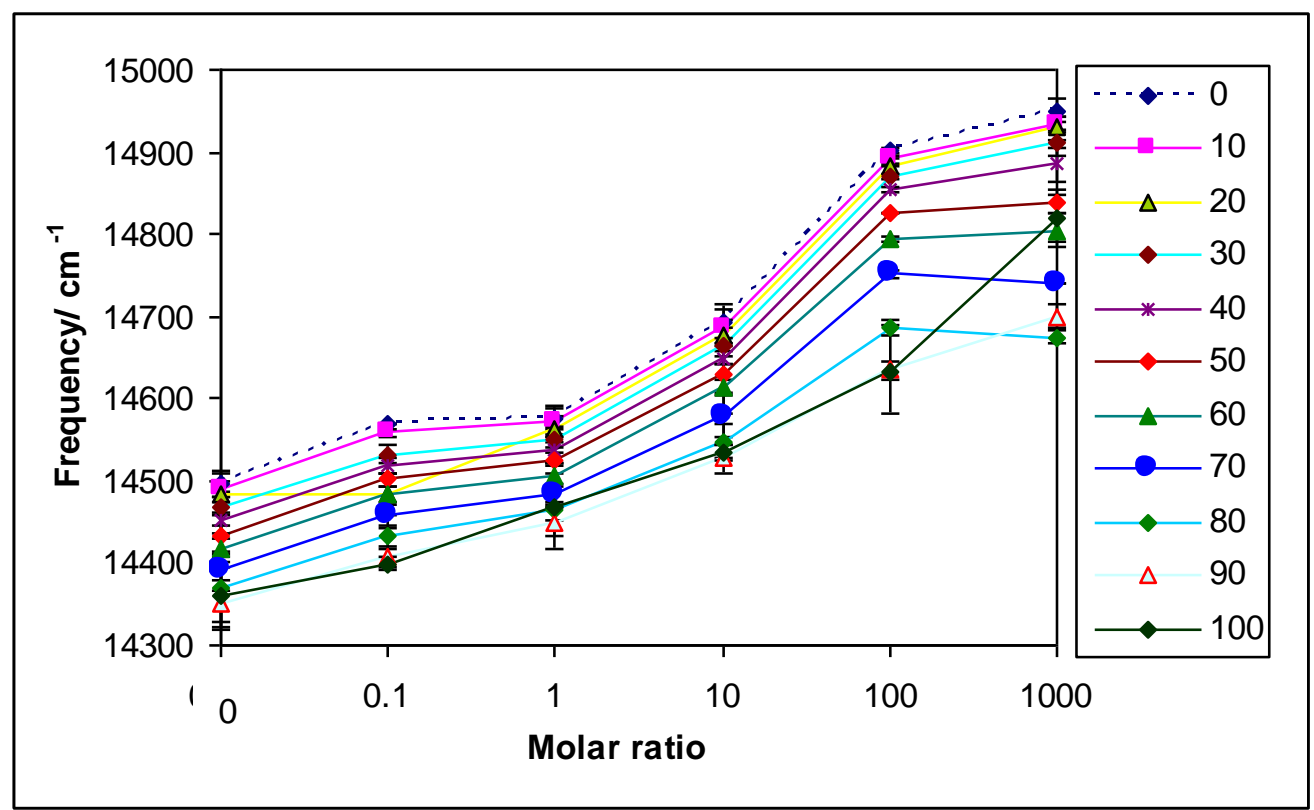

Figure V-24a: Emission energy as a function of maltitol concentration at various temperatures in $\beta$ Lg films. Data replotted from Figure V-2c.

Figure $V-24 b$

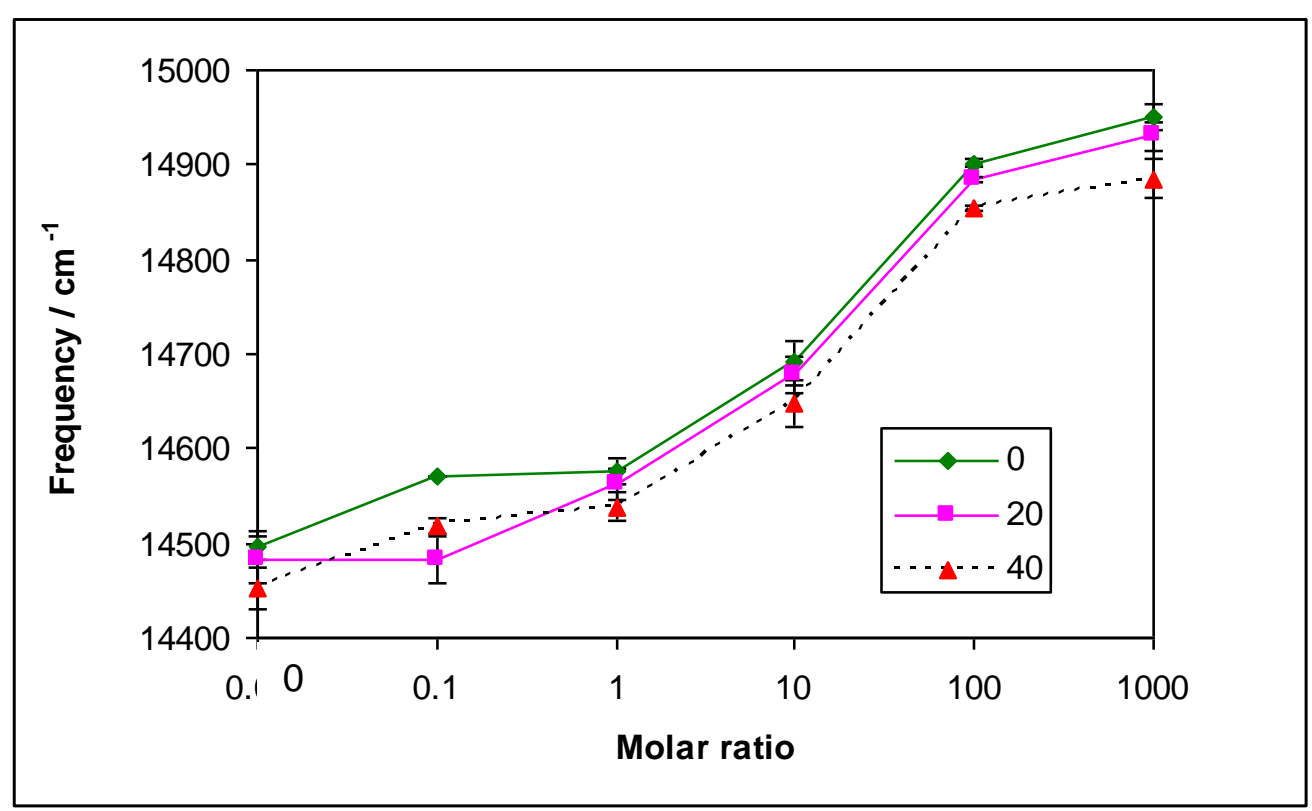

Figure V-24b: Zoom in plot of emission energy as a function of maltitol concentration at various temperatures in $\beta$-Lg films. Data replotted from Figure V-2c. 
Figure V-25a

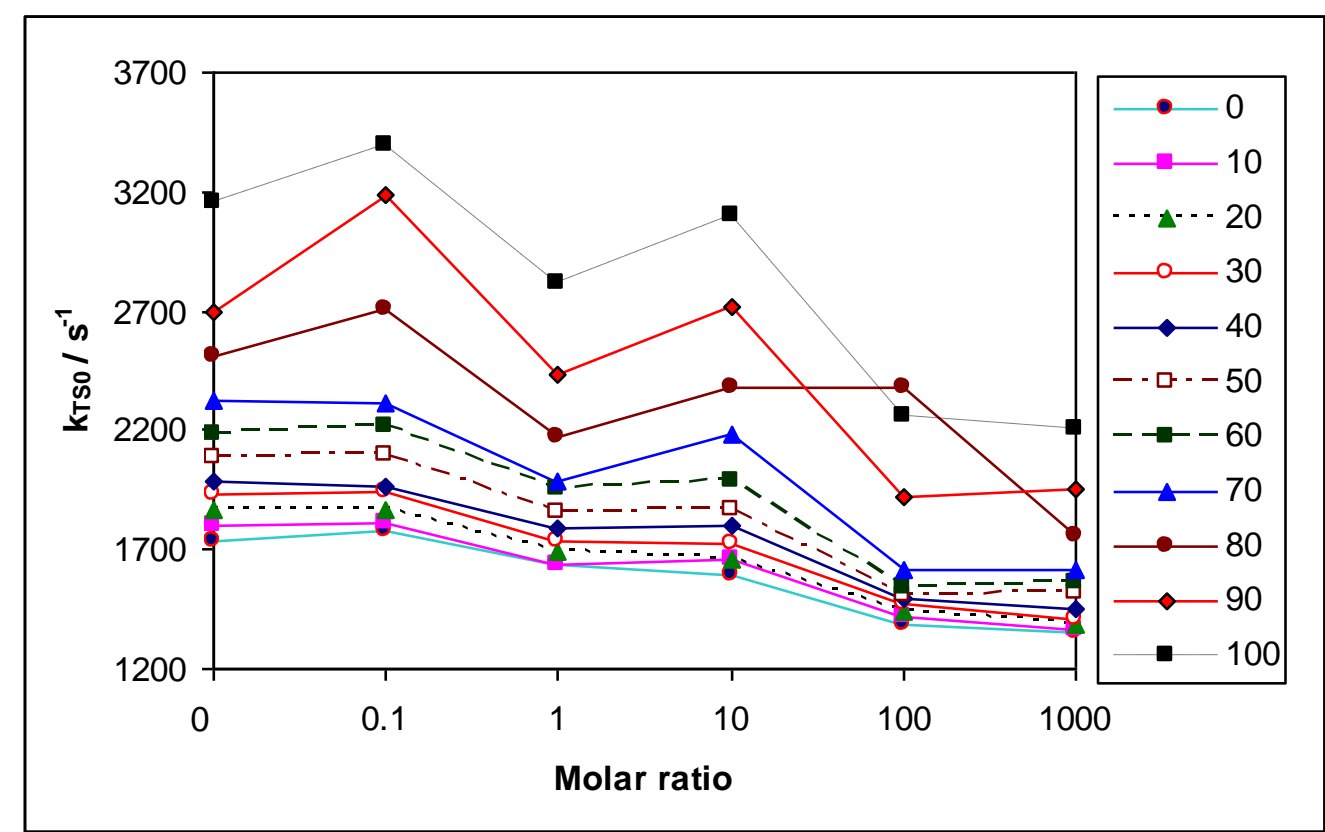

Figure V-25a: A plot of $\mathrm{k}_{\mathrm{TS} 0}$ as a function of maltitol concentration at various temperatures in $\beta$-Lg films. Data replotted from Figure V-13c.

Figure V-25b

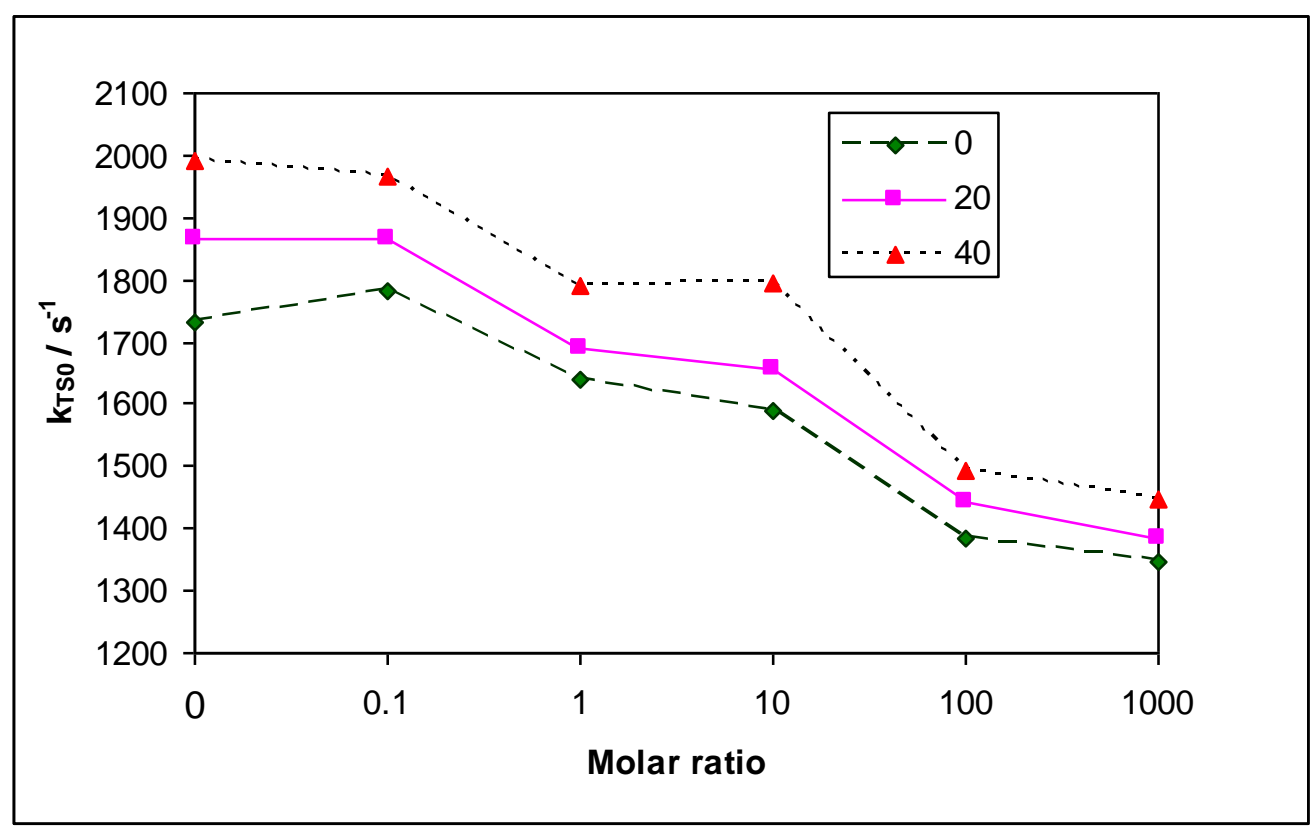

Figure V-25b: Zoom in plot of $\mathrm{k}_{\mathrm{TS} 0}$ as a function of maltitol concentration at various temperatures in $\beta$-Lg films. Data replotted from Figure V-13c. 


\section{Figure V-26a}

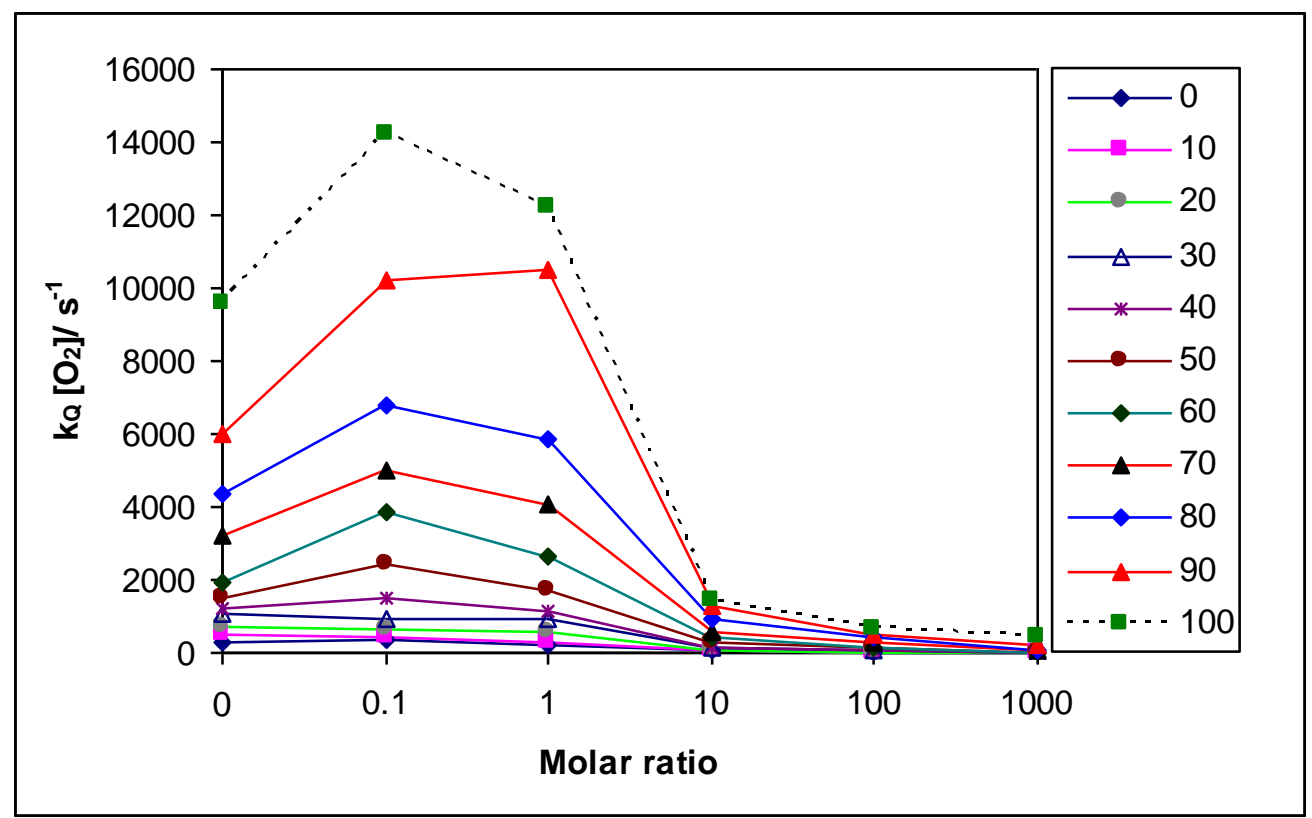

Figure V-26a: $\mathrm{k}_{\mathrm{Q}}\left[\mathrm{O}_{2}\right]$ as a function of maltitol concentration at various temperatures in $\beta$ - $\mathrm{Lg}$ films. Data replotted from Figure V-12c.

Figure V-26b

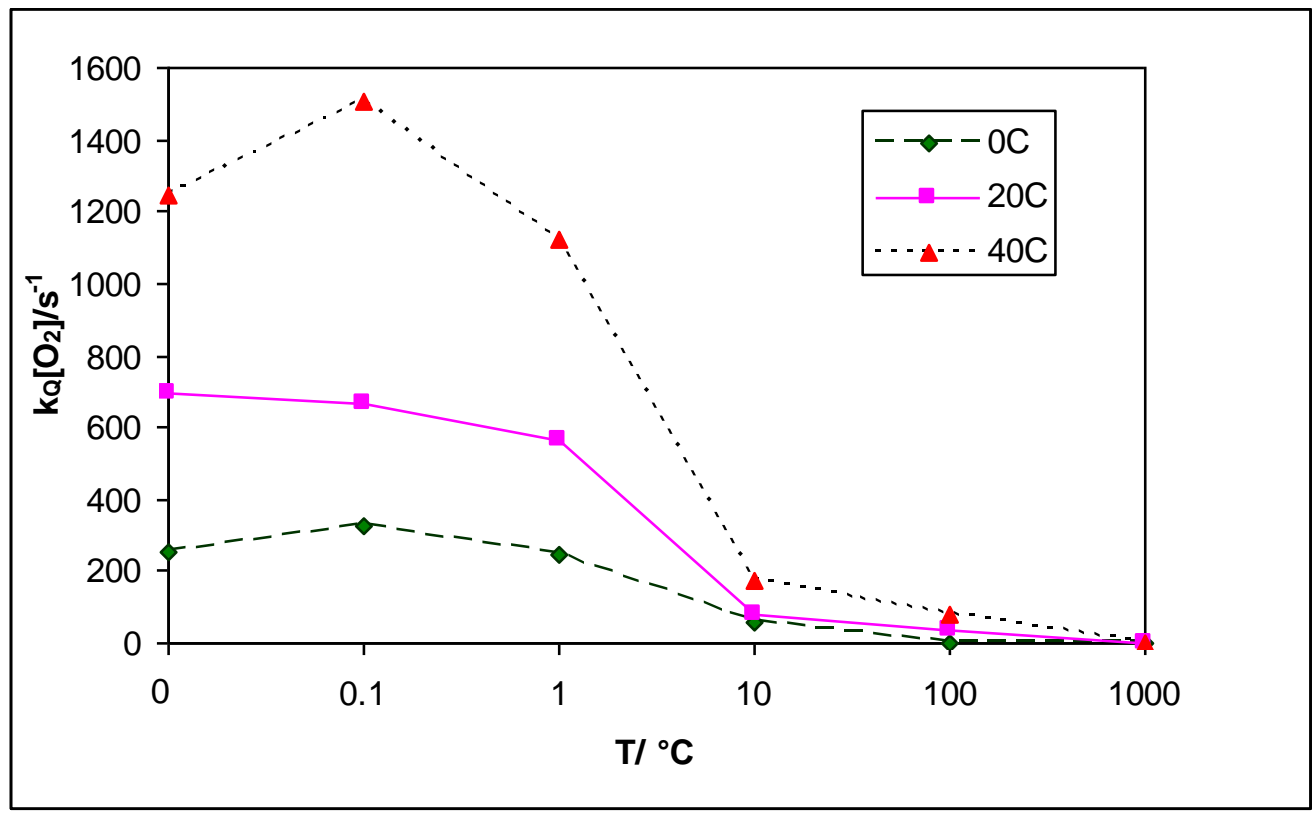

Figure V-26 b: Zoom in plot of $\mathrm{k}_{\mathrm{Q}}\left[\mathrm{O}_{2}\right]$ as a function of concentration of maltitol at various temperatures in $\beta$-Lg films. Data replotted from Figure V-12c. 


\section{Figure V-27a}

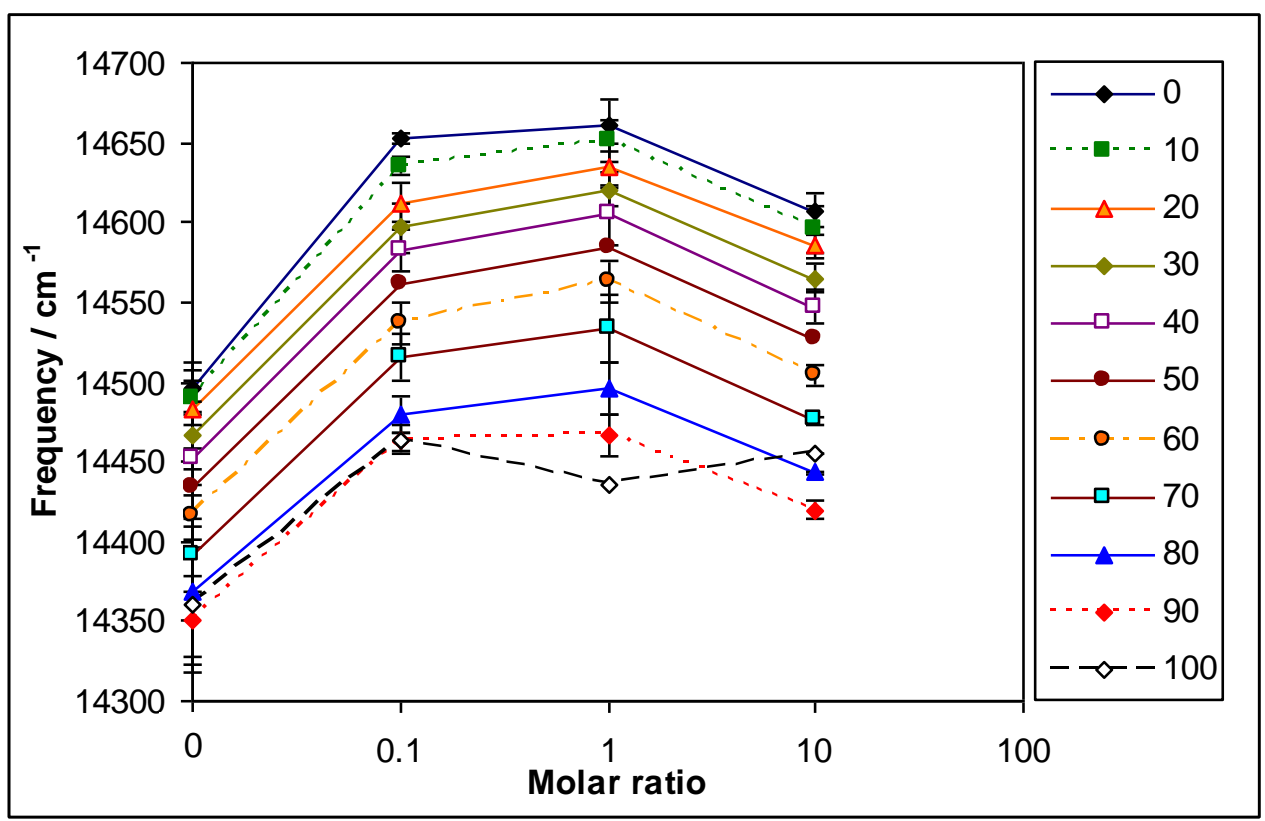

Figure V-27a: Emission energy as a function of PEG concentration at various temperatures in $\beta$ Lg films. Data replotted from Figure V-2d.

Figure V-27b

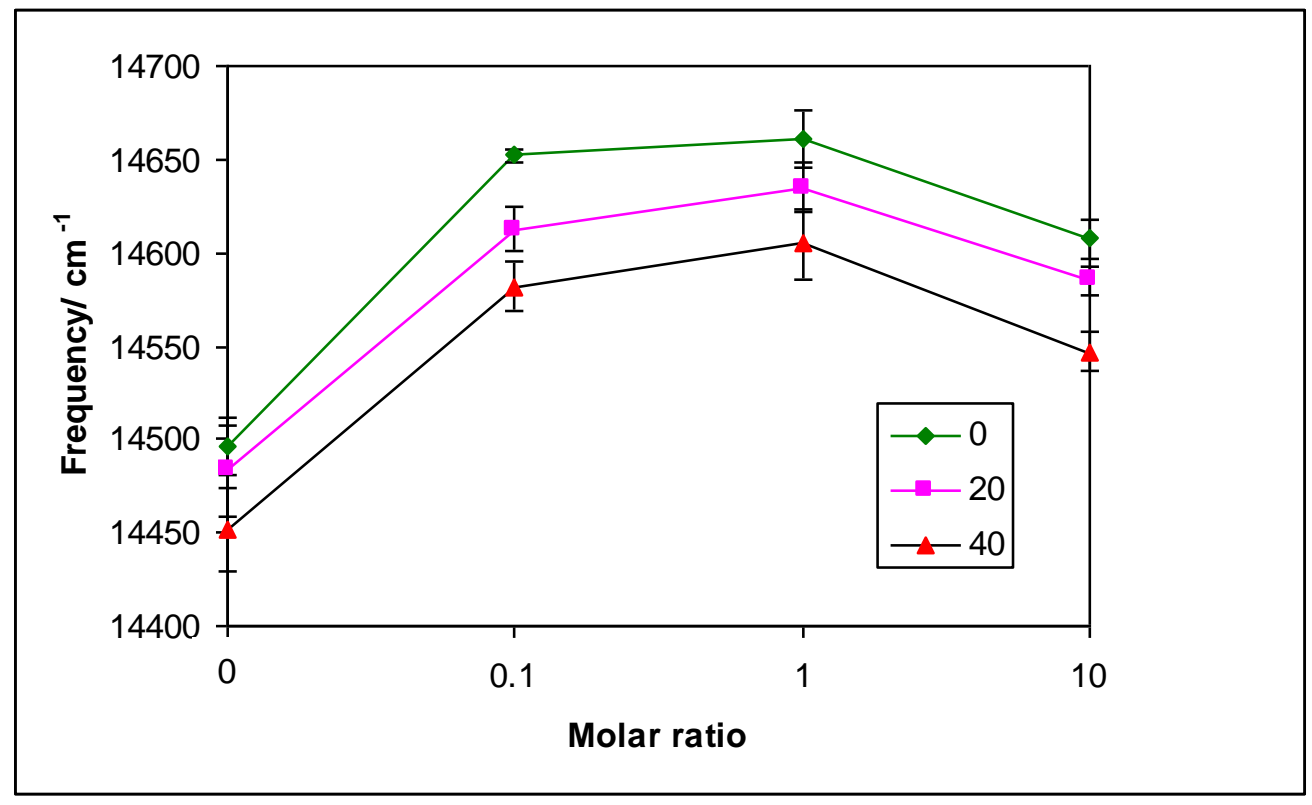

Figure V-27b: Zoom in plot of emission energy as a function of PEG concentration at various temperatures in $\beta$-Lg films. Data replotted from Figure V-2d. 
Figure V-28a

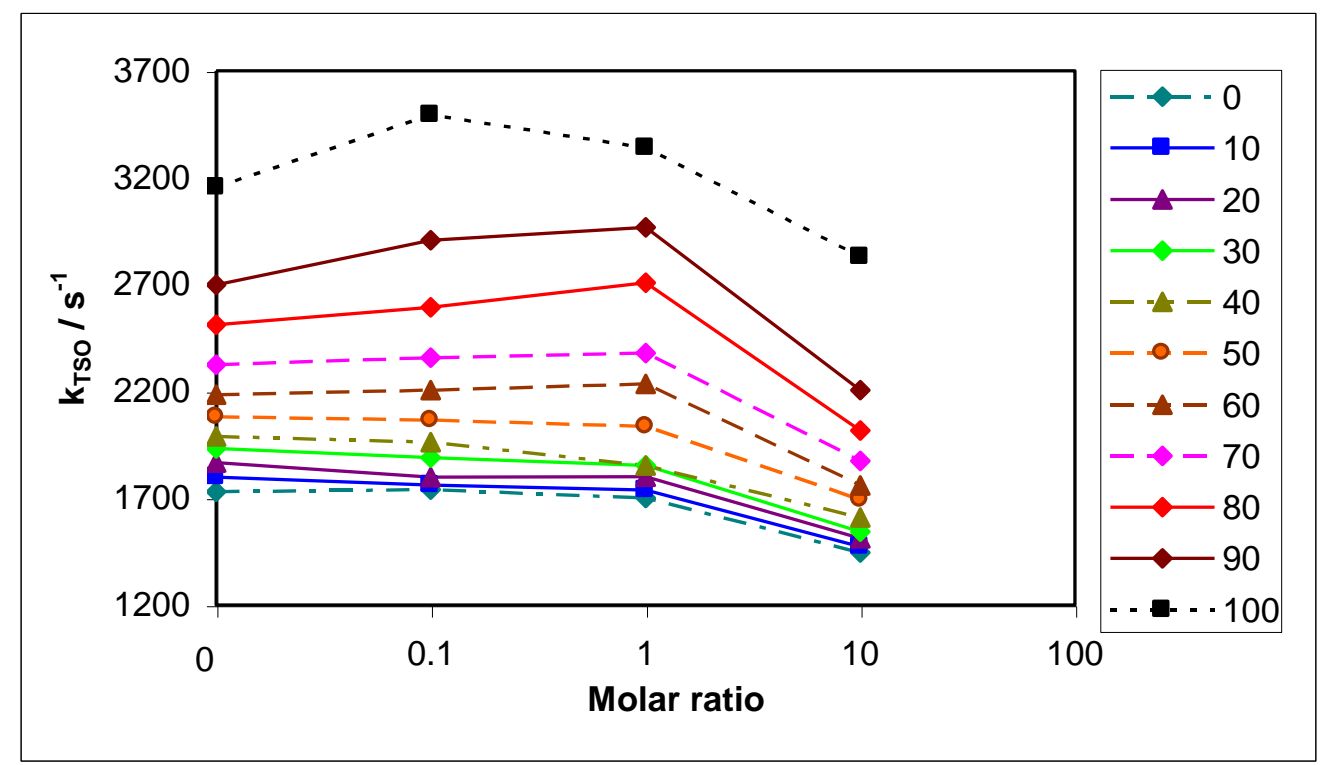

Figure V-28a: A plot of $\mathrm{k}_{\mathrm{TS} 0}$ as a function of PEG concentration at various temperatures in $\beta$ - $\mathrm{Lg}$ films. Data replotted from Figure V-13d.

\section{Figure V-28b}

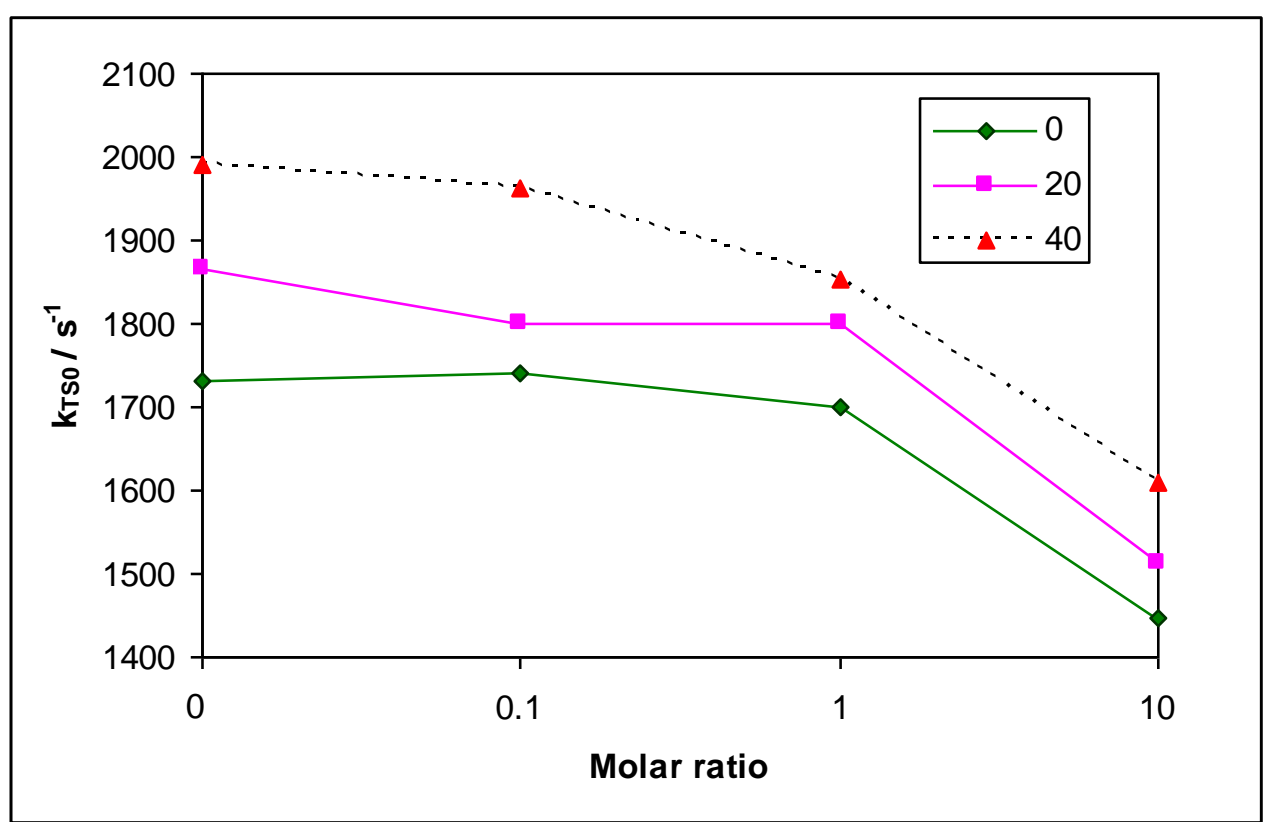

Figure V-28b: Zoom in plot of $\mathrm{k}_{\mathrm{TS} 0}$ as a function of PEG concentration at various temperatures in $\beta$-Lg films. Data replotted from Figure V-13d. 
Figure V-29a

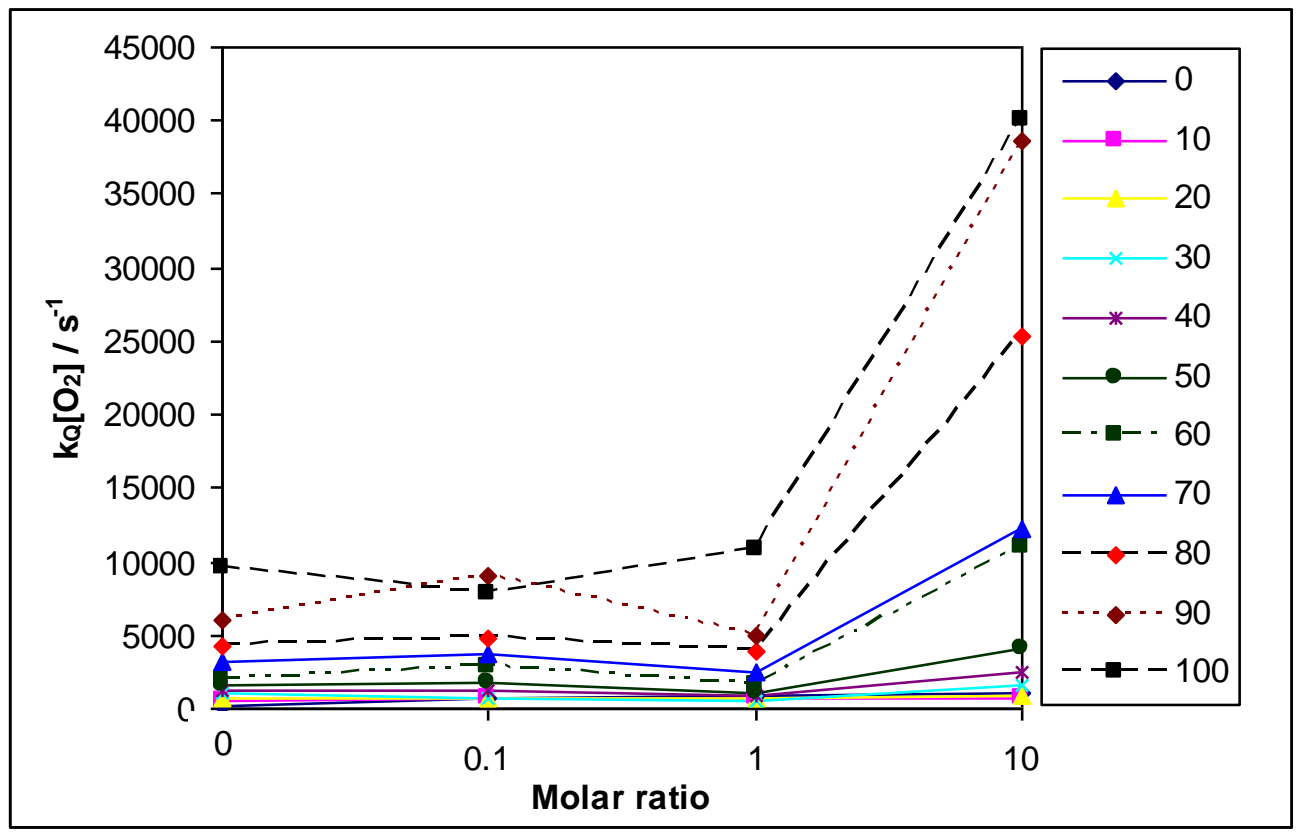

Figure V-29a: $\mathrm{k}_{\mathrm{Q}}\left[\mathrm{O}_{2}\right]$ as a function of PEG 400 concentration at various temperatures in $\beta$-Lg films. Data replotted from Figure $\mathrm{V}-12 \mathrm{~d}$. 


\section{Chapter VI: Molecular Mobility and Oxygen Permeability in Composite Films with $\beta$-Lactoglobulin ( $\beta$-Lg) /Plasticizer mixtures}

\section{$\underline{\text { Introduction }}$}

Since the 1990s, edible films and coatings from biopolymers have received increasing attention. Edible packaging can be used to reduce water vapor, oxygen, lipid, and flavor migration in multi-component food products and between food and surrounding atmosphere (Park et al., 1993; Debeaufort et al., 2002; Fennema et al., 1987).

The use of plasticizers is necessary to improve mechanical properties of proteinor polysaccharide-based edible films (Torres, 1994). Plasticizers are essentially added to $\beta$-Lg films to enhance the intermolecular spacing and thereby reduce the brittleness in the films. Considerable amount of research have focused on the effects of the plasticizers like glycerol, sorbitol, PEG 400, PEG 200, etc on the oxygen permeability of edible films (Sothornvit and Krotcha, 2000; Mc Hugh and Krotcha, 1994). A plasticizer like PEG prevents brittleness in edible films. One of the downsides of its plasticizing effects are it renders the film to be prone to diffusion of small molecules like oxygen (Sothornvit and Krotcha, 2000). A study by Debeaufort and Voilley (1995) revealed that the addition of PEG-400 to methylcellulose films significantly increased the oxygen, water vapor, and aroma transfers and sorption within the films. These can be attributed to the structural changes caused due to the PEG plasticization process.

Chapter III clearly revealed the effects of sucrose on the permeability of the $\beta-\mathrm{Lg}$ matrix. The addition of sucrose (as low as 10 moles of sucrose) to $\beta$-Lg films reduced the permeability by ten folds. 
Glucose hydrogen bond to a high degree with dried lysozyme, but is incapable of inhibiting lyophilization-induced protein unfolding in the absence of an effective cryoprotectant. However, the addition of polyethylene glycol, to glucose protected lysozyme structure during lyophilization (Allison et al., 1999). The synergistic action between sugar (glucose) and polyol (PEG) enhanced biopreservation. Generating innovative composite films through combined use of compatible proteins, sugars and polyols can utilize the functionality of each ingredient in an effective manner. Such complexes in many cases can display better functionality than proteins or plasticizers alone. This study aims on using the anti-plasticization functionality of sucrose discussed in detail in chapter (III) to control the oxygen diffusion in amorphous PEG plasticized $\beta$ Lg films.

Recently, a phenomenon such as "anti-plasticization" behavior has been reported in starch-glycerol (Lourdin et al., 1997), starch-sorbitol (Gaudin et al., 1999; Gaudin et al., 2000), glycerol-sucrose (You and Ludescher, 2007), trehalose-glycerol (Anopchenko et al., 2006) etc.

Although reports of anti-plasticization behavior have been largely confined to mixtures of molecules of dissimilar sizes (and glass transition temperatures), results suggest that such behavior also occurs in mixtures of molecules of nearly identical mass and similar structures. Studies by Shirke et al. (2005) revealed that the mobility of maltose and maltitol display distinct behavior within the glass and around the glass transition temperature and interactions between sugar and sugar alcohol actually lower the molecular mobility within the glassy state in mixtures. In a similar study the 
molecular mobility of a 1:1 (wt/wt) mixture of lactose and lactitol had lower collisional quenching rates in both the glass and the melt than in either of its pure components, indicating that specific interactions among these similar molecules resulted in dynamic synergies that lower the molecular mobility of the components in the mixture, (Shirke et al., 2006). This phenomenon may be a general feature of sugar-sugar alcohols and other carbohydrate mixtures and thus worthy of additional study.

So the present study aimed at utilizing the dynamic synergy of these sugar-sugar alcohols to improvise the permeability characteristics of the $\beta$ - $\mathrm{Lg}$ matrix and also elucidate the effect of these mixtures on the mobility of the $\beta$-Lg matrix. We present here an investigation of the effect of temperature on the molecular mobility and oxygen permeability of $\beta$-Lg films plasticized with mixtures of amorphous maltose and its corresponding sugar alcohol maltitol, using phosphorescence emission and intensity decay from the triplet probe Erythrosin B (tetraiodofluorescein) dispersed within the amorphous matrix. The effectiveness of this technique as a sensitive indicator of molecular mobility has recently been demonstrated in amorphous sucrose (Pravinata et al., 2005), maltose and maltitol (Shirke et al., 2005), lactose and lactitol (Shirke et al., 2006), gelatin (Simon-Lukasik and Ludescher, 2004; Lukasik and Ludescher, 2006a, b) BSA (Nack and Ludescher, 2006) and $\beta-L g$ (Sundaresan and Ludescher, 2007).

In addition to that we also report a comparable phosphorescence study of molecular mobility and oxygen permeability in amorphous thin films of $\beta$-Lg plasticized with PEG 400 and sucrose with a view to judge the critical concentration of sucrose required to control oxygen permeability in the PEG plasticized $\beta$-Lg films. 


\section{$\underline{\text { Materials and Methods }}$}

Sample preparation

Solutions of $\beta-\operatorname{Lg}$ (90\% pure; crystallized and lyophilized Sigma Chemical Co, St.Louis, MO, USA) were prepared in distilled de-ionized water at an approximate concentration of $10 \mathrm{mg} / \mathrm{ml}$. This concentration was selected based on the batch size to ensure maximum recovery after dialysis and to avoid long-term storage. An aqueous $\beta$ Lg solution was transferred to regenerated cellulose dialysis tubing having a $12-14-\mathrm{kDa}$ molecular weight cutoff (Spectrum, Houston, TX). In order to define and minimize the presence of counter ions the solution was extensively dialyzed against $0.1 \mathrm{M}$ potassium chloride for 24 hours with frequent change of buffer every 6 hours. The solution was then dialyzed against distilled de-ionized water for 24 hours with frequent change of water after every 6 hours. The proposed protocol was followed in a very stringent manner in order to control precipitation in the protein during dialysis. The dialyzed solution was then filtered through a $0.2-\mu \mathrm{m}$ membrane to remove particulates. In order to maximize the effectiveness of the dialysis process temperature of the solution was kept at $5^{\circ} \mathrm{C}$ to ensure that the protein remained folded. Several dilutions of a stock solution of dialyzed $\beta$-Lg were prepared. The dye to protein ratio was determined by measuring the absorbance of dilutions in a $1 \mathrm{~cm}$ path length quartz cuvette in a Bausch and Lomb Spectronic 2000 spectrophotometer (Milton Roy company, Analytical Products Division, Rochester, NY). The protein concentration was determined after dialysis by UV absorbance using an extinction coefficient of $0.96 \mathrm{~L} \mathrm{~g} \mathrm{~g}^{-1} \mathrm{~cm}^{-1}$. ErythrosinB (EryB) (tetra iodofluorescein,FD\&C red\#3) from molecular probes (Eugene, OR), was dissolved in spectrophotometric grade N,N-dimethyl formamide (DMF) (Aldrich Chemical, 
Milwaukee, WI) to make $10 \mathrm{mM}$ and $100 \mathrm{mM}$ stock solutions. The solvent DMF was selected for probe stability during long time storage and the concentrations were selected in order to simplify the addition of probe to the protein matrix. After estimating the concentration protein solution was combined with aliquots of dye solution to make solutions with molar ratio of EryB: $\beta$-Lg of 1:4. The dye to protein concentration was sufficient to provide adequate signal /noise in spectroscopic measurements.

Generating composite films:

$\beta$-Lg solution was combined with aliquots of dye solution to make solutions with molar ratio of EryB: $\beta$-Lg of 1:4. Different molar concentrations of sucrose and polyethylene glycol were added to $\beta$-Lg to generate composite films.

The highest concentration of PEG 400 which lead to the formation of intact films with no signs of phase separation and a concentration of plasticizer essentially used to plasticize films in the food industry was selected as the standard concentration of PEG in the $\beta$-Lg films. To this baseline concentration of PEG 400: $\beta-\mathrm{Lg}$, which was 10:1 varying concentrations of sucrose, were added in-order to judge the threshold concentration to control oxygen permeability in these composite films.

$\beta$-Lg films with mixtures

Maltose and maltitol were purchased from Sigma-Aldrich (St. Louis, MO) with a minimum purity of $98 \%$. These components were dissolved in deionized water at a concentration of 66-68 wt \%. Mixtures were prepared from $68 \mathrm{wt} \%$ samples of maltose and maltitol. To prepare a 70:30 mixture, for example, 70 parts of maltose solution was mixed with 30 parts of maltitol solution (weight basis); 50:50 and 30:70 mixtures were prepared similarly. And these solutions were mixed with EryB, $\beta$-Lg solution in the ratio 
of 10:1 (Mixture: $\beta$-Lg). This ratio was chosen because it was seen that both in maltose and maltitol / $\beta$-Lg films, 10:1 was the critical concentration were noticeable changes in permeability were observed.

\section{Preparation of films}

To make glassy films $15 \mu 1$ of protein / plasticizer/ sugar or protein/ mixtures solutions were spread on approximately one third of a quartz slide $(30 \times 13.5 \times 0.6 \mathrm{~mm}$; custom made by NSG Precision Cells, Farmingdale, NY). The films approximately ( 0.05 mm thick when dried) were visually checked for good spreading, uniformity, and consistency. The slides were stored at room temperature in a desiccator containing $\mathrm{P}_{2} \mathrm{O}_{5}$ and drierite for at least 7 days and protected from light to prevent any photobleaching of Erythrosin B prior to luminescence measurements. The slides were checked for crystallization under crossed polarizers using a Nikon Type 102 dissecting microscope (Nikon, Inc., Japan).

\section{Luminescence measurements and analysis}

All luminescence measurements were made on a Cary Eclipse Fluorescence Spectrophotometer (Varian Instruments, Walnut Creek, CA). A slide was fitted diagonally into a standard fluorescence cuvette, which was flushed with oxygen-free $\mathrm{N}_{2}$ gas for at least 15 min prior to measurements. The temperature was controlled using a TLC 50 thermoelectric heating/cooling system (Quantum Northwest, Spokane, WA). For measurements below room temperature, the chamber surrounding the cuvette holder was flushed with dry air to eliminate moisture condensation.

Delayed luminescence emission spectra were collected from 535-800 nm (10nm bandwidth) using excitation of $525 \mathrm{~nm}$ (10nm bandwidth) over the temperature range 
from $0^{\circ} \mathrm{C}$ to $100^{\circ} \mathrm{C}$ (at 10 degrees intervals.) Each data point (1nm interval, $0.1 \mathrm{~s}$ averaging time) was collected from a single flash with a $0.2 \mathrm{~ms}$ delay, $0.5 \mathrm{~ms}$ gate time and $4.0 \mathrm{~ms}$ total decay time.

Intensity decays used excitation at $540 \mathrm{~nm}$ (bandwidth $20 \mathrm{~nm}$ ) and emission collected at $690 \mathrm{~nm}$ (bandwidth $20 \mathrm{~nm}$ ); decays were collected over the temperature range from $0^{\circ} \mathrm{C}$ to $100^{\circ} \mathrm{C}$. The intensity was collected as a function of time following the lamp flash over a window of $4 \mathrm{~ms}$ following a delay time of $0.1 \mathrm{~ms}$ and using a gate time of $0.02 \mathrm{~ms} ; 100$ cycles were summed to generate each decay transient. Before collecting data samples were equilibrated for 15 minutes. The measurements were taken in the presence or absence of air (under a nitrogen purge). All experiments were done several times and the averages were used for data analysis and interpretation. In order to validate the repeatability the standard deviation in between experiments were calculated.

The intensity transients were analyzed using a stretch-exponential decay function:

$$
I(\mathrm{t})=\mathrm{I}(0) \exp \left[-(\mathrm{t} / \tau)^{\beta}\right]+\text { constant }
$$

where $\mathrm{I}(0)$ is the initial intensity at time zero, $\tau$ is the Kohlrausch-Williams-Watts lifetime (William and Watts, 1970) and $\beta$ is the stretching exponent, (Lindsey and Patterson 1980) a parameter that varies from 0 to 1 . Data was analysed using the program Igor (Wavemetrics, Inc., Lake Oswego, OR, USA). We also analyzed some data by fitting with the program Nfit (Island Products, Galveston, TX, USA) to make sure that the results agreed in both fitting programs. All fits gave $\mathrm{R}^{2}$ values in the range of $0.99-$ 1.0 and modified residuals $\left((\right.$ data - fit $) /$ data $\left.^{1 / 2}\right)$ plots that varied randomly about zero( plot not shown) 


\section{Photophysical scheme}

Our analysis of the photophysics of Erythrosin B follows that of Duchowicz et al. (1998) using slightly different nomenclature. The measured emission rate for phosphorescence $\left(\mathrm{k}_{\mathrm{P}}=1 / \tau\right)$ is the sum of all possible de-excitation rates for the triplet state $T_{1}$ : Under conditions whereby oxygen quenching is assumed negligible due to the elimination of oxygen by nitrogen

$$
\mathrm{k}_{\mathrm{P}}=\mathrm{k}_{\mathrm{RP}}+\mathrm{k}_{\mathrm{TS} 0}(\mathrm{~T})+\mathrm{k}_{\mathrm{TS} 1}(\mathrm{~T})
$$

where $\mathrm{k}_{\mathrm{RP}}$ is the rate of radiative emission to the ground state $\mathrm{S}_{0}, \mathrm{k}_{\mathrm{TS} 0}$ is the rate of intersystem crossing to $\mathrm{S}_{0}$ (rate of collisional quenching), and $\mathrm{k}_{\mathrm{TS} 1}$ is the rate of reverse intersystem crossing to the excited singlet $\mathrm{S}_{1}$; only the latter two rate constants vary with temperature.

But in the presence of oxygen the de-excitation of the triplet state is also influenced by the quencher species. The term $\mathrm{k}_{\mathrm{Q}}\left[\mathrm{O}_{2}\right]$ describes the rate of collisional quenching of the excited state of the probe by oxygen. Thus in the presence of oxygen $\mathrm{k}_{\mathrm{P}}(\mathrm{T})$ includes an additional term $\mathrm{k}_{\mathrm{Q}}\left[\mathrm{O}_{2}\right]$.

$$
\mathrm{k}_{\mathrm{P}}(\mathrm{T})=\mathrm{k}_{\mathrm{RP}}+\mathrm{k}_{\mathrm{TS} 1}(\mathrm{~T})+\mathrm{k}_{\mathrm{TS} 0}(\mathrm{~T})+\mathrm{k}_{\mathrm{Q}}\left[\mathrm{O}_{2}\right](\mathrm{T})
$$

The rate of radiative emission $\left(k_{\mathrm{RP}}\right)$ is $41 \mathrm{~s}^{-1}$ and constant. (Duchowicz et al., 1998; Lettinga et al., 2000), The rate of reverse intersystem crossing to $S_{1}\left(k_{T S 1}\right)$ is a thermally activated process. $\mathrm{k}_{\mathrm{TS} 1}$ the rate of reverse intersystem crossing from the excited triplet state to the excited singlet state depends on $\Delta \mathrm{E}_{\mathrm{TS}}$, the energy gap between $\mathrm{S}_{1}$ and $\mathrm{T}_{1}$

$$
\mathrm{k}_{\mathrm{TS} 1}(\mathrm{~T})=\mathrm{k}_{\mathrm{TS} 1}^{\mathrm{o}} \exp \left(-\Delta \mathrm{E}_{\mathrm{TS}} / \mathrm{RT}\right)
$$

$\Delta \mathrm{E}_{\mathrm{TS}}$ is the energy gap between $\mathrm{S}_{1}$ and $\mathrm{T}_{1}, \mathrm{R}=8.314 \mathrm{~J} \mathrm{~K}^{-1} \mathrm{~mol}^{-1}$, and $\mathrm{T}$ is the temperature in Kelvin. The value of $\Delta \mathrm{E}_{\mathrm{TS}}$ is calculated from the slope of a Van't Hoff 
plot of the natural logarithm of the ratio of intensity of delayed fluorescence $\left(\mathrm{I}_{\mathrm{DF}}\right)$ to phosphorescence $\left(\mathrm{I}_{\mathrm{P}}\right)\left(\ln \left(\mathrm{I}_{\mathrm{DF}} / \mathrm{I}_{\mathrm{P}}\right)\right.$ vs $1 / \mathrm{T}$.

Unfortunately, literature values of $\mathrm{k}^{\circ} \mathrm{TS} 1$ for Erythrosin B vary widely, from $0.3 \mathrm{x}$ $10^{7} \mathrm{~s}^{-1}$ in ethanol and $6.5 \times 10^{7} \mathrm{~s}^{-1}$ in water (Duchowicz et al., 1998) to $111 \times 10^{7} \mathrm{~s}^{-1}$ in solid polyvinyl alcohol (Lettinga et al., 2000), and thus provide little guidance. We estimated the maximum possible value for $\mathrm{k}^{\mathrm{o}}$ TS1 in plasticizer / $\beta$ - $\mathrm{Lg}$ films by assuming that $\mathrm{k}_{\mathrm{TS} 1}^{\mathrm{o}}(\mathrm{T})$ cannot result in values for $\mathrm{k}_{\mathrm{TS} 0}$ that decrease with temperature. This procedure thus estimated the minimum possible values of $\mathrm{k}_{\mathrm{TS} 0}(\mathrm{~T})$.

In order to study the oxygen diffusion $\mathrm{k}_{\mathrm{Q}}\left[\mathrm{O}_{2}\right]$ can easily be calculated from the difference between the decay rate in the presence of oxygen and the absence of oxygen

$$
\mathrm{k}_{\mathrm{Q}}\left[\mathrm{O}_{2}\right]=1 / \tau(\text { Air })-1 / \tau_{0}(\text { Nitrogen })
$$

The emission spectra were analyzed by fitting both the delayed fluorescence and the phosphorescence to a lognormal function.

$$
\mathrm{I}(v)=\mathrm{I}_{0} \exp \left\{-\ln (2)\left[\ln \left(1+2 \mathrm{~b}\left(\mathrm{v}-\mathrm{v}_{\mathrm{P}}\right) / \Delta\right) / \mathrm{b}\right]^{2}\right\}
$$

In this equation $I_{0}$ is the maximum emission intensity, $v_{P}$ is the frequency $\left(\mathrm{cm}^{-1}\right)$ of the emission maximum, $\Delta$ is a linewidth parameter, and $b$ is an asymmetry parameter The bandwidth $(\Gamma)$ of the emission band is related to $b$ and $\Delta$ :

$$
\Gamma=\Delta\{\sinh (b) / b\}
$$

Emission spectra were fit using the program Igor (Wavemetrics, Inc., Lake Oswego, OR, USA). We also analyzed some data by fitting with the program Nfit (Island Products, Galveston, TX, USA) to make sure that the results agreed in both fitting programs. 


\section{$\underline{\text { Results }}$}

Previous chapters (III, V) have elucidated the individual effects of sugars and polyols on the amorphous $\beta$-Lg matrix. The major advantages of protein sugar mixtures are they act as excellent oxygen barriers. However, one of the major drawbacks of edible films made from protein-sugar systems are that they are very brittle and difficult to handle (Sothornvit and Krochta, 2000). Edible films generally have a plasticizer to enhance workability but the major disadvantage of these polyols is that they also enhance the permeability of the matrix, which is evident in our previous studies on the effect of plasticizers on the $\beta-\operatorname{Lg}$ matrix. So a food scientist in a practical scenario generally aspires to generate edible films that are less prone to atmospheric oxygen, as well as easy to handle. So this project aimed to solve this real life problem by generating a composite film, which could essentially utilize the functionality of both. PEG 400 is known for ages as an excellent plasticizer, but from our study we see that there was a phenomenal increase in the oxygen permeability in PEG plasticized $\beta$-Lg films. So, this study looked into generating a functional matrix using both PEG 400 and sucrose utilizing the favorable functionality of each of these plasticizers.

\section{Delayed emission spectra}

Figure VI-1 potrays the emission spectra of Erythrosin B in $\beta$-Lg with 10 moles of sucrose and 10 moles of PEG per mole of $\beta$-Lg. Delayed fluorescence and phosphorescence intensity of Erythrosin B dispersed in $\beta$-Lg: PEG: Suc was measured as a function of temperature by exciting the molecules at $525 \mathrm{~nm}$ and collecting emission from $535 \mathrm{~nm}-800 \mathrm{~nm}$. The intensity of delayed fluorescence increased with temperature whereas intensity of phosphorescence decreased as the temperature increased from $0^{\circ} \mathrm{C}$ to 
$100^{\circ} \mathrm{C}$, as expected from a thermally stimulated process and followed a trend very similar to the emission spectra in all other functional matrices. Both delayed fluorescence and phosphorescence bands shifted to longer wavelengths at higher temperatures. The intensity ratio plotted as a Vant Hoff plot of $\ln \left(\mathrm{I}_{\mathrm{DF}} / \mathrm{I}_{\mathrm{P}}\right)$ vs $1 / \mathrm{T}$ was linear over the entire region of the temperature with no systematic deviations $\left(\mathrm{R}^{2}>0.9998\right)$ which indicated that Erythrosin B could be used as an optical thermometer (Pravinata et al ., 2003). In this entire chapter the tertiary matrix comprising of $\beta$-Lg, PEG and sucrose ( $\beta$-Lg:PEG:suc) will be referred as 1:10:10 and 1:10:100. To indicate synergies these tertiary matrices were compared with Peg plasticized $\beta-\mathrm{Lg}$ films (in the ratio of $\beta$-Lg: PEG: Suc 1:10:0) and sucrose/ $\beta$-Lg films ( $\beta$-Lg:PEG: Suc 1:0:10 )respectively. So to facilitate comparison they all will be referred by their respective names as in each case one critical ratio was chosen (Refer Materials and Methods).

Figure VI-2 is a comparison plot of the peak frequencies of the phosphorescence of Erythrosin B in pure $\beta$ - $\mathrm{Lg}$ films, PEG plasticized $\beta$ - $\mathrm{Lg}$ films, sucrose $\beta$ - $\mathrm{Lg}$ films and tertiary matrices with different molar ratios of sucrose 1:10:10 and 1:10:100.

Peak frequencies were plotted at various temperatures from $0^{\circ} \mathrm{C}$ to $100^{\circ} \mathrm{C}$. Peak frequencies were obtained by fitting the phosphorescence intensity to a lognormal function ( $\mathrm{Eq} 6$ Materials and Methods). Over the tested temperature range peak frequency decreased linearly with increase in temperature in all the different matrices. The red shift to lower energy is due to an increase in the rate of relaxation. At high temperatures a slight increase in emission energy may just be due to the inherent difficulty in analyzing data of low intensity. The peak frequency increased with sucrose concentration in the PEG plasticized $\beta$-Lg films. When we compare the different matrices 
we see that the tertiary matrix with a ratio of 1:10:100 had the highest emission energy when compared to the other matrices. For instance, at a temperature of $0^{\circ} \mathrm{C}$ the peak frequency was $14607 \mathrm{~cm}^{-1}$ in PEG plasticized $\beta-\operatorname{Lg}(1: 10: 0)$ and in the tertiary matrix $(1: 10: 100)$ the peak frequency was $14803 \mathrm{~cm}^{-1}$. There was an approximate $200 \mathrm{~cm}^{-1}$ enhancement in the emission energy from PEG plasticized $\beta$-Lg films. These trends suggest that the presence of small molecules like sucrose affects the matrix, in a way that it brings about a rapid decline in the inherent relaxation mechanisms which are otherwise prevalent in the $\beta$-Lg and PEG plasticized films.

The band-width $(\Gamma)$, or Full width at half maximum, $(\mathrm{Eq} 7$ Materials and methods) of the tertiary matrices showed an insignificant increase at low temperatures and a dramatic increase above $80^{\circ} \mathrm{C}$ (Figure VI-3). This phenomenon may be a reflection of the in-homogenous broadening of the spectra due to interactions of Erythrosin B with the surrounding molecules at high temperatures. Sucrose / $\beta$-Lg films showed a noticeable enhancement in the bandwidth above the glass transition temperature of sucrose $\left(62^{\circ} \mathrm{C}\right)$. However, there were no noticeable differences in between the various tertiary matrices until $80^{\circ} \mathrm{C}$. Although the different ratios were distinguishable at $80^{\circ} \mathrm{C}$, a clear trend between ratios was not observed.

\section{Phosphorescence Intensity decays}

The phosphorescence intensity decay of Erythrosin B in $\beta$-Lg with varying ratios of additives was measured over the temperature range from $0^{\circ} \mathrm{C}$ to $100^{\circ} \mathrm{C}$. All decays were well fit using the stretched exponential decay function. The stretched exponential model describes the intensity decay of Erythrosin B phosphorescence in all $\beta$-Lg films. 
The Kohlrausch-Williams-Watts lifetime $(\tau)$ and stretching factor $(\beta)$ are the physically meaningful parameters of the fit intensity decay.

Under both nitrogen and oxygen conditions, the lifetimes decreased biphasically with increasing temperature exhibiting a gradual decrease in low temperatures and more dramatic decrease at high temperatures. Higher lifetimes at low temperatures indicated lower quenching rates. There was an enhancement in the quenching rates at higher temperatures due to increases in the matrix mobility.

Lifetime is an average time a probe spends in the excited triplet state and lifetime values can be thought of as a measure of the rigidity of the matrix. PEG plasticized $\beta-\mathrm{Lg}$ films had a lifetime of $0.66 \mathrm{~ms}$ at $0^{\circ} \mathrm{C}$ and $0.25 \mathrm{~ms}$ at $100{ }^{\circ} \mathrm{C}$. The lifetimes values at a ratio of 1:10:100 ranging from $0.73 \mathrm{~ms}$ at $0^{\circ} \mathrm{C}$ and $0.32 \mathrm{~ms}$ at $100{ }^{\circ} \mathrm{C}$, showed an approximate $10 \%$ increase at $0^{\circ} \mathrm{C}$ and $26 \%$ at $100{ }^{\circ} \mathrm{C}$ when compared to the PEG plasticized $\beta$-Lg films. It is evident that the lifetimes of the tertiary matrices in the ratios of 1:10:10 and 1:10:100 had significantly higher values than the pure $\beta-\mathrm{Lg}$, sucrose $\beta-\mathrm{Lg}$ or PEG plasticized $\beta$-Lg films (Figure VI-4c).

In PEG plasticized $\beta$-Lg films and $\beta$-Lg films, lifetimes in air were significantly lower than that in nitrogen indicating significant oxygen quenching in these films. Results from chapter III clearly indicate that sucrose/ $\beta$-Lg films at a ratio (1:0:10) had comparable lifetimes both in nitrogen and in air indicating that oxygen quenching was barely detectable at this ratio.

Comparison of the Erythrosin B lifetimes in the presence of nitrogen and presence of air in the tertiary matrix in the ratio of 1:10:10 indicated that oxygen quenching was fairly significant in these films at higher temperatures. Further modification of the same 
matrix by adding higher ratios of sucrose and generating tertiary films with a composition of 1:10:100, showed a similarity in the lifetimes in the presence of air and nitrogen due to a dramatic decline in the permeability. These trends suggest that higher amounts of sugar conferred significant rigidity to the matrix, thus preventing the entry of permeant molecules.

Figures VI-5a and VI-5b represent $\beta$ as a function of temperature. The stretching exponent $\beta$ is a measure of heterogeneity of the matrix. High values of $\beta$ indicate less heterogeneity.

Experiments on the effect of the plasticizer PEG on $\beta$-Lg films (Chapter 5) clearly revealed that $\beta$ was indistinguishable between pure $\beta$-Lg and Peg plasticized $\beta$-Lg films in the presence of nitrogen. However, $\beta$ increased significantly in tertiary matrices with ratios of 1:10:10 and 1:10:100 showing that addition of sugar enhanced the homogeneity in the matrix. As $\beta$ essentially reflects the distribution of dynamically distinct probe environments with different values of $\mathrm{k}_{\mathrm{TS} 0}$, large increases in $\beta$ upon addition of sucrose indicated a significant decrease in the range of dynamically distinct probe environments in the glass.

Pure $\beta$-Lg films and PEG plasticized $\beta$-Lg films, had significantly lower $\beta$ values in the presence of air as compared to nitrogen conditions, indicating that in the presence of oxygen the heterogeneity was increased. The tertiary matrices and $\beta$ - $\mathrm{Lg} /$ sucrose films had similar $\beta$ values in nitrogen and in air.

Phosphorescence decay rate constants 
Figure VI-6 depicts $\mathrm{k}_{\mathrm{Q}}\left[\mathrm{O}_{2}\right]$ as a function of temperature. Addition of PEG in the ratio of 1:10:0 brought about a 3-4-fold increase in permeability when compared to the pure $\beta$-Lg matrix. (Chapter V)

Upon addition of 10 moles of sucrose to the PEG plasticized film (1:10:10) the $\mathrm{k}_{\mathrm{Q}}$ $\left[\mathrm{O}_{2}\right]$ values decreased from $987 \mathrm{~s}^{-1}$ to $92 \mathrm{~s}^{-1}$ at $0^{\circ} \mathrm{C}$ and from $38579 \mathrm{~s}^{-1}$ to $18551 \mathrm{~s}^{-1}$ at $100^{\circ}$ C. It is clear that there was a 10-fold decline in the permeability at low temperatures. The magnitude of decline, however, decreased at high temperatures. There was a further decline in the $\mathrm{k}_{\mathrm{Q}}\left[\mathrm{O}_{2}\right]$ values when the sucrose concentration was increased. In the ratio of 1:10:100 we see that the collisional quenching rate constant for oxygen permeability greatly reduced to almost zero. Addition of 100 moles of sucrose to PEG plasticized $\beta \lg$ films brought about a massive (60-70 fold) decline in the collisional quenching rate constants as compared to the PEG plasticized films

The $\mathrm{k}_{\mathrm{TS} 0}$ values for all samples are plotted as $\mathrm{k}_{\mathrm{TS} 0}$ versus temperature in Figure VI 7. At $0^{\circ} \mathrm{C}$, the lowest accessible temperature, $\mathrm{k}_{\mathrm{TS} 0}$ was $1730 \mathrm{~s}^{-1}$ in pure $\beta$-lg films, $1445 \mathrm{~s}^{-1}$ in PEG/ $\beta$-Lg films, $1647 \mathrm{~s}^{-1}$ in sucrose/ $\beta$-Lg films and $1309 \mathrm{~s}^{-1}$ in the tertiary matrix with a ratio of 1:10:100. In the measured temperature range $\mathrm{k}_{\mathrm{Ts} 0}$ followed the rank order of 1:10:100 $<1: 10: 100<$ Peg $\beta$-lg films $<$ sucrose $\beta$-lg films $<\beta$-lg films. The $\mathrm{k}_{\mathrm{Ts} 0}$ values in the tertiary matrices were significantly lower than the PEG plasticized $\beta$-Lg films and sucrose $\beta$-lg films indicating synergistic interactions between sucrose, PEG with $\beta$-lg. We have seen similar synergistic effects in mixtures of maltose and maltitol (Shirke et al., 2005) and lactose and lactitol (Shirke et al., 2006). The rate of molecular mobility ( $\left.\mathrm{k}_{\mathrm{TS} 0}\right)$ is lower in films made from 50:50 mixtures than in those made from either pure compound. 
Although we have yet to correlate these decreases in molecular mobility with functionally relevant changes in the rates of degradative reactions, such studies are certainly promising. Subtle changes in mobility, for example, may have significant implications on the overall shelf life of a product.

There was a clear biphasic correlation between $\mathrm{k}_{\mathrm{TS} 0}$ and $\mathrm{k}_{\mathrm{Q}}\left[\mathrm{O}_{2}\right]$ as illustrated in Figures VI- $8 \mathrm{a}$ and VI $-8 \mathrm{~b}$ in the tertiary matrices indicating that an onset level of mobility was required to activate permeability rather than temperature.

After generating some interesting results with tertiary matrices the investigator aimed to gain a deeper understanding of the effect of sugar and sugar alcohol mixtures on $\beta$-Lg matrix. The dynamic synergy between maltose maltitol mixtures has been well established in our lab and these synergies have led to decreases in matrix mobility when compared to the pure sugar or pure sugar alcohol films (Shirke et al., 2005).

So the present study got its motivation from that study and aimed to make use of these dynamic synergies and study its effect on the matrix mobility and permeability of the $\beta$-Lg matrix. We designed our experiments by making mixtures whereby, mixture 1 had a composition of $\beta$-Lg : maltose : maltitol 1:5:5, Mixture 2 had a ratio of $\beta-\mathrm{Lg}$ : maltose: maltitol 1:3:7 and Mixture 3 was $\beta$-Lg: maltose: maltitol 1:7:3. In the entire chapter the mixtures will referred by their respective ratios

\section{Delayed emission spectra}

The delayed emission spectra of Erythrosin B dispersed in $\beta$-Lg with maltose and maltitol was collected over a range from $0^{\circ} \mathrm{C}$ to $100^{\circ} \mathrm{C}$ and is depicted in Figure VI-9. The probe exhibits emission bands corresponding to both phosphorescence and delayed 
fluorescence. The intensity of phosphorescence decreased with corresponding increase in delayed fluorescence as expected from a thermally stimulated process.

Figure VI-10 depicts the emission energy as function of temperature in the $\beta$-Lg and $\beta-\mathrm{Lg}$ mixtures. The peak frequency of Erythrosin B varied systematically with temperature. The emission peak frequency was approximately constant at low temperatures and decreased at high temperature. The decrease in the emission energy reflects an increase in the average extent of matrix dipolar relaxation around the excited triplet state prior to emission (Lakowicz, 1999: Richert, 2000). A former study on the effect of maltose and maltitol on $\beta$-Lg films revealed that both maltose and maltitol brought about an enhancement in the emission energy of the pure $\beta$-Lg matrix (chapter 3 and chapter 5 respectively). The mixture with the ratio of 1:5:5 had an emission energy of $14580 \mathrm{~cm}^{-1}$ compared to $14742 \mathrm{~cm}^{-1}$ in maltose $\beta-\mathrm{Lg}$ films and $14691 \mathrm{~cm}^{-1}$ in maltitol $\beta$ Lg films at $0{ }^{\circ} \mathrm{C}$. It is clearly evident that addition of the mixture with a ratio of 1:5:5 resulted in a significant increase in the emission energy as compared to the maltose/ $\beta-\mathrm{Lg}$ films or maltitol/ $\beta$-Lg films. This revealed the dynamic synergy in these films similar to that seen in pure maltose maltitol mixtures (Shirke et al., 2005). An interesting observation was made with respect to the mixtures with the ratios of $1: 3: 7$ and $1: 7: 3$. The emission energies were lower than the maltose/ $\beta-\mathrm{Lg}$ and maltitol/ $\beta-\mathrm{Lg}$ films. Intermolecular associations and hydrogen bonding changes are attributed to the inhibition or enhancement of the dipolar relaxations.

The phosphoresecence bandwidth provides a measure of the range of energetically distinct matrix environments seen by Ery B probe within the amorphous $\beta$ Lg matrix. The bandwidth (the full width half maximum) was nearly constant at low 
temperatures and increased at very high temperatures (Figure VI-11). There was no noticeable change in the bandwidth of $\beta$-Lg films with the different maltose/ maltitol ratios.

\section{Phosphorescence Intensity decays}

The phosphorescence emission transients from Ery B in various maltose and maltitol mixtures were collected as a function of temperature from $0^{\circ} \mathrm{C}$ to $100^{\circ} \mathrm{C}$. All intensity decay transients were well fit using the stretched exponential model (Materials and methods Eq 1) in which the Kohlraush- Williams Watts lifetime and the stretching exponent $\beta$ are the physically meaningful parameters. Such a model has been very effective in fitting the data for Ery B dispersed in amorphous matrices (Pravinata et al., 2005; Lukasik et al, 2005; Nack and Ludescher, 2007; Sundaresan and Ludescher, 2007). The fit lifetimes are plotted versus temperature in Figure VI-12a-e. The lifetimes were high at low temperatures and decreased with increasing temperatures. $\beta$-Lg films with the 50:50 mixtures of maltose and maltitol (1:5:5) exhibited the highest rigidity as shown by the high lifetimes of $0.63 \mathrm{~ms}$ and $0.29 \mathrm{~ms}$ at $0^{\circ} \mathrm{C}$ and $100^{\circ} \mathrm{C}$, respectively. $\beta$ - $\mathrm{Lg}$ films incorporated with other mixtures having ratios of 1:3:7 and 1:7:3 exhibited much lower lifetimes than the pure maltose $/ \beta-\operatorname{Lg}$ or maltitol / $\beta$ - $\operatorname{Lg}$ films.

Figure V1-12e is an illustration of the lifetimes of all the films in the presence of air. There was a slight divergence in the lifetimes of nitrogen and oxygen in all the films. In all these films, oxygen seemed to penetrate the matrix and the lifetimes were lower due to additional quenching processes in the presence of oxygen molecules.

$\beta$ reflects the width of an asymmetric lifetime distribution with smaller values indicating increasing distribution width. Figure VI-13a and VI-13b is a representation of 
$\beta$ versus temperature. We observe that the mixture with the ratio of 1:5:5 had the maximum $\beta$, showing that this mixture lowered the dynamic heterogeneity in the films. There was no noticeable difference in the $\beta$ in various films in the presence of nitrogen and in air.

Figure VI-14 depicts $\mathrm{k}_{\mathrm{Q}}\left[\mathrm{O}_{2}\right]$ as a function of temperature in the various films. There was no significant difference in the permeability between maltose, maltitol and maltose/maltitol mixtures. There were slight variations in between the mixtures but they all were similar within error.

Figure VI-15 is a plot of $k_{\mathrm{TS} 0}$ versus temperature. $\mathrm{k}_{\mathrm{TS} 0}$ ranged from $1533 \mathrm{~s}^{-1}$ in Mixture 1(1:5:5), to $1768 \mathrm{~s}^{-1}$ in Mixture 2 (1:3:7) and $1762 \mathrm{~s}^{-1}$ in Mixture 3 (1:7:3) respectively. In the various $\beta$-Lg films $\mathrm{k}_{\mathrm{TS} 0}$ followed a rank order of Mixture 1(1:5:5) < maltitol / $\beta$ - $\operatorname{Lg}(1: 0: 10)<$ Maltose $/ \beta-\operatorname{Lg}(1: 0: 10)<$ Mixture 3 (1:7:3) < Mixture 2(1:3:7). These findings are very similar to the findings by Shirke et al., (2005) who found $\mathrm{k}_{\mathrm{TS} 0}$ was the least in the pure 50:50 mixture of maltitol maltose when compared to the other sugar, sugar alcohol and mixtures. The $\mathrm{k}_{\mathrm{TS} 0}$ of the 70:30 and the 30:70 mixtures were similar to the pure $\beta-\operatorname{Lg}$ matrix.

\section{$\underline{\text { Discussion }}$}

Previous studies have greatly elucidated the strengths of luminescence spectroscopy as a method in accessing important changes pertinent to the matrix and have proven its efficiency in conveying information about the molecular mobility and permeability. The ensemble of experiments described in this chapter involves the application of phosphorescence spectroscopy to study effects of changes in composition of the $\beta$-Lg matrix. Correlations between phosphorescence measures of mobility and 
changes in the film's macroscopic diffusive property with the effects of different kinds of plasticizers and plasticizer mixtures were investigated.

Two different kinds of functional matrices were generated. 1. A composite film comprised of $\beta$-Lg, PEG 400 and sucrose was prepared and the effects of sucrose on the permeability and mobility of polyethylene glycol 400 plasticized $\beta$-Lg films were investigated. We hypothesized that addition of sucrose would result in an improvement in the oxygen barrier properties of the resultant films. 2. Films were prepared using maltose-maltitol mixtures and $\beta$-Lg. The effect of the dynamic synergy between maltosemaltitol mixtures on the mobility and permeability of $\beta$-Lg films was evaluated.

\section{Composite films with $\beta$ - Lg, PEG 400 and sucrose}

Measurements of probe phosphorescence provide valuable information about the molecular events associated with oxygen permeability of sucrose and PEG plasticized $\beta$ Lg films. Generating innovative composite films through combined use of compatible proteins, sugars and polyols can utilize the functionality of each ingredient in an effective and potentially synergistic manner. Such complexes in many cases can display better functionality than proteins or plasticizers alone.

Hydrophilic plasticizers like polyethylene glycol (PEG), which can interfere with protein chain-to-chain hydrogen bonding, are generally used to improve mechanical properties of protein films. One of the downsides of its plasticizing effects are that it renders the film to be prone to diffusion of small molecules like oxygen (Sothornvit and Krotcha, 2000). This study aims on using the anti-plasticization characteristics of sucrose discussed in detail in chapter (III) to control the oxygen diffusion in amorphous films. Intact films with no signs of phase separation and a concentration similar to that used to 
plasticize films in the food industry was selected as the standard concentration of PEG in the $\beta$ - $\mathrm{Lg}$ films. To this concentration of PEG 400 various concentrations of sucrose were added. We used phosphorescence from Ery B to evaluate changes in matrix composition and the effect of these changes on matrix mobility and permeability. Matrix mobility of the tertiary matrix

Phosphorescence emission energy and intensity from Ery B is sensitive to two distinct modes of mobility in amorphous biomaterials: matrix dipolar relaxations around the excited triplet state and matrix collisions that promote intersystem crossing from the excited $T_{1}$ triplet state to the ground $S_{0}$ singlet state (Pravinata et al., 2005). This study illustrated that both these modes of mobility were modulated in these tertiary matrices.

The energy (wavelength) distribution of a luminescence spectrum is modulated by the molecular structure of the probe and by dipolar interactions between the probe and the matrix (Lakowicz, 1999).

Emission energy decreased with increase in temperature in all the tertiary matrices. The decrease in emission energy with temperature suggested an increase in the dipolar relaxation rate. It was clearly evident that emission energy was greatly enhanced upon addition of sucrose to PEG plasticized $\beta$-Lg films. This phenomenon may be due to complex interactions between $\beta$-Lg, sucrose and PEG 400, which resulted in a decrease in the relaxation mechanisms.

Sucrose addition at molar ratios of 10 moles in PEG plasticized $\beta$-Lg films (1:10:10) brought about a noticeable increase in the lifetime. It is clearly evident that addition of sucrose conferred rigidity to the matrix. 
Analysis of the temperature dependence of the lifetime indicates that the rate constant for intersystem crossing to the ground state $\left(\mathrm{k}_{\mathrm{TS} 0}\right)$ is sensitive to the physical state of the amorphous matrix. The magnitude of $\mathrm{k}_{\mathrm{TS} 0}$, the collisional quenching constant, is sensitive to internal factors related to vibrational coupling between the excited $\mathrm{T}_{1}$ state and the $\mathrm{S}_{0}$ ground state (Papp and Vanderkooi, 1989) as well as external factors related to dissipation of the vibrational energy of the excited probe into the surrounding matrix (Fischer et al., 2002). Since the efficiency of this vibrational coupling is related to the overall mobility of the matrix, the magnitude of $\mathrm{k}_{\mathrm{TS} 0}$ provides a direct measure of matrix mobility (Pravinata et al., 2005). Matrix mobility decreased with increase in sucrose concentration in the PEG plasticized $\beta$-Lg films. Plots comparing the matrix mobility of various tertiary matrices showed the existence of a dynamic synergy between sucrose and PEG 400. This synergy was responsible for drastic decline in the mobility of the matrix. A study by Allison et al. (1999) revealed that glucose alone failed to protect the enzymes but worked well in combination with PEG. When they monitored the hydrogen bonding interactions of lysozyme in the presence of glucose and PEG, they found that PEG alone did not hydrogen bond with the protein and was preferentially excluded. But glucose formed direct hydrogen bonding interactions with the protein and the synergistic interactions between glucose and PEG resulted in offering superior protection during lyophilization.

A study by Cicerone et al. (2003) revealed that judicious use of appropriate plasticizers could have dramatic positive effects on the stabilization of biological agents. The study looked into the effect of enzyme stability of peroxidase from horseradish and revealed that addition of inulin and glycerol in a concentration dependent manner 
dramatically offered substantial improvements in the storage lifetime. It was seen that inulin plays a role in linking the plasticizer to the host glass either by enhancing the compatibility or coupling the dynamics of plasticizer to the host.

A study by Poirier-Brulez et al. (2006) on the effect of the plasticizer water and sucrose on starch revealed that addition of sucrose greatly reduced the $\beta$ relaxations and shifted the relaxations to higher temperatures. Carpenter et al. (1993) revealed that in the presence of low concentrations of glucose or trehalose (which alone provided minimal protection) and $1 \%$ PEG (wt/vol), almost full enzyme activity of lactate dehydrogenase and phospho-fructokinase was recovered after freeze-drying and rehydration.

Noticeable decreases in matrix mobility (lower $\mathrm{k}_{\mathrm{TS} 0}$ ) and dipolar relaxations (Increase in emission energy) clearly indicate that polyethylene glycol and sucrose exhibit synergistic interactions resulting in a decline in the mobility of the $\beta-\operatorname{Lg}$ matrix. Oxygen permeability in tertiary films

Comparison of the lifetimes both in the presence and absence of air revealed that addition of 100 moles of sucrose in PEG plasticized $\beta$-Lg films was sufficient to control the permeability in the matrix. The dispersion of lifetimes as depicted by the $\beta$ values followed a trend very close to the lifetime. As shown in our previous study, the PEG plasticized $\beta$-Lg films portrayed a decline in $\beta$ values in the presence of air indicating increases in the width of the distribution of lifetimes. But addition of sucrose led to a reduction in the width of the distribution of lifetimes (reduced heterogeneity). This increase in homogeneity can be attributed to sucrose forming favorable hydrogen bonding interactions, which not only conferred rigidity to the matrix but also reduced the heterogeneity. 
In an effort to quantify the extent of oxygen quenching as a function of temperature, time-resolved intensity decays were measured from $0^{\circ} \mathrm{C}$ to $100^{\circ} \mathrm{C}$ in films equilibrated against nitrogen and against air. The oxygen quenching rate $\mathrm{k}_{\mathrm{Q}}\left[\mathrm{O}_{2}\right]$ was calculated directly from the difference between $\mathrm{k}_{\mathrm{P}}$ in the presence of air and nitrogen (Eq. 5, Materials and methods). Since the oxygen quenching rate is the product of $\mathrm{k}_{\mathrm{Q}}$, which is proportional to the oxygen diffusion rate through the matrix, and $\left[\mathrm{O}_{2}\right]$, the concentration of oxygen in the protein film, is proportional to the permeability of oxygen through the protein film (Nack \& Ludescher, 2006). It is clearly evident that addition of sucrose brought about a phenomenal decline in the oxygen-quenching rate of PEG plasticized $\beta$ Lg films. Studies in the past have revealed that addition of an appropriate plasticizer causes several changes in the physical and dynamic mechanical properties, particularly reduction in small molecule diffusivity (Sefcik et al., 1983).

A general overview of molecular mobility in amorphous materials is related to the amount of free volume, which comes from a sub-optimal efficiency of molecular packing in these disordered solids. Judicious use of plasticizers can drastically improve the permeability of edible films by mainly affecting the availability of free volume. But free volume theory is not applicable in a purely hydrogen bonded matrix. In this matrix a random network of hydrogen bonds primarily holds the amorphous protein matrix together. The presence of PEG enhances the hydrogen bonding sites marked by fewer internal constraints. Complex hydrogen bonding interactions between sucrose- $\beta-\mathrm{Lg}$ may constrain the local molecular mobility and facilitate synergistic interactions (PEG and sucrose). 
Although studies in the past have greatly elucidated the protective effects of the synergy between sugar- polyol mixtures on protein stabilization, it is clearly evident from the present study that sucrose addition has a great effect on the mobility and permeability of the PEG plasticized $\beta$-Lg films. Detailed investigations still need to be done to help us gain a better understanding of the benefits of this synergism between PEG and sucrose on $\beta$ - $\mathrm{Lg}$ films and to understand the mechanisms underlying this synergy.

\section{Influence of maltose -maltitol mixtures on the $\beta-\mathrm{Lg}$ matrix.}

Actual food systems seldom contain a single component. A complex mixture of low molecular weight sugars, polyols, amino acids, fatty acids etc are usually present. These low molecular weight compounds interact with each other and with different macromolecules and such interactions give rise to effects, which are extremely difficult to predict.

Experimental evidence points to the fact that presence of small amounts of diluents in a particular composition exhibit anti-plasticizing effects and decreases free volume. Previous studies by Shirke et al. (2005) have established the dynamic synergy of maltose maltitol mixtures whereby there was reduction in the matrix mobility in these sugars-polyol mixtures. The present study mainly aimed at utilizing this property to generate functional films that can be less permeable to atmospheric oxygen and correlate changes in mobility to permeability. We have used phosphorescence from Erythrosin B to evaluate how these functional films exhibit differences in matrix mobility and permeability. Our estimate of the extent to which these mixtures behave is based on the plots of the spectroscopic parameters plotted vs temperature 
Specific interactions between maltose and maltitol in a specific ratio (50:50 maltose and maltitol) decreased the ability of the matrix to vibrationally dissipate the triplet state energy of Erythrosin B by decreasing the mobility of the amorphous matrix which is clearly revealed by a noticeable decrease in the $\mathrm{k}_{\mathrm{TS} 0}$. This connection was clearly illustrated by the plots of $\mathrm{k}_{\mathrm{TS} 0}$ versus molar ratio of sugar/sugar alcohol (Figure VI-16). This ratio can also be judged as a optimum ratio whereby we see a reduction in the dipolar relaxation as demonstrated by the increase in the peak frequency (Figure VI17).

Spectroscopic measures indicated that the rate and extent of dipolar relaxation and rate of collisional quenching decreased at a ratio of 1:5:5 compared to maltose / $\beta$ - $\mathrm{Lg}$ films or maltitol / $\beta$-Lg films (chapter III and IV). Previous studies have indicated that a decrease in the mobility seems to occur in mixtures of molecules of similar sizes or structures including glycerol in sucrose, (You and Ludescher, 2007), trehalose in glycerol (Anopchenko et al., 2006); maltose with maltitol (Shirke et al., 2005) and lactose with lactitol (Shirke et al., 2006). A model by Liu et al. (1990) viewed the glass as a heterogeneous system containing both high density -low energy and low density- highenergy regions referred to as "islands of mobility" by Johari (1985) with the high-energy regions having greater molecular mobility. The presence of certain diluents tends to relax the high-energy regions thereby allowing rearrangement of the polymer chains and resulting in densification of the glass.

Siew et al. (1999) reviewed that antiplasticization has been hypothesized to involve a combination of several factors. These include a possible reduction in free volume of the polymeric system, polymer-diluent interactions which create steric 
hindrance and decrease segmental mobility. Thus, the presence of rigid plasticizer molecules adjacent to polar groups of the polymer may cause stiffening. You and Ludescher (2007) suggest that free volume may not be a suitable explanation for the phenomenon of anti-plasticization in a hydrogen-bonded matrix. They attribute the antiplasticization of sucrose by glycerol to the presence of stronger, hydrogen bonding interactions in sucrose, in the presence of low concentrations of glycerol.

The suitable explanation for the decreases in mobility in the mixture with a ratio of 1:5:5 is as follows: In this mixture the hydrogen bonding associations between the sugar/polyol mixture and $\beta$-Lg lead to rigidification of the polymer, which is evident, by the decrease in matrix mobility.

Anti-plasticization can also be attributed to a $\beta$ - suppression effect which is ascribed to specific interactions between polymer and diluent molecules which restrict or prevent local rotations of side groups (Butzbach and Wendorff, 1991; Ngai et al., 1991).

It is also interesting to note that the dispersion of lifetimes followed a trend similar to the lifetimes. There was a significant increase in both the lifetime and $\beta$ in the presence of the mixtures with a ratio of 1:5:5. Since the dispersion in lifetimes reflects dispersion in the underlying photophysical rate constants this behavior indicates that not only the mobility decreases but it also results in a decline in the dispersion of lifetimes (portrayed by increases in $\beta$ ).

Modification of the ratios by increasing the individual sugars or sugar alcohols created 1:7:3 and 1:3:7 mixtures. These results were a contrast to what was observed in the ratio of 1:5:5. The resultant mixtures had higher mobility when compared to maltose/ 
$\beta$-Lg or maltitol / $\beta$-Lg films as portrayed by decreases in emission energy and increases in mobility $\mathrm{k}_{\mathrm{TS} 0}$.

It is evident that the anti-plasticization by these mixtures is highly concentration dependent; as appropriately said by Sears and Darby (1982), anti-plasticization occurs over a specific range of diluent concentration. These sugar / polyol mixtures had a complex concentration dependent effect on the $\beta-\operatorname{Lg}$ matrix. These trends are interesting results whereby we notice that maltose and maltitol when added in equal amounts antiplasticize the $\beta$-Lg matrix whereas in unequal amounts plasticize the matrix.

Antiplasticization of some synthetic polymers by certain diluents was accompanied by substantial reductions in permeability to gases (e.g., helium, carbon dioxide and methane), consistent with reduced mobility in the glass (Sefcik et al., 1983; Maeda and Paul, 1987. But in the present study despite the disparity in the $\mathrm{k}_{\mathrm{TS} 0}$ values, the permeabilities $\mathrm{K}_{\mathrm{Q}}\left[\mathrm{O}_{2}\right]$ of films with maltose, maltitol and their respective mixtures were very similar. It is evident that the restrictions in matrix mobility due to antiplasticization effects are insufficient to control the segmental motion necessary for oxygen transport. While some mobility is apparently de-activated by the additive mixtures but cessation in the mobility is in sufficient to induce necessary changes with respect to permeability. 


\section{Conclusions}

The amorphous state of biomaterials is thought to be of critical importance in controlling their stability over long periods. Biophysical studies of the effect of the complex plasticizers on the $\beta$-Lg matrix have provided information about the concentration dependence of permeability and mobility. Certain active modes of mobility are halted or dampened upon addition of various plasticizers. Conversely certain modes of mobility are reactivated by increase in plasticization and permeability. Through complex interactions, addition of other small molecules and synergies may enable man to control and effectively manipulate the functionality of edible films. 


\section{$\underline{\text { References }}$}

Allison, S. D., Chang, B., Randolph, T. W., and Carpenter, J. F. (1999). Hydrogen Bonding between Sugar and Protein Is Responsible for Inhibition of DehydrationInduced Protein Unfolding. Arch. Biochem. Biophys. 365, 289-298.

Anopchenko, A., Psurek, T., VanderHart, D., Douglas, J. F., and Obrzut, J. (2006). Dielectric study of the antiplasticization of trehalose by glycerol. Phys.Rev.E: Stat., Nonlinear, Soft Matter Phys. 74, 031501/1-031501/10.

Butzbach, G. D. and Wendorff, J. H. (1991). Polycarbonate-poly(methyl methacrylate) blends: the role of molecular interactions on miscibility and antiplasticization. Polymer. 32, 1155-1159.

Carpenter, J. F., Prestrelski, S. J., and Arakawa, T. (1993). Separation of freezing- and drying-induced denaturation of lyophilized proteins using stress-specific stabilization. I. Enzyme activity and calorimetric studies. Arch. Biochem. Biophys. 303, 456-464.

Cicerone, M. T., Tellington, A., Trost, L., and Sokolov, A. (2003). Substantially improved stability of biological agents in dried form: the role of glassy dynamics in preservation of biopharmaceuticals. BioProcess Int. 1, 36-38, 40, 42, 44, 46-47.

Debeaufort, F., Quezada-Gallo, J., and Voilley, A. (2002). Edible films and coatings as aroma barriers. Protein-Based Films Coat. 579-600.

Debeaufort, F. and Voilley, A. (1995). Methyl cellulose-based edible films and coatings. Part I. Effect of plasticizer content on water and 1-octen-3-ol sorption and transport. Cellulose (London). 2, 205-213.

Duchowicz, R., Ferrer, M. L., and Acuña , A. U. (1998). Kinetic Spectroscopy of Erythrosin Phosphorescence and Delayed Fluorescence in Aqueous Solution at Room Temperature. Photochemistry and Photobiology. 68, 494-501.

Fennema, O. R., Kamper, S. L., and Kester, J. J. (1987). Edible film barrier resistant to water vapor transfer. PCT Int.Appl. 86-US2641; 85-809531, 29.

Fischer, C. J., Gafni, A., Steel, D. G., and Schauerte, J. A. (2002). The triplet-state lifetime of indole in aqueous and viscous environments: significance to the interpretation of room temperature phosphorescence in proteins. J. Am. Chem. Soc. 124, 10359-10366.

Gaudin, S., Lourdin, D., Forssell, P. M., and Colonna, P. (2000/9). Antiplasticisation and oxygen permeability of starch-sorbitol films. Carbohydrate Polymers. 43, 33-37. 
Gaudin, S., Lourdin, D., Le Botlan, D., Ilari, J. L., and Colonna, P. (1999). Plasticisation and mobility in starch-sorbitol films. Journal of Cereal Science. 29, 273-284.

Johari, G. P. (1985). Low-frequency molecular relaxations in disordered solids. J. Chim. Phys. Phys. -Chim. Biol. 82, 283-291.

Lakowicz, J. R. (1999). Principles of fluorescence spectroscopyKluwer Academic/Plenum Press, Dordrecht/New York.

Lettinga, M. P., Zuilhof, H., and Van Zandvoort, M. A. M. J. (2000). Phosphorescence and fluorescence characterization of fluorescein derivatives immobilized in various polymer matrices. Physical Chemistry Chemical Physics. 2, 3697-3707.

Lindsey, C. P. and Patterson, G. D. (1980). Detailed comparison of the Williams-Watts and Cole-Davidson functions. J. Chem. Phys. 73, 3348-3357.

Liu, Y., Roy, A. K., Jones, A. A., Inglefield, P. T., and Ogden, P. (1990). An NMR study of plasticization and antiplasticization of a polymeric glass. Macromolecules. 23, 968-977.

Lukasik, K. V. and Ludescher, R. D. (2005). Effect of plasticizer on dynamic site heterogeneity in cold-cast gelatin films. Food Hydrocoll. 20, 88-95.

Lukasik, K. V. and Ludescher, R. D. (2005). Molecular mobility in water and glycerol plasticized cold- and hot-cast gelatin films. Food Hydrocoll. 20, 96-105.

Maeda, Y. and Paul, D. R. (1987). Effect of antiplasticization on gas sorption and transport. III. Free volume interpretation. J.Polym.Sci., Part B: Polym.Phys. 25, 1005-1016.

McHugh, T. H. and Krochta, J. M. (1994). Milk-protein-based edible films and coatings. Food Technology (Chicago, IL, United States). 48, 97-103.

Nack, T. J. and Ludescher, R. D. (2006). Molecular Mobility and Oxygen Permeability in Amorphous Bovine Serum Albumin Films. Food Biophysics. 1557-1858.

Ngai, K. L., Rendell, R. W., Yee, A. F., and Plazek, D. J. (1991). Antiplasticization effects on a secondary relaxation in plasticized glassy polycarbonates. Macromolecules. 24, 61-67.

Papp, S. and Vanderkooi, J. M. (1989). Tryptophan phosphorescence at room temperature as a tool to study protein structure and dynamics. Photochem. Photobiol. 49, 775-784. 
Park, H. J., Weller, C. L., Vergano, P. J., and Testin, R. F. (1993). Permeability and mechanical properties of cellulose-based edible films. J. Food Sci. 58, 1361-4, 1370.

Poirier-Brulez, F., Roudaut, G., Champion, D., Tanguy, M., and Simatos, D. (2006). Influence of sucrose and water content on molecular mobility in starch-based glasses as assessed through structure and secondary relaxation. Biopolymers. 81, 63-73.

Pravinata, L. C., You, Y., and Ludescher, R. D. (2005). Erythrosin B Phosphorescence Monitors Molecular Mobility and Dynamic Site Heterogeneity in Amorphous Sucrose. Biophys. J. 88, 3551-3561.

Prestrelski, S. J., Tedeschi, N., Arakawa, T., and Carpenter, J. F. (1993). Dehydrationinduced conformational transitions in proteins and their inhibition by stabilizers. Biophysical Journal. 65, 661-671.

Richert, R. (2000). Triplet state solvation dynamics: Basics and applications. J. Chem. Phys. 113, 8404-8429.

Rischel, C., Madsen, J. C., Andersen, K. V., and Poulsen, F. M. (1994). Comparison of Backbone Dynamics of apo- and holo-Acyl-Coenzyme A Binding Protein Using 15N Relaxation Measurements. Biochemistry (N. Y. ). 33, 13997-14002.

Sears, J. K. and Darby, J. R. (1982). The Technology of Plasticizers. 1166.

Sefcik, M. D., Schaefer, J., May, F. L., Raucher, D., and Dub, S. M. (1983). Diffusivity of gases and main-chain cooperative motions in plasticized poly(vinyl chloride). J.Polym.Sci., Polym.Phys.Ed. 21, 1041-1054.

Seow, C. C., Cheah, P. B., and Chang, Y. P. (1999). Antiplasticization by water in reduced-moisture food systems. J. Food Sci. 64, 576-581.

Shirke, S., Takhistov, P., and Ludescher, R. D. (2005). Molecular Mobility in Amorphous Maltose and Maltitol from Phosphorescence of Erythrosin B. J. Phys. Chem. B. 109, 16119-16126.

Shirke, S. and Ludescher, R. D. (2005). Dynamic site heterogeneity in amorphous maltose and maltitol from spectral heterogeneity in erythrosin B phosphorescence. Carbohydr. Res. 340, 2661-2669.

Shirke, S. and Ludescher, R. D. (2005). Molecular mobility and the glass transition in amorphous glucose, maltose, and maltotriose. Carbohydr. Res. 340, 2654-2660. 
Shirke, S., Takhistov, P., and Ludescher, R. D. (2005). Molecular Mobility in Amorphous Maltose and Maltitol from Phosphorescence of Erythrosin B. J Phys Chem B. 109, 16119-16126.

Shirke, S., You, Y., and Ludescher, R. D. (2006). Molecular mobility and dynamic site heterogeneity in amorphous lactose and lactitol from erythrosin $B$ phosphorescence. Biophys. Chem. 123, 122-133.

Simon-Lukasik, K. V. and Ludescher, R. D. (2004/7). Erythrosin B phosphorescence as a probe of oxygen diffusion in amorphous gelatin films. Food Hydrocolloids. 18, 621-630.

Sothornvit, R. and Krochta, J. M. (2000). Plasticizer effect on oxygen permeability of beta-lactoglobulin films. J. Agric. Food Chem. 48, 6298-6302.

Torres, J. A. (1994). Edible films and coatings from proteins. IFT Basic Symp.Ser. 9, 467-507.

van den Bogaart, G., Hermans, N., Krasnikov, V., de Vries, A. H., and Poolman, B. (2007). On the decrease in lateral mobility of phospholipids by sugars. Biophys. J. 92, 1598-1605.

Williams, G. and Watts, D. C. (1970). Non-symmetrical dielectric relaxation behavior arising from a simple empirical decay function. Trans.Faraday Soc. 66, 80-85.

You Yumin and Ludescher. R. D. (December, 2007). The Effect of Glycerol on Molecular Mobility in Amorphous Sucrose Detected by Phosphorescence of Erythrosin B. Journal Food Biophysics. 2, 133-145. 
Figure VI-1

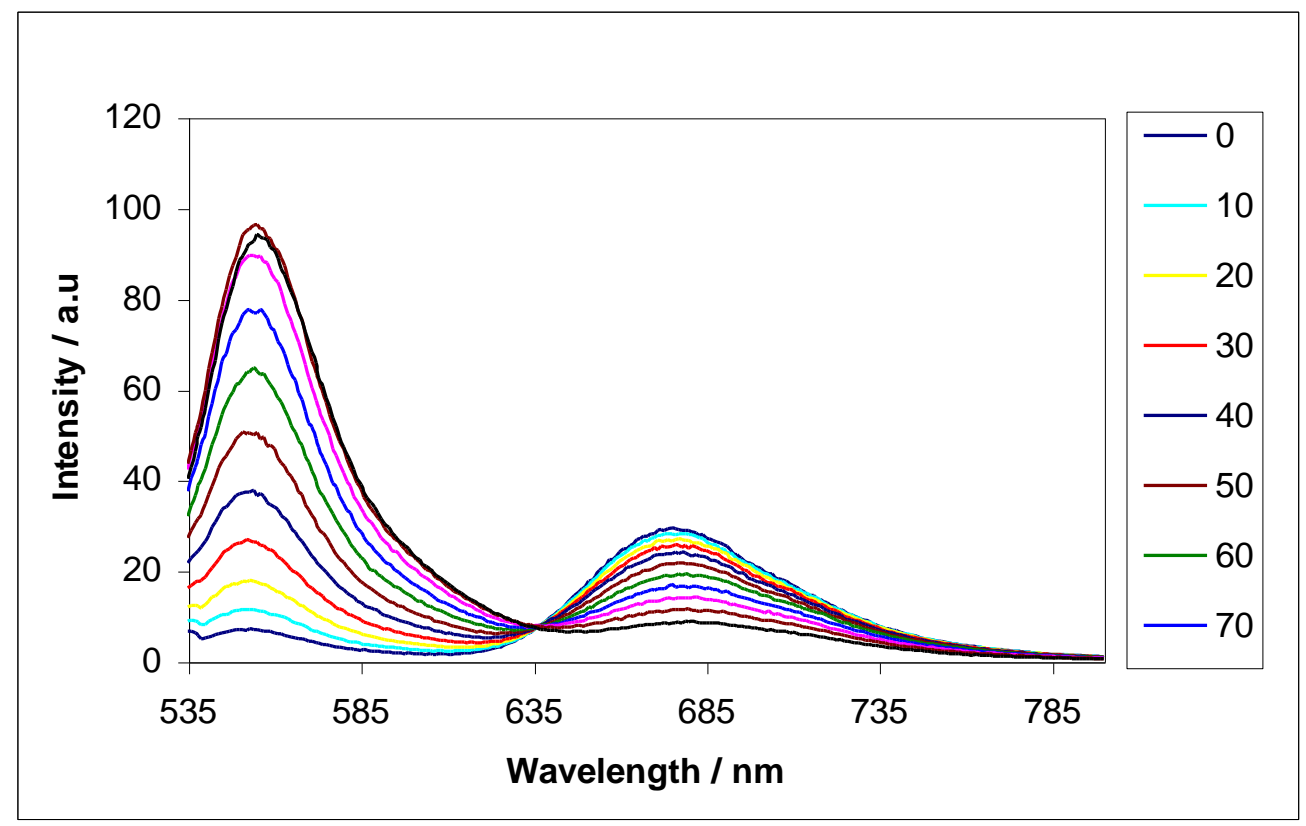

Figure VI-1: The effect of temperature on the delayed emission spectra of Erythrosin B dispersed in $\beta-\mathrm{Lg} / \mathrm{PEG} / \mathrm{suc}$ films ( $\beta-\mathrm{Lg} / \mathrm{PEG} /$ suc at a ratio of 1:10:10). 
Figure VI-2

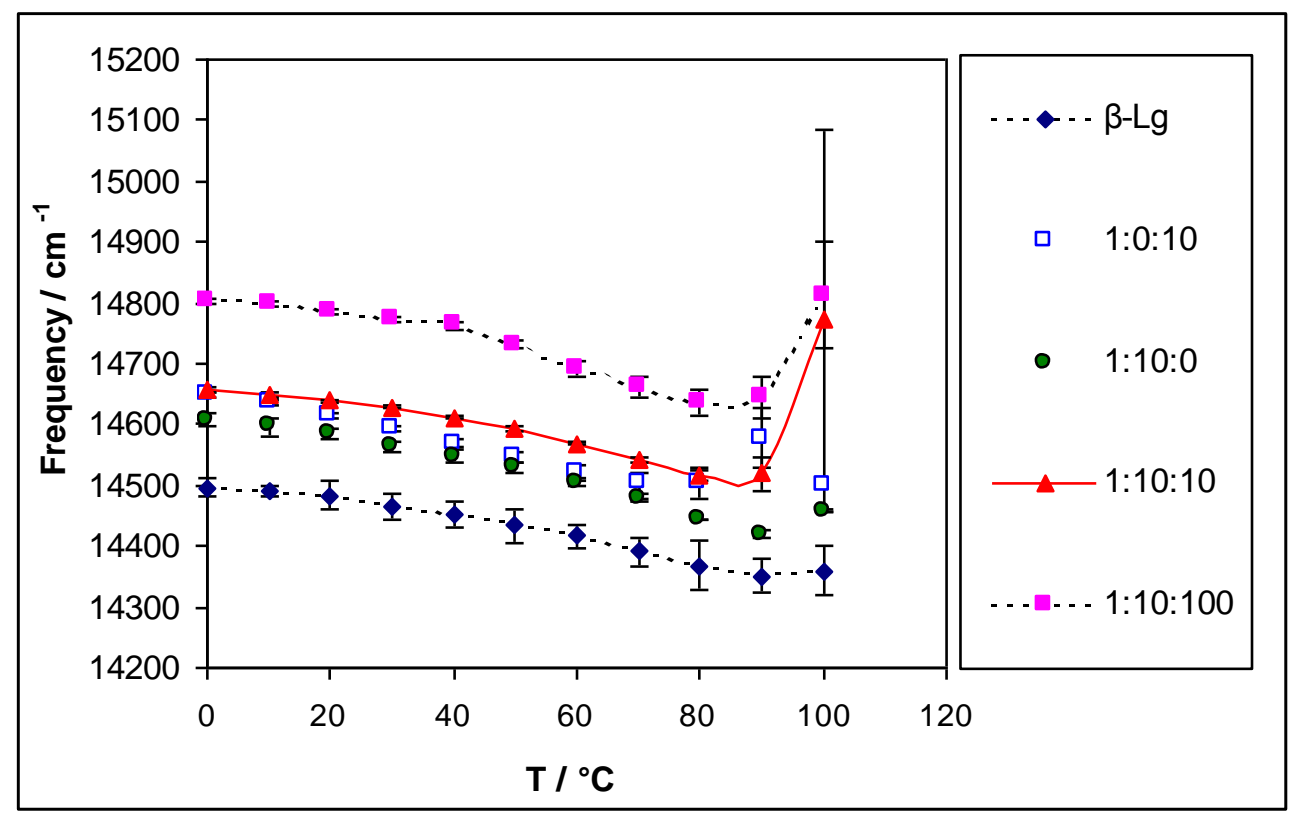

Figure VI-2: The effect of temperature on the emission maximum for phosphorescence from Erythrosin B in $\beta$-Lg/ PEG/ suc films (The ratios in the text box refer to the ratio of $\beta$ Lg: PEG: suc in the films).

Figure VI-3

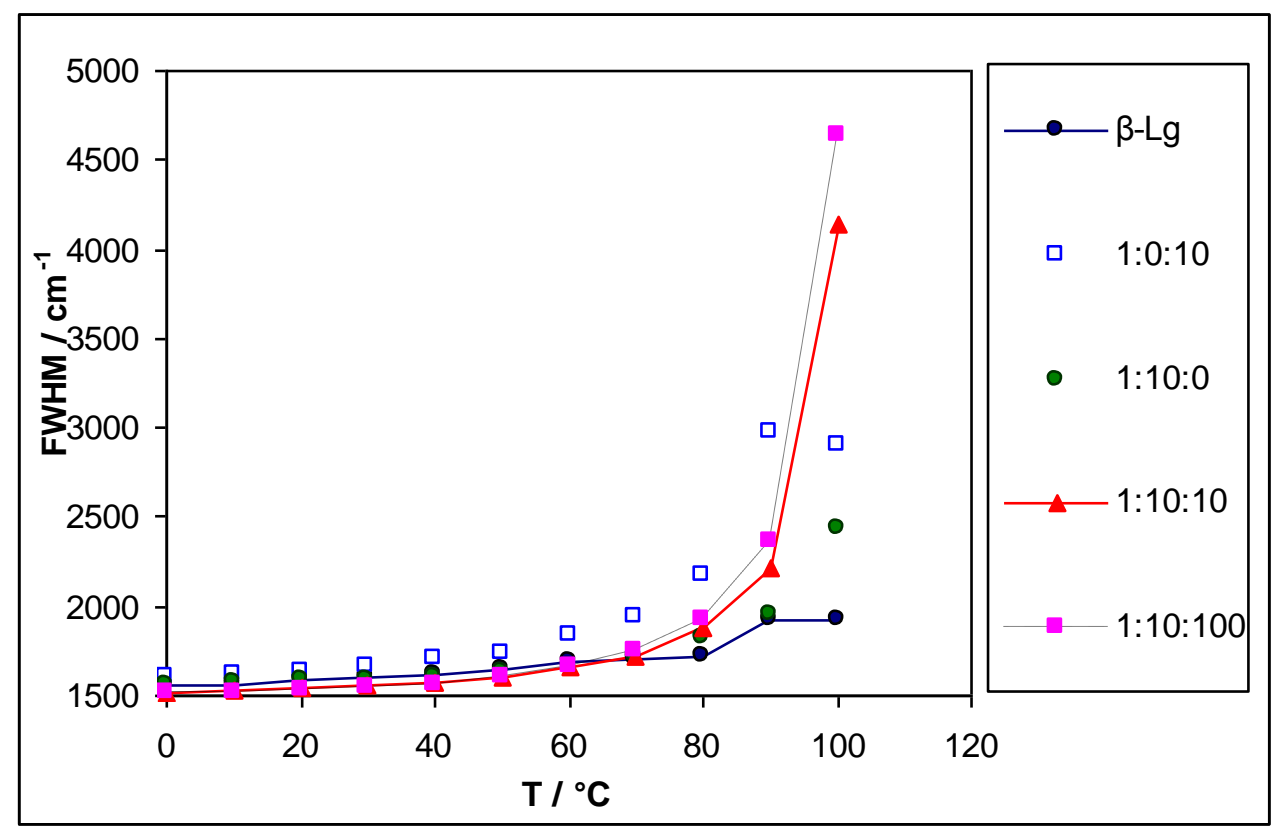

Figure VI-3: The effect of temperature on the bandwidth for phosphorescence from Erythrosin B in $\beta$-Lg/ PEG/ suc films (The ratios in the text box refer to the ratio of $\beta$-Lg : PEG : suc in the films). 
Figure VI-4a

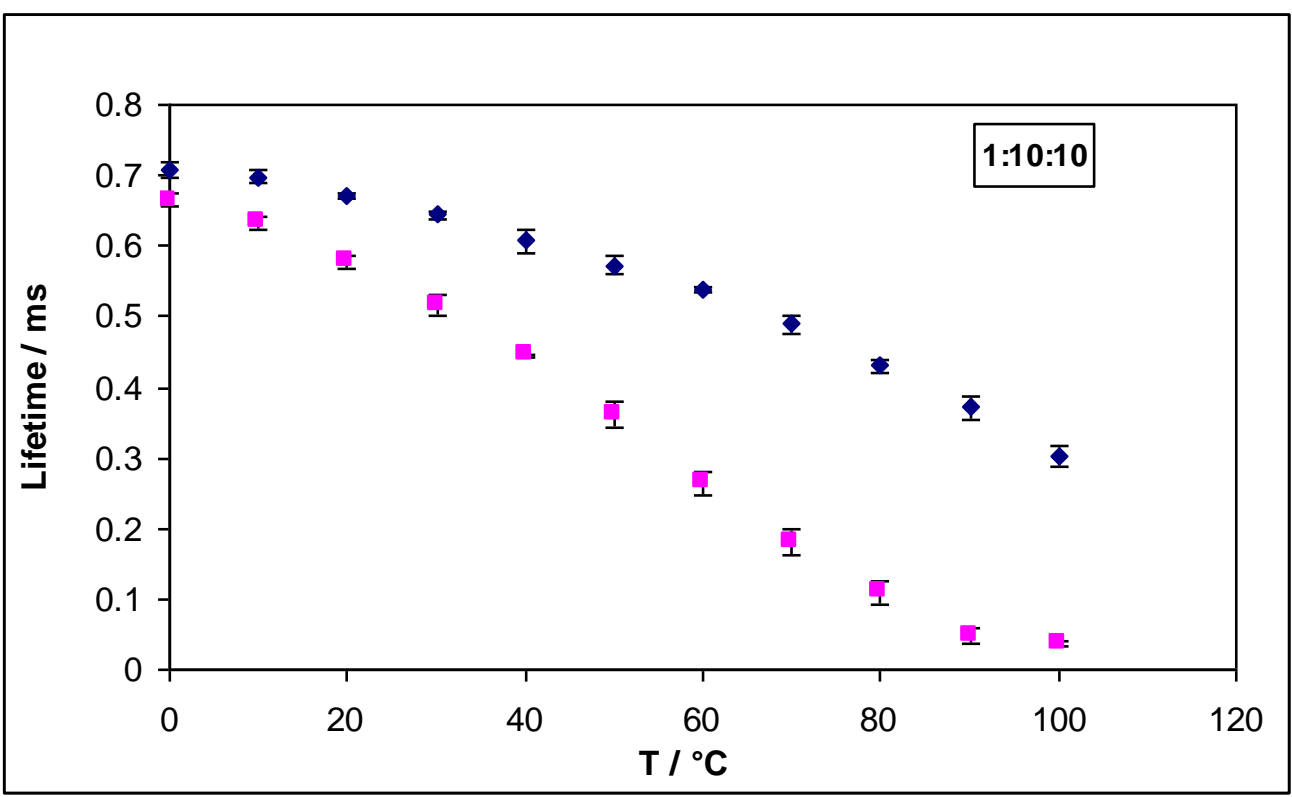

Figure VI-4b

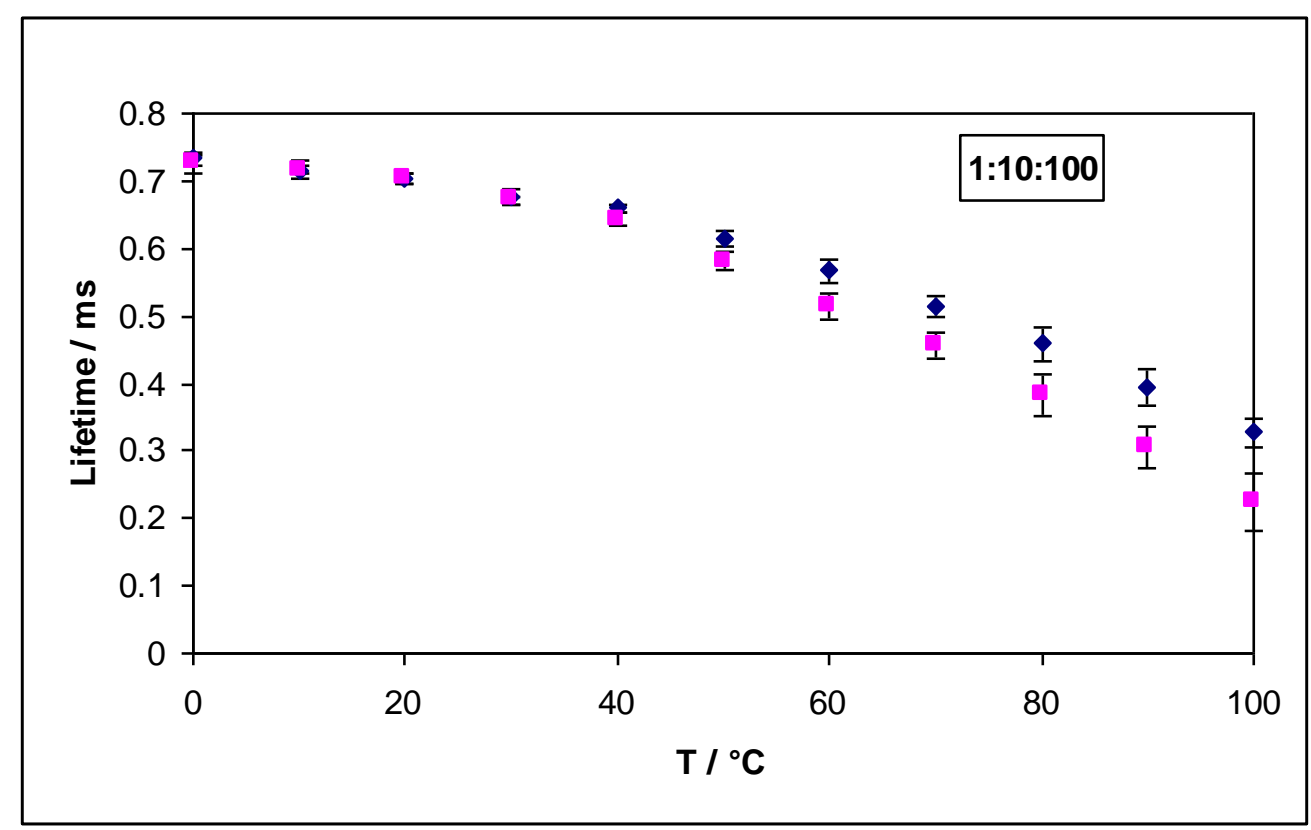

Figure VI-4a, VI-4b The effect of temperature on the lifetime for Erythrosin B dispersed in amorphous $\beta$ $\mathrm{Lg}$ / PEG-suc films in various molar concentrations of sucrose equilibrated against nitrogen $(\bullet)$ and against air $(\square)$; the lifetime was determined from analysis using a stretched exponential decay function. The text box in the graph refers to the molar ratio of $\beta$-Lg:PEG:suc. 
Figure VI-4c

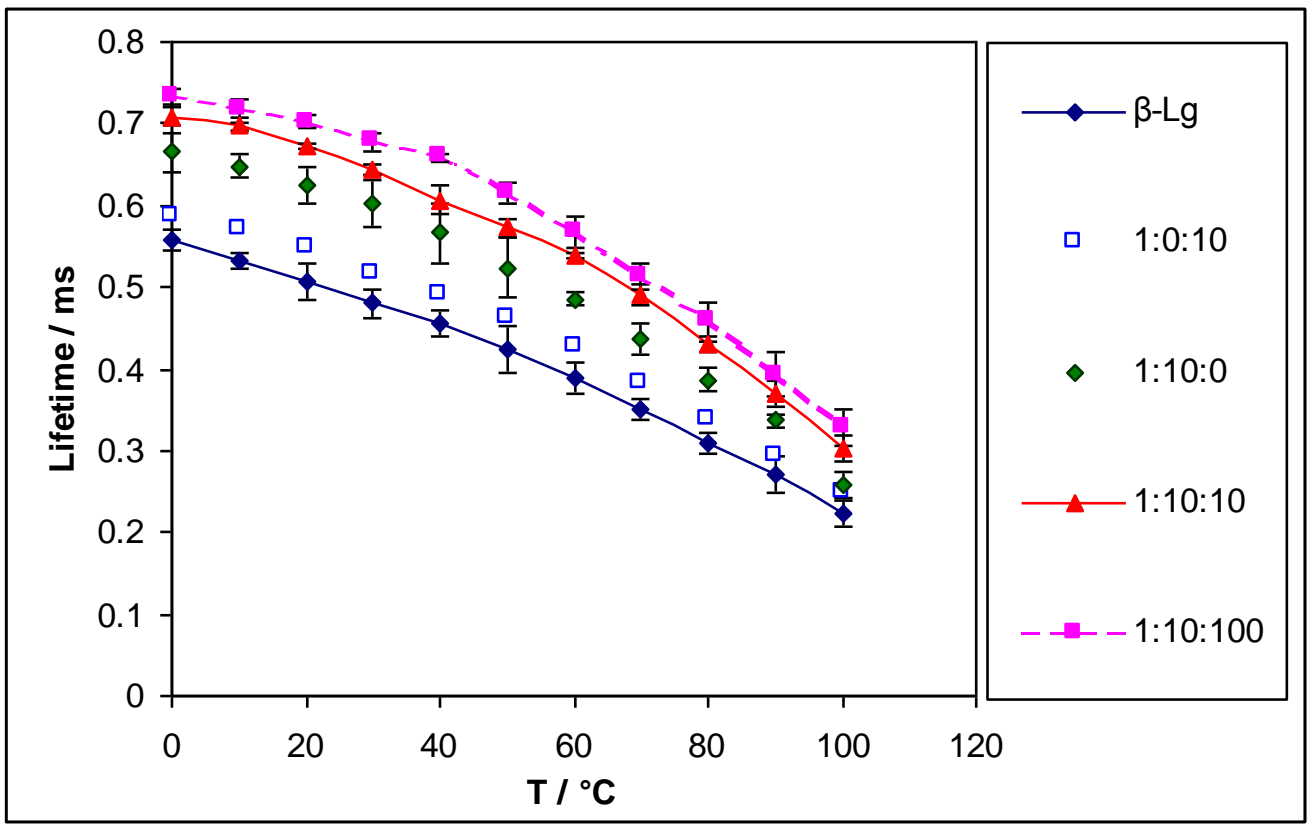

Figure VI-4c Comparison plot depicting the effect of temperature on the lifetime for Erythrosin $\mathrm{B}$ dispersed in $\beta$ - $\mathrm{Lg} / \mathrm{PEG} / \mathrm{suc}$ films equilibrated against nitrogen (The ratios in the text box refer to the ratio of $\beta-\mathrm{Lg}$ : PEG : suc in the films).

Figure VI-4d

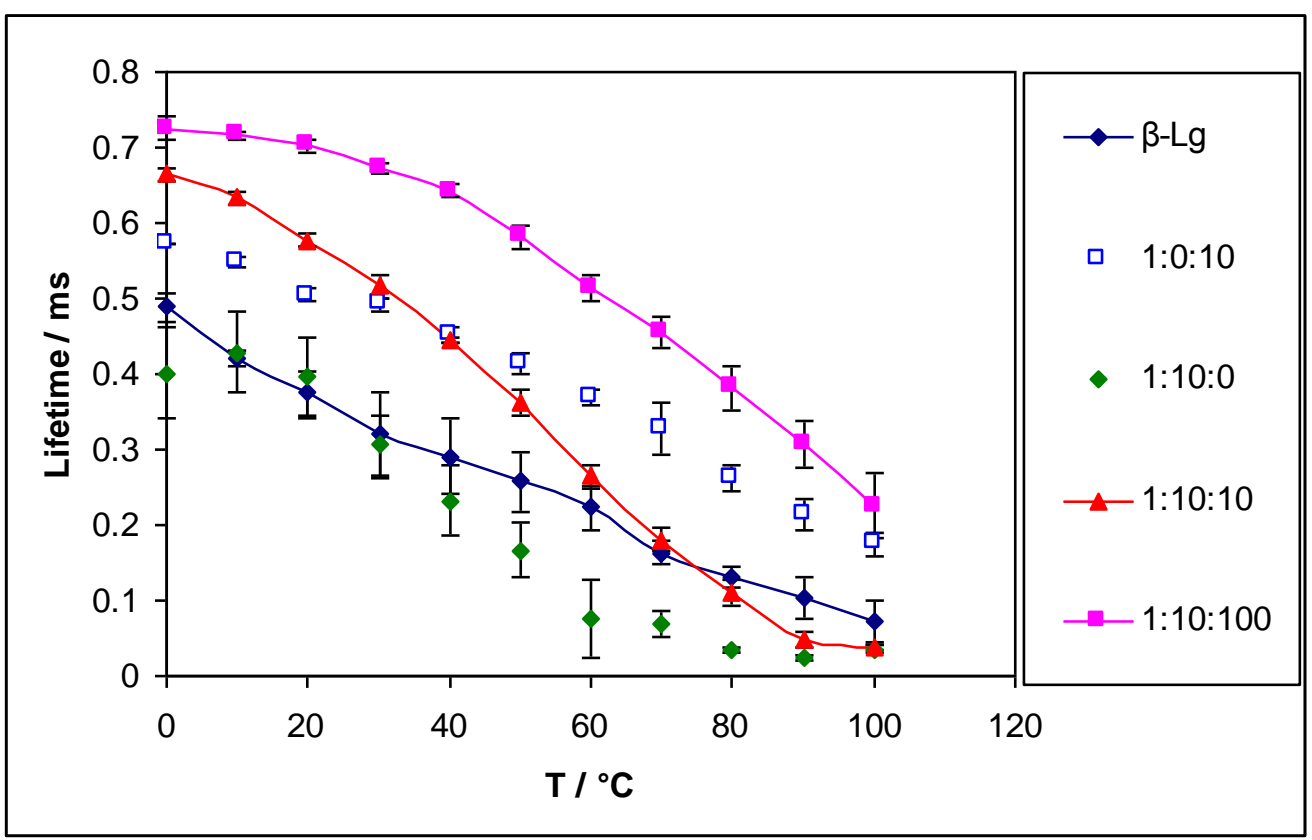

Figure VI-4d: Comparison plot depicting the effect of temperature on the lifetime for Erythrosin $\mathrm{B}$ dispersed in $\beta$-Lg/ PEG/ suc films equilibrated against air (The ratios in the text box refer to the ratio of $\beta-\mathrm{Lg}$ : PEG : suc in the films). 
Figure VI-5a

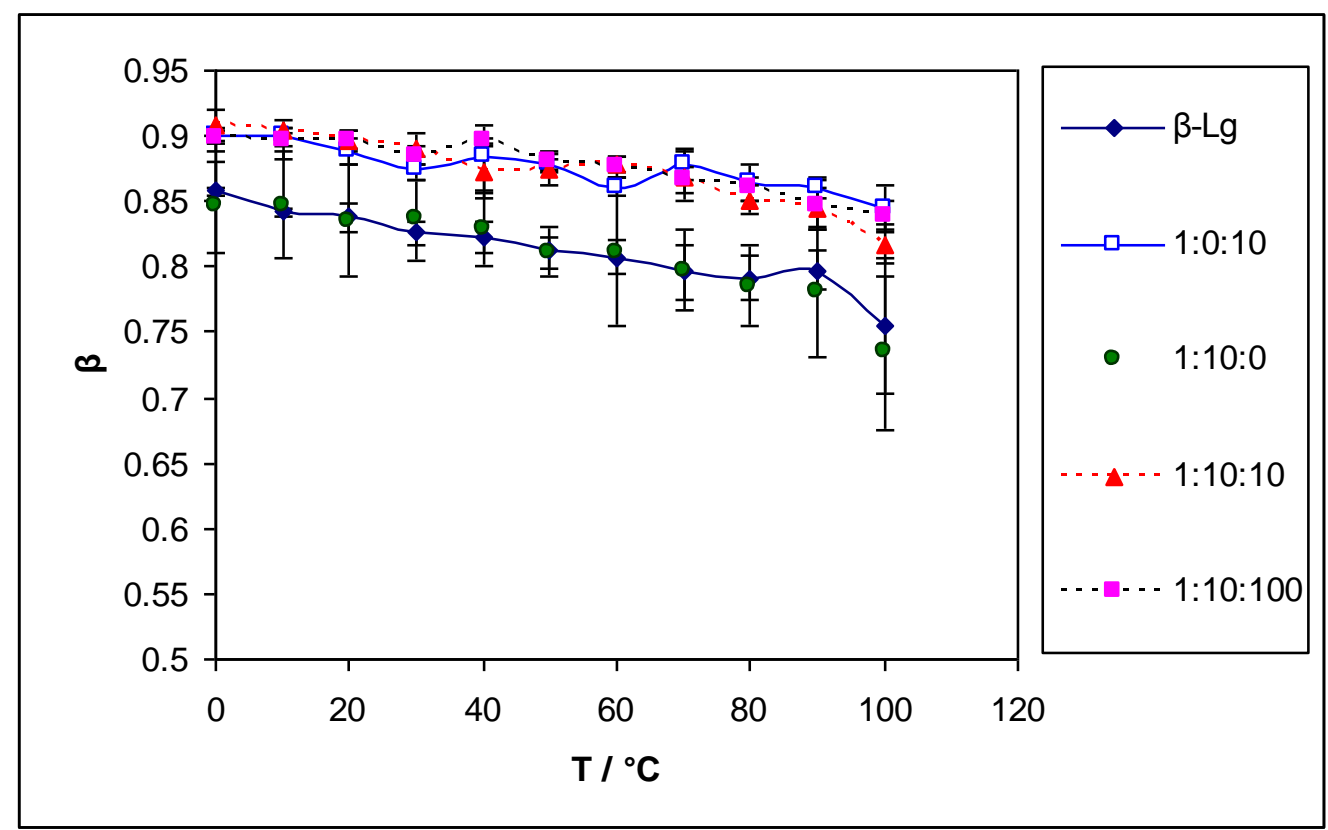

Figure VI-5a: Beta as a function of temperature in $\beta-\mathrm{Lg} / \mathrm{PEG} / \mathrm{suc}$ films equilibrated against nitrogen (The ratios in the text box refer to the ratio of $\beta$-Lg : PEG : suc in the films).

\section{Figure VI-5b}

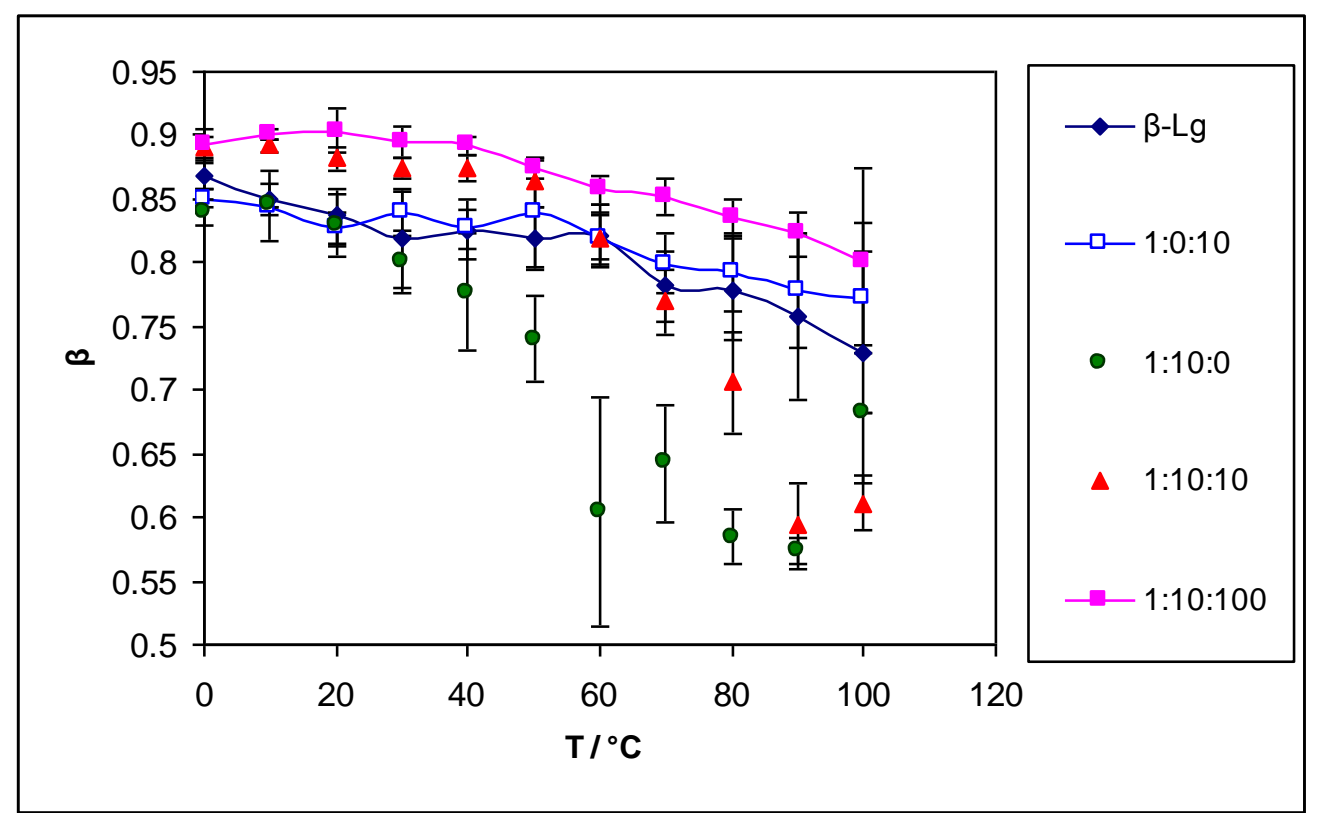

Figure VI-5b: Beta as a function of temperature in $\beta-\mathrm{Lg} / \mathrm{PEG} /$ suc films equilibrated against oxygen (The ratios in the text box refer to the ratio of $\beta-\mathrm{Lg}$ : PEG : suc in the films). 


\section{Figure VI-6}

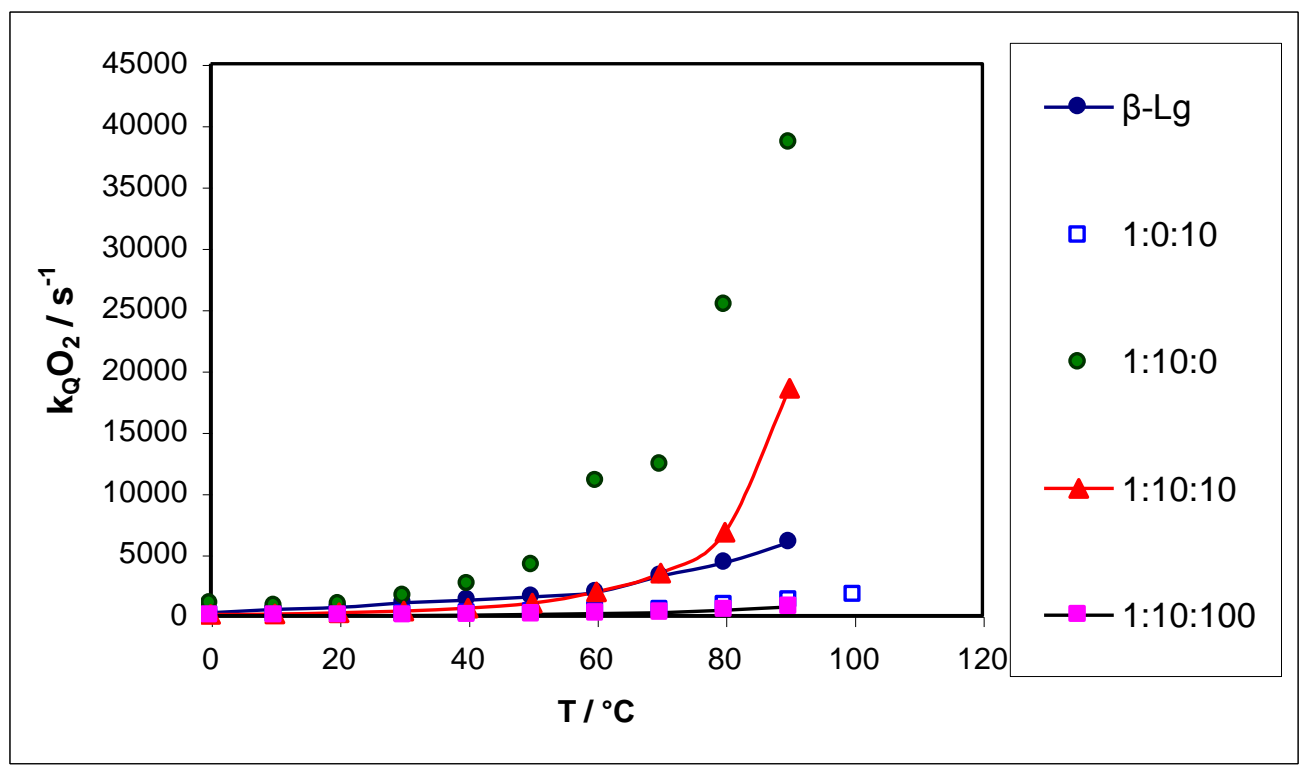

Figure VI-6: The oxygen quenching rate $\mathrm{k}_{\mathrm{Q}}\left[\mathrm{O}_{2}\right]$ as a function of temperature in $\beta-\mathrm{Lg} / \mathrm{PEG} / \mathrm{suc}$ films (The ratios in the text box refer to the ratio of $\beta$-Lg : PEG : suc in the films).

Figure VI-7

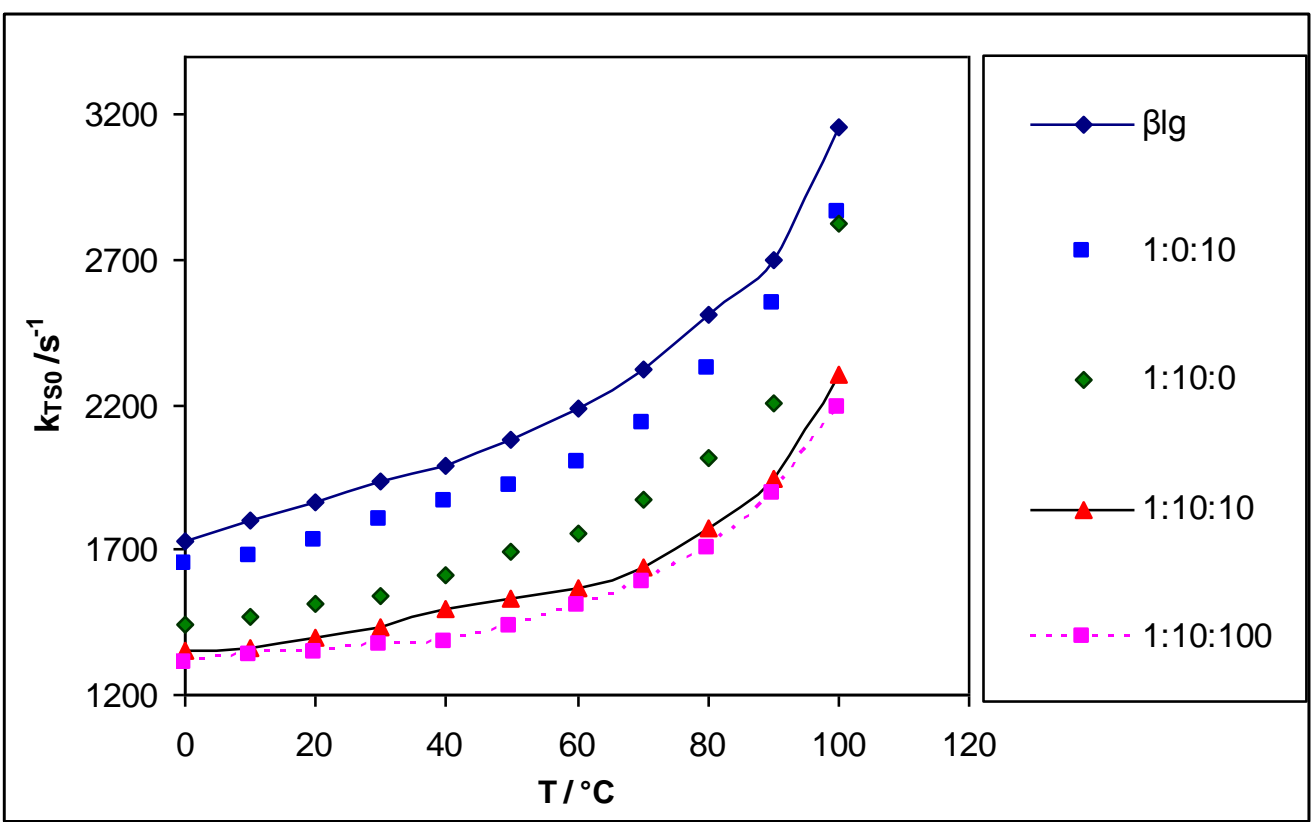

Figure VI-7: Plot of the effect of temperature on non-radiative decay from $T_{1}$ to $S_{0}\left(k_{T S 0}\right)$ in $\beta$ $\mathrm{Lg} / \mathrm{PEG} / \mathrm{suc}$ films (The ratios in the text box refer to the ratio of $\beta$-Lg : PEG : suc in the films). 


\section{Figure VI-8a}

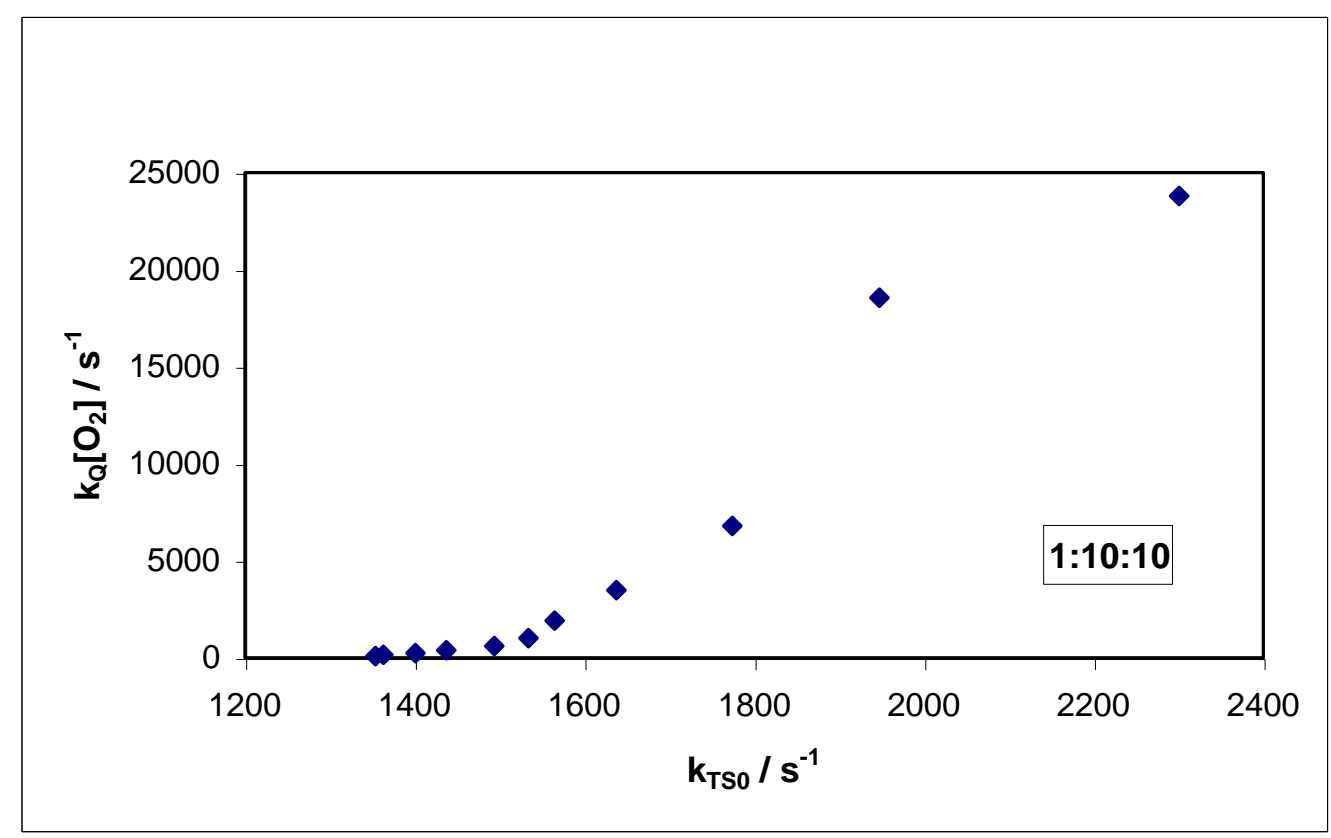

Figure VI- $8 b$

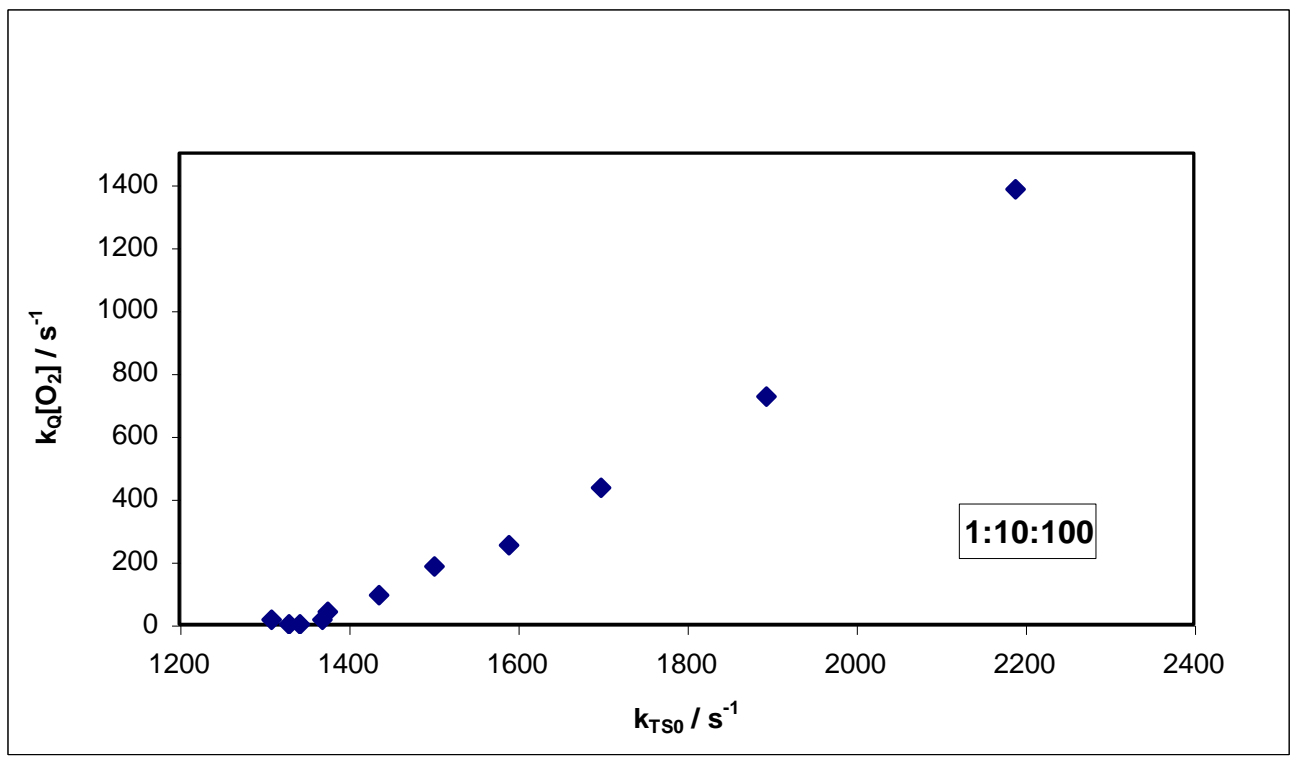

Figure VI-8a, b: Dependence of the oxygen quenching rate $\mathrm{k}_{\mathrm{Q}}\left[\mathrm{O}_{2}\right]$ on the rate of non-radiative quenching $\mathrm{k}_{\mathrm{TS} 0}$ in $\beta$-Lg/ PEG/ suc films (The ratios in the text box refer to the ratio of $\beta$ - $\mathrm{Lg}$ : PEG : suc in the films). 


\section{Figure VI-9}

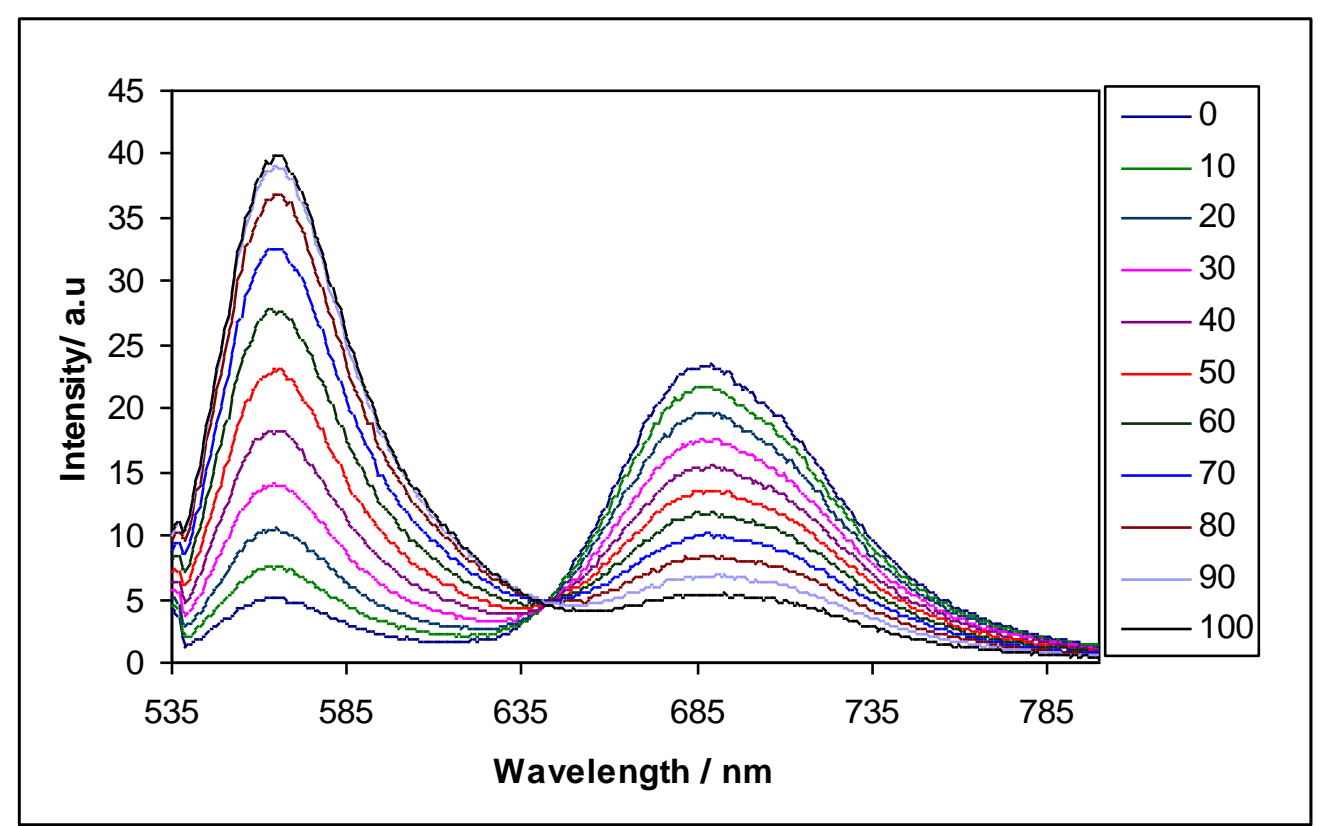

Figure VI-9: The effect of temperature on the delayed emission spectra of Erythrosin B dispersed in amorphous $\beta$-Lg/maltose/ maltitol mixture (Ratio of $\beta$-Lg: maltose: maltitol 1:5:5). 
Figure VI-10

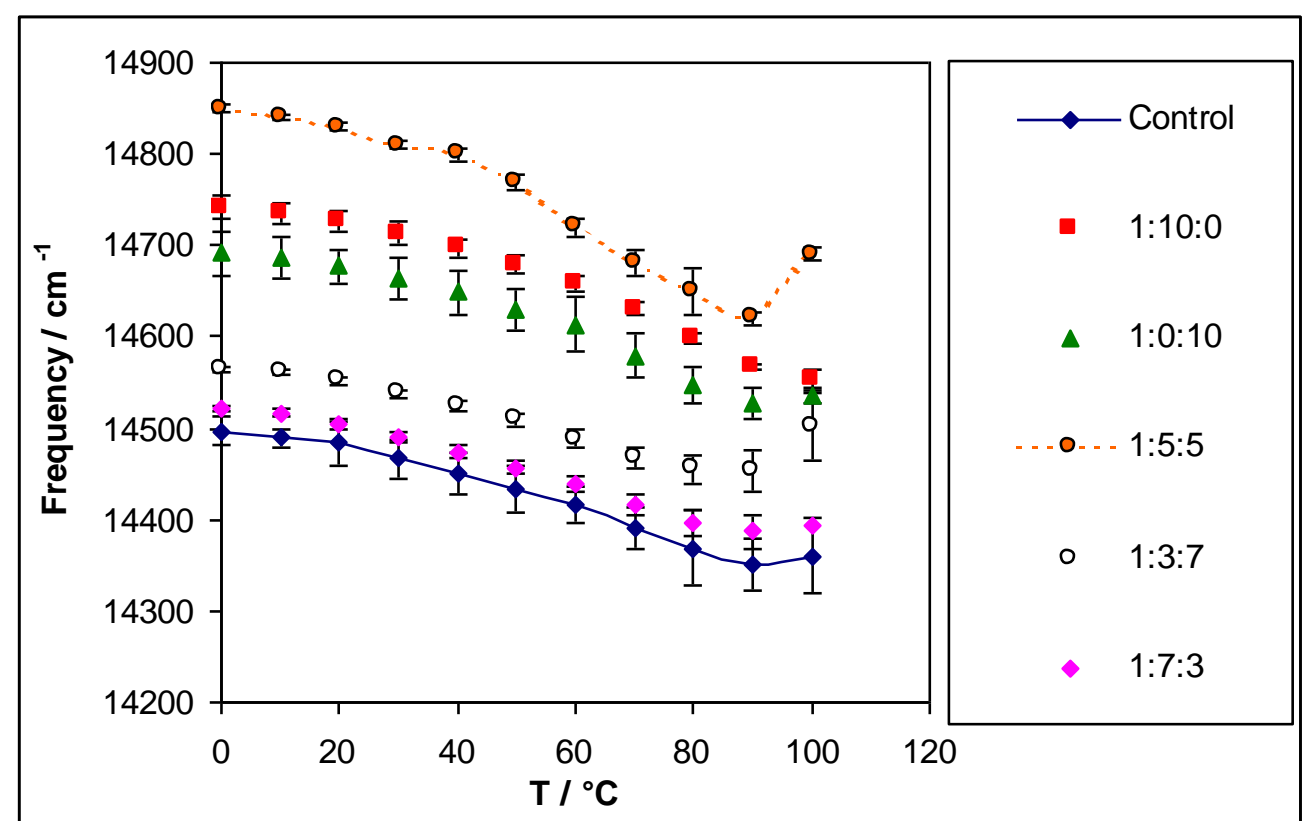

Figure VI-10: The effect of temperature on the emission maximum for phosphorescence from Erythrosin B in composite films from $\beta-\mathrm{Lg} /$ maltose/ maltitol (The ratios in the text box refer to the ratio of $\beta-\mathrm{Lg}$ :maltose : maltitol in the films).

Figure VI-11

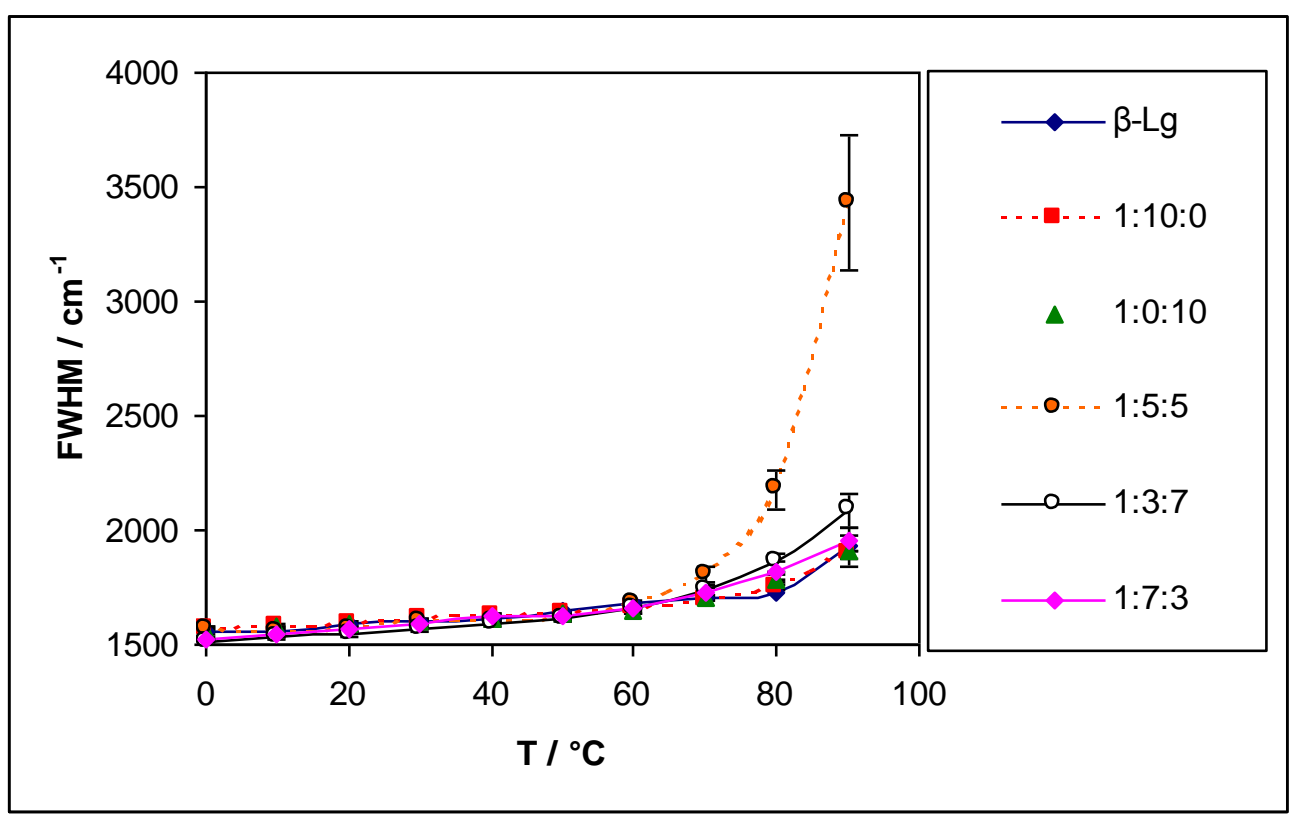

Figure VI-11: The effect of temperature on the band width for phosphorescence from Erythrosin B in composite films from $\beta-\mathrm{Lg}$ / maltose/ maltitol (The ratios in the text box refer to the ratio of $\beta-\mathrm{Lg}:$ maltose : maltitol in the films). 
Figure VI-12a

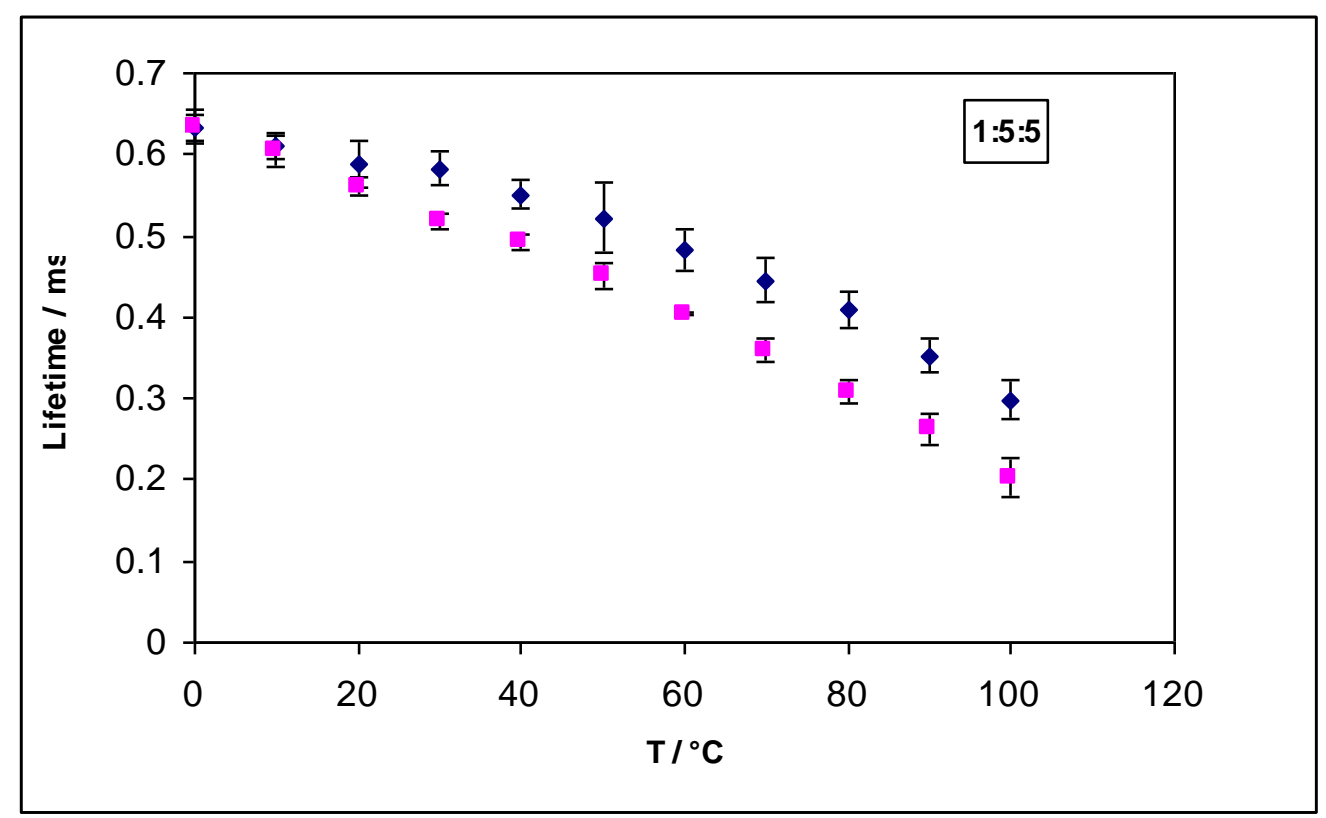

Figure VI-12b

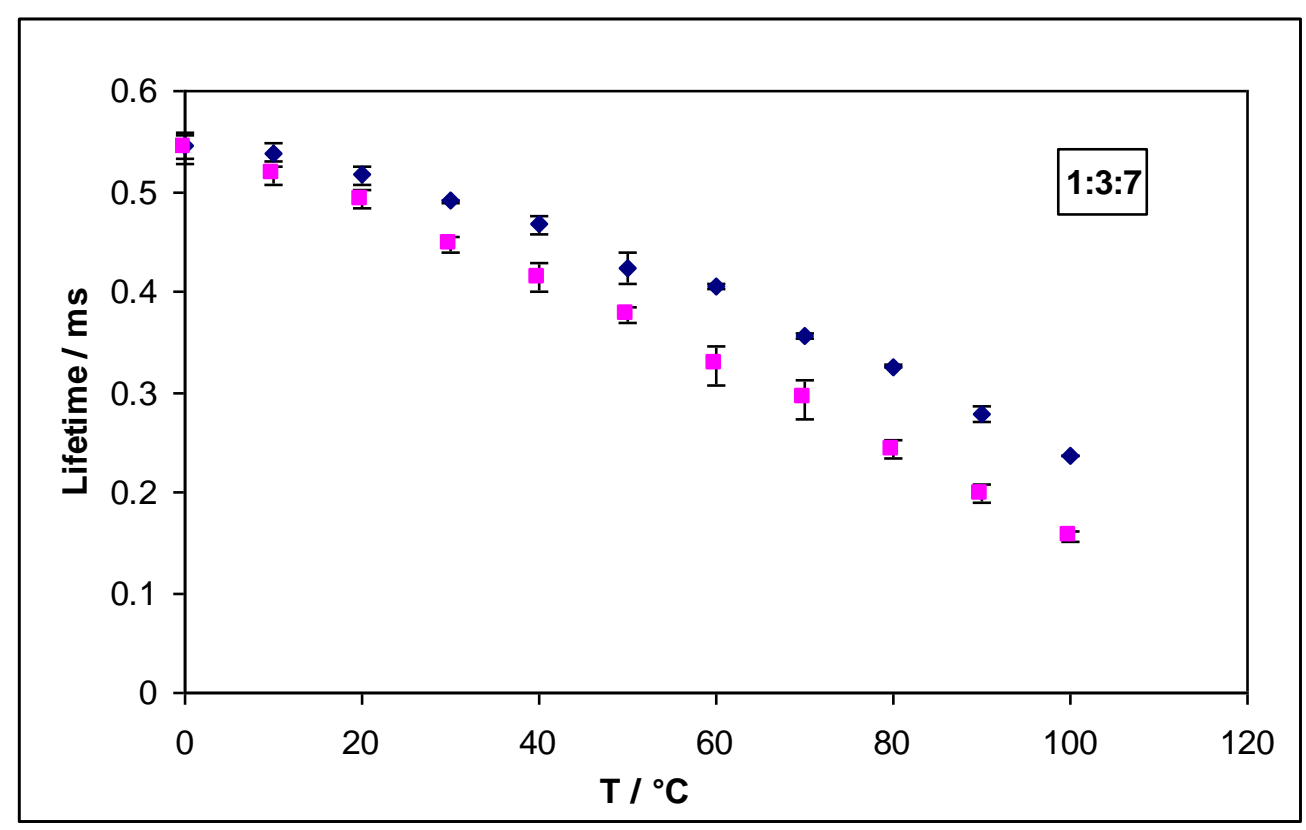


Figure VI-12c

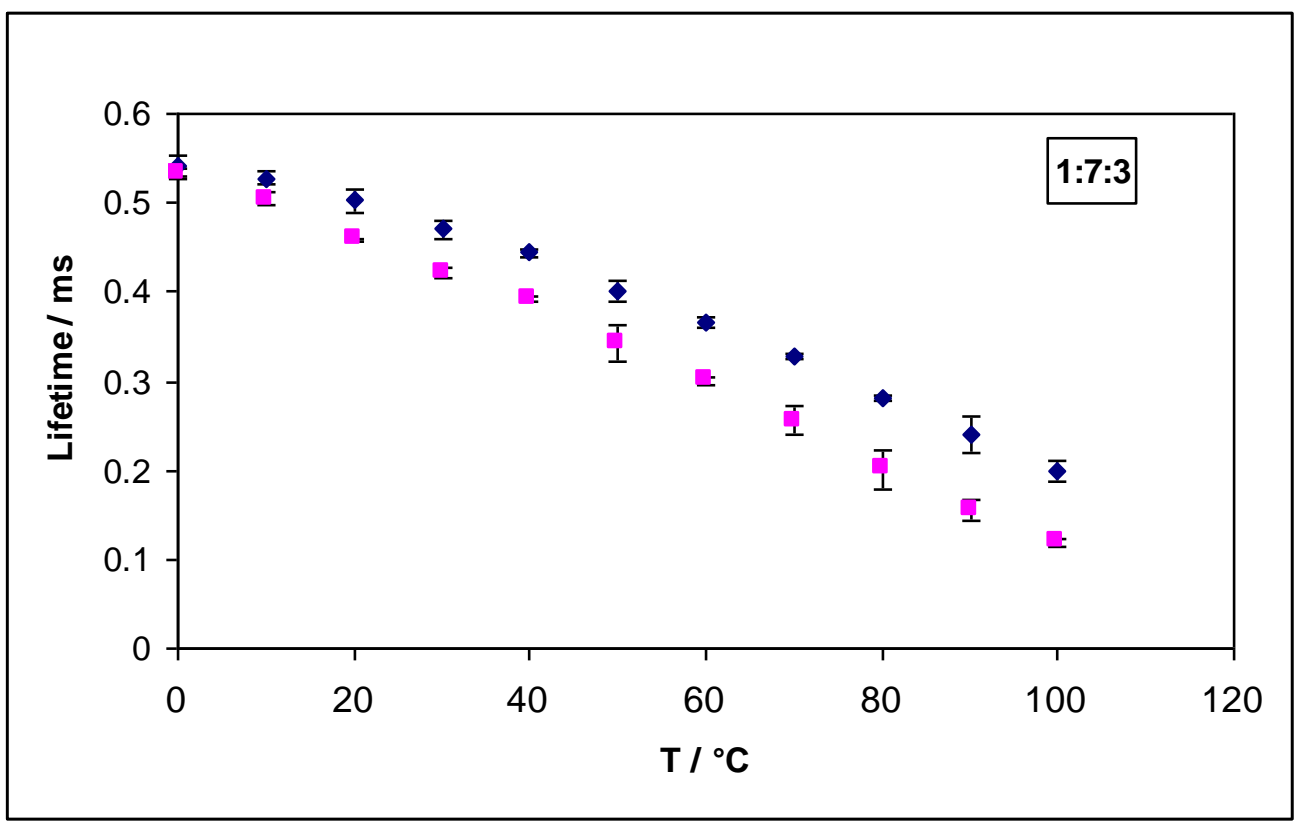

Figures VI-12 a, b, c: The effect of temperature on the lifetime for Erythrosin B dispersed in composite films from $\beta$ - $\mathrm{Lg} /$ maltose/ maltitol mixtures equilibrated against nitrogen $(\bullet)$ and against air $(\square)$; the lifetime was determined from analysis using a stretched exponential decay function. (The ratios in the text box refer to the ratio of $\beta-\mathrm{Lg}$ : maltose : maltitol in the films).

Figure VI-12d

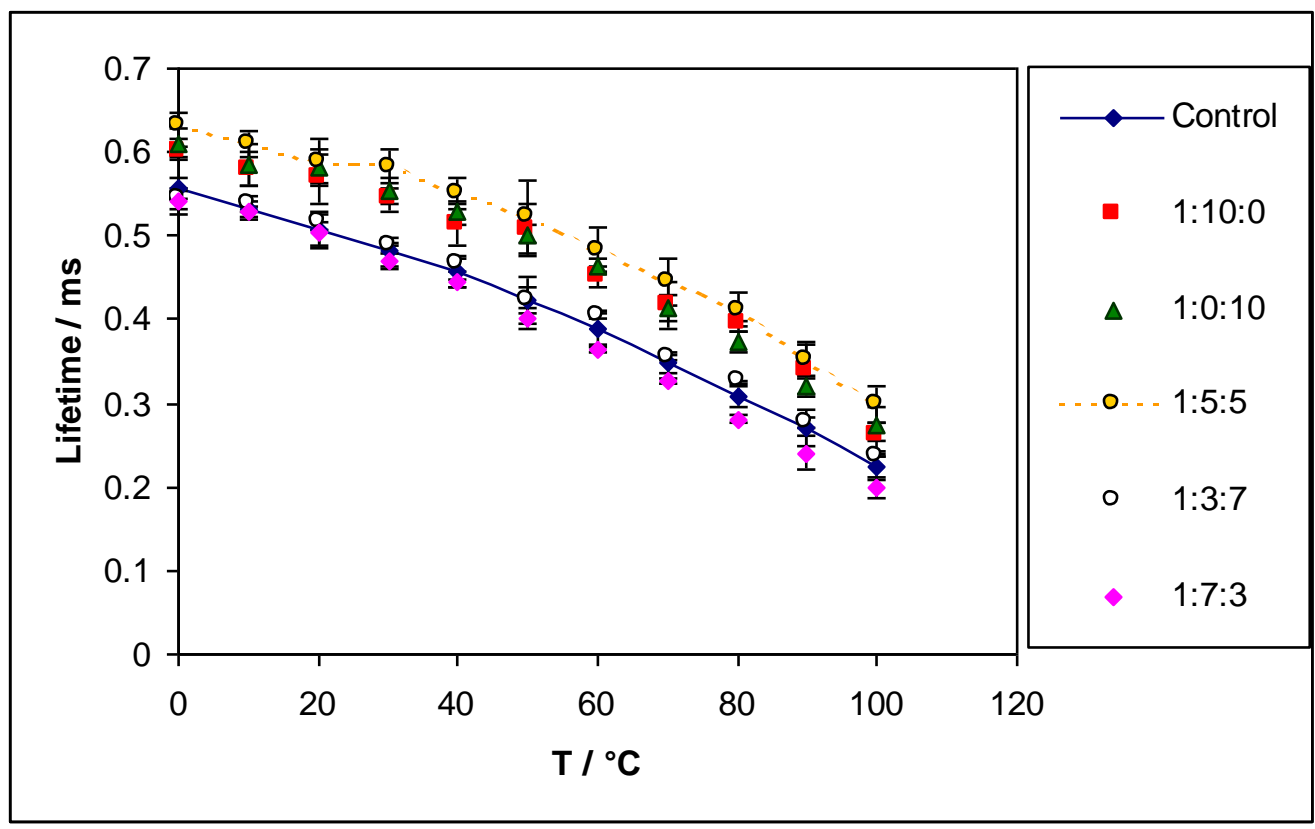

Figure VI-12d: Comparison plot depicting the effect of temperature on the lifetime for Erythrosin B dispersed in composite films from $\beta-\mathrm{Lg} /$ maltose/ maltitol mixtures equilibrated against nitrogen. (The ratios in the text box refer to the ratio of $\beta-\operatorname{Lg}$ : maltose : maltitol in the films). 
Figure VI-12e

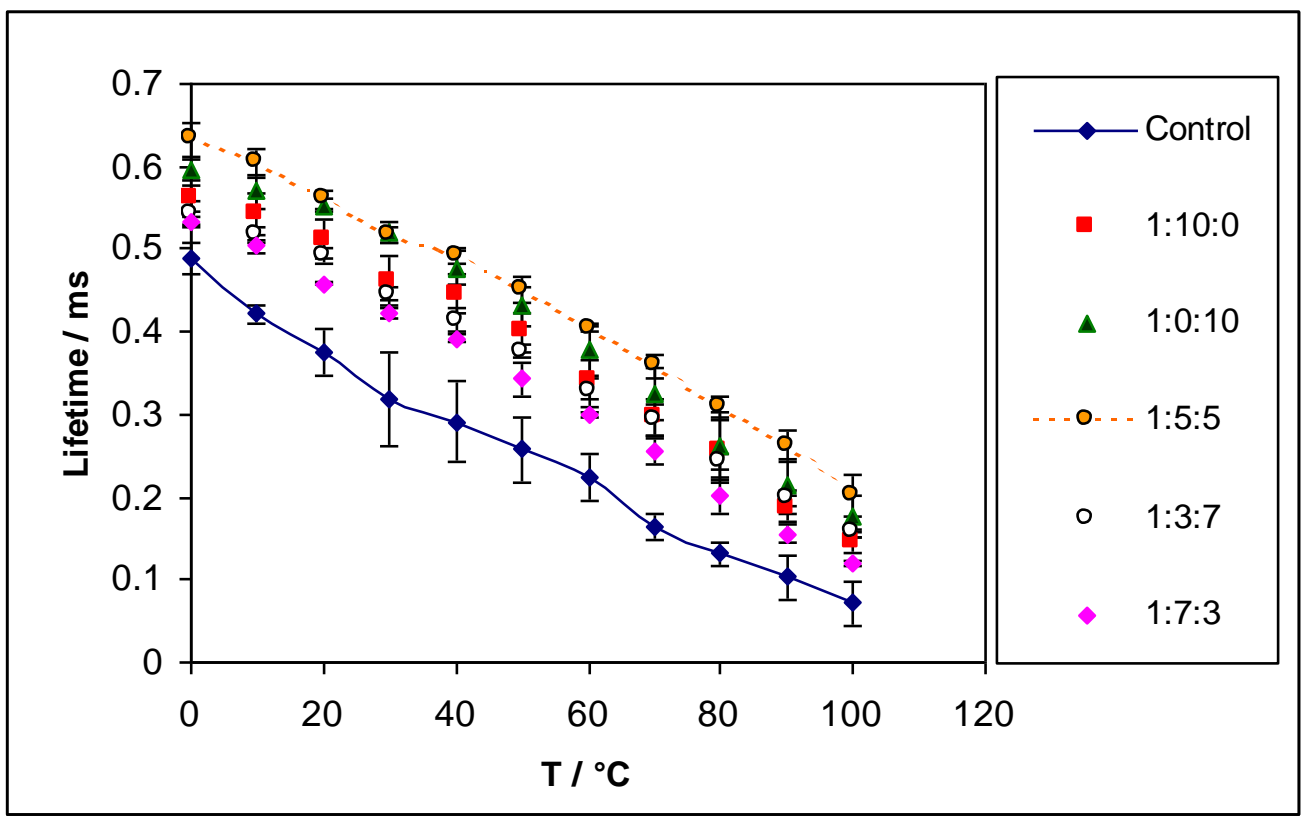

Figure VI-12e: Comparison plot depicting the effect of temperature on the lifetime for Erythrosin B dispersed in composite films from $\beta$ - $\mathrm{Lg} /$ maltose / maltitol mixtures equilibrated against air. (The ratios in the text box refer to the ratio of $\beta-\mathrm{Lg}$ : maltose : maltitol in the films).

Figure VI-13a

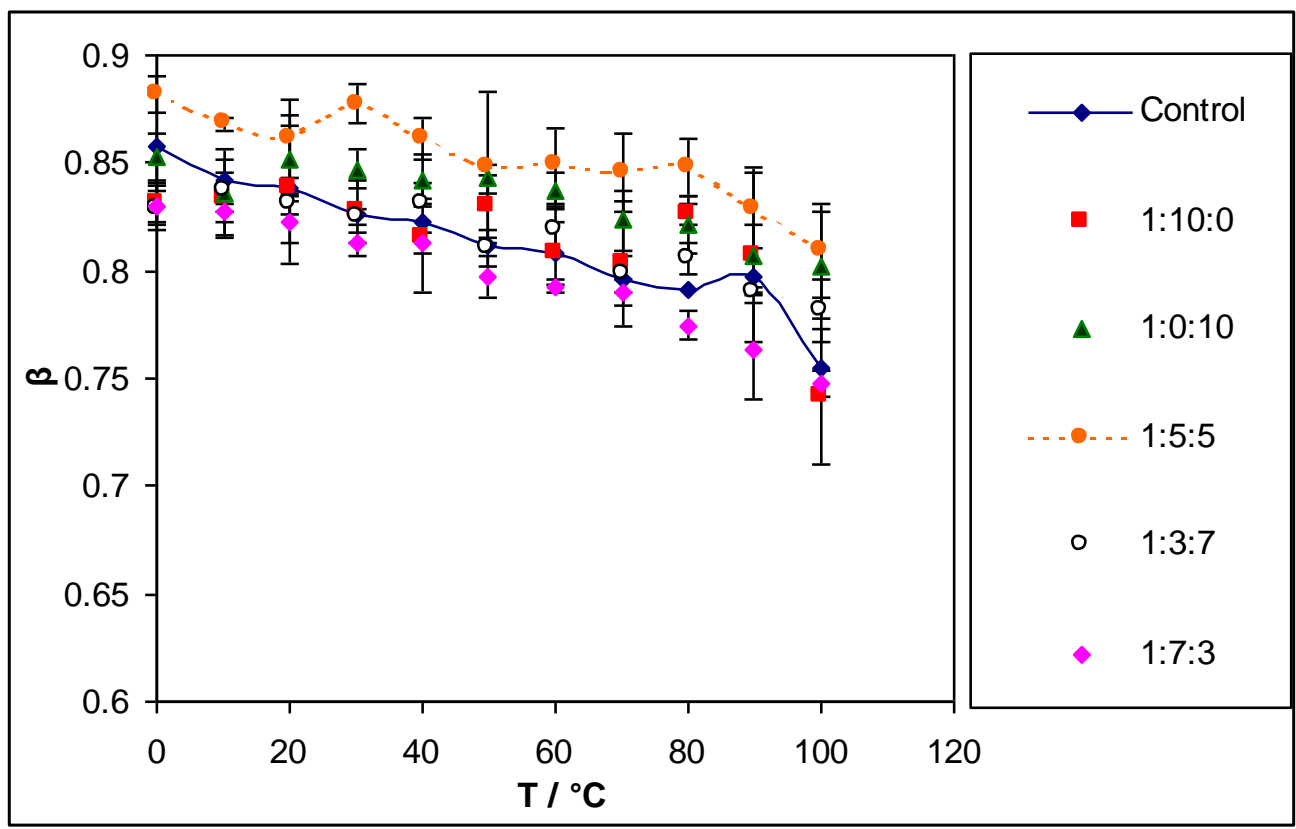

Figure VI-13a: Beta as a function of temperature in composite films from $\beta$-Lg/ maltose/ maltitol mixtures equilibrated against nitrogen (The ratios in the text box refer to the ratio of $\beta$-Lg : maltose : maltitol in the films). 
. Figure VI- 13 b

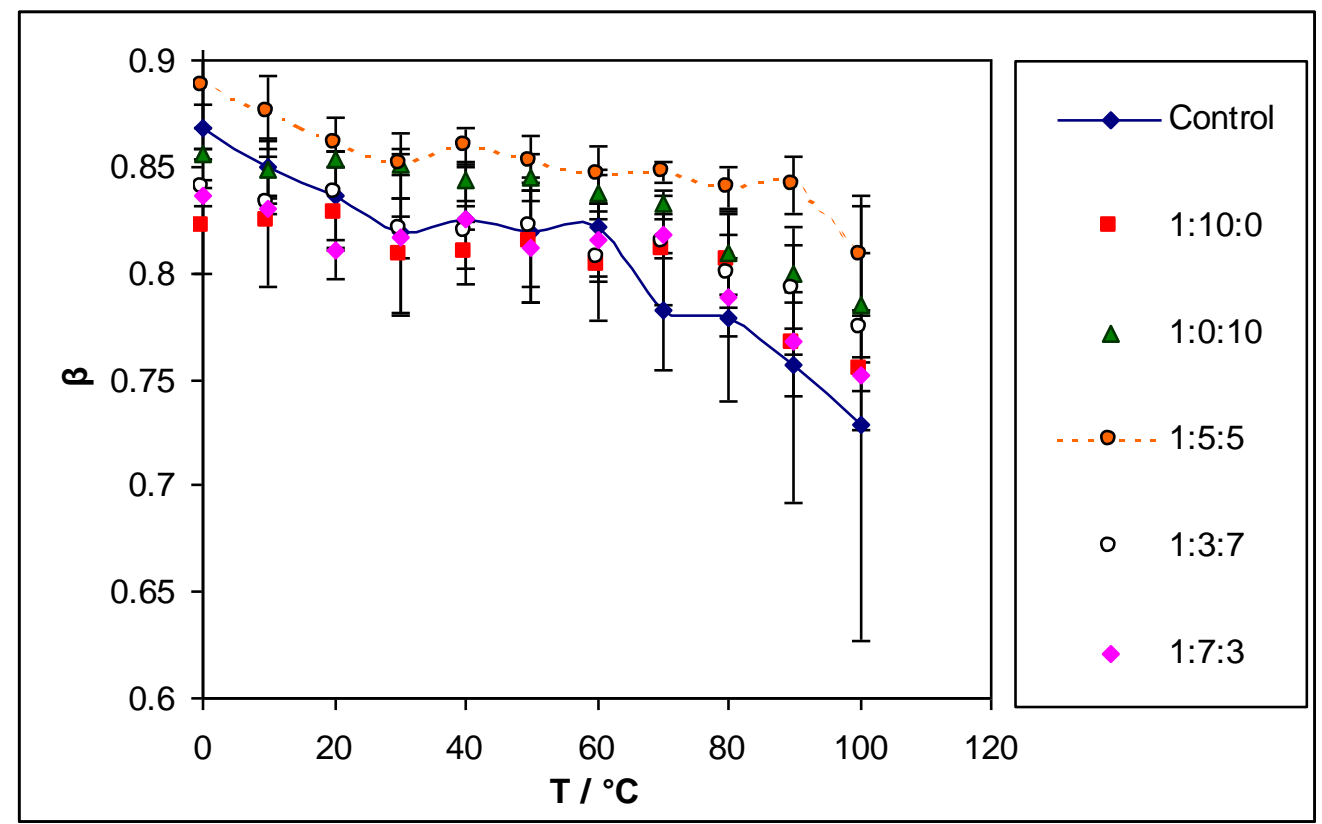

Figure VI-13b: Beta as a function of temperature in composite films from $\beta$-Lg/ maltose/ maltitol equilibrated against air (The ratios in the text box refer to the ratio of $\beta-\mathrm{Lg}:$ maltose : maltitol in the films).

Figure VI-14

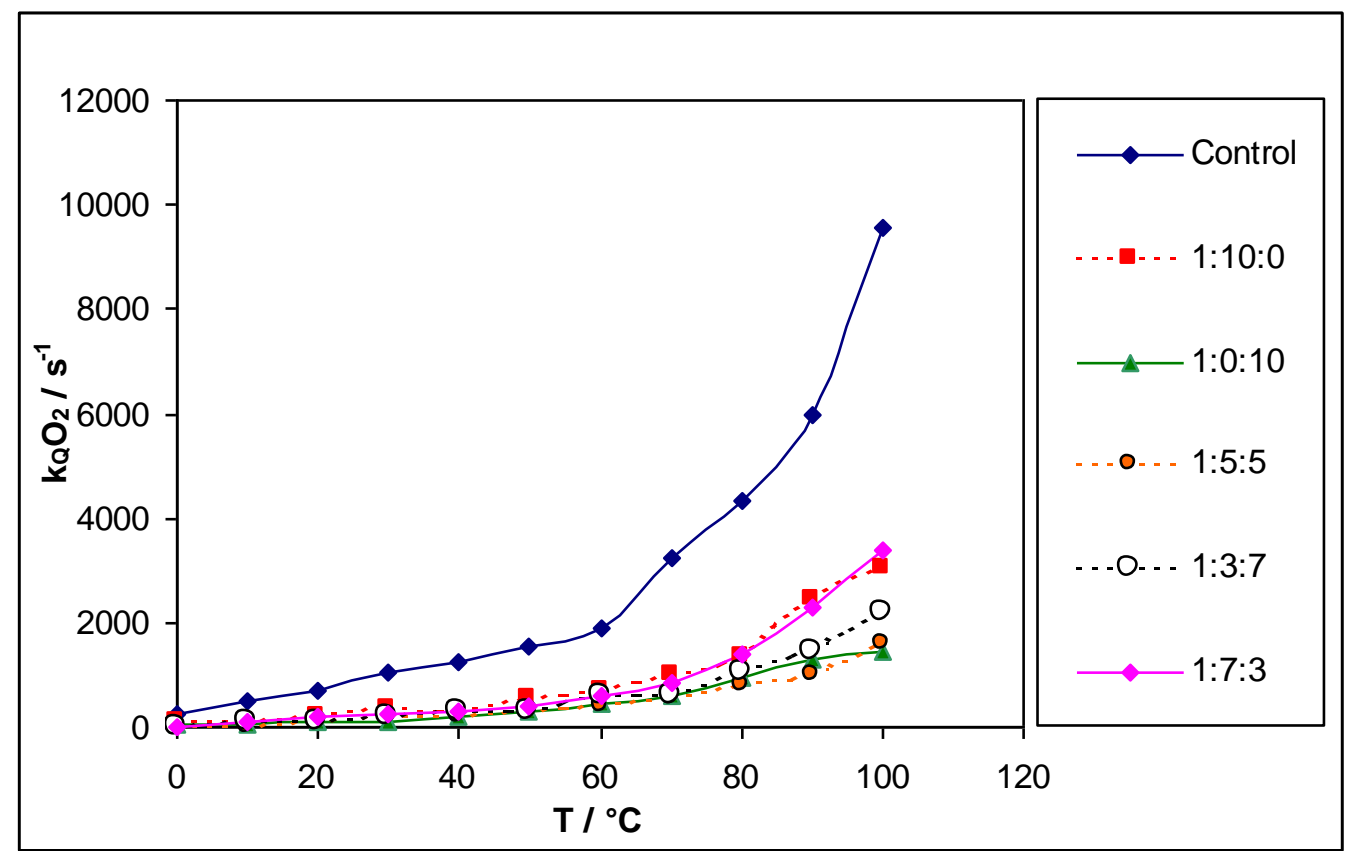

Figure VI-14: The oxygen quenching rate $\mathrm{k}_{\mathrm{Q}}\left[\mathrm{O}_{2}\right]$ as a function of temperature in $\beta$ - $\mathrm{Lg} / \mathrm{maltose} /$ maltitol films. 
Figure VI-15

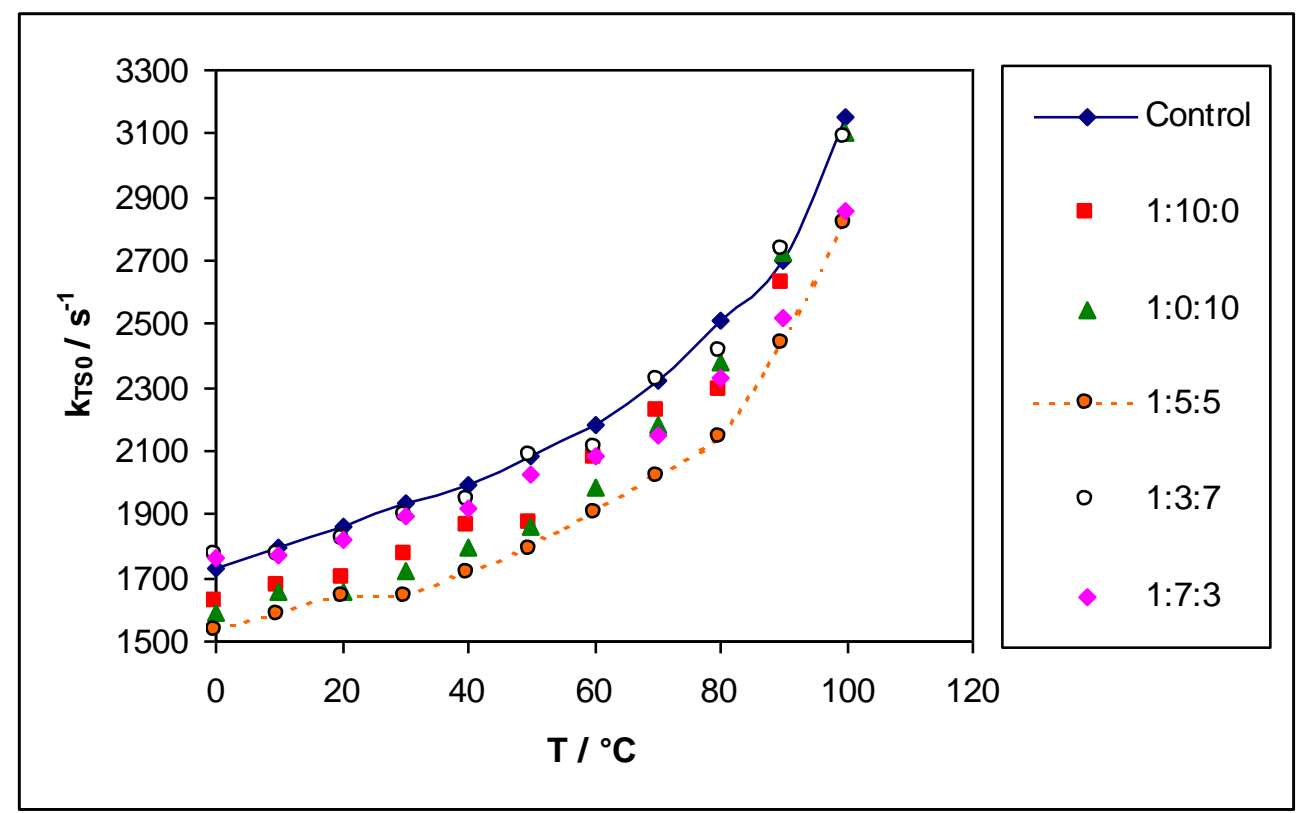

Figure VI-15: Plot of the effect of temperature on non-radiative decay from $\mathrm{T}_{1}$ to $\mathrm{S}_{0}\left(\mathrm{k}_{\mathrm{TS} 0}\right)$ in $\beta-\mathrm{Lg} /$ maltose/ maltitol films.

Figure VI-16

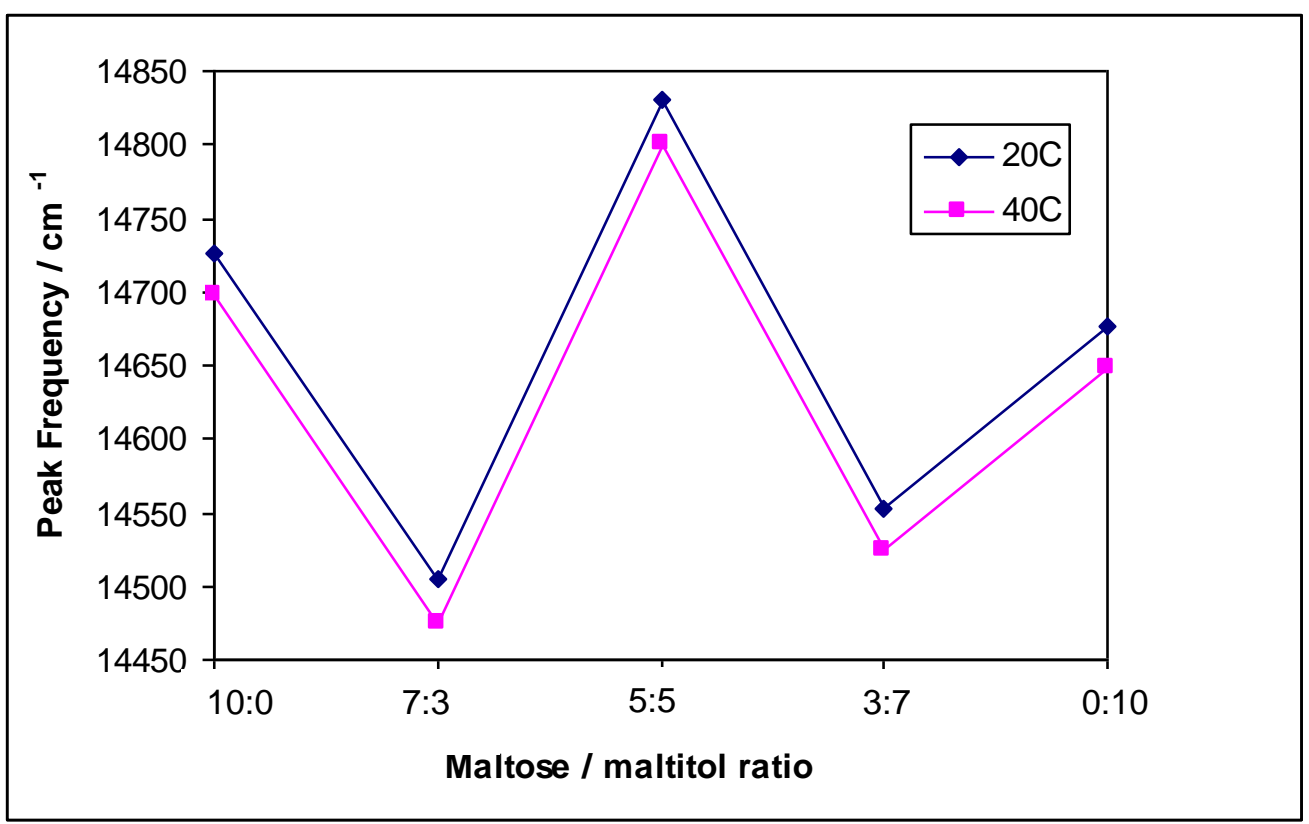

Figure VI-16: Peak frequency as a function of molar ratio of maltose/ maltitol in the $\beta$-lg films. Data re-plotted from Figure VI-10. 
Figure VI-17

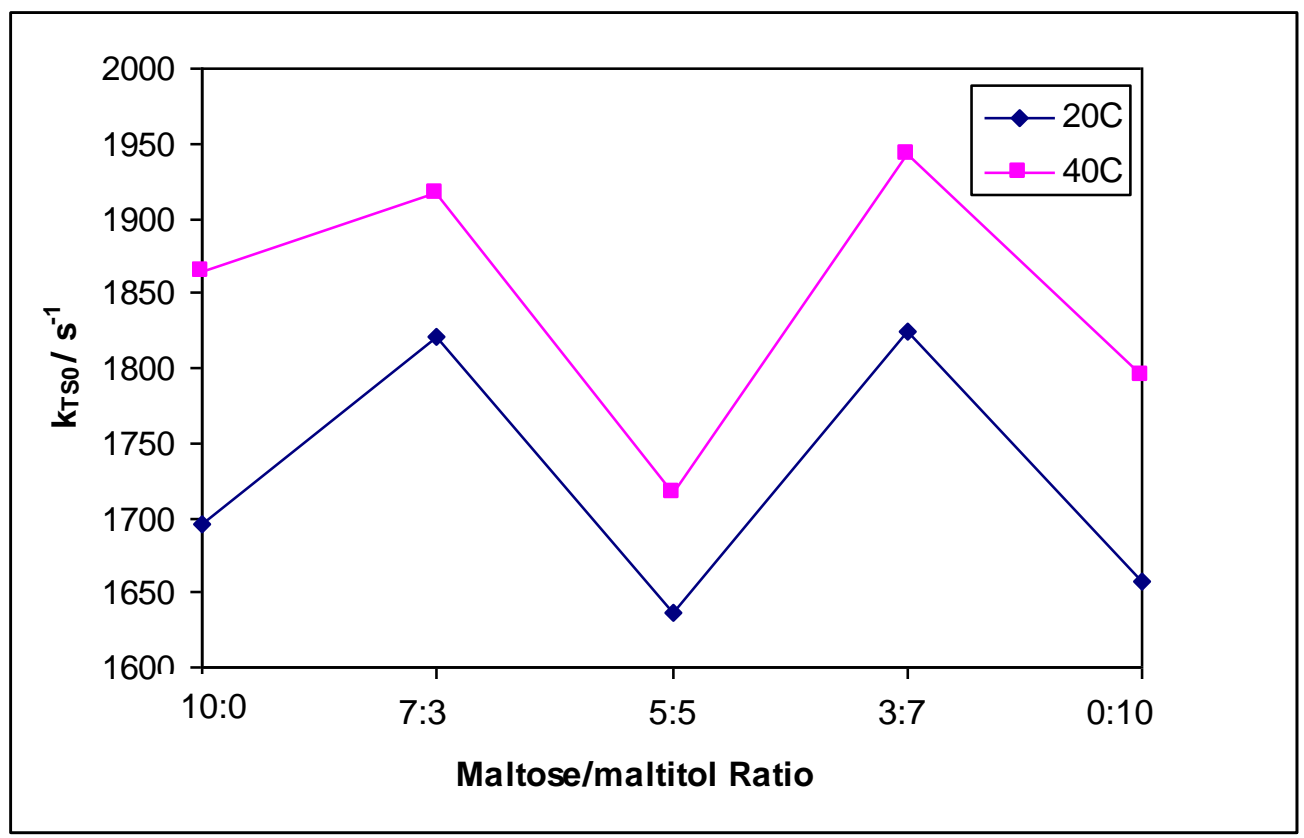

Figure VI-17: $\mathrm{k}_{\mathrm{TS} 0}$ as a function of molar ratio of maltose/ maltitol in the $\beta$-Lg films. Data replotted from Figure VI-14. 


\section{Chapter VII: The effect of bovine serum albumin (BSA) on the molecular mobility and oxygen permeability of the amorphous $\beta$-Lactoglobulin ( $\beta$-Lg) matrix.}

\section{Introduction}

Protein based films are edible, biodegradable, and provide good gas barrier properties at low relative humidity. Due to these properties, protein films are used as selective barrier for gases, vapors and solutes, as well as for mechanical protection in several food systems, in order to extend food shelf life and minimize food quality deterioration.

By mixing two proteins with different structures, new functionalities may be attained. Two proteins being extensively studied in the elaboration of edible films are BSA and $\beta-\mathrm{Lg}$. They are both globular proteins, however, at $\mathrm{pH} 7 \mathrm{BSA}$ is in a monomeric form while $\beta$-Lg exists as a dimer (Kinsella and Whitehead, 1989). The size of BSA is roughly two times that of $\beta$-Lg (BSA MW 66000 and $\beta$-Lg-dimer MW 36000 ). Studies by Nack and Ludescher, 2006 and Sundaresan and Ludescher, 2007 have characterized the molecular mobility of these individual proteins in the solid state. However, a combination of two proteins may affect the packing density and cause changes in the conformational freedom of the resultant mixture and thus may affect the diffusion of small molecules like oxygen.

A number of factors are known to affect protein surface activity. The initial stage of adsorption to an interface is commonly assumed to be diffusion-controlled (Graham et al., 1979). The molecular size and flexibility of a protein molecule has been found to be an important factor governing the incorporation of protein into an interfacial layer. A smaller protein molecule can be easily incorporated into an interfacial film (Suttiprasit, et 
al., 1992; Hunter et al., 1991). The interaction between BSA and $\beta$-Lg in heat-induced gels has been studied previously (Tobitani \& Ross-Murphy, 1997). It was found that the ratio of the two proteins to each other affected the gelation behavior, with the inclusion of BSA accelerating the formation of the heat-set gels. An earlier study (Hines \& Foegeding, 1993) found that BSA increased the elastic modulus of heat-induced gels when added to $\beta$-Lg. These studies however, were done in the solution state, and what governs the macroscopic properties of these proteins in combination in the glassy state can be elucidated with the present study. It is clear that these proteins differ in their surface affinity, size and diffusion coefficients, and therefore the mixtures would be expected to show differences in behavior when mixed together. In the past various mixtures have been shown to establish synergies with respect to mobility, which have long-term implications on a food system for shelf stability and storage.he diffusion of oxygen molecules and temperature, are factors that determine the aging of many materials (Anker et al., 2001). Oxygen can lead to detrimental effects in food system including degradation of vitamins, pigments and lipids with loss of nutritional value and development of off flavors (Fennema, 1996). Oxygen permeability in amorphous proteins is greatly affected by changes in composition and change in temperature (Sundaresan and Ludescher 2008; Nack and Ludescher, 2006). The following study focuses on understanding the effect of a larger sized protein BSA on the oxygen permeability of $\beta$ Lg.

Constant emphasis on the macroscopic properties of amorphous food has essentially overlooked the vital importance of structural heterogeneity in modulating the structural and functional properties of foods. This structural heterogeneity necessarily 
modulates dynamic heterogeneity that is the range of molecular mobility of foods and their influence in affecting the macroscopic properties of the foods. Dynamic heterogeneity in the solid state, a distinctive feature of amorphous synthetic polymers, has received much recent attention (Ediger, 2000 and Richert, 2002). Pure $\beta$-Lg and BSA showed signs of the existence of site heterogeneities (Nack and Ludescher, 2006; Sundaresan and Ludescher, 2008). This study also aims at studying the existence of this phenomenon in amorphous BSA and $\beta-\mathrm{Lg}$ mixtures and thus enables us to draw important connections with respect to heterogeneity and stability.

We have recently demonstrated the use of Erythrosin B as a phosphorescent probe to study the molecular motions in pure $\beta-\operatorname{Lg}$ (Sundaresan and Ludescher, 2008) and BSA (Nack and Ludescher, 2006). We used the same technique to characterize the oxygen permeability and intrinsic molecular mobility in amorphous protein mixtures.

This study aims at investigating the effects of different molar concentrations of BSA on the molecular mobility and oxygen transport through the amorphous $\beta$ - $\mathrm{Lg}$ matrix. These data will provide mobility maps of a matrix comprising of two globular proteins. These maps can be used in the following ways. They will help us understand the functionality of these vital proteins and will answer an important question, do these proteins complement each other in controlling oxygen diffusion. Creating films with a combination of milk proteins may help us discover various interactions in these proteins and thus create more avenues for developing functional edible films with requisite properties. 


\section{Materials and Methods}

Preparation of $\beta$-Lactoglobulin solution

$\beta$-Lactoglobulin ( $\beta$-Lg) was purchased from Sigma Chemical Co (St. Louis, MO); solutions were prepared in distilled de-ionized water at a concentration of $10 \mathrm{mg} / \mathrm{mL}$. In order to define and minimize the presence of counter ions, this solution was extensively dialyzed using 12-14 kDa molecular weight cutoff cellulose tubing (Spectrum, Houston, $\mathrm{TX})$ at $5^{\circ} \mathrm{C}$, first against $0.1 \mathrm{M} \mathrm{KCl}$ and then against distilled de-ionized water. The dialyzed solution was then filtered through a $0.2 \mu \mathrm{m}$ Acrodisc membrane filter (Pall Corporation, Ann Arbor, MI) to remove particulate matter. The protein concentration was determined after dialysis by measuring the absorbance at $280 \mathrm{~nm}$ using an extinction coefficient of $0.96(\mathrm{~g} / \mathrm{L})^{-1} \mathrm{~cm}^{-1}$.

Preparation of BSA solution

Purified BSA was obtained from Sigma (St. Louis, MO, USA). To define and minimize counter-ions, BSA was dissolved in distilled deionized water at $10 \mathrm{mg} / \mathrm{ml}$, placed in a cellulose dialysis tube having a $12-14 \mathrm{kDa}$ molecular weight cutoff (Spectrum, Houston, TX, USA), dialyzed against $0.1 \mathrm{M}$ potassium chloride for at least $36 \mathrm{~h}$ with frequent changes of buffer, and then dialyzed extensively against distilled deionized water. All dialysis was carried out at room temperature. The solution was then filtered sterilized through a 0.2- $\mu \mathrm{m}$ Acrodisc filter (Pall Gellman Laboratory, Ann Arbor, MI, USA). The concentration of the protein after dialysis was determined by absorbance at $280 \mathrm{~nm}$ using a molar absorptivity of $4.3 \times 10^{-4} \mathrm{M}^{-1} \mathrm{~cm}^{-1}$ (Fasman, 1989).

Erythrosin B (Ery B; tetraiodofluorescein) was purchased from Molecular Probes, Inc. Erythrosin B (Eugene, OR), was dissolved in spectrophotometric grade N, N- 
dimethylformamide (DMF; Aldrich Chemical, Milwaukee, WI) to make either $10 \mathrm{mM}$ or100 mM stock solutions. The solvent DMF was selected for probe stability during long time storage and the concentrations were selected in order to simplify the addition of probe to the protein solutions. Aliquots of dye stock were added to protein solutions to give a molar ratio of 1:4 (Ery B: $\beta$ - $\mathrm{Lg}$ ) / (EryB: BSA). The resultant BSA and the $\beta$ - $\mathrm{Lg}$ solutions were mixed together in a molar concentration basis. Three mixtures were prepared, Mixture I - Equi-molar mixture of (1 $\beta$-Lg : 1 BSA), Mixture II comprised of ( 2 moles of $\beta-\mathrm{Lg}$ and 1 mole of BSA) and mixture III consisted of ( 1 mole of $\beta-\mathrm{Lg}$ and 2 moles of BSA).

Preparation of protein films

To make glassy films, $15 \mu \mathrm{l}$ of protein / Erythrosin B solution at room temperature were spread on approximately one third of a quartz slide $(30 \times 13.5 \times 0.6 \mathrm{~mm}$; custom made by NSG Precision Cells, Farmingdale, NY). The protein / Erythrosin films, $0.05 \mathrm{~mm}$ thick when dried, were visually checked for good spreading, uniformity, and consistency. The slides were stored at room temperature against the desiccant DrieRite and kept in an atmosphere of $\mathrm{P}_{2} \mathrm{O}_{5}$ in order to maintain $0 \% \mathrm{RH}$. The slides were stored in the desiccator for at least one week and protected from light to prevent any photo bleaching of Erythrosin B. The desiccant was refreshed as necessary.

\section{Luminescence measurements}

All measurements were conducted using a Cary Eclipse spectrophotometer (Varian Instruments, Walnut Creek, CA). The temperature was controlled using a TLC 50 thermoelectric heating/cooling system (Quantum Northwest, Spokane, WA) with a sample compartment fitted with a jacketed cover. For luminescence measurements 
necessitating the absence of oxygen (which is a phosphorescence quencher) the samples were flushed with nitrogen gas for at least 15 minutes prior to collecting data. A virtually oxygen-free nitrogen stream was generated by passing high purity nitrogen through a Supelco (Bellefonte, PA) carrier gas purifier. This gas line was routed into the sample compartment and directly into the quartz fluorescence cuvette that held the slide. The cuvette was capped with a lid having inlet and outlet ports for the gas line, so all experiments were performed at constant pressure. The cuvette was flushed for at least $30 \mathrm{~min}$ to ensure that oxygen was eliminated.

Quartz slides containing protein film were placed on-end on the diagonal of a standard $1 \mathrm{~cm} \times 1 \mathrm{~cm}$ quartz fluorescence cuvette, which was capped with a lid having inlet and outlet, ports for gas lines. The outside of the cuvette was flushed with dry air to prevent condensation on the faces of the cuvette for experimental conditions below room temperature.

Delayed luminescence emission spectra were collected from 535-800 nm (10 nm bandwidth) using excitation of $525 \mathrm{~nm}$ (10nm bandwidth) over the temperature range from $0^{\circ} \mathrm{C}$ to $100^{\circ} \mathrm{C}$. For the scan, each datum point (collected at $1 \mathrm{~nm}$ interval, $0.1 \mathrm{~ms} 8$ averaging time) was collected from a single lamp flash with a $0.2 \mathrm{~ms}$ delay, $0.5 \mathrm{~s}$ gate time and $4.0 \mathrm{~ms}$ total decay time. For lifetime measurements as a function of temperature samples were excited at $540 \mathrm{~nm}(20 \mathrm{~nm}$ bandwidth $)$ and emission transients collected at $690 \mathrm{~nm}(20 \mathrm{~nm}$ bandwidth $)$ at temperatures ranging from $0^{\circ} \mathrm{C}$ to $100^{\circ} \mathrm{C}$. Before collecting data, films were equilibrated at each temperature for 15 minutes. The measurements were taken in the presence or absence of air (under a nitrogen purge). Each time-resolved decay transient was the average of 100 cycles and, for each cycle, data was collected 
from a single lamp flash with a delay of $0.1 \mathrm{~ms}$, a $0.02 \mathrm{~ms}$ gate time, and $4.0 \mathrm{~ms}$ total decay time.

Intensity decays were collected as a function of emission wavelength over the emission range from $640 \mathrm{~nm}$ to $740 \mathrm{~nm}$ at 10-nm increments with an excitation wavelength of $540 \mathrm{~nm}$. The temperature range was $0-100^{\circ} \mathrm{C}$, and the excitation and emission bandwidths monochromators were both set at $20 \mathrm{~nm}$ band pass, with a delay time of $0.1 \mathrm{~ms}$, gate time of $0.02 \mathrm{~ms}$, and a total decay time of $4.0 \mathrm{~ms}$. All experiments were done multiple times and the averages were used for data analysis and interpretation; the reported errors are standard deviations between experiments.

\section{Data Analysis}

Phosphorescence lifetimes were determined by nonlinear least squares analysis with the program NFIT (Island Products, Galveston, TX). Data was also fit using the program Igor (Wavemetrics, Inc., Lake Oswego, OR, USA) to make sure that the results agreed in both fitting programs. Fits were judged acceptable if fit parameters had satisfactory fit errors and if data points were randomly distributed about the fit curve; most data sets had $\mathrm{R}^{2}$ in the range of $0.99-1.0$. Intensity decays $(\mathrm{I}(\mathrm{t})$ ) were clearly nonexponential and were analyzed using a stretched exponential model function.

$$
I(t)=I_{0} \exp \left[-(t / \tau)^{\beta}\right]+c
$$

Where $\mathrm{I}_{0}$ is the initial intensity at time zero, $\tau$ is the Kohlrausch-Williams-Watt lifetime, $\beta$ is the stretching exponent, and a term characterizing the distribution of decay times, and $\mathrm{c}$ is a constant. The heterogeneity in the matrix is modeled by $\beta$; as $\beta$ decreases below unity, the intensity decay is less single exponential and the distribution of decay times becomes broader (Lindsey and Patterson, 1980; Richert, 2000). 
Delayed luminescence spectra collected from 535-800 $\mathrm{nm}$ were converted to intensity versus frequency $\left(\mathrm{I}(\mathrm{v})\right.$, with $v$ in $\mathrm{cm}^{-1}$ ) and fit to a sum of two log-normal functions using the program NFIT; all fit parameters were independent for each emission band. The lognormal fitting function (Maroncelli and Fleming, 1987) is as follows:

$$
\mathrm{I}(\mathrm{v})=\mathrm{I}_{0} \exp \left\{-\ln (2)\left[\ln \left(1+2 \mathrm{~b}\left(\mathrm{v}-\mathrm{v}_{\mathrm{P}}\right) / \Delta\right) / \mathrm{b}\right]^{2}\right\}
$$

In this equation, $\mathrm{I}_{0}$ is the maximum emission intensity, $v_{\mathrm{P}}$ is the frequency $\left(\mathrm{in} \mathrm{cm}^{-1}\right.$ ) of the emission maximum, $\Delta$ is a linewidth parameter, and $\mathrm{b}$ is an asymmetry parameter. The bandwidth ( $\Gamma$, full width at half maximum) of the emission band is related to $b$ and $\Delta$.

$$
\Gamma=\Delta\{\sinh (\mathrm{b}) / \mathrm{b}\}
$$

\section{Photophysical scheme}

The phosphorescence lifetimes were used to calculate the rate constants associated with the various processes that depopulate the triplet state. Our analysis of the delayed emission is similar to the photo-physical scheme for Erythrosin B outlined by Duchowicz et al., (1998) using slightly different nomenclature. The measured phosphorescence lifetime $(\tau)$ is the inverse sum of all possible de-excitation rates for the triplet state $\mathrm{T}_{1}$.

$$
1 / \tau=\mathrm{k}_{\mathrm{P}}(\mathrm{T})=\mathrm{k}_{\mathrm{RP}}+\mathrm{k}_{\mathrm{TS} 1}(\mathrm{~T})+\mathrm{k}_{\mathrm{TS} 0}(\mathrm{~T})+\mathrm{k}_{\mathrm{Q}}\left[\mathrm{O}_{2}\right](\mathrm{T})
$$

Here $\mathrm{k}_{\mathrm{RP}}$ is the rate of radiative decay to the ground state, $\mathrm{k}_{\mathrm{TS} 1}$ is the rate of reverse Intersystem crossing to $\mathrm{S}_{1}, \mathrm{k}_{\mathrm{TS} 0}$ is the rate of intersystem crossing to the singlet manifold followed by vibrational relaxation to $\mathrm{S}_{0}$, and $\mathrm{k}_{\mathrm{Q}}\left[\mathrm{O}_{2}\right]$ is the rate of oxygen quenching (Assumed negligible in the absence of oxygen). The radiative decay rate has a value of $41 \mathrm{~s}^{-1}$ for Erythrosin B (Duchowicz et al., 1998; Lettinga, et al., 2000). Reverse 
intersystem crossing is a thermally activated process that has an exponential dependence on the energy gap $\Delta \mathrm{E}_{\mathrm{TS}}$ between $\mathrm{T}_{1}$ and $\mathrm{S}_{1}$.

$$
\mathrm{k}_{\mathrm{TS} 1}(\mathrm{~T})=\mathrm{k}^{\circ} \mathrm{TS} 1 \exp \left(-\Delta \mathrm{E}_{\mathrm{TS}} / \mathrm{RT}\right)
$$

The ratio of the intensity of delay fluorescence $\left(\mathrm{I}_{\mathrm{DF}}\right)$ to phosphorescence $\left(\mathrm{I}_{\mathrm{P}}\right)$, where $\mathrm{I}_{\mathrm{DF}}$ and $I_{P}$ are determined from analysis of emission spectra using the log normal function (equation 2), is proportional to the rate of reverse intersystem crossing (Duchowicz et al., 1998). A plot of $\ln (\mathrm{IDF} / \mathrm{IP})$ versus $1 / \mathrm{T}$ thus has slope of $-\mathrm{E}_{\mathrm{TS}} / \mathrm{R}$. We estimated the maximum possible value for $\mathrm{k}_{\mathrm{TS} 1}^{\mathrm{o}}$ in $\beta$ - $\mathrm{Lg} / \mathrm{BSA}$ films by assuming that $\mathrm{k}_{\mathrm{TS} 1}(\mathrm{~T})$ cannot result in values for $\mathrm{k}_{\mathrm{TS} 0}$ that decrease with temperature. This procedure thus estimated the minimum possible values of $\mathrm{k}_{\mathrm{TS} 0}(\mathrm{~T})$. (Duchowicz et al., 1998).

The magnitude of $\mathrm{k}_{\mathrm{Q}}\left[\mathrm{O}_{2}\right]$ was calculated from the difference between the decay rates $(\tau)$ in the presence and absence of oxygen:

$$
\mathrm{k}_{\mathrm{Q}}\left[\mathrm{O}_{2}\right]=1 / \tau(\text { Air })-1 / \tau_{0}(\text { Nitrogen })
$$

\section{$\underline{\text { Results }}$}

The ensemble of experiments in this chapter essentially elucidates the effect of mixing a larger sized globular protein BSA on the molecular mobility and permeability of the $\beta$-Lg matrix. As these proteins are essentially found together in nature so this study will be of utmost practical importance and will essentially portray the behavior of a real food system. Revelations from this study may enable us to answer important questions related to shelf stability and implications of the functionality of these proteins in everyday life. Three types of mixtures were generated: Mixture I was an equi-molar mixture of 1 mole $\beta$-Lg and 1 mole BSA; Mixture II contained 2 moles $\beta$-Lg and 1 mole BSA; and Mixture III had 1 mole $\beta$-Lg and 2 moles of BSA. 


\section{Delayed emission spectra}

Delayed emission spectra of Ery B dispersed in optically clear amorphous mixtures of BSA: $\beta-\mathrm{Lg}$ at a molar ratio of 0.25 probes per protein, were collected over the temperature range from $0^{\circ} \mathrm{C}$ to $90^{\circ} \mathrm{C}$ (Figure VII- 1). The delayed emission spectra from Ery $\mathrm{B}$ in amorphous BSA and $\beta$-Lg mixtures include a phosphorescence band at long wavelengths (corresponding to the $\mathrm{S}_{0} \leftarrow \mathrm{T}_{1}$ transition) and a thermally activated delayed fluorescence band at short wavelengths (corresponding to the $S_{0} \leftarrow S_{1} \leftarrow T_{1}$ transition). These spectra showed a decrease in the phosphorescence intensity $\left(\mathrm{I}_{\mathrm{P}}\right)$ and increase in the delayed fluorescence intensity $\left(\mathrm{I}_{\mathrm{DF}}\right)$ with increasing temperature as expected for a thermally stimulated process.

Van't Hoff plots of $\ln \left(\mathrm{I}_{\mathrm{DF}} / \mathrm{I}_{\mathrm{P}}\right)$ versus $1 / \mathrm{T}$ (using the maximum intensity determined from fitting spectra to a log-normal function) were linear with $\mathrm{R}^{2} \geq 0.995$ (data not shown); the slope provides a measure of the $\mathrm{S}_{1} \leftarrow \mathrm{T}_{1}$ energy gap. As shown in previous studies pure $\beta$ - $\mathrm{Lg}$ and $\mathrm{BSA}$ had similar $\Delta \mathrm{E}_{\mathrm{TS}}$ values. $\Delta \mathrm{E}_{\mathrm{TS}}$ in $\beta-\mathrm{Lg}$ was $33.6 \pm 0.8 \mathrm{~kJ} \mathrm{~mol}^{-1}$ (Sundaresan and Ludescher, 2007) and 32.9 $\pm 0.5 \mathrm{~kJ} \mathrm{~mol}^{-1}$ in BSA (Nack and Ludescher, 2006). However, values of $\Delta \mathrm{E}_{\mathrm{TS}}$ in mixtures of $\beta-\mathrm{Lg}$ and BSA were significantly higher than that of the pure $\beta$ - $\mathrm{Lg}$ and BSA films. Mixture I having a composition of $1: 1 \beta$-Lg to BSA had $\Delta \mathrm{E}_{\mathrm{TS}}$ values of $35.8 \mathrm{~kJ} \mathrm{~mol}^{-1} ; \Delta \mathrm{E}_{\mathrm{TS}}$ in mixture II $(2: 1 \beta$-Lg to BSA) was 35.3 $\mathrm{kJ} \mathrm{mol}^{-1}$ and $36.4 \mathrm{~kJ} \mathrm{~mol}^{-1}$ in mixture III $(1: 2 \beta-\mathrm{Lg}$ to BSA). Despite the striking similarity in the $\Delta \mathrm{E}_{\mathrm{TS}}$ values among these protein mixtures the values were significantly different from those for Ery $B$ in amorphous sucrose $\left(31.6 \pm 0.4 \mathrm{~kJ} \mathrm{~mol}^{-1}\right)$, in ethanol $\left(28.5 \pm 2.5 \mathrm{~kJ} \mathrm{~mol}^{-1}\right.$; Duchowicz et al., 1998), in polyvinyl alcohol $\left(41.2 \pm 0.4 \mathrm{~kJ} \mathrm{~mol}^{-1}\right.$; Lettinga et al., 2000), in maltose $\left(32.7 \pm 1.1 \mathrm{~kJ} \mathrm{~mol}^{-1}\right)$ and in maltitol $\left(34.2 \pm 0.9 \mathrm{~kJ} \mathrm{~mol}^{-1}\right)$ 
(Shirke et al., 2005), and in lactose $\left(34.1 \pm 0.3 \mathrm{~kJ} \mathrm{~mol}^{-1}\right)$ and lactitol $\left(34.0 \pm 0.3 \mathrm{~kJ} \mathrm{~mol}^{-1}\right)$ (Shirke, You, \& Ludescher, 2006), suggesting that matrix properties somewhat modulate the singlet-triplet energy gap. Hereafter, in the entire document the mixtures will be referred as mixture I, II and III

The peak frequency and bandwidth were determined by fitting the delayed emission spectra to a sum of two log-normal functions (Materials and Methods, Eq 2). The temperature effect on frequency is illustrated in Figure VII- 2. The peak frequency decreased linearly and gradually at low temperature and more steeply at higher temperatures in $\beta$-Lg and the mixtures, contrasting the obvious biphasic behavior seen in BSA, which showed a dynamic transition in emission energy.

A decrease in the emission energy with temperature can be attributed to an increase with temperature in the rate of dipolar relaxation around the excited triplet state (Pravinata et al., 2005; Richert, 2000).

The $\Delta v_{\mathrm{P}}$ (change in frequency with temperature from $0-100^{\circ} \mathrm{C}$ ) was slightly lower in the mixtures when compared to the pure proteins. Pure BSA and $\beta$-Lg films had very similar frequency change with respect to temperature whereby $\Delta v_{\mathrm{P}}$ was $165.3 \mathrm{~cm}^{-1}$ and $145.4 \mathrm{~cm}^{-1}$ respectively for the two pure globular proteins, whereas mixture I had a $\Delta v_{\mathrm{P}}$ of $136 \mathrm{~cm}^{-1}, \Delta v_{\mathrm{P}}$ of mixture II was $110 \mathrm{~cm}^{-1}$ and Mixture III had peak frequency change of only $6 \mathrm{~cm}^{-1}$ within the temperature range from $0^{\circ} \mathrm{C}-90^{\circ} \mathrm{C}$.

Mixtures I, II and III had comparable emission energies of $14692 \mathrm{~cm}^{-1}, 14675 \mathrm{~cm}^{-}$ ${ }^{1}, 14706 \mathrm{~cm}^{-1}$ respectively at $0^{\circ} \mathrm{C}$. It is clearly evident that the emission energy was higher in the mixtures when compared to the pure proteins. 
A plot of emission bandwidth as a function of temperature is depicted in Figure VII- 3. The emission bandwidth, a measure of the extent of inhomogeneous broadening due to the presence of probes in multiple sites with different emission energies, increased with increasing temperature. The curves of bandwidth for the mixtures merged with that of the pure proteins until $90^{\circ} \mathrm{C}$. Dramatic increases in bandwidth were observed after $90^{\circ} \mathrm{C}$. Similar findings were noticed with respect to bandwidth with other plasticizer / $\beta$ Lg and sugar/ $\beta$-Lg mixtures.

Analysis of emission lifetimes

In an effort to quantify the extent of oxygen quenching as a function of temperature, time-resolved phosphorescence intensity decays were measured from $0^{\circ} \mathrm{C}$ to $100^{\circ} \mathrm{C}$ and were equilibrated against nitrogen and against air. The stretched exponential decay model provided a satisfactory fit for data collected at all temperatures. The stretched exponential decay has been successfully used to study various amorphous matrices like sucrose (Pravinata et al., 2005); gelatin (Lukasik and Ludescher, 2004, 2005); BSA (Nack and Ludescher, 2006); $\beta$-Lg (Sundaresan and Ludescher, 2007) and sugar alcohols (Shirke et al., 2005a, b ). The stretched exponential function is appropriate for fitting complex relaxation processes that depopulate the excited triplet state (Richert, 1997). The physically meaningful parameters lifetime $(\tau)$ and $(\beta)$ are highly sensitive to change in temperature and molecular environment of the matrix (You and Ludescher, 2007; Nack and Ludescher, 2006; Sundaresan and Ludescher, 2008). The stretched exponential lifetime $\tau$ of various films are depicted in Figure VII- 4 a, b, c. It is clearly evident that over the temperature interval from 0 to $100^{\circ} \mathrm{C}$, the lifetimes decreased gradually in nitrogen and more steeply in air. The decrease in lifetime with temperature 
in nitrogen reflects an increase in $\mathrm{k}_{\mathrm{P}}$, the rate of non-radiative decay of the excited $\mathrm{T}_{1}$ state, caused by increases in the rate $\mathrm{k}_{\mathrm{TS} 1}$ of reverse intersystem crossing to the excited $\mathrm{S}_{1}$ state and the rate $\mathrm{k}_{\mathrm{TS} 0}$ of intersystem crossing to the ground state $\mathrm{S}_{0}$ because of collisional quenching, whereas the lower lifetimes when oxygen is present are caused by the additional collisional quenching by oxygen with rate $\mathrm{k}_{\mathrm{Q}}\left[\mathrm{O}_{2}\right]$. A plot of the lifetimes (Figure VII- 4d) clearly revealed that the lifetimes of pure $\beta$ - Lg were lower than that seen in either BSA or the amorphous protein mixtures. However, the measured lifetimes of the mixtures were not significantly different from each other and had values very similar to BSA. The probe Erythrosin B binding specifically to BSA (Garland and Moore, 1979; Nack and Ludescher, 2006) also cannot be ruled out.

The lifetimes in the presence of air were significantly lower (Figure VII-4e) than in nitrogen in all the mixtures, clearly indicating that oxygen quenching was significant in these matrices. Also the lifetimes in the presence of air were comparable to each other showing that in air the matrices had far more dynamic complexity and quenching processes dominated and caused a decrease in the lifetimes.

The stretching exponent $\beta$ is a measure of the dynamic heterogeneity in the matrix; values of $\beta$ close to 1 indicate that the matrix is dynamically homogenous while smaller values of $\beta$ indicate increasing dynamic heterogeneity (Lindsey and Paterson, 1980). It is clearly evident from Figure VII-5a, b that the decay kinetics of Ery B in pure protein and protein mixtures became more heterogeneous with increasing temperature and in the presence of oxygen. The values of $\beta$ in BSA and mixtures were significantly higher than in the pure $\beta$-Lg films. But within the scope of the error this increase in $\beta$ was insignificant. The stretching exponent $\beta$ in air had comparable and much lower values in 
all the pure protein and protein mixtures. The lower values may be attributed to increase in the heterogeneous environments in the presence of oxygen as the quencher species.

\section{Photophysical rate constants}

Additional analysis of lifetimes with respect to various photo physical rate constants can throw light on the various non-radiative processes that quench the excited triplet state. These non-radiative processes can be important indicators of the mobility and permeability in amorphous matrices. Since the oxygen-quenching rate is the product of $\mathrm{k}_{\mathrm{Q}}$, which is proportional to the oxygen diffusion rate through the matrix, and $\left[\mathrm{O}_{2}\right]$, the concentration of oxygen in the protein film, it is proportional to the permeability of oxygen through the protein film (Nack \& Ludescher, 2006).

The rate constant for quenching the triplet state by oxygen, $\mathrm{k}_{\mathrm{Q}}\left[\mathrm{O}_{2}\right]$, is calculated from the difference in inverse lifetime in air and in nitrogen. The oxygen quenching rate $\mathrm{k}_{\mathrm{Q}}\left[\mathrm{O}_{2}\right]$, increased gradually until $40^{\circ} \mathrm{C}$, where there was a more drastic increase with temperature increase. It is clearly evident from Figure VII-6 that BSA, $\beta$-Lg and the mixtures had identical permeabilities over the entire range of temperature $\left(0-100^{\circ} \mathrm{C}\right)$. Thus it is clear that mixing differently sized proteins in varying molar ratios didn't bring significant changes to the permeability.

The temperature effect on $\mathrm{k}_{\mathrm{Q}}\left[\mathrm{O}_{2}\right]$ was further analyzed by Arrhenius analysis of $\mathrm{k}_{\mathrm{Q}}\left[\mathrm{O}_{2}\right]$ (Figure VII-7). From these plots, activation energies $\left(\mathrm{E}_{\mathrm{A}}\right)$ were calculated. Table VII- 1 illustrates the $\mathrm{E}_{\mathrm{A}}$ values for $\mathrm{k}_{\mathrm{Q}}\left[\mathrm{O}_{2}\right]$ in various matrices. It is evident that there were minor differences in the activation energies between the Mixture I and mixture II at low $\left(0-20^{\circ} \mathrm{C}\right)$ and high temperatures $\left(80-100^{\circ} \mathrm{C}\right)$. However, mixture III had significantly lower activation energy at low temperatures. It is clear that the activation energies for 
oxygen quenching were significantly lower in the mixtures when compared to the pure proteins.

The Arrhenius plot of oxygen quenching (FigureVII-7) was biphasic in all the mixtures with lower break point temperatures than in pure $\beta$ - $\mathrm{Lg}(\beta-\mathrm{Lg}$ break point at $\sim 75^{\circ} \mathrm{C}$ ). Break in the plots were observed at $39.5^{\circ} \mathrm{C}$ in mixture I and $37.5^{\circ} \mathrm{C}$ in mixture II. However, the transition temperatures in mixture III was significantly lower at $26.4^{\circ} \mathrm{C}$. These break point temperatures calculated from the arrhenius plots, correlated well with the observed increase in $\mathrm{k}_{\mathrm{Q}}\left[\mathrm{O}_{2}\right]$ in the various mixtures as depicted in Figure VII-6.

The decrease in lifetime with temperature essentially reflects an increase in the non-radiative decay of the excited triplet state $\mathrm{T}_{1}$ (Eq 4 Materials and Methods) due to an increase in both the rate of non- radiative decay to the ground state $\mathrm{S}_{0}\left(\mathrm{k}_{\mathrm{TS} 0}\right)$ and reverse intersystem crossing to $\mathrm{S}_{1}\left(\mathrm{k}_{\mathrm{TS} 1}\right)$. Based on the maximum physically reasonable value of $\mathrm{k}_{\mathrm{TS} 1}$, an estimate of the lower limit of $\mathrm{k}_{\mathrm{TS} 0}$ was calculated and is plotted versus temperature in Figure VII- 8 . The $\mathrm{k}^{\circ}$ TS1 values used were $4 * 10^{7} \mathrm{~s}^{-1}, 2 * 10^{7} \mathrm{~s}^{-1}$ and $6.5 *$ $10^{7} \mathrm{~s}^{-1}$ in mixture I, mixture II and mixture III, respectively.

The quenching rate $\mathrm{k}_{\mathrm{TS} 0}$ in the mixtures increased with increase in temperature but with a lower magnitude and lesser slope than the pure proteins indicating lower mobility in the mixtures.

Mixture I, mixture II and mixture III had $\mathrm{k}_{\mathrm{TS} 0}$ values of $1535 \mathrm{~s}^{-1}, 1550 \mathrm{~s}^{-1}$ and $1518.7 \mathrm{~s}^{-1}$ respectively at $0^{\circ} \mathrm{C}$. These values were comparable to the pure BSA, but, significantly lower than $\beta$-Lg. However, with increase in temperature above a certain temperature (around $60^{\circ} \mathrm{C}$ ), mixture II had $\mathrm{k}_{\mathrm{Ts} 0}$ values similar to the pure $\beta$-Lg matrix. It 
exhibited the behavior of the protein BSA at low temperatures and that of $\beta-\mathrm{Lg}$ at high temperatures.

The activation energy of motions can be calculated from the Arrhenius plot of temperature dependence for $\mathrm{k}_{\mathrm{TS} 0}$ as shown in Figure VII- 9. Mixture I containing an equimolar ratio of BSA and $\beta$ - Lg yielded a single activation energy $\left(\mathrm{E}_{\mathrm{A}}\right)$ of $3.07 \mathrm{KJ}$ with no signs of dynamic transition. In Mixtures II and III, $\mathrm{k}_{\mathrm{TS} 0}$ was a biphasic linear function of temperature. Linear fits to the Arrhenius plot at low $\left(0-40^{\circ} \mathrm{C}\right)$ and high temperatures $(50-$ $\left.100^{\circ} \mathrm{C}\right)$, gave activation energies $\left(\mathrm{E}_{\mathrm{A}}\right)$ of the mixtures and these values are illustrated in Table VII-1. The activation energies of the mixtures were comparable to the protein $\beta$ $\mathrm{Lg}$ at low temperatures. However, at high temperatures, activation energies of $\beta$ - Lg were significantly larger then the mixtures. The activation energies for $\mathrm{k}_{\mathrm{Q}}\left[\mathrm{O}_{2}\right]$ were significantly larger than the activation energies for $\mathrm{k}_{\mathrm{TS} 0}$.

The break in the Arrhenius plot indicated a dynamic transition at $60^{\circ} \mathrm{C}$ in mixture II and $58^{\circ} \mathrm{C}$ in mixture III. These activation energies ie origins of so-called dynamic transition reflects the onset of large-scale collective motions. These transition temperatures with respect to $\mathrm{k}_{\mathrm{TS} 0}$ do not correlate with the breaks in the $\mathrm{k}_{\mathrm{Q}}\left[\mathrm{O}_{2}\right]\left(\sim 40^{\circ} \mathrm{C}\right)$ data, in the mixtures.

\section{Spectral heterogeneity}

Phosphorescence intensity decays of Erythrosin B in equi-molar mixture of 1:1 $\mathrm{BSA} / \beta-\mathrm{Lg}$ were measured as a function of emission wavelength at $0^{\circ} \mathrm{C}-100^{\circ} \mathrm{C}$ (Figure VII-10). All decays were well analyzed using a stretched exponential model. It is clearly evident that the lifetimes varied greatly over the emission wavelength in the protein mixture. Lifetimes were higher at the blue edge, and lower at the red edge. For instance, 
at $0^{\circ} \mathrm{C}$ the lifetimes varied from a high of $0.62 \mathrm{~ms}$ to a low of $0.53 \mathrm{~ms}$ at $740 \mathrm{~nm}$. Lifetimes increased at the blue edge to a maximum at $650-670 \mathrm{~nm}$ and then decreased at higher wavelengths. Similar variation in the decay kinetics have been previously reported in amorphous sucrose (Pravinata et al., 2005), maltose and maltitol (Shirke et al., 2005), lactose and lactitol (Shirke et al., 2006), and BSA (Nack and Ludescher, 2006). This behavior suggests that there were regions of different mobility and Erythrosin B was able to detect these site heterogeneities. As expected, lifetimes values for all emission wavelengths decreased with increase in temperature. Further experiments comparing the variation of lifetime across the emission wavelength showed no difference between the protein and protein mixtures envisaging that the composition didn't alter the heterogeneity in the matrix (Figure VII-11).

The stretching exponent $\beta$ also varied systematically as a function of emission wavelength. Figure VII-12 depicts the variation of $\beta$ with change in emission wavelength. $\beta$ values were clearly lower at the red $(740 \mathrm{~nm})$ and the blue edges $(640 \mathrm{~nm})$. $\beta$ increased with wavelength at the blue edge and had a maximum value at $680 \mathrm{~nm}$ and then decreased at higher wavelengths. It is clearly evident that there were widespread differences in $\beta$ indicating greater dynamic heterogeneity, within the intensity decays collected at the red and blue edges of the emission spectra. $\beta$ decreased systematically over the temperature range, once again suggesting that at higher temperatures the matrix becomes more heterogeneous. There was no change in the variation of $\beta$ across the emission band in the respective proteins and their mixture (Figure VII-13).

Phosphorescence emission spectra of Erythrosin B were collected as function of delay time by varying the delay time from $0.1,0.6,1.1$ and $1.6 \mathrm{~ms}$ at various 
temperatures $\left(0^{\circ} \mathrm{C}, 25^{\circ} \mathrm{C}\right.$ and $\left.65^{\circ} \mathrm{C}\right)$. Figure VII-14 is a plot of emission spectra for Erythrosin B in equimolar mixture of BSA and $\beta-\mathrm{Lg}$ as a function of delay time. The emission spectra at all temperatures blue -shifted with increase in delay time. At short time delay $(0.1 \mathrm{~ms})$ the spectrum reflected emission from all chromophores, whereas at long time delay (1.6 ms), the measured spectrum only reflected emission from long lived chromophores. The emission peak frequency $\left(v_{\mathrm{P}}\right)$ and bandwidth $\left(\Gamma_{\mathrm{P}}\right)$ for these spectra (calculated using Eq 2 and 3 Materials and Methods) are plotted versus delay time in Figures VII-15 and VII-16

The peak frequency increased with delay time at all temperatures. These shifts were approximately linear. Spectral shifts were consistent at all temperatures $\left(\sim 120 \mathrm{~cm}^{-}\right.$ ${ }^{1}$ ). These trends correlated with the experiments with respect to lifetime as a function of emission wavelength.

These data provide independent evidence that the emission from Erythrosin B in these amorphous protein mixtures is spectrally correlated such that probes in environments with shorter lifetimes have red-shifted emission spectra and probes in environments with longer lifetimes have blue-shifted emission spectra. These trends were clearly reflective of the spectral trends observed in other amorphous matrices.

The bandwidth decreased initially with increase in delay time. However after a time delay of $0.6 \mathrm{~ms}$ the bandwidth increased rapidly. The width reflects the inhomogenous broadening of the spectra due to presence of energetically different environments.

The lifetime decreased with an increase in temperature at all emission wavelengths. The apparent activation energy $\left(\mathrm{E}_{\mathrm{A}}\right)$ for de-excitation of the triplet state, calculated from the 
slope of a $\ln \left(\mathrm{k}_{\mathrm{TS} 0}\right)$ vs. 1/T curve, also decreased systematically with an increase in emission wavelength at all temperatures (Figure VII-17)

\section{$\underline{\text { Discussion }}$}

Why study the effect of molecular mobility and oxygen permeability of BSA $\beta-\mathrm{Lg}$ mixtures?

The interaction between BSA and $\beta-\mathrm{Lg}$ in heat-induced gels has been studied previously. It was found that the ratio of the two proteins to each other affected the gelation behavior with the inclusion of BSA accelerating the formation of heat-set gels (Tobitani and Ross-Murphy, 1997). Many studies in the past have elucidated this synergy with respect to various other physical parameters but have overlooked the effect of mixing these two differently sized proteins pertaining to the permeability and mobility of the matrix. The synergistic behavior with respect to permeability of these two differently sized proteins is of importance to the food industry. So the present study looked into the matrix mobility and permeability of these mixtures using phosphorescence spectroscopy.

Phosphorescence from Erythrosin B provides information about three different aspects of molecular mobility and dynamic heterogeneity within the BSA $\beta$-Lg mixtures. First, the emission energy sense changes in the rate of dipolar relaxation around the excited triplet state (Pravinata et al., 2005). Second, the emission lifetime $(\tau)$ provides a direct measure of the rate of non-radiative coupling to the ground state $\left(\mathrm{k}_{\mathrm{TS} 0}\right)$ due to matrix collisions with the probe (Papp and Vanderkooi, 1989; Pravinata et al., 2005; Vanderkooi and Berger, 1989), and third molecular oxygen, which is a potent diffusional 
quencher of the triplet state, can provide a direct measure of the rate of oxygen permeability through the matrix (Simon-Lukasik and Ludescher, 2004).

\section{Effect of matrix mobility on BSA $\quad \beta$-Lg mixtures.}

Phosphorescence emission energy is mainly influenced by the $T_{1}$ energy and thus the dipolar relaxations of the polar groups around the excited $T_{1}$ state within the matrix (Pravinata et al., 2005). For Ery B dispersed in a protein film, dipolar relaxation is primarily a result of reorientation of side-chain hydroxyl, amino, and carboxyl groups on the surface of the protein. The thermal response of the peak emission energy and bandwidth reflects the effect of temperature on the rate and extent of dipolar relaxation around the excited triplet state. The phosphorescence peak frequency decreased linearly and gradually in $\beta$-Lg films at low temperatures and more steeply at high temperatures as compared to BSA whereby the evidence of a dynamic transition was indicated by a biphasic decline in the emission energy (Nack and Ludescher, 2006). The phosphorescence peak frequency in the mixtures however did not show obvious biphasic behaviour over the entire temperature range. The linear relationship in these plots indicated that, unlike BSA, the mixtures did not show any signs of the presence of a dynamical transition. A comparative plot of the emission energies of the globular proteins BSA and $\beta-\mathrm{Lg}$ and their respective mixtures illustrated that the emission energy increased in the following order $\beta$ - $\operatorname{Lg}<\mathrm{BSA}<\beta$-Lg BSA Mixtures. These results were surprising as one would anticipate that BSA, owing to its larger size, greater conformational freedom in solution and lower denaturation temperature than $\beta$ - $\mathrm{Lg}$ would have faster dipolar relaxations and thus lower emission energy (Denaturation temperature for BSA is $68^{\circ} \mathrm{C}$ and $78^{\circ} \mathrm{C}$ in $\beta-\mathrm{Lg}$, Michnik, 2003; Murayama and Tomida, 2004 ). 
But what governs the relaxations of proteins in solution may not necessarily be the same in the solid state. In solution all the complex processes revolve around water. However, in solid state the entire gamut of reactions may be totally different. The higher emission energy in protein mixtures when compared to pure BSA and $\beta$ - $\mathrm{Lg}$ is suggestive of a dynamic coupling between these two differently sized proteins. When these two proteins are mixed together they affect the local packing. BSA being a larger globular protein and $\beta$-Lg being a smaller protein affects the local conformational dynamics and the packing density leading to a much tighter network and slower dipolar relaxations. $\beta$ Lg molecules seem to participate in specific interactions with the hydroxyl, amino, and carboxyl groups of BSA forming an intermolecular network. As a result the relaxation rate decreased due to limited mobility of surface dipolar groups. Increase in emission energy in mixtures is indicative of a decrease in the dipolar relaxation rate due to reduced mobility of the polar groups around the probe molecule.

The magnitude of $\mathrm{k}_{\mathrm{TS} 0}$ reflects both internal factors related to the manner in which the excited $T_{1}$ state of Ery $B$ is vibrationally coupled to the $S_{0}$ ground state as well as external factors apparently related to the manner in which the ground state vibrational energy can dissipate from the excited probe into the surrounding matrix. Because the efficiency of this vibrational coupling is related to the overall mobility of the matrix, the magnitude of $\mathrm{k}_{\mathrm{TS} 0}$ provides a measure of matrix mobility (Pravinata et al., 2005).

Compared with the pure proteins $\mathrm{k}_{\mathrm{TS} 0}$ in the mixtures were significantly lower indicating more restricted mobility. The mobility curves from mixture I and III were super-imposable at low temperatures and showed differences at higher temperatures. 
The temperature dependence of $\mathrm{k}_{\mathrm{TS} 0}$, is plotted in an Arrhenius format. These activation energies ie origins of so-called dynamic transition reflects the onset of large-scale collective motions. The small value of EA in the glass suggests that the motions that activate vibrational relaxation are small amplitude, localized modes of molecular mobility; in the rubber, on the other hand, the significantly larger value of EA suggests that the modes of protein motion involve significantly larger segments of the protein structure. The activation energies for $\mathrm{k}_{\mathrm{TS} 0}$ were clearly lower in the mixtures when compared to the pure proteins indicating that BSA and $\beta-\mathrm{Lg}$ when mixed together had a tighter structure, the tightly packed structure inhibited the large scale motions and shifted it to more localized small scale modes of mobility.

The break in the Arrhenius plot indicated a dynamic transition at $60^{\circ} \mathrm{C}$ in mixture II and $58^{\circ} \mathrm{C}$ in mixture III. These values were significantly lower than the pure proteins whereby a break in the Arrhenius plot occurred at $81^{\circ} \mathrm{C}$ in $\beta-\mathrm{Lg}$ film and $68^{\circ} \mathrm{C}$ in BSA. A recent study by Kehoe et al. (2007) revealed that when $\beta$-Lg was partially replaced with BSA, the denaturation temperature of the resultant mixture shifted to lower temperatures. However, in the present study this change in the dynamic transition cannot be attributed to denaturation occurring in solution as the curves between heating and cooling were super-imposable in these mixtures (data not shown). For reasons clearly indicated in Chapter II this temperature cannot be compared with denaturation occurring in protein solutions, however, may be reflective of a dynamic transition resembling a glass transition in these mixtures

It is clearly indicated that the proteins when mixed together bring about a decrease in the local relaxation mechanisms, however, the onset temperature for the 
origin of large scale motions or dynamic transition is drastically lowered in these mixtures when compared to the pure proteins.

Mixture II containing 2 moles of $\beta$-Lg and 1 mole BSA portrayed a noticeable biphasic behavior with respect to matrix mobility; whereby we could see that after the transition temperature $\left(60^{\circ} \mathrm{C}\right)$ there was an enhancement of $\mathrm{k}_{\mathrm{TS} 0}$ and the values were nearly identical to the pure $\beta$-Lg matrix. This particular matrix showed behavior similar to BSA at low temperatures and similar to $\beta$ - $\mathrm{Lg}$ at high temperatures, reflecting that the effect of composition and temperature had an effect on the mobility of the matrix.

Lower dipolar relaxation (higher emission energy), lower matrix mobility (longer lifetime, lower $\mathrm{k}_{\mathrm{Ts} 0}$ ) clearly suggest the dynamic synergy between these differently sized proteins and the stabilization effect with respect to mobility. In BSA and $\beta$-Lg mixtures the resultant protein- protein interactions affects the packing density and results in a tighter rigid structure and thus leading to a decrease in the matrix mobility and dipolar relaxations.

Studies by Garland and Moore (1979) have revealed that the probe Erythrosin B can specifically bind to BSA. Another suitable explanation for the increase in emission energy and decrease in matrix mobility $\left(\mathrm{k}_{\mathrm{Ts} 0}\right)$ is based on the assumption, that the probe Ery B may be bound specifically to BSA, and $\beta$-Lg molecules may alter the conformational dynamics and flexibility of the probe located in the BSA matrix.

The mobility contours of these mixtures reveal two important kind of information. These mixtures when mixed together bring a decrease in the local relaxations dynamics, however, the onset temperature of the origin of large scale collective motions or dynamic transition is drastically lowered in these mixtures when compared to the pure proteins. 


\section{Effect on Matrix permeability}

Despite differences in the $\mathrm{k}_{\mathrm{TS} 0}$ values and emission energies reflecting changes in mobility of the matrix, the permeability $\mathrm{k}_{\mathrm{Q}}\left[\mathrm{O}_{2}\right]$ of BSA, $\beta$ - $\mathrm{Lg}$ and the mixtures were very similar. It is evident that the restrictions in matrix mobility are insufficient to control the segmental motion necessary for oxygen transport. While some mobility is apparently deactivated by the protein mixtures, but the decrease in mobility is in sufficient to induce necessary changes with respect to a macroscopic diffusive property. In part, this difference must reflect the qualitatively different processes that are being monitored: $\mathrm{k}_{\mathrm{TS} 0}$ measures the coupling of vibrational motions in the probe with local motions in the protein matrix, whereas $\mathrm{k}_{\mathrm{Q}}\left[\mathrm{O}_{2}\right]$ measures the coupling of translational motion of an oxygen molecule with collective motions of the protein matrix (Nack and Ludescher, 2006).

The rate of oxygen quenching had a much higher $\mathrm{E}_{\mathrm{A}}$ value based on the linear fit to the data of an Arrhenius plot of $\mathrm{k}_{\mathrm{Q}}\left[\mathrm{O}_{2}\right]$, when compared to $\mathrm{k}_{\mathrm{TS} 0}$ values. The significantly larger activation energies for $\mathrm{k}_{\mathrm{Q}}\left[\mathrm{O}_{2}\right]$, show that the molecular motions to activate diffusion appears to involve more delocalized complex collective motions of the proteins. The break in plots of $\mathrm{k}_{\mathrm{Q}}\left[\mathrm{O}_{2}\right]$ do not correlate with the break in the $\mathrm{k}_{\mathrm{TS} 0}$ values in the mixtures $\left(\sim 60^{\circ} \mathrm{C}\right)$. This leads to the conclusion that these processes are not related to each other.

The linear relationship between $\mathrm{k}_{\mathrm{TS} 0}$ and $\mathrm{k}_{\mathrm{Q}}\left[\mathrm{O}_{2}\right]$ is evident in pure $\beta-\mathrm{Lg}$ and BSA films. But mixing two proteins generates a binary system and complexities arise due to changes in the composition and thus lead to differences in behavior. So to test the 
hypothesis and to find the relationship between the $\mathrm{k}_{\mathrm{Q}}\left[\mathrm{O}_{2}\right]$ and $\mathrm{k}_{\mathrm{TS} 0}$ we generated plots of oxygen permeability versus mobility.

Despite the massive disparities in activation energies for oxygen permeability (ten fold larger) and matrix mobility ( $\mathrm{k}_{\mathrm{Ts} 0}$ ), the plots depicted in Figure VII 18 a, b, c display linear correlations between $\mathrm{k}_{\mathrm{Q}}\left[\mathrm{O}_{2}\right]$ and $\mathrm{k}_{\mathrm{TS} 0}$ in all the mixtures. It is also evident from these plots that, a certain basal level of molecular mobility and temperature was required to bring about massive increases in permeability. Comparing these plots to amorphous protein model systems, we observed a direct linear dependence of oxygen permeability on molecular mobility in the BSA matrix (Nack and Ludescher, 2006) and a biphasic curve in $\beta$ - $\mathrm{Lg}$ (Sundaresan and Ludescher, 2008). The relative similarities in the plots of the pure proteins and the mixtures signify that the molecular motions underlying permeability and mobility are modulated in a specific direct manner in pure proteins and their mixtures.

\section{Spectral Heterogeneity in the BSA mixtures}

As reviewed by Ediger (2000) when a liquid is cooled below its melting point there is a drastic reduction in the molecular dynamics resulting in qualitative changes in the character of molecular motion with slowing of dynamics. There are regions with different dynamics one region can be orders of magnitude faster than the other region. This dynamic heterogeneity is important in understanding the transport and kinetic properties of chemical reactions in amorphous biomaterials.

Erythrosin B phosphorescence emission bandwidth, variation in lifetime across the emission bands and the emission decay-stretching exponent all provide information about site heterogeneity. These heterogeneities provide insight in to the local 
environments within the amorphous matrix. Previous studies in our lab have established dynamic site heterogeneity in various amorphous solids composed of pure sugars (Pravinata et al., 2005), sugar alcohols (Shirke and Ludescher, 2005; Shirke et al ., 2006) and proteins (Nack and Ludescher, 2006), suggesting that heterogeneity is a common feature of amorphous solid foods and biomaterials.

The emission bandwidth (FWHM) changed insignificantly within the pure proteins and the mixtures over the entire temperature range. Overlooking, the slight increase at high temperatures, altering the composition didn't bring any changes in the width of the distribution of energetically distinct matrix environments.

Phosphorescence intensity decays of Ery B in $\beta-\mathrm{Lg} / \mathrm{BSA}$ mixtures were measured as a function of emission wavelength at a temperature range of $0-100^{\circ} \mathrm{C}$ and the respective mixtures were measured as a function of emission wavelength at various temperatures. The findings from this and previous studies are not consistent with the standard homogenous relaxation model in which longer lifetime probes have red shifted emission due to extensive dipolar relaxation around the longer lived excited states (Pravinata, 2005; Richert, 2000). The decreases in lifetime and corresponding increase in $\mathrm{K}_{\mathrm{TS} 0}$ across the emission band in the mixtures essentially were consistent with the findings with respect to the dynamic site heterogeneity model, described by Pravinata et al. (2005) in amorphous sucrose.

In the dynamic site heterogeneity model, probes with blue shifted emission have longer lifetimes and thus have smaller values of $\mathrm{k}_{\mathrm{TS} 0}$ while probes in sites with red shifted emission have shorter lifetimes and thus larger values of $\mathrm{k}_{\mathrm{TS} 0}$. Blue shifted sites thus 
have lower rates of molecular collisions that dissipate the vibrational energy of the probe while red shifted sites have faster rates of collisions.

There was no change in the variation in lifetime (and thus $\mathrm{k}_{\mathrm{TS} 0}$ ) between the BSA, $\beta$-Lg and their mixture, across the emission bands. Since this variation of lifetime with wavelength reflects the broad continuum of local matrix sites that vary in terms of their overall molecular mobility, mixing BSA and $\beta-\mathrm{Lg}$ in different molar ratios doesn't seem to influence this variation across this continuum.

The wavelength dependence of the apparent activation energies $\Delta \mathrm{E}_{\mathrm{A}}$ for matrix quenching can be calculated from the Arrhenius plot of $\mathrm{k}_{\mathrm{TS} 0}$ versus temperature at respective wavelength. The activation energy $\Delta \mathrm{E}_{\mathrm{A}}$ also exhibited spectral heterogeneity in amorphous proteins and their mixture. The mixture displayed single activation energy over the entire temperature range as opposed to the pure BSA and $\beta-\mathrm{Lg}$ which displayed separate activation energies at high and low temperatures. However, the trend across the emission band remained the same with the blue shifted sites displaying higher activation energies for molecular mobility and the red shifted sites showing lower activation energies.

Shirke and Ludescher, 2005 correlated higher activation at blue sites to a strongly interacting matrix that has lower molecular mobility, probably due to strong hydrogen bonding interactions, whereas the red sites are characterized by faster collisional quenching rates with weakly interacting molecules and higher overall molecular mobility due to less extensive hydrogen bonding. Nack and Ludescher 2006, attributed higher activation energies at the blue sites to the collective motions of larger regions of the protein matrix. 
The term $\beta$ reflects the distribution of lifetimes and thus the corresponding distribution of dynamically distinct probe environments with different values of $\mathrm{k}_{\mathrm{TS} 0}$ (Pravinata et al., 2005). Mixtures of BSA and $\beta$-Lg had different sites with variable heterogeneity as reflected by the variation in $\beta$. High value of $\beta$ at around $680 \mathrm{~nm}$ reflected a significant decrease in the range of dynamically distinct probe environments in that particular wavelength. Also low values of $\beta$ in the red and blue edge reflected the presence of broader distribution of $\mathrm{k}_{\mathrm{TS} 0}$ at these sites. The variation of $\beta$ across the emission band in BSA, $\beta-\mathrm{Lg}$ and the mixtures were very similar.

The BSA/ $\beta$-Lg mixture showed a consistent increase in the peak frequency with an increase in delay time. These trends were similar to that found in the pure proteins (Nack and Ludecher 2006; Sundaresan and Ludescher, 2007). The short delay times correspond to average emission energies for all chromophores distributed throughout the matrix and the longer delay times measure the emission energies from local high-energy sites that have long lived chromophores. These findings substantiate that their exists a physical coupling between dipolar relaxations and collisional quenching in the mixture whereby Ery B probes reside in distinct sites within the matrix, such that sites with higher emission energy have longer lifetime (thus lower mobility).

\section{$\underline{\text { Conclusion }}$}

This study used the phosphorescence of Erythrosin B to generate detailed mobility maps of amorphous BSA $\beta-\operatorname{Lg}$ mixtures. BSA is a large globular protein and approximately two times the size of the $\beta$-Lg molecule. It is clearly evident from this study that the resulting mixture of these two proteins significantly lowered the local 
relaxation dynamics due to favorable inter-molecular protein interactions resulting in the lowering of the dipolar relaxations. The packing density and reduction in the conformational freedom due to specific interactions between these differently sized proteins may be the cause of to these changes in dynamics. However the interactions between these proteins do not seem to affect the permeability of the resultant matrix. These findings aid in the understanding of complex molecular interactions in amorphous protein mixtures and it is worthy mapping out the relationship between these proteins with respect to mobility. Dynamic site heterogeneity is also observed in this matrix and this can create a new perspective and provide us with deeper insight into the understanding of complex dynamic properties of these milk proteins.

Our hypothesis that these proteins will complement each other by affecting the packing density was supported by the reductions in the matrix mobility; however, reductions in packing density were insufficient to hamper the entry of the permeant oxygen species. The synergistic response between these proteins may be useful to a food technologist. This information is useful in designing edible films as these mixtures will confer superior stability when compared to the pure matrices. 


\section{$\underline{\text { References }}$}

Anker, M., Stading, M., and Hermansson, A. (2001). Aging of Whey Protein Films and the Effect on Mechanical and Barrier Properties. J. Agric. Food Chem. 49, 989995.

Birks, J. B. (1970). Photophysics of Aromatic Molecules (Wiley Monographs in Chemical Physics). 704.

Duchowicz, R., Ferrer, M. L., and Acuña , A. U. (1998). Kinetic Spectroscopy of Erythrosin Phosphorescence and Delayed Fluorescence in Aqueous Solution at Room Temperature. Photochemistry and Photobiology. 68, 494-501.

Ediger, M. D. (2000). Spatially heterogeneous dynamics in supercooled liquids. Annu. Rev. Phys. Chem. 51, 99-128.

Fasman, G. D. and Editor. (1989). Practical Handbook of Biochemistry and Molecular Biology. 624.

Fennema, O. R. and Editor. (1996). Food Chemistry, Third Edition. [In: Food Sci. Technol. (N. Y.), 1996; 76]. 1089.

Garland, P. B. and Moore, C. H. (1979). Phosphorescence of protein-bound eosin and erythrosin: a possible probe for measurements of slow rotational mobility. Biochem. J. 183, 561-572.

Graham, D. E., Neustadter, E. L., Stockwell, A., Whittingham, K. P., and Cairns, R. J. R. (1979). Kinetics of interfacial phenomena in emulsion resolution. Surf.Act.Agents, Symp. 127-136.

Hines, M. E. and Foegeding, E. A. (1993). Interactions of alpha -lactalbumin and bovine serum albumin with beta -lactoglobulin in thermally induced gelation. J. Agric. Food Chem. 41, 341-346.

Hunter, J. R., Carbonell, R. G., and Kilpatrick, P. K. (1991). Coadsorption and exchange of lysozyme/b-casein mixtures at the air/water interface. J. Colloid Interface Sci. 143, 37-53.

Kehoe, J. J., Morris, E. R., and Brodkorb, A. (2007). The influence of bovine serum albumin on beta -lactoglobulin denaturation, aggregation and gelation. Food Hydrocoll. 21, 747-755.

Kinsella, J. E. and Whitehead, D. M. (1989). Proteins in whey: chemical, physical, and functional properties. Adv. Food Nutr. Res. 33, 343-438. 
Lettinga, M. P., Zuilhof, H., and Van Zandvoort, M. A. M. J. (2000). Phosphorescence and fluorescence characterization of fluorescein derivatives immobilized in various polymer matrices. Physical Chemistry Chemical Physics. 2, 3697-3707.

Lindsey, C. P. and Patterson, G. D. (1980). Detailed comparison of the Williams-Watts and Cole-Davidson functions. J. Chem. Phys. 73, 3348-3357.

Lukasik, K. V. and Ludescher, R. D. (2005a). Effect of plasticizer on dynamic site heterogeneity in cold-cast gelatin films. Food Hydrocoll. 20, 88-95.

Lukasik, K. V. and Ludescher, R. D. (2005b). Molecular mobility in water and glycerol plasticized cold- and hot-cast gelatin films. Food Hydrocoll. 20, 96-105.

Maroncelli, M. and Fleming, G. R. (1987). Picosecond solvation dynamics of coumarin 153: the importance of molecular aspects of solvation. J. Chem. Phys. 86, 62216239.

Michnik, A. (2003). Thermal stability of bovine serum albumin: DSC study. J.Therm.Anal.Calorim. 71, 509-519.

Murayama, K. and Tomida, M. (2004). Heat-induced secondary structure and conformation change of bovine serum albumin investigated by Fourier transform infrared spectroscopy. Biochemistry (N. Y. ). 43, 11526-11532.

Nack, T. J. and Ludescher, R. D. (2006). Molecular Mobility and Oxygen Permeability in Amorphous Bovine Serum Albumin Films. Food Biophysics. 1557-1858.

Papp, S. and Vanderkooi, J. M. (1989). Tryptophan phosphorescence at room temperature as a tool to study protein structure and dynamics. Photochem. Photobiol. 49, 775-784.

Paulsson, M. and Dejmek, P. (1992). Surface film pressure of b-lactoglobulin, alactalbumin and bovine serum albumin at the air/water interface studied by Wilhelmy plate and drop volume. J. Colloid Interface Sci. 150, 394-403.

Pravinata, L. C., You, Y., and Ludescher, R. D. (2005). Erythrosin B Phosphorescence Monitors Molecular Mobility and Dynamic Site Heterogeneity in Amorphous Sucrose. Biophys. J. 88, 3551-3561.

Richert, R. (2002). Heterogeneous solvent dynamics and time-resolved optical linewidths. J.Non-Cryst.Solids. 307-310, 50-56.

Richert, R. (2000). Triplet state solvation dynamics: Basics and applications. J. Chem. Phys. 113, 8404-8429. 
Rischel, C., Madsen, J. C., Andersen, K. V., and Poulsen, F. M. (1994). Comparison of backbone dynamics of apo- and holo-acyl-coenzyme A binding protein using $15 \mathrm{~N}$ relaxation measurements. Biochemistry (N. Y. ). 33, 13997-14002.

Shirke, S. and Ludescher, R. D. (2005 a). Dynamic site heterogeneity in amorphous maltose and maltitol from spectral heterogeneity in erythrosin B phosphorescence. Carbohydr. Res. 340, 2661-2669.

Shirke, S. and Ludescher, R. D. (2005 b). Molecular mobility and the glass transition in amorphous glucose, maltose, and maltotriose. Carbohydr. Res. 340, 2654-2660.

Shirke, S., Takhistov, P., and Ludescher, R. D. (2005). Molecular Mobility in Amorphous Maltose and Maltitol from Phosphorescence of Erythrosin B. J Phys Chem B. 109, 16119-16126.

Shirke, S., You, Y., and Ludescher, R. D. (2006). Molecular mobility and dynamic site heterogeneity in amorphous lactose and lactitol from erythrosin B phosphorescence. Biophys. Chem. 123, 122-133.

Simon-Lukasik, K. V. and Ludescher, R. D. (2004/7). Erythrosin B phosphorescence as a probe of oxygen diffusion in amorphous gelatin films. Food Hydrocolloids. 18, 621-630.

Sundaresan, K. V. and Ludescher, R. D. (2007). Molecular mobility and oxygen permeability in amorphous beta -lactoglobulin films. Food Hydrocoll. 22, 403413.

Suttiprasit, P., Krisdhasima, V., and McGuire, J. (1992). The surface activity of alactalbumin, b-lactoglobulin, and bovine serum albumin. I. Surface tension measurements with single-component and mixed solutions. J. Colloid Interface Sci. 154, 316-326.

Tobitani, A. and Ross-Murphy, S. B. (1997). Heat-Induced Gelation of Globular Proteins. 2. Effect of Environmental Factors on Single-Component and Mixed-Protein Gels. Macromolecules. 30, 4855-4862.

Vanderkooi, J. M. and Berger, J. W. (1989). Excited triplet states used to study biological macromolecules at room temperature. Biochimica et Biophysica Acta, Bioenergetics. 976, 1-27. 
Table VII-1. Activation energies of pure $\beta-\mathrm{Lg}$, BSA and BSA $\beta$-Lg, mixtures.

\begin{tabular}{|c|c|c|c|c|c|c|}
\hline \multirow[t]{2}{*}{ Matrix } & \multicolumn{2}{|c|}{$\begin{array}{l}\Delta E a \text { of } k_{T S O} \\
\text { KJ mol }^{-1}\end{array}$} & \multirow[t]{2}{*}{$\begin{array}{l}\text { Break } \\
\text { Point }\end{array}$} & \multicolumn{2}{|c|}{$\begin{array}{l}\Delta E a \text { of } k_{Q}\left[O_{2}\right] \\
\mathrm{KJ} \mathrm{mol}^{-1}\end{array}$} & \multirow[t]{2}{*}{$\begin{array}{l}\text { Break } \\
\text { Point }\end{array}$} \\
\hline & Low $T$ & High T & & Low $\mathrm{T}$ & $\operatorname{High} T$ & \\
\hline$\beta-\mathrm{Lg}$ & 2.35 & 21.4 & 81 & 20.8 & 54.8 & 75 \\
\hline BSA* & 1.4 & 7.9 & 68 & \multicolumn{2}{|c|}{29.9} & No \\
\hline Mixture 1 & \multicolumn{2}{|c|}{3.07} & No & 14.25 & 37.63 & 39.5 \\
\hline Mixture II & 2.8 & 12.6 & 60 & 14.35 & 37.26 & 37.5 \\
\hline Mixture III & 2.2 & 8.9 & 58 & 5.85 & 36.9 & 26.4 \\
\hline
\end{tabular}

* Values used with permission from Nack and Ludescher, 2006 


\section{Figure VII-1}

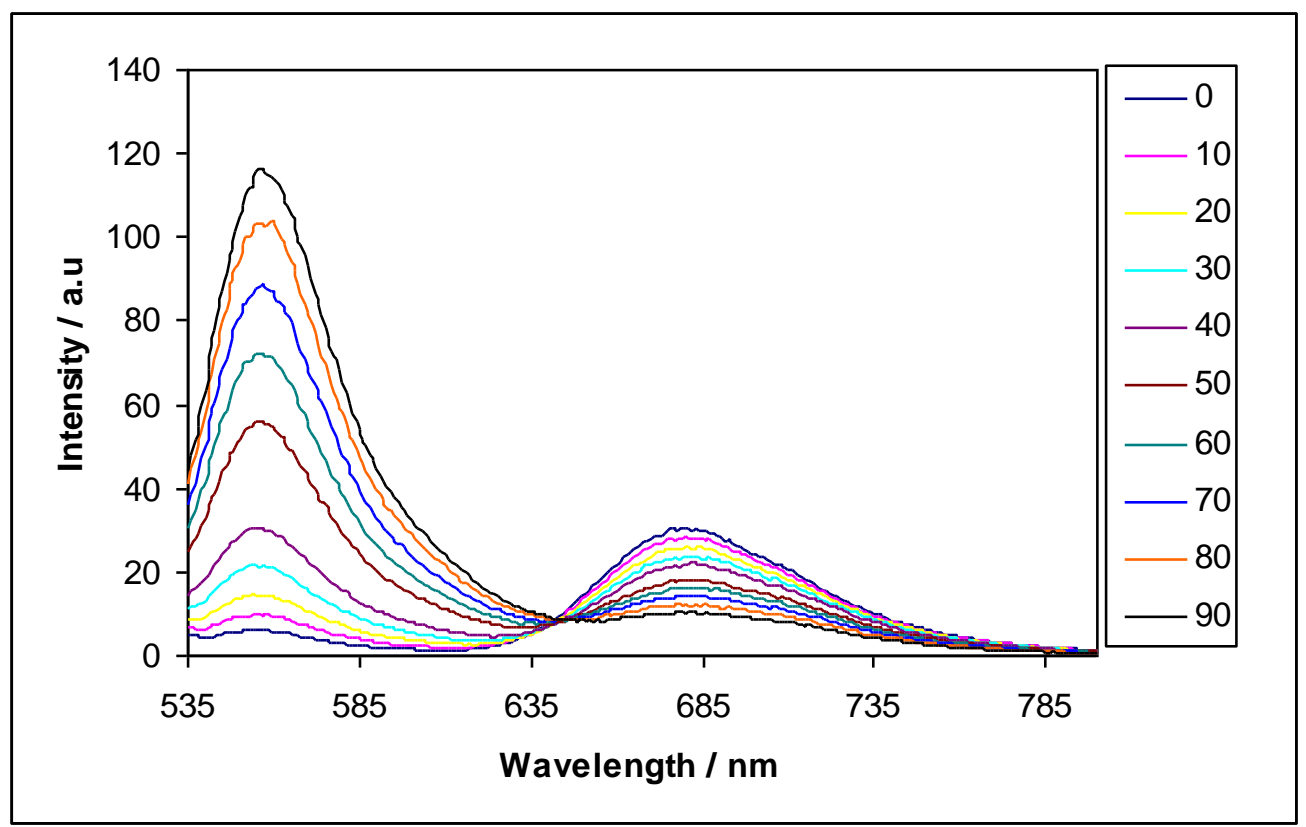

Figure VII-1: The effect of temperature on the delayed emission spectra of Erythrosin B dispersed in amorphous BSA/ $\beta-\operatorname{Lg}$ films (BSA: $\beta-\operatorname{Lg} 1: 1)$. 


\section{Figure VII-2}

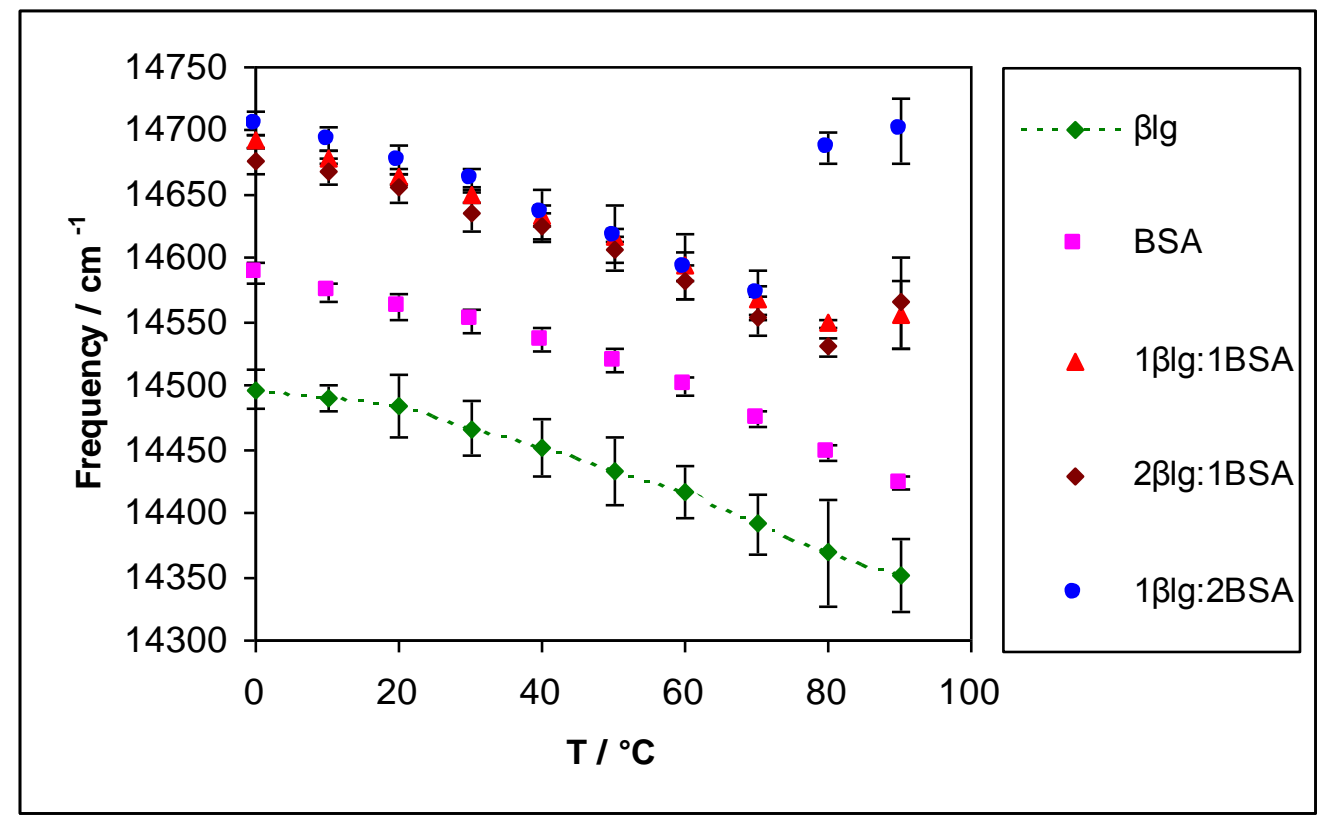

Figure VII-2: The effect of temperature on the emission maximum for phosphorescence from ErythrosinB in amorphous BSA/ $\beta$-Lg films.

\section{Figure VII-3}

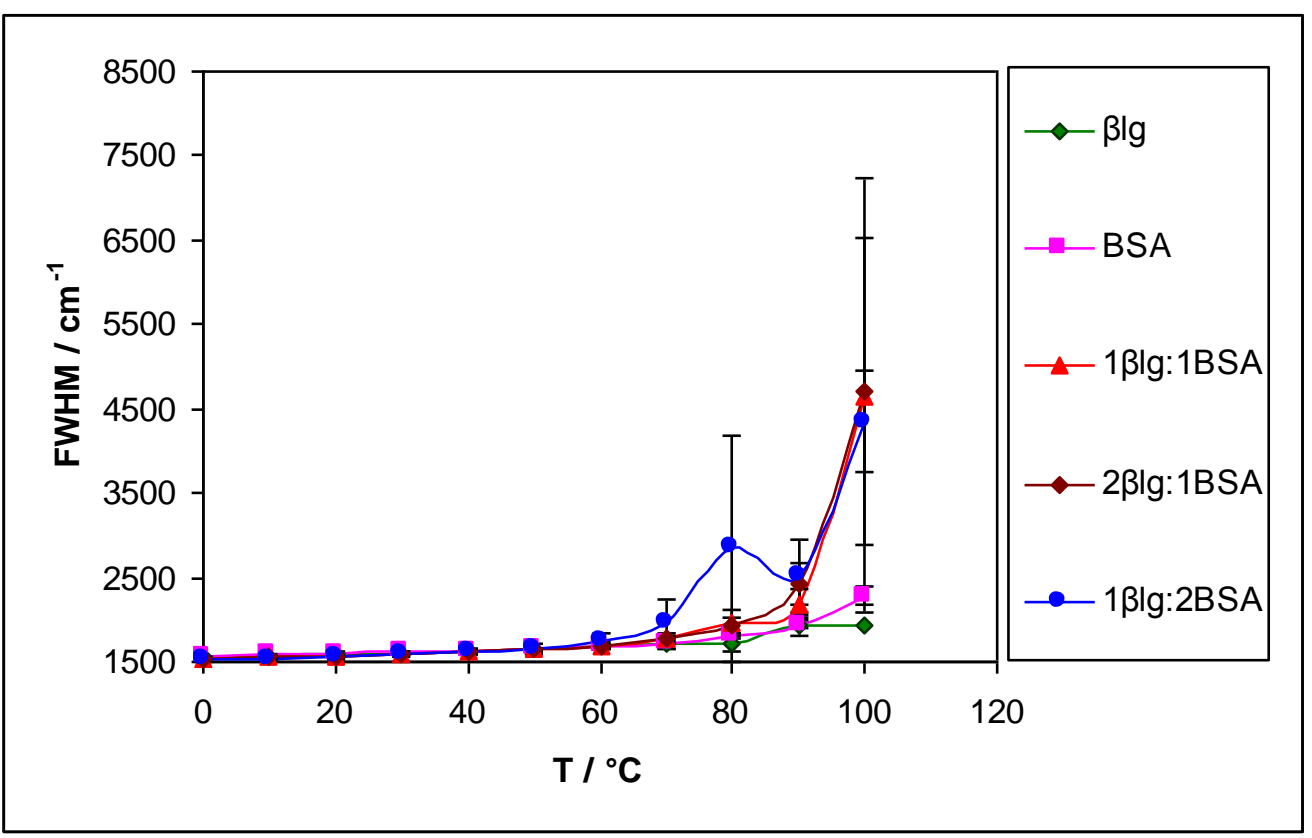

Figure VIII-3: The effect of temperature on the bandwidth for phosphorescence from Erythrosin B in amorphous BSA/ $\beta$-Lg films. 
Figure VII-4a

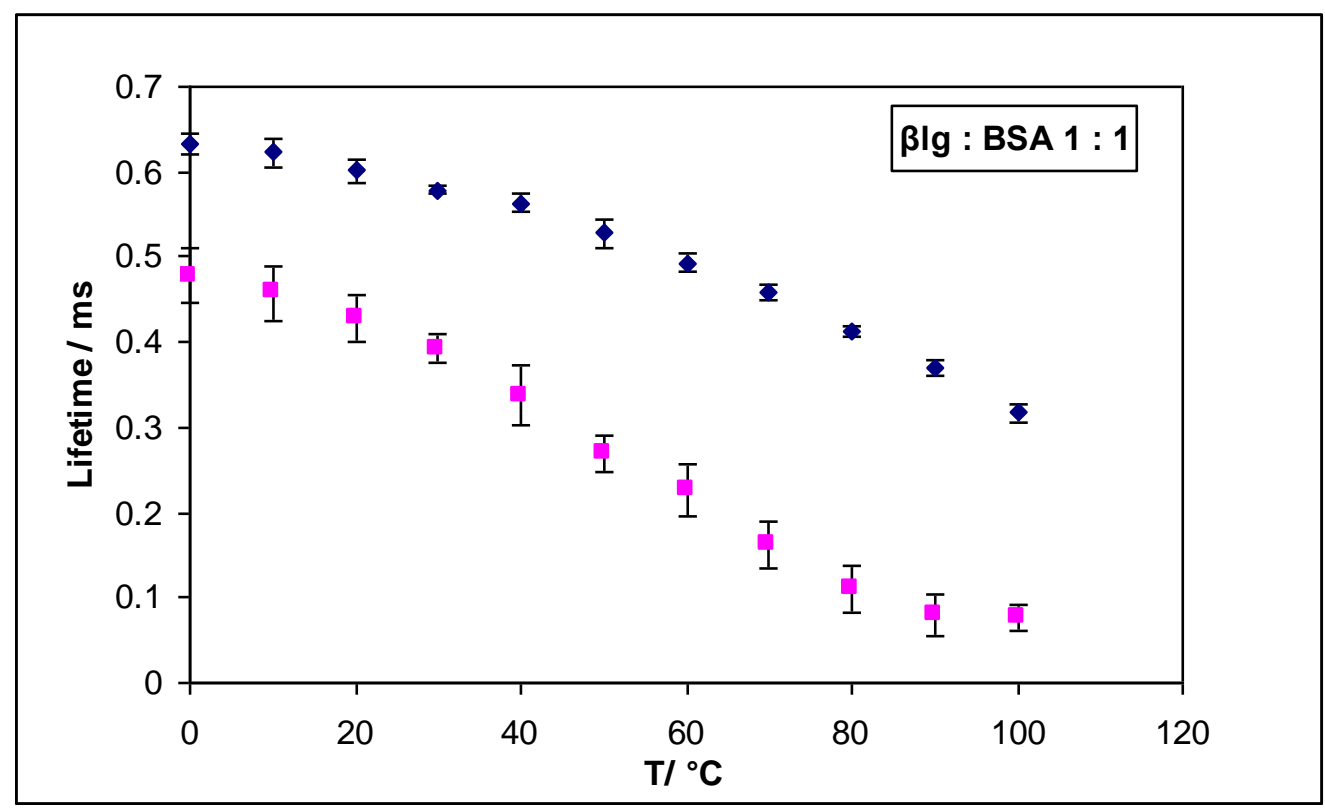

Figure VII-4b

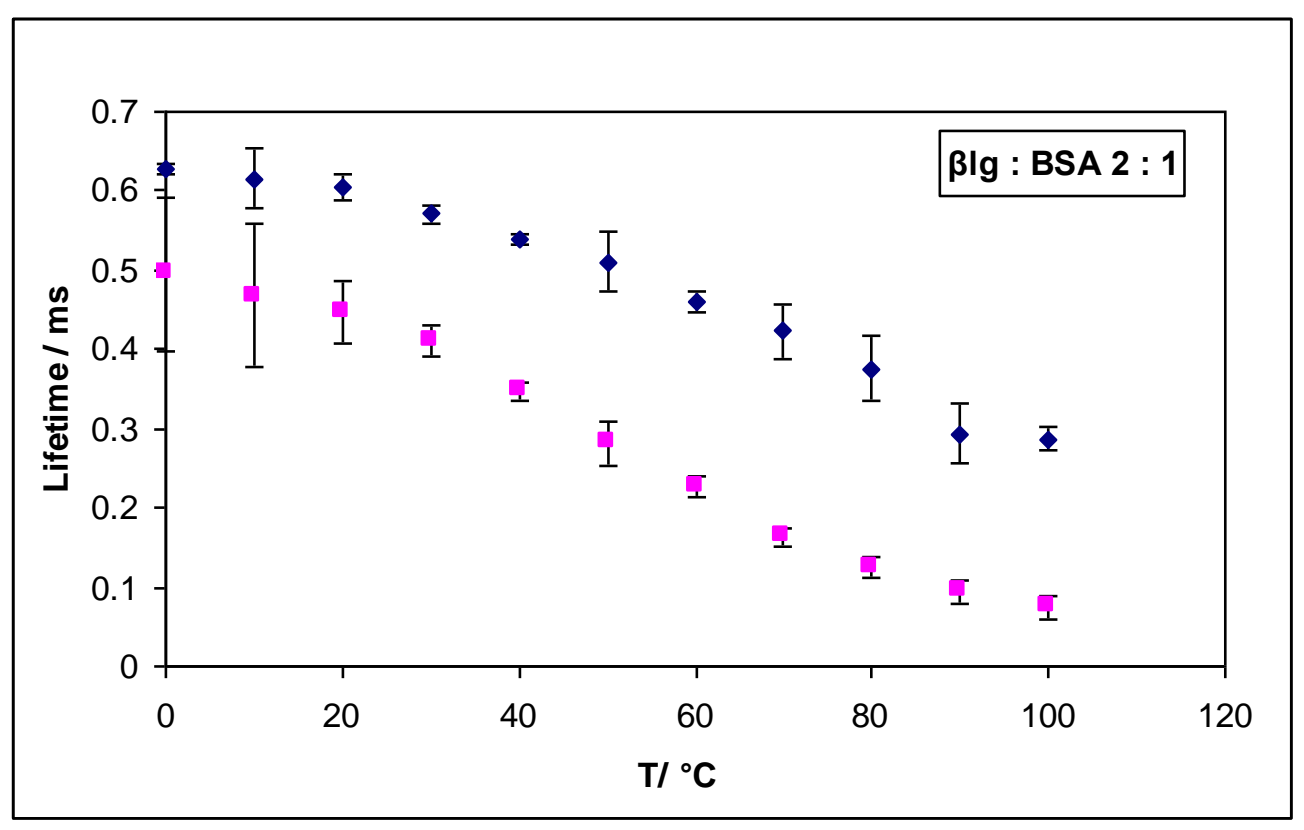




\section{Figure VII-4c}

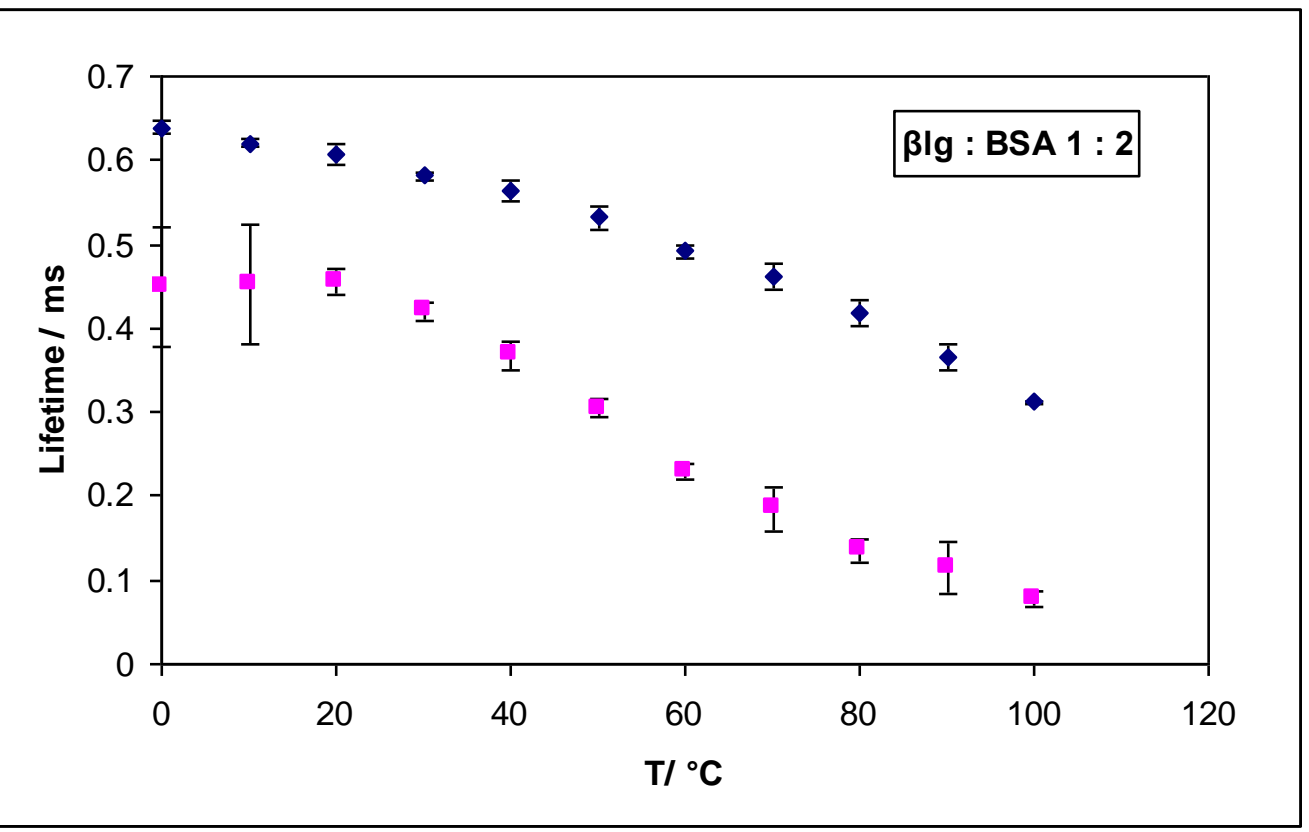

FigureVII-4a, $b$, c: Lifetime as function of temperature in amorphous BSA $\beta-\mathrm{Lg}$ films equilibrated against N2 $(\bullet)$ and air $(\square)$ : The lifetime was determined from analysis using a stretched exponential decay function.

Figure VII-4d

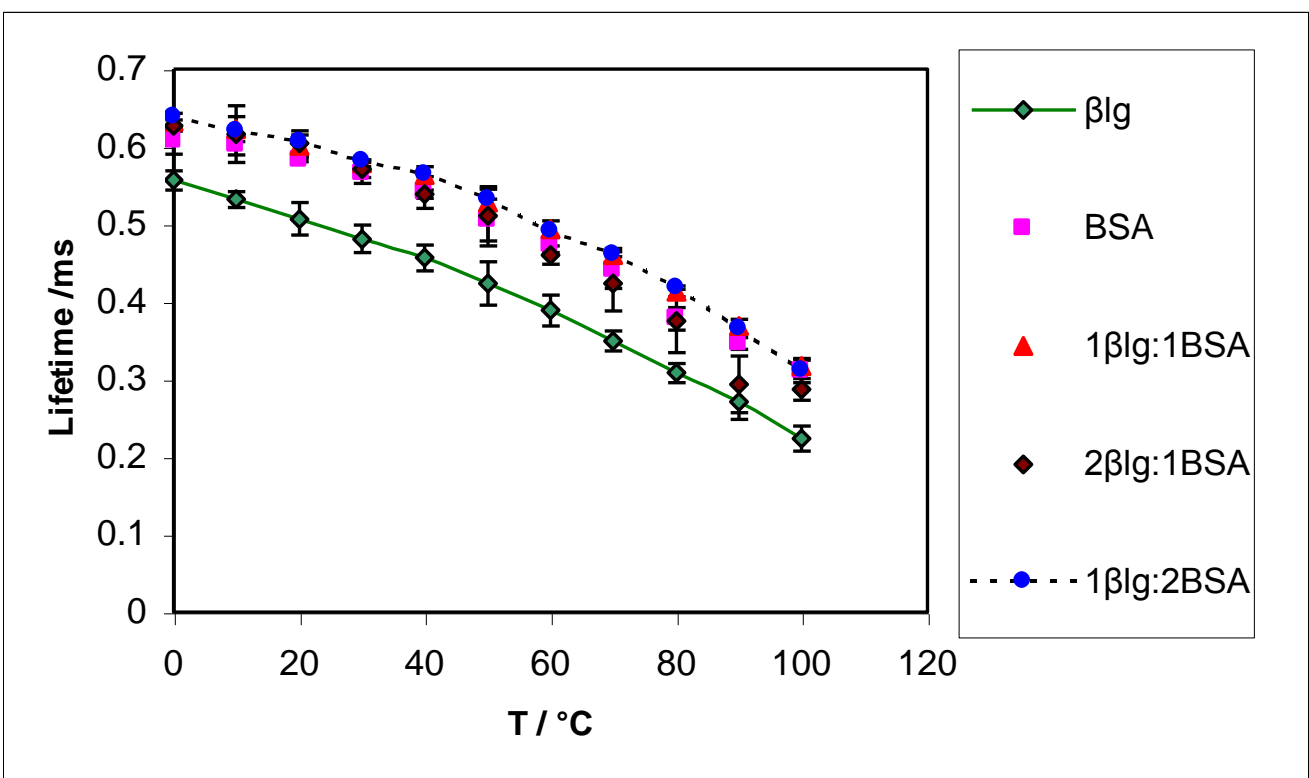

Figure VII-4d: Comparison plot depicting the effect of temperature on the lifetime of Erythrosin B dispersed in BSA/ $\beta$-Lg films equilibrated against nitrogen. The lifetime was determined from analysis using a stretched exponential decay function. 
Figure VII-4e

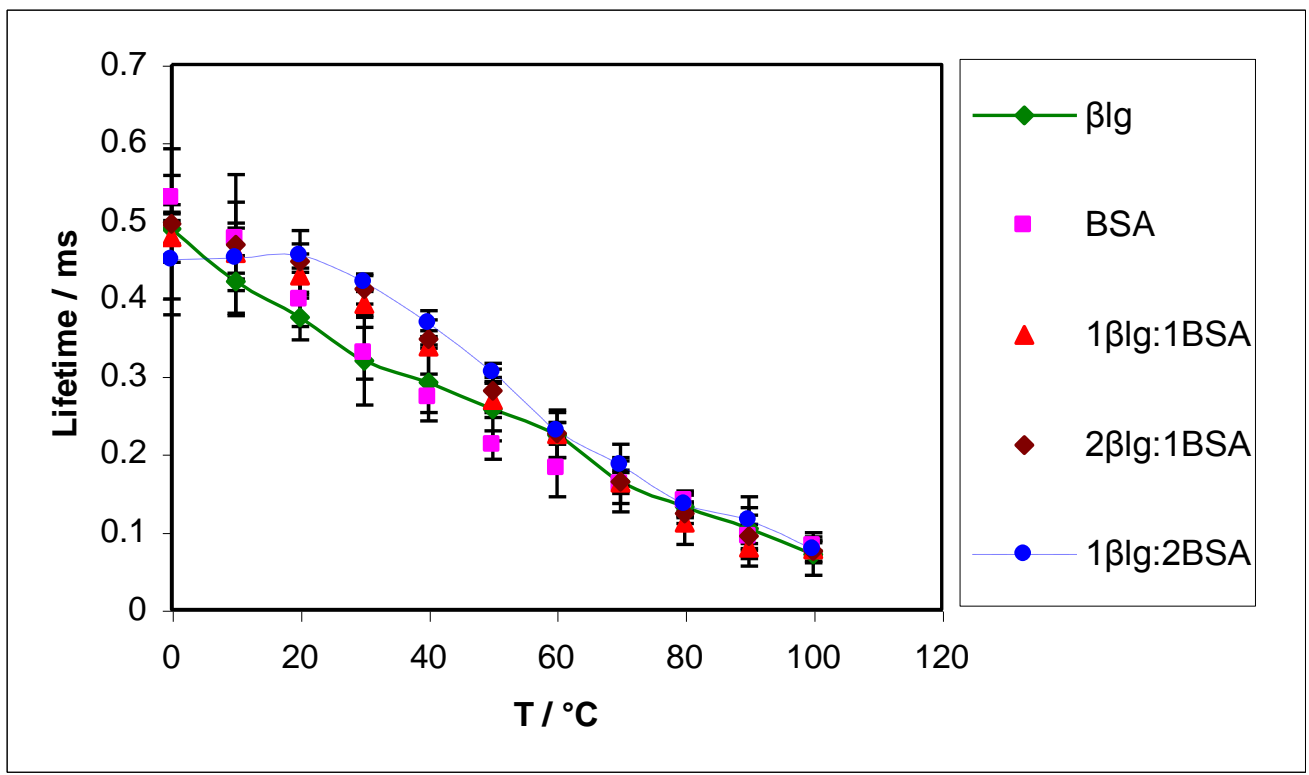

Figure VII-4e Comparison plot depicting the effect of temperature on the lifetime of Erythrosin $\mathrm{B}$ dispersed in BSA/ $\beta$ - $\mathrm{Lg} /$ films equilibrated against air. The lifetime was determined from analysis using a stretched exponential decay function. 
Figure VII-5a

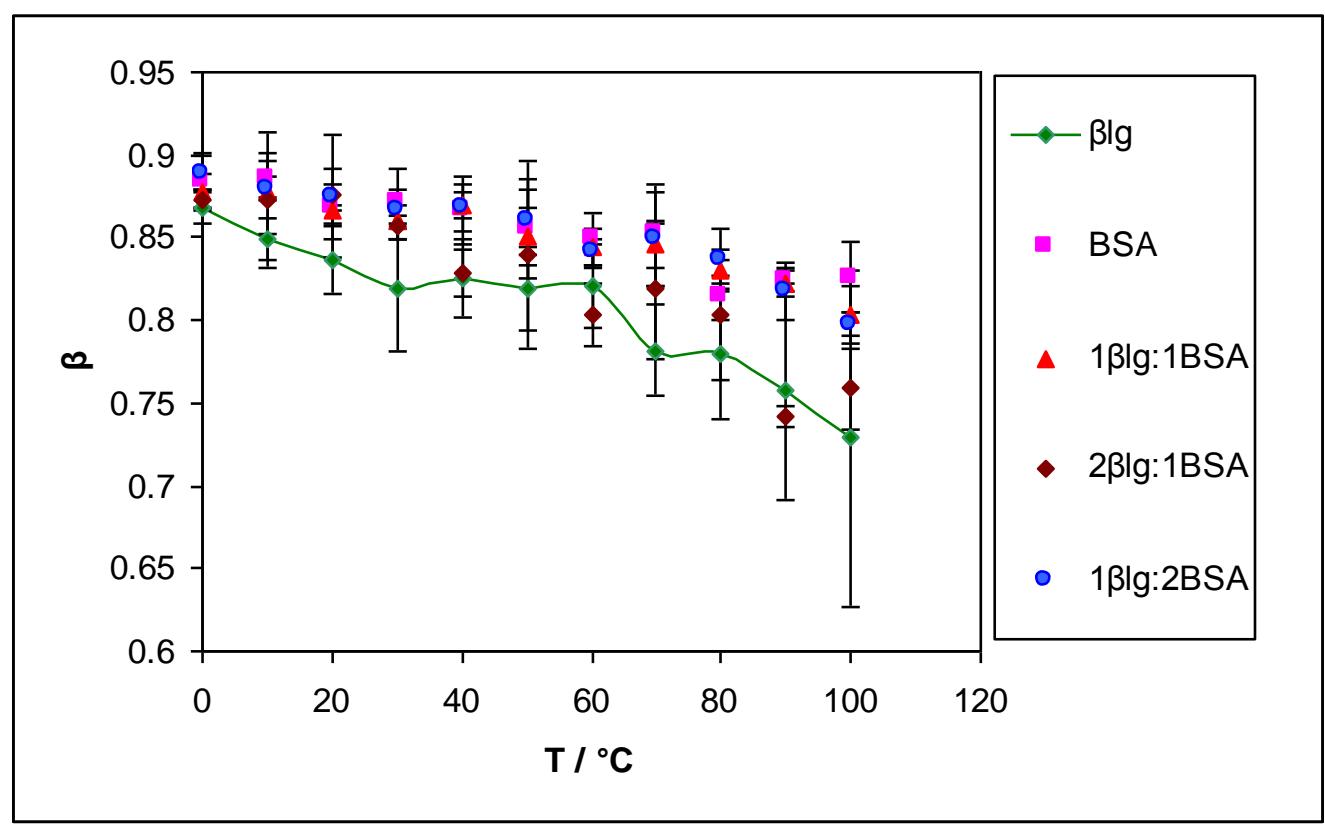

Figure VII-5a: The effect of temperature on the stretching exponent $\beta$ for Erythrosin B dispersed in BSA/ $\beta$-Lg films equilibrated against nitrogen. $\beta$ was determined from analysis using a stretched exponential decay function.

\section{Figure VII-5b}

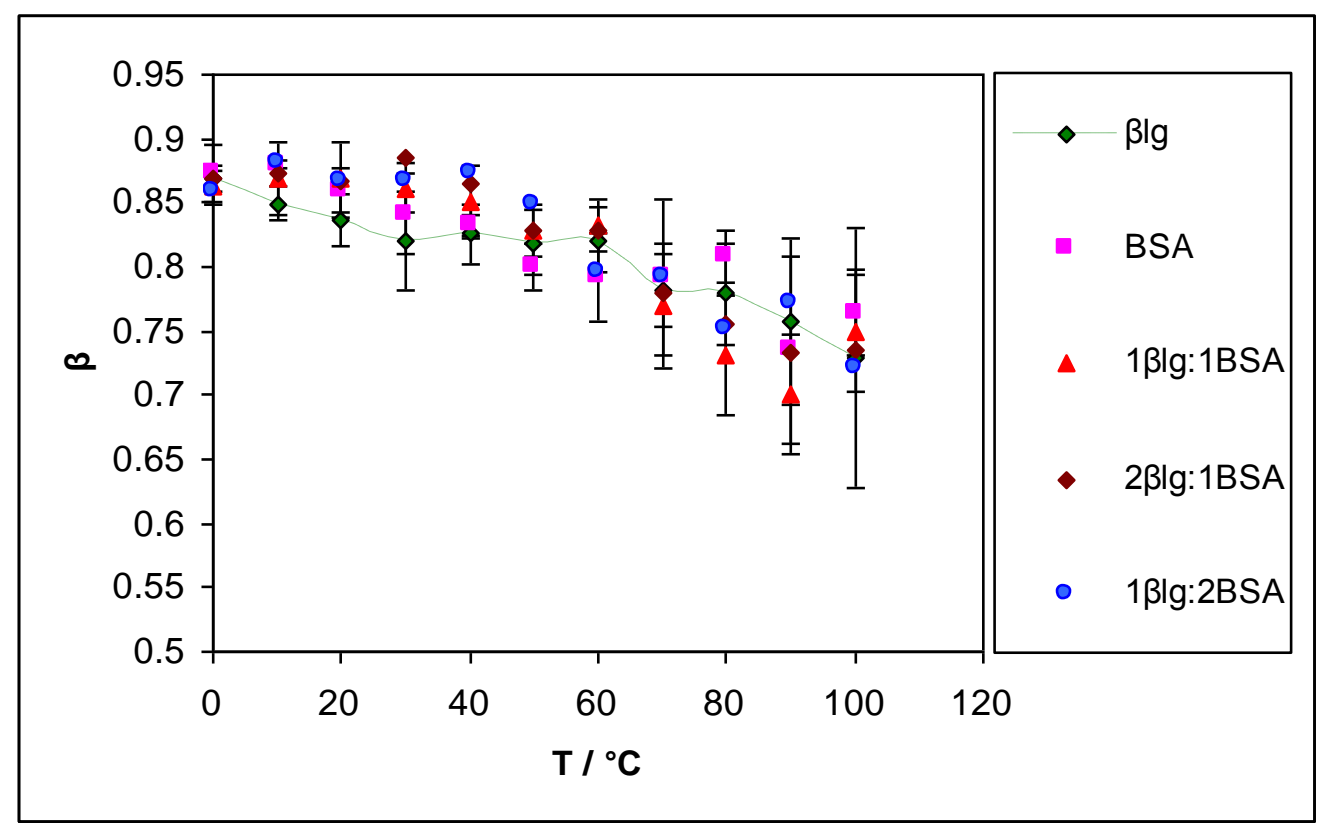

Figure VII-5b: The effect of temperature on the stretching exponent $\beta$ for Erythrosin B dispersed in BSA/ $\beta$ - $\mathrm{Lg}$ films equilibrated against nitrogen. $\beta$ was determined from analysis using a stretched exponential decay function. 
Figure VII-6

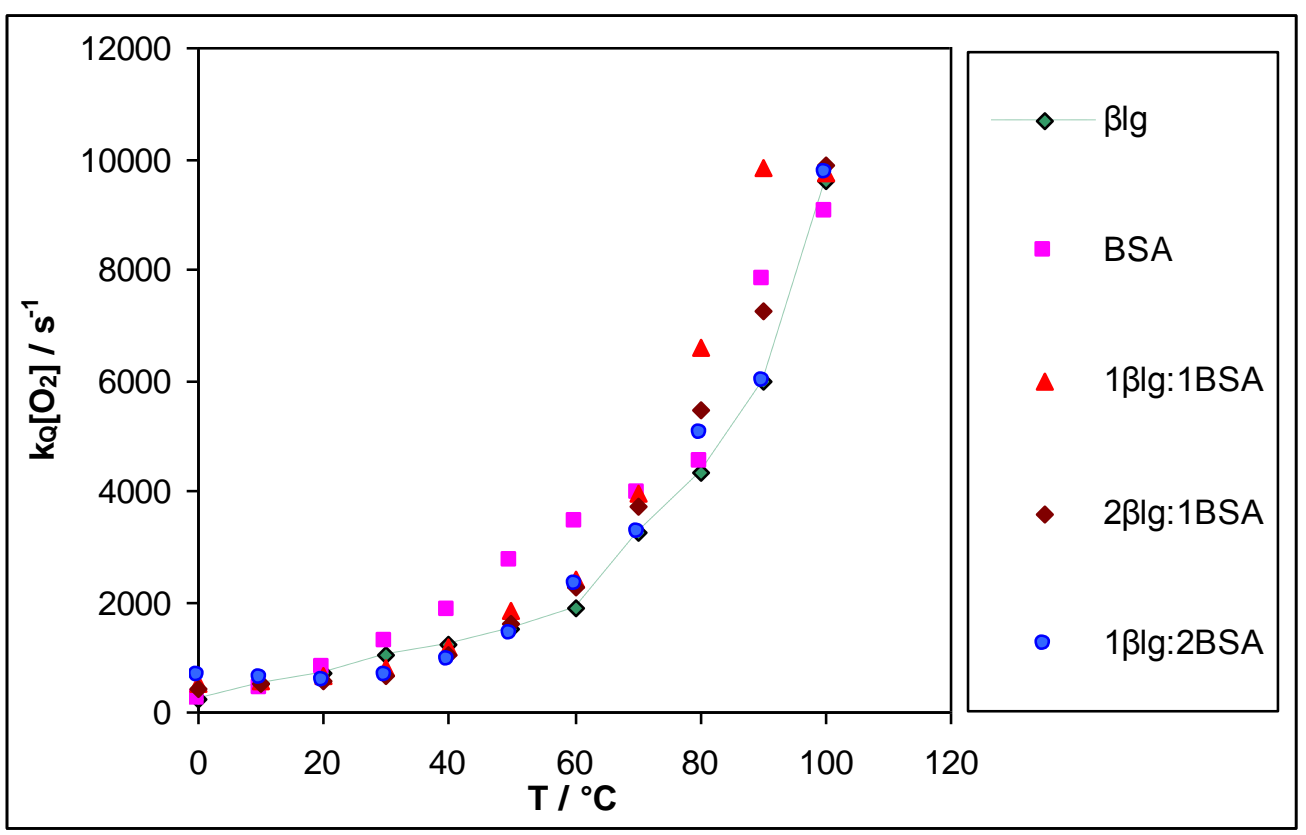

Figure VII-6: The oxygen quenching rate $\mathrm{k}_{\mathrm{Q}}\left[\mathrm{O}_{2}\right]$ as a function of temperature in $\beta$-Lg and BSA mixtures.

Figure VII-7

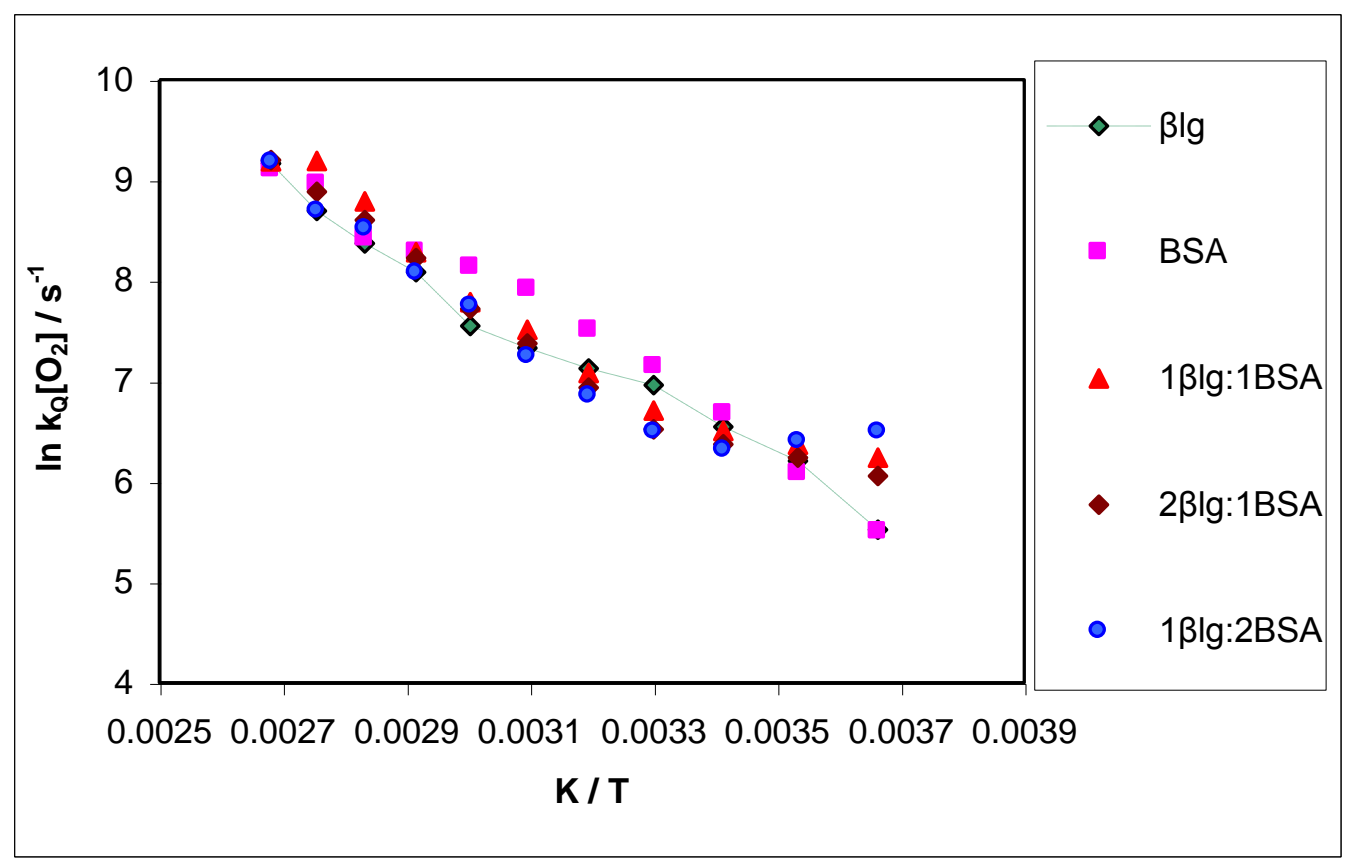

Figure VII-7: Arrhenius plot of the effect of temperature on oxygen quenching rate $\mathrm{k}_{\mathrm{Q}}\left[\mathrm{O}_{2}\right]$ in amorphous BSA/ $\beta$-Lg films. 
Figure VII-8

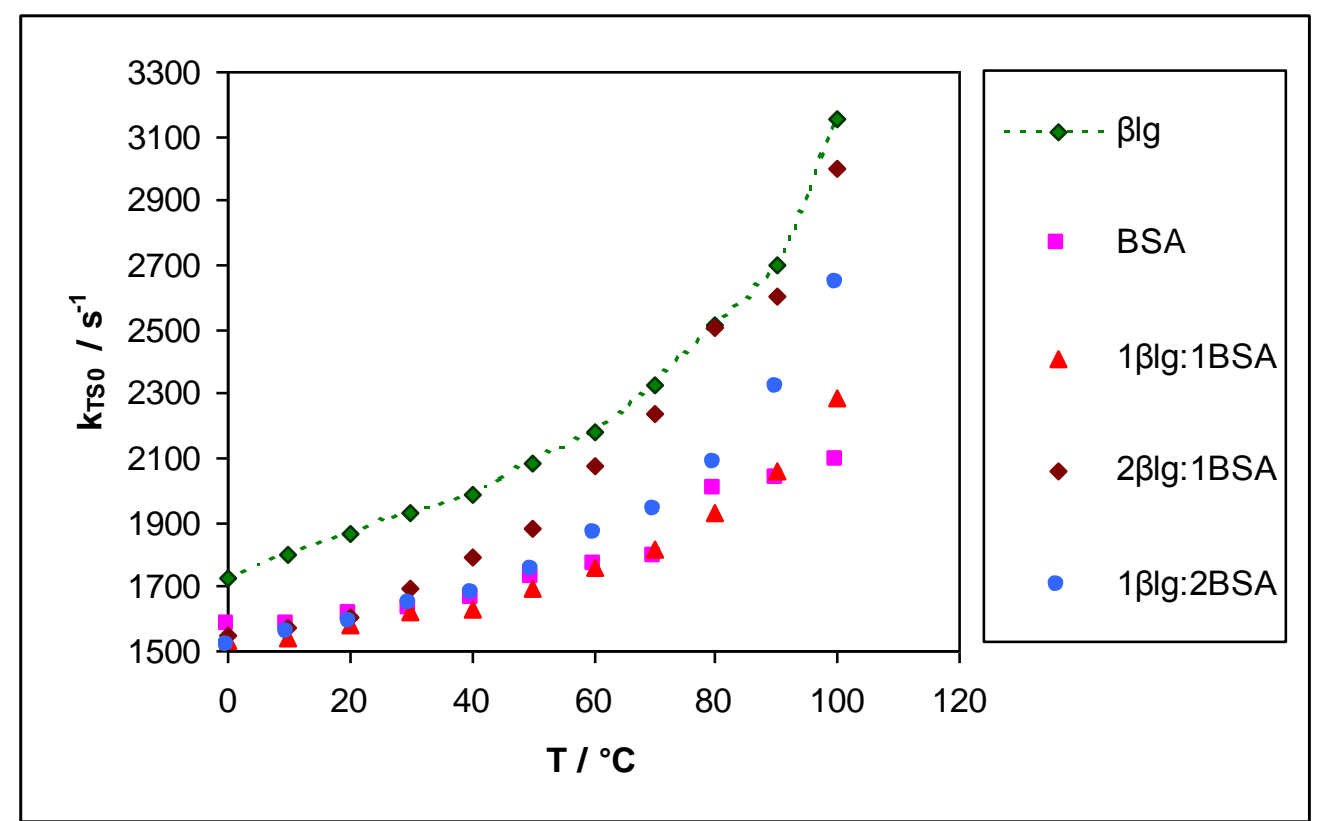

Figure VII-8: Plot of the effect of temperature on non radiative decay from $\mathrm{T}_{1}$ to $\mathrm{S}_{0}\left(\mathrm{k}_{\mathrm{TS} 0}\right)$ in amorphous BSA/ $\beta$-Lg films.

\section{Figure VII-9}

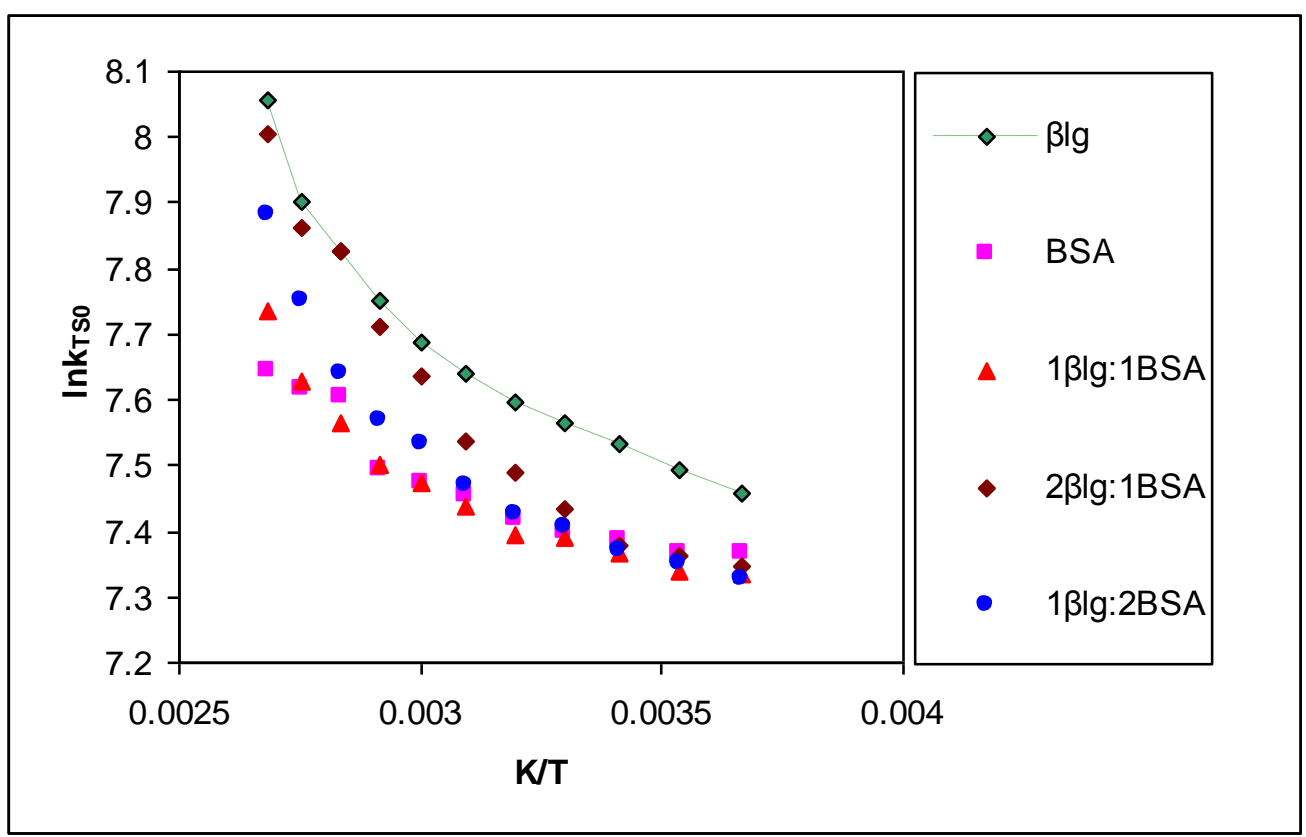

Figure VII-9: Arrhenius plot of the effect of temperature on non radiative decay from $\mathrm{T}_{1}$ to $\mathrm{S}_{0}\left(\mathrm{k}_{\mathrm{TS} 0}\right)$ in amorphous BSA/ $\beta$ - Lg films. 
Figure VII-10

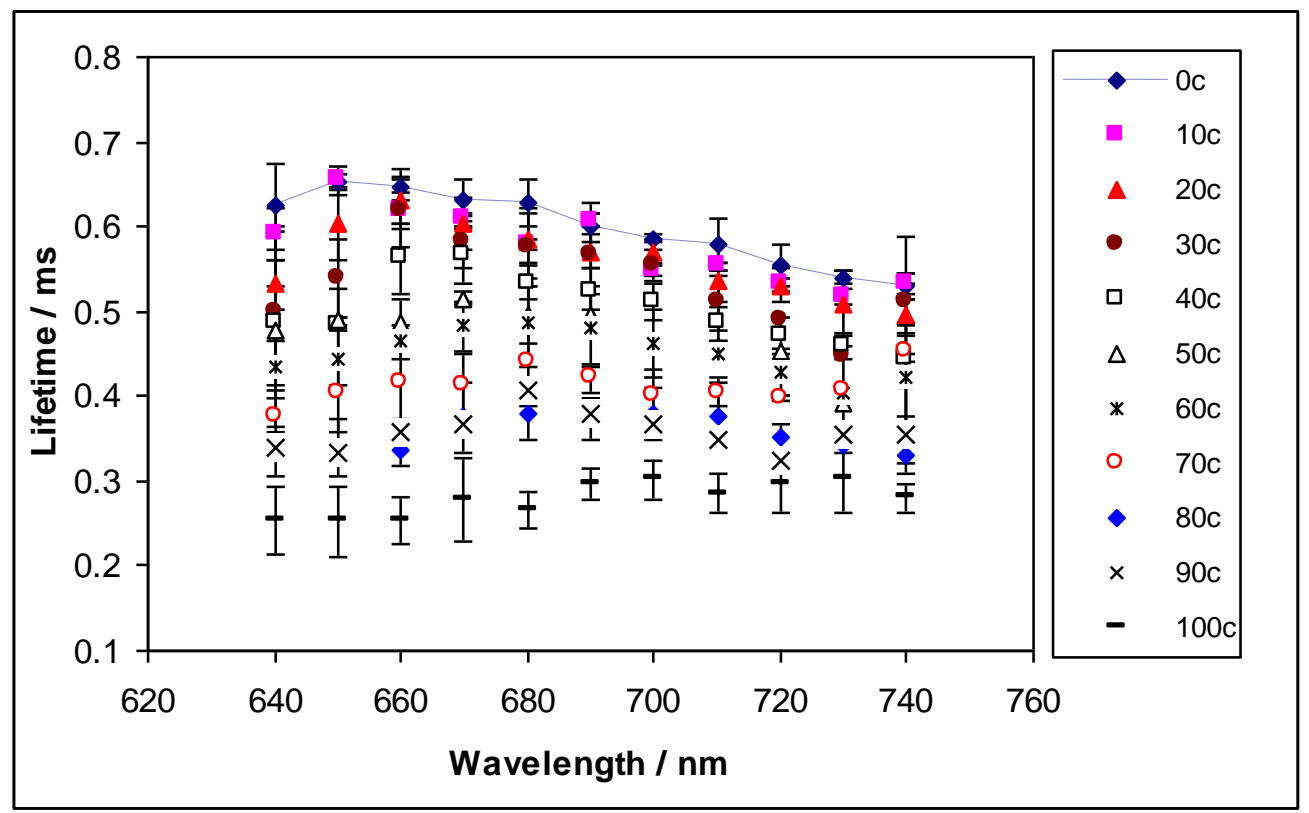

Figure VII-10: Effect of emission wavelength on the Erythrosin B phosphorescence lifetimes in amorphous BSA/ $\beta$-Lg mixtures determined from analysis using a stretched exponential model.

Figure VII-11

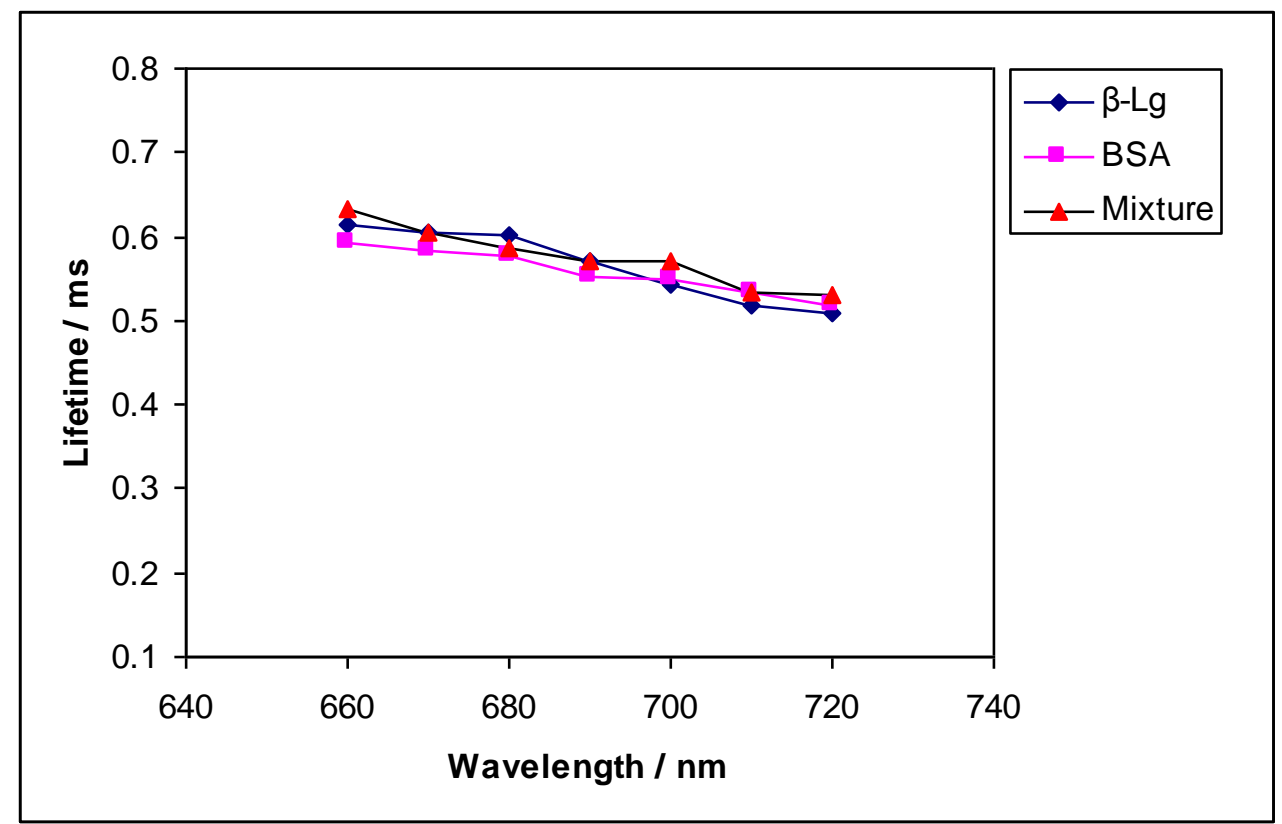

Figure VII-11: Comparitive plot of the effect of emission wavelength on the Erythrosin B phosphorescence lifetimes in amorphous $\beta$ - $\mathrm{Lg}$, BSA and BSA/ $\beta-\mathrm{Lg}$ mixtures determined from analysis using a stretched exponential model at $20^{\circ} \mathrm{C}$. 
Figure VII-12

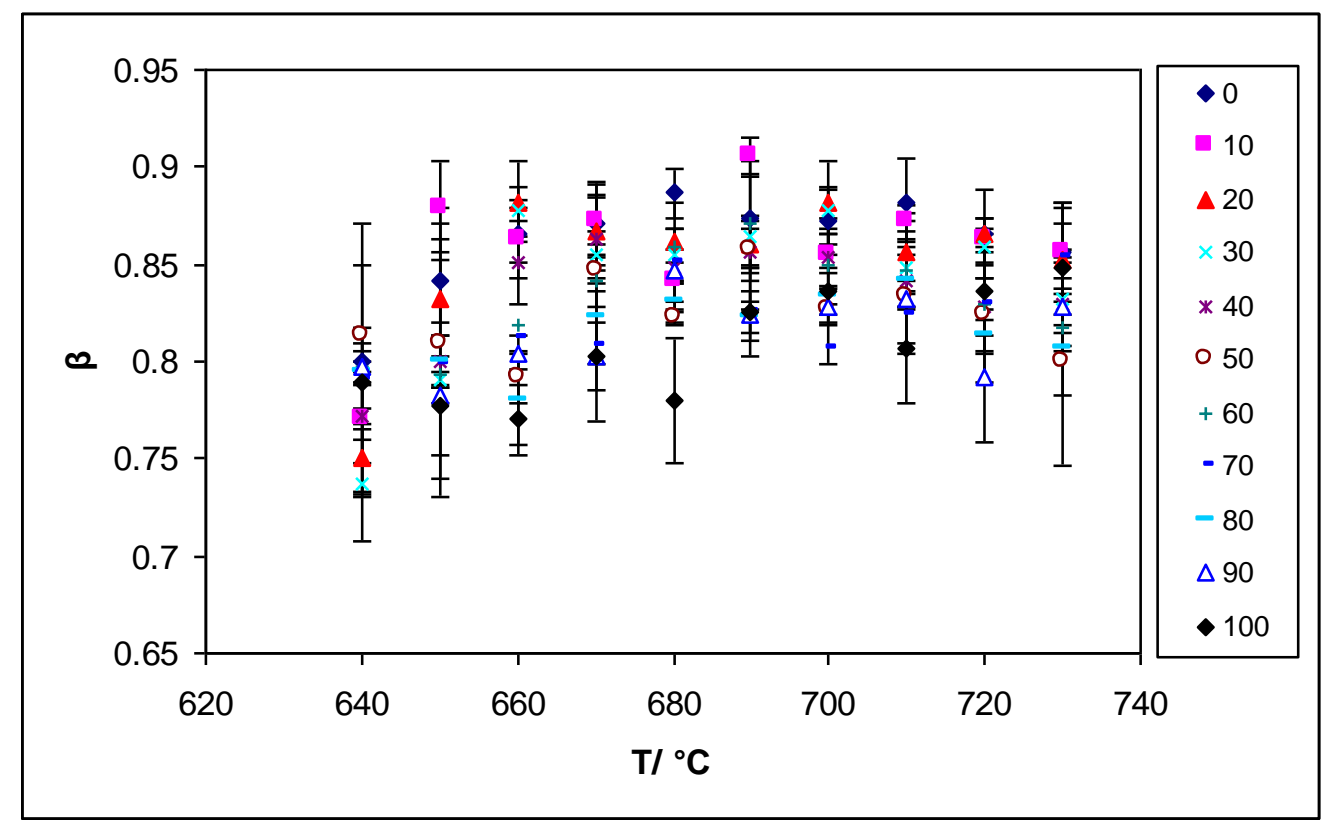

Figure VII-12: Effect of emission wavelength on the stretching exponent $(\beta)$ characterizing the intensity decay transients of Erythrosin B phosphorescence in amorphous BSA $\beta$-Lg mixtures determined from analysis using a stretched exponential model.

Figure VII-13

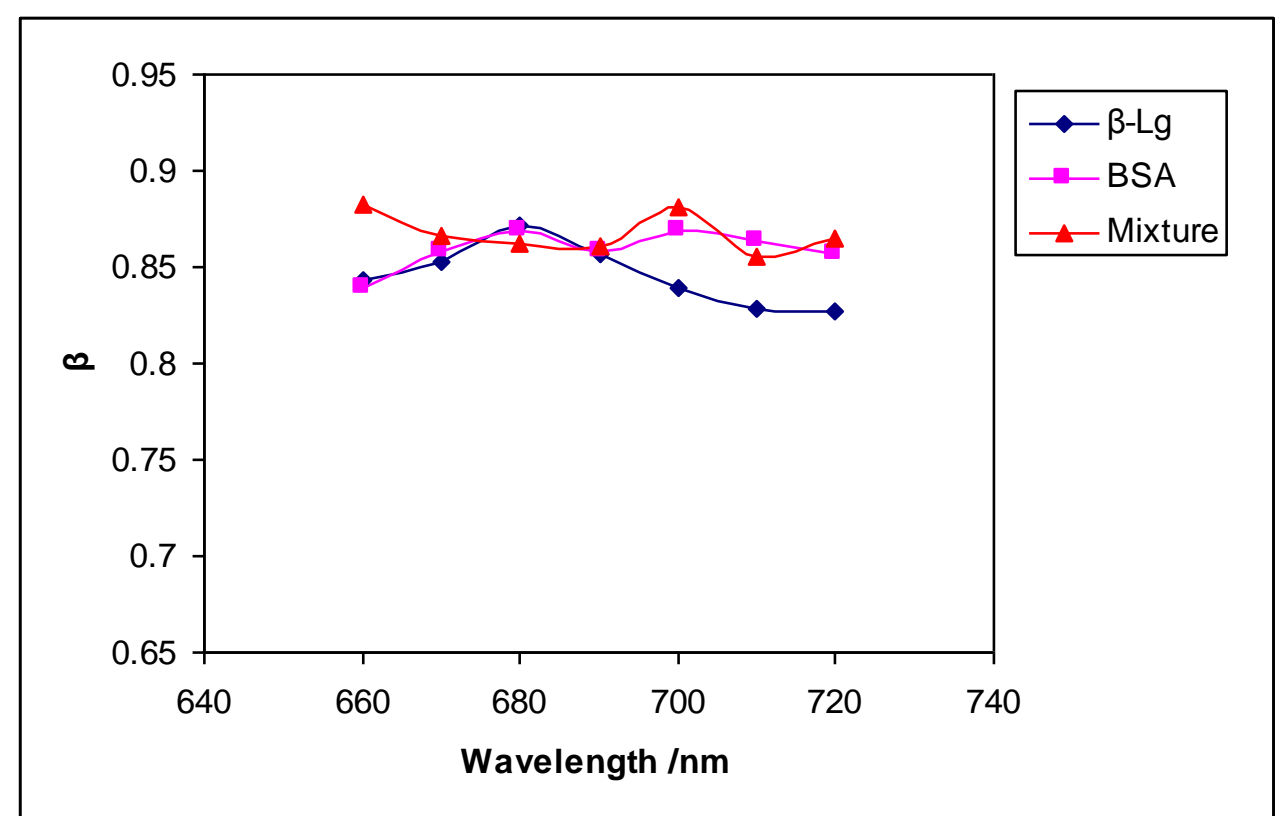

Figure VII-13: Effect of emission wavelength on the stretching exponent $(\beta)$ characterizing the intensity decay transients of Erythrosin B phosphorescence in amorphous BSA $\beta$-Lg mixtures determined from analysis using a stretched exponential model. 
Figure VII-14

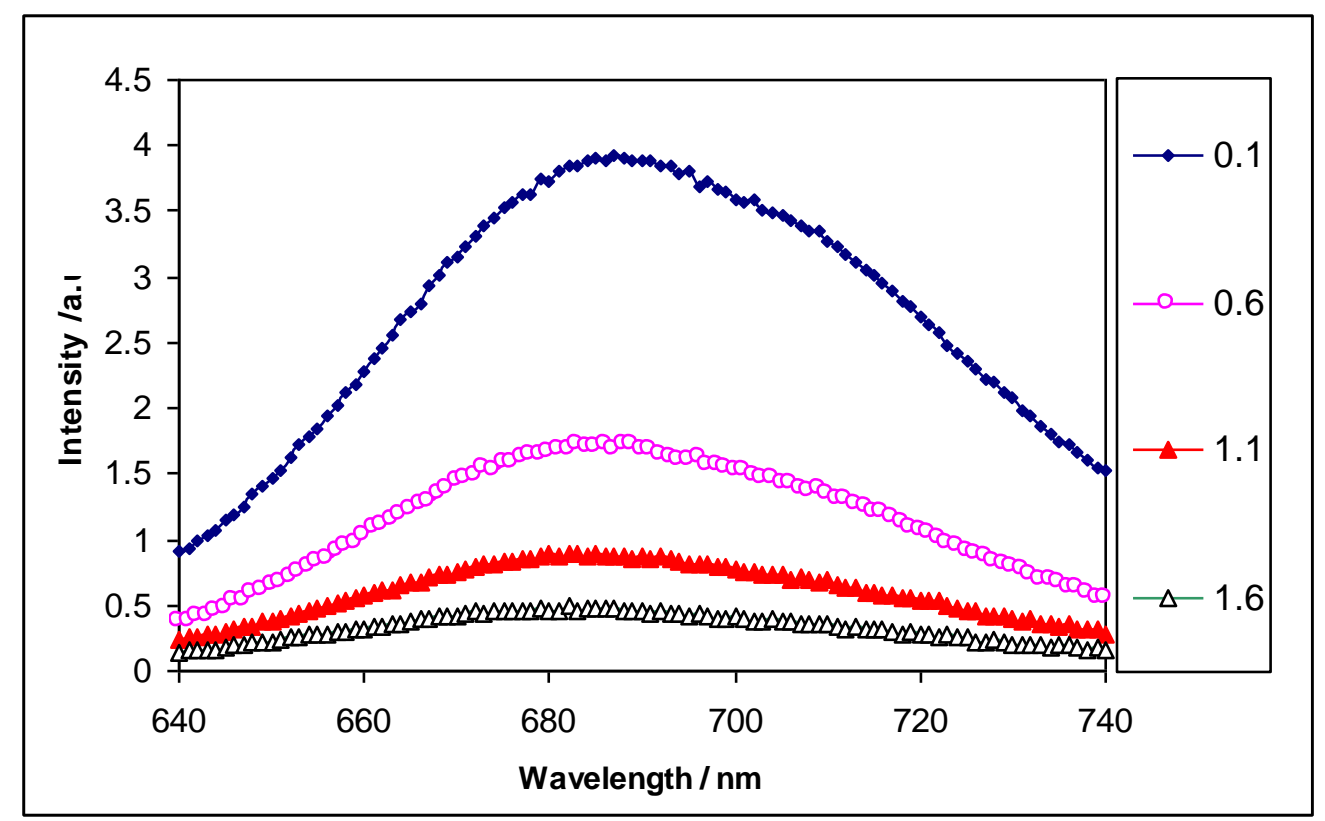

Figure VII-14: Phosphorescence emission spectra for Erythrosin B in amorphous BSA/ $\beta$-Lg mixtures collected as a function of delay time.

\section{Figure VII-15}

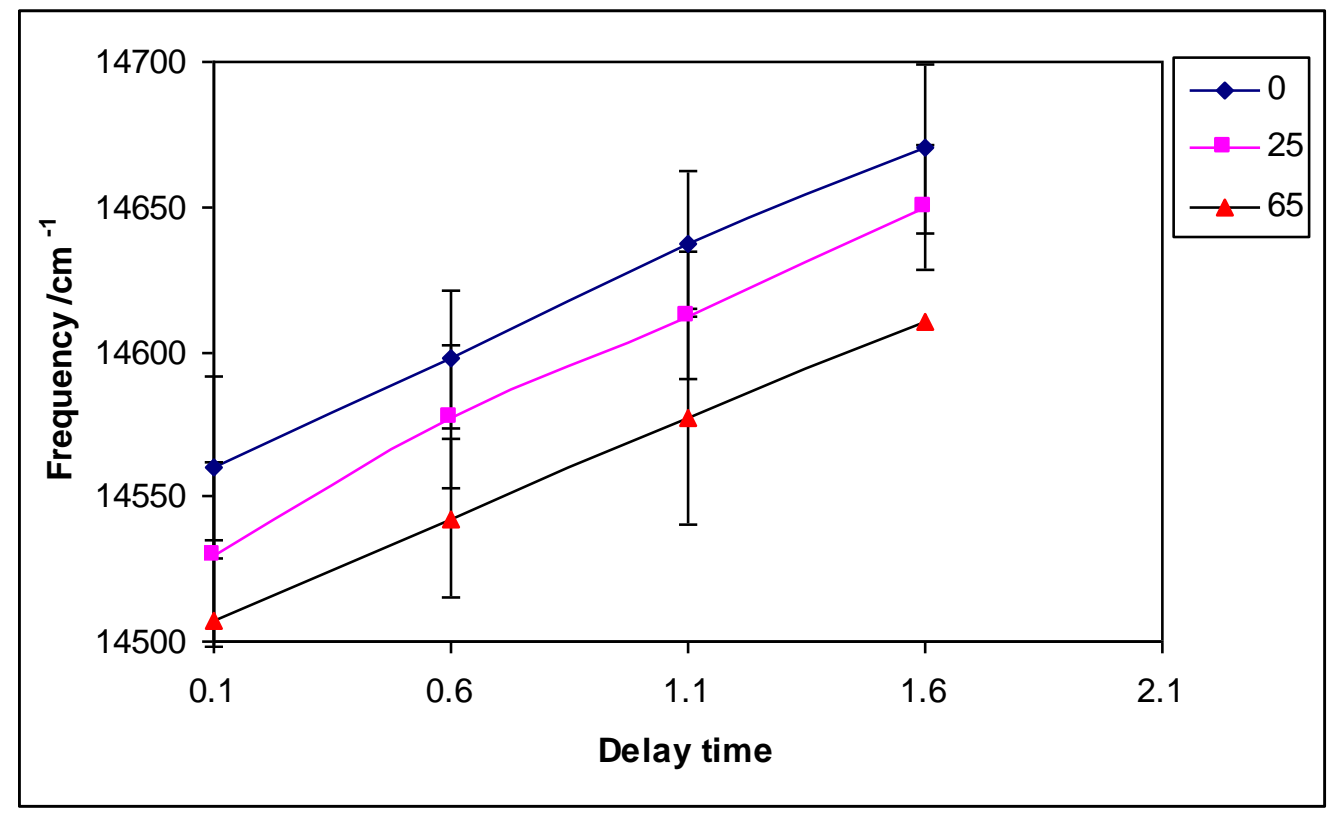

Figure VII-15: Evolution of phosphorescence emission peak frequency with time following excitation in amorphous $\mathrm{BSA} / \beta-\mathrm{Lg}$ mixtures. 
Figure VII-16

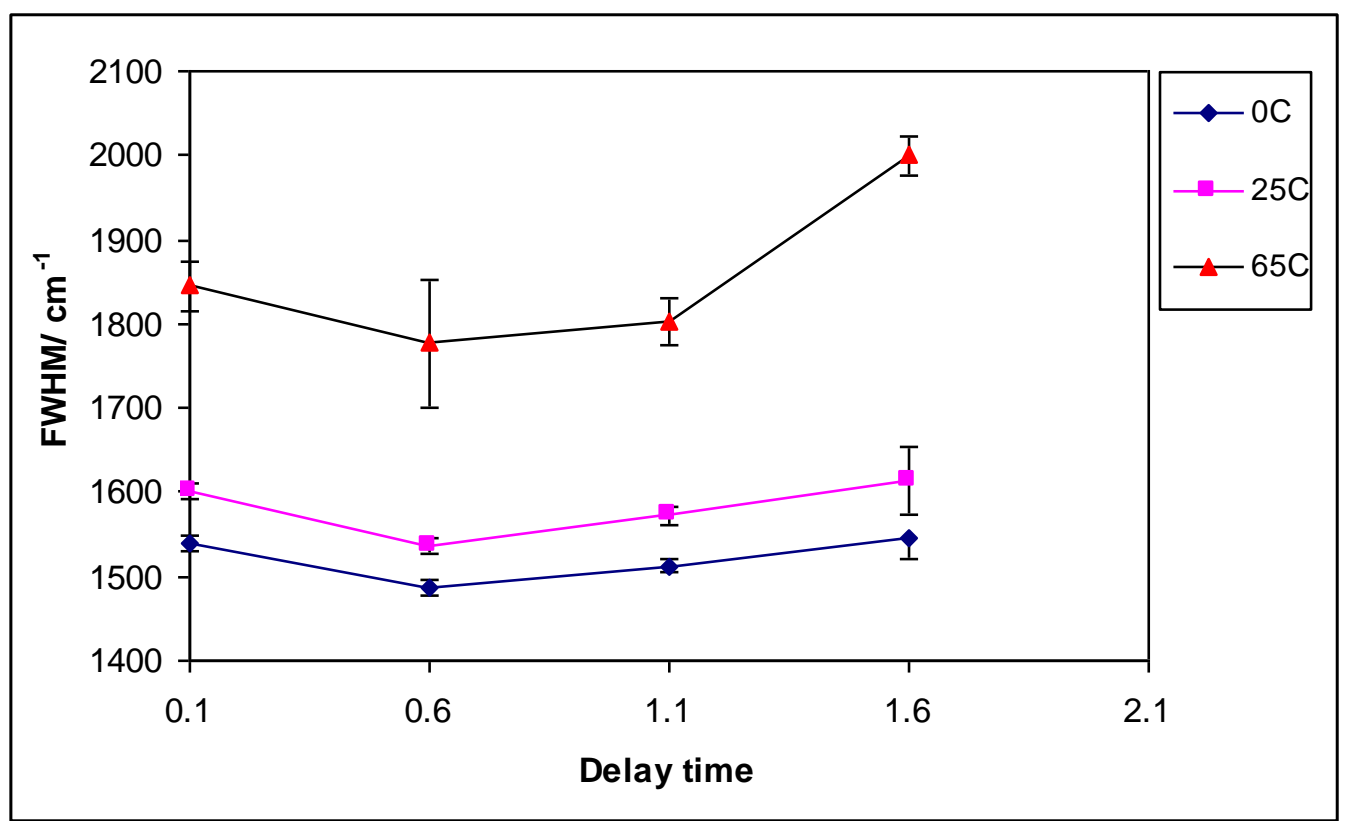

Figure VII-16: Evolution of phosphorescence emission bandwidth with time following excitation in amorphous BSA/ $\beta-\operatorname{Lg}$ mixtures. 
Figure VII-17

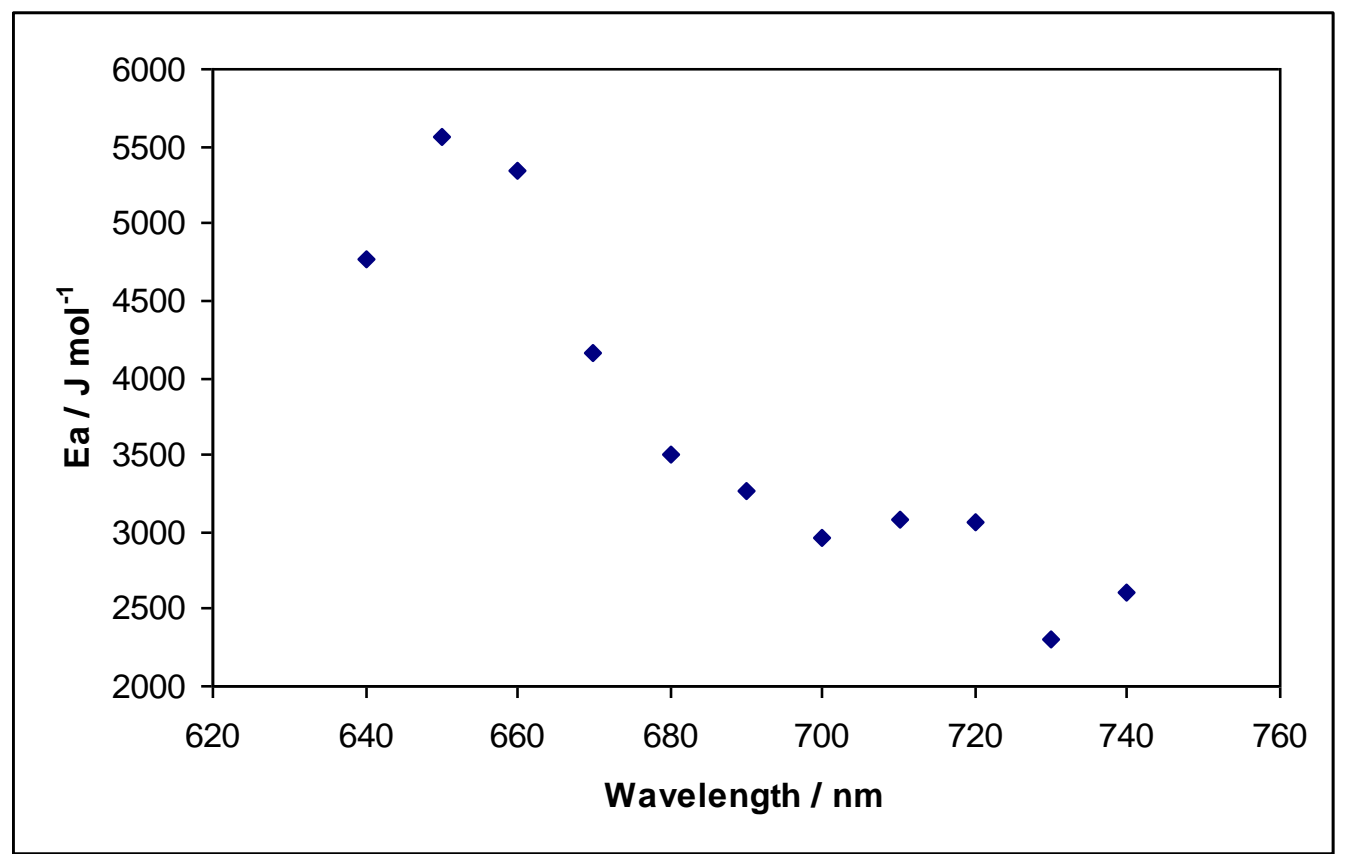

Figure VII-17: Variation of activation energy $\left(\mathrm{E}_{\mathrm{A}}\right)$ for quenching of phosphorescence lifetime of Erythrosin B in amorphous BSA/ $\beta-\mathrm{Lg}$ mixtures at $0^{\circ} \mathrm{C}-100^{\circ} \mathrm{C}$. 
Figure VII-18a

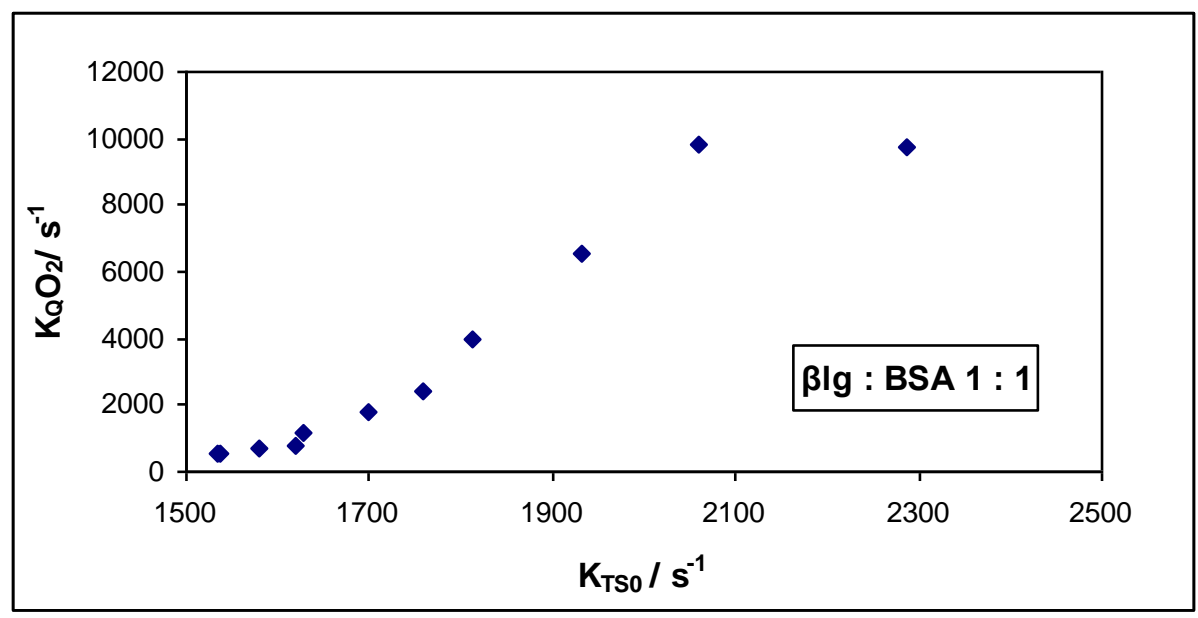

Figure VII-18b

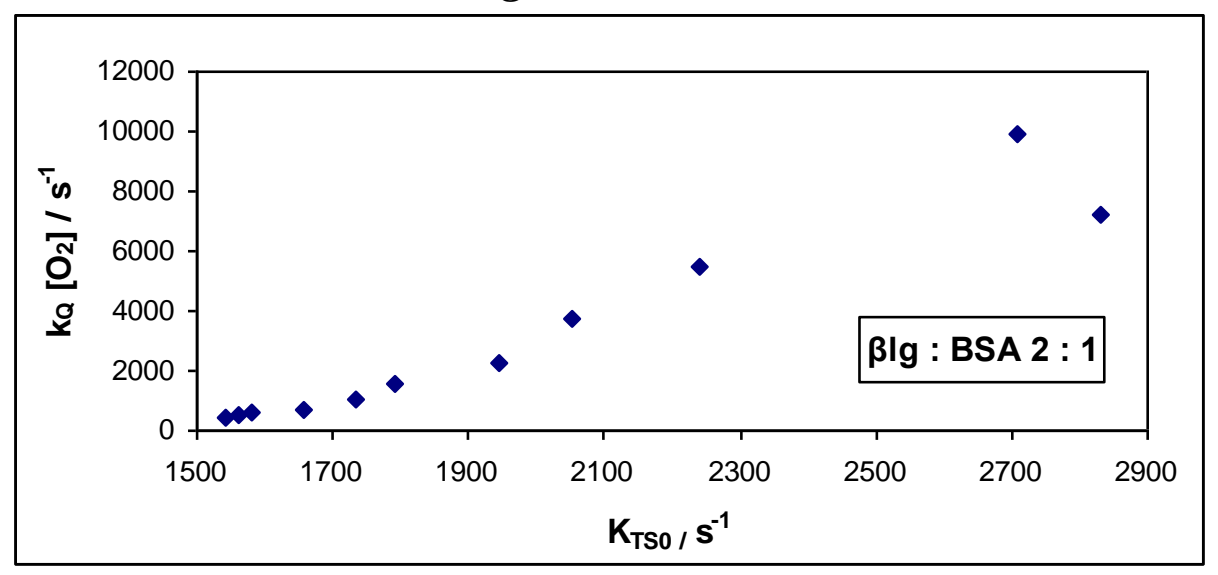

Figure VII-18c

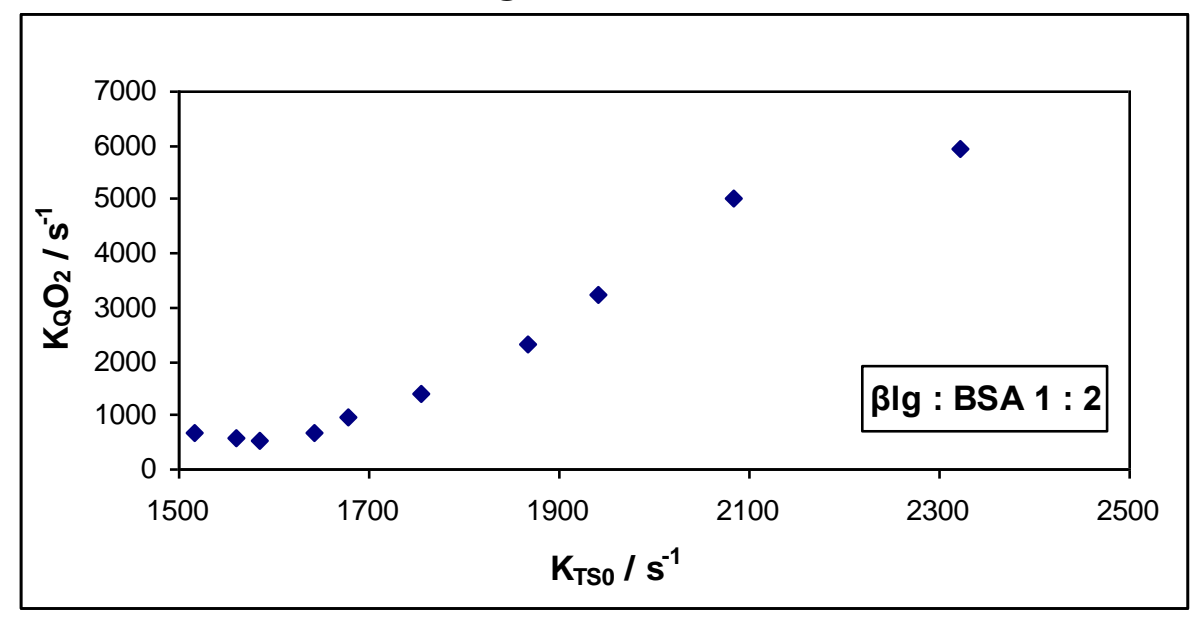

Figure VII-18a, b, c: Plot of the dependence of the radiative collisional quenching rate $\mathrm{k}_{\mathrm{TS} 0}$ to oxygen quenching rate $\mathrm{k}_{\mathrm{Q}}\left[\mathrm{O}_{2}\right]$ in $\mathrm{BSA} / \beta$ - $\mathrm{Lg}$ films. 


\section{Chapter VIII: Effect of fatty acids on the molecular mobility and permeability of amorphous $\beta$-Lactoglobulin $(\beta-\mathrm{Lg})$ films.}

\section{$\underline{\text { Introduction }}$}

Bovine $\beta$-Lg is a well-known member of the lipocalin family (Kontopidis et al., 2004). $\beta$ - Lg consists of 162 amino acid residues (18 kDa), and contains two disulfide bonds (Cys66-Cys160 and Cys106-Cys119) and a free thiol (Cys121). The protein structure contains a $\beta$-barrel with eight anti-parallel $\beta$-strands with $(+1)_{8}$ topology, labelled A-H. Strands A-D form one surface of the barrel while strands E-H form the other (Brownlaw et al., 1997; Kontopidis et al., 2002; Perez et al., 1989).

The $\beta$-barrel assumes a flattened calyx (or cone) shape with a large cavity lined with hydrophobic residues, which is accessible to bulk solvent. $\beta$-Lg binds a variety of hydrophobic ligands like fatty acids, retinol, etc. (Perez et al., 1989; Spector and Fletcher, 1970; Narayan and Berlinger, 1998), protoporphyrin (Dufour et al., 1990) and heme-CO complexes (Marden et al., 1994).

It has been long established that $\beta$ - $\mathrm{Lg}$ strongly binds 1 mole of long-chain fatty acids (myristic, palmitic, stearic acid) per mole of monomeric protein (Spector \& Fletcher, 1970; Frapin et al., 1993; Dufour et al., 1994). This binding is sensitive to $\mathrm{pH}$ in that the protein is unable to bind fatty acids at the primary fatty acid binding site below $\mathrm{pH}$ 3.5. X-ray crystallography of $\beta$ - $\mathrm{Lg}$ in complexes with various ligands revealed that the ligands lie within the central cavity of the protein, indicating that the cavity is the primary binding site of ligands (Kontopdis et al., 2002). Retinoids and fatty acids have been hypothesized (Frapin et al., 1993; Dufour et al., 1994) and later proven to bind independently and simultaneously to $\beta-\operatorname{Lg}$ (Narayan and Berliner, 1997). Fatty acids 
with longer aliphatic chains (palmitate and laurate) are found to display higher affinities for the protein (Ragona et al., 2000; Collini et al., 2003). Two potential binding sites have been postulated for small hydrophobic molecules, an interior cavity within the beta barrel and a surface cleft located in a groove between the helix and the barrel (Frapin et al., 1993; Dufour et al., 1994; Ragona et al., 1997). However, bovine $\beta$-Lg displays only one high affinity-binding site for palmitate with an apparent dissociation constant of $1 \times 10^{-7}$ M. The strength of the binding decreased in the following way: palmitate > stearate > myristate $>$ arachidate $>$ laurate. Caprylic and capric acids are not bound at all.

A very low-binding specificity of caprylic acid to $\beta$-Lg was observed, possibly due to its minor sterical hindrance, thus conferring a large conformational freedom within a cavity that preferentially hosts the endogenous longer-chain fatty acids (Perez et al., 1989). But these observations were made in the solution state and what governs the properties in the solid state may be totally different.

It is commercially important to produce whey proteins films with constant functionalities. But in the past binding of small hydrophobic ligands has been shown to cause significant changes to $\beta$-Lg functionalities like altering the gelation behavior, denaturation temperature, etc (Puyol et al., 1998; Puyol, 1994). It has also been reported that the binding of fatty acids increases resistance to proteolytic degradation (Puyol., et al., 1993). Several other proteins have been observed to undergo significant changes in backbone mobility upon ligand binding. Examples include FK506 binding protein (Cheng et al., 1994), acyl-coenzyme A binding protein (Rischel et al., 1994), and HIV protease (Nicholson et al., 1995). These findings suggest that the binding of ligands have an important effect on the protein structure and cause significant changes to its properties. 
So it will be of importance to study the effect of these small hydrophobic molecules on the $\beta$-Lg matrix.

Although there is wealth of information about the binding of ligands to $\beta-\mathrm{Lg}$ in solution, there are virtually no studies that have established a connection between the underlying molecular mobility and the macroscopic diffusive property in the solid state. The solid-state biophysics may throw light on various important events and may be significant in exploring edible film technology more effectively.

The main properties of edible films are their barrier properties (permeability to oxygen, water vapor, aroma, etc). Barrier properties, especially oxygen permeability are directly related to the chemical structure of the backbone, degree of crystallinity and orientation of molecular chains and the nature of additives. Certain additives may cause conformational changes to the polymer backbone and thus bring about changes in the gas permeability.

We have recently demonstrated the efficacy of the probe Erythrosin B in studying molecular details pertaining to mobility and permeability in various amorphous matrices (Pravinata et al., 2005; Shirke and Ludescher, 2005; Shirke et al., 2005, 2006; Lukasik and Ludescher, 2006a, 2006b; Lukasik and Ludescher, 2004; Nack and Ludescher, 2006; Sundaresan and Ludescher, 2008). In the present study phosphorescence from Erythrosin B was used to measure the matrix mobility and permeability of amorphous fatty acid / $\beta$ Lg mixtures. The temperature dependence of mobility was measured in palmitic acid / $\beta$ Lg and caprylic acid / $\beta$-Lg films and comparisons were made with the pure protein to investigate the effect of fatty acids on the molecular mobility and permeability. Important questions related to stability could be inferred from the present study. 


\section{$\underline{\text { Materials and Methods }}$}

Sample preparation

Solutions of $\beta-\operatorname{Lg}$ (90\% pure; crystallized and lyophilized from Sigma Chemical Co, St. Louis, MO, USA) were prepared in distilled de-ionized water at an approximate concentration of $10 \mathrm{mg} / \mathrm{ml}$. An aqueous $\beta-\mathrm{Lg}$ solution was transferred to regenerated cellulose dialysis tubing having a 12-14kDa molecular weight cutoff (Spectrum, Houston, TX). In order to define and minimize the presence of counter ions the solution was extensively dialyzed against $0.1 \mathrm{M}$ potassium chloride for 24 hours with frequent change of buffer every 6 hours. The solution was then dialyzed against distilled de-ionized water for 24 hours with frequent change of water after every 6 hours. The dialyzed solution was then filtered through a $0.2-\mu \mathrm{m}$ membrane to remove particulates. In order to maximize the effectiveness of the dialysis process the temperature of the solution was kept at $5^{\circ} \mathrm{C}$ to ensure that the protein remained folded. Several dilutions of a stock solution of dialyzed $\beta-\mathrm{Lg}$ were prepared. The dye to protein ratio was determined by measuring the absorbance of dilutions in a $1 \mathrm{~cm}$ path length quartz cuvette in a Bausch and Lomb Spectronic 2000 spectrophotometer (Milton Roy company, Analytical Products Division, Rochester, NY). The protein concentration was determined after dialysis by UV absorbance using an extinction coefficient of $0.96 \mathrm{~L} \mathrm{~g} \mathrm{~g}^{-1} \mathrm{~cm}^{-1}$.

\section{Preparation of $\beta$-Lg: Fatty acid solution}

Palmitic acid (Hexadecanoic acid 99\% pure, molecular weight 256.42g / mole) was purchased from Sigma - Aldrich (St. Louis, MO). A solution of $\beta$-Lg - palmitic acid mixture was made according to the specifications of Ragonna et al. (2000). Palmitic acid at a molar concentration ratio of 1:1 with respect to the protein monomer was weighed. 
The fatty acid was then dissolved in chloroform and dispensed in two glass tubes. After that the organic solvent was evaporated under nitrogen atmosphere. Solutions of $\beta$ $\mathrm{Lg}$ were added to the tubes and the mixtures were incubated overnight at $37^{\circ} \mathrm{C}$. In previous studies this procedure ensured that the palmitic acid was bound to $\beta$-Lg. Preparation of samples with higher concentration of fatty acid led to opaque films, which failed to give sufficient spectroscopic signal.

Caprylic acid (Octanoic acid molecular weight $144.1 \mathrm{~g} / \mathrm{mole}$, 99\% pure) was purchased from Sigma- Aldrich. Caprylic acid at a molar concentration ratio of 1:1 with respect to the protein monomer was weighed and dissolved in $98 \%$ pure ethanol. The organic solvent was then evaporated under nitrogen atmosphere. Solutions of $\beta$-Lg were added as above.

Erythrosin B (EryB) (tetra iodofluorescein, FD\&C red \#3) from Molecular Probes (Eugene, OR), was dissolved in spectrophotometric grade $\mathrm{N}, \mathrm{N}$-dimethyl formamide (DMF) (Aldrich Chemical, Milwaukee, WI) to make $10 \mathrm{mM}$ and $100 \mathrm{mM}$ stock solutions. The solvent DMF was selected for probe stability during long time storage and the concentrations were selected in order to simplify the addition of probe to the protein matrix. Aliquots of dye solution were combined with the protein fatty acid solution to achieve a molar ratio of EryB: $\beta-\operatorname{Lg}$ fatty acid of $1: 4$. The dye to protein concentration was sufficient to provide adequate signal / noise in spectroscopic measurements.

Preparation of $\beta$-Lg Ifatty acid films

To make glassy films, $15 \mu 1$ of $\beta$-Lg / fatty acid solution was spread on approximately one third of a quartz slide $(30 \times 13.5 \times 0.6 \mathrm{~mm}$; custom made by NSG 
Precision Cells, Farmingdale, NY). The $\beta$-Lg / fatty acid films approximately $(0.05 \mathrm{~mm}$ thick when dried) were visually checked for good spreading, uniformity, and consistency. The slides were stored at room temperature in a desiccators containing $\mathrm{P}_{2} \mathrm{O}_{5}$ and DrieRite for at least 7 days and protected from light to prevent any photo bleaching of Erythrosin B prior to luminescence measurements.

\section{Luminescence Measurements}

All measurements were conducted using a CARY Eclipse spectrophotometer (Varian Instruments, Walnut Creek, CA). The temperature was controlled by using a TLC 50 thermoelectric heating / cooling system (Quantam Northwest, Spokane, WA). The TLC-50 sample compartment was fitted with a jacketed cover. For luminescence measurements necessitating the absence of oxygen (luminescence quencher) the samples were flushed with nitrogen gas for at least 15 minutes to free the environment from oxygen. An oxygen free nitrogen stream was generated by passage of high purity nitrogen through a Supelco (Bellefonte, PA) carrier gas purifier.

Quartz slides were placed on end on the diagonal of a standard $1 \mathrm{~cm} \times 1 \mathrm{~cm}$ quartz fluorescence cuvette which was capped with a lid having inlet and outlet ports for gas lines, thus all experiments were conducted at constant total pressure. Dry air was flushed to prevent condensation on the faces of the cuvette at experimental conditions below room temperature.

Delayed luminescence emission spectra were collected from 535-800 nm (10nm bandwidth) using excitation of $525 \mathrm{~nm}$ (10nm bandwidth) over the temperature range from $0^{\circ} \mathrm{C}$ to $100^{\circ} \mathrm{C}$ (at 10 degrees intervals). Each data point ( $1 \mathrm{~nm}$ interval, $0.1 \mathrm{~s}$ 
averaging time) was collected from a single flash with a $0.2 \mathrm{~ms}$ delay, $0.5 \mathrm{~ms}$ gate time and $4.0 \mathrm{~ms}$ total decay time.

For lifetime measurements as a function of temperature, samples were excited at $540 \mathrm{~nm}$ (20nm bandwidth) and emission transients collected at 690nm (20nm bandwidth) at temperature ranging from $0^{\circ} \mathrm{C}-100^{\circ} \mathrm{C}$. Before collecting data samples were equilibrated for 15 minutes. The measurements were conducted in the presence or absence of air (under a nitrogen purge). Each time-resolved decay transient was the average of 100 cycles and for each cycle data was collected from a single flash with a delay of $0.1 \mathrm{~ms}$, a 0.02 gate time and $4.0 \mathrm{~ms}$ total decay time. All experiments were done several times and the averages were used for data analysis and interpretation. In order to validate the repeatability the standard deviation between experiments was calculated.

\section{Basic photophysics}

Luminescence is a general term used to describe what occurs when a probe molecule absorbs photons of higher energy (excitation) and subsequently releases photons of lower energy (emission). This phenomenon is a function of a molecule's structure, which in luminescent molecules is usually characterized by an extended delocalized $\pi$-orbital system. The Jablonski energy level diagram summarizes the various photophysical events occurring during luminescence. Following light absorption the ground state molecule makes an essentially instantaneous transition $\left(\sim 10^{-15} \mathrm{~s}\right)$ to one of the many vibrational levels of the first excited state $\left(S_{1}\right)$; excess vibrational energy is rapidly $\left(\sim 10^{-12} \mathrm{~s}\right)$ dissipated to the solvent matrix to relax to the lowest vibrational level of $\mathrm{S}_{1}$. Along with fluorescence (rate $\mathrm{k}_{\mathrm{RF}}$ ), the excited state may engage in several deexcitation processes including non-radiative decay to $S_{0}$ through exchange of vibrational 
energy to the matrix (rate constant $\mathrm{k}_{\mathrm{SS} 0}$ ), intersystem crossing from $\mathrm{S}_{1}$ to $\mathrm{T}_{1}$ (rate $\mathrm{k}_{\mathrm{ST}}$ ) and collisional quenching rate $\mathrm{k}_{\mathrm{q}}\left[\mathrm{O}_{2}\right]$. Along with phosphorescence (rate $\mathrm{k}_{\mathrm{RP}}$ ) occurs the deexcitation processes including non-radiative decay from $\mathrm{T}_{1}$ to $\mathrm{S}_{0}$ (rate $\mathrm{k}_{\mathrm{TS} 0}$ ) reverse intersystem crossing from $\mathrm{T}_{1}$ to $\mathrm{S}_{1}\left(\right.$ rate $\mathrm{k}_{\mathrm{TS} 1}$ ) and collisional quenching by oxygen $\mathrm{k}_{\mathrm{Q}}\left[\mathrm{O}_{2}\right]$. The rate constants $\mathrm{k}_{\mathrm{RF}}, \mathrm{k}_{\mathrm{RP}}$ and $\mathrm{k}_{\mathrm{TS} 1}$ are fixed by the probe structure and largely unaffected by environment (Turro, 1991; Birks, 1970).

\section{Emission intensity}

Luminescence emission intensity is directly proportional to the emission quantam yield, $\Phi=$ photons emitted/photons absorbed. The emission quantam yield reflects the relative rates of the several de-excitation pathways available to the excited state of a chromophore. The quantam yield for fluorescence is given by the following ratio of rate constants (Lakowicz, 1999). The term $\mathrm{k}_{\mathrm{q}}\left[\mathrm{O}_{2}\right]$ describes the rate of collisional quenching of the excited state of the probe by oxygen.

$$
\Phi_{\mathrm{F}}=\mathrm{k}_{\mathrm{RF}} /\left(\mathrm{k}_{\mathrm{RF}}+\mathrm{k}_{\mathrm{SS} 0}+\mathrm{k}_{\mathrm{ST}}+\mathrm{k}_{\mathrm{q}}\left[\mathrm{O}_{2}\right]\right)
$$

In the absence of specific quenchers in the local environment, the typical situation for fluorescence, Eq. (1) simplifies to:

$$
\Phi_{\mathrm{F}}=\mathrm{k}_{\mathrm{RF}} /\left(\mathrm{k}_{\mathrm{RF}}+\mathrm{k}_{\mathrm{SS} 0}+\mathrm{k}_{\mathrm{ST}}\right)
$$

In the case of phosphorescence, the quantam yield for phosphorescence $\Phi_{\mathrm{P}}$ is the product of the quantam yield for $T_{1}$ formation $\left(\Phi_{T}\right)$ times the probability of emission from $T_{1}\left(q_{P}\right)$ (Hurtubise, 1990):

$$
\Phi_{\mathrm{P}}=\Phi_{\mathrm{T}} \mathrm{q}_{\mathrm{P}}=\left\{\mathrm{k}_{\mathrm{ST}} /\left(\mathrm{k}_{\mathrm{RF}}+\mathrm{k} \mathrm{k}_{\mathrm{SS} 0}+\mathrm{k}_{\mathrm{ST}}+\mathrm{k}_{\mathrm{q}}\left[\mathrm{O}_{2}\right]\right)\right\}\left\{\mathrm{k}_{\mathrm{RP}} /\left(\mathrm{k}_{\mathrm{RP}}+\mathrm{k}_{\mathrm{TS} 0}+\mathrm{k}_{\mathrm{TS} 1}+\mathrm{k}_{\mathrm{Q}}\left[\mathrm{O}_{2}\right]\right)\right\}(3)
$$

The quantam yield for delayed fluorescence in the absence of oxygen is the product of 
the quantam yield of fluorescence and the probabilities of intersystem crossing from $S_{1}$ to $\mathrm{T}_{1}$ and also from $\mathrm{T}_{1}$ to $\mathrm{S}_{1}$ (Duchowicz et al., 1998)

$$
\Phi_{\mathrm{DF}}=\Phi_{\mathrm{F}}\left\{\mathrm{k}_{\mathrm{ST}} /\left(\mathrm{k}_{\mathrm{RF}}+\mathrm{k}_{\mathrm{SS} 0}+\mathrm{k}_{\mathrm{ST}}\right)\right\}\left\{\mathrm{k}_{\mathrm{TS} 1} /\left(\mathrm{k}_{\mathrm{RP}}+\mathrm{k}_{\mathrm{TSO}}+\mathrm{k}_{\mathrm{TS} 1}\right)\right\}
$$

By comparing the emission intensities of delayed fluorescence and phosphorescence it is possible to obtain information about the rate constants for intersystem crossing $\mathrm{k}_{\mathrm{TS} 1}$.

$$
\mathrm{I}_{\mathrm{DF}} / \mathrm{I}_{\mathrm{P}}=\Phi_{\mathrm{F}}\left\{\mathrm{k}_{\mathrm{ST}} / \mathrm{k}_{\mathrm{F}}\right\}\left\{\mathrm{k}_{\mathrm{TS} 1} / \mathrm{k}_{\mathrm{P}}\right\} /\left\{\mathrm{k}_{\mathrm{ST}} / \mathrm{k}_{\mathrm{F}}\right\}\left\{\mathrm{k}_{\mathrm{RP}} / \mathrm{k}_{\mathrm{P}}\right\}=\Phi_{\mathrm{F}} \mathrm{k}_{\mathrm{TS} 1} / \mathrm{k}_{\mathrm{RP}}
$$

where $\mathrm{k}_{\mathrm{F}}$ and $\mathrm{k}_{\mathrm{P}}$ are the total decay rates for the de-excitation of the singlet and triplet state respectively.

The slope of the Van't Hoff plot of the natural log of the ratio of delayed fluorescence $\left(\mathrm{I}_{\mathrm{DF}}\right)$ to phosphorescence $\left(\mathrm{I}_{\mathrm{P}}\right)$ intensity versus inverse temperature provides a measure of $\Delta \mathrm{E}_{\mathrm{TS}}$ (Duchowicz, et al., 1998). This ratio is highly temperature dependent due to the additional energy required to go from $T_{1}$ to $S_{1}$ energy level and can be described by the following equation where $\Delta \mathrm{E}_{\mathrm{TS}}$ is the effective energy gap between the triplet and singlet states.

$$
\Phi_{\mathrm{F}} \mathrm{k}_{\mathrm{TS} 1} / \mathrm{k}_{\mathrm{R} P}=\left\{\Phi_{\mathrm{F}} / \mathrm{k}_{\mathrm{RP}}\right\} \mathrm{k}^{\circ}{ }_{\mathrm{TS} 1} \exp \left(-\Delta \mathrm{E}_{\mathrm{TS}} / \mathrm{RT}\right)
$$

Phosphorescence intensity decays are non-exponential and a stretched exponential decay function has been shown to be appropriate to describe the wide distribution of relaxation times (Champion et al., 2000; Richert, 2000).

$$
\mathrm{I}(\mathrm{t})=\mathrm{I}_{0} \exp \left[-(\mathrm{t} / \tau)^{\beta}\right]+\mathrm{c}
$$

Where $\mathrm{I}_{0}$ is the initial amplitude, $\tau$ is the stretched exponential lifetime, $\beta$ is an exponent varying from 0 to 1 and characterizing the distribution of lifetimes. The use of a stretched exponential model provides a direct measurement of a continuous distribution of lifetimes, which is appropriate for describing the decay in a complex glass possessing a 
distribution of relaxation times for the dynamic molecular processes. The phosphorescence lifetimes were used to calculate the rate constants associated with the various processes that depopulate the excited triplet state. In an amorphous solid in the absence of oxygen the temperature dependence of $\mathrm{k}_{\mathrm{P}}$ can be written as follows:-

$$
\mathrm{k}_{\mathrm{P}}(\mathrm{T})=\mathrm{k}_{\mathrm{RP}}+\mathrm{k}_{\mathrm{TS} 1}(\mathrm{~T})+\mathrm{k}_{\mathrm{TS} 0}(\mathrm{~T})
$$

But in the presence of oxygen the de-excitation of the triplet state is also influenced by the quencher species. The term $\mathrm{k}_{\mathrm{Q}}\left[\mathrm{O}_{2}\right]$ describes the rate of collisional quenching of the excited state of the probe by oxygen. Thus in the presence of oxygen $\mathrm{k}_{\mathrm{P}}(\mathrm{T})$ includes an additional term $\mathrm{k}_{\mathrm{Q}}\left[\mathrm{O}_{2}\right]$.

$$
\mathrm{k}_{\mathrm{P}}(\mathrm{T})=\mathrm{k}_{\mathrm{RP}}+\mathrm{k}_{\mathrm{TS} 1}(\mathrm{~T})+\mathrm{k}_{\mathrm{TS} 0}(\mathrm{~T})+\mathrm{k}_{\mathrm{Q}}\left[\mathrm{O}_{2}\right](\mathrm{T})
$$

Equation 9 can be used to calculate $\mathrm{k}_{\mathrm{TS} 0}$, the rate of collisional quenching to the ground state when $\mathrm{k}_{\mathrm{RP}}$ and $\mathrm{k}_{\mathrm{TS} 1}$, are known. $\mathrm{k}_{\mathrm{RP}}$ the radiative decay rate of the triplet state, is $41 \mathrm{~s}^{-1}$ for Erythrosin B (Duchowicz et al.,1998; Lettinga et al., 2000).

$\mathrm{k}_{\mathrm{TS} 1}$ the rate of reverse intersystem crossing from the excited triplet state to the excited singlet state depends on $\Delta \mathrm{E}_{\mathrm{TS}}$, the energy gap between $\mathrm{S}_{1}$ and $\mathrm{T}_{1}$

$$
\mathrm{k}_{\mathrm{TS} 1}(\mathrm{~T})=\mathrm{k}^{\mathrm{o}} \mathrm{TS} 1 \exp \left(-\Delta \mathrm{E}_{\mathrm{TS}} / \mathrm{RT}\right)
$$

A plot of $\ln \left(\mathrm{I}_{\mathrm{DF}} / \mathrm{I}_{\mathrm{P}}\right)$ versus $1 / \mathrm{T}$ showed linearity and has slope of $-\mathrm{E}_{\mathrm{TS}} / \mathrm{R}$ and thus $\Delta \mathrm{E}$ for the $\beta$ - $\mathrm{Lg}$ matrix was calculated. Unfortunately, literature values of $\mathrm{k}^{\circ}{ }_{\mathrm{TS} 1}$ for Erythrosin $\mathrm{B}$ vary widely, from $0.3 \times 10^{7} \mathrm{~s}^{-1}$ in ethanol and $6.5 \times 10^{7} \mathrm{~s}^{-1}$ in water (Duchowicz et al., 1998) to $111 \times 10^{7} \mathrm{~s}^{-1}$ in solid polyvinyl alcohol (Lettinga et al., 2000), and thus provide little guidance. We estimated the maximum possible value for $\mathrm{k}^{\mathrm{o}} \mathrm{TS} 1$ in $\beta-\mathrm{Lg}$ sucrose films by assuming that $\mathrm{k}_{\mathrm{TS} 1}(\mathrm{~T})$ cannot result in values for $\mathrm{k}_{\mathrm{TS} 0}$ that decrease with temperature. This procedure thus estimated the minimum possible values of $\mathrm{k}_{\mathrm{TS} 0}(\mathrm{~T})$. 
In order to study the oxygen diffusion in the $\beta-\mathrm{Lg}$ matrix $\mathrm{k}_{\mathrm{Q}}\left[\mathrm{O}_{2}\right]$ can easily be calculated from the difference between the decay rate in the presence of oxygen and the absence of oxygen

$$
\mathrm{K}_{\mathrm{Q}}\left[\mathrm{O}_{2}\right]=1 / \tau(\mathrm{Air})-1 / \tau(\mathrm{N} 2)
$$

\section{Data analysis}

\section{Emission energy as a function of Temperature}

Delayed luminescence spectra collected from $535 \mathrm{~nm}$ to $800 \mathrm{~nm}$ were fit using the program NFIT (Island Products) to a sum of distinct log-normal functions for delayed fluorescence $\left(\mathrm{I}_{\mathrm{DF}}(v)\right)$ and phosphorescence $\left(\mathrm{I}_{\mathrm{P}}(v)\right)$ in which all fit parameters were independent for each emission band. The energy of the emission maximum $\left(v_{\mathrm{P}}\right)$ and the full width at half maximum $(\Gamma)$ of the emission bands were determined from the function (Maroncelli and Fleming, 1987). Some of the data were fit using the program Igor (Wavemetrics, Inc., Lake Oswego, OR)

$$
\mathrm{I}(v)=\mathrm{I}_{0} \exp \left\{-\ln (2)\left[\ln \left(1+2 \mathrm{~b}\left(v-v_{\mathrm{P}}\right) / \Delta\right) / \mathrm{b}\right]^{2}\right\}
$$

In this equation $\mathrm{I}_{0}$ is the maximum emission intensity, $v_{\mathrm{P}}$ is the frequency $\left(\mathrm{cm}^{-1}\right)$ of the emission maximum, $\Delta$ is a linewidth parameter, and $\mathrm{b}$ is an asymmetry parameter The bandwidth $(\Gamma)$ of the emission band is related to $\mathrm{b}$ and $\Delta$ :

$$
\Gamma=\Delta\{\sinh (\mathrm{b}) / \mathrm{b}\}
$$

Phosphorescent intensity decay and rate calculations

Intensity decays were clearly non exponential and were analyzed using a stretched exponential function. Phosphorescence lifetimes were determined by nonlinear leastsquares analysis with the program NFIT (Island Products, Galveston, TX). Fits were judged acceptable if they had satisfactory fit errors and if data points were randomly 
distributed about the fit curve; most data sets had $\chi^{2} \leq 1.0$ and $\mathrm{R}^{2}$ in the range of $0.99-1.0$. Data were well analyzed with a stretched exponential decay model

\section{$\underline{\text { Results }}$}

Composite films of proteins and lipids are meant to combine the good structural and oxygen barrier properties of protein films. In the present study we investigated the effect of binding of fatty acids on the molecular mobility and oxygen permeability of the amorphous $\beta$-Lg matrix. Studies in the past have looked into other properties but have overlooked the finer molecular detail and effect of ligand binding on the permeability of oxygen. Graphs in chapter VIII mainly depict the effect of temperature, and effect of change in composition due to binding of fatty acids on the molecular mobility and oxygen permeability of $\beta$-Lg films. Fatty acids essentially bind to $\beta$ - $\operatorname{Lg}$ at a molar ratio of 1:1 Fatty acid/ $\beta$-Lg. This was the chosen concentration to investigate the effect of fatty acids on $\beta$ - $\operatorname{Lg}$ films.

\section{Delayed emission spectra}

Figures VIII -1a, b depicts the emission spectra of Erythrosin B dispersed in amorphous palmitic acid / $\beta$ - $\operatorname{Lg}$ and caprylic acid/ $\beta$ - $\operatorname{Lg}$ films as a function of temperature (Fatty acid / $\beta$-Lg: 1:1). The long wavelength emission band reflects phosphorescence from the triplet state whereas the short wavelength emission band reflects delayed fluorescence from the singlet state that has been repopulated by reverse intersystem crossing from the triplet state (Parker, 1968). Delayed emission spectra collected over the temperature range from $0^{\circ} \mathrm{C}$ to $100^{\circ} \mathrm{C}$ showed an decrease in phosphorescence $\left(\mathrm{I}_{\mathrm{P}}\right)$ and increase in delayed fluorescence $\left(\mathrm{I}_{\mathrm{DF}}\right)$ intensity at higher temperatures as expected for a thermally stimulated process in xanthene dyes (Parker, 1968). The emission spectra were 
very similar to the spectra obtained by dispersing in Erythrosin B in various amorphous matrices. $\Delta \mathrm{E}_{\mathrm{TS}}$ the energy gap between the $\mathrm{T}_{1}$ and $\mathrm{S}_{1}$ states, was calculated from the slope of a Van't Hoff plot of $\ln \left(\mathrm{I}_{\mathrm{DF}} / \mathrm{I}_{\mathrm{P}}\right)$ vs. 1/T (Eq. 6, Materials and Methods). The values for the intensity of delayed fluorescence $\left(\mathrm{I}_{\mathrm{DF}}\right)$ and phosphorescence $\left(\mathrm{I}_{\mathrm{P}}\right)$ were determined from fitting the spectra to a sum of two lognormal functions (Eq. 12, Materials and Methods) (data not shown). The Van't Hoff plot was linear over the entire range of temperatures with no systemic variations $\left(\mathrm{R}^{2}>0.9998\right)$. The value of $\Delta \mathrm{E}_{\mathrm{TS}}$ in palmitic acid / $\beta$-Lg films was $34.3 \pm 0.6 \mathrm{kJmol}^{-1}$. This value was significantly smaller than the values in water $\left(36.9 \pm 0.6 \mathrm{~kJ} \mathrm{~mol}^{-1}\right)$ or $66 \mathrm{wt} . \%$ aqueous sucrose $\left(36.9 \pm 0.1 \mathrm{~kJ} \mathrm{~mol}^{-1}\right)$ and in polyvinyl alcohol $\left(41.2 \pm 0.4 \mathrm{~kJ} \mathrm{~mol}^{-1}\right)$, and larger than the value in ethanol (28.5 $\left.\pm 2.5 \mathrm{~kJ} \mathrm{~mol}^{-1}\right)$. Caprylic acid / $\beta$-Lg films had higher $\Delta \mathrm{E}_{\mathrm{Ts}}$ value $\left(35.4 \pm 0.9 \mathrm{~kJ} \mathrm{~mol}{ }^{-1}\right)$ than palmitic acid / $\beta$-Lg films. These trends suggest that solvent (matrix) properties modulate the singlet-triplet energy gap of Erythrosin B.

The phosphorescence emission band shifted to longer wavelength (lower energy) with increasing temperature. The peak emission energy $v_{\mathrm{P}}$ was determined by fitting the emission bands to a lognormal function (Eq. 12, Materials and Methods).

Figure VIII- 2 is a plot of emission energy as a function of temperature. The phosphorescence peak frequency provides a measure of the average energy of emission. The phosphorescence emission energy decreased with increasing temperature from $0^{\circ} \mathrm{C}$ to $90^{\circ} \mathrm{C}$; the decrease in emission energy reflects an increase with temperature in the extent of matrix dipolar relaxation around the excited triplet state prior to emission. Over the tested temperature range the emission energy decreased linearly and gradually at low temperatures and steeply at higher temperatures in palmitic acid / $\beta$-Lg and caprylic acid / 
$\beta$-Lg films. It can be seen that addition of fatty acid led to increases in the emission energy. In caprylic acid / $\beta$ - Lg films the peak frequency was $14707 \mathrm{~cm}^{-1}$ at $0^{\circ} \mathrm{C}$ and $14554 \mathrm{~cm}^{-1}$ at $80^{\circ} \mathrm{C}$ and for palmitic acid $/ \beta-\mathrm{Lg}$ films the frequency change from $0^{\circ} \mathrm{C}$ $80^{\circ} \mathrm{C}$ was from $14659 \mathrm{~cm}^{-1}$ to $14516 \mathrm{~cm}^{-1}$. These values were significantly higher than those in the pure $\beta-\mathrm{Lg}$ film $\left(14496 \mathrm{~cm}^{-1}\right.$ at $0^{\circ} \mathrm{C}$ and $14369 \mathrm{~cm}^{-1}$ at $\left.80^{\circ} \mathrm{C}\right)$.

Figure VIII-3 is a representation of the bandwidth of fatty acid incorporated $\beta$-Lg films as a function of temperature. The emission bandwidth increased with increasing temperature. This increase was gradual at low temperature and more dramatic with increasing temperature, indicating that the increase in dipolar relaxation rate was accompanied by an increase in the width of the distribution of energetically distinct environments. Addition of fatty acids did not significantly influence the bandwidth over the temperature range $\left(0^{\circ} \mathrm{C}-80^{\circ} \mathrm{C}\right)$ indicating that the change in composition had no obvious change in the width of the distribution of energetically distinct matrix environments. However above $80^{\circ} \mathrm{C}$ (the softening temperature of $\beta$ - $\mathrm{Lg}$ ) (Sundaresan and Ludescher, 2007), palmitic acid incorporated films exhibited a noticeable increase in bandwidth, indicating a massive increase in the width of energetically distinct environments. These results with fatty acids were comparable to the effects with other additives.

Phosphorescence Decay Kinetics

The phosphorescence emission intensity decay transients from Erythrosin B in fatty acid / $\beta-\mathrm{Lg}$ films were collected as a function of temperature over the temperature range from $0^{\circ} \mathrm{C}$ and $100^{\circ} \mathrm{C}$. All intensity decay transients were well fit using the stretched exponential model decay model (Eq 7 Materials and Methods) in which the KWW 
lifetime and the stretching exponent $\beta$ are the physically meaningful parameters. In this model $\beta$ reflects the width of an asymmetric lifetime distribution with smaller values corresponding to increasing distribution width while the lifetime essentially reflects the maximum value of the lifetime distribution (Lindsey and Patterson, 1980). The fit lifetimes and $\beta$ for palmitic acid and caprylic acid $\beta$-Lg films were plotted versus temperature in Figures VIII- 4 a, b and 5 a, b, respectively

Lifetime is the average time a probe spends in the excited triplet state and measured lifetimes can be indicative of the various complex processes occurring in the matrix. The lifetimes decreased linearly in caprylic acid $/ \beta$ - Lg films ranging from 0.60 ms at $0^{\circ} \mathrm{C}$ to $0.28 \mathrm{~ms}$ at $100^{\circ} \mathrm{C}$ in the presence of $\mathrm{N}_{2}$. Palmitic acid $/ \beta-\mathrm{Lg}$ films had a similar linear decrease with temperature with lifetimes ranging from $0.59 \mathrm{~ms}$ at $0^{\circ} \mathrm{C}$ to $0.29 \mathrm{~ms}$ at $100^{\circ} \mathrm{C}$. It is clearly indicated that there was a slight but significant increase in the lifetimes upon addition of fatty acids. A study by Buettner, (1964) has shown that comparison of the probe emission lifetimes in the presence and absence of oxygen is a facile indicator of the quenching of oxygen in the matrix. So, in order to detect oxygen quenching, lifetime measurements were made in the presence of air. Lifetimes in the presence of air were significantly different from those in nitrogen indicating that in the presence of fatty acids the $\beta$ - Lg matrix was highly permeable to atmospheric oxygen.

The stretching exponent $\beta$ is a measure of the dynamic heterogeneity of the matrix. $\beta$ was constant at low temperatures and decreased at high temperatures in all the films in the presence of nitrogen. It is also clearly evident from Figure VIII 5a that increasing palmitic acid concentration brought about an increase in $\beta$ suggesting that in the presence of palmitic acid the presence of dynamically different environments were 
lowered. Addition of caprylic acid also led to a slight increase in $\beta$. These trends essentially suggest that fatty acid binding had an effect on the mobility of the matrix.

$\beta$ reflects the distribution of lifetimes and thus the corresponding distribution of dynamically distinct probe environments with different values of $\mathrm{k}_{\mathrm{Ts} 0}$ (Pravinata et al ., 2005). Small increase in $\beta$ with addition of fatty acids indicated significant decrease in the range of dynamically distinct probe environments in the entire temperature range.

It is clearly evident that in the presence of air, $\beta$ confirmed the existence of a greater number of different environments, which is illustrated by a noticeable decrease in $\beta$. Trends in lifetimes and $\beta$, clearly indicate that the fatty acid $/ \beta-\mathrm{Lg}$ matrix is highly permeable to atmospheric oxygen.

The term $\mathrm{k}_{\mathrm{Q}}\left[\mathrm{O}_{2}\right]$ describes the rate of oxygen quenching of the excited triplet state of the probe by oxygen. The rate constant for quenching the triplet state by oxygen, $\mathrm{k}_{\mathrm{Q}}\left[\mathrm{O}_{2}\right]$, is calculated from the difference in inverse lifetime in air and in nitrogen (Eq 11 Materials and Methods). $\mathrm{k}_{\mathrm{Q}}\left[\mathrm{O}_{2}\right]$ values in the various fatty acid / $\beta$-Lg films are depicted in Figure VIII-6. It is clearly evident from the study that addition of small amounts of palmitic acid and caprylic acid had virtually no impact on the permeability of the $\beta$ - $\operatorname{Lg}$ matrix. Although these fatty acids in such low concentrations had an impact on the dipolar relaxations with subtle differences between the fatty acids, but the macroscopic property of permeability remained unaffected.

The decrease in lifetime with temperature reflects an increase in the rate of nonradiative decay of the excited triplet state $T_{1}$ due to an increase in both the rate of non radiative decay to the ground state $\mathrm{S}_{0}\left(\mathrm{k}_{\mathrm{TS} 0}\right)$ and reverse intersystem crossing to $\mathrm{S}_{1}\left(\mathrm{k}_{\mathrm{TS} 1}\right)$. Based on the maximum reasonable values of $\mathrm{k}_{\mathrm{TS} 1}$, an estimate of the lower limits of $\mathrm{k}_{\mathrm{TS} 0}$ 
was calculated from Equation 8 (Materials and Methods) and was plotted versus temperature in Figure VIII-7.

$\mathrm{k}_{\mathrm{TS} 0}$ increased with temperature for the fatty acid $\beta$-Lg films, which was expected since the matrices become more mobile as temperature increases. The $\mathrm{k}_{\mathrm{TS} 0}$ in palmitic acid/ $\beta-\mathrm{Lg}$ and caprylic acid/ $\beta-\mathrm{Lg}$ at $0^{\circ} \mathrm{C}$ was $1639 \mathrm{~s}^{-1}$ and $1614 \mathrm{~s}^{-1}$ respectively. These values were significantly smaller than that in pure $\beta$ - Lg film $\left(1730 \mathrm{~s}^{-1}\right)$. At higher temperatures $\left(100^{\circ} \mathrm{C}\right)$ the values of $\mathrm{k}_{\mathrm{TS} 0}$ were $2562 \mathrm{~s}^{-1}$ and $2752 \mathrm{~s}^{-1}$ respectively for palmitic acid/ $\beta$-Lg and caprylic acid / $\beta$-Lg compared to $3154 \mathrm{~s}^{-1}$ in pure $\beta-\mathrm{Lg}$. Both fatty acids at low temperatures had a similar effect on the mobility of the $\beta$ - $\mathrm{Lg}$ matrix, as evident by the decline in $\mathrm{k}_{\mathrm{TS} 0}$ values (5\% decline in palmitic acid and $7 \%$ in caprylic acid at $0^{\circ} \mathrm{C}$ ). However, when compared to caprylic acid, palmitic acid had a greater effect on the mobility at high temperatures showing an approximate $19 \%$ decline in the $\mathrm{k}_{\mathrm{TS} 0}$ values. Compared to the pure $\beta$ - $\mathrm{Lg}$ films quenching rate $\mathrm{k}_{\mathrm{TS} 0}$ was considerably lower in the presence of fatty acids. Specific interactions between fatty acids and $\beta$-Lg (probably binding of fatty acid to the protein) decreased the ability of the matrix to vibrationally dissipate the triplet state energy of Erythrosin B by decreasing the mobility of the amorphous matrix.

It is clear that the fatty acids had an impact on the overall mobility of the matrix. A look into the combinatorial effect of temperature and mobility revealed that in the presence of fatty acids the decline in $\mathrm{k}_{\mathrm{TS} 0}$ was magnified at high temperature showing that the fatty acids exerted a greater influence on the mobility at high temperatures. 


\section{$\underline{\text { Discussion }}$}

Why study the effect of fatty acid on $\beta$-lactoglobulin films?

$\beta$ - $\mathrm{Lg}$ is a small globular whey protein found in the milk and has been known for its excellent film forming capabilities. Much effort in the past has been devoted in delineating the various ligand binding sites in $\beta$-Lg (Frapin et al., 1993; Creamer, 1995). It was previously demonstrated that 1 mole of $\beta$ lactoglobulin could approximately bind one mole of apolar substance (Hidalgo and Kinsella, 1989). Compared to other whey proteins $\beta$-Lg exhibited the maximum affinity to fatty acids (Lemeste et al., 1991). When a ligand is introduced in a protein matrix, that small hydrophobic molecule can have dramatic effects on the conformational dynamics of the protein thereby affecting the mobility and physical properties of the matrix. Reference the present study throws light on the effect of fatty acid binding on the $\beta$-Lg matrix.

The spectroscopic data indicate that the phosphorescence of Erythrosin B is sensitive to the distinct modes of molecular mobility in amorphous $\beta-\operatorname{Lg}$ and $\beta-\operatorname{Lg}$ fatty acid mixtures. Measurements of temperature dependence of the emission energy and lifetime of the probe dispersed in amorphous $\beta$-Lg with fatty acids provide insight into the solid-state biophysics of amorphous $\beta$ - $\operatorname{Lg}$ in the presence of fatty acids. Effect of fatty acids on the matrix mobility

Phosphorescence emission wavelength and intensity of Erythrosin B is sensitive to two kinds of molecular mobility, dipolar relaxation and collisional quenching. This study mainly looks into the effect of two fatty acids, palmitic acid (which is bound 
specifically in solution) and caprylic acid (which is not bound) on the molecular mobility and permeability of the $\beta$-Lg. The phosphorescence emission energy reflects the average $\mathrm{T}_{1} \leftarrow \mathrm{S}_{0}$ energy gap; it is primarily modulated by the $\mathrm{T}_{1}$ energy and thus the average extent of relaxation of the dipolar groups around the excited $\mathrm{T}_{1}$ state prior to emission. From this study it is evident that very low concentrations of palmitic acid and caprylic acid have a significant effect on the local mobility of the matrix. Binding of ligands to proteins generally stabilizes protein structure. Examples include fatty acid bound to BSA (Gumpen et al., 1979), $\mathrm{Ca}^{+2}$ bound to $\alpha$ - lactalbumin (Relkin et al., 1993), or $\mathrm{Fe}^{+3}$ bound to lactoferrin (Sanchez et al., 1992).

We saw that addition of small amounts of fatty acids at molar ratio of 1:1 ( $\beta$ - $\mathrm{Lg}$ : fatty acid) brought about an enhancement in the emission energy. Creamer (1995) demonstrated enhanced stability when $\beta$-Lg was complexed with either palmitic acid or low concentrations of SDS, and, moreover, palmitic acid offered superior stability when compared to the other fatty acids in solution.

However in our study contrary, to our expectations, palmitic acid, which is specifically bound in the central cavity in the protein, had a lesser effect on the emission energy as compared to caprylic acid. This anomalous trend cannot be explained with the current postulated theories. However, we propose an explanation, which needs to be further substantiated with other instrumental techniques. Based on solution studies, literature suggest that caprylic acid is not bound to $\beta$-Lg (Frapin et al., 1993) but the possibility of it binding in the solid state cannot be ruled out.

Collini et al. (2003) studied the binding of caprylic acid to $\beta$ - $\mathrm{Lg}$ solution. He found that caprylic acid had a weak-binding constant $\left(\mathrm{K}_{\mathrm{F}}\left(* 10^{2}\right) \mathrm{M}^{-1}=255 \pm 50\right)$ at $\mathrm{pH} 8$. 
However, in the solid state the concentration of fatty acid increases greatly. So the probability of caprylic acid binding to the central cavity of the $\beta-\mathrm{Lg}$ is very high. This eight-carbon fatty acid may bind in the protein in such a way that the specific interactions may be stronger leading to higher emission energy. Palmitic acid on the other hand also brought about a significant enhancement in the emission energy but it was not as pronounced as caprylic acid. Significant increase in the lifetimes in the presence of fatty acids and relative similarities further substantiated that both fatty acids were bound in the hydrophobic cavity of the protein in the solid state. Ligand binding has been shown to confer stability to the molecules and this enhanced lifetime may be an indication of enhanced rigidity conferred by fatty acid binding to the protein.

The phosphorescence lifetime is directly modulated by the rate of intersystem crossing $\mathrm{k}_{\mathrm{TS} 0} ; \mathrm{k}_{\mathrm{TS} 0}$ in turn is modulated by the physical state of the amorphous matrix. The magnitude of $\mathrm{k}_{\mathrm{TS} 0}$ reflects both internal factors related to the manner in which the excited $\mathrm{T}_{1}$ state of Ery B is vibrational coupled to the $\mathrm{S}_{0}$ ground state, as well as external factors apparently related to the manner in which the ground-state vibrational energy can dissipate from the excited probe into the surrounding matrix. Because the efficiency of this vibration coupling is related to the overall mobility of the matrix, the magnitude of $\mathrm{k}_{\mathrm{TS} 0}$ provides a direct measure of matrix mobility. Lower $\mathrm{k}_{\mathrm{TS} 0}$ values in the presence of fatty acids are a clear indication that the matrix mobility is greatly restricted in the presence of fatty acids. This may be attributed to the effects of ligand binding. These findings were quite similar for emission energy and lifetimes.

The binding of fatty acids to $\beta-\mathrm{Lg}$ increases its conformational stability to trypsin degradation. A study by Puyol et al. (1994) compared the relative efficiency of the 
ligand binding to fatty acids and retinal and it clearly revealed that the binding of fatty acids to $\beta$-Lg rendered greater stability when compared to other ligands

A study by Hidalgo and Kinsella (1989) observed that the interaction of $\beta$-Lg with linoleic acid 13-hydroperoxide induced a decrease in the free amino groups and that the blockage of protein amino-groups prevented the deteriorative changes which accompanied this interaction. A study by Lemeste et al. (1991) revealed that in the presence of fatty acids the rotational mobility of the more mobile side chains in $\beta$-Lg was greatly reduced..

Arhenius plots of the mobility parameter $\mathrm{k}_{\mathrm{TS} 0}$ versus temperature in caprylic acid/ $\beta$-Lg films (FigureVIII-8) had activation energies of $1.9 \mathrm{~kJ}$ at low temperatures and 10.3 $\mathrm{kJ}$ and high temperatures respectively and $1.6 \mathrm{~kJ}$ and $9.3 \mathrm{~kJ}$ in palmitic acid/ $\beta$-Lg films. These values were significantly lower than the pure $\beta$-Lg matrix $\left(2.35\right.$ and $\left.21.4 \mathrm{~kJ} \mathrm{~mol}^{-1}\right)$. The small value of $\mathrm{E}_{\mathrm{A}}$ at low temperature suggests that the motions that activate vibrational relaxation are small amplitude, localized modes of molecular mobility; on the other hand, at high temperatures, the significantly larger value of EA suggests that the modes of protein motion involve significantly larger segments of the protein structure. Lower activation energies in the presence of fatty acids clearly illustrate that in the presence of fatty acids the mobility modes in the $\beta$ - $\mathrm{Lg}$ matrix are shifted to small scale molecular vibrations.

Transition temperatures calculated from the intersection of the linear fits at low and high temperatures were $62^{\circ} \mathrm{C}$ and $64^{\circ} \mathrm{C}$ for caprylic acid/ $\beta$-Lg films and palmitic acid/ $\beta$-Lg, films respectively, which were lower than the pure protein films $\left(81^{\circ} \mathrm{C}\right)$. Lower transition temperatures may be reflective of the early origins of large-scale 
motions in the amorphous matrix. This anomalous behavior needs to investigate further to derive a suitable explanation.

Effect of fatty acids on the permeability of the matrix

$\beta$ the stretching exponent and lifetimes in the presence of air and nitrogen clearly indicated the fact that addition of caprylic acid and palmitic acid to the $\beta$ - $\mathrm{Lg}$ matrix didn't bring any changes in the permeability of the matrix.. These findings were further substantiated by the calculation of the collisional quenching rate constant for oxygen diffusion $\mathrm{k}_{\mathrm{Q}}\left[\mathrm{O}_{2}\right]$.

Comparison of the collisional constant for oxygen quenching $\mathrm{k}_{\mathrm{Q}}\left[\mathrm{O}_{2}\right]$ revealed that the change in composition and ligand binding had an insignificant effect on the permeability of the $\beta$-Lg matrix. This behavior can be explained as follows:

Fatty acids essentially bind to $\beta$-Lg at a molar ratio of $1: 1$ Fatty acid/ $\beta$-Lg. In this present study this was the chosen concentration to investigate the effect of fatty acids on $\beta$-Lg films. The weight percentages of fatty acids palmitic acid and caprylic acid in $\beta-\mathrm{Lg}$ films were only $1.3 \%$ and $0.77 \%$ respectively. Research studies in the past have shown that addition of lipid causes an increase in the oxygen permeability of edible films. However, the concentrations of fatty acids used in those studies were much higher than the present study. Gennadios et al. (1993) compared the oxygen permeability of protein films (gluten, gluten/soy protein isolate, zein) with that of composite films of polysaccharides and lipids, and observed that the films without the addition of lipid were less permeable to oxygen. Ayranci and Tunc. (2003) produced composite methylcellulose films with added stearic acids and observed that increasing the stearic acid content of the film (15 g/100 g of methyl cellulose) enhanced the oxygen permeability. Similar results 
were reported by Park et al. (1993) in cellulose based edible films. They found that the oxygen permeability of laminated films increased with increasing fatty acid content. Although showing better water vapor barrier properties the film containing added stearic acid showed an increase in oxygen permeability as the amount of fatty acid was increased $(10 \%-40 \%)$. In the present study, however, matrix in the presence of fatty acids was predominantly a $\beta$-Lg matrix (98\%) and so the permeability characteristics of the composite film primarily reflected the pure $\beta$-Lg matrix behavior. Such low concentrations were insufficient to cause substantial changes in the macroscopic diffusive property of the matrix as the composition was altered very minimally.

\section{Oxygen Permeability and Molecular mobility}

Fatty acids palmitic acid and caprylic acid brought significant decreases in the mobility of the matrix, but the permeability of the matrix remained unaffected. These differences may be attributed to the fundamental difference in these two processes. The indicator of matrix mobility, $\mathrm{k}_{\mathrm{TS} 0}$ is a measure of the coupling of vibrational motions in the probe with local vibrational motions in the protein matrix while $\mathrm{k}_{\mathrm{Q}}\left[\mathrm{O}_{2}\right]$ measures the coupling of translational motion of an oxygen molecule with collective motions of the protein matrix (Nack and Ludescher, 2006). Despite the differences in the manner in which the fatty acids affect mobility $\left(\mathrm{k}_{\mathrm{TS} 0}\right)$ and oxygen permeability $\mathrm{k}_{\mathrm{Q}}\left[\mathrm{O}_{2}\right]$, there existed a correlation between mobility and permeability (Figure VIII-9a, b). This correlation suggested that the basic mechanisms underlying these processes were the same in pure $\beta$ $\mathrm{Lg}$ and the fatty acid $\beta$ - $\mathrm{Lg}$ films. Similar correlations between $\mathrm{k}_{\mathrm{Q}}\left[\mathrm{O}_{2}\right]$, and $\mathrm{k}_{\mathrm{TS} 0}$ were observed in amorphous BSA and $\beta$-Lg (Nack and Ludescher, 2006; Sundaresan and Ludescher, 2007). 


\section{Conclusions}

Molecular mobility in amorphous solids can be modulated by temperature and change in composition. Phosphorescence from ErythrosinB can tell us about changes in mobility. Ligand binding had a profound effect on the local relaxation dynamics. Incorporation of fatty acid exerted two kinds of stabilization effects primarily by decreasing the mobility, inhibiting the local relaxation mechanisms and conferring rigidity effects. The results from this study clearly indicate that both dipolar relaxation and collisional quenching $\left(\mathrm{k}_{\mathrm{TS} 0}\right)$ in $\beta$ - $\mathrm{Lg}$ films with fatty acid essentially reflect the stabilization conferred to the protein matrix by the binding of ligands. Permeability was unaffected due to minimal alterations in the composition. Higher concentrations of fatty acids when incorporated in the film generated opaque films, which could not yield accurate spectroscopic results. 


\section{$\underline{\text { References }}$}

Ayranci, E. and Tunc, S. (2002). A method for the measurement of the oxygen permeability and the development of edible films to reduce the rate of oxidative reactions in fresh foods. Food Chem. 80, 423-431.

Birks, J. B. (1970). Photophysics of Aromatic Molecules (Wiley Monographs in Chemical Physics). 704.

Brownlow, S., Cabral, J., Cooper, R., Flower, D., Yewdall, S., Polikarpov, I., North, A., and Sawyer, L. (1997). Bovine $\beta$-lactoglobulin at $1.8 \AA$ resolution - still an enigmatic lipocalin. Structure, 5, 481-495.

Buettner, A. V. (1964). Flash photolysis in thin films of gelatin and other polymers. Journal of Physical Chemistry. 68, 3253-3259.

Champion, D., Le Meste, M., and Simatos, D. (2000/2). Towards an improved understanding of glass transition and relaxations in foods: molecular mobility in the glass transition range. Trends in Food Science \& Technology. 11, 41-55.

Cheng, J., Lepre, C. A., and Moore, J. M. (1994). 15N NMR Relaxation Studies of the FK506 Binding Protein: Dynamic Effects of Ligand Binding and Implications for Calcineurin Recognition. Biochemistry (N. Y. ). 33, 4093-4100.

Collini, M., D'Alfonso, L., and Baldini, G. (2000). New insight on beta -lactoglobulin binding sites by 1-anilinonaphthalene-8-sulfonate fluorescence decay. Protein Sci. 9, 1968-1974.

Collini, M., D'Alfonso, L., Molinari, H., Ragona, L., Catalano, M., and Baldini, G. (2003). Competitive binding of fatty acids and the fluorescent probe 1-8-anilinonaphthalene sulfonate to bovine beta -lactoglobulin. Protein Sci. 12, 1596-1603.

Creamer, L. K. (1995). Effect of Sodium Dodecyl Sulfate and Palmitic Acid on the Equilibrium Unfolding of Bovine beta -Lactoglobulin. Biochemistry (N. Y. ). 34, 7170-7176.

Duchowicz, R., Ferrer, M. L., and Acuña, A. U. (1998). Kinetic Spectroscopy of Erythrosin Phosphorescence and Delayed Fluorescence in Aqueous Solution at Room Temperature. Photochemistry and Photobiology. 68, 494-501.

Dufour, E., Genot, C., and Haertle, T. (1994). beta -Lactoglobulin binding properties during its folding changes studied by fluorescence spectroscopy. Biochim.Biophys.Acta, Protein Struct.Mol.Enzymol. 1205, 105-112.

Dufour, E., Marden, M. C., and Haertle, T. (1990). beta -Lactoglobulin binds retinol and protoporphyrin IX at two different binding sites. FEBS Lett. 277, 223-226. 
Frapin, D., Dufour, E., and Haertle, T. (1993). Probing the fatty acid binding site of beta lactoglobulins. J. Protein Chem. 12, 443-449.

Gennadios, A., Weller, C. L., and Testin, R. F. (1993). Temperature effect on oxygen permeability of edible protein-based films. J. Food Sci. 58, 212-14, 219.

Gumpen, S., Hegg, P. O., and Martens, H. (1979). Thermal stability of fatty acid-serum albumin complexes studied by differential scanning calorimetry. Biochim.Biophys.Acta, Lipids Lipid Metab. 574, 189-196.

Hidalgo, F. J. and Kinsella, J. E. (1989). Changes induced in beta -lactoglobulin B following interactions with linoleic acid 13-hydroperoxide. J. Agric. Food Chem. 37, 860-866.

Hurtubise, R. J. (1990). Phosphorimetry:Theory,Instrumentation and applications. .

Kontopidis, G., Holt, C., and Sawyer, L. (2004). beta -Lactoglobulin: binding properties, structure, and function. J. Dairy Sci. 87, 785-796.

Kontopidis, G., Holt, C., and Sawyer, L. (2002). The ligand-binding site of bovine beta lactoglobulin: evidence for a function? J. Mol. Biol. 318, 1043-1055.

Lakowicz, J. R. (1999). Principles of fluorescence spectroscopyKluwer Academic/Plenum Press, Dordrecht/New York.

Le Meste, M., Closs, B., Courthaudon, J. L., and Colas, B. (1991). Interactions between milk proteins and lipids. A mobility study. ACS Symp. Ser. 454, 137-147.

Lettinga, M. P., Zuilhof, H., and Van Zandvoort, M. A. M. J. (2000). Phosphorescence and fluorescence characterization of fluorescein derivatives immobilized in various polymer matrices. Physical Chemistry Chemical Physics. 2, 3697-3707.

Lindsey, C. P. and Patterson, G. D. (1980). Detailed comparison of the Williams-Watts and Cole-Davidson functions. J. Chem. Phys. 73, 3348-3357.

Lukasik, K. V. and Ludescher, R. D. (2005 a). Effect of plasticizer on dynamic site heterogeneity in cold-cast gelatin films. Food Hydrocoll. 20, 88-95.

Lukasik, K. V. and Ludescher, R. D. (2005 b). Molecular mobility in water and glycerol plasticized cold- and hot-cast gelatin films. Food Hydrocoll. 20, 96-105.

Marden, M. C., Dufour, E., Christova, P., Huang, Y., Leclerc-L'Hostis, E., and Haertle, T. (1994). Binding of heme-CO to bovine and porcine beta -lactoglobulins. Arch. Biochem. Biophys. 311, 258-262.

Maroncelli, M. and Fleming, G. R. (1987). Picosecond solvation dynamics of coumarin 153: the importance of molecular aspects of solvation. J. Chem. Phys. 86, 6221-6239. 
Nack, T. J. and Ludescher, R. D. (2006). Molecular Mobility and Oxygen Permeability in Amorphous Bovine Serum Albumin Films. Food Biophysics. 1557-1858.

Narayan, M. and Berliner, L. J. (1998). Mapping fatty acid binding to beta -lactoglobulin: ligand binding is restricted by modification of Cys 121. Protein Sci. 7, 150-157.

Narayan, M. and Berliner, L. J. (1997). Fatty Acids and Retinoids Bind Independently and Simultaneously to beta -Lactoglobulin. Biochemistry (N. Y. ). 36, 1906-1911.

Nicholson, L. K., Yamazaki, T., Torchia, D. A., Grzesiek, S., Bax, A., Stahl, S. J., Kaufman, J. D., Wingfield, P. T., Lam, P. Y. S., and et al. (1995). Flexibility and function in HIV-1 protease. Nat. Struct. Biol. 2, 274-280.

Park, H. J., Weller, C. L., Vergano, P. J., and Testin, R. F. (1993). Permeability and mechanical properties of cellulose-based edible films. J. Food Sci. 58, 1361-4, 1370.

Parker, C. A., Ed. (1968.). Photoluminescence of SolutionsElsevier Publishing, Amsterdam, The Netherlands.

Perez, M. D., Diaz de Villegas, C., Sanchez, L., Aranda, P., Ena, J. M., and Calvo, M. (1989). Interaction of fatty acids with beta -lactoglobulin and albumin from ruminant milk. $J$. Biochem. 106, 1094-1097.

Pravinata, L. C., You, Y., and Ludescher, R. D. (2005). Erythrosin B Phosphorescence Monitors Molecular Mobility and Dynamic Site Heterogeneity in Amorphous Sucrose. Biophys. J. 88, 3551-3561.

Puyol, P., Perez, M. D., Burgos, J., and Calvo, M. (1998). Effect of the binding of palmitic acid to beta -lactoglobulin on its gelation properties. Int. Dairy J. 8, 119-123.

Puyol, P., Perez, M. D., Mata, L., Ena, J. M., and Calvo, M. (1993). Effect of retinol and fatty acid binding by bovine beta -lactoglobulin on its resistance to trypsin digestion. Int. Dairy J. 3, 589-597.

Puyol, P., Perez, M. D., Peiro, J. M., and Calvo, M. (1994). Effect of binding of retinol and palmitic acid to bovine beta -lactoglobulin on its resistance to thermal denaturation. $J$. Dairy Sci. 77, 1494-1502.

Qin, B. Y., Bewley, M. C., Creamer, L. K., Baker, H. M., Baker, E. N., and Jameson, G. B. (1998). Structural Basis of the Tanford Transition of Bovine \&\#x03B2;-Lactoglobulin. Biochemistry. 37, 14014-14023.

Ragona, L., Fogolari, F., Zetta, L., Perez, D. M., Puyol, P., De Kruif, K., Lohr, F., Ruterjans, H., and Molinari, H. (2000). Bovine beta -lactoglobulin: interaction studies with palmitic acid. Protein Sci. 9, 1347-1356. 
Ragona, L., Pusterla, F., Zetta, L., Monaco, H. L., and Molinari, H. (1997). Identification of a conserved hydrophobic cluster in partially folded bovine beta -lactoglobulin at $\mathrm{pH} 2$. Folding Des. 2, 281-290.

Relkin, P., Launay, B., and Eynard, L. (1993). Effect of sodium and calcium addition on thermal denaturation of apo-alpha -lactalbumin: a differential scanning calorimetric study. $J$. Dairy Sci. 76, 36-47.

Richert, R. (2000). Triplet state solvation dynamics: Basics and applications. J. Chem. Phys. 113, 8404-8429.

Rischel, C., Madsen, J. C., Andersen, K. V., and Poulsen, F. M. (1994). Comparison of Backbone Dynamics of apo- and holo-Acyl-Coenzyme A Binding Protein Using 15N Relaxation Measurements. Biochemistry (N. Y. ). 33, 13997-14002.

Sanchez, L., Peiro, J. M., Castillo, H., Perez, M. D., Ena, J. M., and Calvo, M. (1992). Kinetic parameters for denaturation of bovine milk lactoferrin. J. Food Sci. 57, 873-879.

Sawyer, L. and Kontopidis, G. (2000). The core lipocalin, bovine $\beta$-lactoglobulin. Biochimica et Biophysica Acta (BBA) - Protein Structure and Molecular Enzymology,. 1482, 136-148.

Shirke, S., Takhistov, P., and Ludescher, R. D. (2005). Molecular Mobility in Amorphous Maltose and Maltitol from Phosphorescence of Erythrosin B. J. Phys. Chem. B. 109, 16119-16126.

Shirke, S. and Ludescher, R. D. (2005). Dynamic site heterogeneity in amorphous maltose and maltitol from spectral heterogeneity in erythrosin B phosphorescence. Carbohydr. Res. 340, 2661-2669.

Shirke, S. and Ludescher, R. D. (2005). Molecular mobility and the glass transition in amorphous glucose, maltose, and maltotriose. Carbohydr. Res. 340, 2654-2660.

Shirke, S., Takhistov, P., and Ludescher, R. D. (2005). Molecular Mobility in Amorphous Maltose and Maltitol from Phosphorescence of Erythrosin B. J Phys Chem B. 109, 16119-16126.

Simon-Lukasik, K. V. and Ludescher, R. D. (2004/7). Erythrosin B phosphorescence as a probe of oxygen diffusion in amorphous gelatin films. Food Hydrocolloids. 18, 621-630.

Spector, A. A. and Fletcher, J. E. (1970). Binding of long chain fatty acids to beta -lactoglobulin. Lipids. 5, 403-411.

Sundaresan, K. V. and Ludescher, R. D. (2007). Molecular mobility and oxygen permeability in amorphous beta -lactoglobulin films. Food Hydrocoll. 22, 403-413.

Turro, N., Ed. (1991). Modern molecular photochemistry, Sausalito,CA. 
Yu, L., Zhu, C., Tse-Dinh, Y., and Fesik, S. W. (1996). Backbone dynamics of the C-terminal domain of Escherichia coli topoisomerase $\mathrm{I}$ in the absence and presence of singlestranded DNA. Biochemistry (N. Y. ). 35, 9661-9666. 


\section{Figure VIII-1a}

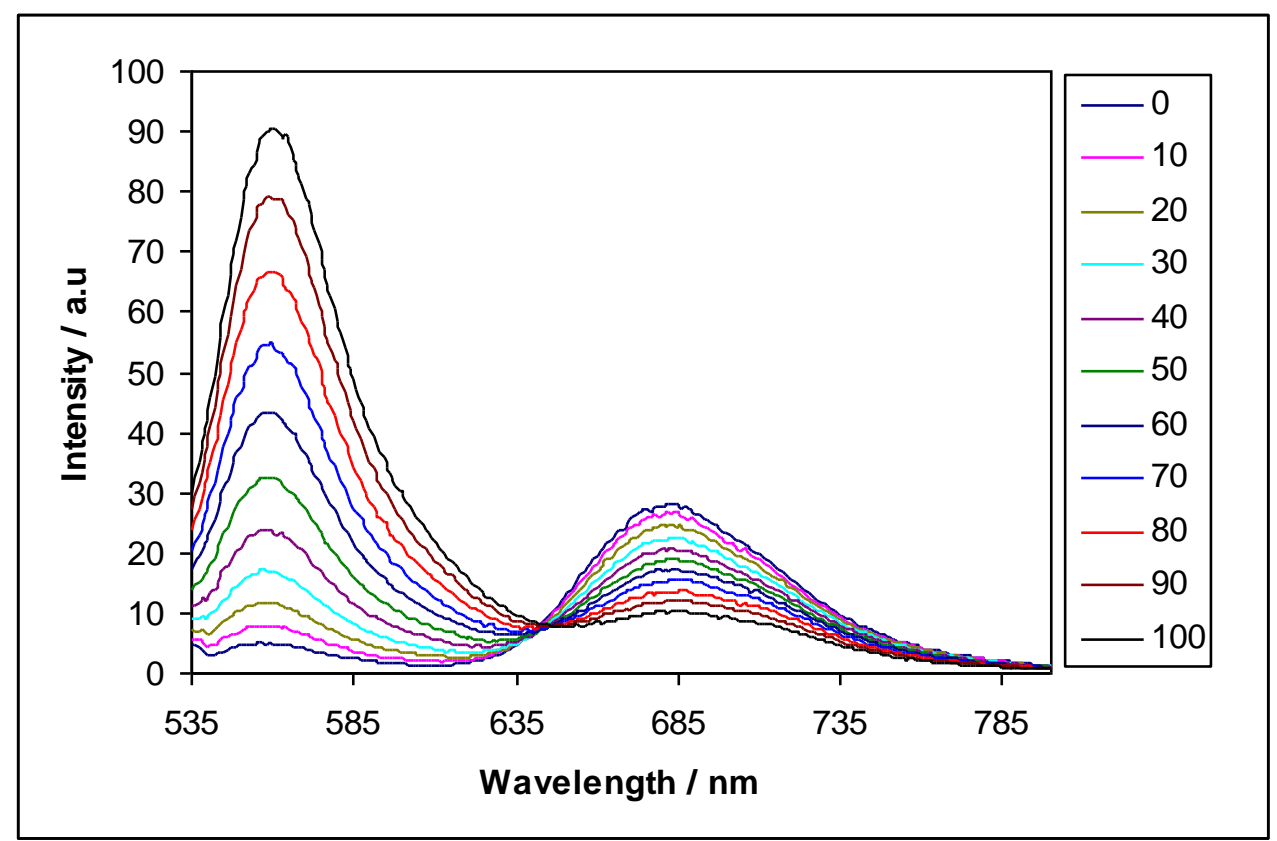

Figure VIII-1a: The effect of temperature on the delayed emission spectra of Erythrosin B dispersed in amorphous $\beta$-Lg- palmitic acid films ( $\beta$ - $\mathrm{Lg}$ : palmitic acid 1:1).

\section{Figure VIII-1b}

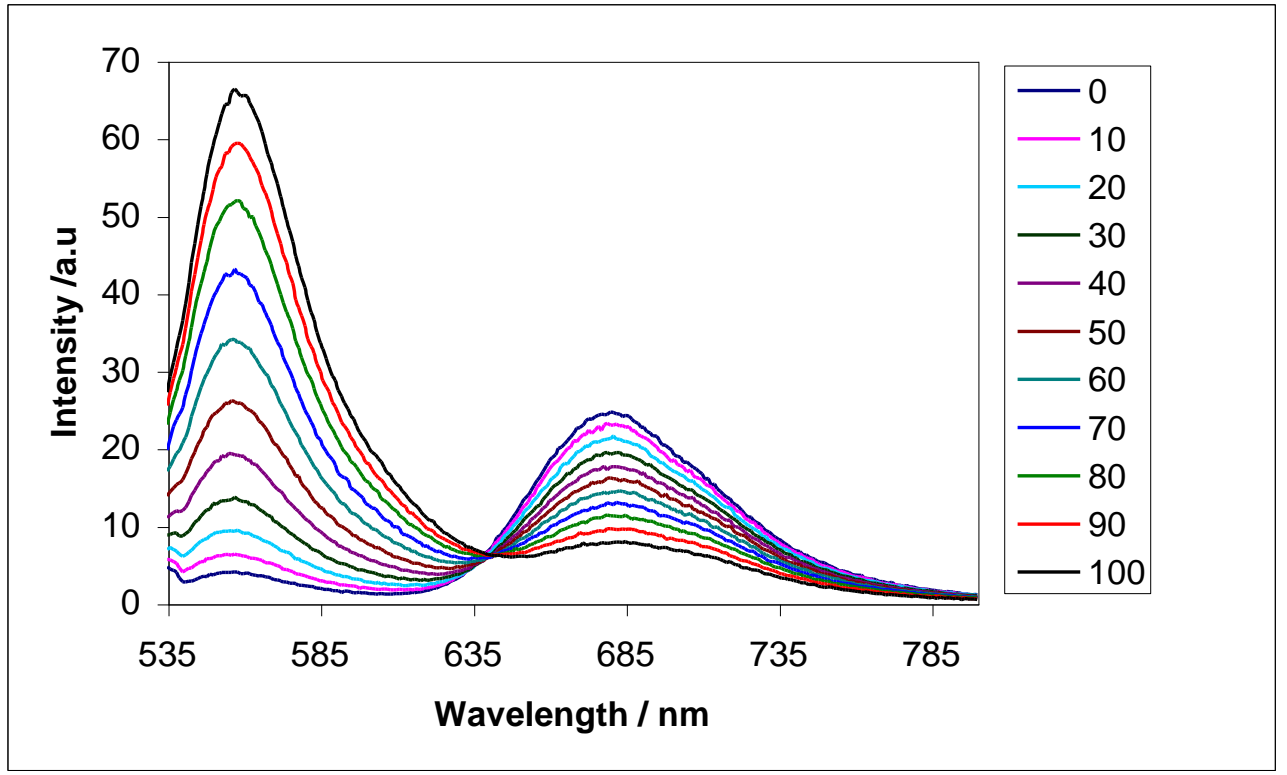

Figure VIII-1b: The effect of temperature on the delayed emission spectra of Erythrosin B dispersed in amorphous $\beta$-Lg- caprylic acid films ( $\beta$-Lg:caprylic acid 1:1). 
Figure VIII-2

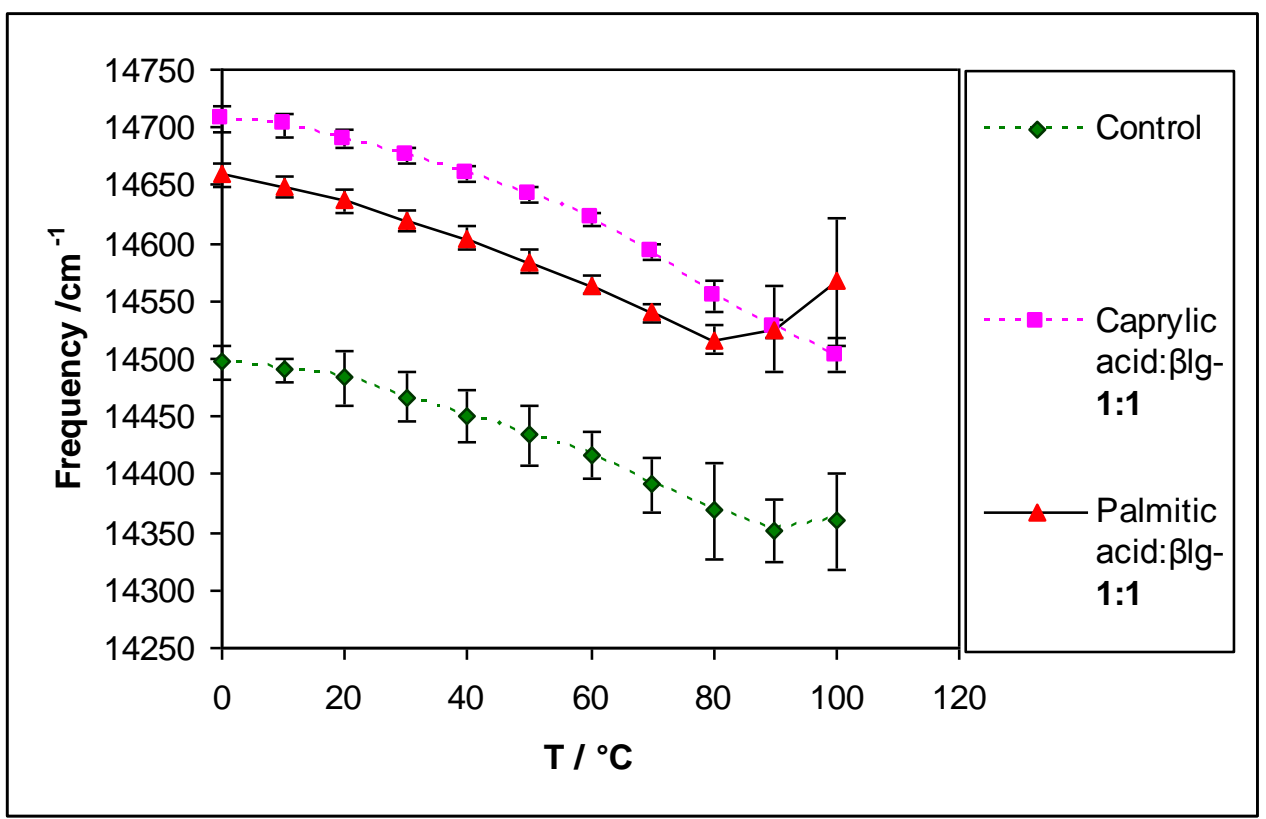

Figure VIII-2: The effect of temperature on the emission maximum for phosphorescence from Erythrosin B in amorphous fatty acid/ $\beta$ - Lg films.

Figure VIII-3

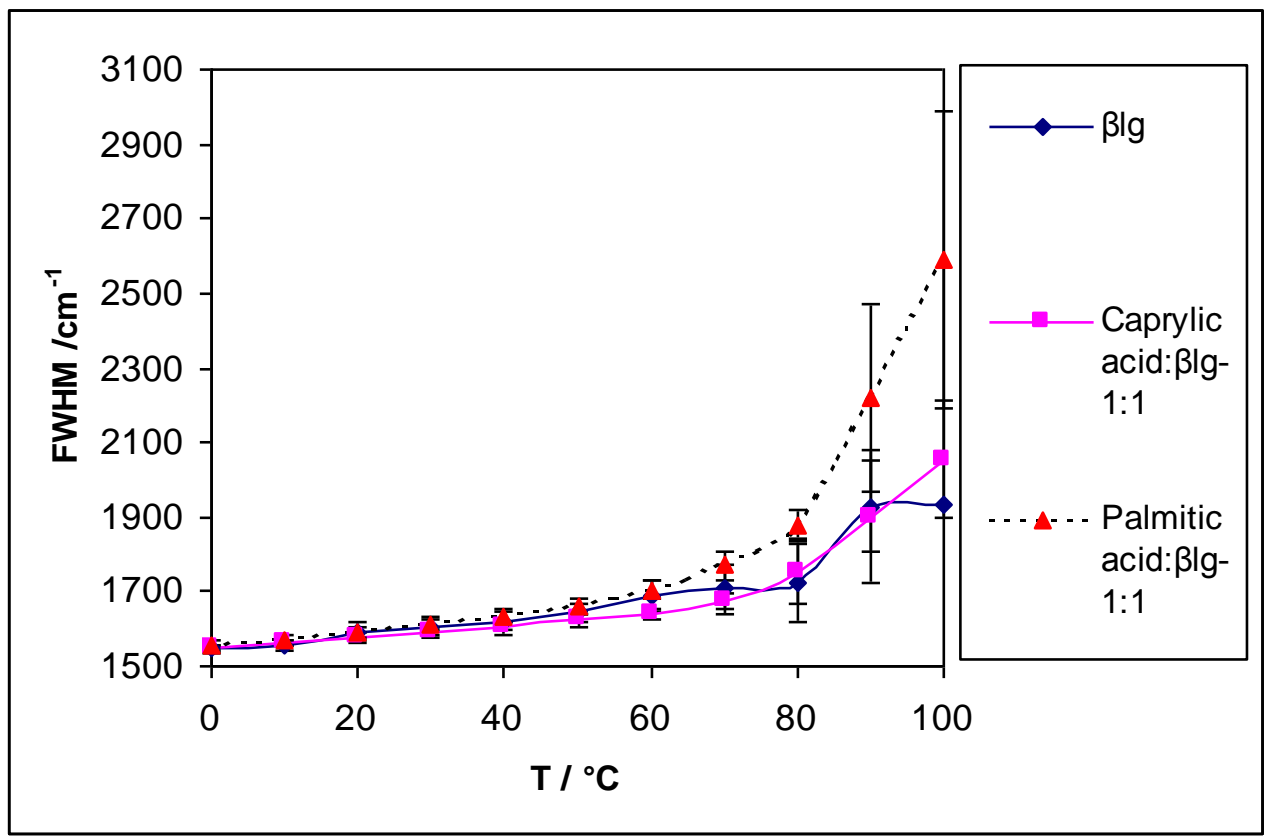

Figure VIII-3: The effect of temperature on the bandwidth for phosphorescence from Erythrosin B in amorphous fatty acid/ $\beta-\mathrm{Lg}$ films. 
Figure VIII-4a

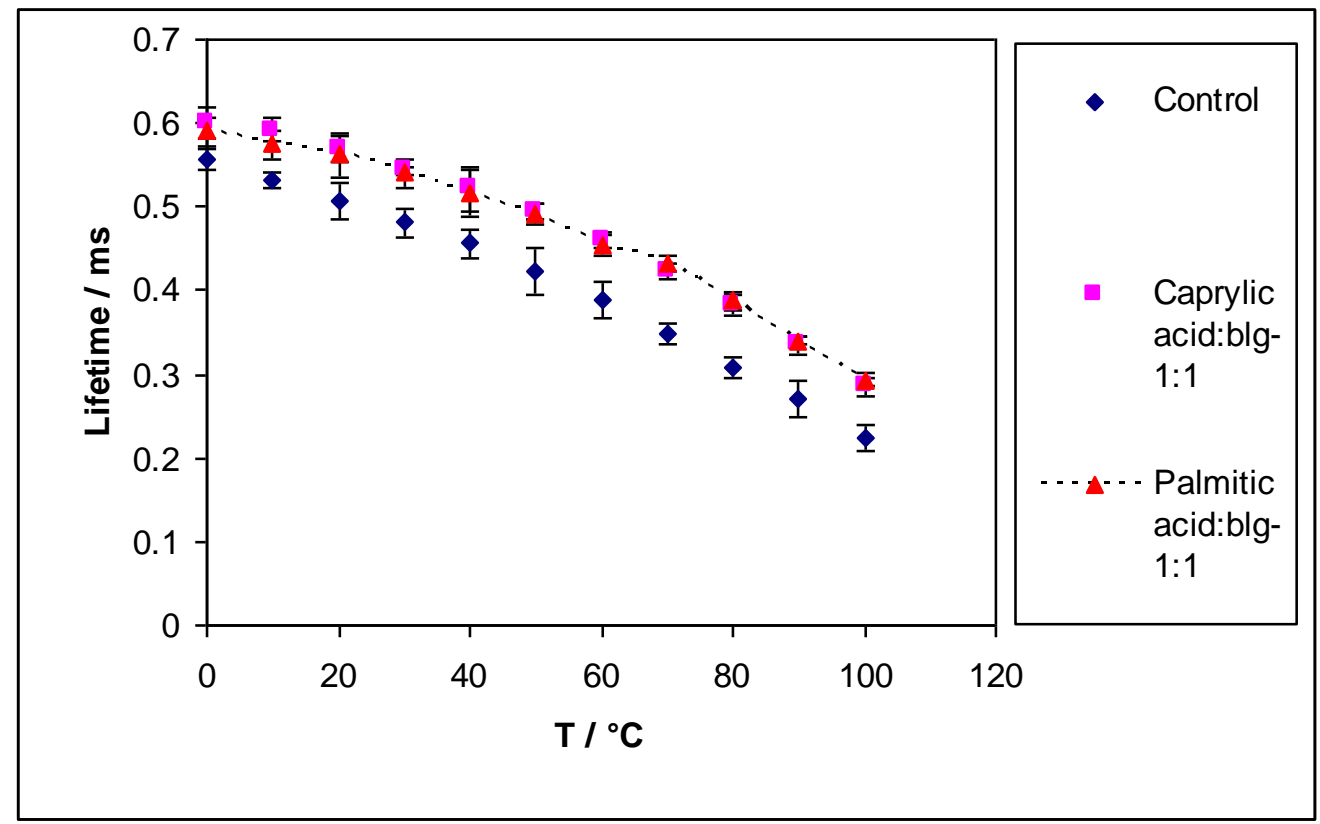

Figure VIII-4a: Comparison plot depicting the effect of temperature on the lifetime of Erythrosin B dispersed in fatty acid/ $\beta-\mathrm{Lg}$ films equilibrated against nitrogen.

Figure VIII-4b

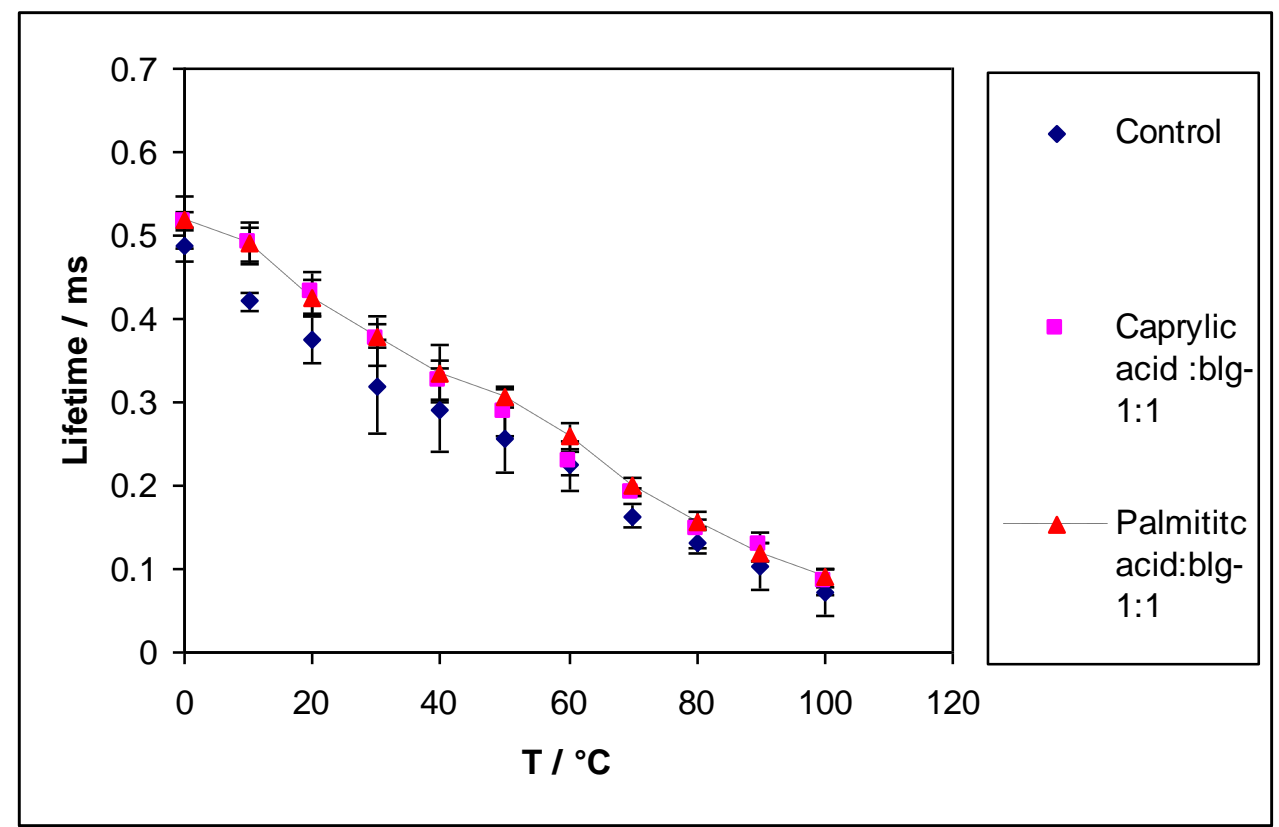

Figure VIII-4b: Comparison plot depicting the effect of temperature on the lifetime of Erythrosin B dispersed in fatty acid/ $\beta$ - Lg films equilibrated against air. 


\section{Figure VIII-5a}

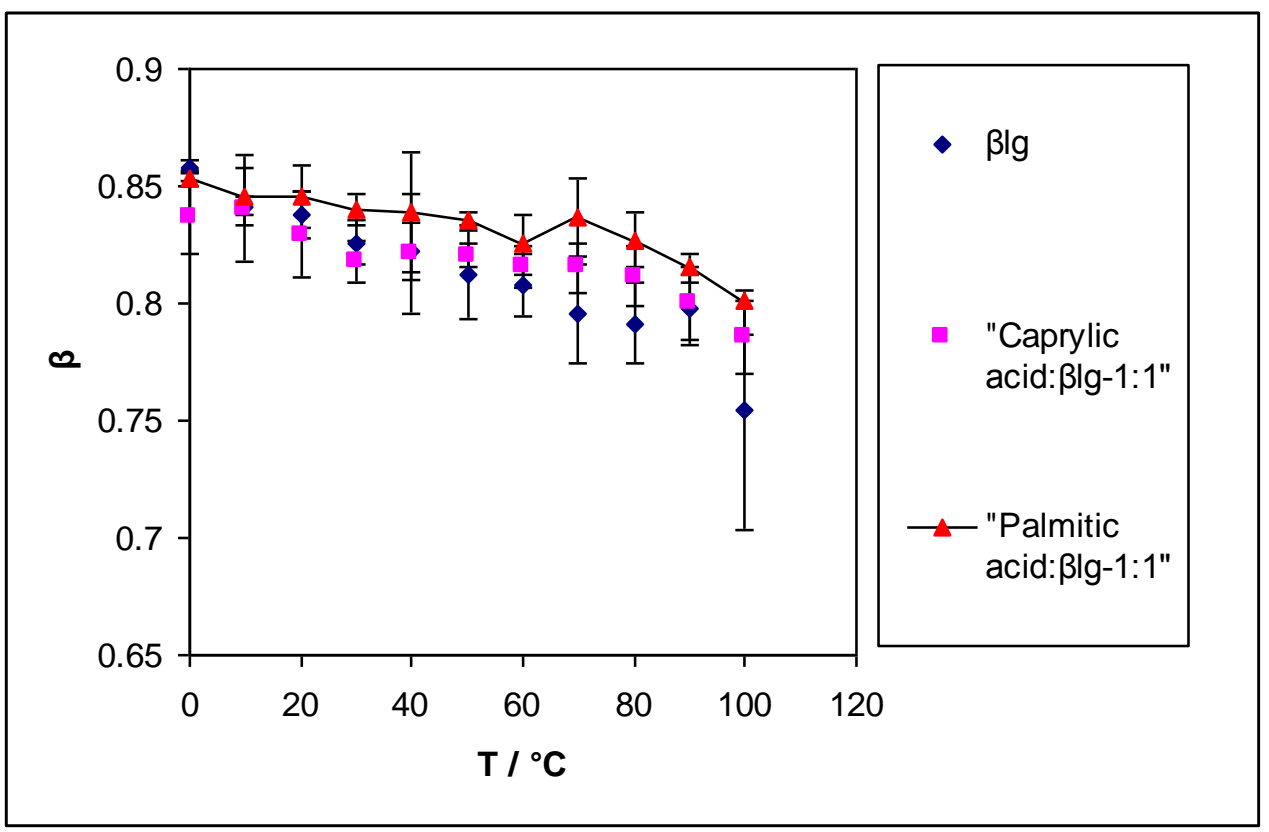

Figure VIII-5a: The effect of temperature on the stretching exponent $\beta$ for Erythrosin B dispersed in fatty acid/ $\beta$ - Lg films equilibrated against nitrogen. $\beta$ was determined from analysis using a stretched exponential decay function.

Figure VIII-5b

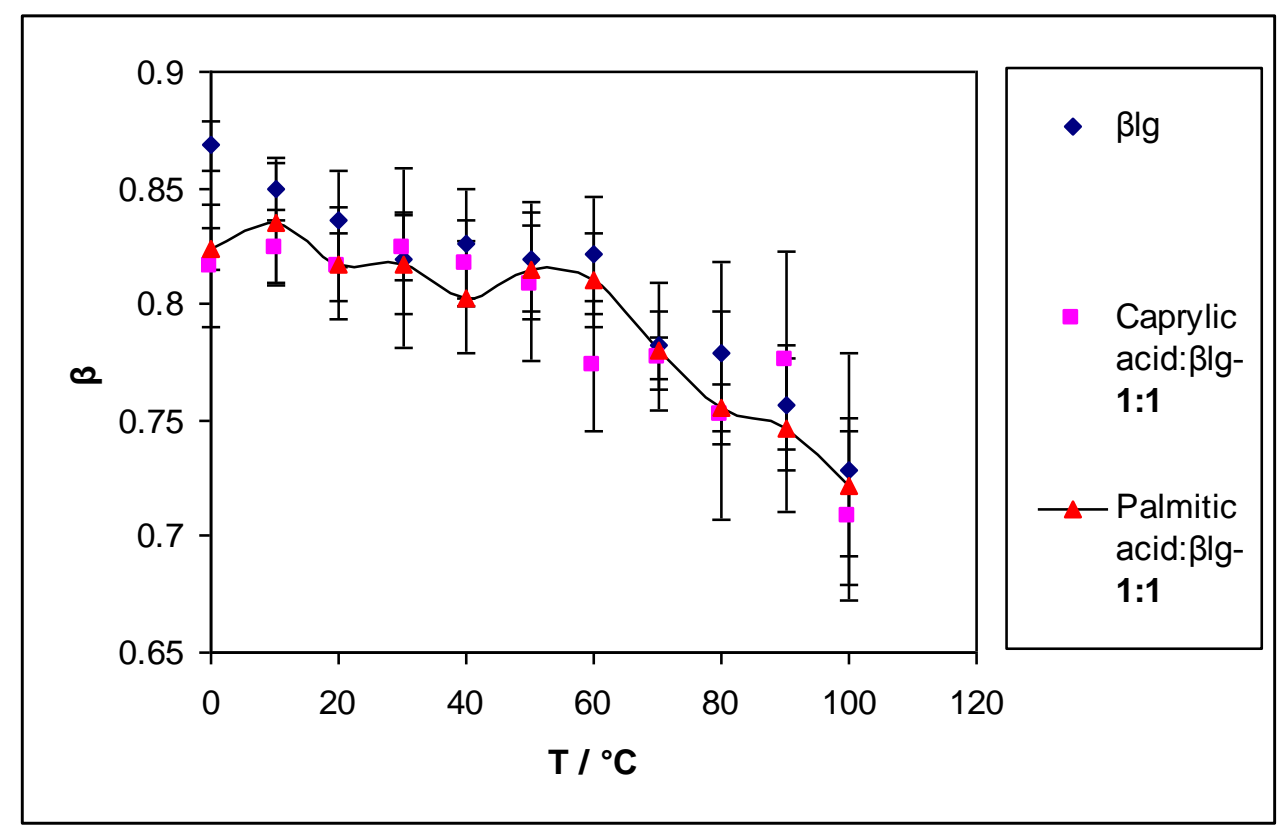

Figure VIII-5b: The effect of temperature on the stretching exponent $\beta$ for Erythrosin B dispersed in fatty acid/ $\beta$-Lg films equilibrated against nitrogen. $\beta$ was determined from analysis using a stretched exponential decay function. 


\section{Figure VIII-6}

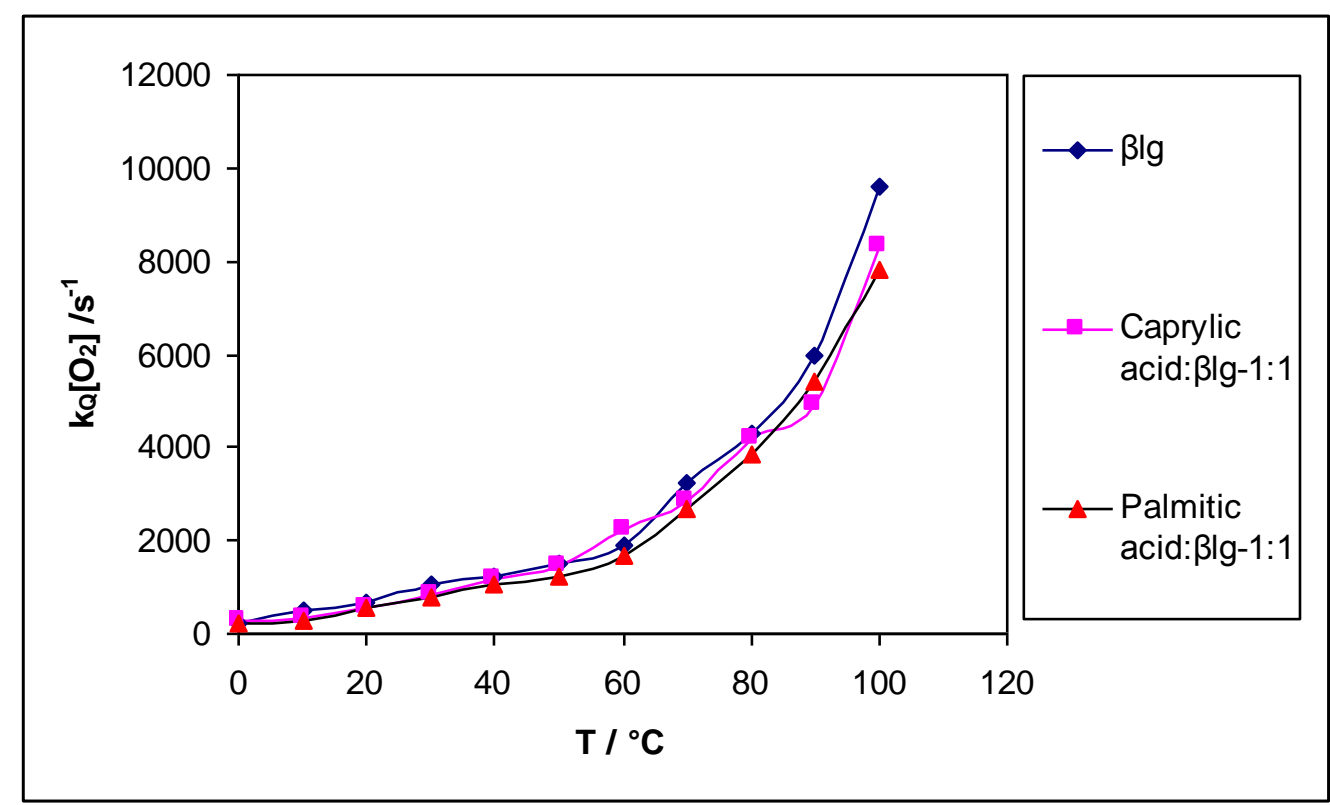

Figure VIII-6: The oxygen quenching rate $\mathrm{k}_{\mathrm{Q}}\left[\mathrm{O}_{2}\right]$ as a function of temperature in $\beta$-Lg and fatty acid/ $\beta$-Lg mixtures.

\section{Figure VIII-7}

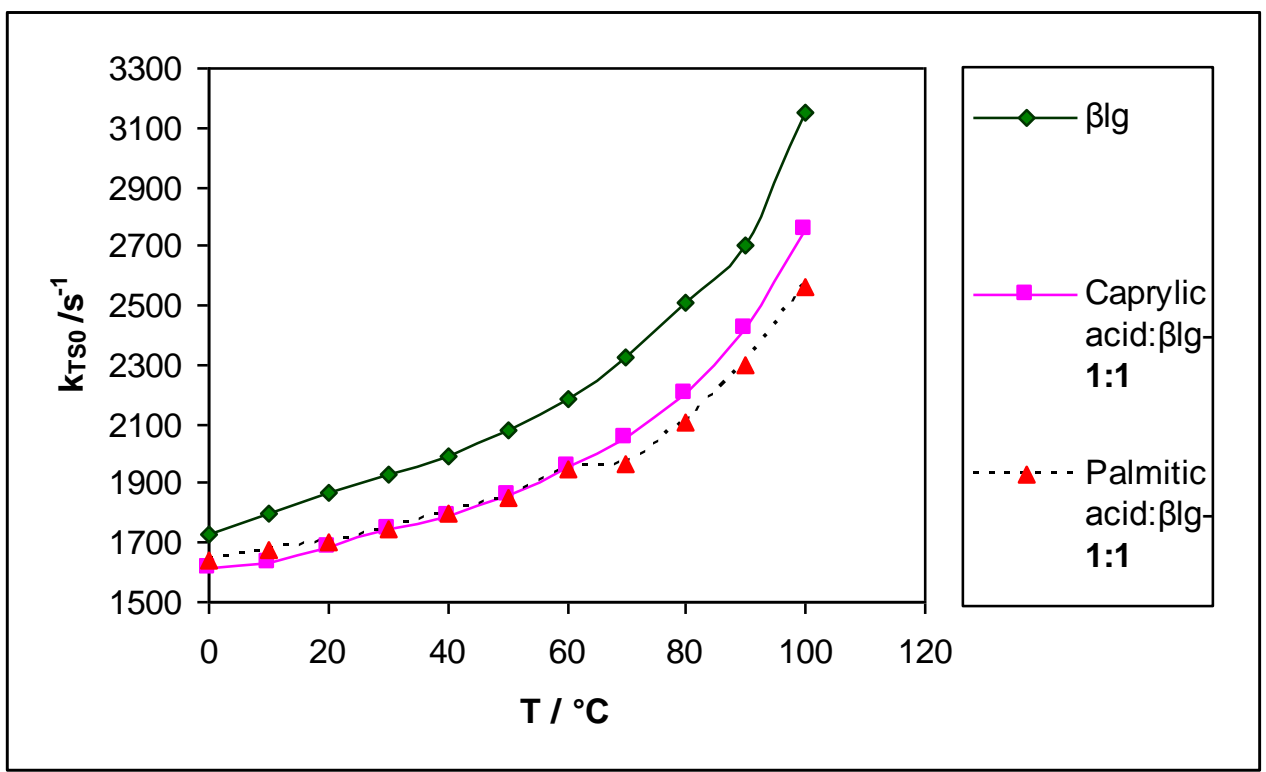

Figure VIII-7: Plot of the effect of temperature on non radiative decay from $T_{1}$ to $S_{0}\left(k_{T S 0}\right)$ in amorphous fatty acid/ $\beta$ - Lg films. 
Figure VIII-8

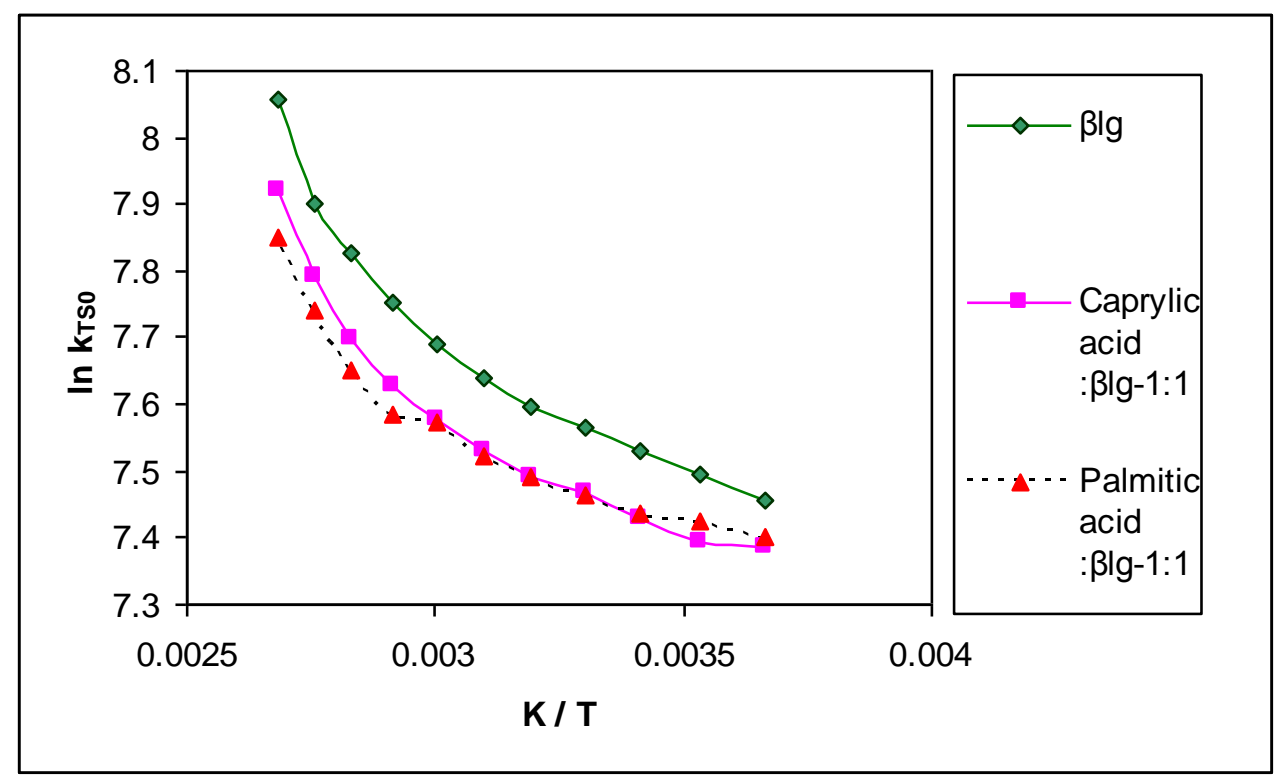

Figure VIII-8: Arrhenius plot of the effect of temperature on non radiative decay from $\mathrm{T}_{1}$ to $\mathrm{S}_{0}\left(\mathrm{k}_{\mathrm{TS} 0}\right)$ in amorphous fatty acid/ $\beta$ - Lg films. 
Figure VIII-9a

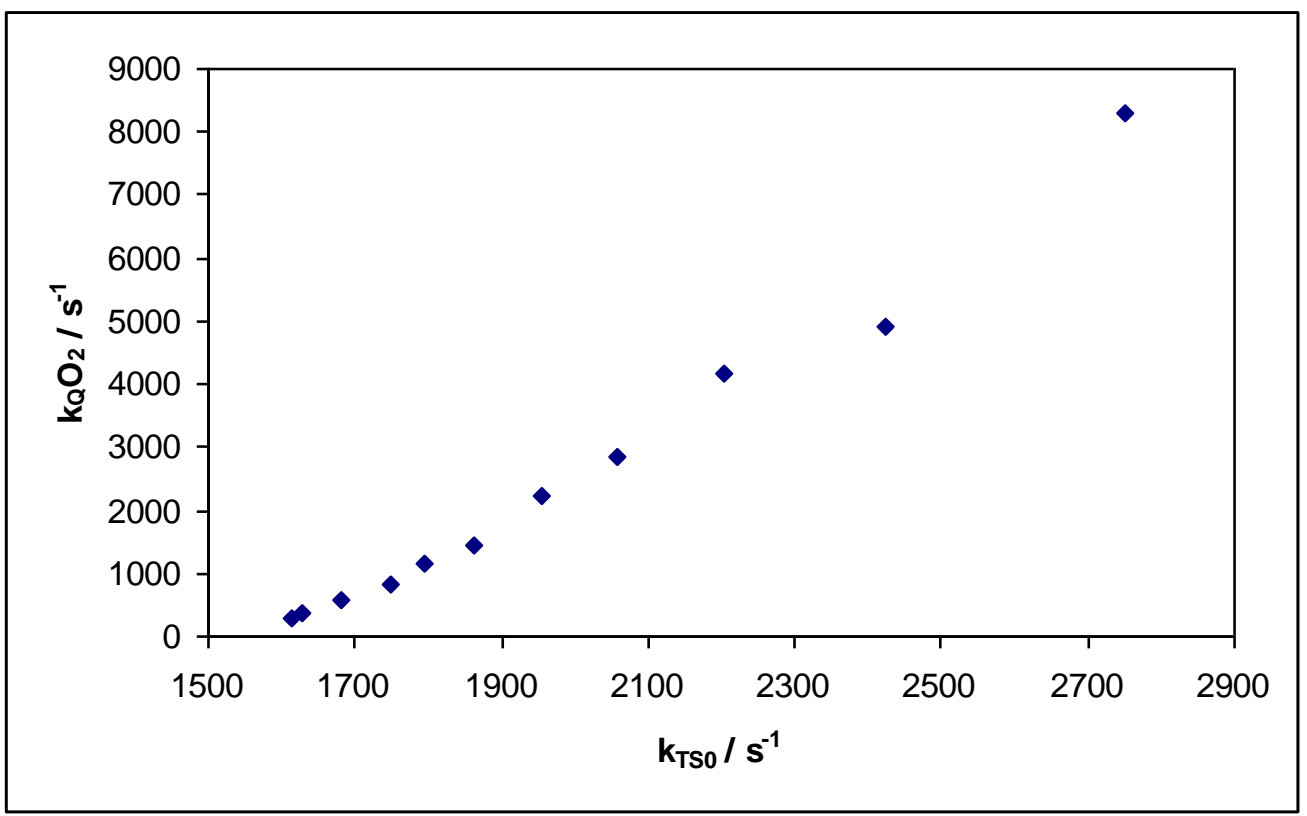

FigureVIII-9a: Dependence of the oxygen quenching rate $\mathrm{k}_{\mathrm{Q}}\left[\mathrm{O}_{2}\right]$ on the rate of nonradiative quenching $\mathrm{k}_{\mathrm{TS} 0}$ in caprylic acid/ $\beta$-Lg films.

Figure VIII $9 b$

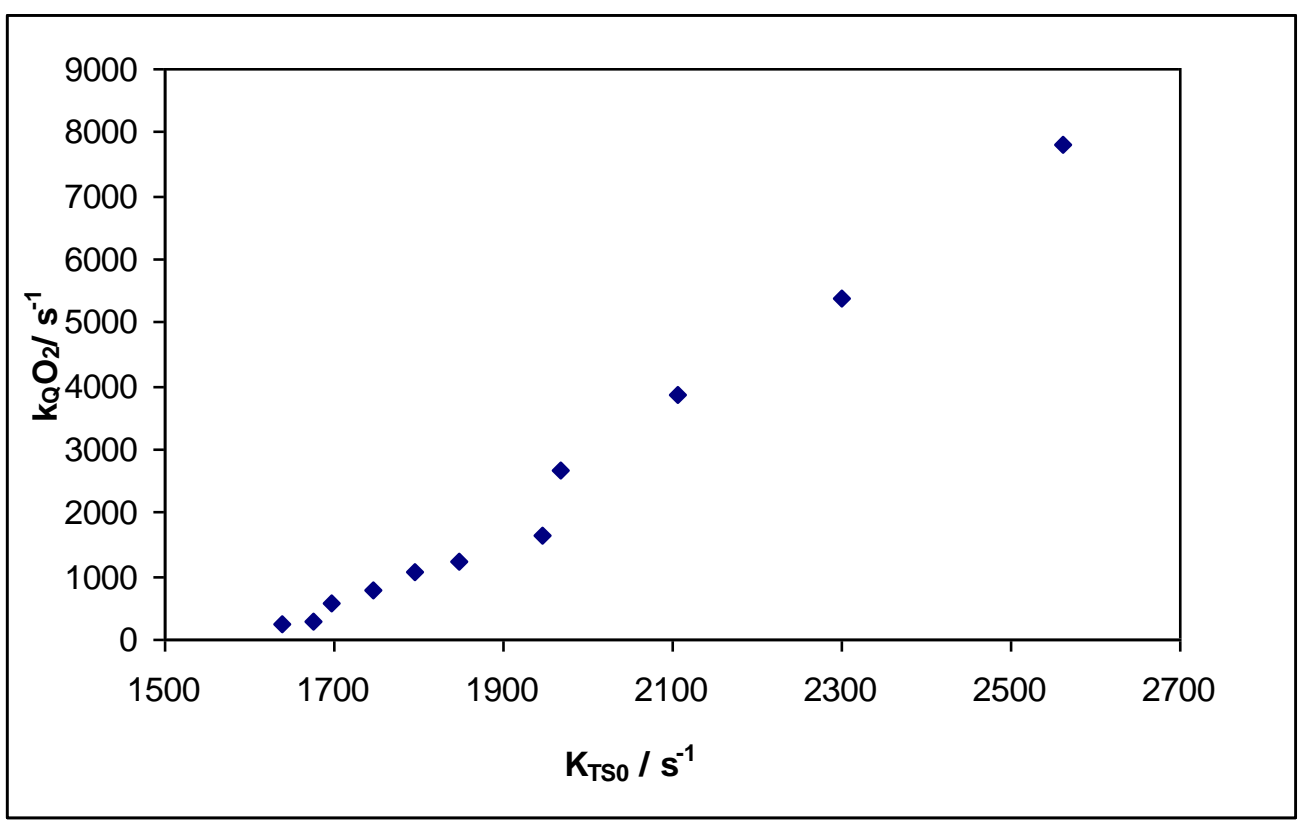

FigureVIII-9b: Dependence of the oxygen quenching rate $\mathrm{k}_{\mathrm{Q}}\left[\mathrm{O}_{2}\right]$ on the rate of nonradiative quenching $\mathrm{k}_{\mathrm{TS} 0}$ in palmitic acid/ $\beta$ - Lg films. 
Chapter IX: Study to characterize the use of retinol and retinal as phosphorescent probes in the amorphous $\beta$-lactoglobulin $(\beta-\mathrm{Lg})$ matrix

\section{Introduction}

Retinol has a polyene chromophore which is weakly fluorescent. It has a low quantum efficiency in solvents like hexane (fluorescent decay time of $4.2 \mathrm{~ns}$ ). The binding of retinol to retinol binding protein causes an enhancement of the fluorescence decay time to $8 \mathrm{~ns}$; and an increase in the emission anisotropy $(\mathrm{A}=0.29)$ (Georghiou and Churchich, 1975). Two molecules of retinol are bound per $\beta$-Lg dimer, with a binding constant of $2 \times 10^{-8} \mathrm{M}$. The fluorescence lifetime of retinol increases dramatically on binding to $\beta$ - $\mathrm{Lg}$, from approximately 2 to $10 \mathrm{~ns}$, as does the fluorescence quantum yield. The retinol binding to $\beta$ - $\mathrm{Lg}$ does not show $\mathrm{pH}$ dependence and the binding site is hydrophobic (Fugate and Song, 1980).

The carrier property, for retinol in neonates along with its remarkable acid stability, makes $\beta$ - $\mathrm{Lg}$ an attractive candidate for protein engineering whereby $\beta$ - $\mathrm{Lg}$ (or other proteins) could be modified or designed to serve as transporters for delivering important nutrients to specific targets in the alimentary tract (Sawyer et al., 1985).

Much effort has been devoted in the past in delineating the various ligand binding

sites on $\beta$-Lg (Frapin et al., 1993; Creamer, 1995). It has been shown that retinol is bound within the $\beta$-barrel of the molecule (Cho et al., 1994). Previous studies have explored the binding of retinol using phosphorescence of tryptophan (Subramanium et al., 1996). However, there are virtually no studies exploring the phosphorescence properties of retinol bound to the protein $\beta$-Lg. This study aims at looking into the possibility of using retinol as a possible phosphorescent probe to study the amorphous $\beta$ - $\mathrm{Lg}$ matrix. The 
retinol phosphorescence is of great interest since it arises from a chromophore bound at a unique binding site, the intactness of which should be a prerequisite for the maintenance of the active conformation of the protein. Hence, the retinol molecule can act as a reporter group in the protein. In this study films of $\beta$ - $\mathrm{Lg}$ will be made with retinol at various molar concentrations and the threshold concentration of retinol required to induce phosphorescence will be determined. Also the excitation wavelength will specifically excite the extrinsic probe retinol at $325 \mathrm{~nm}$ as opposed to the intrinsic tryptophan commonly studied in previous studies. A probable connection with binding of retinol and its effects on matrix properties will be further evaluated.

Retinal, technically called "retinaldehyde", is a light-sensitive molecule found in the photoreceptor cells of the retina. Retinal is the fundamental chromophore involved in the transduction of light into visual signals, i.e. nerve impulses, in the visual system of the central nervous system. These molecules consist of a chain of alternating single and double carbon-carbon bonds ending in an aldehyde group.

UV-visible absorption spectra and photostationary state of retinal embedded in the binding cavity of $\beta-\mathrm{Lg}$ are consistent, with the view that the carbonyl group of these polyenes are hydrogen-bonded with the protein host, most likely with the lone protonated lysine residue in the binding pocket ( $\mathrm{Li}$ and Liu, 1995). From its composition one would judge this chromophore to be a relatively simple one but the characterization of its triplet state still presents a challenge to spectroscopists, not the least because phosphorescence has not been observed (Das and Becker, 1982). Studies by Groenan et al. (1992) showed that the electronic structure of the lowest triplet state of retinal resembles that of a polyenal with a total number of six double bonds. The double bond of retinal that is part 
of the methyl-substituted cyclohexene ring apparently fully participates in the triplet excitation, which suggests that the chromophoric part of retinal is more or less planar in $\mathrm{T}_{1}$. So based on the speculation about the possible existence of a triplet state in retinal, this study explored the possibility of room temperature phosphorescence of retinal in the solid state. A probable connection with binding of retinal and its effects on matrix properties will be further evaluated.

\section{Materials and methods}

Solutions of $\beta-\operatorname{Lg}$ (90\% pure; crystallized and lyophilized Sigma Chemical Co, St.Louis, MO, USA) were prepared in distilled de-ionized water at an approximate concentration of $10 \mathrm{mg} / \mathrm{ml}$. This concentration was selected based on the batch size to ensure maximum recovery after dialysis and to avoid long-term storage. An aqueous $\beta$ Lg solution was transferred to regenerated cellulose dialysis tubing having a $12-14 \mathrm{kDa}$ molecular weight cutoff (Spectrum, Houston, TX). In order to define and minimize the presence of counter ions the solution was extensively dialyzed against $0.1 \mathrm{M} \mathrm{Kcl}$ and water with frequent change of buffer every 6 hours. The dialyzed solution was then filtered through a $0.2-\mu \mathrm{m}$ membrane to remove particulates. In order to maximize the effectiveness of the dialysis process, temperature of the solution was kept at $5^{\circ} \mathrm{C}$, to ensure that the protein remained folded. Several dilutions of a stock solution of dialyzed $\beta$-Lg were prepared. The dye to protein ratio was determined by measuring the absorbance of dilutions in a $1 \mathrm{~cm}$ path length quartz cuvette in a Bausch and Lomb Spectronic 2000 spectrophotometer (Milton Roy company, Analytical Products Division, Rochester, NY). The protein concentration was determined after dialysis by UV absorbance using an extinction coefficient of $0.96 \mathrm{~L} \mathrm{~g}^{-1} \mathrm{~cm}^{-1}$. 
All trans retinal and All trans retinol were obtained from Sigma Chemical Co. (St. Louis, MO). All trans retinol purchased in sealed ampoules were opened under nitrogen. The sample was then immediately dissolved in ethanol and stock solutions at millimolar concentrations were prepared.

Weighing of the sample was done in the dark. $10 \mathrm{mM}$ and $100 \mathrm{mM}$ stock solutions were prepared by dissolving retinol and retinal in $95 \%$ ethanol with a nitrogen purge in order to prevent possible oxidation, isomerization and photo bleaching. The solvent ethanol was selected for probe stability during long time storage and the concentrations were selected in order to simplify the addition of probe to the protein matrix. Absorption spectra of these probe molecules were measured to rule out any possible isomerization.

After estimating the concentration, protein solution was combined with aliquots of dye solution to make solutions with molar ratio of 1:4 (Retinol: $\beta$-Lg) and 1:1 (Retinol: $\beta-\mathrm{Lg}$ ). Similar dye to protein concentrations was selected for all trans retinal. The dye to protein concentration was sufficient to provide adequate signal /noise in spectroscopic measurements.

\section{Preparation of films}

To make glassy films $15 \mu \mathrm{l}$ of protein solutions were spread on approximately one third of a quartz slide $(30 \times 13.5 \times 0.6 \mathrm{~mm}$; custom made by NSG Precision Cells, Farmingdale, NY). The films approximately $(0.05 \mathrm{~mm}$ thick when dried) were visually checked for good spreading, uniformity, and consistency. The slides were stored at room temperature in a desiccator containing $\mathrm{P}_{2} \mathrm{O}_{5}$ and DrieRite for at least 7 days and protected from light to prevent any photo bleaching of retinol prior to luminescence measurements. All luminescence measurements were made on a Cary Eclipse Fluorescence 
Spectrophotometer (Varian Instruments, Walnut Creek, CA). A slide was fitted diagonally into a standard fluorescence cuvette, which was flushed with oxygen-free $\mathrm{N}_{2}$ gas for at least 15 min prior to measurements. As the triplet state is quickly quenched by collision with solvent and oxygen molecules, it is essential to remove the oxygen thoroughly. So, all the experiments were conducted in $\mathrm{N}_{2}$ atmosphere

To detect phosphorescence and to elucidate the probe behavior in detail various excitation and emission parameters were varied. In order to gain an in-depth understanding of the possible spectroscopic details, variation in parameters like gate time, delay time etc were also studied. All measurements were made in room temperature.

\section{$\underline{\text { Results }}$}

\section{Can retinol be used as a phosphorescent probe?}

This study aimed at characterizing the phosphorescent properties of the probe retinol in amorphous $\beta$-Lg films. This section presents a summary of this investigation

Prior to making films, absorption of the probe all trans retinol was measured in ethanol. Absorption was measured in order to determine the absorption wavelength, and rule out any possible isomerization of the probe. The absorption spectra (data not shown) did not show any signs of isomerization as the probe absorbed at a wavelength of 325 $330 \mathrm{~nm}$. As outlined by Morton, (1975) these fell in the specifications for all trans retinol. This was used as a guideline and the dry films were excited at $325 \mathrm{~nm}$ in order to detect the phosphorescence of retinol. Exhaustive optimizations of the instrumental conditions, which may affect the phosphorescence emission, and may help us gain a deeper insight onto the characteristics of these probe molecules, were conducted. In all optimizations the experimental conditions were varied within certain limits searching for a better 
sensitivity in the phosphorescence determinations. The selection of optimum values was carried out following a univariant method (each variable was optimized to maintain the rest of variables constant) and observing the change of the intensity/noise ratio.

The applied photo multiplier voltage influences directly both instrumental sensitivity and background. An increase in the detector voltage from 400V - $800 \mathrm{~V}$ was studied. The best signal to noise ratio for all retinol/ $\beta$-Lg films were obtained for 20/20 nm excitation/emission slits and a detector voltage of 600V. In phosphorescence measurements using an instrument based on a pulsed excitation source it is necessary to ensure a delay time $\left(t_{d}\right.$, time elapsed between the flash of the excitation source and the start of data acquisition) which should be long enough to discriminate the phosphorescence emission from all possible delayed fluorescence and from the emission light from the pulsed excitation source. After an appropriate delay time, a gate time $\left(\mathrm{t}_{\mathrm{g}}\right)$ should also be set which is the acquisition time after the delay time. Both instrumental parameters have a great influence on the measurement of the phosphorescence emission and must be studied and selected carefully for optimum analysis of phosphorescence. Table IX $1 \mathrm{a}, \mathrm{b}, \mathrm{c}$ are a summary of the instrumental parameters used to characterize the phosphorescent properties of the probes.

To study the effect of change in gate time, phosphorescence emission spectra was collected from $350 \mathrm{~nm}$ to $650 \mathrm{~nm}$ (bandwidth $20 \mathrm{~nm}$ ) using excitation at $325 \mathrm{~nm}$ (bandwidth $20 \mathrm{~nm}$ ) and delay time of $0.1 \mathrm{~ms}$. Gate times were varied from $1.0 \mathrm{~ms}$ and incremented to $10.0 \mathrm{~ms}$. Figure XI-1 a reflects the emission spectra of retinol / $\beta$-Lg films as a function of gate time at $25^{\circ} \mathrm{C}$. Small peaks were observed and the intensity of these peaks increased with increase in gate time until a gate time of $5 \mathrm{~ms}$ was reached, after 
which the intensity started decreasing. To further analyze whether these peaks were reflective of phosphorescence of retinol, we measured the emission spectra of the blank protein slide with similar gate times and subtracted the blank protein with the probe retinal (Figure XI-1b). There was no intensity detected which proved that there was no phosphorescence in retinol and the peaks observed in Figure IX 1a were a mere reflection of possible tryptophan interference.

Variations in delay time can also reveal some important aspects. Some probes may exhibit phosphorescence with longer time delay. Figure IX 2a depicts the emission spectra of retinol with change in delay time from $0.1 \mathrm{~ms}-0.6 \mathrm{~ms}$. We see that optimum delay time for this experiment was $0.1 \mathrm{~ms}$ and further subtraction with the blank firmly proved that retinol is not phosphorescent at room temperature (Figure IX-2b). These experiments were based on the assumption that the solvent (ethanol used in minimal amounts) won't bring about any changes in the spectroscopic measurements. So, the effect of different solvents normally used for characterization of probes in phosphorescence measurements was not studied.

Figure IX-3a depicts the emission spectra as function of excitation wavelength Emission spectra of retinol in $\beta$ - $\mathrm{Lg}$ were collected from $350 \mathrm{~nm}$ to $700 \mathrm{~nm}$ (bandwidth $20 \mathrm{~nm}$ ) using excitation wavelengths of $310,320,325,330 \mathrm{~nm}$ (all with bandwidth of $20 \mathrm{~nm}$ ) at $25^{\circ} \mathrm{C}$ with delay time $0.1 \mathrm{~ms}$ and gate time of $5 \mathrm{~ms}$ and at $600 \mathrm{~V}$. The absorption wavelength of all trans retinal is $325 \mathrm{~nm}$ (Morton 1975). Upon exciting the samples between $310 \mathrm{~nm}$ to $330 \mathrm{~nm}$ small peaks were observed. As tryptophan in $\beta$ Lg gets excited at $280 \mathrm{~nm}$ it may interfere with the spectra at longer wavelengths. To find out whether these peaks were from phosphorescence of retinol in $\beta-\mathrm{Lg}$ or from 
tryptophan, we ran emission spectra of the protein blank as a function of excitation wavelength. The emission spectra of retinol in $\beta-\mathrm{Lg}$ and the blank protein slide, merged with each other. As depicted by Figure IX-3b, the subtracted emission spectra varied between zero amplitude.

Different molar ratios of retinol were tried, in order to elucidate the effect of increase in concentration of retinol. Figures IX-3b and IX-4b clearly prove that change in concentration of retinol had no effect; clearly emphasizing the fact that retinol indeed cannot be used as a phosphorescent probe. Studies at lower temperatures $0^{\circ} \mathrm{C}$ didn't yield any positive results (data not shown).

\section{Can retinal be used as a phosphorescent probe?}

Retinal is another probe, which has been widely studied for its fluorescent properties. There is evidence from flash photolytic studies that the triplet state is indeed populated upon illumination of retinal (Grellman et al., 1962; Dawson and Abrahamson, 1962). Unfortunately, the population of this state has not been directly observed, that is neither phosphorescence nor singlet-triplet absorption has been observed. Energy transfer to all-trans-retinal has been shown to take place from a series of compounds of known triplet energy and lifetime (Guzzu et al., 1969). These studies have been done in solution whereby non-radiative processes dominate and may quench any possible phosphorescence. But what governs the properties in the solid state may be totally different from that in solution. So the present study using these studies as guidelines looked into the possible phosphorescence of retinal in the solid state. All trans retinal in solution has been shown to have an absorption of $375 \mathrm{~nm}$ (Morton, 1975). The absorption spectra of all trans retinal were studied in ethanol to rule out any possible formation of 
isomers. The retinal solutions in the present study did not show any signs of isomerization. Instrument optimizations similar to those performed for retinol $\beta$ - $\mathrm{Lg}$ films to characterize the phosphorescence were performed. The best signal to noise ratio for all retinal/ $\beta$-Lg films was obtained for $20 / 20 \mathrm{~nm}$ excitation/emission slits and a detector voltage of $600 \mathrm{~V}$. All experiments were done in the absence of oxygen.

To study the effect of change in gate time, emission spectra of retinal in $\beta$ - $\mathrm{Lg}$ was collected from $400 \mathrm{~nm}$ to $650 \mathrm{~nm}$ (bandwidth $20 \mathrm{~nm}$ ) using excitation at $375 \mathrm{~nm}$ (bandwidth $20 \mathrm{~nm}$ ) with delay time of $0.1 \mathrm{~ms}$. Gate times were varied from $1.0 \mathrm{~ms}$ and incremented to $10.0 \mathrm{~ms}$. Emission spectra of the protein blank were also measured as a function of gate time. Variation of gate times with retinal in the ratio 1: 4 retinal: $\beta$ - $\mathrm{Lg}$ is depicted in Figure IX 5a. It is clearly evident from Figure IX 5b, that phosphorescence is not evident in retinal, in the solid state at room temperature.

Emission spectra of retinal as a function of delay time are illustrated in Figure IX6a. The excitation wavelength used was $375 \mathrm{~nm}$. The trends revealed that the optimum delay time to conduct this experiment (based on changes in intensity) was $0.1 \mathrm{~ms}$, which was used for all other experiments. We were unable to detect any phosphorescence in this probe as the curves for blank protein and probe with protein merged with each other. Figure IX 7a is a plot of emission spectra as a function of excitation wavelength. Further analysis with the blank (Figure IX-7b) disproves our hypothesis about possible phosphorescence in retinal. Change in concentration of the probe also did not yield any positive results.

It is clearly evident from this brief study that phosphorescence is not detected at room temperature in retinol and retinal in the solid state. However this present study can 
be used as speculative trials for future experiments. The possibility of detection of phosphorescence in these probes at very low temperatures $(77 \mathrm{~K})$ cannot be ruled out. The instrumental parameters characterized in this present study could be of potential use to study the solid-state biophysics of these important classes of probe molecules. 


\section{Table IX-1a}

Instrumental parameters used to characterize the phosphorescent properties of the probes retinol and retinal. Variation of delay time with emission wavelength (a) Variation of gate time with emission wavelength (b) Variation of excitation wavelength with emission wavelength (c)

\begin{tabular}{|l|l|l|l|l|l|}
\hline Probe & Delay time & Gate time & $\begin{array}{l}\text { Excitation } \\
\text { wavelength }\end{array}$ & $\begin{array}{l}\text { Detector } \\
\text { voltage }\end{array}$ & $\begin{array}{l}\text { Phosphorescence } \\
\text { Detected }\end{array}$ \\
\hline Retinol & $0.1-0.6 \mathrm{~ms}$ & $5 \mathrm{~ms}$ & $325 \mathrm{~nm}$ & $600 \mathrm{~V}$ & None \\
\hline Retinal & $0.1-0.6 \mathrm{~ms}$ & $5 \mathrm{~ms}$ & $375 \mathrm{~nm}$ & $600 \mathrm{~V}$ & None \\
\hline
\end{tabular}

Table IX-1b

\begin{tabular}{|l|l|l|l|l|l|}
\hline Probe & Delay time & Gate time & $\begin{array}{l}\text { Excitation } \\
\text { wavelength }\end{array}$ & $\begin{array}{l}\text { Detector } \\
\text { voltage }\end{array}$ & $\begin{array}{l}\text { Phosphorescence } \\
\text { Detected }\end{array}$ \\
\hline Retinol & $0.1 \mathrm{~ms}$ & $1-10 \mathrm{~ms}$ & $325 \mathrm{~nm}$ & $600 \mathrm{~V}$ & None \\
\hline Retinal & $0.1 \mathrm{~ms}$ & $1-10 \mathrm{~ms}$ & $375 \mathrm{~nm}$ & $600 \mathrm{~V}$ & None \\
\hline
\end{tabular}

Table IX-1c

\begin{tabular}{|l|l|l|l|l|l|}
\hline Probe & $\begin{array}{l}\text { Delay } \\
\text { time }\end{array}$ & $\begin{array}{l}\text { Gate } \\
\text { time }\end{array}$ & Excitation wavelength & $\begin{array}{l}\text { Detector } \\
\text { voltage }\end{array}$ & $\begin{array}{l}\text { Phosphorescence } \\
\text { Detected }\end{array}$ \\
\hline Retinol & $0.1 \mathrm{~ms}$ & $5 \mathrm{~ms}$ & $\begin{array}{l}310 \mathrm{~nm}, 320 \mathrm{~nm}, 325 \mathrm{~nm}, \\
330 \mathrm{~nm}\end{array}$ & $600 \mathrm{~V}$ & None \\
\hline Retinal & $0.1 \mathrm{~ms}$ & $5 \mathrm{~ms}$ & $\begin{array}{l}330 \mathrm{~nm}, 340 \mathrm{~nm}, 350 \mathrm{~nm}, \\
360 \mathrm{~nm}, 370 \mathrm{~nm}, 375 \mathrm{~nm}\end{array}$ & $600 \mathrm{~V}$ & None \\
\hline
\end{tabular}




\section{$\underline{\text { References }}$}

Cho, Y., Batt, C. A., and Sawyer, L. (1994). Probing the retinol-binding site of bovine beta -lactoglobulin. J. Biol. Chem. 269, 11102-11107.

Creamer, L. K. (1995). Effect of Sodium Dodecyl Sulfate and Palmitic Acid on the Equilibrium Unfolding of Bovine beta -Lactoglobulin. Biochemistry (N. Y. ). 34, 7170-7176.

Das, P. K. and Becker, R. S. (1982). Spectroscopy of polyenes. 6. Absorption and emission spectral properties of linear polyenals of the series $\mathrm{CH} 3-(\mathrm{CH}: \mathrm{CH}) n-$ CHO. J. Phys. Chem. 86, 921-927.

Dawson, W. and Abrahamson, E. W. (1962). Population and decay of the lowest triplet state in polyenes with conjugated heteroatoms: retinene. J. Phys. Chem. 66, 25422547.

Frapin, D., Dufour, E., and Haertle, T. (1993). Probing the fatty acid binding site of beta lactoglobulins. J. Protein Chem. 12, 443-449.

Fugate, R. D. and Song, P. (1980). Spectroscopic characterization of beta -lactoglobulinretinol complex. Biochim.Biophys.Acta, Protein Struct. 625, 28-42.

Georghiou, S. and Churchich, J. E. (1975). Nanosecond spectroscopy of retinol bound to proteins. Int.J.Quantum Chem., Quantum Biol.Symp. 2, 331-337.

Grellman, K. H., Livingston, R., and Pratt, D. (1962). A flash-photolytic investigation of rhodopsin at low temperatures. Nature (London, U.K.). 193, 1258-1260.

Groenen, E. J. J., Kok, P., and Ros, M. (1992). From polyenals to retinal: an electronspin-echo study of the triplet state. Pure Appl. Chem. 64, 833-839.

Guzzo, A. V. and Pool, G. L. (1969). Energy transfer to the triplet level of all-trans retinal. J. Phys. Chem. 73, 2512-2515.

Li, X. and Liu, R. S. H. (1995). Photostationary state compositions of retinal and related compounds included in beta -lactoglobulin. Effects of protein host on isomer distribution of polyene substrates. Photochem. Photobiol. 62, 361-366.

Morton, R. A. (1975). Biochemical Spectroscopy, Adam Hilger Ltd.

Papiz, M. Z., Sawyer, L., Eliopoulos, E. E., North, A. C. T., Findlay, J. B. C., Sivaprasadarao, R., Jones, T. A., Newcomer, M. E., and Kraulis, P. J. (1986). The structure of beta -lactoglobulin and its similarity to plasma retinol-binding protein. Nature (London). 324, 383-385. 
Sawyer, L., Papiz, M. Z., North, A. C. T., and Eliopoulos, E. E. (1985). Structure and function of bovine beta -lactoglobulin. Biochem. Soc. Trans. 13, 265-266.

Subramaniam, V., Gafni, A., and Steel, D. G. (1996). Time-resolved tryptophan phosphorescence spectroscopy: a sensitive probe of protein folding and structure. IEEE J.Sel.Top.Quantum Electron. 2, 1107-1114. 


\section{Figure IX-1a}

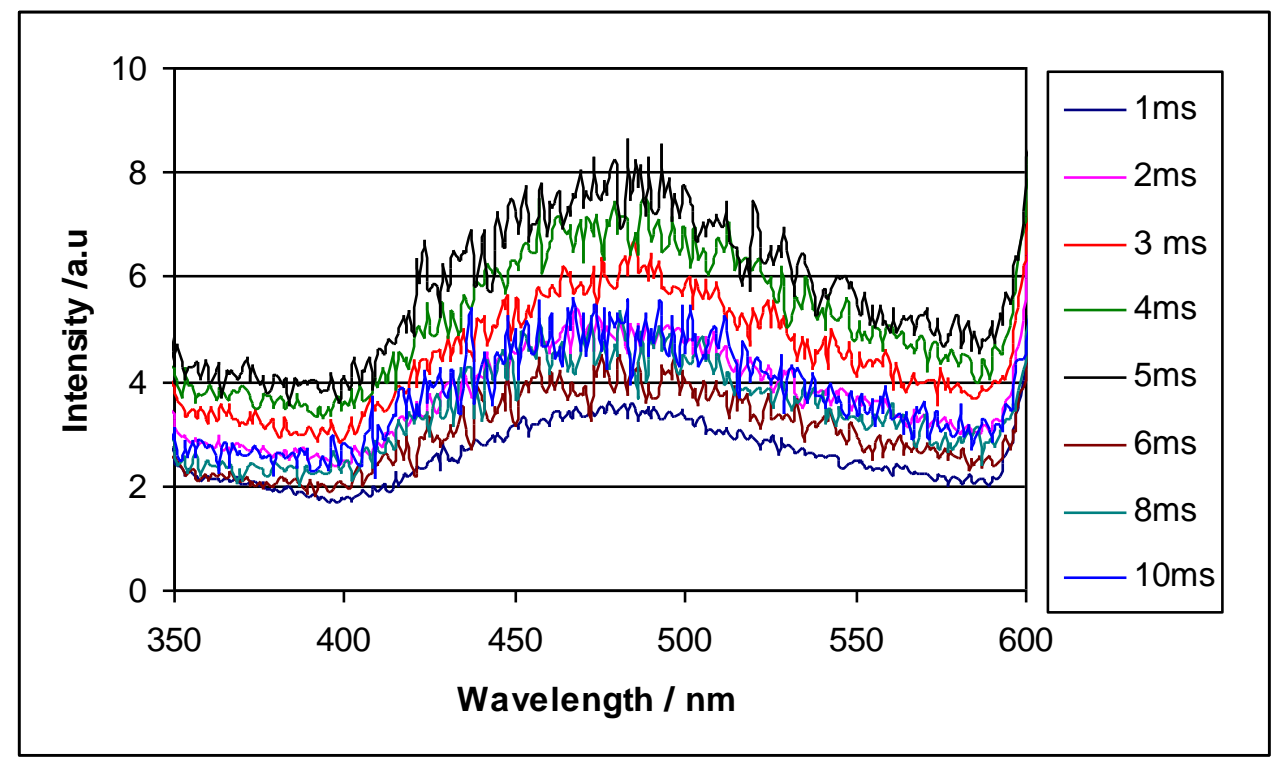

Figure IX-1a: Emission spectrum of retinol in $\beta-\operatorname{Lg}(1: 4)$ as a function of gate time

Figure IX-1b

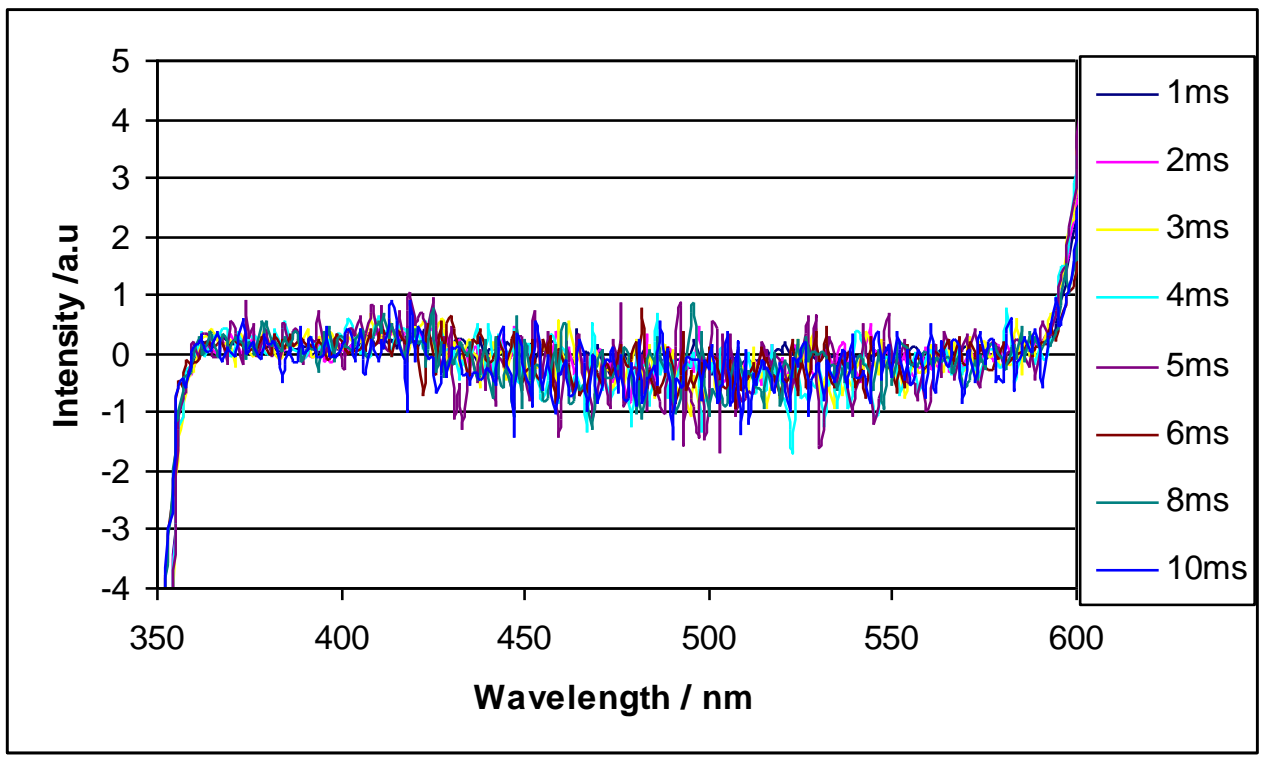

Figure IX-1b: Emission spectrum of retinol in $\beta-\operatorname{Lg}(1: 4)$ (pure $\beta$-Lg subtracted) as a function of gate time 


\section{Figure IX-2a}

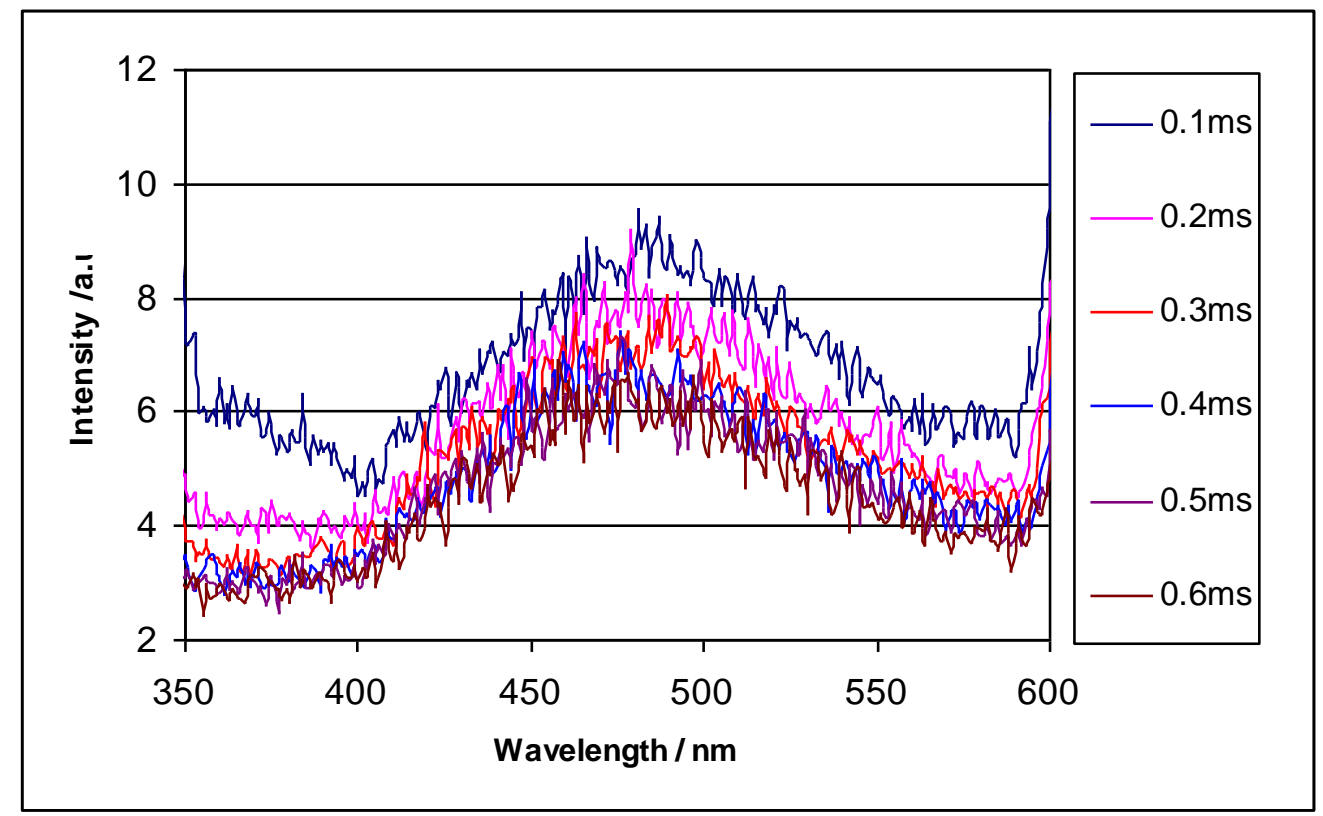

Figure IX-2a: Emission spectrum of retinol in $\beta-\operatorname{Lg}(1: 4)$ as a function of delay time

\section{Figure IX-2b}

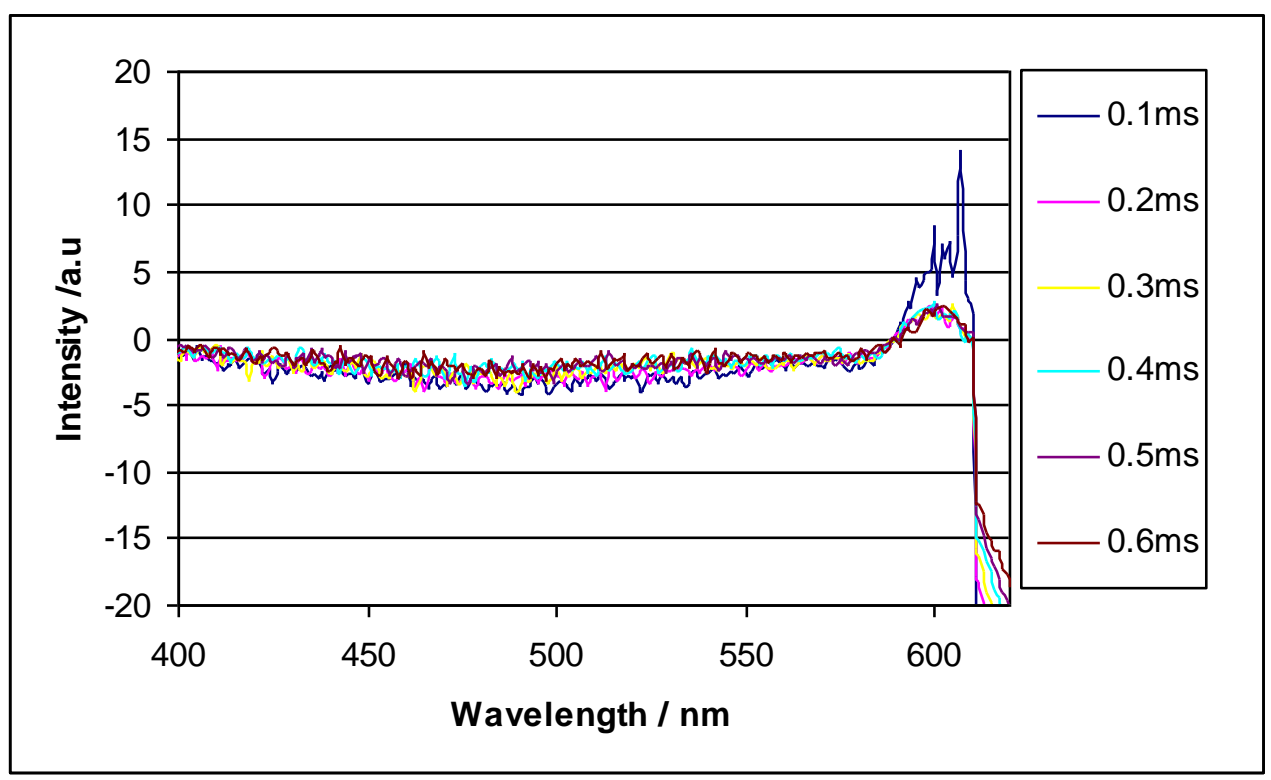

Figure IX-2b: Emission spectrum of retinol in $\beta$ - $\operatorname{Lg}(1: 4)$ (pure $\beta$-Lg subtracted) as a function of delay time 


\section{Figure IX-3a}

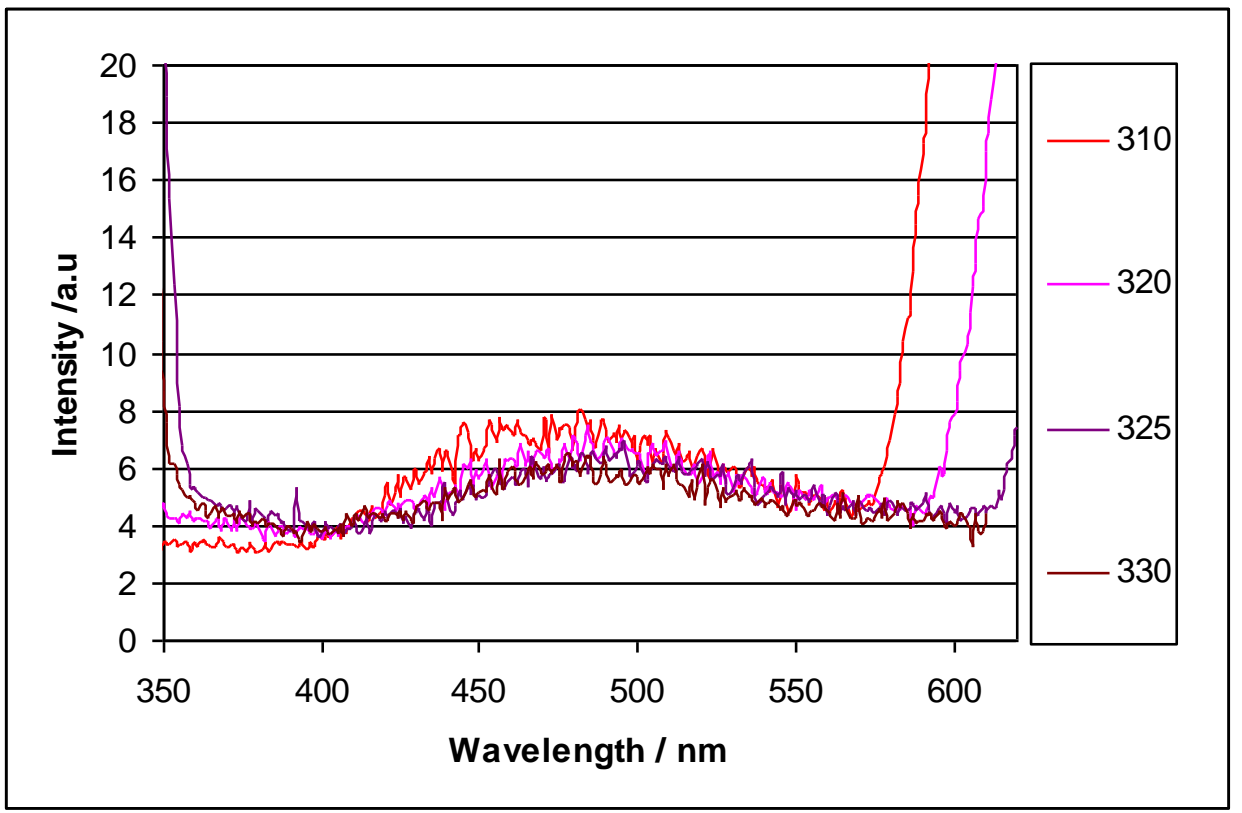

Figure IX-3a: Emission spectrum of retinol in $\beta-\operatorname{Lg}(1: 1)$ as a function of excitation wavelength

\section{Figure IX-3b}

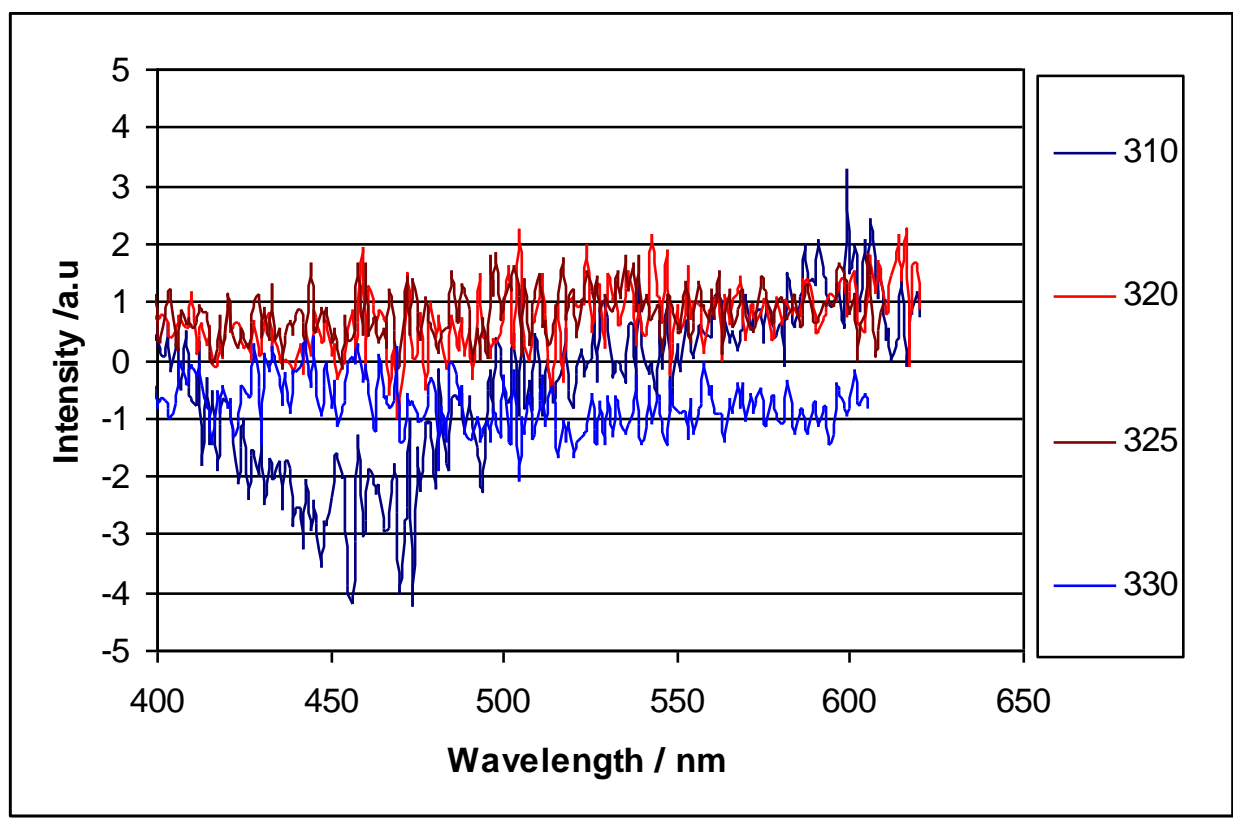

Figure IX-3b: Emission spectrum of retinol in $\beta-\operatorname{Lg}(1: 1)$ (pure $\beta-\operatorname{Lg}$ subtracted) as a function of excitation wavelength 


\section{Figure IX-4a}

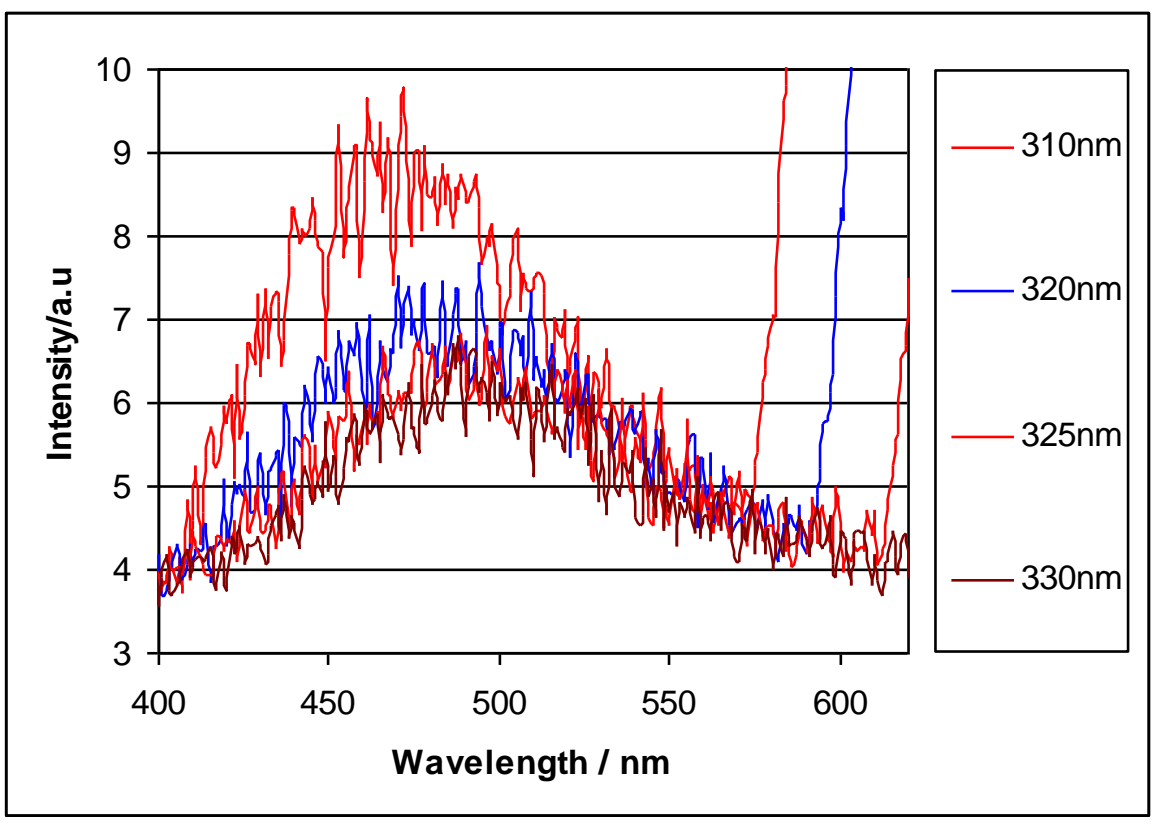

Figure IX-4a: Emission spectrum of retinol in $\beta$ - $\operatorname{Lg}(1: 4)$ as a function of excitation wavelength

Figure IX-4b

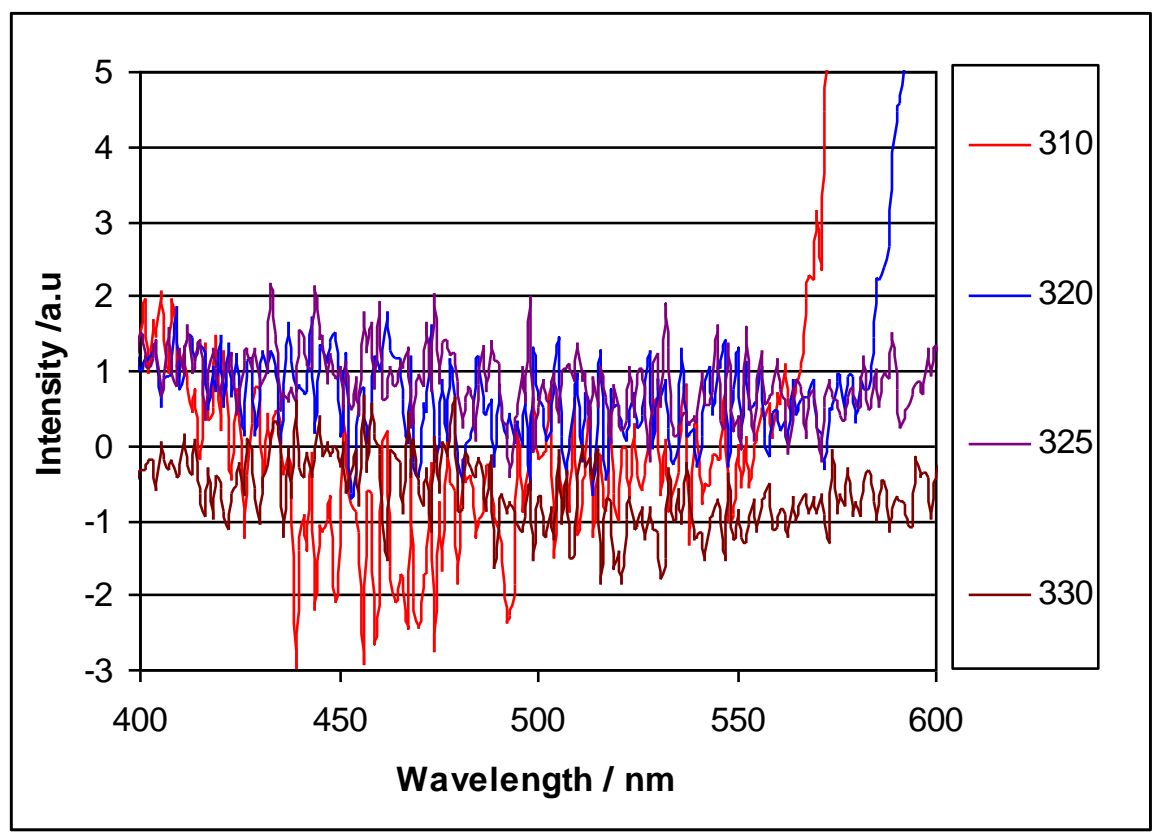

Figure IX-4b: Emission spectrum of retinol in $\beta-\operatorname{Lg}(1: 4)$ (pure $\beta-\mathrm{Lg}$ subtracted) as a function of excitation wavelength 


\section{Figure IX-5a}

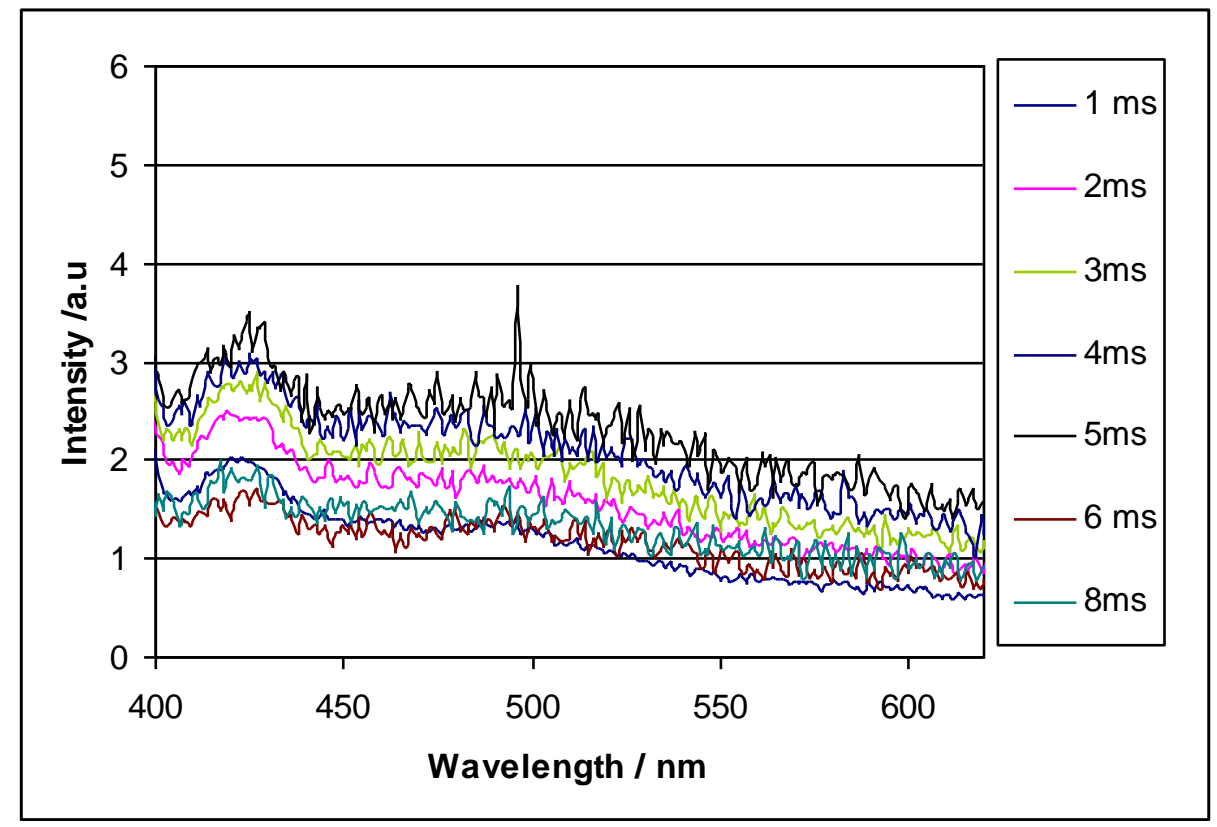

Figure IX-5a: Emission spectrum of retinal in $\beta \lg (1: 4)$ as a function of gate time

Figure IX-5b

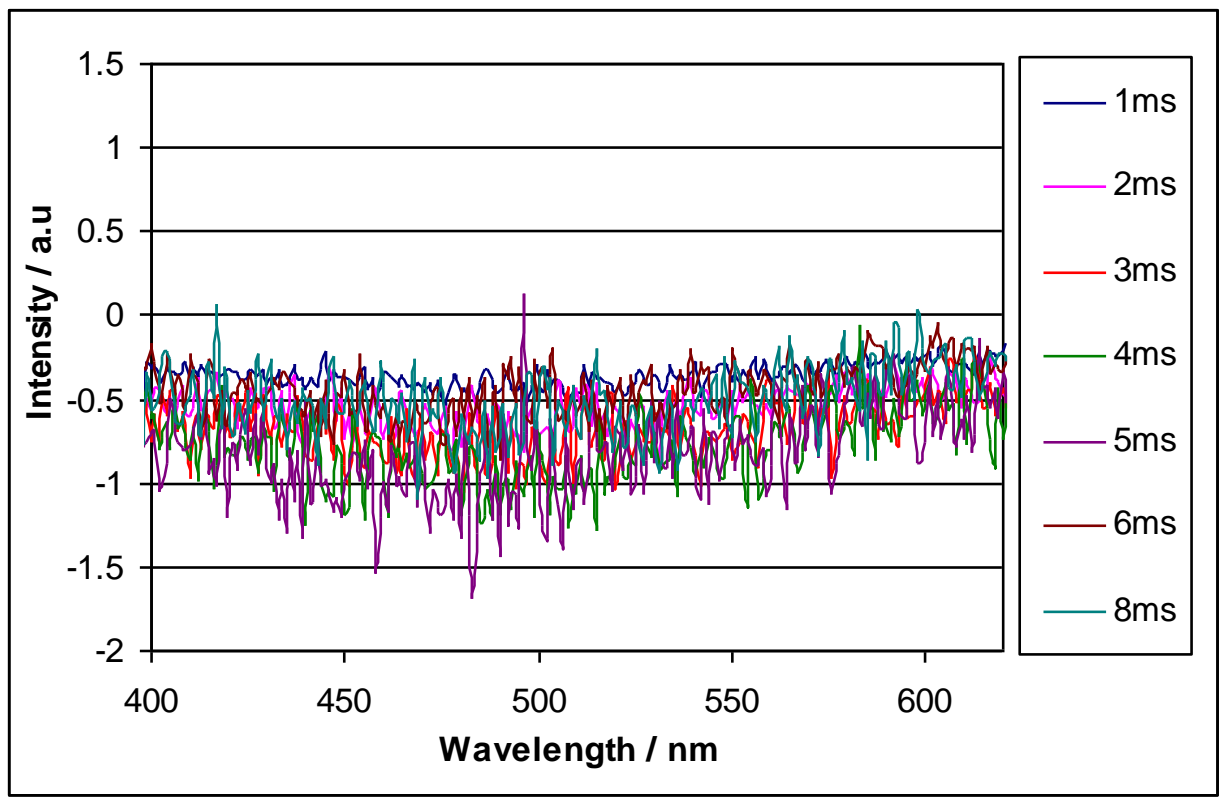

Figure IX-5b: Emission spectrum of retinal in $\beta \lg (1: 4)$ (pure $\beta-\mathrm{Lg}$ subtracted) as a function of gate time 


\section{Figure IX-6a}

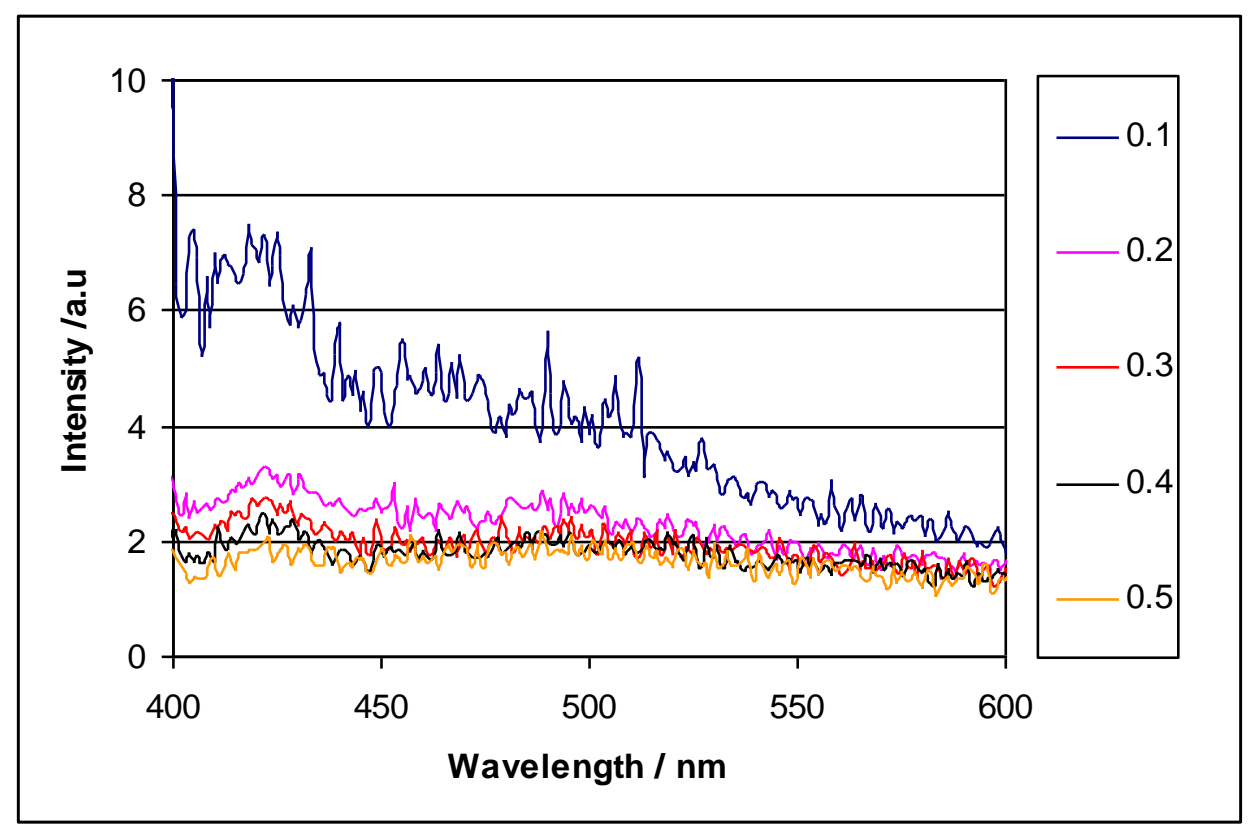

Figure IX-6a: Emission spectrum of retinal in $\beta-\operatorname{Lg}(1: 4)$ as a function of delay time

\section{Figure IX-6b}

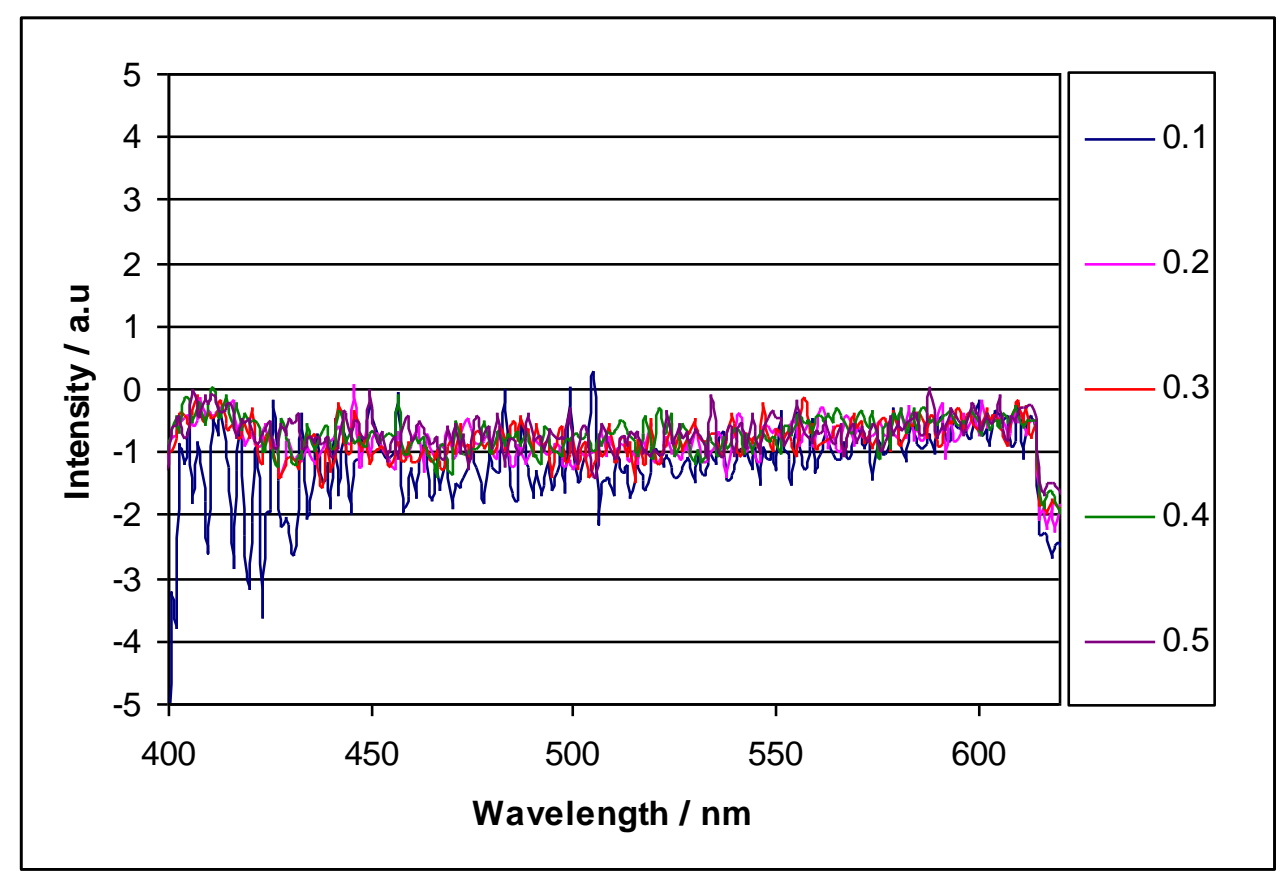

Figure IX-6b: Emission spectrum of retinal in $\beta-\operatorname{Lg}(1: 4)$ (pure $\beta-\operatorname{Lg}$ subtracted) as a function of delay time 


\section{Figure IX-7a}

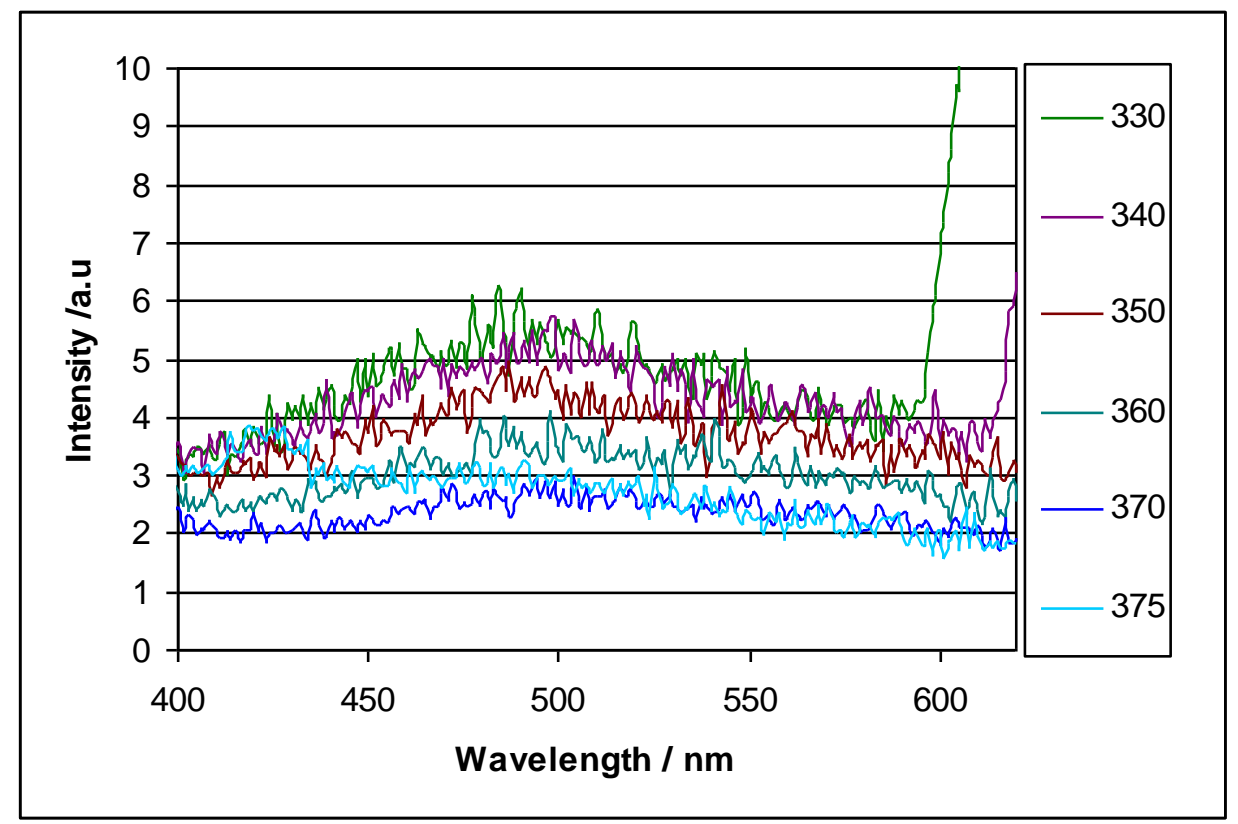

Figure IX-7a: Emission spectrum of retinal in $\beta-\operatorname{Lg}(1: 1)$ as a function of excitation wavelength

Figure IX-7b

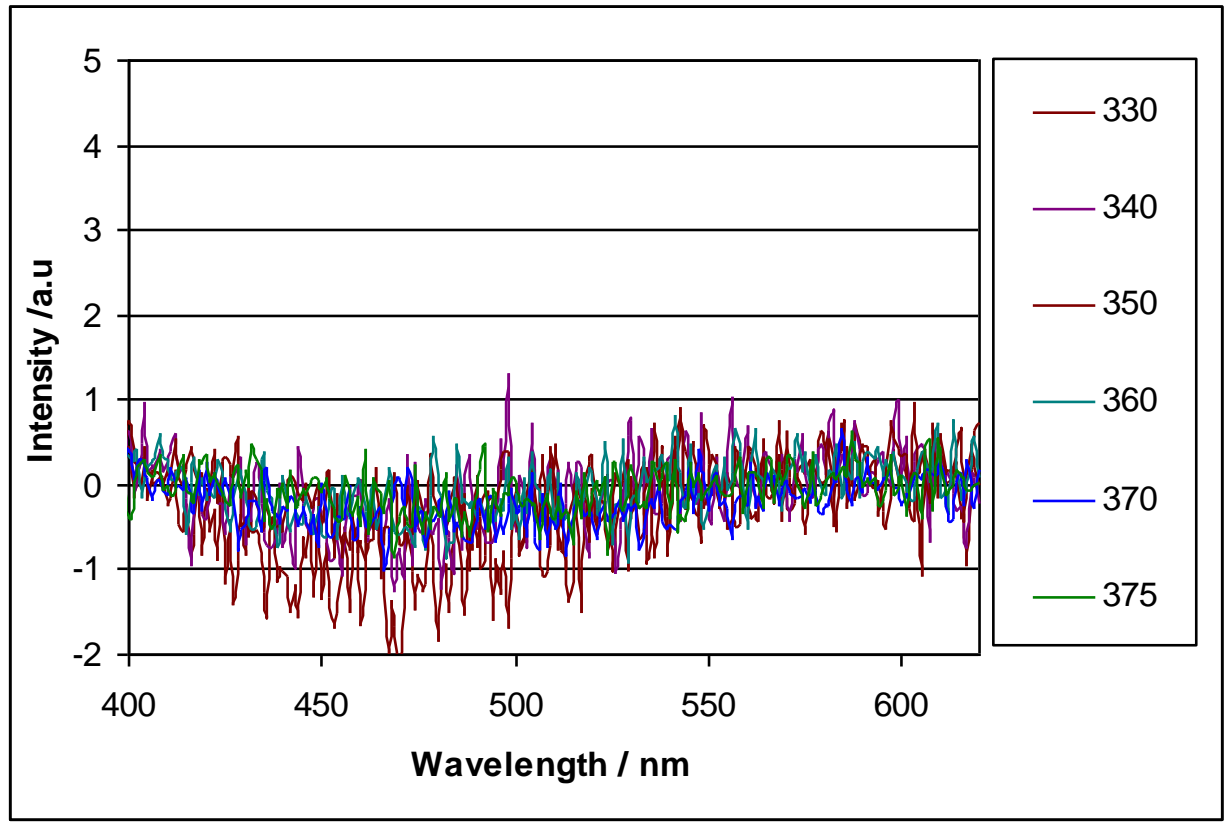

Figure IX-7b: Emission spectrum of retinal in $\beta-\mathrm{Lg}(1: 1)$ (pure $\beta$-Lg subtracted) as a function of excitation wavelength 
Figure IX-8a

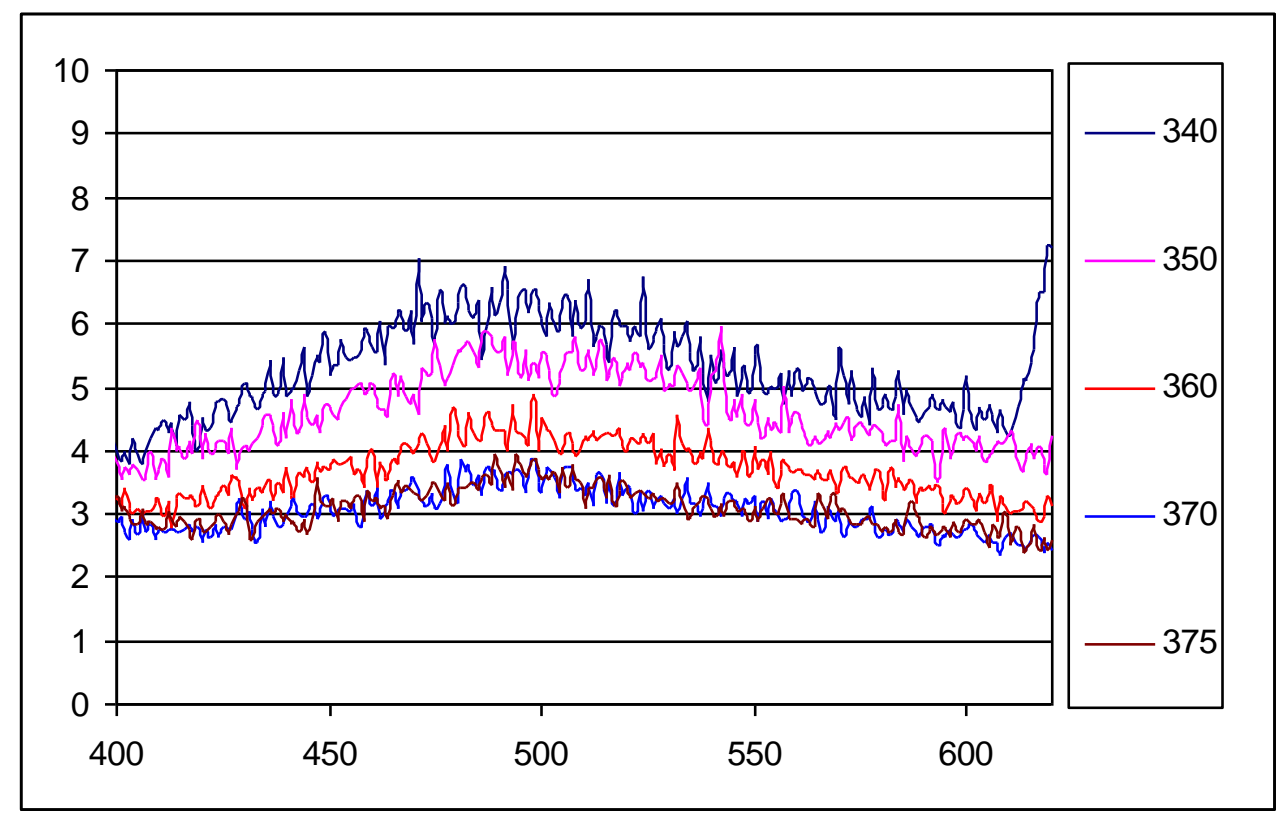

Figure IX-8a: Emission spectrum of retinal in $\beta-\operatorname{Lg}(1: 4)$ as a function of excitation wavelength

Figure IX-8b

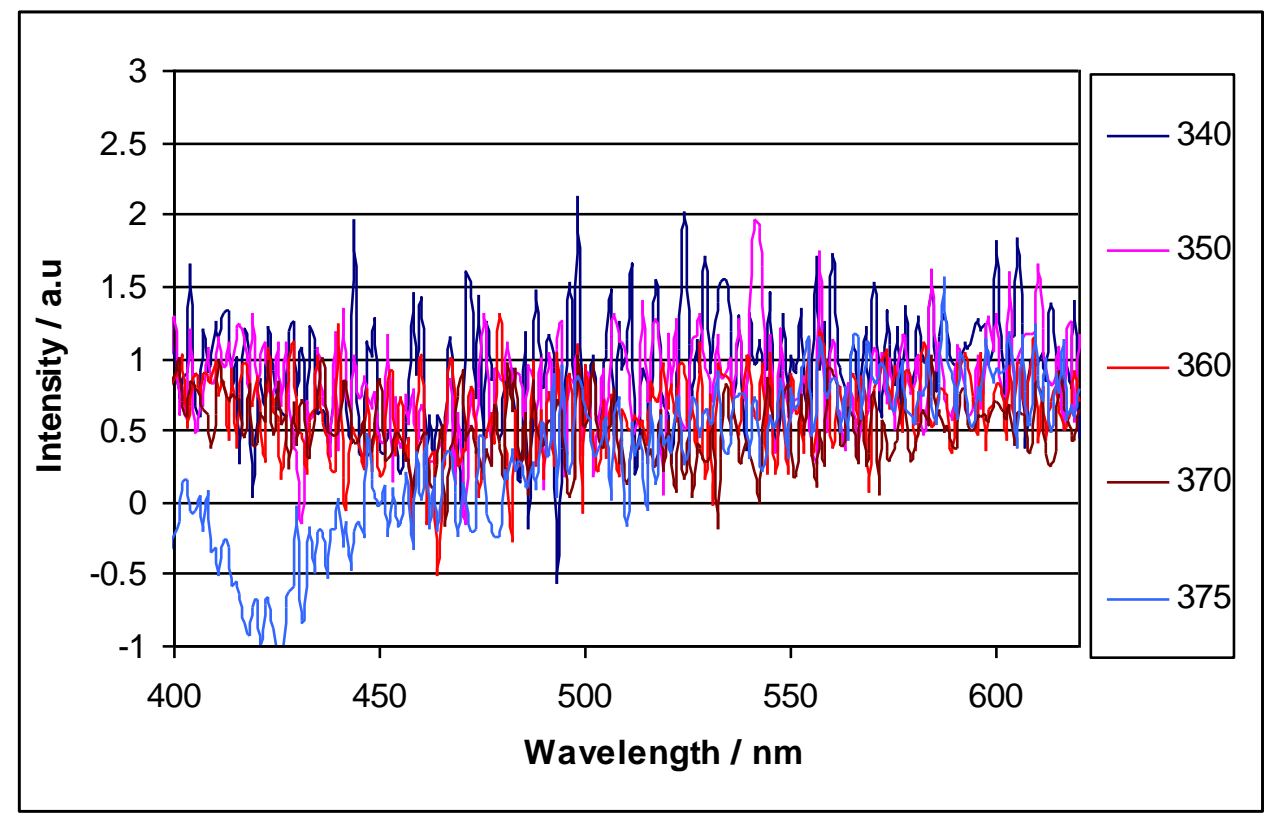

Figure IX-8b: Emission spectrum of retinal in $\beta \lg (1: 4)$ (pure $\beta$-Lg subtracted) as a function of excitation wavelength 


\section{Chapter X: Summary and future work.}

Molecular mobility and diffusion in amorphous solids are important for food quality, stability and shelf life. The amorphous state of biomaterials is thought to be of critical importance in controlling the stability over long periods of time. Various studies of macromolecules and their interaction with small molecules have provided perspective on the time frames in which the dynamic events occur in solution, but there is considerably less information available about the same events in the amorphous state. Certain active modes of mobility at the molecular level may be halted and certain latent modes of mobility may be reactivated by an increase in temperature or plasticization. Edible films and coatings can prevent quality deterioration and increase the shelf life of food products by acting as barriers to control the transfer of moisture, oxygen, carbon dioxide, lipids, and flavor compounds. The macroscopic functional properties of edible films made from proteins are modulated by mobility in the rubbery state. Scientific studies have clearly emphasized the role of local molecular mobility in modulating the macroscopic reactivity and thus the overall stability of many foods. Edible films necessarily contain certain additives to manipulate their overall functionality. These additives like plasticizers, sugars, lipids etc. essentially modulate the molecular mobility and permeability in these films.

This dissertation established a vital connection between molecular mobility and an underlying macroscopic diffusive property in amorphous $\beta$ - Lg films. Luminescence from the triplet probe Erythrosin B (Ery B) provides spectroscopic characteristics that are sensitive to molecular mobility of the local environment. Probe luminescence can be 
characterized by its lifetime and emission energy (wavelength). Each of these signals provides a commentary of the molecular environment around the probe.

Molecular mobility in amorphous $\beta$-lg films

Spectroscopic data provided by the luminescent probe Ery B dispersed throughout a thin film of amorphous $\beta$-Lg provide insight into the molecular mechanism(s) controlling oxygen transport through the protein matrix. Analysis of the phosphorescent lifetime of the probe in the absence and presence of oxygen gives the rate of nonradiative decay, $\mathrm{k}_{\mathrm{TS} 0}$, a quantitative measure of the matrix molecular mobility, and the rate of oxygen quenching, $\mathrm{k}_{\mathrm{Q}}\left[\mathrm{O}_{2}\right]$, which is proportional to the oxygen permeability. The thermal behavior of these two rates indicates that the $\beta-\operatorname{Lg}$ matrix undergoes a dynamic, softening transition near $80^{\circ} \mathrm{C}$, which does not appear to be related to protein denaturation. The linear dependence of $\mathrm{k}_{\mathrm{Q}}\left[\mathrm{O}_{2}\right]$ on $\mathrm{k}_{\mathrm{TS} 0}$ above a threshold value and ranging over nearly 3 -orders of magnitude change in the amplitude of oxygen quenching provides compelling evidence that oxygen transport is controlled by the local molecular mobility of the matrix. The variation of lifetime with wavelength in $\beta$ - $\mathrm{Lg}$ films shows evidence of the presence of dynamic site heterogeneity.

Edible films made of $\beta$ - $\mathrm{Lg}$ are usually brittle and are highly permeable to oxygen at room temperatures, so this study sought to elucidate the effect of different kinds of additives on the amorphous $\beta$ - $\mathrm{Lg}$ matrix and draw vital connections between underlying molecular mobility and the macroscopic diffusive property. Binary mixtures containing $\beta$-Lg and various additives like sugars (maltose, sucrose, trehalose), plasticizers (glycerol, sorbitol, polyethylene glycol), sugar alcohols (maltitol), fatty acids (caprylic acid and palmitic acid) and a globular protein (BSA) and composite films containing $\beta$-Lg, sucrose 
and PEG 400 and $\beta$-Lg maltose and maltitol mixtures were selected to investigate how variation in composition affects the molecular mobility and oxygen permeability in the amorphous $\beta$-Lg matrix.

\section{Influence of sugars on the $\beta$-Lg matrix.}

Increase in emission energy due to decrease in the mobility parameter $\mathrm{k}_{\mathrm{TS} 0}$ with increase in sugar concentration in the $\beta$ - Lg matrix provide compelling evidence that addition of sugars increases the local rigidity in the matrix and decreases the rate of the local relaxation mechanisms. $\beta$ values increased significantly with addition of sugars. These trends suggest that the local distribution of dynamic environments decreased in the presence of sugars due to matrix rigidification. However, at very high concentrations of sucrose (100:1 and 1000:1), as the glass transition of sucrose was approached, an enhancement of the mobility was observed. As the glass transition temperature of maltose and trehalose are high we did not see a noticeable change in these films with regard to the glass transition, as it was not within the functional temperature range of this study. Adding sugars in ratios ranging from 0.1:1 to 1000:1 sugar: $\beta-\mathrm{Lg}$, we found that oxygen permeability was inversely related to the amount of sugar added.

The sugars sucrose, maltose and trehalose exhibited similarities in the way they reduced the mobility of the $\beta-\mathrm{Lg}$ matrix. But the sugar/ $\beta-\mathrm{Lg}$ matrices exhibited significant differences in permeability. This variability can be attributed to differences in hydrogen bonding which needs to further investigated. Among the disaccharides studied, sucrose was the most effective in reducing the permeability and trehalose was the least effective. 
The crux of this dissertation is to answer an important question: Is their a relationship between mobility and permeability? These binary systems displayed both linear and biphasic correlations between $\mathrm{k}_{\mathrm{TS} 0}$ and $\mathrm{k}_{\mathrm{Q}}\left[\mathrm{O}_{2}\right]$, depending on the ratio. At higher sugar ratios there was no noticeable trend observed between $\mathrm{k}_{\mathrm{TS} 0}$ and $\mathrm{k}_{\mathrm{Q}}\left[\mathrm{O}_{2}\right]$ suggesting that permeability may not correlate with mobility in more complex binary systems.

\section{Effect of plasticizers on the $\beta$-Lg matrix}

Trends in emission energy, $\mathrm{k}_{\mathrm{TS} 0}, \beta$ and lifetimes clearly indicate that the primary effect of glycerol is to plasticize the $\beta$ - $\operatorname{Lg}$ matrix by increasing the matrix mobility. However, at a low molar ratio of 1:1 glycerol: $\beta$-Lg we see that all these parameters showed an opposite trend of decreasing mobility clearly indicating that glycerol exhibited signs of anti- plasticization at this ratio. However, glycerol at all ratios had no clear effect on the permeability of the $\beta-\operatorname{Lg}$ matrix .

Sorbitol on the other hand showed a complex concentration dependent effect on the mobility of the $\beta$-Lg matrix and an exact trend could not be predicted. Sorbitol plasticized $\beta-\mathrm{Lg}$ films showed some anomalous trends with respect to the mobility. We attribute these differences in mobility to possible presence of "sorbitol clusters". Complementary techniques can also throw light on some of the anomalous trends observed. Sorbitol plasticized $\beta$ - $\mathrm{Lg}$ films, however, showed a straightforward concentration dependency on permeability. At a critical molar ratio of 10:1 sorbitol : $\beta$ Lg (9 wt \% sorbitol) dramatic decreases in permeability were observed. Ratios higher than the critical concentration of sorbitol showed minimal oxygen permeability. It is clear that both sorbitol and glycerol affected the mobility and permeability of the $\beta$-Lg matrix 
in a distinct manner. Sorbitol proved to be a superior oxygen barrier when compared to glycerol at very low concentrations

Addition of maltitol, a sugar alcohol had a strong "rigidification effect" similar to that of the sugars in the $\beta$ - $\mathrm{Lg}$ matrix (decreasing both mobility and permeability).

Addition of PEG 400 to $\beta$-Lg showed some anamolous trends. At higher PEG concentrations, where a drastic increase in permeability (10 fold) was noticed, the mobility parameters decreased. It is difficult to derive a suitable scientific explanation for this kind of behavior and complementary techniques should be used to further explain these trends.

Despite exhibiting different trends in mobility and permeability all the studied plasticizers exhibited a direct linear correlation between mobility and permeability. The similarities in the relationship between oxygen permeability and molecular mobility between $\beta$-Lg and plasticizer/ $\beta$-Lg mixtures suggest that the basic mechanisms controlling oxygen permeability are similar in these matrices.

Actual food systems seldom contain a single component. Complex mixtures of low molecular weight sugars, polyols, amino acids, fatty acids, etc., are usually present. These low molecular weight compounds interact with each other and with different macromolecules and such interactions give rise to effects, which are extremely difficult to predict.

The present study looked in to the effects of the plasticizer PEG and sucrose on the $\beta$-Lg matrix to get close to the interactions in a real food system. It is clearly evident that sucrose addition had a great effect on the mobility and permeability of PEG plasticized $\beta$-Lg films. The dynamic synergy between sucrose and PEG is evident by the 
decrease in the mobility $\left(\mathrm{k}_{\mathrm{TS} 0}\right)$ and decrease in $\mathrm{k}_{\mathrm{Q}}\left[\mathrm{O}_{2}\right]$ elucidating that the specific interactions between these molecules can have a profound effect on the mobility and permeability of the matrix. Allison et al, (2002) has clearly shown that PEG is preferentially excluded in a protein matrix and sugar form hydrogen bonds to the host protein matrix. We hypothesize that the dynamic synergy between sucrose and PEG is a result of the hydrogen bonding interactions between sucrose specifically with the host protein $\beta$-Lg and the specific interactions between PEG and sucrose creating a dynamic synergy. A detailed investigation of the mechanical properties of the edible film with these components will throw light on the positive effects of these synergies on the mechanical properties and thus formulators can use this synergy to successfully modulate both the diffusive and mechanical properties of the film.

Experimental evidence points to the fact that presence of small amounts of diluents in a particular composition exhibit anti-plasticizing effects. We were able to detect dynamic synergies in $\beta$-Lg maltose and maltitol mixtures, whereby these sugarpolyol mixtures at equal ratios anti-plasticized the $\beta$-Lg matrix and at unequal ratios plasticized the matrix. Specific interactions between $\beta-\mathrm{Lg}$, maltose and maltitol in a specific ratio (1: $5: 5 \beta$-Lg: maltose: maltitol) decreased the ability of the matrix to vibrationally dissipate the triplet state energy of Ery B by decreasing the mobility of the amorphous $\beta$-Lg matrix. These synergies can be of immense importance to the food and pharmaceutical industry and formulators can successfully manipulate the functionality of edible films utilizing these synergies.

BSA is a large globular protein and approximately two times the size of the $\beta$-Lg matrix. It is clearly evident from this study that the resultant mixture of these two 
proteins significantly lowered the local relaxation dynamics marked by decreases in $\mathrm{k}_{\mathrm{TS} 0}$ and increase in emission energy. However the interaction between these proteins did not affect the permeability of the resultant matrix. The packing density and reduction in the conformational freedom due to specific interactions between these differently sized proteins may be attributed to these changes in mobility; the reduction in mobility apparently was not sufficient to hamper the entry of per meant molecules. We hypothesize that in the BSA $\beta-\operatorname{Lg}$ mixtures the matrix is essentially composed of large enough voids thus allowing the oxygen to permeate through these "channels" or "voids" easily. Local relaxation mechanisms are reduced by the synergy between these proteins but the channels through which oxygen can permeate is still large enough for oxygen to permeate through this matrix. Detailed mechanistic studies needs to be undertaken to clearly indicate the possible mechanisms affecting permeability and mobility.

Ligand binding had a profound effect on the local relaxation dynamics of the $\beta$ - $\mathrm{Lg}$ matrix. Incorporation of fatty acids palmitic acid and caprylic acid exerted two kinds of stabilization effects primarily by decreasing the mobility and inhibiting the local relaxation mechanisms. The results from this study clearly indicate that both dipolar relaxation and collisional quenching $\left(\mathrm{k}_{\mathrm{TS} 0}\right)$ in $\beta$-Lg films with fatty acid reflect the stabilization conferred to the protein matrix by the binding of the ligands. Although showing a decrease in mobility there was no effect on the macroscopic diffusive property of the matrix, due to minimal alterations in the composition. This decrease in mobility can have great implications for the storage stability of food and drug formulations where presence of a small amount of lipid can confer greater stabilization. Due to the similarities in the way in which these fatty acids affect the mobility in the $\beta$-Lg we 
hypothesize that caprylic acid which has a very low binding specificity to $\beta$ - $\mathrm{Lg}$ is bound in the solid state.

It is clearly evident that all these additives have a distinct effect on the molecular mobility of the $\beta$ - Lg matrix. The effect mainly originates from complex interactions between the protein and additive molecules. These additives differ considerably in their structure, conformation and thus exhibit differences in the way they affect the matrix dynamics of the protein $\beta-\mathrm{Lg}$.

\section{Influence on dynamic site heterogeneity}

In the pure $\beta$-Lg matrix, the decrease in emission energy across the emission band is consistent with the dynamic site heterogeneity model in which probes are distributed in sites, which differ in mobility. The measurement of dynamic site heterogeneity is an important indicator of matrix stability. Sucrose increased the homogeneity of the $\beta-\mathrm{Lg}$ matrix. However BSA, $\beta$-Lg mixtures had no effect on site heterogeneity. The dynamic site heterogeneity is associated with the packing degree of local regions in the matrix. Molecular confirmation thus has a significant effect on these heterogeneities. Sucrose affects the local conformational dynamics of the protein $\beta$ - $\mathrm{Lg}$ by decreasing the heterogeneity whereas it is clear that the protein BSA has no effect.

Such mechanistic studies will be useful in developing rational methods for manipulating and controlling oxygen transport through protein and other biomolecular matrixes that are based on detailed biophysical mechanisms of the transport process.

\section{Future work}

The experiments conducted in $\beta$-Lg films have a great potential for future exploration and can be used to effectively to understand edible films technology. The 
model systems studied in the present study are simple model systems but significant differences in actual food systems may arise due to differences in sample preparation, casting and storage techniques. Further factors, which deserve attention, include a detailed study on the effect of protein preparation, casting methods and conditions of film equilibration. Luminescence spectroscopy is versatile technique for detecting detailed information about the subtle micro-structural elements in a matrix, but it would be useful to have complementary data from other methods like DSC, AFM and FTIR. Hydrogen bonding interactions are of utmost importance and are hypothesized to be responsible for most of the trends observed in protein sugar matrices. These measurements will enable us to understand the complex interactions occurring in these matrices. Other measurements like analyzing the mechanical properties can help us further understand edible film technology in a better manner. These techniques can throw light on the plasticization mechanisms of plasticizers on edible films. They can also give us a detailed overview of the beneficial effects of the synergies between additives. Edible films are often exposed to changes in relative humidity. Further study of the effect of change of relative humidity will be helpful in establishing the effect of humidity on the mobility and permeability of the matrix. Other possible additives useful in the formulation of edible films like polysaccharides, protein (gelatin), salts etc should be studied inorder to gain a comprehensive understanding of the effect of different kinds of additives on the mobility and permeability of the $\beta-\mathrm{LG}$ matrix.

The choice of available luminescent probes is virtually unlimited due to decades of research in chemical and biochemical fields. Many luminescent molecular species naturally occur naturally in foods. Identification of such luminescent species in foods 
could aid in elucidating reaction mechanism based on the time frame of the probe. Future work should thus involve the study of probes with lifetimes longer than erythrosin B.

The main objective of this research was to find a relationship between mobility and permeability. But specific questions still remain unanswered. Is there a connection between mobility and permeability? Further future experiments need to be undertaken to derive a suitable explanation. Actual measurements of diffusion through these edible films are relevant for applications involving both packaging materials and edible films. An important question to address is whether micro scale heterogeneity (such as that evident in room temperature phosphorescence lifetime data across the probe emission band) manifests itself in functional differences. So, future work should throw light on macro scale heterogeneity in these edible films, which can reveal important information about the possible mechanisms occurring in the matrix. 


\section{Curriculum Vita}

Kasi Visalakshi Sundaresan

\section{$\underline{\text { Education }}$}

Rutgers, The State University of New Jersey

Major: Food Science with an emphasis in Food Chemistry.

January 2004 - January 2008

Degree: Ph.D. Food Science, January 2008

Avinashilingam University

Major: Food service management and dietetics

August 1999-August 2000

Degree: M.Phil, Food service management and dietetics, August 2000

Bangalore University

Major: Food service management and dietetics

August 1996-August 1998

Degree: MS, Food service management and dietetics, August 1998

Bangalore University

Major: Nutrition and Dietetics

August 1993-August 1996

Degree: BS, Nutrition and Dietetics, August 1996

\section{$\underline{\text { Publication }}$}

Sundaresan Kasi Visalakshi and Richard D. Ludescher. 2007 "Molecular mobility and oxygen permeability in amorphous $\beta$-Lactoglobulin films" Food hydrocolloids, 22(3) 403-413 